\title{
Treuhänderische Übernahme und Verwahrung
}

International und interdisziplinär betrachtet

\section{Vienna University Press}

Beschlagnahme

Treuhänderische Übernahme Gerechte und faire Lösung Woshington Principles

Raubgut 명 Rückgabe

今. 선 Raubkunst

co

ट

今.

옹 


\section{VÉR Academic}




\section{Bibliothek im Kontext}

Band 3

Herausgegeben von

Stefan Alker-Windbichler, Murray G. Hall und Markus Stumpf

Wissenschaftlicher Beirat:

Andreas Brandtner, Ursula Georgy, Hans-Christoph Hobohm, Frank Möbus ( $\dagger$ ), Rudolf Mumenthaler, Oliver Rathkolb, Ulrich Johannes Schneider, Konrad Umlauf

Die Bände dieser Reihe sind peer-reviewed. 
Olivia Kaiser / Christina Köstner-Pemsel / Markus Stumpf (Hg.)

\section{Treuhänderische Übernahme und Verwahrung}

International und interdisziplinär betrachtet

Mit 43 Abbildungen

V\& R unipress

Vienna University Press 



\section{ZukunftsFonds WIEN口 $\square$ der Republik Österreich KULTUR D}

Bibliografische Information der Deutschen Nationalbibliothek

Die Deutsche Nationalbibliothek verzeichnet diese Publikation in der Deutschen Nationalbibliografie; detaillierte bibliografische Daten sind im Internet über http://dnb.d-nb.de abrufbar.

ISSN 2366-0244

ISBN 978-3-7370-0783-2

Weitere Ausgaben und Online-Angebote sind erhältlich unter: www.v-r.de

Veröffentlichungen der Vienna University Press erscheinen im Verlag V\& R unipress GmbH.

Gedruckt mit freundlicher Unterstützung des Bundeskanzleramts der Republik Österreich, der Vereinigung Österreichischer Bibliothekarinnen und Bibliothekare (VÖB), des Nationalfonds der Republik Österreich für die Opfer des Nationalsozialismus, des Zukunftsfonds der Republik Österreich, der Kulturabteilung der Stadt Wien (MA 7), des Rektorats der Universität Wien, der HistorischKulturwissenschaftlichen Fakultät der Universität Wien und der Universitätsbibliothek Wien.

(C) 2018, V\&R unipress GmbH, Robert-Bosch-Breite 6, D-37079 Göttingen / www.v-r.de Dieses Werk ist als Open-Access-Publikation im Sinne der Creative-Commons-Lizenz BY-NC-ND International 4.0 („Namensnennung - Nicht kommerziell - Keine Bearbeitungen“) unter dem DOI 10.14220/9783737007832 abzurufen. Um eine Kopie dieser Lizenz zu sehen, besuchen Sie https://creativecommons.org/licenses/by-nc-nd/4.0/.

Jede Verwertung in anderen als den durch diese Lizenz zugelassenen Fällen bedarf der vorherigen schriftlichen Einwilligung des Verlages.

Titelbild: (c) Hannah Alker-Windbichler 


\section{Inhalt}

Geleitwort der Vizerektorin für Infrastruktur der Universität Wien . . . . 9

Grußbotschaft der Generalsekretärin des Nationalfonds der Republik Österreich für Opfer des Nationalsozialismus . . . . . . . . . . . . . 13

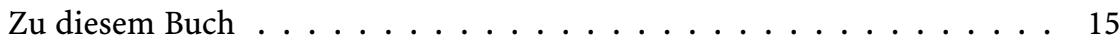

James D. Bindenagel

Artworks Looted During the Holocaust: The Unfinished Story of The Washington Principles on Nazi-Confiscated Art - Just and Fair Solutions

Markus Stumpf / Christina Köstner-Pemsel / Olivia Kaiser

„Treuhänderisch“ - Themenaufriss im Kontext der

NS-Provenienzforschung . . . . . . . . . . . . 37

Sebastian Spitra

Recht und Metapher: Die „treuhänderische“ Verwaltung von „Kulturgut"



Leonhard Weidinger

The Mauerbach Stock - Where Did the So-Called Ownerless Objects

Come From? . . . . . . . . . . . . . . . . . . . 71

Michael Wladika

Die Beanspruchung von Kunst- und Kulturgegenständen durch die Sammelstellen 1959-1972 f . . . . . . . . . . . . . 85

Alexandra Caruso / Anneliese Schallmeiner

Das Bundesdenkmalamt und der Bestand der sogenannten „1960er Jahre

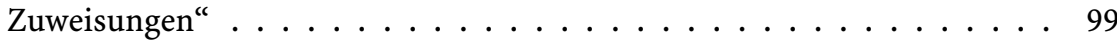


Jana Kocourek

„Offene Vermögensfragen“ - von der Suche nach sogenannten

Schlossbergungsbeständen in der SLUB Dresden

Christian George

Bücher als „Danaergeschenk“. Nachkriegszugänge der UB Mainz durch

die französische Militärregierung . . . . . . . . . . . . 129

Michal Bušek

Provenance Research in the Book Collection of the Jewish Museum in Prague ........................... 145

Marcela Strouhalová

Provenance Research in the National Library of the Czech Republic . . . 155

Johana Prouzová

Die Sammlung „Pollák“ in den Prager Museen . . . . . . . . . . . . 171

Monika Mayer

„Treuhänderische“ Übergaben von Kunstwerken an die Österreichische

Galerie im Kontext der aktuellen Provenienzforschung . . . . . . . . . . 187

Monika Löscher

Die „1963er Zuweisungen“ an das Kunsthistorische Museum. Einige

Fallbeispiele ....................... 201

Christian Mertens

„[...] ich kann Sie versichern, daß ich Ihnen das Paket mit den biogr.

Schriften mit dem größten Vergnügen aufhebe“. „Treuhänderisch“

übernommene Sammlungen in der Wienbibliothek . . . . . . . . . . 221

Philipp Mettauer

„Den neuen Mietern zur treuhändigen Verwahrung übergeben“. Die

Räumungen von „Judenwohnungen“ im Auftrag von „Vugesta“ und

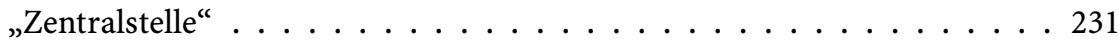

Johannes Gramlich

Kunstwerke aus NS-Besitz auf dem Weg in die Bayerischen

Staatsgemäldesammlungen - Amerikanische Restitutionspolitik und bayerische Treuhänderschaft . . . . . . . . . . . . . . . . 245 
Meike Hopp

"In Trusteeship" or "Guilty Secret”? The "Rudolf von Alt Aktion" 1938, the "Collection" of Martin Bormann and the "Fiduciary" Transfers of "Former Nazi Property" to the Bavarian State after 1945 . . . . . . . . . 261

Stephan Kellner

Abgabe der Alliierten: Die Bibliothek der NS-Ordensburg Sonthofen in der Bayerischen Staatsbibliothek . . . . . . . . . . . . 279

Julia Stepnowska / Kamil Zeidler

The Case of Polish Museums Holding Cultural Objects "in Trust" after

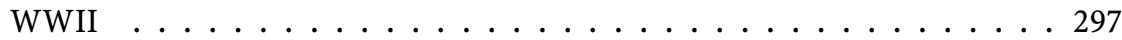

Nawojka Cieślińska-Lobkowicz

Umgang mit dem sogenannten „postjüdischen“ Kulturgut in Polen von

1945 bis heute . . . . . . . . . . . . . . . . . 303

Lara Lempertienè

Looted? Abandoned? Saved? The Provenance and Status of Jewish

Documents in the State Document Repositories of Lithuania . . . . . . 313

Ekaterina Oleshkevich

Rediscovering the Schneerson Collection: Historical Aspects and Challenges of Provenance Research . . . . . . . . . . . . . . 321

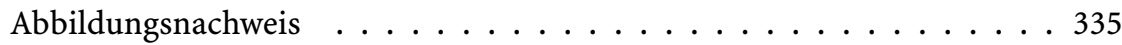

Kurzbiographien der Autorinnen und Autoren . . . . . . . . . . . 339 
Open-Access-Publikation im Sinne der CC-Lizenz BY-NC-ND 4.0

(c) 2018, V\&R unipress GmbH, Göttingen

ISBN Print: 9783847107835 - ISBN E-Lib: 9783737007832 


\section{Geleitwort der Vizerektorin für Infrastruktur der Universität Wien}

Seit 2004 werden an der Universität Wien die Bestände der Universitätsbibliothek Wien aus den Jahren 1938-1945 auf bedenkliche Erwerbungen überprüft. Ziel ist es, Klarheit über unrechtmäßige Bestände aus NS-Raubgut $\mathrm{zu}$ schaffen und diese gegebenenfalls zu restituieren.

Die Tagung „Treuhänderische“ Übernahme und Verwahrung - international und interdisziplinär betrachtet vom 2.-4. Mai 2017 an der Universität Wien behandelte ein bislang wenig beachtetes Thema in der Provenienzforschung und lieferte wertvolle Erkenntnisse im Umgang mit treuhänderisch erworbenen Raubkulturgütern. Für den Eröffnungsvortrag der Tagung konnte Prof. James D. Bindenagel, der frühere US-Botschafter für Holocaust Issues, gewonnen werden.

Der Zeitpunkt der Tagung war gut gewählt - sie fand in zeitlicher Nähe internationaler und nationaler Gedenk- und Feiertage statt, wie der israelische Nationalfeiertag und Gedenktag Jom haScho'a (24. April 2017), an dem der Opfer des Nationalsozialismus und der jüdischen WiderstandskämpferInnen gedacht wird. Am 5. Mai 2017 wurde in Österreich der Gedenktag gegen Gewalt und Rassismus im Gedenken an die Opfer des Nationalsozialismus in einer Sondersitzung des Österreichischen Nationalrats begangen. Die Gedenkrede wurde von der in Wien geborenen Holocaust-Überlebenden und Historikerin Prof. Dr. Gertrude Schneider gehalten. Bereits im Jahr 2014 wurde eine Ausstellung zu ihrer Bibliothek an der Fachbereichsbibliothek Zeitgeschichte der Universität Wien gezeigt. Insbesondere in Ausstellungen zeigt sich die wichtige Vermittlerfunktion zwischen universitärer Wissenschaft und Öffentlichkeit.

Seit einigen Jahren setzt die Universität Wien vielfältige Initiativen, die Dimensionen des Nationalsozialismus an der eigenen Institution aufzuarbeiten. Zahlreiche Projekte wurden bereits umgesetzt, so z. B. das Forschungsprojekt „Vertreibung der Studierenden 1938“, die historische und künstlerische Kontextualisierung des „Siegfriedskopfes“ oder die Gedenkveranstaltung anlässlich der Aberkennung und Wiederverleihung akademischer Grade. Die Universität Wien realisierte auch das „Gedenkbuch für die Opfer des Natio- 
nalsozialismus an der Universität Wien 1938“ mit der damit verbundenen Online-Datenbank. Zuletzt wurden 2017 die Rektorenfasten kontextualisiert. Das Kunstprojekt will die Diskussion anregen, die eine lebendige Gedenkkultur ermöglicht. Die Rektorate der Universität Wien unterstützen seit den späten 1990er Jahren aktiv Projekte zur wissenschaftsspezifischen Auseinandersetzung mit der NS-Zeit. Zum Stand der Forschung sei hier auf die Webplattformen http://geschichte.univie.ac.at und http://forum-zeitgeschichte. univie.ac.at hingewiesen.

Der Arbeitsbereich NS-Provenienzforschung an der Universitätsbibliothek Wien ist ein integraler Bestandteil der vielfältigen Forschungs- und Gedenkprojekte an der Universität Wien.

Bereits in 24 Fällen konnten Bücher und Objekte (mehr als 2.000 Druckschriftenbände, ein Nachlassfragment und fünf Objekte) zwischen 2009 und 2018 restituiert werden. Als eine der ersten Universitäten weltweit widmet sich die Universität Wien im Rahmen der NS-Provenienzforschung zudem den universitären Sammlungen und erweitert so ihren Forschungsgegenstand.

Nicht in allen Fällen ist es möglich, ErbInnen oder Rechtsnachfolger ausfindig zu machen. Aus diesem Grund wurde im April 2017 im Vorfeld der Tagung der Vertrag über sogenanntes „erbloses“ Raubgut mit dem Nationalfonds der Republik Österreich für die Opfer des Nationalsozialismus unterzeichnet. Jene Bücher und Objekte, für die keine Rechtsnachfolger gefunden werden können, werden dem Nationalfonds zur Verwertung übergeben. In weiterer Folge werden diese von der Universität angekauft, um mit dem Erlös im Sinne des Nationalfonds Opfer des Nationalsozialismus zu unterstützen. Erstmals wurde diese Vorgehensweise nun in den Fällen der Bibliothek der All Peoples' Association (APA) und der Objekte aus der Ägyptologischen Sammlung an Gipsabdrücken umgesetzt. Damit konnte ein „fairer und gerechter" Weg zur Umsetzung der Forderung der Washingtoner Prinzipien aufgezeigt werden.

Im österreichischen Gedenk- und Jubiläumsjahr 2018 begeht die Republik Österreich nicht nur ihren 100. Geburtstag, sondern muss sich auch 80 Jahre nach dem „Anschluss“ an das Deutsche Reich kritisch mit ihrer Geschichte auseinandersetzen. Zum nachwirkenden Erbe dieser Zeit gehört im Jahr 2018 auch das 20jährige Jubiläum der Washingtoner Prinzipien und des österreichischen Kunstrückgabegesetzes. Daher versammelt der vorliegende Band nicht nur die Ergebnisse der Tagung zu „treuhänderisch“verwahrten Kulturund Raubgütern im internationalen und interdisziplinären Kontext und trägt damit zur Weiterentwicklung der Provenienzforschung bei, sondern ist auch als ein Beitrag der Universität Wien zum Gedenk- und Jubiläumsjahr zu sehen. 
Alle Beiträge zu diesem Band wurden international begutachtet und entsprechen den hohen Qualitätskriterien der Vienna University Press. Ich darf daher allen AutorInnen sowie den HerausgeberInnen sehr herzlich für ihre Beiträge danken!

Wien, im Februar 2018

Univ.-Prof. Dr. Regina Hitzenberger Vizerektorin der Universität Wien 
Open-Access-Publikation im Sinne der CC-Lizenz BY-NC-ND 4.0

(c) 2018, V\&R unipress GmbH, Göttingen

ISBN Print: 9783847107835 - ISBN E-Lib: 9783737007832 


\section{Grußbotschaft anlässlich Eröffnung und Festakt der Tagung "Treuhänderische" Übernahme und Verwahrung - international und interdisziplinär betrachtet, Universitätsbibliothek der Universität Wien, 2. Mai 2017}

Sehr geehrte Damen und Herren!

Seit mehr als zwanzig Jahren ist die Arbeit des Nationalfonds der Anerkennung und Unterstützung von Opfern des Nationalsozialismus gewidmet. Dabei wurde und wird immer aufs Neue deutlich, wie viel nationalsozialistisches Unrecht allzu lange unerkannt, vergessen, verdrängt und daher unaufgearbeitet geblieben ist - bis heute. Die Spuren und Auswirkungen sind in Österreich immer noch gegenwärtig und prägen die Gesellschaft, mögen auch die ursprünglichen Ereignisse Jahrzehnte zurückliegen. Aus diesem Grund ist es für den Nationalfonds seit seinen Anfängen eine zentrale Aufgabe, dem Sog des Vergessens entgegenzuwirken und verdrängte Geschichte in das Bewusstsein der Menschen zu holen.

Wir unterstützen unterschiedlichste Projekte, die dem Bewahren der Erinnerung oder der wissenschaftlichen Erforschung des Nationalsozialismus und des Schicksals seiner Opfer dienen.

Dabei konnten wir in den vergangenen Jahren feststellen, dass der Wille zum Lernen aus Geschichte in vielen Bereichen der Gesellschaft stärker geworden ist: Heute erkunden Schülerinnen und Schüler die Geschichte ihrer Schulen, an vielen Orten in Österreich entstehen lokale Projekte, die die Vergangenheit von Gemeinden oder Stadtvierteln beleuchten, und auch große Unternehmen und Institutionen stellen sich ihrer Geschichte in den Jahren 1938-1945.

Was geraubtes Kulturgut betrifft, so wird heute die nationalsozialistische „Verwertungs- und Bereicherungs-Maschinerie“, in die auch zahlreiche Museen und andere Akteure der österreichischen Kulturlandschaft in der einen oder anderen Form verstrickt waren, weit intensiver erforscht als noch vor einigen Jahren.

Es ist ein positives Signal, dass sich viele Institutionen zu einer aktiven Provenienzforschung bekennen und um zügige Aufarbeitung bemüht sind - unter ihnen Museen, Archive und Bibliotheken -, nicht zuletzt die Parlamentsbibliothek, die 2009 unter der damaligen Nationalratspräsidentin Barbara Prammer (1954-2014) eine umfassende Provenienzforschung einleitete. 
Der Nationalfonds war und ist immer wieder gern bei der Suche nach berechtigten ErbInnen entzogener Objekte behilflich. Die Online-Kunst-Datenbank des Nationalfonds, die aktuell fast 9.000 Objekte enthält, ermöglicht unter www.kunstdatenbank.at eine Suche nach geraubten Objekten. In einigen Fällen ist es bereits gelungen, verschollene Berechtigte ausfindig zu machen. Es freut mich, dass wir dank der ausgezeichneten Kooperation mit Institutionen, unter ihnen die Universität Wien, bereits zu einigen erfolgreichen Rückgaben beitragen konnten.

Auch für jene Objekte, für die keine ErbInnen ausfindig gemacht werden können, hat das Kunstrestitutionsgesetz 1998 Vorsorge getroffen. Der Nationalfonds ist beauftragt, „erblose“ Kunstgegenstände aus öffentlichem Besitz zu verwerten und die Erlöse Opfern des Nationalsozialismus zukommen zu lassen. Wie Sie sicherlich wissen, ist es gerade bei Büchern und Druckschriften oft besonders schwierig bis unmöglich, die ursprünglichen EigentümerInnen zu identifizieren.

Die erste Übereignung, die nach dem Kunstrückgabegesetz an den Nationalfonds erfolgte, betraf 8.363 herrenlose Druckschriften aus dem Bestand der Österreichischen Nationalbibliothek - sie wurden 2009 dem Nationalfonds zur Verwertung übergeben und von der Österreichischen Nationalbibliothek zurückgekauft. Aus den Mitteln, die wir aus diesem Rückkauf erhalten haben, konnten wir seither mehr als 20 Überlebende unterstützen.

Vor kurzem fand die zweite Übereignung statt: Die Universität Wien hat im April 2017 als erste Universität Österreichs Bücher und Objekte an den Nationalfonds übereignet und zurückgekauft. Auch die daraus erlösten Mittel kommen überlebenden Opfern zugute. Es ist zu hoffen, dass diesem Beispiel noch weitere folgen werden.

\section{Sehr geehrte Damen und Herren!}

Die Tagung „Treuhänderische“ Übernahme und Verwahrung und der hier vorliegende Tagungsband stellen eine wichtige Etappe in der interdisziplinären wissenschaftlichen Zusammenarbeit in Fragen des Umgangs mit geraubten Kulturgütern dar. Die Ergebnisse sind ein fruchtbarer Beitrag auf dem Weg zu einer systematischen, akkordierten Rückgabepolitik und zu mehr Klarheit über die Bestände unserer Bibliotheken. Ich freue mich, dass der Nationalfonds mit seiner Unterstützung einen Beitrag zur Realisierung dieses gemeinsamen Forums leisten konnte.

Mag. ${ }^{a}$ Hannah M. Lessing Generalsekretärin des Nationalfonds der Republik Österreich für Opfer des Nationalsozialismus 


\section{Zu diesem Buch}

NS-Provenienzforscher und NS-Provenienzforscherinnen haben es sich zur Aufgabe gemacht, Bestände zu analysieren, zu autopsieren und zu beforschen. Die Rückgabe an die rechtmäßigen VorbesitzerInnen, ErbInnen oder Nachfolgeinstitutionen ist das Ziel. Es ist viel Zeit vergangen, bis Institutionen in einer strukturierten Art und Weise begonnen haben, sich mit den eigenen Beständen kritisch auseinanderzusetzen. Die Washingtoner Prinzipien aus dem Jahr 1998 stellen einen Meilenstein dar, wenn es um faire und gerechte Lösung für durch die Nationalsozialisten geraubtes Kulturgut geht.

Oftmals übersehen und nicht thematisiert wurde dabei die Frage nach dem Umgang mit ,treuhänderisch“ verwahrtem Kulturgut bzw. Raubgut in Bibliotheken, Archiven und Museen sowie jüdischen Institutionen. Während die gängigen Erwerbungsarten in Kultureinrichtungen wie Kauf, Geschenk, Pflicht und Tausch übliche Geschäftsvorgänge darstellen und im Rahmen der NS-Provenienzforschung kritisch untersucht werden, sind mit Auflagen versehene Übernahmen und Verwahrungen wie Treuhand, Leihgaben oder Legate, aber auch staatliche Zuweisungen im Kontext von NS-Kulturgutraub und staatlichen Transformationsprozessen bislang wenig beachtet worden.

An der Universitätsbibliothek der Universität Wien (UB Wien) gaben mehrere „treuhänderisch“ verwahrte Bestände Anstoß zur Tagung "Treuhänderische“ Übernahme und Verwahrung - international und interdisziplinär betrachtet, die vom 2.-4. Mai 2017 vom Arbeitsbereich NS-Provenienzforschung ${ }^{1}$ an der Uni-

1 NS-Provenienzforschung wird an der UB Wien seit 2004 systematisch betrieben. Informationen zum Arbeitsbereich NS-Provenienzforschung der Universitätsbibliothek Wien unter der Leitung von Markus Stumpf finden Sie auf unserer Website URL: http://bibliothek.univie. ac.at/provenienzforschung.html (abgerufen am 10.11.2017). Für die Unterstützung des Arbeitsbereiches ist den beiden Rektoraten der Universität Wien unter Georg Winckler und Heinz W. Engl, hier v. a. den jeweilig zuständigen VizerektorInnen: dem kürzlich verstorbenen Johann Jurenitsch (1947-2017), Susanne Weigelin-Schwiedrzik, Karl Schwaha und der amtierenden Vizerektorin Regina Hitzenberger sowie der Leiterin des Bibliotheks- und Archivwesens Maria Seissl zu danken. Ebenfalls bedanken möchten wir uns bei den ModeratorInnen der Tagung: Reinhard Buchberger, Murray G. Hall, Maria Kesting, Bertrand Perz, 
versität Wien veranstaltet wurde. Neben der sogenannten „Sammlung Tanzenberg“, die nach 1945 „treuhänderisch“ übernommen wurde und aktuell noch Gegenstand der Forschung ist, galt es für zwei „erblose“ Bestände, der Bücherei des englischen Kulturvereins All Peoples’ Association (APA) und für Gipsobjekte der ägyptologischen Sammlung der Universität Wien, die aufgrund der entsprechenden Rückgabeentscheidungen ebenfalls „treuhänderisch“ verwahrt wurden, im Sinne der Washingtoner Prinzipien eine „faire und gerechte Lösung“ zu finden. ${ }^{2}$

International gelten in jedem Fall die Washingtoner Prinzipien als Basis für die Bemühungen, NS-Provenienzforschung zu betreiben und daher ist jeder „Kultur“-Institution aus den Bereichen Bibliothek, Archiv, Museum und Wissenschaft die Frage nach ihrem „fairen und gerechten“ Umgang mit NS-Raubgut nicht nur zumutbar, sondern sollte auch wesentlicher Teil ihres Selbstverständnisses sein.

Für Österreich ist diesbezüglich jedenfalls historisch ein „schlampiges“ Verhältnis zu konstatieren: Von eilig abgewickelten „frühen“ Rückgaben, über die Rückstellungsgesetze (1946 und 1949) mit kurzen Meldefristen, bis hin zu unfairen Ausfuhrverboten für zu restituierende Kunstwerke; der Schaffung von Sammelstellen, um - oftmals scheinbar - erblose Kunst- und Kulturobjekte treuhänderisch aufzubewahren, bis sich ErbInnen finden würden. Mit den Kunst- und Kulturgüterbereinigungsgesetzen $(1969,1971,1985)$ versuchte die Republik Österreich aufs Neue einen Umgang mit - oftmals scheinbar - erblosem Gut zu finden.

Mit dem 1998 in Österreich erlassenem Kunstrückgabegesetz waren staatliche Institutionen ab sofort gefordert, aktiv Provenienzforschung zu betreiben und auf ErbInnen zuzugehen. Dieses Gesetz, das 2009 novelliert wurde, stellt einen Paradigmenwechsel im Umgang mit NS-Raubgut in Österreich dar. Wenngleich es zunächst nur für Bundeseinrichtungen und ab 2009 auch für Bundessammlungen gültig ist, bemühen sich Institutionen wie die UB Wien, als ehemalige „Staatsbibliothek ${ }^{\text {“3 }}$, um eine analoge Vorgehensweise zu den Bundesmuseen.

Oliver Rathkolb, Pia Schölnberger und Monika Schreiber. Und wir möchten uns für die aktive Mithilfe bei der Tagung besonders bei den KollegInnen des Arbeitsbereiches NS-Provenienzforschung Karin Lach, Monika Schreiber und Susanne Wicha sowie den KollegInnen der Fachbereichsbibliotheken Zeitgeschichte und Romanistik Günter Bräuhofer, Marc Drews, Volkmar Neuer, Sophie Radler und Birgit Rajabi bedanken!

2 Siehe dazu den Beitrag von Stumpf/Köstner-Pemsel/Kaiser „,Treuhänderisch ` - Themenaufriss im Kontext der NS-Provenienzforschung" in diesem Band.

3 Vgl. Markus Stumpf: Kontaminierte Bücher - Exemplarspezifika und Eigentumsnachweise in den Büchern der Universitätsbibliothek Wien. In: Mitteilungen der VÖB 68 (2015), Nr. 3/4, S. 546-565; Christina Köstner-Pemsel, Markus Stumpf: Ein Spiegelbild machtpolitischer Umbrüche - Die Universitätsbibliothek Wien. In: Reflexive Innensichten aus der Universität. Disziplinengeschichten zwischen Wissenschaft, Gesellschaft und Politik. Hg. von Karl Anton 
Was kann aber eine international aufgestellte NS-Provenienzforschung leisten? Wie weit sollen die Forschungen und Recherchen gehen? Mit der Aufforderung eines Schlussstrichs unter der Auseinandersetzung mit dem Nationalsozialismus sieht sich unser Fach nicht selten konfrontiert. Gleich ob sich der/die VorbesitzerIn identifizieren lässt - der nationalsozialistische Raub war und bleibt Unrecht.

Provenienzforschung geht vom Objekt aus und beforscht darüber hinaus historische Kontexte, Personen und Institutionen - und stellt damit ein Stück Erinnerungsarbeit dar. „Alles, was mir lieb war, wurde mir 1938 genommen“. Diese Worte stammen aus den 2012 im Zsolnay Verlag erschienenen Erinnerungen Ari heißt Löwe von Ari Rath (1925-2017), der als Kind aus Wien vor dem NS-Regime flüchten musste. Der ehemalige Chefredakteur und Herausgeber der Jerusalem Post drückt dabei aus, um was es in der Provenienzforschung geht: dass die Dinge einen persönlichen und daher unschätzbaren Wert besitzen können und einen Teil der persönlichen Geschichte darstellen, insbesondere im Zusammenhang mit der Geschichte vor der Verfolgung und der Ermordung von Familienmitgliedern.

Und: Diese Erinnerungsarbeit will nicht als „Wiedergutmachung“verstanden werden. Das erfahrene Leid kann nicht wieder gut gemacht werden - als Kulturinstitutionen können und müssen wir uns jedoch der historischen Verantwortung stellen.

Die Anerkennung des Unrechts, das Menschen aufgrund von politischer, rassistischer oder weltanschaulicher Verfolgung durch die Nationalsozialisten und seinen Kollaborateuren angetan wurde, vermag die NS-Provenienzforschung jedenfalls ein Stück weit zu leisten: Auch durch die Anerkennung von „treuhänderisch“ übernommenem und verwahrtem Kulturgut in den Kulturinstitutionen.

Mit dem Thema „,Treuhänderische Übernahme und Verwahrung“ widmete sich die Tagung einem Spezialthema des Faches NS-Provenienzforschung. In sieben Panels wurden rechtliche und historische Aspekte, Fallbeispiele und Möglichkeiten des Umgangs mit „treuhänderischen“ Übernahmen vor und nach 1945 erörtert. Dies wird beispielhaft in den Beiträgen von Christian Mertens, Michal Bušek, Marcela Strouhalová und Lara Lempertienè beschrieben.

Dabei haben wir den Begriff „Treuhand“ aus seiner strengen juristischen Definition herausgelöst und um „erblose“ Objekte oder um Leihgaben erweitert - u. a. überantworteten verfolgte Personen einer Institution ihre Sammlung, um sie vor den Nazis zu schützen (und wurden schließlich ermordet), wie der Beitrag

Fröschl, Gerd B. Müller, Thomas Olechowski und Brigitta Schmidt-Lauber (= 650 Jahre Universität Wien - Aufbruch ins neue Jahrhundert, hg. von Friedrich Stadler u. a., Band 4). Göttingen: Vienna University Press bei V\&R unipress 2015, S. 513-528. 
von Johana Prouzová aufzeigt. Sebastian Spitra erläutert den Begriff „Treuhand“ aus juristischer Sicht, während Kamil Zeidler und Julia Stepnowska in ihrem Beitrag von der Situation in Polen berichten.

Obwohl der Fokus auf NS-entzogenem Raubgut liegt, werden u. a. auch Fragen zu Besitztransfers im Zuge der Bodenreform in der Sowjetischen Besatzungszone im Beitrag von Jana Kocourek sowie im Rahmen von staatlichen Transformationsprozessen des 20. Jahrhunderts in den Beiträgen von Nawojka Cieślińska-Lobkowicz und Ekaterina Oleshkevich behandelt.

An den Bibliotheken, Archiven und Museen rücken damit jene geraubten Kunst- und Kulturgüter in das Zentrum des Forschungsinteresses, die in der NSZeit oder nach 1945 weltweit an öffentliche Einrichtungen übergegangen sind. Wie diese insbesondere mit treuhänderischen Übernahmen von Sammlungen früherer NS-Institutionen umgehen, wird in den Beiträgen von Johannes Gramlich, Meike Hopp und Stephan Kellner erläutert.

Philipp Mettauer geht auf die Zuweisungen von NS-Stellen in Wien zwischen 1938-1945 im Zuge von Delogierungen ein. Das Thema von Besitztransfers im Zuge der Rückgabebemühungen von Sammelstellen wird in den Beiträgen von Michael Wladika, Leonhard Weidinger und Alexandra Caruso und Anneliese Schallmeiner behandelt, auf Zuweisungen im Kontext von NSKulturgutraub gehen die Beiträge von Christian George, Monika Löscher und Monika Mayer ein.

An dieser Stelle ist den Fördergebern und KooperationspartnerInnen zu danken, ohne die die Tagung und der vorliegende Band nicht umsetzbar gewesen wären: Der Kommission für Provenienzforschung beim Bundeskanzleramt der Republik Österreich, dem Arbeitskreis Provenienzforschung e.V., dem Nationalfonds der Republik Österreich für Opfer des Nationalsozialismus, dem Zukunftsfonds der Republik Österreich, der Kulturabteilung der Stadt Wien, dem Institut für Zeitgeschichte der Universität Wien, der Vereinigung Österreichischer Bibliothekarinnen und Bibliothekare (VÖB), der Gesellschaft für Buchforschung in Österreich, dem Arbeitskreis Provenienzforschung und Restitution - Bibliotheken, der Historisch-Kulturwissenschaflichen Fakultät der Universität Wien und last but not least der Universitätsbibliothek Wien.

Wien, im Februar 2018

Olivia Kaiser, Christina Köstner-Pemsel und Markus Stumpf 


\title{
Artworks Looted During the Holocaust: The Unfinished Story of The Washington Principles on Nazi-Confiscated Art - Just and Fair Solutions
}

\begin{abstract}
Zusammenfasssung
Während des Holocaust geraubte Kunstwerke: Die unvollendete Geschichte der Washingtoner Prinzipien zu von den Nazis beschlagnahmter Kunst - Gerechte und faire Lösungen Der Beitrag beschäftigt sich mit den jahrzehntelangen Bemühungen um „faire und gerechte Lösungen“ für die im NS-Regime geraubten Kunstwerke. In Anerkennung, dass Privateigentum von Kulturgütern in einer zivilisierten Gesellschaft notwendig ist, einigten sich 1998 in Washington D.C. 44 Länder auf die Washingtoner Prinzipien. Obwohl nicht rechtsverbindlich, stellen sie eine Basis für die globalen Bemühungen um Gerechtigkeit und die zu implementierenden Gesetze bezüglich Restiutionsforderungen dar. NSRaubgut, das heute noch nicht zurückgegeben wurde, bedeutet eine Ungerechtigkeit zu dulden. Die Restitution von Raubgut bringt Gerechtigkeit und schafft die Möglichkeit, zukünftige Genozide und andere Massengräueltaten zu verhindern.
\end{abstract}

Schlagwörter

NS-Raubkunst, Kunstraub, Gerechtigkeit, Faire Lösung

\begin{abstract}
This contribution addresses decades-long efforts for just and fair solutions to National Socialist-looted art. Recognizing that civilized society compels the public protection of privately held cultural assets, forty-four countries gathered in Washington in 1998 and agreed to the Washington Principles on Nazi-confiscated art in an organized, albeit nonbinding, global effort designed to bring justice and to be implemented in law based on the merits of claims. Today, Nazi-looted art that has not been returned to its rightful owners constitutes injustice. The return of looted art is about delivering justice and setting a precedent that will help prevent genocide and mass atrocity crimes.
\end{abstract}

Keywords

Nazi-Confiscated Art, Looted Art, Justice, Fair Solutions 


\section{Introduction}

I am pleased to address the unfinished story of National Socialist looted art at this Conference on Trusteeship Custody - international and interdisciplinary standpoints. It is an honor to address this distinguished gathering of scholars dedicated to the search for justice for National Socialist victims.

My contribution to the scholarly study and research is based on my experience in the negotiation and implementation of the Washington Principles on Naziconfiscated Art. ${ }^{1}$ I am hopeful this lecture will help inform your exploration of 'just and fair' solutions to restitution efforts.

William Penn, in a 1693 book, said: "To delay justice is injustice." 2 Today, injustice continues in the form of Nazi-confiscated art that was looted from victims of the National Socialists that has not been returned to rightful owners.

My lecture supports the continuation of decades-long efforts by the international community in the search for just and fair solutions to looted art. The 1998 Washington Principles are grounded in the London Declaration of 1943 and Allied Military Law Number 59. It is certainly appropriate that this conference revisits long-standing international efforts to find just and fair solutions in the resolution of ownership of looted art.

As we have seen, the law can facilitate or block 'just and fair' solutions. The Washington Principles are norms designed to bring justice and to be implemented in law.

For the sake of the future - and for the remembrance of the victims of past wrongdoing - Europe should set norms for just and fair solutions that embrace transitional justice principles relating to transparency, memorialization, commemoration, and legal rules about Holocaust-looting.

Well-established principles of transitional justice demand that the international response to systematic or widespread violations of human rights - such as Holocaust-looted art - recognize the gross injustices suffered by victims and promote peace, reconciliation, and the rule of law in the form of just and fair solutions. Implementing the Washington Principles on Nazi-Confiscated Art helps establish normative principles that will undermine the will to loot in the future. Making clear the legal inability of Holocaust-looting to transfer good title to anyone beyond the rightful owner - including the State - is an essential deterrent against future looting. The time has come to make these solutions normative.

1 James D. Bindenagel: Washington Conference Principles on Nazi-Confiscated Art. The Washington Conference on Holocaust Era Assets (1998). URL: https://2001-2009.state.gov/p/ eur/rt/hlcst/23231.htm (accessed on May 19, 2017).

2 William Penn: Some Fruits of Solitude (1693). London: Headly 1905, p. 86. 
Recent cases - like those involving works by Gustav Klimt, the vast Gurlitt Trove and Wassily Kandinsky's Das Bunte Leben - confirm that the fight for just and fair solutions is long from resolution.

There is no question that Holocaust-era looting from victims of Nazi persecution violated established principles of law. Acknowledgment of that fact under international law is internationally accepted. The principles are embodied in The Hague Conventions, the Nuremberg Charter, the London Declaration, and various post-World War II peace and armistice treaties.

No nation possesses more than a custodial interest in Holocaust-loot art; and each nation has an affirmative obligation to identify, locate and return Holocaust-looted art to its original owner. It is important to recognize that the Allies never stated (or conceivably intended) that states should acquire title to Holocaust-looted art. ${ }^{3}$ As such, the State can be no more than a custodian of Holocaust-looted private property. It is not an owner and has no primacy of title - and that is true even as to unclaimed property.

It is important the State never act as if it could "acquire" title to Holocaustlooted private property. Indeed, to act otherwise would be for the State to become complicit in the initial crime because the State would be "cleansing" the Holocaust-looted property of the wrongful acts that provide its special status and "memory."

The history and nature of Holocaust-era looting led to the Washington Principles' negotiation and the reluctant implementation of the Principles since then. The Washington Principles enumerated a set of voluntary commitments for governments. They are based on the moral principle that art and cultural property confiscated by the Nazis from Holocaust victims should be returned to them or their heirs, in a manner consistent with national laws and regulations as well as international obligations, to achieve just and fair solutions. ${ }^{4}$

\section{Initial Efforts at "Just and Fair Solutions"}

At war's end, the Allies set up a comprehensive system to identify, catalog and return stolen assets. The scope was vast, with over 20 million objects ultimately passing through Allied collection points. ${ }^{5}$ The Allies sent tangible assets such as

3 Inter-Allied Declaration against Acts of Dispossession Committed in Territories under Enemy Occupation or Control. London 1943.

4 Washington Conference Principles on Nazi-Confiscated Art. The Washington Conference on Holocaust Era Assets, 1998. URL: https://2001-2009.state.gov/p/eur/rt/hlcst/23231.htm (accessed on May 19, 2017).

5 Lynn Nicholas: The Rape of Europa. The Fate of Europe's Treasures in the Third Reich and the Second World War. New York: Vintage Press, December 1994. 
art back to their countries of origin, where national governments were expected to return assets to owners or their heirs. That system was accepted and partially implemented in Western Europe, ${ }^{6}$ but not in Eastern Europe where Soviet-installed communist governments ruled and returned art was nationalized as state property - spoils of war.

In the West, governments returned some art. For example, France returned some 61,000 works of art from Monuments Men collection points in Germany. There were no owners or heirs for 15,000 artworks and of that number only 2,050 were considered "most important." ${ }^{\text {" }}$ French museums held and continue to hold some of these assets - but not necessarily as a custodian for unidentified or unlocated true owners.

Nonetheless, given the rapid collapse of Germany in 1944-45, the pressing issues of economic reconstruction and refugee resettlement and relief - and the onset of the Cold War, the Allies never created a comprehensive European-wide approach. The policy remained to ensure that individual nations receiving art from Allied collection points returned that property to true owners or their families. As such, the "restitution" process continued only for about five years, but then stopped almost completely with significant work unfinished.

By 1950, the West was no longer focused on World War II and "keeping Germany in check." The Second World War was replaced by the Cold War, and keeping the Soviet Union in check became a top priority. ${ }^{8}$ Except for a few Jewish agencies, the restitution of assets stolen by the Nazis would have to await the fall of the Berlin Wall in 1989 and the collapse of the Soviet Union in 1991. When

6 Evelien Campfens: Fair and Just Solutions? Alternatives to Litigation in Nazi-Looted Art Disputes: Status Quo and New Developments. The Hague: Eleven International Publishing 2015, p. 20.

7 See generally Konstantin Akinsha, Grigorii Kozlov and Sylvia Hochfeld: Stolen Treasure: The Hunt for the World's Lost Masterpieces. London: Weidenfeld \& Nicolson 1995 (published in the United States as "Beautiful Loot: The Soviet Plunder of Europe's Art Treasures"); Elizabeth Simpson: The Spoils of War - World War II and Its Aftermath. The Loss, Reappearance and Recovery of Cultural Property. New York: Abrams 1997; Hector Feliciano: The Lost Museum. The Nazi Conspiracy to Steal the World's Greatest Works of Art. New York: Basic Books 1997; Jonathan G. Petropoulos: The Faustian Bargain. The Art World in Nazi Germany. Oxford: Oxford University Press 2000; Jonathan G. Petropoulos: Art as Politics in the Third Reich. Chapel Hill: University N.C. Press 1996; Owen C. Pell: The Potential for a Mediation/Arbitration Commission to Resolve Disputes Relating to Artworks Stolen or Looted During World War II. In: DePaul Journal of Art, Technology \& Intellectual Property (200), and pp. 29-30. For an exhaustive bibliography of literature on Holocaust-related looting by the Germans, see also Nancy H. Yeidi, Konstantin Akinsha and Amy Walsh: The American Association of Museums Guide to Provenance Research. Washington: American Alliance of Museums Press 2001.

8 James D. Bindenagel: Fighting Impunity and Promoting International Justice, Post Conflict Justice - The German Experience. In: The Pursuit of International Criminal Justice - A World Study on Conflicts, Victimization, and Post-Conflict Justice. Ed. by M. Cherif Bassiouni. Cambridge: Intersentia 2010. 
those historical events happened, and mindful that Holocaust survivors were rapidly aging, there was renewed interest in finding measures of justice so long denied.

\section{Revisiting the Search for Justice - Negotiations for the Washington Principles}

In 1995, President William Clinton asked Stuart Eizenstat, while he was the U.S. Ambassador to the European Union, to engage the U.S. government in a renewed effort to assist Holocaust victims and to seek redress for Nazi injustices. ${ }^{9} \mathrm{He}$ continued this work after becoming Under Secretary of State and then Deputy Treasury Secretary.

Three days after Egon Schiele's exhibit closed in 1998, New York prosecutor, Robert Morgenthau, reignited the search for justice in art when he seized the Egon Schiele painting "Portrait of Wally" from the Museum of Modern Art. The painting had been loaned from the Leopold Museum in Vienna. Although a court of appeals held the seizure violated New York's anti-seizure statute, that same day a Federal Magistrate Judge issued a seizure warrant for the work based on probable cause that Dr. Leopold had violated the U.S. National Stolen Property Act. $^{10}$

How did one painting shake the foundations of the art world? Its story is a story of Nazi-confiscated art. Nazis seized it from a private Jewish collection; American Forces recovered it at the end of the Second World War and returned it to the Austrian Government; a private collector purchased it and loaned it to the Museum of Modern Art; the New York prosecutor seized it as stolen property and American federal authorities held it until a settlement was reached; finally the portrait was displayed at the Museum of Jewish Heritage in New York before returning to Austria 13 years after it was seized. ${ }^{11}$

Museums were worried that claims would arise everywhere and their collections would be emptied. Holocaust survivors and heirs hoped to reclaim their lost treasures. Civil disputes over art could also be criminal complaints of theft.

In response to the Egon Schiele painting's seizure MoMA Director Glen Lowry

9 Stuart E. Eizenstat, US EU Ambassador informed James Bindenagel, U.S. Embassy. Bonn 1995.

10 Raphael Contel, Giulia Soldan and Alessandro Chechi: Case Portrait of Wally - United States and Estate of Lea Bondi and Leopold Museum. In: Platform ArThemis (2012), Art-Law Centre, University of Geneva.

11 Colleen Long: Painting stolen by Nazis goes up at Jewish Museum. In: Desert News, July 29, 2010. URL: http://www.deseretnews.com/article/700052023/Painting-stolen-by-Nazis-goesup-at-Jewish-Museum.html (accessed on May 19, 2017). 
convened a meeting of the Association of Art Museum Directors (AAMD) in June 1998 at the Harriman estate in New York. Among the participants was Craig Smythe, a Monuments Man who had participated in the heroic efforts to collect stolen art objects at the end and in the aftermath of the Second World War. AAMD members agreed on guidelines for such cases, and the guidelines became the basis for international negotiations that led to the Washington Principles on Nazi-confiscated Art. ${ }^{12}$

The negotiations in 1998 sought a mandate that Holocaust-looted works be identified through public exhibitions and broadly available archival information so that claimants may assess their rights to ownership. The goal was and remains to reduce the burden on claimants to prove ownership, given that the Holocaust and subsequent efforts to hide looted art, complicates efforts to prove claims. Most important, the Washington Principles direct national governments to create processes for "just and fair solutions" that are based on the merits of claims, not on technical legal defenses that may penalize claimants for failing to locate assets until too much time has passed.

The negotiating partners addressed Nazi-era injustices. When it came to art they agreed on principles premised on the idea that wherever possible art looted from victims of Nazi persecution during the Nazi era (January 30, 1933, to May 9, 1945) whether by actual theft, plunder, or through forced sale, be returned to rightful owners. The Washington Principles provide a road map to bring some measure of justice to survivors and their families.

In the early stages of planning for the Washington Conference on Holocaustera Assets ${ }^{13}$ with the State Department and the Holocaust Memorial Museum, we realized that success depended on forging an international consensus. We had no leverage to compel nations to deal with their histories, other than to simply appeal for justice and, more practically, common sense. Negotiating an international agreement was out for this reason. On the other hand, we needed to ensure that even if the Conference would have "non-binding" results, it would nonetheless compel tangible, positive change in the way nations deal with Holocaust asset issues. How to do this was the question.

12 Author participated in the MoMa Conference.

13 Proceedings - Washington Conference on Holocaust-Era Assets. Ed. by James D. Bindenagel, November 30-December 3, 1998. U.S. Government Printing Office: April 1999. URL: https:// fcit.usf.edu/holocaust/resource/assets/index.HTM (accessed on May 19, 2017). 


\section{How to Resolve Claims}

I was tasked to internationalize the AAMD guidelines and that led to the Washington Principles. When I set out to Europe, the proposal I made met with fierce resistance to the idea of art restitution so long after the Second World War, even after the guidelines by art museum directors were agreed. I explored with governments and museums their willingness to create international principles to guide restitution.

The Netherlands held in trust thousands of unclaimed looted art objects for discussion. The French MNR collection included art objects held in trust that were returned to them at the end of the war. The conversations were difficult. The Netherlands, the U.K., Germany, and other countries fiercely resisted any American-imposed principles. ${ }^{14}$

Good faith purchase laws and statutes of limitations were among some of the objections to the proposed Principles, notwithstanding significant issues of fairness with respect to property that had not been transparently identified to true owners and their families and heirs.

Russia was deeply involved. The Red Army's looting of public, religious and private artworks from Nazi Germany was considered spoils of war by the Russian government, which treated all that property as nationalized state property - and still does to this day. Although the Duma created an exception for victims to recover art, inventories of captured art have never been fully available to victims.

It was immediately evident in those talks that restitution cannot be accomplished without knowledge of potentially looted art and cultural property. The importance for all stakeholders to have access to intensified systematic provenance research cannot be stressed enough. Research in both public and private archives and publicizing the results of this study, including ongoing updates, is critical for restitution.

After I had returned from Europe, transparency in provenance research became a major theme of a conference seminar on June 30,1998, and spurred the preparations for the November 1998 Holocaust-era Assets Conference. From June until the opening of the Holocaust Era Assets Conference in November,

14 See, e.g., partial lists of unclaimed property: Ministry of Culture of the Czech Republic, Database of Works of Art from Property of Victims of the Holocaust, URL: http://www. restitution-art.cz (accessed on May 19, 2017), listing over 2,600 Holocaust-looted works in Czech museums; Musees Nationaux Recuperation (“MNR"), URL: http://www.culture.gouv. fr/documentation/mnr/pres.htm (accessed on May 19, 2017), listing over 16,000 Holocaustlooted objects in French national museums; Stichting Nederlands Kunstbezit, NK Collection, listing 4,217 objects in the custody of the Netherlands Institute for Cultural Heritage; The Lost Art Internet Database, URL: http://www.lostart.de (accessed on May 19, 2017), listing over 16,000 Holocaust-looted objects turned over to German authorities and unclaimed. 
Europeans rejected any direct endorsement of the AAMD guidelines. Our negotiating team drafted an alternative, which became the Washington Principles on Nazi-confiscated Art that were considered and agreed at the November Washington conference.

The American debate included the voices of Earl "Rusty" Powell of the National Gallery, Chair of the MOMA Board Ron Lauder, Michael Kurtz of the National Archives, and others, all of whom made the case that hundreds of thousands of artworks remained lost to their rightful owners. During the negotiations the Director of Metropolitan Museum of Art and chair of the Association of Art Museum Directors Task Force on Spoliation of Art, Philippe De Montebello, rightly proclaimed of these Washington Principles: "The art world will never be the same."

The hallmark of the Washington Conference was the way in which the Principles grew out of the cooperative effort of many different parties: American, as well as European museum directors, dialogued continuously and various governments compromised at the very highest levels to achieve consensus.

Recognizing that civilized society compels the public protection of privately held cultural assets, those gathered in Washington in 1998 pledged they would make an organized, albeit non-binding, global effort. That effort would ideally involve researching provenance, uncovering looted art, publicizing its existence and encouraging just and fair solutions to conflicting claims of ownership principles then embodied in the Washington Principles.

During the conference, Stuart Eizenstat sought a compromise with the different legal systems in Europe and the U.S. in a preamble to the Principles that read:

"In developing a consensus on non-binding principles to assist in resolving issues relating to Nazi-confiscated art, the Conference recognizes that among participating nations there are differing legal systems and that countries act within the context of their laws." 15

Article XI would set the course for national legislation that would change the art world.

U.S. Congressman James Leach of Iowa, whose Congressional Hearings played a significant role, led the fight for the legal implementation of the Washington Principles when he modified the eleventh article at the Conference to include:

"Nations are encouraged to develop national processes to implement these principles, particularly as they relate to alternative dispute resolution mechanisms for resolving ownership issues."16

15 Washington Conference Principles on Nazi-Confiscated Art (Fn. 4).

16 Ibid. 
The Austrian Government of Wolfgang Schüssel was incensed at the New York seizure of the Egon Schiele paintings. Culture Minister Elisabeth Gehrer led the conference debate and the Austrians enacted (and amended in 2009) an Art Restitution Law (Kunstrückgabegesetz) on November 30 while the conference was in session.

At the end of the meeting, Conference Chairman Abner Mikva declared that forty-four governments had agreed to a set of international principles - The Washington Principles on Nazi-confiscated Art.

\section{Washington Principles}

Most important, the Washington Principles direct national governments to create processes for "just and fair solutions" that are based on the merits of claims, not on technical legal defenses that may penalize claimants for failing to locate assets until too much time has passed. All stakeholders were called to ensure that their legal systems or alternative processes while taking into account the different legal traditions, facilitate just and fair solutions. ${ }^{17}$

Also, States were to make certain that claims to recover such art are resolved expeditiously and based on the facts and merits of the claims and all the relevant documents submitted by all parties. Governments should consider all relevant issues when applying various legal provisions that may impede the restitution of art and cultural property, to achieve just and fair solutions, as well as alternative dispute resolution, where appropriate under the law. ${ }^{18}$

Following the Washington Conference, an international consensus for consistent and efficient resolution of claims began to develop. In 1999, the International Council of Museums called on its members to follow the Washington Principles. The 1999 Council of Europe Resolution 1205, the 2000 Vilnius Forum Declaration, the 2003 Hearings of the European Parliament and, ultimately, the 2003 European Parliament Resolution 408 all called for action to facilitate methods to resolve claims. ${ }^{19}$ The Permanent Court of Arbitration/Peace Palace

17 Ibid.

18 Proceedings of the Washington Conference (Fn. 13).

19 See European Parliament Resolution A5-0408/2003 (Freedom of movement and ownership of goods) [hereinafter European Parliament Resolution 408]; see further text accompanying note 35 infra; see generally Report on a Legal Framework for Free Movement Within the Internal Market of Goods Whose Ownership is Likely to be Contested, Commission on Legal Affairs and the Internal Market (Rapporteur Willy C.E.H. De Clercq), A5-0408/2003 (Nov. 26, 2003). Resolution 1205, Looted Cultural Property, Parliamentary Assembly of the Council of Europe, Nov. 4, 1999, reproduced as Annex X in this volume [hereinafter Resolution 1205]. Vilnius Forum Declaration, Vilnius International Forum on Holocaust Era Looted Cultural Assets, Vilnius, Oct. 3-5, 2000. URL: http://www.vilniusforum.lt (accessed on May 19, 2017). 
Papers 2003 proposed arbitral tribunals relating to Holocaust-looted art. ${ }^{20}$ The 2009 Terezin Declaration renewed the call for just and fair solutions.

\section{What Are "Just and Fair Solutions"?}

Evelien Campfens' reader Fair and Just Solutions? sets about to explain norms for the assessment of ownership claims to Nazi-looted art as codified in the 1998 Washington Principles on Nazi-confiscated Art. ${ }^{21}$ Prominent settlements have shown various ways to achieve just and fair solutions.

Justice comes in complicated ways. Consider these four examples of the Principles and the reality of dispute resolution.

\section{Negotiation-Settlement}

When the North Carolina Museum of Art discovered its prized possession, Madonna and Child in a Landscape by Lucas Cranach was looted by the Nazis and taken to Hitler in 1943, they ultimately set about contacting the rightful owners and negotiating with them. Eventually, after much discussion, a settlement was reached that kept the painting in North Carolina with a portion of the painting's value being donated back to the museum. ${ }^{22}$ Thus, rather than stand on legality and litigate, the museum chose to resolve the matter in less time and in a way that recognized the rights of the true owners.

Another outstanding example of a settlement that keeps the artwork on public display is The Lighthouse with Rotating Beam in the Bundeskunsthalle Bonn, which agreed to pay heirs of Alfred Flechtheim half of its market value to keep the painting in the museum. ${ }^{23}$

European Parliament, Public Hearing, A legal framework for free movement within the internal market of goods whose ownership is likely to be contested, Mar. 18, 2003. See Programme for the hearing, European Parliament (Mar. 18, 2003). URL: http://www.euro parl.europa.eu/hearings/20030318/juri/programme_en.pdf (accessed on May 2, 2018). See Report, European Parliament (Nov. 26, 2003). URL: http://www.europarl.europa.eu/sides/ getDoc.do?pubRef=-//EP//TEXT +REPORT +A5-2003-0408+0+DOC+XML+V0//EN (accessed on May 3, 2018).

20 Owen C. Pell: Using Arbitral Tribunals to Resolve Disputes Relating to Holocaust-Looted Art. Resolution of Cultural Property Disputes. The Permanent Court of Arbitration/Peace Palace Papers 2003.

21 Evelien Campfens: Fair and Just Solutions? (Fn. 6).

22 Ibid., pp. 196-197.

23 Anne Laure Bandle, Raphael Contel and Marc-André Renold: Case Lighthouse with Rotating Beam - Flechtheim Heirs and Kunstmuseum Bonn. In: Platform ArThemis (2012), Art-Law Centre, University of Geneva, p. 4. 
2. Judicial Claim - Negotiation

Heirs of Paul Rosenberg commenced a lawsuit against the Seattle Art Museum seeking the return of the Odalisque or Oriental Woman Seated on the Floor by Henri Matisse. The claim ultimately was resolved without a trial when the museum, using the ample evidence provided during discovery, decided to return the work, having confirmed that the Nazis stole it from Paul Rosenberg's collection during World War II. ${ }^{24}$

\section{Judicial Claim - Negotiation - Settlement Agreement}

The dispute over Landscape with Smokestacks by Edgar Degas in the Art Institute of Chicago headed for court after costing hundreds of the thousands of dollars in fees. The parties agreed to a cash settlement in 1998, under which the painting remained in the Art Institute. ${ }^{25}$

4. Conciliation, International Facilitator, Judicial Claim, Negotiation, Settlement or Arbitration and Arbitral Award

The art collection of Jacques Goudstikker looted and acquired by Herman Göring, was recovered by Allied Forces and returned to The Netherlands government, which decreed it as Dutch National Property and divided the art among Dutch museums. The Dutch government forced a partial settlement with Goudstikker's wife in 1952 in which she relinquished claim to the remaining artworks. A 1998 claim by Marei Von Saher, the sole surviving heir, was denied by the Dutch government citing the 1952 agreement, which was upheld in court. ${ }^{26}$

A Dutch Restitution Committee established in 2002 reviewed the Von Saher claim and finally in 2005 recommended restitution of 202 of the 267 paintings Saher claimed; 200 were eventually returned. The family donated to the Dutch Government one of the paintings - Child on Deathbed by Bartholomeus Van der Helst. $^{27}$

24 Alessandro Chechi, Raphael Contel and Marc-André Renold: Case Odalisque Painting - Paul Rosenberg Heirs and Seattle Art Museum. In: Platform ArThemis (2012), Art-Law Centre, University of Geneva, p. 5.

25 Evelien Campfens: Fair and Just Solutions? (Fn. 6), pp. 198-199.

26 Anne Laure Bandle, Alessandro Chechi and Marc-André Renold: Case 200 Paintings Goudstikker Heirs and the Netherlands. In: Platform ArThemis (2012), Centre of Art-Law, University of Geneva, p. 1.

27 Ibid. p. 1. 


\section{Klimt}

Then there is the Klimt case. First claims were denied by Austria, then a case was introduced in U.S. Court, and finally the fate of the paintings was decided in arbitration that tested the Austrian law. Austria had established in 1998 a Restitution Committee. Maria Altman made a claim, which was denied by the Austrian Government based on its assessment that the government acquired the paintings before the Nazi era. The Austrians refused to consider Arbitration. Legal proceedings were initiated in the U.S. to avoid the bond requirement to access the Austrian court system. In the U.S. court case, the Austrians sought sovereign immunity, which was denied by the U.S. Supreme Court. ${ }^{28}$

Finally, the Austrian Government accepted arbitration to avoid a court battle in the U.S. The arbitration panel ruled that the paintings were not transferred to Austria under the terms of the will of Ferdinand Bloch-Bauer in 1925. To obtain export permits for the rest of the estate, heirs had earlier accepted the Austrian assertion of ownership. In the end, Austria was obligated to return to the rightful owner all six Klimt paintings. The Lady in Gold movie tells the story. ${ }^{29}$

\section{Principles and the Reality}

What is the case today?

"The Principles call for the identification of confiscated and looted artworks. [...] Sharing provenance data by putting it on the internet would help to reduce repetitive research. [...] Countries should take the lead by doing more provenance research on the collections held in publicly owned museums. [...] Some countries have taken commendable if small initiatives, but all countries need to do significantly better in this area." ${ }^{30}$

Indeed, public museums remain remarkably secretive about their collections and how works entered them.

"Many museums and collections are unaware of what was looted because they have not developed the complete provenance of their holdings that were in Europe from 1933 to 1945. Except for a few countries, most have not undertaken thorough provenance research and published the results." ${ }^{31}$

28 Caroline Renold, Alessandro Chechi, Anne Laure Bandle, and Marc-André Renold: Case Six Klimt Paintings - Maria Altmann and Austria: Platform ArThemis (2012), Art-Law Centre, University of Geneva.

29 Ibid.

30 Stuart E. Eizenstat: Opening Plenary Session Remarks. Prague Holocaust Era Assets Conference (2009).

31 Ibid. 
In Germany, which has conducted such research, Minister of State for Cultural Affairs Bernd Neumann, said in December 2008 that Germany has "thousands and thousands" pieces of looted art in their museums today. ${ }^{32}$

Although the German news magazine Der Spiegel has reported on how little German authorities have done since 1945 to investigate ${ }^{33}$ and return 20,000 looted items known to be still in the hands of German agencies and museums, new German efforts are underway. ${ }^{34}$ In regard to Russia, Stuart E. Eizenstat furthermore stated that:

"Russia may have the largest number of Nazi-looted artworks, but despite an art restitution law passed by the Duma, the Russian Government has done little to publicize its inventories, implement its law, and has not created adequate claims processes. Israel should do more systematic provenance research, as should museums in the United States."

Thus, "the record of implementing the Washington Principles is at best uneven, and the vision of an art world that gives the Principles a high priority remains elusive."36

"Some have been critical of the Principles because they do not include an enforcement mechanism." ${ }^{37}$ But in transitional justice terms, that perceived weakness is simply a call for governments to overcome the resistance of countries to join in creating legal mechanisms. The Principles embodied concepts of fundamental fairness that are part of U.S. and European law, which might over time allow them, to some degree, to work, including by creating a policy basis for victims to engage with museums and with legislators on ways to implement the Principles at national levels. ${ }^{38}$

Alternative solutions in the U.S. are on-going. As part of Christie's willingness to be as open and approachable as possible the auction house developed guidelines in 2009 with principles for Christie's, as an experienced and expert intermediary, to help resolve claims between the current holders of looted art and claimants through exchanging information and documentation so that informed decisions can be achieved. Essentially Christie's promotes a non-litigious and amicable approach to just and fair solutions to claims based on hearing historical

32 Ibid.

33 Ulrike Knöfel: Balky Bavarians. US Congress Demands Action on Nazi Looted Art. In: Der Spiegel, November 26, 2015.

34 James D. Bindenagel, Owen Pell: How to Handle That Nazi - Era Art Trove. Germany Must Ensure that the Treasures Found in a Munich Apartment are Restored to their Rightful Owners. In: The Wall Street Journal, November 18, 2013.

35 Stuart E. Eizenstat: Opening Plenary Session Remarks (Fn. 30).

36 Ibid.

37 Ibid.

38 Ibid. 
facts of claims balanced with the position of the current holder, who may well be the unwitting possessor of looted art, having no knowledge of its prior history. Restitution claims are always sensitive and often complex and sometimes to get to common ground, the courts are not always the right forum for claims. This sensitivity is especially acute for those claims connected to works of relatively modest financial value. Indeed probably $95 \%$ of Nazi-era claims presented to Christie's are resolved using dialogue and without litigation to reach successful settlements. ${ }^{39}$

\section{Private Collections}

In a welcome complement to these government/public institutions examples, some private individuals and institutions holding art of questionable provenance have been amenable to discussing claims and resolving issues in an amicable manner. A recent example is the Oetker Collection (Kunstsammlung Rudolf-August Oetker $\mathrm{GmbH}$ ), which owns hundreds of works of art, which are mainly kept from public view.

The Oetker Collection is engaging in systematic research to determine which of the pieces it holds were looted or expropriated by the Nazi regime and returning those stolen artworks to their rightful heirs. A partner at Rowland \& Petroff, Rowland represented the rightful heirs to a Hans Thoma painting restituted by the Oetker Collection in January 2017. The victim's representative stated the Oetker example is one of how things should be done. ${ }^{40}$

\section{The Gurlitt Art Trove Case}

International interest in Nazi-looted art was stirred again in 2013 when the Bavarians discovered a trove of some 1,400 artworks. The collection belonged to Cornelius Gurlitt, a reclusive 80-year-old man whose father Hildebrand Gurlitt was among the few art dealers favored by the Nazi's to handle "Degenerate Art" and to collect art for Adolf Hitler and the National Socialists. The German government estimates that some of these works were looted from Jews. ${ }^{41}$

39 See Christie's Guidelines for Dealing with Nazi-era Art Restitution Issues June 2009. URL: http://www.christies.com/pdf/services/2010/christies-guidelines-for-dealing-with-restitu tion-issues.pdf (accessed on May 19, 2017).

40 Isaac Kaplan: Dr. Oetker Is Showing the World What to Do with Nazi-Looted Art. Artsy (2017). URL: https://www.artsy.net/article/artsy-editorial-frozen-food-company-showingnazi-looted-art (accessed on May 19, 2017).

41 Evelien Campfens: Fair and Just Solutions? (Fn. 6), pp. 87-88. 
The Gurlitt Collection includes works owned by Cornelius Gurlitt before the Nazi era and works of so-called "Degenerate Art" sold off by German museums under Nazi laws enacted after 1936. But it also includes works subject to forced sales by Jews attempting to flee Germany and works looted from German and non-German Jews, including after the start of World War II. ${ }^{42}$

\section{Germany's Constitutional Mandate to Protect and Promote Human Dignity}

Soon after the Gurlitt Art Trove was made public, Owen Pell and I wrote for the Wall Street Journal an article: "How to handle that Nazi Art Trove." ${ }^{43}$ Our suggestion recommended Germany follow the Washington Principles on Naziconfiscated Art and implement them in law. This case now provides Germany with the chance to remind the world that it has changed and had become a defender of human dignity. Germany has created an open and transparent process that will enhance the likelihood that looted works in the Gurlitt Collection are returned to their rightful owners.

Bavarian Minister of Justice Winfried Bausback asserted at the time:

"This voluntary agreement of a private collector strengthens the value of the Washington Principles as a set of guidelines to resolve rightful ownership issues. The entire world is watching to see how we will answer these questions, and this agreement is a real solution." ${ }^{\prime 4}$

German Minister of State Monika Grütters added:

"This (Gurlitt) agreement creates the necessary basis for just and fair solutions, in particular using restitution, as Mr. Gurlitt has now explicitly stated [...] is so important that it sends a clear signal to Germany, and beyond that, we [Germans] will not let Nazi injustice to stand, even 70 years after World War II." ${ }^{45}$

The Gurlitt Trove provides Germany with the chance to show the world that it has the political will to find justice in returning looted art, including through the planned public exhibit at the Bundeskunsthalle in Bonn in November 2017. The University of Bonn is addressing provenence research and the implementation of

42 James D. Bindenagel, Owen Pell: How to Handle That Nazi (Fn. 34).

43 Ibid.

44 Winfried Bausback, Bavarian Minister of Justice, in Joint Press Release BKM, Bavarian Ministry of Justice and Christoph Edel, Lawyer for Cornelius Gurlitt, April 7, 2014. URL: http:// www.lootedart.com/QMWQYX761931_print;Y (accessed on May 19, 2017).

45 Joint Press Release 64/2014, Bayerisches Staatsministerium der Justiz und die Beauftragte der Bundesregierung für Kultur und Medien, pp. 1-2. 
the Washington Principles in a newly established program, initiated by State Minister Monika Grütters from the office of the Federal Chancellor.

\section{Thoughts on the Future of Just and Fair Solutions of Nazi-Confiscated Art Restitution}

I remain concerned by the tendency of holders of disputed art to seek refuge in statutes of limitation and laches defenses in order to block otherwise meritorious claims even in situations where the claimant has not been provided with provenance information.

Recent cases and the recognition that States continue to hold a significant number of objects that could be classified as Holocaust-looted art highlights this inflection point as a critical moment. I testified to the European Parliament in 2003 urging the European Union to create a title-clearing process for Holocaustrelated artworks. Now is the time for that process. The central tenets of a titleclearing process would be that these artworks are held in trust by the State, pending resolution of ownership issues, with an eye toward facilitating restitution. ${ }^{46}$ The German government should urge other European nations and the EU to enact similar legislation, especially on States waiving any possessory interest in Holocaust-looted property.

The key portions of this type of the law would be as follows: ${ }^{47}$

1. States at the federal and provincial level must renounce any possessory interest beyond that of trust or custodianship in any cultural property that was owned by persecuted persons and which came into State hands from January 30, 1933, to May 9, 1945.

2. State agencies, universities, and museums should be required to inventory and report to the State all art objects now in their custody that were not in State hands prior to January 30, 1933, or were acquired after the end of Allied Occupation in the 1950s, but changed hands during the period January 30, 1933 to May 9, 1945 - "Unassigned Property". Unassigned Property would carry a presumption that it was looted from its true owner.

3. Creating a title-clearing process for Unassigned Property, for it to be inventoried, photographed and uploaded onto internet accessible databases. Any object not claimed after a defined period (18-24 months) would be

46 James D. Bindenagel, Testimony before the European Parliament, "The Washington Principles on Nazi-confiscated Art", March 18, 2003.

47 James D. Bindenagel, Owen Pell: How to Handle That Nazi (Fn. 34); see also James D. Bindenagel: Speech on the Washington Principles on Nazi-confiscated Art: Ten Years and Promises of the Washington Principles Holocaust Era Assets Conference, Prague and Terezin, Czech Republic, June 26-30, 2009. 
deemed heirless property as to which the Conference on Jewish Material Claims would have presumptive title. Religious property and other Judaica should be put in the hands of some organization chosen by the government and the Claims Conference, which agency will loan such objects for use around the world.

4. Authorizing a special administrative tribunal to process claims that are received as to Unassigned Property, which claims would be resolved based on the Washington Principles with respect to lowered burdens of proof, and to presumptions of ownership running in favor of claimants who had listed property as listing since World War II. The administrative tribunal also would have the authority to issue documents of title that would constitute a judgment that would be recognized regarding such property.

5. Authorizing governments and the Claims Conference to undertake a program to assign certain of the Unassigned Property to museums for exhibitions associated with Holocaust remembrance and education, and to allow other Unassigned Property to be auctioned with the proceeds to be used to fund Holocaust-related compensation and reparation programs, and Holocaust education programs in Europe to ensure that past genocides do not recur.

6. Putting in place tax regulations designed to prevent taxation relating to property being returned to its rightful owners. States also would waive the right to designate Holocaust-looted property in any way that would restrict the sale or export of such works. ${ }^{48}$

\section{Conclusion}

The Gurlitt Art Trove vividly illustrates that the vast economic crimes perpetrated by the Nazis still have not been fully or satisfactorily addressed. The way forward for just and fair solutions is to use the Washington Principles, which provide a road map to bringing some measure of justice through just and fair solutions for survivors and their families. Until those steps are taken, the public, and the international community simply won't know if justice is being served.

As I began, let me also conclude: "To delay justice is injustice." Today, injustice continues in the Holocaust-looted art that has not been returned to its rightful owners. Remember, this search is not only about art; it is about delivering justice and setting a precedent that will affect how future States may act, including in response to genocide and mass atrocity crimes. As such, this process will reveal us as the people we truly are. It is never too late to do the right thing.

48 James D. Bindenagel, Owen Pell: How to Handle That Nazi (Fn. 34). 
Open-Access-Publikation im Sinne der CC-Lizenz BY-NC-ND 4.0

(c) 2018, V\&R unipress GmbH, Göttingen

ISBN Print: 9783847107835 - ISBN E-Lib: 9783737007832 


\title{
Markus Stumpf / Christina Köstner-Pemsel / Olivia Kaiser
}

\section{„Treuhänderisch“ - Themenaufriss im Kontext der NS-Provenienzforschung}

\begin{abstract}
Zusammenfassung
In diesem Beitrag wird die Problematik der treuhänderischen Übernahme und Verwahrung beispielhaft anhand von zwei Themenkomplexen aus der Universitätsbibliothek der Universität Wien dargestellt und die Frage nach dem „fairen und gerechten“ Umgang im Sinne der Washingtoner Prinzipien gestellt. Dabei werden neue Erkenntnisse zu den treuhänderisch übernommenen Restbeständen aus der sogenannten „Sammlung Tanzenberg" präsentiert und ein Ansatz für den Umgang mit sogenannten „erblosen“, aber zur Restitution vorgesehenen treuhänderisch zu verwahrenden erblosen Büchern und Objekten aus der Bücherei der All Peoples' Association (APA) und der ägyptischen Sammlung der Universität Wien vorgestellt.

Schlagwörter

Universitätsbibliothek Wien, NS-Provenienzforschung, Bücherraub, Treuhand, Sammlung Tanzenberg, All Peoples’ Association, Ägyptische Sammlung der Universität Wien
\end{abstract}

\begin{abstract}
"Fiduciary" - An Outline of Topics Related to Provenance Research into the Nazi Period The following article deals with the issue of fiduciary or holding in trust with the focus on two exemplary cases involving the Vienna University Library and the question of achieving a "fair and just" solution along the lines of the Washington Principles on Nazi-Confiscated Art. The aim is to present new findings concerning remnants of the so-called "Tanzenberg Collection", which were held in trust, and describe a possible approach to dealing with socalled "heirless" books and objects from the library of the All Peoples' Association and the Egyptian Collection of the University of Vienna, which, though held in trust, are scheduled to be restituted.

Keywords

University Library Vienna, Provenance Research, Book Looting, Trusteeship, Tanzenberg Collection, All Peoples' Association, Egyptian Collection at the University of Vienna
\end{abstract}


Mit treuhänderisch übernommenen und zu verwahrenden Beständen wurde in der Vergangenheit insbesondere im Kontext von NS-Raubgut oft sorglos umgegangen. Selten kam es bei diesem schwierigen Thema zur Einhaltung der rechtlichen Bedingungen, die an solch eine Übernahme geknüpft waren, geschweige denn zu einer „fairen und gerechten“ Lösung im Sinne der Washingtoner Prinzipien.

In diesem Beitrag wird die Problematik der treuhänderischen Übernahme und Verwahrung beispielhaft anhand von zwei Themenkomplexen mit Fallbeispielen aus der Universitätsbibliothek der Universität Wien (UB Wien) dargestellt:

- treuhänderisch übernommene Restbestände aus der sogenannten „Sammlung Tanzenberg" und

- zur Restitution vorgesehene treuhänderisch zu verwahrende erblose Bücher anhand der Bücherei der All Peoples' Association (APA) und fünf Gipsrepliken aus der ägyptologischen Sammlung.

\section{Die „Sammlung Tanzenberg“ an der UB Wien}

Als ein zentrales Beispiel für eine „treuhänderische“ Übernahme an der Universitätsbibliothek Wien gilt die sogenannte "Sammlung Tanzenberg“, die in gewisser Weise den Beginn der NS-Provenienzforschung an der UB Wien markiert. 2002 erschien Evelyn Adunkas Buch Der Raub der Bücher, in dem die Autorin u. a. erstmals die Geschichte des Klosters Tanzenberg in der NS-Zeit und der dort gelagerten Bücher thematisierte und auf unrechtmäßige Bestände in der UB Wien hinwies. ${ }^{1}$ Im Jahr 2004 begann die NS-Provenienzforschung in der Hauptbibliothek und 2005 im sogenannten „Außenbereich“, den weiteren Teilbibliotheken der UB Wien. ${ }^{2}$ Im Zuge der ersten Recherchen konnte festgestellt werden, dass neben den Erwerbungen der Jahre 1938 bis 1945 auch die Nachkriegserwerbungen untersucht werden müssen. Denn aus heutiger Sicht kam erst nach 1945 ein großer Teil der möglicherweise restitutionswürdigen Bücher an die Hauptbibliothek der UB Wien. ${ }^{3}$

1 Siehe Evelyn Adunka: Der Raub der Bücher. Plünderung in der NS-Zeit und Restitution nach 1945. Wien: Czernin Verlag 2002 (= Bibliothek des Raubes 9), S. 15-70, bes. S. 64.

$2 \mathrm{Zu}$ den bisherigen Ergebnissen der Forschung siehe die Website des Arbeitsbereichs NS-Provenienzforschung der UB Wien, URL: http://bibliothek.univie.ac.at/provenienzfor schung.html (abgerufen am 15.01.2018).

3 Festgehalten werden muss, dass die UB Wien auch während der NS-Zeit zahlreiche Raubgutbestände erhielt, so etwa insg. knapp 1.900 Bände vor allem französische Bücher, die 1942/ 1943 von der Gestapo Wien übernommen wurden. Vgl. Markus Stumpf: Ergebnisse der Provenienzforschung an der Universitätsbibliothek Wien. In: NS-Provenienzforschung an österreichischen Bibliotheken. Anspruch und Wirklichkeit. Hg. von Bruno Bauer, 
Bereits im Studienjahr 2004/2005 beschäftigte sich eine Projektgruppe im Rahmen des Universitätslehrgangs „Library and Information Studies“ mit der „Sammlung Tanzenberg“. ${ }^{4}$ Es folgte das Teilprojekt an der Hauptbibliothek, dessen Zwischenergebnisse nun vom Arbeitsbereich NS-Provenienzforschung ergänzend beforscht und entsprechende Restitutionen umgesetzt werden. ${ }^{5}$

Ein Motiv für die Bestrebungen des 1945 wiederernannten UB-Direktors Johann Gans (1886-1956) Buchgeschenke für die UB Wien zu erhalten, waren die großen Bestandsverluste während des Zweiten Weltkrieges. ${ }^{6}$ Es gelang ihm nicht nur Geschenke des „British Council“ oder der „Schweizer Bücherhilfe“ zu erhalten, sondern Gans überzeugte das Unterrichtsministerium auch davon, der UB Wien vermeintlich besitzlose Bücher aus der so genannten Büchersortierungsstelle zugutekommen zu lassen. ${ }^{7}$ Diese Büchersortierungsstelle, die von 1949 bis 1952 in Räumen der Österreichischen Nationalbibliothek (ÖNB) tätig war, wurde von Alois Jesinger (1886-1964), dem Direktor der UB Wien von 1940

Christina Köstner-Pemsel und Markus Stumpf. Graz-Feldkirch: W. Neugebauer 2011 (=Schriften der Vereinigung Österreichischer Bibliothekarinnen und Bibliothekare 10), S. 113-132, hier S. 129-132, URL: http://eprints.rclis.org/17777/ (abgerufen am 15.01.2018); Christina Köstner-Pemsel: „28 sacks“ of French books for the Vienna University Library - a current case of provenance research. In: Où sont les bibliothèques spoliées par les nazis? Tentatives d'identification et de restitution, un chantier en cours. Ed. by Martine Poulaine. Paris 2018 (in Vorbereitung).

4 Vgl. die historisch auf dem Stand des Lehrgangsprojektes eingefrorene Website URL: http://bi bliothek.univie.ac.at/tanzenberg/ (abgerufen am 24.06.2017) und resümierend den Beitrag von Angelika Zdiarsky: Stempelspuren in der NS-Vergangenheit. Die „Sammlung Tanzenberg 1951 “ an der Universitätsbibliothek Wien. In: Mitteilungen der Gesellschaft für Buchforschung in Österreich 2006-1, S. 19-26.

5 Vgl. dazu u. a. Peter Malina: „Werke, denen keine große Wichtigkeit beizumessen ist“? Zur Provenienzforschung und Restitutionsarbeit an der Hauptbibliothek der Universitätsbibliothek Wien. In: Bibliotheken in der NS-Zeit. Provenienzforschung und Bibliotheksgeschichte. Hg. von Stefan Alker, Christina Köstner und Markus Stumpf. Göttingen: V\& R unipress 2008, S. 237-255; Peter Malina: Die Gestapo als Bücherlieferant. Vorläufige Ergebnisse der Provenienzforschung an der Universitätsbibliothek Wien. In: Mitteilungen der Gesellschaft für Buchforschung in Österreich 2006-2, S. 30-40; Peter Malina: Die „Sammlung Tanzenberg“: „Ein riesiger Berg verschmutzter mit Schnüren verpackter Bücher“. In: NS-Provenienzforschung an österreichischen Bibliotheken. Anspruch und Wirklichkeit. Hg. von Bruno Bauer, Christina Köstner-Pemsel, Markus Stumpf. Graz-Feldkirch: W. Neugebauer 2011 (=Schriften der Vereinigung Österreichischer Bibliothekarinnen und Bibliothekare 10), S. 133-154.

6 Die UB Wien hatte rund ein Zehntel ihres Bestandes verloren. Vgl. dazu Walter Pongratz: Geschichte der Universitätsbibliothek. Wien [u. a.]: Böhlau 1977, S. 152; Murray G. Hall, Christina Köstner-Pemsel: Kriegsbergungen der großen Wiener Bibliotheken. Die Nationalbibliothek Wien und die Universitätsbibliothek Wien. In: Bergung von Kulturgut im Nationalsozialismus. Hg. von Pia Schölnberger, Sabine Loitfellner. Wien-Köln-Weimar: Böhlau 2016, S. 307-330.

7 Ebd. 
bis 1945, geleitet. $^{8}$ So schrieb Otto Starnbacher (1885-1953) für das Unterrichtsministerium bereits 1950 an Jesinger:

„Bücher, deren Provenienz nicht feststellbar ist (herrenlos), werden treuhändig stückzahlmäßig an die Universitäts- und Nationalbibliothek übergeben. Erstere erhält wegen ihrer großen Verluste (etwa 80.000 Bände) den Vorzug. Nach Aussortierung der in diesen Bibliotheken verbleibenden Bestände werden die Verwahrer davon Listen anlegen und diese in zweifacher Ausfertigung dem Bundesministerium für Finanzen, Sektions [sic] Vermögensicherung, übermitteln. “9

In der Büchersortierungsstelle wurden vor allem konfiszierte Bestände aus ehemaligen Gemeinde-, Pfarr- und Vereinsbüchereien, die in der Nationalbibliothek gelagert wurden, aus dem Dorotheum, der Gestapobücherei in Wien sowie der Zentralbibliothek der Hohen Schule in Tanzenberg zusammengetragen. Aus den vier Provenienzgruppen sollten nur jene Bücher an Bibliotheken weitergegeben werden, die keine Besitzvermerke enthielten. So wurden aus dem Bestand des Dorotheums 38.629/38.634 Bände ${ }^{10}$, von der ehemaligen Bücherei der Gestapoleitstelle Wien 2.933 Bände, von der Nationalbibliothek 897 Bände und aus Tanzenberg 108.974 Bände an die Universitätsbibliothek Wien transferiert. Die Nationalbibliothek bekam im Vergleich dazu insgesamt „nur“ 32.155 Bände von der Büchersortierungsstelle zugesprochen.

Über 151.000 Bände, die später als „Sammlung Tanzenberg“ bezeichnet wurden, kamen 1951 von der Büchersortierungsstelle an die UB Wien. Darüber hinaus wurden ebenfalls die Verwaltungsaufzeichnungen der aufgelösten Zentralbibliothek und zwölf Kisten mit den Karteikatalog-Kästchen übergeben. Leider sind diese für die Provenienzforschung wichtigen Quellen bislang weder in der Bibliothek noch im Archiv aufgetaucht und gelten als verschollen. ${ }^{11}$

Die Übernahme der Bücher wurde an Bedingungen geknüpft, die damals und im späteren Verlauf der Einarbeitung der „Sammlung“ nicht durchgängig befolgt wurden. So heißt es zum Beispiel unter mehrfacher Verwendung des Begriffes „treuhändig“ in der Übernahmeerklärung der Bestände der Gestapo Wien:

„[...] bis zur Klärung der Eigentumsfrage, bzw. endgültigen Zuweisung in treuhändige Verwahrung.

Der Verwahrer verpflichtet sich:

$8 \mathrm{Zu}$ Jesingers Rolle in und nach der NS-Zeit bedarf es noch weiterer Forschungen.

9 Archiv der Universität Wien (UAW), Bestand UB Wien, UB A.70 Kurrentakten 1950, Mappe 1-1950 Bundesministerium für Unterricht (BMfU), Zl. 1/15/1950, Min.R. Dr. Otto Starnbacher an Alois Jesinger, 27.06.1950.

10 Die Zahlen variieren hier in den Akten.

11 Alois Jesinger erwähnt diese Unterlagen in seinem Abschlussbericht. UAW, Bestand UB Wien, UB A.79 Kurrentakten 1959, Mappe 19-1959 Rückgabe israelitischer Bücher, Zl. 19/7/1959, „Rückgabe israelitischer Bücher“, Alois Jesinger, Büchersichtung in der Neuen Hofburg, Abschluss, 18.11.1951. 
1.) die übernommenen Werke umgehend listenmässig zu erfassen und 4 Ausfertigungen des Verzeichnisses dem Bundesministerium für Unterricht vorzulegen, welches 2 Exemplare dem Bundesministerium für Finanzen übermitteln wird,

2.) für die ordnungsgemässe Verwahrung und Verwaltung der übernommenen Bestände Sorge zu tragen,

3.) die Haftung für alle Schäden und Verluste, insbesondere die Schad- und Klagloshaltung gegenüber allfälligen Rückstellungswerbern zu übernehmen,

4.) Werke, die nach der Übernahme als Restitutionsgut agnosziert werden sollten, umgehend wieder zurückzugeben.

Der Verwahrer ist seinerseits berechtigt, die treuhändig übernommenen Werke im Rahmen der internen Vorschriften unter Vorkehrung ausreichender Sicherheitsmassnahmen der Öffentlichkeit bis auf weiteres zur Verfügung zu stellen.

Wien, am 9. Nov. 1951

Übergeber: Dr. Jesinger

Übernehmer: Dr. Joh. Gans ${ }^{\text {“12 }}$

Nach dieser Verteilung schaltete sich die Israelitische Kultusgemeinde Wien ein und erwirkte eine Anmeldung des entzogenen Vermögens beim zuständigen Magistratischen Bezirksamt. Erst im November 1955 kam es zu einem endgültigen Übereinkommen zwischen der Republik Österreich, der Israelitischen Kultusgemeinde und der Jewish National and University Library (JNUL) in Jerusalem, wonach die Bücher in einem Verhältnis $60 \mathrm{zu} 40$ zwischen der UB Wien und der JNUL aufgeteilt werden sollten. Gleichzeitig einigte man sich darauf, dass keine Listen der übernommenen Bücher erstellt werden müssen, wie in der Übernahmeerklärung festgehalten wurde. ${ }^{13}$

Trotzdem dauerte es weitere drei Jahre bis die tatsächliche Aufteilung des Tanzenberg-Bestandes zwischen Wien und Jerusalem vorgenommen wurde. Schließlich unterzeichneten am 30. November 1959 Shlomo Shunami (1897-1984) im Namen der JNUL und UB-Direktor Rudolf Dettelmeier (1903-1991) ein Protokoll, in dem sie erklärten, dass die vorgesehenen Teilungsmodalitäten eingehalten worden seien und aus diesen Buchbeständen „keine gegenseitigen Forderungen mehr bestehen“. Shunami bestätigte gleichzeitig, den der JNUL zustehenden Teil im Einvernehmen mit der Israelitischen Kultusgemeinde in Wien übernommen zu haben. ${ }^{14}$

12 UAW, Bestand UB Wien, UB A.71 Kurrentakten 1951, Mappe 7-1951 Geschenke, ad Zl. 511/ 1951, Übernahme-Erklärung, betreffend 2.932 Bände der ehemaligen Bibliothek der Staatspolizeileitstelle Wien, unterzeichnet von Johann Gans und Alois Jesinger, 13.11.1951 (Hervorherbung im Original, Anm.).

13 UAW, Bestand UB Wien, UB A.80 Kurrentakten 1960, Mappe 28-1960 Rückgabe israelitischer Bücher, Zl. 28/11/1960 Aktennotiz vom 08.11. 1955 über Besprechungen im Bundesministerium für Finanzen (Min.rat Dr. Kramsal), betreffend Rückgabe von Büchern aus ehemals jüdischem Besitz.

14 UAW, Bestand UB Wien, UB A.79 Kurrentakten 1959, Mappe 19-1959 Rückgabe israelitischer Bücher, o.Zl., Protokoll betreffend „Aufteilung des Buchbestandes aus der ehemaligen Bi- 
Damit begann die UB Wien acht Jahre nach der Übernahme im Jahr 1959 mit der Einarbeitung der Bücher. Sie wurden mit dem Stempel „Sammlung Tanzenberg“ versehen und im Laufe der nächsten zehn Jahre in den Bestand aufgenommen.

Bisher wurde jedoch in der Forschung nicht berücksichtigt, dass es in weiterer Folge im Schriftverkehr zwischen der UB Wien und der JNUL nicht nur um die fehlenden Bestandslisten ging, sondern dass die gesamte Aufteilungsangelegenheit seitens des Finanzministeriums bereits im Oktober 1956 mit der Bedingung versehen worden war, dass die damals im Entwurf des Fünften Rückstellungsgesetz vorgesehene „Sammelstelle für erbloses jüdisches Vermögen in Wien“, die dann 1957 auch gebildet wurde, diesem Übereinkommen über die Aufteilung des Buchbestandes und die Übertragung an die UB Wien und Israelitische Kultusgemeinde zustimmen hätte müssen. ${ }^{15}$

Folgerichtig fragte das Bundesministerium für Finanzen im Mai 1960 bei der UB Wien nach:

„Das vorliegende Protokoll vom 30.11.1959 enthält jedoch keinen Hinweis, daß die inzwischen konstituierten Sammelstellen dem Übereinkommen zugestimmt hätten. Das Bundesministerium für Finanzen ersucht daher um Aufklärung und sollte die Befassung der Sammelstellen bisher unterblieben sein, so wird empfohlen, sich nachträglich mit den Sammelstellen in Verbindung zu setzen, um die Zustimmung zu diesem Übereinkommen zu erreichen. “16

Der Direktor der UB Wien, Rudolf Dettelmaier, wandte sich umgehend an den Direktor der JNUL, Curt D. Wormann (1900-1991), und liefert folgende Lösungsvorschläge:

„Diese Zustimmungserklärung der ,Sammelstelle für erbloses jüdisches Vermögen in Wien' habe ich leider vergessen einzuholen und auch Herr Professor Shunami hat sie nicht erbracht. Als Nichtjuristen sahen wir beide nicht das formal Juristische. Da Ihre Bibliothek im Einvernehmen mit der Israelitischen Kultusgemeinde die Bücher von hier übernahm, möchte ich Sie, sehr geehrter Herr Direktor, bitten, der Universitätsbibliothek Wien nachträglich eine der folgenden Erklärungen zu beschaffen:

A) Die Sammelstelle für erbloses Vermögen in Wien stimmt dem Abkommen [...] vom 12. Juni $1956[\ldots] \mathrm{zu}$.

B) Die Sammelstelle für erbloses jüdisches Vermögen in Wien erklärt, daß sie verzichtet, aus der Masse der Bücher, welche sich in der Universitätsbibliothek Wien

bliothek „Tanzenberg“. Beendigung der Arbeiten“, 30.11.1959. Siehe dazu auch Malina: Die „Sammlung Tanzenberg“ (Anm. 5), S. 133-154, hier S. 142.

15 UAW, Bestand UB Wien, UB A.79 Kurrentakten 1959, Mappe 19-1959 Rückgabe israelitischer Bücher, Zl. 310/1960, Rudolf Dettelmaier an C. Wormann, 01.06.1960.

16 UAW, Bestand UB Wien, UB A.80 Kurrentakten 1960, Mappe 7-1960 BMfU, Zl. 32.916-1/ 1960, Österreichisches Unterrichtsministerium an die UB Wien, 25.05.1960. 
befindet und von welcher ein Teil als entzogenes Vermögen gilt oder zu gelten hat, Rückstellungsanträge zu stellen. “17

Wormann antwortete, dass er die IKG informiert habe und diese gebeten habe sich mit Dettelmaier in Verbindung zu setzen. Und weiters:

„Ich habe Herrn Direktor Krell [Anm.: Wilhelm Krell war Amtsdirektor der IKG Wien] eine Abschrift Ihres Briefes zugesandt, und ich hoffe sehr, dass er eine der von Ihnen erwünschten Erklärungen (A oder B) der Universitätsbibliothek Wien beschaffen wird. “18

Schließlich wandte sich Dettelmaier im November 1960 an die Sammelstelle für erbloses Vermögen und bat um Ausstellung der Verzichtserklärung:

„Unter Bezugnahme auf das gestern geführte Ferngespräch und das [sic] vor ca. 6 Wochen stattgefundene Vorsprache des Bibliothekars Shunami [...] bei Herrn Dr. Georg Weiß bitte ich um Ausstellung einer Verzichtserklärung beiliegenden Musters. Zur Erklärung sei festgehalten, daß 1955 zwischen der Republik Österreich und der Israelitischen Kultusgemeinde ein Abkommen über die Teilung der ,Bibliothek Tanzenberg' unterzeichnet wurde. In einer diesbezüglichen Aktennotiz [...] heißt es im Punkt C, cc:

,Dieses Abkommen würde jedoch unter einer aufschiebenden Bedingung erfolgen, nämlich unter der Bedingung, daß die Sammelstelle für erbloses Vermögen, welche auf Grund des Staatsvertrages sicherlich zu Beginn des nächsten Jahres errichtet werden wird, diesem Abkommen entweder ausdrücklich zustimmt oder, was noch einfacher ist, eine Verzichtserklärung abgibt, wonach sie die Absicht habe, aus der Masse der Bücher, welche sich in der Universitätsbibliothek befinden und von welchen ein Teil als entzogenes Vermögen gilt oder zu gelten hat, Rückstellungsanträge zu stellen.

Im Punkt D wird festgehalten, ,daß die Israelitische Kultusgemeinde Wien ... einen wesentlichen Teil der erhaltenen Bücher der Jüdischen National- und Universitätsbibliothek in Jerusalem zur Verfügung stellen wird.' [...] Leider habe ich und auch Herr Shunami vor der Durchführung des Abkommens vergessen, die ,Verzichtserklärung der ,Sammelstelle' einzuholen, sodaß ein Eckpfeiler der juridischen Voraussetzungen noch aussteht. $[\ldots]^{\text {«19 }}$

Dr. Georg Weis (1898-1992), Leiter der Sammelstelle A, stellte schließlich mit Schreiben vom 23. November 1960 die benötigte Verzichtserklärung aus:

„In Erledigung Ihres Schreibens [...] teile ich Ihnen namens der Sammelstelle A (Gesetz vom 13. März j1957 Nr. 73/57) höflichst mit, dass diese wegen der Bücher der ehe-

17 UAW, Bestand UB Wien, UB A.80 Kurrentakten 1960, Mappe 28-1960 Rückgabe israelitischer Bücher, Zl. 28/1/1960, Zl. 310/1960, Rudolf Dettelmaier an C. Wormann, 01.06.1960.

18 UAW, Bestand UB Wien, UB A.80 Kurrentakten 1960, Mappe 28-1960 Rückgabe israelitischer Bücher, Zl. 28/3/1960, C. Wormann an Rudolf Dettelmaier, 29.06.1960.

19 UAW, Bestand UB Wien, UB A.80 Kurrentakten 1960, Mappe 28-1960 Rückgabe israelitischer Bücher, Zl. 28/1960, Rudolf Dettelmaier an die Sammelstelle für erbloses Vermögen, 17.11. 1960. 
maligen ,Bibliothek Tanzenberg', welche sich in der Universitätsbibliothek befinden, keine Rückstellungsansprüche geltend machen und keine Rückstellungsanträge stellen wird. ${ }^{\text {20 }}$

Dettelmaier informierte Shunami umgehend und bat um eine Liste der wichtigsten übernommenen Bestände:

„Durch diese Liste [...] soll bei der vorgesetzten Behörde dokumentiert werden, daß die wichtigsten Bestände, von Ihnen übernommenen Bestände erfaßt wurden, es sich aber für keine der beteiligten Bibliotheken lohnt, größere Massen kaum verwertbarer Literatur zu katalogisieren. “21

Schließlich informierte Dettelmaier auf Druck des Finanz- sowie des Unterrichtsministeriums ${ }^{22}$ über die seiner Meinung nach damit hergestellte „rechtliche Fundierung“ das Unterrichtsministerium. Dabei nahm er die Antwort Shunamis vorweg und erklärte, dass ein Verzeichnis über die „wichtigsten und wertvollsten von der JNUL übernommen Werke“ angelegt wurde:

„Es sind dies vorwiegend Periodika und Sammelwerke oder umfangreichere Schriften von bleibendem Wert. Für die große Masse von nicht selten belanglosen Monographien, antiquierten Klassikerausgaben und Lexika, veralteter Sammelschriften und Sonderdrucken etc. etc. wurden keine Verzeichnisse angelegt. Für beide Teilungspartner war dies aus Raum-, Zeit- und Personalgründen unmöglich. Vom wirtschaftlichen Standpunkt gesehen, wäre dies für beide Teile ein ausgesprochenes Verlustgeschäft gewesen. “23

Für das Unterrichts- sowie für das Finanzministerium war damit die Angelegenheit erledigt.

Obwohl die Bücher bereits 1951 in die UB Wien transferiert wurden, ließ die tatsächliche Aufnahme der Bestände 14 Jahre auf sich warten. Rudolf Dettelmaier war sich der Problematik offenbar bewusst, denn in einem Bericht 1956 konstatierte er, richtig gelegen zu sein, mit der Sichtung und Katalogisierung der Tanzenberg-Bestände auf den Abschluss der Staatsvertragsverhandlungen zu warten. ${ }^{24}$ Im Sommer 1963 wurden fünf neue MitarbeiterInnen,

20 UAW, Bestand UB Wien, UB A.80 Kurrentakten 1960, Mappe 28-1960 Rückgabe israelitischer Bücher, Zl. 28/6/1960, Sammelstelle A an die Universitätsbibliothek Wien, 23.11.1960.

21 UAW, Bestand UB Wien, UB A.80 Kurrentakten 1960, Mappe 28-1960 Rückgabe israelitischer Bücher, Zl. 28/7/1960, Rudolf Dettelmaier an Shlomo Shunami, 16.12.1960.

22 UAW, Bestand UB Wien, UB A.80 Kurrentakten 1960, Mappe 7-1960 BMfU, Zl. 7/85/1960, Erinnerungsschreiben des Bundesministeriums für Unterricht an die Direktion der Universitätsbibliothek in Wien, 30.12.1960.

23 UAW, Bestand UB Wien, UB A.80 Kurrentakten 1960, Mappe 28-1960 Rückgabe israelitischer Bücher, Zl. 28/1/1961, Rudolf Dettelmaier an das Bundesministerium für Unterricht, 09.01. 1961.

24 UAW, Bestand UB Wien, UB A.79 Kurrentakten 1959, Mappe 17-1959 Akademischer Senat 
darunter die spätere Direktorin der UB Wien, Ilse Dosoudil, eingestellt, um die Aufnahme der Tanzenberg-Bestände rascher vorantreiben zu können. ${ }^{25}$

Shlomo Shunami antwortete aus nicht bekannten Gründen erst im Jänner 1966 auf Dettelmaiers Anfrage und inkludierte ein Verzeichnis von 150 Zeitschriftentiteln, das allerdings keine Bestandsangaben enthielt. ${ }^{26}$

Zusammenfassend kann festgehalten werden, dass eine Suche nach den ursprünglichen BesitzerInnen der treuhänderisch übernommen Bestände nie weiter verfolgt wurde. ${ }^{27}$ Die Bücher wurden wie „herrenlose“ Güter behandelt und auf die Einhaltung der Auflagen bzw. ethischer Bedenken wurde kein Wert gelegt. Schließlich erfolgte die weitere Transformation hin zu einer geschichtsund gesichtslosen Büchermasse, bei der eine nähere Befassung ökonomisch nicht gerechtfertigt schien.

\section{Zwischenergebnisse der Provenienzforschung zur „Sammlung Tanzenberg“}

Der von der Büchersortierungsstelle prinzipiell treuhänderisch übernommene Bestand wurde eingearbeitet, abgegeben, getauscht oder entsorgt, so dass im Rahmen des Provenienzforschungsprojekts „nur“ insgesamt 8.242 Titelsätze in der Hauptbibliothek der UB Wien mit dem Vermerk bzw. mit dem bei der Einarbeitung vergebenen Stempel „Sammlung Tanzenberg“ erfasst werden konnten. ${ }^{28}$ Ähnlich wie bei der Causa Mauerbach ${ }^{29}$ war den Spuren von VorbesitzerInnen im Zuge der treuhänderischen Übernahme in keinster Weise nachgegangen worden. Der Arbeitsbereich NS-Provenienzforschung beschäftigt sich seit Jahren mit der Aufarbeitung insbesondere dieser Bücher und konnte einige Restitutionen von Büchern aus der „Sammlung Tanzenberg“ bereits abschließen. So wurden 2009 elf Bände aus dem Besitz von Oscar Ladner restituiert und

Jahresberichte, Zl. 423/1956, Bericht Rudolf Dettelmaier an den Akademischen Senat der Universität Wien, 29.09.1956.

25 UAW, Bestand UB Wien, UB A.83 Kurrentakten 1963, Ordner UB Akten 1963 (313-732), Zl. 378/1963, Laufer betr. Ordenverleihung, Beförderungen, Überstellungen, Neueinstellungen, Prüfungen im Jahre 1963, 10.07.1963.

26 UAW, Bestand UB Wien, UB A.80 Kurrentakten 1960, Mappe 28-1960 Rückgabe israelitischer Bücher, Zl. 28/7/1960, Shlomo Shunami an Rudolf Dettelmaier, 10.01.1966.

27 Vgl. Markus Stumpf: „Stille“ Restitution. NS-Provenienzforschung im Spannungsfeld von universitärer Erinnerungsarbeit und Öffentlichkeitswirksamkeit. In: „Was bleibt? Bibliothekarische NS-Provenienzforschung und der Umgang mit ihren Ergebnissen“. Hg. von Markus Helmut Lenhart und Birgit Scholz. Graz: Clio 2018 (=Veröffentlichungen der Forschungsstelle Nachkriegsjustiz 5), S. 79-90.

28 Bereits im Rahmen des anfangs erwähnten ULG-Projekts wurden erste Hinweise in den damaligen Bibliothekskatalog eingetragen. Im Oktober 2009 waren es 355 Bücher, die mit Bestand „Tanzenberg“ nachgewiesen waren.

29 Vgl. dazu auch die Beiträge von Monika Mayer und Leonhard Weidinger in diesem Band. 
weitere neun Bände von vier Vorbesitzern als zur Restitution vorgesehen eingestuft werden.

Als „wahrscheinliches Raubgut“ wurden gut 350 Bände eingestuft, deren Indizien noch überprüft werden müssen. Viele der weiteren Bücher tragen zwar einen Sammlung Tanzenberg-Stempel oder einen Stempel der Zentralbibliothek der Hohen Schule, jedoch sonst keine weiteren Hinweise auf VorbesitzerInnen. Alles in allem zeigen diese Zahlen, dass in den letztlich an der UB Wien verbliebenen und bei der Autopsie wiedergefundenen Büchern aus der Sammlung Tanzenberg trotz der Sichtung an der Büchersortierungsstelle Hinweise auf VorbesitzerInnen enthalten sind.

Dass den Spuren in den Büchern nicht nachgegangen wurde, lässt sich ebenso in den Akten nachvollziehen. So hatte Jesinger bezeichnenderweise in seinem an Johann Gans gerichteten Begleitschreiben zu seinem Schlussbericht von 1951 bereits ausdrücklich darauf hingewiesen, dass er seinen Schlussbericht „absichtlich“ als „Statistische Abrechnung“ ohne kritische Analyse der gesichteten Bestände gemacht habe. ${ }^{30}$ Eine intensive Suche nach Vorbesitzern hatte Jesinger jedenfalls auch gar nicht vor. So finden sich auch keinerlei Hinweise auf ihm notwendig erscheinende weiterführende Recherchen - es ging ihm vor allem um die Sicherung der bibliothekarischen Nutzung der nun so reichhaltig zur Verfügung stehenden Bücher. Die Bedingungen der „treuhänderischen“ Bestandsvermehrung nahmen Jesinger und später auch alle anderen DirektorInnen bis zum Einsetzen der NS-Provenienzforschung im Jahr 2004 nicht ernst.

\section{Treuhänderisch zu verwahrende erblose Objekte und Bücher}

Heute hingegen stellt sich die UB Wien dieser Verantwortung, einerseits in der Aufarbeitung der sogenannten „Sammlung Tanzenberg“ und andererseits in Fällen, in denen eine treuhänderische Verwahrung im Zuge der Provenienzforschung notwendig wird.

In der Praxis wird der Universitätsleitung, vertreten durch die Leitung der UB Wien, ein Dossier über die als Raubgut klassifizierten Bestände zur Entscheidung vorgelegt. Wird entschieden, dass die Bestände an die EigentümerInnen bzw. deren RechtsnachfolgerInnen zurückzustellen sind, beginnt die Erbensuche.

Bis diese gefunden werden und die Abwicklung der Restitution erfolgt, können oft Jahre vergehen. In diesem Zeitraum ist die jeweilige Institution zwar im Besitz der Bücher und Objekte, verwahrt diese aber für eine „unbekannte“

30 UAW, Bestand UB Wien, UB A.79 Kurrentakten 1959, Mappe 19-1959 Rückgabe israelitischer Bücher, o.Zl., Alois Jesinger an Johan Gans, 29.11.1951. 
Person/Institution sozusagen „treuhänderisch“, also zu treuen Händen. Mit anderen Worten: ab dem Zeitpunkt der Entscheidung zur Restitution ist die jeweilige Institution im Sinne der Washingtoner Prinzipien nicht mehr Eigentümerin der Bücher und/oder Objekte.

Das ist für die verwahrende Institution eine herausfordernde Phase, denn die Geschäftsprozesse und Abläufe in einer Universitätsbibliothek wie der UB Wien sind - anders als in Museen und National- bzw. Staatsbibliotheken - nicht primär auf das Verwahren und Erhalten, sondern auf die Informationsversorgung von Forschung und Lehre ausgerichtet. Das führt zu Fragen der Verwaltung, der Ausweisung dieser Bestände und des Benutzungs- und Rechtemanagements einzelner Buchexemplare. Letztlich bedeutet dies einen Lernprozess für eine Universitätsbibliothek, Bücher (wieder) als museale Objekte zu sehen. Für die UB Wien, die bei etwa 100.000 Universitätsangehörigen - davon über 90.000 Studierende - als Massenuniversität gilt, stellen sich dabei in der Gestaltung dieser Prozesse größere Herausforderungen.

Anhand von zwei Fallbeispielen aus der UB Wien, der Bücherei der All Peoples' Association (APA) und fünf Gipsrepliken aus der ägyptologischen Sammlung, soll dieser treuhänderischen Verwahrung nachgegangen werden. In beiden Fällen erwiesen sich die Objekte und Bücher letztlich als „erblos“, womit sich die Frage, wie weiter damit umzugehen sei und wie eine „faire und gerechte“ Lösung im Sinne der Washingtoner Prinzipien aussehen könnte, stellte und einer Lösung bedurfte.

\section{Fallbeispiel All Peoples' Association (APA)}

Über die Raubgeschichte der Bücherei der All Peoples’ Association wurde bereits bei der von der UB Wien gemeinsam mit der Wienbibliothek im Rathaus im Jahr 2008 veranstalteten Tagung ${ }^{31}$ und im ersten Band der Schriftenreihe der Kommission für Provenienzforschung ${ }^{32}$ berichtet.

31 Monika Löscher: Provenienzforschung an der Universitätsbibliothek Wien - Der dezentrale Bereich. In: Bibliotheken in der NS-Zeit. Provenienzforschung und Bibliotheksgeschichte. Hg. von Stefan Alker, Christina Köstner und Markus Stumpf. Göttingen: V\& R unipress 2008, S. 257-271, hier S. 264-266. URL: http://www.v-r.de/_files_media/mediathek/downloads/ 810/9783899714500_Bibliotheken_in_der_NS-Zeit_OA.pdf (abgerufen am 15.01.2018).

32 Monika Löscher, Markus Stumpf: „... im wesentlichen unbeschädigt erhalten geblieben ...“. Provenienzforschung an der Universitätsbibliothek Wien am Beispiel der Fachbereichsbibliothek Anglistik und Amerikanistik. In: ... wesentlich mehr Fälle als angenommen. 10 Jahre Kommission für Provenienzforschung. Hg. von Gabriele Anderl, Christoph Bazil, Eva Blimlinger, Oliver Kühschelm, Monika Mayer, Anita Stelzl-Gallian und Leonhard Weidinger. Wien, Köln, Weimar: Böhlau 2009 (= Schriftenreihe der Kommission für Provenienzforschung 1), S. 281-297, hier S. 294-297. 


\section{ALtPROPHES ACSOCHFIOA-(APA)}

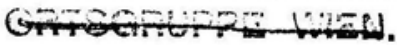

Abb. 1: APA-Stempel

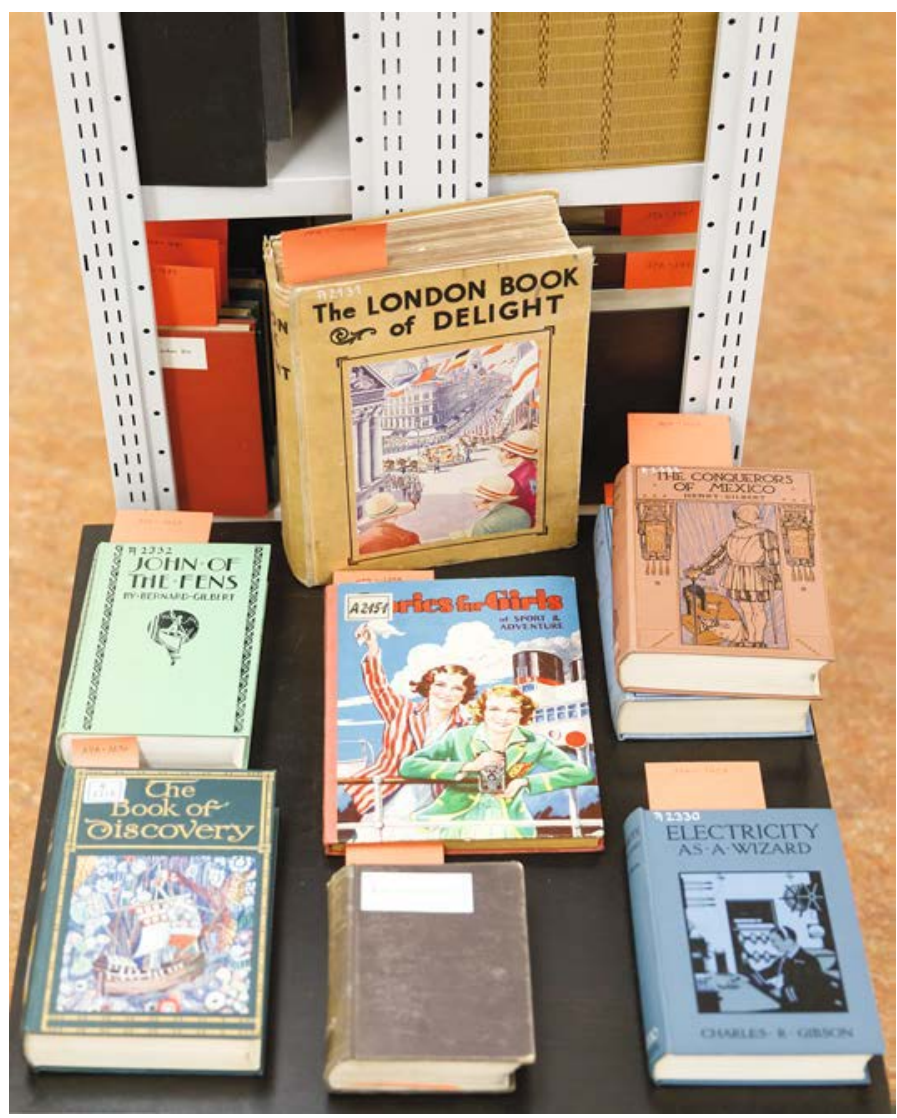

Abb. 2: Werke aus der APA-Bücherei

Das Seminar für Englische Philologie der Universität Wien erhielt während der NS-Zeit einige Bestände von den durch die NS-Verwaltung zwangsaufgelösten englischen und amerikanischen Kulturvereinen. Dies betraf etwa die Bibliothek der Deutsch-Englischen Gesellschaft - Zweigstelle Wien (ca. 1.800 Bände), die Bibliothek des American Women's Club (mehr als 2.000 Bände), die Bibliothek des Amerika-Hauses (dazu sind leider keine Bestandsangaben überliefert) und die Bibliothek der APA.

Die APA war im Jahr 1930 als eine weltweite Vereinigung in London gegründet worden. Mit Vorträgen, Sprachkursen und dem Aufbau von Bibliotheken wollten 
seine Mitglieder einen Beitrag zur „Völkerverständigung“ leisten. ${ }^{33}$ Die Wiener Zweigstelle wurde Anfang Februar 1935 gegründet $^{34}$, in deren Satzungen auch eine Bibliothek für die Mitglieder angeführt wird. Während der Londoner Hauptsitz 1936 aufgelöst wurde, bestand der Wiener Zweig weiter und wurde erst im Nationalsozialismus im Juni 1939 zwangsaufgelöst, wobei das Vermögen, inklusive der Bibliothek, zu gleichen Teilen zwischen dem Stillhaltekommissar für Vereine, Organisationen und Verbände und der NSDAP, GAU Wien aufgeteilt wurde. ${ }^{35}$ Dem Englischen Seminar der Universität Wien wurde schließlich der APA-Bestand zunächst als „Leihgabe“ zugeteilt. Obwohl eine endgültige Zuteilung nach heutigem Quellenbestand nie erfolgte und der Formalakt der Übertragung daher nicht abgeschlossen war, wurde wenig später ab 1942 die APABücherei am Englischen Seminar bereits als Eigenbestand geführt. ${ }^{36}$

Im Jahr 2010 konnte als Ergebnis der Provenienzforschung zunächst ein Bestand von etwa 1.600 Bänden der APA identifiziert werden. Als eindeutiges Raubgut klassifiziert, wurde von der Universitätsleitung, respektive der Leitung der UB Wien, entschieden, die Bibliothek an die EigentümerInnen bzw. RechtsnachfolgerInnen zurückzugeben und den Fall in die Kunstdatenbank des Nationalfonds in der Hoffnung auf zusätzliche Informationen einzutragen.

Danach wuchs durch begleitende Recherchen der identifizierte Bestand auf insgesamt 1.830 Druckschriftenbände an, wobei zumindest 15 Bände aus der Fachbereichsbibliothek Translationswissenschaften und ein Band aus der Hauptbibliothek der APA zugeordnet werden konnten. Während die Translationswissenschaft die Bestände als Geschenke vom Anglistischen Seminar erhalten hatten, entstammte das Exemplar aus der Hauptbibliothek dem Stempel zufolge aus der „Sammlung Tanzenberg“ ${ }^{37}$ Auch fanden seither einige ergän-

33 The APA: new home in London. In: The Times, Wednesday, Feb 14, 1934.

34 Wiener Stadt- und Landesarchiv (WStLA), M.Abt. 119 - A32-1066/35, Ernst Ritter von Streeruwitz an die MA2, 01.02.1935.

35 WStLA, M.Abt. 119 - A32-1066/35, Reichskommissar für die Wiedervereinigung Österreichs mit dem Deutschen Reich, Stab Stillhaltekommissar für Vereine, Organisationen und Verbände, Schlußbericht vom 26.06.1939.

36 Einer der zentralen Akteure am Englischen Seminar war Professor Friedrich Wild (1888-1966) der gemeinsam mit seiner Frau auch in den Raubgutfällen von Helene und Elise Richter sowie Melanie Adler beteiligt war. Siehe dazu beispielhaft Christiane Hoffrath: Bücherspuren. Das Schicksal von Elise und Helene Richter und ihrer Bibliothek im „Dritten Reich“. 2., erg. Aufl. Köln, Weimar, Wien: Böhlau 2010 (= Schriften der Universitäts- und Stadtbibliothek Köln 19) und Markus Stumpf: Raub und Rückgabe der Bibliothek und des Nachlasses Guido Adlers - Anmerkungen und Aktualisierungen. In: Guido Adlers Erbe. Restitution und Erinnerung an der Universität Wien. Hg. von Markus Stumpf, Herbert Posch und Oliver Rathkolb. Göttingen: Vienna University Press bei V\&R unipress, 2017 (= Bibliothek im Kontext 1), S. 83-202. Open Access: URL: http://www.vr-elibrary.de/doi/pdf/10. 14220/9783737007214.83 (abgerufen am 15.01.2018).

37 Unklar ist dabei, ob der Band als Geschenk des Anglistischen Seminars oder aus der Büchersortierungsstelle an die Hauptbibliothek kam. Im ersten Fall wäre er irrtümlich mit den 
zende wissenschafts- und institutionengeschichtliche Forschungen statt, die aber allesamt über die Bibliotheksbestände keine wesentlichen zusätzlichen Informationen brachten. ${ }^{38}$

Wie bereits 2010 angenommen worden war, konnte auch für den Trägerverein der APA in London, der ja bereits vor der Wiener Zweigstelle 1936 aufgelöst worden war, kein Rechtsnachfolger festgestellt werden, so dass die APA-Bücherei schließlich 2016 als erbloses Gut eingestuft wurde. In weiterer Folge wurde ein unabhängiges Schätzgutachten eingeholt. Darin wurde festgestellt, dass die inhaltlichen Schwerpunkte der APA-Bibliothek in erster Linie literarische bzw. belletristische Werke darstellen und v.a. Werke vertreten sind, die kulturgeschichtlich interessierte LeserInnen ansprechen.

Fallbeispiel Gipsrepliken aus der archäologischen Schausammlung des Instituts für Ägyptologie der Universität Wien

Ende Juli 1938 waren zwölf Objekte von der Gestapo an das damalige Institut für Ägyptologie und Afrikanistik der Universität Wien übergeben worden, was auch bei der Inventarisierung akribisch festgehalten wurde. So heißt es bei den Inventarnummern 133 bis 144 im Erwerbungsvermerk „Von der Gestapo übergeben am 30. Juli 1938“. Von diesen ursprünglich zwölf Objekten erhielten sich allerdings über die folgenden Jahrzehnte nur fünf. 2010 wurden diese fünf $\mathrm{Ob}$ jekte eindeutig als Raubgut klassifiziert und die Restitution empfohlen. ${ }^{39}$

Den Spuren auf den Objekten wurde schließlich 2016 im Rahmen der NSProvenienzforschung an den Sammlungen der Universität Wien nachgegangen. Einerseits war auf manchen Objekten der Stempel „Staatliche Museen Berlin Gipsformerei“ zu identifizieren, andererseits gab es eingekratzte Nummern. Durch die Forschungen konnte eindeutig festgestellt werden, dass es sich dabei

Tanzenberg-Stempel versehen worden, im zweiten Fall würde dies bedeuten, dass nicht der gesamte Bestand der APA-Bücherei von den Nationalsozialisten an das Anglistische Seminar verbracht wurde - es also auch andere Wege der Verteilung gegeben haben müsste.

38 Vgl. Ramon Pils: „Ein Gelehrter ist kein Politiker.“ Die Professoren der Wiener Anglistik im Kontext des Nationalsozialismus. In: Geisteswissenschaften im Nationalsozialismus. Das Beispiel der Universität Wien. Hg. von Mitchel G. Ash, Wolfram Nieß und Ramon Pils. Göttingen: Vienna University Press bei V\&R unipress 2010, S. 455-485; Ramon Pils: Disziplinierung eines Faches: Zur Englischen Philologie in Wien im frühen 20. Jahrhundert. In: Reflexive Innensichten aus der Universität. Disziplinengeschichten zwischen Wissenschaft, Gesellschaft und Politik. Hg. von Karl Anton Fröschl, Gerd B. Müller, Thomas Olechowski und Brigitta Schmidt-Lauber. Göttingen: Vienna University Press bei V\&R unipress 2015 $(=650$ Jahre Universität Wien - Aufbruch ins neue Jahrhundert, hg. von Friedrich Stadler u. a., Band 4), S. 539-549.

39 Internes Archiv des Arbeitsbereichs NS-Provenienzforschung der UB Wien, Markus Stumpf: Dossier GESTAPO-Sammlung Ägyptologie, Stand: 27.02.2010. 
um die Formnummern der Gipsrepliken aus der Gipsformerei der Staatlichen Museen zu Berlin, Preußischer Kulturbesitz, handelte. Da die Gipsrepliken dort auch heute noch produziert werden, bestand damit die vage Hoffnung, dass eventuell erhaltene Verkaufsunterlagen zum ursprünglichen Käufer führen und so eine Hinweiskette auf den Vorbesitzer gebildet werden könnte. Diese Hoffnungen ließen sich leider nicht bestätigen.

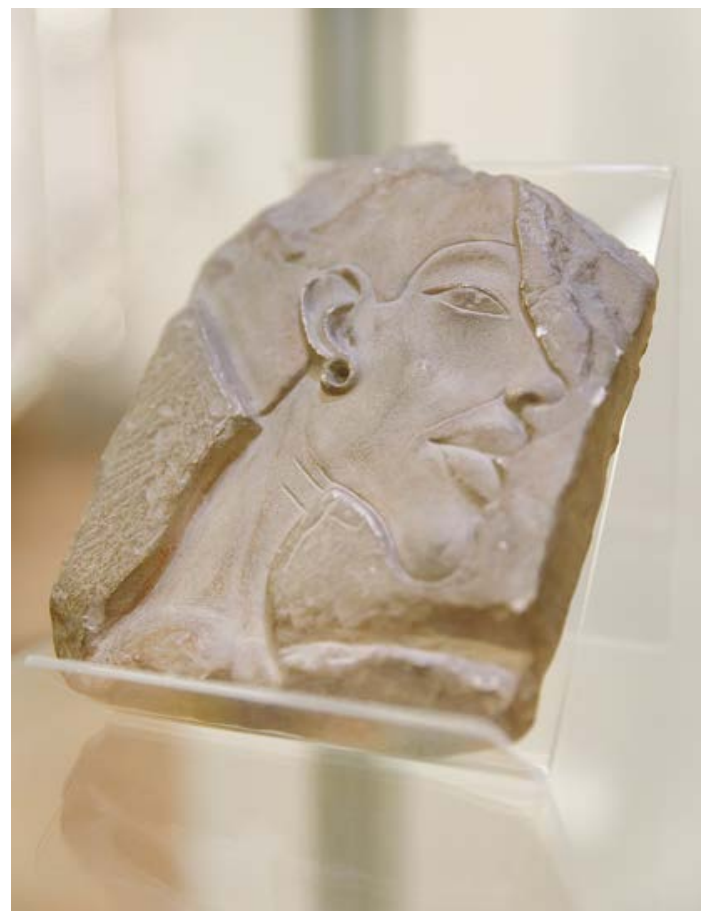

Abb. 3: Von der Gestapo übergebenes Gipsrelief Armenophis IV

Auf Basis dieser Erkenntnisse wurden, da die Hinweise auf den Objekten keine Zuordnung zu bestimmten VorbesitzerInnen zulassen, die Objekte selbst keinen unikalen Charakter haben und auch keine weiterführenden Informationen etwa durch zusätzliche Quellenfunde zu erwarten waren, die Objekte als erbloses Gut klassifiziert. Der derzeitige monetäre Wert war hingegen aufgrund des derzeit gültigen Verkaufskataloges der Gipsformerei der Staatlichen Museen zu Berlin Preußischer Kulturbesitz ${ }^{40}$ eindeutig bestimmbar. ${ }^{41}$

40 Aegypten. Freiplastik und Reliefs. Gipsformerei, Staatliche Museen zu Berlin Preußischer Kulturbesitz (= Katalog der Originalabgüsse Heft 1/2). URL: http://ww2.smb.museum/GF/ index.php mode $=$ browse $\&$ cat $=1$ (abgerufen am 20.06.2017).

41 Internes Archiv des Arbeitsbereichs NS-Provenienzforschung der UB Wien, Monika 


\section{Rückgabe und Wiederankauf „erbloser“ Bücher und Objekte}

Mit der Klassifizierung als sogenannte „erblose“ Objekte und Bücher sowie den Wertbestimmungen konnte der Frage nach dem weiteren Umgang mit diesen Beständen nachgegangen werden. Nach zahlreichen Gesprächen von Markus Stumpf mit Ministerien, Nationalfonds, Kommission für Provenienzforschung, Universitätsleitung und BibliotheksdirektorInnen konnte als Lösung der Problematik für beide Fälle von der Universität Wien ein Vertrag mit dem Nationalfonds geschlossen werden, bei dem zunächst sowohl die APA als auch die Gipsrepliken dem Nationalfonds zur Verwertung übertragen und im April 2017 von der Universität Wien - diesmal legal - wiedererworben wurden. Der Nationalfonds hingegen sorgt dafür, dass diese Mittel den überlebenden Opfern des Nationalsozialismus zugutekommen. ${ }^{42}$

Damit konnte in diesen Fällen die Phase der „treuhänderischen“ Verwahrung für die unbekannten Eigentümer abgeschlossen und die Phase der begleitenden reflektierenden Erinnerungsarbeit auf anderer Ebene weitergeführt werden. Waren die Bibliotheksbestände der APA bereits seit 2010 im Bibliothekssystem als Raubgut ausgewiesen und sie zusätzlich so wie auch die Gipsrepliken in der Kunstdatenbank eingetragen worden, so können beide Bestände nun aufgrund des Vertrages wieder in die jeweilige Bibliothek und Sammlung integriert werden. Für die zukünftige Nutzung bedeutet dies, dass auf die Herkunft und Erwerbungsgeschichte und die besondere Verantwortung der Universität Wien bei diesen Büchern und Objekten spezifisch hingewiesen werden kann und damit auch eine Vermittlung der Problematik innerhalb der Universität stattfinden kann.

Während die Gipsrepliken im Rahmen des 17. Sammlungstreffens Anfang Juni 2017 wieder an die Schausammlung des Instituts für Ägyptologie übergeben wurden $^{43}$, wird die APA-Bücherei im Jahr 2018 in der Fachbereichsbibliothek Anglistik und Amerikanistik frei zugänglich aufgestellt werden. Damit kann die Bibliothek - entsprechend dem ursprünglichen Vereinszweck der All Peoples' Association - wieder einen „Beitrag zur Völkerverständigung“ leisten.

Schreiber: Ergänzungsdossier zur Schausammlung des Instituts für Ägyptologie, Stand: 09.03.2016.

42 Vgl. die Pressemitteilung der Universität Wien vom 24.04.2017. Restitution: Universität Wien unterzeichnet Vertrag mit dem Nationalfonds. URL: http://medienportal.univie.ac.at/ presse/aktuelle-pressemeldungen/detailansicht/artikel/restitution-universitaet-wien-unter zeichnet-vertrag-mit-dem-nationalfonds/ (abgerufen am 20.06.2017).

43 Vgl. Irene Kaplan, Markus Stumpf: Objekt des Monats: Gipsabguss eines Reliefs von Amenophis IV. URL: http://bibliothek.univie.ac.at/sammlungen/objekt_des_monats/0124 05.html (abgerufen am 20.06.2017); Bericht über das 17. Sammlungstreffen: Provenienzforschung. URL: http://bibliothek.univie.ac.at/sammlungen/sammlungstreffen_17.html (abgerufen am 20.06.2017). 
In beiden Fällen ergibt sich damit die Möglichkeit die Vermittlungsarbeit zu NS-entzogenem Raubgut auf neuen, bislang nicht zugänglichen Ebenen anzugehen. Wie an diesen Beispielen ersichtlich bedarf es für NS-Provenienzforschung einen langen Atem - so haben die Forschungen zu den APA-Beständen bereits 2006 und zu den Gipsrepliken 2008 begonnen. 2017 - elf bzw. neun Jahre später - konnte eine - wie wir meinen - „faire und gerechte Lösung“ im Sinne der Washingtoner Prinzipien erreicht werden. Für die Tanzenberg-Bestände wird es voraussichtlich noch länger dauern. 
Open-Access-Publikation im Sinne der CC-Lizenz BY-NC-ND 4.0

(c) 2018, V\&R unipress GmbH, Göttingen

ISBN Print: 9783847107835 - ISBN E-Lib: 9783737007832 


\title{
Sebastian Spitra
}

\section{Recht und Metapher: Die „treuhänderische“ Verwaltung von „Kulturgut“ mit NS-Provenienz}

\section{Zusammenfassung}

Die Formel von der „treuhänderischen Verwaltung von Kulturgut“ beschreibt einerseits eine rechtliche Begebenheit. Sie ist JuristInnen so vertraut, dass sie dem Sprachbild zumeist keinen Wert mehr beimessen. Im juristischen Sinn spricht man von einer Treuhand, wenn jemandem Rechte anvertraut sind, die er oder sie nicht im eigenen Interesse, sondern im Interesse anderer ausüben soll. Andererseits handelt es sich beim Wort „Treuhand“ aber auch um eine rhetorische Figur, eine Metapher, und der Gehalt dieses Begriffs ist von verschiedenen Kontexten und Interessen geprägt. Zu ihnen gehören auch problematische Konnotationen, die auf die NS-Zeit zurückgehen. Der vorliegende Text beabsichtigt, das juristische Regelungsinstrument kritisch mit den Methoden einer historischen Rechtsmetaphorologie zu beleuchten.

\section{Schlagwörter}

Treuhand, Rechtsmetapher, Kulturgut, Restitution, NS-Recht, Rechtsgeschichte der NSZeit

\begin{abstract}
Law and Metaphor: The Holding in "Trust" of "Cultural Property" with Nazi Provenance On the one hand, the phrase "holding objects in trust" describes a legal situation. Lawyers are so familiar with it that they do not ascribe a distinct meaning to that expression any more. In a legal sense, a trust is defined as the right to hold title to property or assets for the benefit of a third party. On the other hand, the word "trust" is also a rhetorical figure, a metaphor, as the content of this term depends on its different interests and contexts. These include connotations going back to usage during and after the Nazi era. This text intends to comment on "trust" as a legal regulatory instrument and to analyze it critically from the perspective of historical legal metaphorology.
\end{abstract}

Keywords

Trust, Legal Metaphor, Cultural Property, Restitution, Nazi Law, Legal History of the Third Reich 


\section{Einleitung: Die Treuhand zwischen Recht und Metapher}

Im juristischen Sinn spricht man von einer Treuhand, „wenn jemandem Rechte anvertraut sind, über die er selbst verfügen kann, die er aber nicht im eigenen Interesse, sondern im Interesse anderer Personen oder objektiver Zwecke ausüben soll“. ${ }^{1}$ Benutzt man diesen Begriff für die Verwaltung von Kulturgut mit NSProvenienz, schwingen hier subkutan außerrechtliche Bedeutungsebenen mit, die bereits in den problematischen Strukturen des Anvertrauens jener Güter und dem unbekannten Subjekt liegen, zu dem ein Treueverhältnis betont wird. Aber schon die formalrechtliche Bedeutung des Instituts der Treuhand ist nicht immer klar. In der Rechtswissenschaft wird mitunter die Ansicht vertreten, dass es keinen einheitlichen Begriff des Treuhänders in der Rechtssprache gibt. ${ }^{2}$ Umso wichtiger ist es, die rechtliche von den verschiedenen metaphorischen Ebenen zu trennen, gleichzeitig aber die metaphorische Ebene in der rechtlichen Betrachtung ernst zu nehmen und in der Analyse mit einzubeziehen.

Die völkerrechtlichen und innerstaatlichen Dimensionen unterstreichen die Bedeutung des Rechtsinstituts der Treuhand für Kulturgüter mit NS-Provenienz. Seit der Londoner Erklärung im Jahr 1943 drängten die Alliierten offiziell auf die Rückgabe von entzogenen Vermögenswerten. ${ }^{3}$ Kunst und Kulturgüter nehmen dabei aufgrund ihrer relativ einfachen Identifizierbarkeit und des hohen Wiedererkennungsgrades neben Immobilien in Restitutionsangelegenheiten einen besonderen Platz ein. ${ }^{4}$ Erste Restitutionsbemühungen wurden in Deutschland durch die treuhänderische Verwahrung und Verteilung von „Kulturgut“ durch die Central Collecting Points gesetzt. In Österreich mündeten die rechtlichen Anstrengungen um die Rückgabe vor allem im Nichtigkeitsgesetz $1946^{5}$, dem Dritten Rückstellungsgesetz aus $1947^{6}$ sowie dem 1998 erlassenen Kunstrückgabegesetz $^{7}$, das zuletzt 2009 (BGBl. I Nr. 117/2009) novelliert wurde.

Jenseits dieser rechtlichen Betrachtung soll außerdem die Metaphorik der

1 Karl Otto Scherner: Treuhand. In: Handwörterbuch zur Deutschen Rechtsgeschichte. Bd. 5. Berlin: Erich Schmidt Verlag 1998, Sp. 341-343.

2 Nachweise bei Rudolf Strasser: $\$ 1002$ ABGB. In: Kommentar zum Allgemeinen bürgerlichen Gesetzbuch. Hg. von Peter Rummel. Wien: Manz 2000, Rz. 42.

3 Wilfried Fiedler: Die Alliierte (Londoner) Erklärung vom 5.1.1943: Inhalt, Auslegung und Rechtsnatur in der Diskussion der Nachkriegsjahre. In: Private Law in the International Arena. Privatrecht in der internationalen Arena. From National Conflict Rules Towards Harmonization and Unification, Liber Amicorum Kurt Siehr. Hg. von Jürgen Basedow, Isaak Meier, Anton K. Schnyder, Talia Einhorn und Daniel Girsberger. Den Haag: T.M.C. Asser Press 2000, S. 197-218.

4 Alfred J. Noll: Fortschritt und Versäumnis. Kunstrückgabe in Österreich. In: juridikum (2003), H. 1, S. 31-33.

5 BGBl. I Nr. 106/1946.

6 BGBl. Nr. 54/1947.

7 BGBl. I Nr. 181/1998, zuletzt geändert durch BGBl. I Nr. 117/2009. 
„treuhänderischen“ Verwaltung von „Kulturgut“ in den Blick genommen werden, um Problemfelder aufzuzeigen, die durch die historischen Semantiken und die Zusammenhänge, in denen das Wort „Treuhand“, aber auch „Kulturgut“ genutzt wurde, entstehen können. Denn in rechtlichen Kontexten wurde vor allem der Begriff der Treuhand im 20. Jahrhundert verschieden verwendet. Sei es im Dienste der Zivilisation wie bei der Wendung „a sacred trust of civilization “8 für Mandatsgebiete des Völkerbunds oder in der Figur der Treuhandschaft des gemeinsamen Kulturerbes der Menschheit. ${ }^{9}$

\section{Die Treuhand als Metapher des Rechts}

Bei der Treuhand handelt es sich um eine Rechtsfigur, die ihre Ursprünge in der Antike hat und ihre Bedeutung zunächst im Familien-, Erb- und Schuldrecht entfaltete. ${ }^{10}$ Ihre wesentliche und für das moderne Wirtschaftsleben relevante Ausprägung erfuhr die Treuhand aber erst gegen Ende des 19. Jahrhunderts im kontinentaleuropäischen Rechtssystem. ${ }^{11}$ Trotzdem war und ist in keinem der "großen“ europäischen Zivilgesetzbücher die Treuhand explizit geregelt. Sie beruht meistens auf Rechtsanalogien, Richterrecht oder wird als gewohnheitsrechtlich gültig anerkannt. ${ }^{12}$

Was also als „Treuhand“ bezeichnet wurde und wird, ist zwar historisch gewachsen, rechtlich gesehen jedoch flexibel. Klare Normen dazu finden sich weder im heute noch gültigen Allgemeinen Bürgerlichen Gesetzbuch (ABGB) aus 1812 noch dem deutschen Bürgerlichen Gesetzbuch (BGB) von 1900. Der Auftrag, die sogenannte Geschäftsführung ohne Auftrag und die Regeln zur Stellvertretung sind im heutigen Obligationenrecht jene Rechtsgeschäfte, die dem gewöhnlichen Verständnis einer Treuhand am nächsten kommen und die zur Analogiebildung herangezogen werden. ${ }^{13}$ Die Privatautonomie im zivilrechtlichen Vertragsrecht überlässt es dabei dem Ermessen der Parteien, welche

8 Article 22 Covenant of the League of Nations; zum Mandatssystem siehe Susan Pedersen: The Guardians. The League of Nations and the Crisis of Empire. Oxford: Oxford University Press 2015.

9 Barbara Genius-Devime: Bedeutung und Grenzen des Erbes der Menschheit im völkerrechtlichen Kulturgüterschutz. Baden-Baden: Nomos Verlagsgesellschaft 1996, S. 353-358.

10 Peter Apathy: Die Treuhandschaft aus rechtshistorischer Sicht. In: Die Treuhandschaft. Hg. von Peter Apathy. Wien: Manz'sche Verlags- und Universitätsbuchhandlung 1995, S. 1-17.

11 Helmut Coing: Europäisches Privatrecht. 19. Jahrhundert. Überblick über die Entwicklung des Privatrechts in den ehemals gemeinrechtlichen Ländern. Bd. 2. München: C.H. Beck'sche Verlagsbuchhandlung 1989, S. 423-427.

12 Daniel Rubin: $\$ 1002$ ABGB. In: ABGB-ON - Kommentar zum Allgemeinen bürgerlichen Gesetzbuch. Hg. von Andreas Kletečka, Martin Schauer. Wien: Manz 2015, Rz. 90-220.

13 Manfred Umlauft: Die Treuhandschaft aus zivilrechtlicher Sicht. In: Die Treuhandschaft. Hg. von Peter Apathy. Wien: Manz'sche Verlags- und Universitätsbuchhandlung 1995, S. 18-68. 
Geschäfte als Treuhand bezeichnet werden. ${ }^{14}$ Jedoch wird zur Erklärung wie zur Lösung von konkreten Rechtsfragen immer wieder auf einen Treuhandgedanken rekurriert. $^{15}$

Im gewissen Sinne kann das rechtliche Institut der Treuhand damit als eine Metapher des Rechts betrachtet werden: eine Konstruktion, die eine bestimmte Semantik suggeriert, aber anhand der konkreten Umstände bestimmt werden muss. Der Begriff Metapher bezeichnet hier das Übertragen einer sinnlichen Begebenheit in eine geistige Bedeutung. ${ }^{16}$ Ein Rechtsgeschäft, einen Rechtsvorgang und eine Rechtssituation als Treuhand oder als treuhänderisch zu beschreiben, ist somit aus einer rein dogmatischen Perspektive eine Zuschreibung, die sich nicht auf ein Gesetz stützen kann, sondern stets im rechtlichen Diskurs erschlossen werden muss. Die epistemologische Grundlage, um die Rechtsfigur der Treuhand zu erkennen, stellt zu einem wichtigen Teil auch auf das Alltagsverständnis ab. Darin liegt die sinnliche Wirklichkeit der Treuhand, die in rechtliche Tatbestände transformiert wurde.

Bei der Beschäftigung mit dem Begriff der „treuhänderischen Verwaltung“ von Kulturgut mit NS-Provenienz macht es daher Sinn, zunächst den zeitgenössischen Treuhandbegriff einzugrenzen. Für die Zeit des Nationalsozialismus sowie die unmittelbaren Nachkriegsjahre findet sich in Trübners deutsches Wörterbuch ein Indiz für eine solche historische Semantik der Treuhand. Ab 1934 wurde das Wörterbuch zu Ehren des Verlegers Karl J. Trübners geplant. Bis in die Nachkriegszeit der 1950er Jahre hinein wird dieses Wörterbuch mit einschlägig ideologischer Ausrichtung herausgegeben. ${ }^{17}$ Zwar gibt es darin kein eigenes Lemma zur Treuhand, doch der Eintrag zu Treue zeigt das breite Spektrum des Begriffsverständnisses von einer besonders historisch aufgeladenen Semantik („Nibelungentreue“) bis hin zu einem Verständnis als moralische Grundhaltung (Treue als „allgemeines Verhalten“). ${ }^{18}$

Diese ideologische Semantik trifft dabei auf die bewegliche und argumentative Grunddisposition des Rechtsinstituts der Treuhand, welches keinen festen

14 Helmut Koziol, Rudolf Welser und Brigitta Zöchling-Jud: Grundriss des bürgerlichen Rechts. Bd. 2. Wien: Manz 2015, S. 13-15.

15 Nils Jansen: $\$ \$ 677-687$ Geschäftsführung ohne Auftrag. In: Historisch-kritischer Kommentar zum BGB. Schuldrecht: Besonderer Teil $\$ \$ 433-853$. [Bd.] 3. Hg. von Mathias Schmoeckel, Joachim Rückert und Reinhard Zimmermann. Tübingen: Mohr Siebeck 2013, S. 1755 f, Rz. 86 und S. 1816f, Rz. 25.

16 Jörg Michael Schindler: Rechtsmetaphorologie - Ausblick auf eine Metaphorologie der Grundrechte (= Schriften zur Rechtstheorie 281). Berlin: Duncker \& Humblot 2016, S. $27 \mathrm{f}$.

17 Wenke Mückel: „Trübners Deutsches Wörterbuch“ (Band 1-4) - ein Wörterbuch aus der Zeit des Nationalsozialismus. Eine lexikografische Analyse der ersten vier Bände (erschienen 1939-1943). Tübingen: Max Niemeyer Verlag 2005, S. 5-20.

18 Treue. In: Trübners deutsches Wörterbuch. Bd. 7. Hg. von Walther Mitzka. Berlin: De Gruyter 1956, S. 113-115. 
positivistischen Kern in einem Gesetz findet. Das macht den rechtlichen Ausdruck Treuhand mit seinen außerrechtlichen Konnotationen besonders offen für die Indienstnahme von verschiedenen Seiten mit ihren jeweiligen Interessen.

So wurde der Begriff „treuhänderische Übernahme“ bereits in der Verwaltungspraxis der NS-Bürokratie benutzt. Er bezeichnete etwa die Bemächtigung des Mobiliars und der Wertgegenstände all jener, die in Sammelwohnungen gezwungen und später deportiert wurden. ${ }^{19}$ Ebenso wurde in besetzten polnischen Gebieten die Haupttreuhandstelle Ost oder der Generaltreuhänder beauftragt, Kulturgüter und Wertgegenstände zu requirieren. ${ }^{20}$ Die entsprechende Verwendung des Wortes Treuhand reicht über das Ende des Kriegs hinaus. Österreichische Behörden benannten im Jahr 1951 die Verwaltung des aus Depots und Bergungsorten stammenden „Kulturguts“ als „treuhändig“. ${ }^{21}$

In Deutschland gründete das Auswärtige Amt 1952 die Treuhandverwaltung von Kulturgut München, welche die Bestände des Central Collecting Points München übernahm, die zuvor der Bayrische Ministerpräsident treuhänderisch verwaltete. ${ }^{22}$ Aus dem Central Collecting Point Wiesbaden wurden ebenfalls Bestände an die Treuhandverwaltung in München im Jahr 1952 übergeben. ${ }^{23}$ Für die rituellen-jüdischen Gegenstände wurde die Jewish Cultural Reconstruction, Inc. treuhändig mit der Verwaltung erbenloser Kulturgüter jüdischer Provenienz betraut, die sich in der deutschen Besatzungszone unter amerikanischer Militärverwaltung befanden. ${ }^{24}$ In den französischen und britischen Sektoren wurden außerdem jeweils die Jewish Trust Corporations gegründet.

Alle diese Sachverhalte konkret als „Treuhand“ zu bezeichnen oder gerade nicht als Treuhand zu bezeichnen, impliziert Vorannahmen, die Fragen aufwerfen: Weshalb spielte das Konstrukt der Treuhand eine zentrale Rolle? Wem gegenüber bestand und besteht ein Treueverhältnis? Welche Verpflichtungen

19 Siehe den Beitrag von Philipp Mettauer in diesem Band.

20 Für diesen Hinweis danke ich Nawojka Cieślińska-Lobkowicz. Siehe Michael H. Kater: Das „Ahnenerbe“ der SS 1935-1945. Ein Beitrag zur Kulturpolitik des Dritten Reiches. 4. Aufl. München: R. Oldenbourg Verlag 2006, S. 149.

21 Gerhard Sailer: Rückbringung und Rückgabe: 1945-1966. In: Kunstraub, Kunstbergung und Restitution in Österreich 1938 bis heute. Hg. von Theodor Brückler. Wien-Köln-Weimar: Böhlau Verlag 1999, S. 33.

22 Iris Lauterbach: Der Central Collecting Point in München. Berlin-München: Deutscher Kunstverlag 2015, S. 185-191.

23 Tanja Bernsau: Besatzer als Kuratoren? Der Central Collecting Point Wiesbaden als Drehscheibe für einen Wiederaufbau der Museumslandschaft nach 1945. Berlin: Lit Verlag 2013, S. 336-342.

24 Elisabeth Gallas: Das Leichenhaus der Bücher. Kulturrestitution und jüdisches Geschichtsdenken nach 1945. Göttingen: Vandenhoeck \& Ruprecht 2013, S. 134-173; Katharina Rauschenberger: The Restitution of Jewish Cultural Objects and the Activities of Jewish Cultural Reconstruction Inc. In: Leo Baeck Institute Yearbook 53 (2008), S. 193-211. 
bringt eine solche Treuhandschaft mit sich? Woraus ergeben sich diese Verpflichtungen des Treuhänders?

Hier lässt sich die multinormative Dimension der Fragestellung erkennen, ${ }^{25}$ denn es fließen zumindest völkerrechtliche, innerstaatliche und ethische Erwägungen in die Beantwortung dieser Fragen mit ein. ${ }^{26}$

\section{Vermeidung des Treuhandbegriffs in Österreich}

Eine Vorreiterrolle zur Klärung der Verhältnisse um die Kulturgüter nach dem Zweiten Weltkrieg nahm die völkerrechtliche Ebene ein. Die wichtige Rolle des Völkerrechts zeigte schon Thomas Armbruster in seiner Dissertation zur westlich-alliierten Besatzungszone in Deutschland auf. ${ }^{27}$ Die grundlegenden Instrumente waren hier zunächst die besatzungsrechtlichen Rückerstattungsgesetzgebungen, wobei in der amerikanischen Zone besondere Bemühungen unternommen wurden.

In Österreich war die Ausgangslage anders. Hier diente Artikel 26 Abs. 2 des Staatsvertrags von Wien 1955 (StV) als wichtiger Ausgangspunkt für die Verpflichtung Österreichs, die sogenannten „erblosen“ Vermögen zu übernehmen und zugunsten von NS-Opfern zu verwerten. ${ }^{28}$ Nach Abschluss des Staatsvertrags 1955 wurden im Zuge des Auffangorganisationsgesetzes Sammelstellen für Vermögen gegründet, denen das Vermögen der Opfer von nationalsozialistischen Verfolgungsmaßnahmen übertragen wurde. ${ }^{29}$ Andere Länder sind ähnli-

25 Miloš Vec: Multinormativität in der Rechtsgeschichte. In: Berlin-Brandenburgische Akademie der Wissenschaften (vormals Preußische Akademie der Wissenschaften). Jahrbuch 2008. Berlin: Akademie Verlag 2009, S. 155-166; Thomas Duve: Was ist „Multinormativität“? - Einführende Bemerkungen. In: Rechtsgeschichte. Zeitschrift des Max-Planck-Instituts für europäische Rechtsgeschichte 25 (2017), S. 88-101.

26 Andeutungen zu einem kulturgeschichtlichen Ansatz gegenüber Rechtsinstituten, wie er in diesem Beitrag geübt wird, sind zu finden bei Miloš Vec: Kulturgeschichte als implizite Praxis der Rechtsgeschichte. Selbstwahrnehmungen, Bekenntnisse, Vorbehalte. In: Zeitschrift für Neuere Rechtsgeschichte 36 (2014), Nr. 3/4, S. 261-287.

27 Thomas Armbruster: Rückerstattung der Nazi-Beute. Die Suche, Bergung und Restitution von Kulturgütern durch die westlichen Alliierten nach dem Zweiten Weltkrieg. Berlin: De Gruyter 2008.

28 Zur völkerrechtlichen Auslegung der Norm siehe Bruno Simma, Hans-Peter Folz: Restitution und Entschädigung im Völkerrecht. Die Verpflichtungen der Republik Österreich nach 1945 im Lichte ihrer außenpolitischen Praxis. Wien-München: Oldenbourg Verlag 2004, S. 258-260.

29 Ein Überblick findet sich bei Margot Werner, Michael Wladika: Die Tätigkeit der Sammelstellen. Wien-München: Oldenbourg Verlag 2004 (= Veröffentlichungen der Österreichischen Historikerkommission. Vermögensentzug während der NS-Zeit sowie Rückstellungen und Entschädigungen seit 1945 in Österreich 28). 
che Verpflichtungen eingegangen, um das „herrenlose“ und „erblose“ Vermögen von Vertriebenen nicht an den Staat heimfallen zu lassen. ${ }^{30}$

Diese normativ geführte Debatte über den Geltungsbereich von Artikel 26 Abs. 2 StV hatte ihre ethischen Implikationen. Während Österreich die von den Rückstellungsgesetzen umfassten Gegenstände als ausreichend für die Sammelstellen erachtete, drängten jüdische Organisationen und die Westalliierten auf eine Ausdehnung dieses Anwendungsbereichs, um mehr Objekte zu erfassen. Als Argument wurde vor allem angeführt, dass es ethisch nicht vertretbar wäre, das Vermögen der Vertriebenen gerade jenem Staat zu überlassen, in dem sich solch düstere Ereignisse abgespielt haben. Hier kollidierte somit die innerstaatliche Praxis nicht bloß mit einer weiten Auslegung des völkerrechtlichen Artikels 26 Abs. 2 StV, sondern auch mit dem ethischen Anspruch an die Zweite Republik.

Das Instrument der Treuhand stand zudem im Widerspruch mit dem Heimfallsrecht des Staates an erblosen Verlassenschaften. ${ }^{31}$ Vor allem aber betont die Metapher der Treuhand in diesem Kontext eine doppelte Verantwortung des Treuhänders: sie besteht einerseits dem in Gewahrsam genommenen Gut gegenüber und andererseits gegenüber den Opfern und Nachfolgeorganisationen wie etwa des Committee for Jewish Claims on Austria. ${ }^{32}$

In der Gesetzessprache wählte man stattdessen scheinbar neutrale Metaphern zur Bezeichnung für die involvierten Institutionen. Anstatt von „Treuhand“ war die Rede von „Auffangorganisationen“ und „Sammelstellen“, auch wenn das rechtliche Konstrukt einer Treuhand ganz ähnlich war. Erwähnenswert erscheint ebenso die Metapher der Reinigung im Kunst- und Kulturgutbereinigungsgesetz. Das Bild der Reinigung mutmaßlich unsauberer Eigentumsverhältnisse spricht eine deutliche Sprache. Es appellierte an ein Reinheits-Verständnis, das diese transkulturell und über viele Epochen hinweg als erstrebenswerten Zustand begreift. $^{33}$

Im Verwaltungsgebrauch wurde der Terminus „Treuhand“ jedoch durchaus

30 Ernst Féaux de la Croix: Interalliierte Reparationsagentur. In: Wörterbuch des Völkerrechts. Bd. 2. Hg. von Karl Strupp, Hans-Jürgen Schlochauer. Berlin: Walter de Gruyter \& Co. 1961, S. 29-31; Ignaz Seidl-Hohenveldern: Kriegsentschädigung. In: Wörterbuch des Völkerrechts. Hg. von Karl Strupp, Hans-Jürgen Schlochauer. Berlin: Walter de Gruyter \& Co. 1961, S. 337-343.

$31 \S 760$ ABGB eingeführt durch RGBl. 1914/276.

32 Brigitte Bailer: Österreich-Israel nach 1945: die Entschädigungsverhandlungen mit dem Committee for Jewish Claims on Austria. In: Israel - Geschichte und Gegenwart. Wien: Braumüller 2009, S. 53-64.

33 Michael Stolleis: Über Reinheit. In: ders., Margarethe und der Mönch. Rechtsgeschichte in Geschichten. München: C.H. Beck 2016, S. 258-274; Discourses of Purity in Transcultural Perspective (300-1600). Hg. von Matthias Bley, Nikolas Jaspert und Stefan Köck. LeidenBoston: Brill 2015. 
verwendet, wie von vielen Beiträgen in diesem Band belegt wird. Für eine solche selektive Wortwahl gab es wohl mannigfache Gründe und außenpolitische Argumente. Ebenso könnte die Übernahme von jener bereits oben erwähnten NSTerminologie wie etwa der „Haupttreuhandstelle Ost“ eine Rolle gespielt haben.

Diese Wortwahl in Österreich stellt sich gegen den Trend zum Treuhandbegriff, der sich vor allem unter den Westalliierten in Deutschland verfolgen lässt. Für das Staatsgebiet der Bundesrepublik wurden bereits die Jewish Trust Corporation und die Treuhandverwaltung von Kulturgut in München erwähnt. Auch in Frankreich wählte die Regierung explizit das Konstrukt einer öffentlichen Treuhand für die Verwaltung jener Kulturgüter, die während der NS-Zeit geraubt wurden und sich im Besitz Frankreichs befinden. ${ }^{34}$ Probleme bei der Restitution solcher Objekte bestehen jedoch bis in das 21 . Jahrhundert. ${ }^{35}$ Hier zeigt sich, dass die Treuhandkonstruktion allein kein Garant für eine entsprechende Genugtuung und Entschädigung ist und war. ${ }^{36}$ Jedoch impliziert(e) sie zumindest das Bewusstsein einer Verantwortung für solche Gegenstände, die in der österreichischen Gesetzessprache nicht zum Ausdruck kommt.

\section{Die Treuhand als Metapher des Kulturgüterschutzes}

Die Metapher der Treuhand hat eine weitere Bedeutung für die Verwahrung sowie Restitution von Kulturgütern mit NS-Provenienz. Ihren Ausdruck fand diese Bedeutungsvariante in einer ambivalenten Praxis zum dritten Rückstellungsgesetz, die lange Zeit prägend war. Diese bestand darin, dass zwar das Eigentum an geraubten Kulturgütern übertragen wurde und sie damit in gewisser Weise restituiert wurden. Jedoch durften diese oftmals nicht oder nur aufgrund von Gegengeschäften mit den rechtmäßigen EigentümerInnen außer Landes gebracht werden. ${ }^{37}$ Dies ist auf die behördliche Anwendung des entsprechenden Ausfuhrgesetzes zurückzuführen. Georg Graf spricht daher von einer „akquisitorischen Verwendung des Ausfuhrverbotsgesetzes“. ${ }^{38}$ Dieses

34 Isabelle Masne de Chermont, Didier Schulmann: Le pillage de l'art en France pendant l'occupation et la situation des 2000 œuvres confiées aux musées nationaux. Paris: La documentation Française 2000.

35 Philippe Dagen: La difficile restitution des œuvres d'art pillées par les nazis. In: Lemonde.fr, 24.04.2014, URL: http://www.lemonde.fr/culture/article/2014/04/24/des-uvres-deux-foisvolees_4406968_3246.html (abgerufen am 12.05.2017).

36 Hannes Hartung: Kunstraub in Krieg und Verfolgung. Die Restitution der Beute- und Raubkunst im Kollisions- und Völkerrecht. Berlin: De Gruyter 2005, S. 5.

37 Claire Fritsch: Überblick Kunstrestitution in Österreich. In: Handbuch Kunstrecht. Hg. von Alexander Pfeffer, Roman Alexander Rauter. Wien: Manz 2014, S. 243-263.

38 Georg Graf: Überlegungen zum Anwendungsbereich des $\$ 1$ Z 2 KunstrückgabeG. Exem- 
Gesetz wurde ursprünglich von der Provisorischen Nationalversammlung 1918 mit der Intention erlassen, die Ansprüche der verschiedenen Nachfolgestaaten der Habsburgermonarchie auf die in österreichischen Sammlungen befindlichen Kunstwerke abzuwehren. ${ }^{39}$ Schon die Bezugnahme auf die Krongüter und das sogenannte hofärarische Vermögen im Gesetz deuten dies eindeutig an. ${ }^{40}$

Der grundsätzliche Gedanke solcher Ausfuhrgesetze lautet, dass gewisse Kulturgüter integraler Bestandteil für die Identität der Bevölkerung sind. Er entwickelte sich im Laufe des 19. Jahrhunderts. ${ }^{41}$ Die konkrete rechtliche Begründung ist vielfältig und umstritten. ${ }^{42}$ In jedem Fall agiert der Staat hier als Treuhänder dieser Kulturgüter, insofern er sie vor der Ausfuhr bewahrt. Dies alles ungeachtet dessen, wer ihr Eigentümer ist. Dasselbe Gesetz diente nach dem Zweiten Weltkrieg dazu, die Ausfuhr der von den Nationalsozialisten ihren rechtmäßigen EigentümerInnen entzogenen Kunstwerke zu vereiteln. Diese anerkannte kulturelle Aufgabe des Staates wurde in der Nachkriegszeit von Behörden und der Regierung missbraucht, um sich der Wiedergutmachungsverantwortung zu entziehen. ${ }^{43}$

Nach dem Ende des Kalten Kriegs kam die Debatte um Entschädigung, Wiedergutmachung und Restitution des NS-Unrechts von Neuem auf. ${ }^{44}$ Von diplomatischer Ebene angeregt verlieh das Völkerrecht den ethischen For-

plarisch entwickelt anhand des Falles Klimt/Bloch-Bauer. In: Österreichische Notariatszeitung 79 (2005), Nr. 11, S. 321-337.

39 Einen guten Überblick über das Thema bietet Yves Huguenin-Bergenat: Kulturgüter bei Staatensukzession. Die internationalen Verträge Österreichs nach dem Zerfall der österreichisch-ungarischen Monarchie im Spiegel des aktuellen Völkerrechts. Berlin: De Gruyter 2010.

$40 \S 2$ Gesetz der Provisorischen Nationalversammlung vom 5. Dezember 1918, betreffend das Verbot der Ausfuhr und der Veräußerung von Gegenständen von geschichtlicher, künstlerischer oder kultureller Bedeutung, RGBl. 90/1918.

41 Thilo Franke: Die Nationalität von Kunstwerken. Berlin: de Gruyter 2012; Erik Jayme: Die Nationalität des Kunstwerks als Rechtsfrage. In: Internationaler Kulturgüterschutz. Hg. von Gerte Reichelt. Wien: Manz 1992, S. 7-30.

42 Stefan Turner: Das Restitutionsrecht des Staates nach illegaler Ausfuhr von Kulturgütern. Eigentumsordnung und völkerrechtliche Zuordnung. Berlin-New York: Walter de Gruyter 2002.

43 Eva Blimlinger: Rückstellungen und Entschädigungen in Österreich 1945 bis 2008. Ein Überblick. In: ...wesentlich mehr Fälle als angenommen. 10 Jahre Kommission für Provenienzforschung. Hg. von Gabriele Anderl, Christoph Bazil, Eva Blimlinger, Oliver Kühschelm, Monika Mayer, Anita Stelzl-Gallian und Leonhard Weidinger. Wien-Köln-Weimar: Böhlau Verlag 2009 (=Schriftenreihe der Kommission für Provenienzforschung 1), S. 17-33, hier S. 21.

44 Ein Überblick über die seither erschienene Literatur zum NS-Kunstraub ist zu finden bei Christian Welzbacher: Kunstschutz, Kunstraub, Restitution. Neue Forschungen zur Geschichte und Nachgeschichte des Nationalsozialismus. In: H-Soz-Kult, 13.12.2012, URL: http://hsozkult.geschichte.hu-berlin.de/index.asp $? \mathrm{id}=1296 \& \mathrm{view}=\mathrm{pdf} \& \mathrm{pn}=$ forum $\&$ type $=$ forschungsberichte (abgerufen am 12.05.2017). 
derungen nach Entschädigung positivrechtlichen Ausdruck. ${ }^{45}$ In Washington wurden am 3. Dezember 1998 die nicht bindenden Grundsätze zum Umgang mit Kunstwerken erlassen, die von Nationalsozialisten beschlagnahmt worden waren. ${ }^{46}$ Der Europarat verabschiedete am 4. November 1999 die Resolution Nr. 1205 zu geraubten jüdischen Kulturgütern. Innerstaatlich wurde in Österreich 1998 das Kunstrückgabegesetz erlassen und in der Folge die Provenienzforschung institutionalisiert. ${ }^{47}$ Außerdem wurde dem Nationalfonds der Republik Österreich für die Opfer des Nationalsozialismus Verwertungsaufgaben übertragen, um „erblose“ Kunstgegenstände aus öffentlichem Besitz zugunsten von NS-Opfern zu veräußern. Auf rechtlicher Ebene wurden damit Fortschritte erzielt, obwohl die Beschränkung des Anwendungsbereichs auf Bundeseigentum sowie das Fehlen von Verfahrensrechten nach wie vor zu Recht kritisiert werden. ${ }^{48}$ Jedoch wurden restituierte Güter mit dem zweiten Kunst- und Kulturgutbereinigungsgesetz 1986 sowie dem Kunstrückgabegesetz von 1998 von der staatlichen Treuhand ausgeschlossen. Wenn Kulturgüter restituiert werden sollen, sind sie nunmehr vom Anwendungsbereich des Ausfuhrgesetzes sowie des Denkmalschutzgesetzes ausgenommen. ${ }^{49}$

Parallel dazu entwickelte sich auf der Ebene der Staatengemeinschaft spätestens ab der Zwischenkriegszeit der Gedanke einer Treuhand des Staates nicht nur im eigenen innerstaatlichen Interesse, sondern für die internationale $\mathrm{Ge}$ meinschaft. ${ }^{50}$ Nach dem Zweiten Weltkrieg legt die Haager Konvention zum Schutz von Kulturgut bei bewaffneten Konflikten aus 1954 in ihrer Präambel eine ähnliche Verpflichtung der Treuhandschaft des einzelnen Staates fest. ${ }^{51}$ Der Staat

$45 \mathrm{Zu}$ den historischen Wurzeln der Indienstnahme des Völkerrechts für ethische Zwecke: Kristina Lovrić-Pernak: Morale internationale und humanité im Völkerrecht des späten 19. Jahrhunderts. Bedeutung und Funktion in Staatenpraxis und Wissenschaft. BadenBaden: Nomos 2013.

46 Washington Conference on Holocaust-Era Assets Proceedings. November 30-December 3, 1998. Hg. von James D. Bindenagel. Washington: U.S. Government Printing Office 1999.

47 Siehe hierzu den Sammelband: ...wesentlich mehr Fälle als angenommen. 10 Jahre Kommission für Provenienzforschung. Hg. von Gabriele Anderl, Christoph Bazil, Eva Blimlinger, Oliver Kühschelm, Monika Mayer, Anita Stelzl-Gallian und Leonhard Weidinger. Wien, Köln, Weimar: Böhlau Verlag 2009 (= Schriftenreihe der Kommission für Provenienzforschung 1).

48 Eindringliche Kritik ist etwa zu finden bei Alfred J. Noll: Abnehmende Anwesenheit. Ein Pamphlet zur Kunstrückgabe in Österreich. Wien: Czernin Verlag 2011.

49 Georg Graf: Die österreichische Rückstellungsgesetzgebung. Eine juristische Analyse. Wien, München: Oldenbourg Verlag 2003, S. 480-483.

50 Charles de Visscher: La Protection Internationale des Objets d'Art et des Monuments Historique. In: Revue de droit international et de législation comparée 16 (1935), S. 32-74 und S. 246-288; Sabine [von] Schorlemer: Internationaler Kulturgüterschutz: Ansätze zur Prävention im Frieden sowie im bewaffneten Konflikt. Berlin: Duncker \& Humblot 1992, S. 564-566.

51 Kerstin Odendahl: Kulturgüterschutz - Entwicklung, Struktur und Dogmatik eines ebenenübergreifenden Normensystems. Tübingen: Mohr Siebeck 2005, S. 118-121. 
wird demnach als eine Art Treuhänder jener auf seinem Staatsgebiet befindlicher Kulturgüter betrachtet, für dessen Schutz und Erhaltung er Sorge tragen soll. Die Verantwortlichkeit besteht dabei in erster Linie in Friedenszeiten zur Vorbereitung auf den Kriegsfall sowie im bewaffneten Konflikt selbst. Das Verständnis einer Treuhand für die Weltgemeinschaft entfaltete sich aber trotz des speziellen Geltungsbereichs der Haager Konvention 1954 weiter und erfuhr in der Welterbe-Konvention der UNESCO im Jahr 1972 noch eine weitere Stufe der Verrechtlichung.

Die Metapher und Figur der „Treuhand“ spielt eine wichtige Rolle im Kulturgüterschutz. Sowohl zur Begründung der Verpflichtungen der internationalen Gemeinschaft sowie der eigenen Bevölkerung gegenüber ist die Treuhand eine oft gewählte Metapher. Besonders die Konstruktion des Staates als Treuhänder oder Hüter spezieller Gegenstände, deren Erhaltung im künstlerischen, geschichtlichen oder kulturellen Interesse des Staates liegen, kann problematisch sein. Diese grundsätzlich legitime Einschränkung des privaten sowie öffentlichen Eigentums durch den Staat war Ausgangspunkt für eine rechtliche Rechtfertigungsstrategie. Dabei halfen den Behörden die großzügigen Formulierungen des 1918 erlassenen Ausfuhrgesetzes, dessen Intention bei der Erlassung es war, Ansprüche auf Herausgabe und Übereignung von Kulturgütern aus den ehemaligen Kronländern legistisch abzuwehren. Im Völkerrecht werden jedoch auch Positionen vertreten, die für den Verkehr mit Kulturgütern überhaupt keine Beschränkungen fordern. ${ }^{52}$ Problematisch sind die Implikationen solcher Forderungen aber ebenso.

\section{Die Metapher des „Kulturguts“}

Die rechtliche Definition des Begriffs Kulturgut ist weit davon entfernt, einheitlich zu sein. ${ }^{53}$ Innerstaatliche wie völkerrechtliche Begriffsbestimmungen aus verschiedenen Regelungsbereichen entwerfen jeweils ihr eigenes Verständnis von Kulturgut. In früheren wie heutigen rechtlichen Debatten wird dieser Begriff oft verwendet, dennoch gibt es jedoch kaum Untersuchungen zu seinen historischen Ursprüngen.

Der theoretische Anstoß zu einer Debatte über Kulturgut erfolgte in der Zwischenkriegszeit. In der Rechtssprache war der Begriff des Kulturguts dabei zeitgenössisch neu. Zuvor wurde in der Gesetzessprache entweder der Begriff

52 John Henry Merryman: Cultural Property Internationalism. In: International Journal of Cultural Property 12 (2005), S. 11-39.

53 Sabine [von] Schorlemer: Internationaler Kulturgüterschutz: Ansätze zur Prävention im Frieden sowie im bewaffneten Konflikt. Berlin: Duncker \& Humblot 1992, S. 46-48. 
von Gegenständen mit geschichtlicher, wissenschaftlicher, künstlerischer oder kultureller Bedeutung ${ }^{54}$ oder der Begriff des Denkmals ${ }^{55}$ verwendet. In der völkerrechtlichen Haager Landkriegsordnung 1907 war in Artikel 56 die Rede von "geschichtlichen Denkmälern, Werken der Kunst und Wissenschaft“. Außerdem wurden die Schutzgegenstände über die Zugehörigkeit zu speziellen Sammlungen oder Institutionen bestimmt, die öffentlichen und wohltätigen Zwecken dienen.

Nicht aus rechtlichen Debatten, sondern aus der Völkerkunde ging der Begriff des Kulturguts hervor. Er wurde maßgeblich vom deutschen Mediävisten und Völkerkundler sowie späteren Nationalsozialisten Hans Naumann (1886-1951) und seiner Theorie der gesunkenen Kulturgüter eingeführt. Naumann versucht in seinem ursprünglich 1922 erschienenen Werk zu zeigen, dass die „primitiven Volksgemeinschaften“ durch die „Kultur einer gebildeten Oberschicht“ geprägt sind. ${ }^{56}$ Hans Naumann unterteilt Kultur in „primitives Gemeinschaftsgut“ und die „wirkliche Kultur“, die im sozialen Oben entsteht und von dort nach unten absinkt. Diese Konzeption ist antagonistisch angelegt und beinhaltet zugleich ein Evolutionsmodell: Volkskultur, die sich verändert, ist Naumann zufolge durch von oben gesunkenes Kulturgut gebildet. Die Frage nach dem „gesunkenen Kulturgut" sowie dessen kritische Rezeption eröffnete in der deutschen Volkskunde die Möglichkeit, den Kultur-Begriff systematisch und kritisch zu erörtern. ${ }^{57}$

Kurze Zeit nach Naumann wendet sich ebenso der deutsche neukantianische Philosoph Heinrich Rickert der Kultur und dem Begriff des Kulturguts zu. Diesen akzentuiert er aber verschieden zu Naumann. Zunächst stellt Rickert fest: „Kultur ist der Inbegriff der Güter, die wir um ihrer Werte willen pflegen. ${ }^{\text {“58 }}$ Damit steht für Rickert die Frage zu beantworten, „wie alle die verschiedenen Kulturwerte und Kulturgüter des wissenschaftlichen, staatlichen und wirtschaftlichen, des künstlerischen und des religiösen Lebens sich zu einem einheitlichen Kulturganzen zusammenschließen. ${ }^{\text {“59 }}$ Das Wort Kulturgut wurde von

54 Ausfuhrgesetz, RGBl. 90/1918 (Anm. 40); Verordnung über den Schutz von Denkmalen und Kunstwerken, dtRGBl. 104/1920, S. 913.

55 In Deutschland Art. 150. Weimarer Reichsverfassung dtRGBl. 152/1919; in Österreich: Bundesgesetz vom 25. September 1923, betreffend Beschränkungen in der Verfügung über Gegenstände von geschichtlicher, künstlerischer oder kultureller Bedeutung (Denkmalschutzgesetz), BGBl. 533/1923.

56 Hans Naumann: Grundzüge der deutschen Volkskunde. Leipzig: Verlag von Quelle \& Meyer 1922, S. 1-6.

57 Wolfgang Kaschuba: Einführung in die Europäische Ethnologie. München: C.H. Beck 2012, S. 62.

58 Heinrich Rickert: Kant als Philosoph der modernen Kultur. Ein geschichtsphilosophischer Versuch. Tübingen: J. C. B. Mohr (Paul Siebeck) 1924, S. 7.

59 Ebd. 
Rickert 1924 in einem umfassenden Sinn gemeint und ist auf alle aufgezählten Bereiche Wissenschaft, Staat, Wirtschaft, Kunst und Religion anwendbar.

Der Begriff des Kulturguts wurde im deutschen Sprachraum in der weiteren Zwischenkriegszeit oft rezipiert und in verschiedenen anderen Kontexten verwendet. Seine Bedeutung wurde dabei zumeist, wie etwa bei Rickert, diffus gehalten und war funktionell bestimmt. „Kulturgut“ blieb damit für mehrere Gebiete und Disziplinen attraktiv und anwendbar, namentlich Religion, Pädagogik, Archäologie, Ethnologie, Orientalistik, Philologie und Recht. ${ }^{60}$

Der Staatsrechtler Hermann Heller nahm den Begriff des Kulturguts in seinem hegelianisch geprägten Buch zur Staatslehre auf und traf dabei die Unterscheidung der bloßen Materialität und der Wirklichkeit von Kulturgütern. ${ }^{61}$ Ist das Kulturgut bloß materiell vorhanden, entspräche das nicht automatisch der Wirklichkeit, da die Kultur, für die das Kulturgut eine Rolle gespielt hat, auch nicht mehr vorhanden sein kann. Nur in der Übereinstimmung der Äußerung des objektiven Geistes in der Materialität und der inneren Komponente durch den subjektiven Geist besteht nach Heller die Wirklichkeit des Kulturguts. ${ }^{62}$ Diese Definition legt nicht nur die Bindung von Kulturgut an den Staat nahe, sondern kündet auch von einem treuhänderischen Verständnis der staatlichen Verwaltung von Kulturgut.

Zur selben Zeit findet die rechtliche Normierung des Begriffs „Kulturgut“ durch den NS-Staat statt, nämlich in der ersten Verordnung zur Durchführung des Reichskulturkammergesetzes. ${ }^{63}$ Darin hieß es 1933:

\section{„5. Kulturgut}

Kulturgut im Sinne dieser Verordnung ist:

1. jede Schöpfung oder Leistung der Kunst, wenn sie der Öffentlichkeit übermittelt wird,

2. jede andere geistige Schöpfung oder Leistung, wenn sie durch Druck, Film oder Funk der Öffentlichkeit übermittelt wird. “64

60 Siehe beispielsweise Katholisches Kulturgut als Bildungsstoff: eine Reihe von Abhandlungen. Hg. von Friedrich Schneider. Paderborn: Schöningh Verlag 1925; Arthur Hoffmann: Kulturgut und Schule. Erfurt: Verlag von Kurt Stenger 1925; Eckhard Unger: Altindogermanisches Kulturgut in Nordmesopotamien. Leipzig: Otto Harrassowitz 1938.

61 Siehe zu Hermann Heller im Allgemeinen: Michael Stolleis: Geschichte des öffentlichen Rechts in Deutschland. Dritter Band 1914-1945. München: C.H. Beck 1999, S. 183-186.

62 Hermann Heller: Staatslehre. Leiden: A.W. Sijthoff's Uitgeversmaatschappij N.V. 1934, S. 37-48.

63 Für zeitgenössische Schriften zur Reichskulturkammer siehe Günther Gentz: Handbuch der Reichskulturkammer. Hg. von Hans Hinkel. Berlin: Deutscher Verlag für Politik und Wirtschaft 1937; Karl-Friedrich Schrieber: Die Reichskulturkammer. Organisation und Ziele der deutschen Kulturpolitik. Berlin: Junker und Dünnhaupt Verlag 1934.

64 RGBl. I 1933, S. 797. 
Der Begriff des „Kulturguts“ ging in der Folge in weitere NS-Vorschriften und Anordnungen ein, etwa in die „Reichskulturkammer der bildenden Künste“ oder in exekutive Anweisungen der SS-Verwaltung. Oft wird in Aufzählungen dargelegt, welche Gegenstände unter „Kulturgut“ zu verstehen sind, womit er gleichzeitig zum Rechtsbegriff wird. ${ }^{65}$ Naumanns Theorie der gesunkenen Kulturgüter steht dabei im Hintergrund dieser rechtlichen Normierung, da die Übermittlung an die Öffentlichkeit das zentrale Kriterium ist. Dies wird etwa in den verschiedenen Anordnungen zum sogenannten Berufsschutz der Angehörigen der Reichskulturkammer festgehalten. In Naumannscher Terminologie gesprochen versuchte die Kammer damit, eine „Oberschicht“ in sich zu vereinen und zu kontrollieren. Die Vermittlung an die Öffentlichkeit konnte dann als „Sinken“ der Kulturgüter verstanden werden.

Aus diesen kurzen Andeutungen einer Begriffsgeschichte wird bereits deutlich, dass „Kulturgut“ nicht nur ein Begriff des Rechts ist, sondern „Kulturgüterschutz" als Rechtsinstitution mitunter in problematischer Weise das Gemeininteresse an gewissen Objekten betont und überzeichnet. Aktuelle Darstellungen des Kulturrechts nehmen diese problematische Vergangenheit des Begriffs nicht immer wahr. ${ }^{66}$

Nicht zuletzt spiegelt sich hier auch die „Treuhand“ als Metapher des Kulturgüterschutzes wieder. Der scheinbare neutrale Begriff „Kulturgut“, wie er etwa seit 1949 im deutschen Grundgesetz (heute in Artikel 73 Abs. 1 Z. 5a „den Schutz deutschen Kulturgutes gegen Abwanderung ins Ausland“) festgeschrieben ist, indiziert die staatliche Aufgabe, für die Kultur seines Volks Sorge zu tragen. ${ }^{67}$ Der Konflikt von Einzelinteressen mit einem Gemeininteresse ist dem Kulturgüterschutz damit konzeptionell eingeschrieben. Durch die Übernahme des Terminus in den allgemeinen wie wissenschaftlichen Sprachgebrauch wird die Wahrnehmung weniger auf Eigentumsverhältnisse als auf das öffentliche Interesse an jenen Gegenständen geleitet.

65 Siehe z. B. Erste Anordnungen des Präsidenten der Reichskammer der bildenden Künste betr. den Schutz des Berufes und die Berufsausübung der Kunst- und Antiquitätenhändler vom 4. August 1934, abgedruckt in: Das Recht der Reichskulturkammer. Hg. von Karl-Friedrich Schrieber, Alfred Metten und Herbert Collatz. Bd. 1. Berlin: De Gruyter 1943, RdbK VII, 1.

66 Siehe etwa die meines Erachtens demzufolge verkürzte Darstellung bei: Sophie-Charlotte Lenski: Öffentliches Kulturrecht. Materielle und immaterielle Kulturwerke zwischen Schutz, Förderung und Wertschöpfung. Tübingen: Mohr Siebeck 2013, S. 29-35; Kerstin Odendahl: Kulturgüterschutz. Entwicklung, Struktur und Dogmatik eines ebenenübergreifenden Normensystems. Tübingen: Mohr Siebeck 2005, S. 70-83.

67 Grundgesetz für die Bundesrepublik Deutschland vom 23. Mai 1949, dtBGBl. S. 1. Zuletzt geändert durch Artikel 1 des Gesetzes vom 23.12.2014, dtBGBl. I S. 2438. 


\section{Conclusio: (Re)Interpretation der Treuhandmetapher}

Die „Treuhand“ ist eine Metapher des Rechts, die nicht gesetzlich normiert ist. Als Metapher konstruiert sie weitreichende, moralisch gegründete Verantwortlichkeitsverhältnisse. Ihre Bedeutung sowie die positivrechtlichen Implikationen ergeben sich jeweils aus dem Kontext. Im Fall der treuhänderischen Verwaltung von Kulturgut mit NS-Provenienz kommt es zu einem Zusammenwirken von Normen auf einer völkerrechtlichen und innerstaatlichen Ebene, die mit ethischen Ansprüchen in einem spannungsreichen Diskurs stehen.

Eine generelle Bewertung der Treuhandmetapher ist nicht möglich, denn ihre Funktionen hängen jeweils vom Anwendungskontext ab. Es sind die konkreten Interessen, die hinter der Nutzung der Treuhandmetapher stehen, welche ihre konkrete Ausgestaltung am meisten beeinflussen. Was es also heißt, „treuhänderisch“ zu verwalten, wem gegenüber ein Treueverhältnis bestehen soll und welche Verpflichtungen sie begleiten, hängt jeweils vom Zusammenhang ab.

Für den Bereich des Völkerrechts und innerstaatlichen Rechts in Österreich und Deutschland wurde dargestellt, wie die verschiedenen Verständnisse von Treuhand miteinander kollidierten oder sich gar offen widersprochen haben. Sowohl der Verzicht als auch das Bestehen auf treuhänderischer Verwaltung konnte dabei - abhängig vom Kontext - zu denselben Resultaten führen. So war es die völkerrechtlich anerkannte Aufgabe des Staates, die wesentlichen Kulturgüter des Landes treuhändig zu verwalten und $\mathrm{zu}$ binden, was sich im innerstaatlichen österreichischen Recht etwa in den Regelungen zur Kulturgutausfuhr widerspiegelte. Obwohl sie unter anderen historischen Kontexten entstanden sind, prägen sie noch immer das Rechtsverständnis. Der Treuhandschaftscharakter ist der Geschichte des heute so gängigen (Rechts)Begriffs „Kulturgut“ mit seiner Betonung von Gemeininteressen besonders stark eingeschrieben, wie die historische Analyse dieser Metapher gezeigt hat.

Mit dem $₫ 4$ des Kunstrückgabegesetzes 1998, der festlegt, dass zu restituierende Gegenstände von den Ausfuhrbestimmungen des Denkmalschutzgesetzes ausgenommen sind, wurde jedoch die Treuhand des Staates über „seine“ Kulturgüter durchbrochen. Diese Normen, die Beschlüsse und Gesetze auf Ebene der Bundesländer sowie die internationalen Bemühungen etwa in Form der Washingtoner Erklärung, legen schließlich die (Re)Interpretation der Treuhandmetapher nahe. Statt einer treuhänderischen Verwaltung, welche die Ausfuhr der betroffenen Kulturgüter verhüten will, soll die Verantwortung gegenüber den Opfern des Nationalsozialismus in den Fokus kommen. Eine solche (Re)Interpretation des staatlichen Handelns als Treuhand würde auch die moralische Selbstverpflichtung Österreichs verstärken und möglicherweise rechtspolitischer Anlass für weitere Bemühungen um Restitution sein. 
Open-Access-Publikation im Sinne der CC-Lizenz BY-NC-ND 4.0

(c) 2018, V\&R unipress GmbH, Göttingen

ISBN Print: 9783847107835 - ISBN E-Lib: 9783737007832 


\title{
Leonhard Weidinger
}

\section{The Mauerbach Stock - Where Did the So-Called Ownerless Objects Come From?}

\begin{abstract}
Zusammenfassung
Der Mauerbach-Bestand - Woher stammen die so genannten erblosen Objekte?

Kunst- und Kulturgüter aus dem ehemaligen Central Collecting Point in München, die an Österreich übergeben worden waren, und solche, die in Österreich nach dem Ende des NSRegimes und des Zweiten Weltkriegs „übriggeblieben“ waren, wurden vom Bundesdenkmalamt in treuhänderische Verwaltung übernommen. Sie bildeten ab 1966 den sogenannten Mauerbach-Bestand. Der kleinere Teil der Objekte wurde nach den Kunst- und Kulturgutbereinigungsgesetzen 1 und 2 restituiert, der größere Teil 1996 in der Mauerbach-Auktion zugunsten von NS-Opfern versteigert. Dass es möglich gewesen wäre, viele der früheren EigentümerInnen zu ermitteln und Objekte zu restituieren, ist bekannt. In diesem Beitrag sollen die Provenienzen der Objekte des Mauerbach-Bestands in einen breiteren Blick genommen werden.
\end{abstract}

Schlagwörter

Mauerbach-Bestand, erblose Objekte, Mauerbach-Auktion, Central Collecting Point München

\begin{abstract}
The Federal Monument Authority took over "in trust" cultural objects from the former Central Collecting Point in Munich that were handed over to Austria, as well as others that were left behind after the end of the Nazi regime and World War II. From 1966 onwards, they formed the so-called Mauerbach stock. A small number of these objects were restituted following the Art and Cultural Assets Settlement Law, whereas the bulk of the objects were sold in the Mauerbach Auction for the benefit of Nazi victims in 1996. It is a fact that it would have been possible to identify many of the previous owners and to restitute objects. This talk attempts a broader look at the provenance of the objects of the Mauerbach stock.
\end{abstract}

Keywords

Mauerbach Stock, Ownerless Objects, Mauerbach Auction, Central Collecting Point Munich 
The translocation of works of art was an important topic in the Third Reich: The objects mainly taken from Jewish collectors, but also from other "enemies" of the National Socialists - political opponents, religious institutions, foreigners from wartime enemy countries, etc. - had to be stored, inventoried, and distributed. The "Sonderauftrag Linz" brought together art from all over Europe for the socalled "Führermuseum" Hitler wanted to have built in Linz after the victory of the Third Reich. The Wehrmacht and Nazi organizations like "Dienststelle Mühlmann" and "Einsatzstab Reichsleiter Rosenberg" plundered works of art in the occupied countries. As the air raids on German cities constantly increased during World War II, objects from museums were transferred to cellars inside cities or to castles, abbeys and houses in the countryside, where they would be safe from bombs. ${ }^{1}$ When the war ended in May 1945 many depots filled with works of art were spread all over the defeated Third Reich, and also the reestablished Republic of Austria, whose eastern part was then occupied by the Soviet Union, while the western and southern provinces lay under control of the British, the French and the US Army. To administer the works of art in the depots in the American occupation zone the Monuments, Fine Arts, and Archives Section of the US Army set up Central Collecting Points (CCP). Objects from the Austrian part of the American occupation zone, especially those thousands that had been designated to be on display at the "Führermuseum" in Linz and stored in the salt mine of Altaussee, were transported to the Central Collecting Point in Munich. In the following years many objects from the CCP in Munich were handed over to the Republic of Austria with the order to restitute them to the former owners. But Austria demanded more objects - on the one hand, because they had been deposited in Altaussee, on Austrian territory, and on the other hand, to compensate war losses. ${ }^{2}$ Although these claims were criticized especially by Germany, ${ }^{3}$ over 900 objects were transported from Munich to Salzburg and brought to a depot of the Austrian Federal Monuments Authority in January 1952. On what basis these pieces had been selected is not really clear. The Federal Monuments Authority didn't seem to have a concept on what to do with them and simply stored them.

By the second half of the 1940s the art depots in Austria had been vacated. Just one depot, the castle of Immendorf, had been destroyed completely in May 1945;

1 Cf. Bergung von Kulturgut im Nationalsozialismus. Mythen - Hintergründe - Auswirkungen. Ed. by Pia Schölnberger, Sabine Loitfellner. Vienna-Cologne-Weimar: Böhlau 2016 (= Schriftenreihe der Kommission für Provenienzforschung 6).

2 Cf. Iris Lauterbach: Der Central Collecting Point in München. Berlin-Munich: Deutscher Kunstverlag 2015 (= Veröffentlichungen des Zentralinstituts für Kunstgeschichte in München 34), pp. 151-159.

3 For instance N.N.: Die Eigentumsfrage der 967 Kunstwerke. In: Die Weltkunst, February 1, 1952, p. 5. The author thanks Meike Hopp for this article. 
others had been damaged and plundered. All in all the total loss of objects due to war damage was not very high. ${ }^{4}$ Until 1949, the Federal Monuments Authority coordinated the return transport from the depots. Most of the objects had to be brought back to the museums they belonged to; some came from private collections - and there were also seized pieces. From 1945 on the Republic of Austria set legal measures, in particular seven restitution laws, to return property that had been seized by or under the Nazi regime. Many works of art were restituted in the following years, although a number of objects were not allowed to leave the country in accordance with export laws. ${ }^{5}$ For some objects there were no claims they stayed behind and through the years more and more "ownerless" items came under the administration of the Federal Monuments Authority.

On July 27, 1955, the State Treaty for the re-establishment of an independent and democratic Austria, signed by the United States, the United Kingdom, France, the Soviet Union, and Austria, came into force. Article 26 of the treaty obligated the Austrian Republic to restitute property seized under the Nazi regime in the cases where this had not already happened. If there were no heirs and/or claims, the items were to be given, within 18 months, to an organization supporting victims of the Third Reich and its confederates. ${ }^{6}$ So in 1957 the "Sammelstellen" were established. ${ }^{7}$ But these institutions were not responsible for the "ownerless" works of art. As the limits set by the earlier restitution laws had expired, there was no legal possibility for Nazi victims to claim the restitution of these objects.

In 1966 the remaining "ownerless" works of art and cultural objects were brought together in the former Carthusian monastery of Mauerbach, northwest of Vienna. Nobody was allowed to see these objects - only the officials of the

4 Especially compared to the losses of museums in Germany. Although Burgenland, Lower Austria, Vienna, and parts of Upper Austria were under Soviet control there were no so-called trophy commissions that transferred works of art to the Soviet Union like in the later GDR. Just one case has been documented: In 194739 paintings were taken away from Thürntal castle by the Soviets. Cf. Theodor Brückler: Schloß Thürntal als Kunstgut-Bergungsort während des Zweiten Weltkriegs. In: Jahrbuch für Landeskunde von Niederösterreich 63/64 (1997/98). Tradition im Fortschritt. Hermann Riepl zum 60. Geburtstag, pp. 205-224, here p. 221.

5 Cf. Clemens Jabloner, Eva Blimlinger: The Regulation of the Restitution of Artworks in Austria. In: Verantwortung wahrnehmen. NS-Raubkunst - Eine Herausforderung an Museen, Bibliotheken und Archive / Taking Responsibility. Nazi-looted Art - A Challenge for Museums, Libraries and Archives. Magdeburg: Koordinierungsstelle für Kulturgutverluste 2009 (=Veröffentlichungen der Koordinierungsstelle für Kulturgutverluste 7), pp. 225-243.

6 Österreichischer Staatsvertrag. Wikisource. URL: https://de.wikisource.org/wiki/Österreichi scher_Staatsvertrag (accessed on August 15, 2017).

7 Cf. Margot Werner, Michael Wladika: Die Tätigkeit der Sammelstellen. Vienna-Munich: Oldenbourg 2004 (= Veröffentlichungen der Österreichischen Historikerkommission. Vermögensentzug während der NS-Zeit sowie Rückstellungen und Entschädigungen seit 1945 in Österreich 28). 
Federal Monuments Authority who administered the depot. This led to the myth of the Mauerbach "treasure": Nazi looted art hidden from the public eye.

As early as 1965 Simon Wiesenthal (1908-2005) sent a letter to the Austrian government demanding a solution for "ownerless" works of art under control of the state. This seems to have been the final impulse for the Austrian officials to set up a new law: On June 27, 1969 the Austrian parliament passed the Art and Cultural Assets Settlement Act. ${ }^{8}$ In September 1969 a list of 1,231 positions covering 8,422 objects was published in the Wiener Zeitung, the Austrian State Gazette. Copies of this list were posted at Austria's diplomatic missions to inform Nazi victims and their heirs who had fled from the Third Reich and lived abroad. The descriptions of the objects were taken from the existing transport protocols and inventories, and were therefore rather condensed. The officials presented this as a possible way for proving the validity of claims: A former owner of an object should know his property, and be able describe it in detail. ${ }^{9}$ The concept did not work: many objects were demanded by two or more claimants, and the judges often could not decide who was the original owner and settled the lawsuits, or they dismissed the claims altogether. ${ }^{10}$ By December 31, 1972343 persons had claimed 1,231 positions, but only 72 positions containing 269 objects were restituted. ${ }^{11}$ The remaining 1,159 positions with 8,153 objects became the property of the Republic of Austria, which had paid five million Schillings to the "Sammelstellen". The Federal Monuments Authority still administered the remaining objects in Mauerbach, no longer as a trustee, but as representative of the new owner, the Republic of Austria.

The fact that only a small portion of the Mauerbach stock had been restituted was not a satisfying result. In December 1984 the magazine ARTnews published Andrew Decker's article "A Legacy of Shame". ${ }^{12}$ It focussed once again on the Mauerbach objects, and claimed to have a solution. International debates on the

8 Bundesgesetz vom 27. Juni 1969 über die Bereinigung der Eigentumsverhältnisse des im Gewahrsam des Bundesdenkmalamtes befindlichen Kunst- und Kulturgutes. In: Bundesgesetzblatt für die Republik Österreich, (1969), I. 74, Nr. 294, pp. 1533-1535. Also online, URL: https://www.ris.bka.gv.at/Dokumente/BgblPdf/1969_294_0/1969_294_0.pdf (accessed on June 10, 2017).

9 Cf. Kurt Haslinger: Mauerbach und der lange Weg bis zur Auktion: 1969-1996. In: Kunstraub, Kunstbergung und Restitution in Österreich 1938 bis heute. Ed. by Theodor Brückler. Vienna-Cologne-Weimar: Böhlau 1999 (= Studien zu Denkmalschutz und Denkmalpflege XIX), pp. 39-52, here p. 42.

10 Sophie Lillie: "Herrenlos"? Die ungeklärte Akte Mauerbach. In: Recollecting. Raub und Restitution. Ed. by Alexandra Reininghaus. Vienna: Passagen Verlag 2009, pp. 211-223, here p. 217.

11 Cf. Haslinger: Mauerbach und der lange Weg bis zur Auktion (Fn. 9), pp. 43 f. A list of all the cases is published in Otto Fritscher: Kontroversen um den "Mauerbachschatz". Die Restitutionsverfahren von 1969 bis 1986 . Vienna: new academic press 2012, pp. 457-463.

12 Cf. Andrew Decker: A Legacy of Shame. In: ARTnews 83 (December 1984), pp. 55-75. 
topic followed and led to the Second Art and Cultural Assets Settlement Act that was passed by the Austrian parliament on December $13,1985 .{ }^{13}$ The procedure was the same as in 1969: The list of the objects was published in the Wiener Zeitung and posted at Austria's diplomatic missions. It was not really surprising that a system that had not worked properly the first time was not successful when repeated with nearly no changes.

Over 140 positions from the list were restituted on behalf of the Second Art and Cultural Assets Settlement Act. The remaining objects stayed in Mauerbach until 1994, then they were transferred to a depot at Schönbrunn Palace in Vienna. They were to be sold at an auction for the benefit of victims of the NationalSocialist regime. ${ }^{14}$ The amendment of the Second Art and Cultural Assets Settlement Act from August 4, 1995 set the stage for the sale. ${ }^{15}$ The Republic of Austria handed over the remaining objects to the Federal Jewish Community Organization. Christie's Auction House was commissioned to prepare the sale, and to publish a catalogue. ${ }^{16}$ The auction took place on October 29 and 30, 1996 at the MAK - Austrian Museum of Applied Arts in Vienna. The international response to the Mauerbach Auction was mainly positive in 1996. Even critics acknowledged that Austria had - better late than never - made a step toward facing up to its historical responsibility. The revenues of the auction were paid into the Mauerbach Fund that supported Nazi victims.

The year 1998 brought important changes in the approach to seized works of art: In Austria, the Commission for Provenance Research was established, and the Art Restitution Law $^{17}$ was adopted. In December 1998 representatives from 44 states signed the Washington Conference Principles on Nazi-Confiscated Art. ${ }^{18}$

13 Bundesgesetz vom 13. Dezember 1986 über die Herausgabe und Verwertung ehemals herrenlosen Kunst- und Kulturgutes, das sich im Eigentum des Bundes befindet (2. Kunst- und Kulturgutbereinigungsgesetz). In: Bundesgesetzblatt für die Republik Österreich, (1986), I. 2, Nr. 2, pp. 313-316. Also online, URL: https://www.ris.bka.gv.at/Dokumente/BgblPdf/ 1986_2_0/1986_2_0.pdf (accessed on June 10, 2017).

14 Cf. Haslinger: Mauerbach und der lange Weg bis zur Auktion (Fn. 9), pp. 50 f.

15 Bundesgesetz, mit dem das 2. Kunst- und Kulturgutbereinigungsgesetz geändert wird. In: Bundesgesetzblatt für die Republik Österreich, (1995), I. 168, Nr. 515, pp. 6669-6672. Also online, URL: https://www.ris.bka.gv.at/Dokumente/BgblPdf/1995_515_0/1995_515_0.pdf (accessed on June 10, 2017).

16 Mauerbach. Items Seized by the National Socialists to Be Sold for the Benefit of the Victims of the Holocaust. Ed. by Christie's. MAK - Österreichisches Museum für angewandte Kunst Vienna, 29 and 30 October 1996. Vienna: Christie's 1996, p. 39.

17 Bundesgesetz über die Rückgabe von Kunstgegenständen aus den Österreichischen Bundesmuseen und Sammlungen. In: Bundesgesetzblatt für die Republik Österreich, Teil I (1998), Nr. 181, pp. 2045f. Also online, URL: https://www.ris.bka.gv.at/Dokumente/ BgblPdf/1998_181_1/1998_181_1.pdf (accessed on June 10, 2017).

18 Cf. Anneliese Schallmeiner: 1998 - die Kommission für Provenienzforschung und der Weg zum Kunstrückgabegesetz. In: ... wesentlich mehr Fälle als angenommen. 10 Jahre Kommission für Provenienzforschung. Ed. by Gabriele Anderl, Christoph Bazil, Eva Blimlinger, 
Looking back on the Mauerbach stock from this newly developed point of view, it was soon evident that it would have been possible to find many of the original owners, if there had been pro-active provenance research into the works of art. In the exhibition Recollecting, that took place from December 2008 to February 2009 at the MAK, the Austrian Museum of Applied Arts in Vienna, Sophie Lillie and Arye Wachsmuth showed a video installation based on photos of provenance marks on the back sides of paintings from the Mauerbach stock. In additional texts and in the catalogue Sophie Lillie documented that in many cases of Mauerbach objects, it would have been possible to find out to whom the "ownerless" objects had belonged - and who their heirs were.

But there was another reason why it was complicated for former owners of Mauerbach items to find their property: Nearly all the reports, documentations, articles, etc. about the Mauerbach stock since the 1960s suggested that the objects kept in the monastery originally came from Austria, where they had been seized from Jews in the Nazi era from 1938 to 1945. In an article in ARTnews from 1984 Andrew Decker introduced his article with four examples of Nazi victims who had lived in Vienna in 1938 and had lost works of art under the Nazi regime. And the New York Times' article on the Mauerbach Auction stated: "Most of the art sold over the two days was owned by the approximately 65,000 Austrian Jews who did not survive the Holocaust." 19 But did the objects really come from Austria? And so: Where did the objects that became the Mauerbach stock in 1966 come from? When regarding the provenance, one can roughly categorise the over 8,400 objects in four groups:The first group of assets were objects from Hohenfurt Abbey, today Vyšší Brod in the Czech Republic. After the occupation of the Sudetenland in October 1938, Hohenfurth lay within the Third Reich. In 1941 the abbey was dissolved, and its buildings were used as a camp, a military hospital, and as a depot for the "Sonderauftrag Linz". After the war over 3,300 coins and medals, documents, and one painting from Hohenfurt Abbey came under the administration of the Austrian Federal Monuments Authority. Although there were clear indications of the abbey's ownership, just three registers and a book were restituted. ${ }^{20}$ The better part was sold in the Mauerbach Auction the numismatic collection as lots $801-1033$, books as lots 1034-1045, documents

Oliver Kühschelm, Monika Mayer, Anita Stelzl-Gallian and Leonhard Weidinger. ViennaCologne-Weimar: Böhlau 2009 (= Schriftenreihe der Kommission für Provenienzforschung 1), pp. 34-47.

19 The Associated Press: Art Stolen From Jews Is Auctioned in Vienna. In: The New York Times, October 31, 1996. URL: http://www.nytimes.com/1996/10/31/arts/art-stolen-from-jews-isauctioned-in-vienna.html (accessed on June 15, 2017).

20 Cf. Fritscher: Kontroversen um den "Mauerbachschatz" (Fn. 11), pp. 154 f. 
within the lots $738-748$, and the $18^{\text {th }}$ century painting Portrait of the Abbot of Hohenfurt Quirin Mickl as lot $93 .^{21}$

The second group of assets was also provided for the museum in Linz, specifically for its library. It was a collection of nearly 3,000 volumes of theatre literature, which the antiquary Rudolf Engel (1882-1955) had sold to Paul Heigl (1887-1945), the director general of the National Library in Vienna, in 1942. It was brought first to the depot at the former Kremsmünster Abbey, then to the Altaussee salt mine. In 1949 the books were handed over to the Republic of Austria by the US Property Control and Restitution Branch, and they became part of the Mauerbach stock. ${ }^{22}$ The collector Hans Ernst Weidinger bought the complete collection at the auction in 1996, where the books were offered as lots $749-760 .{ }^{23}$ From 2003 to 2006 the Da Ponte Institute in Vienna was in charge of the documentation, digitisation, and provenance research of the collection, in 2007 the Don Juan Archiv Wien took over the project, which is still in progress. ${ }^{24}$

The third group of assets consisted of approximately 1,200 objects that remained after the clearing of several depots: Fischhorn Castle near Zell am See, that was used by the SS at the end of World War II, depots that had been established from 1939 on to save objects from museums and collections from air raids and that were administered by the Federal Monuments Authority after the war, depots of the financial administration of Vienna, Lower Austria and Burgenland, and headquarters and other offices of the Allied troops after their withdrawal in 1955.

This group of 1,200 objects contained, except for one painting, all the pieces Sophie Lillie has identified as coming from the seized collections of Wilhelm Freund (1915-1963), Arthur Fuchs (1862-1940), Rudolf Gutmann (1880-1966), and others. Still, this group also included objects that were not seized under the Nazi regime, but came under control of the state for other reasons, for instance to save items from private collections from air-raids. Some of them "lost their provenance" in the often chaotic situation at the end of war, and could not be attributed to their original owners. Seven miniatures from the collection of Eugen Czernin (1892-1955) that were stored in the salt mine of Lauffen were presumed to be lost. As Imma Walderdorff found out, these miniatures were

21 Mauerbach (Fn. 16), pp. 70f; 338f; 349-381. Cf. Lillie: "Herrenlos"? (Fn. 10), p. 214.

22 Cf. Brigitte Dalinger: Die Theatersammlung "Komplex Mauerbach": Bericht von einer Spurensuche. In: Mitteilungen der Gesellschaft für Buchforschung in Österreich 2013-2, pp. 37-48, here pp. 39-43. Also online, URL: http://www.buchforschung.at/pdf/MB2013-2. pdf (accessed on August 16, 2017).

23 Mauerbach (Fn. 16), pp. 339f.

24 Cf. Dalinger: Die Theatersammlung "Komplex Mauerbach" (Fn. 22), pp. 37-48. The publication "Eine Theatersammlung 'aus fachmännischem Besitze' zwischen 'Führerbibliothek' und Mauerbach Benefit Sale" that was announced for 2014 in this text (p. 37) was not released until 2017. 
found in a private flat in 1946. They were handed over to the Federal Monuments Authority, where they were not recognized as the property of Eugen Czernin but rather kept as unclaimed good, resulting in them being brought to Mauerbach in 1966. Four miniatures were restituted following the Art and Cultural Assets Settlement Act - obviously not to their original owner. ${ }^{25}$ In 1996 three miniatures were sold in the Mauerbach Auction as lots 20, 96 and $412 \mathrm{f}^{26}$ But also objects from the Paintings Gallery of the Academy of Fine Arts in Vienna were part of the Mauerbach stock. The painting The Morra Players by Thomas Wick was restituted following the first Art and Cultural Assets Settlement Act - again not to its original owner - and subsequently sold to an art dealer who identified the work as property of the Academy of Fine Arts that finally bought the painting. The Martyrdom of Saint Peter, a copy after Titian, was sold in the Mauerbach Auction as lot 116. In the catalogue the provenance was described as "Akademie der bildende Künste, Vienna, before 1945."27 The Academy of Fine Arts Vienna or the Republic had the chance to claim the painting before the auction, but they didn't - it was sold.

The fourth group of assets contained the objects which the Federal Monuments Authority had taken over from the Munich Central Collecting Point. In January 1952 about 960 objects from the CCP were transported to a depot in Salzburg. The basis for this proceeding was the Inter-Allied Declaration against Acts of Dispossession committed in Territories under Enemy Occupation of Control that had been adopted in London on January 5, 1943. ${ }^{28}$ It declared all acquisitions in territories occupied by "the Enemy Powers" ${ }^{29}$ to be illegal. ${ }^{30}$ As the Declaration covered all categories of property, the rules for the restitution of works of art were specified in the following years. Following this concept, the Central Collecting Points were not to hand over the items to their original

25 Cf. Imma Walderdorff: Verschollene Miniaturen aus der Czerninschen Gemäldegalerie. Ausgelagert - gestohlen/verloren - wiedergefunden. In: Bergung von Kulturgut im Nationalsozialismus. Ed. by Schölnberger, Loitfellner (Fn. 1), pp. 383-400.

26 Mauerbach (Fn. 16), pp. 26; 70; 206.

27 Ibid., p. 82.

28 Inter-Allied Declaration against Acts of Dispossession committed in Territories under Enemy Occupation of Control (with covering Statement by His Majesty's Government in the United Kingdom and Explanatory Memorandum issued by the Parties to the Declaration) London, January 5, 1943. Commission for Looted Art in Europe. URL: http://www.loote dartcommission.com/inter-allied-declaration (accessed on June 15, 2017).

29 The Declaration did not name "the enemy Powers", just once they are defined as "Axis".

30 Inter-Allied Declaration against Acts of Dispossession committed in Territories under Enemy Occupation of Control (with covering Statement by His Majesty's Government in the United Kingdom and Explanatory Memorandum issued by the Parties to the Declaration) London, January 5. 1943. Commission for Looted Art in Europe. URL: http://www.loote dartcommission.com/inter-allied-declaration (accessed on June 15, 2017). Cf. Lauterbach: Der Central Collecting Point in München (Fn. 2), p. 21. 
owners. The works of art were given to the countries from which they had been acquired during the Third Reich, and it was considered to be those countries' duty to restitute the items. Restitutions to Austria were seen rather critically by German as well as US officials, as Austria- although accepted as a victim of the Third Reich since the Moscow Declaration of 1943 - and Austrians had played significant roles within the Nazi regime. Between 1946 and 1949 the Austrian Federal Monuments Authority sent representatives to the Munich CCP for several weeks or even months, and it took even more than two years to reach the final decision and send the items to Austria. ${ }^{31}$ The criteria for the selection of the objects to be handed over to are not really clear. In 1952 the Federal Monuments Authority received not only the objects, but also the related Property Cards, on which the results of the research of the Central Collecting Point were recorded who sold an object and when it was sold to the German Reich or Adolf Hitler. These file cards - and their information - were available to Austrian officials at the latest in 1952.

Today these file cards, over 900 in total, are kept in the archive of the Federal Monuments Authority, and can be found online via the Central Collecting Point Munich database on the website of Deutsches Historisches Museum. ${ }^{32}$ The comparison between the file cards and the record of delivery ${ }^{33}$ from January 1952 documents that all objects except six were brought to the Carthusian monastery of Mauerbach in 1966. The exceptions were six paintings: one of them was apparently not taken over, there is also no related file card in the archive of the Federal Monuments Authority. Four paintings were seized by the Einsatzstab Reichsleiter Rosenberg, and were therefore restituted in 1953 and 1955 to France. One painting was handed over to the Kurpfälzisches Museum Heidelberg as a loan. ${ }^{34}$ None of the paintings that were taken over from the Munich Central Collecting Point in January 1952 were handed over to an Austrian federal museum or institution.

More than half of the 909 file cards - exactly 485 - contain information about the provenance of the items. 23 pieces, including the five restituted paintings, came from Einsatzstab Reichsleiter Rosenberg. 462 objects were bought from art dealers or private persons within the German Reich including Austria. 35 file cards give

31 Cf. Lauterbach: Der Central Collecting Point in München (Fn. 2), pp. 152-154.

32 URL: http://www.dhm.de/datenbank/ccp/dhm_ccp.php? seite=9 (accessed on June 15, 2017).

33 Archiv des Bundesdenkmalamts, Restitutionsmaterialien, Box 12/4, Folder 1 "Depot Dorotheum Salzburg”, fol. 7-46: “Gesamtverzeichnis der am 18. und 22. Jänner 1952 überstellten Objekte".

34 Dagmar Hirschfelder, head of the paintings and graphic department of Kurpfälzisches Museum der Stadt Heidelberg informed the author on June 25, 2017, via email that there is no information if this painting ever had been in the Kurpfälzisches Museum Heidelberg. 
information about acquisitions in Austria. The items were bought from the art dealers Herbert Barth-Wehrenalp (1904-1990), ${ }^{35}$ Oskar Hamel (1889-1949), ${ }^{36}$ Karl Löscher (1903-1989), ${ }^{37}$ and Otto Schatzker (1885-1959), ${ }^{38}$ others from establishments like the Dorotheum, ${ }^{39}$ Neue Galerie, ${ }^{40}$ L. T. Neumann, ${ }^{41}$ Galerie Sanct Lucas, ${ }^{42}$ and Strudlhofgalerie, ${ }^{43}$ all based in Vienna. "A. Schultze, Altaussee" and "Theuermann, Vienna" could not be identified. The art dealer C. F. Ernst Schmidt (data unknown) was localized on one file card in Berlin and in Vienna. ${ }^{44}$ His company was originally in Berlin but moved to Vienna around 1944. His business address was then the Grand Hotel. ${ }^{45}$

Two Property Cards mention the name Ernst Schulte Strathaus (1881-1968). Seated at the headquarters of the NSDAP in Munich and responsible for art and cultural issues Schulte Strathaus was sent to Vienna to buy drawings and paintings by Rudolf von Alt (1812-1905), his brother Franz Alt (1824-1914), and their father Jakob Alt (1789-1872) in $1938 .{ }^{46} 52$ paintings by members of the Alt family, resp. their circle, were part of the stock the Central Collecting Point sent to Salzburg in 1952. Why Austria did not also take over the more than 600 re-

35 Herbert Barth-Wehrenalp started his business in the early 1940s. Cf. Ernst Köller: Alt-Wiener Malerei des 19. Jahrhunderts - Die Galerie Herbert Barth-Wehrenalp. In: alte und moderne kunst, 7 (1962), I. 54/55, pp. 29-31.

36 Oskar Hamel was antiquary since the early 1920s but really "successful" in the Nazi era. Cf. Gabriele Anderl: “Am Wiener Platz": Schlaglichter auf die Rolle des Wiener Kunsthandels während der NS-Zeit. In: NS-Kunstraub in Österreich und die Folgen. Ed. by Gabriele Anderl, Alexandra Caruso. Innsbruck-Wien-Bozen: Studien Verlag 2005, pp. 171-211, here pp. 174-181.

37 Karl Löscher was art dealer in Vienna, Spiegelgasse 8, 1939-1977.

38 Otto Schatzker had his salesroom at the Philipphof that was destroyed by bombs in February 1945. Cf. Anderl: “Am Wiener Platz" (Fn. 36), pp. 182-191.

39 The auction house Dorotheum was one of the main suppliers of the "Sonderauftrag Linz". Cf. Stefan August Lütgenau, Alexander Schröck and Sonja Niederacher: Zwischen Staat und Wirtschaft. Das Dorotheum im Nationalsozialismus. Vienna-Munich: Oldenbourg 2006.

40 The Neue Galerie was founded in 1923 by Otto Kallir-Nirenstein (1894-1978), who fled from Vienna in 1938. Kallirs associate Vita Künstler managed the Neue Galerie 1938-1952.

41 L.T. Neumann, founded in 1833, was managed 1930-1970 by August Eymer (1894-1978).

42 Founded in 1919 the Galerie Sanct Lucas was managed by Adolf Fritz Mondschein since 1925. Mondschein was forced to sell his company share in 1938. He was followed by Robert Herzig who managed the gallery until 1974.

43 The Strudlhofgalerie (not "Strudelhofgalerie" as falsely on the Property Card Mü 9770) existed circa 1940-1952.

44 Central Collecting Point Munich database on the website of Deutsches Historisches Museum, Property Card Mü 45071.

45 The author thanks Monika Löscher for this information.

46 Cf. Meike Hopp: "Weiß gar nicht, wo sie alle hingerathen sind". Der Münchner Bestand der Werke Rudolf von Alts und die "Sammlung Bormann" - Eine Herausforderung für die Provenienzforschung. In: Rudolf von Alt ... genial, lebhaft, natürlich und wahr. Der Münchner Bestand und seine Provenienz. Ed. by Andreas Strobl. Berlin-Munich: Deutscher Kunstverlag 2015, pp. 147-190, here pp. 162-168. 
maining Alt works from the Munich Central Collecting Point - or at least some of them - cannot really be answered. The Alt family had close connections to Austria and especially to Vienna: they painted mostly within the Habsburg monarchy; their works were collected and exhibited nearly exclusively in Austria. $^{47}$

427 Property Cards document acquisitions on the art market respectively from private persons in the German Reich excluding Austria. Over 360 of these file cards refer to Munich. Maria Almas Dietrich (1892-1971) was mentioned 82 times, Jakob Scheidwimmer (1895-?), who had aryanised Hugo Helbing's company, 60 times. There are sales from Eugen Brüschwiler (data unknown), the galleries Arnold and Stuffler, from Hitler's photographer Heinrich Hoffmann (1885-1957), and others. The largest number of acquisitions from one company - 108 - came from the auction house Weinmüller. Adolf Weinmüller (18861958), art dealer in Munich since 1921, and member of the NSDAP since 1931, expedited his career after the Nazis had assumed power in Germany in 1933. In 1936 he started his auction house in Munich, and in 1938 he aryanised the auction house S. Kende in Vienna. After 1945 Weinmüller declared that his complete business records had been destroyed at the end of the war. ${ }^{48}$ So there seemed to be no chance to investigate where the objects that were sold in Weinmüller auctions had come from - until the annotated catalogues of Weinmüller's auction houses in Munich and Vienna were found in 2013, and subsequently digitized and analysed under the supervision of Meike Hopp. Now it is possible to research the sellers of many of the Weinmüller auctions. Eleven of the 108 objects had been brought to auction by the Gestapo, very likely most of them by the Gestapo Prague. One of them was a Dutch River Landscape with Boats that was attributed to C. J. Decker on the Property Card. As there was no possibility to find out who the former owner the work was - now attributed to the circle of Cornelis Simonsz. van der Schalke ${ }^{49}-$ it was sold in the Mauerbach Auction as lot number $39 . .^{50}$

Back to the objects from the Central Collecting Point: 851 of the 909 Property Cards which were handed over to the Austrian Federal Monuments Authority in 1952 provided no indication of a provenance or of a relation to Austria. Why these objects were handed over to Austria, remains unknown. In

47 The exhibition "Rudolf von Alt ... genial, lebhaft, natürlich und wahr" of the Staatliche Graphische Sammlung München at the Pinakothek der Moderne in Munich from July 23 to October 11, 2015, was the first Alt exhibition outside Austria.

48 Cf. Meike Hopp: Kunsthandel im Nationalsozialismus: Adolf Weinmüller in München und Wien. Vienna-Cologne-Weimar: Böhlau 2012.

49 Alternative writing: Cornelis Simonsz van der Schalke bzw. Cornelis Symonsz van der Schalcke.

50 Mauerbach (Fn. 16), p. 39. 
only one case it has been verified that an item was seized in Austria under the Nazi regime. Sophie Lillie found out that the painting Mother with Child in a Meadow by Hermann Wilhelm Soltau had belonged to Philipp Gomperz (1860-1948). ${ }^{51}$ It was seized by the Gestapo in 1940, and was sold to the "Sonderauftrag Linz" by Oskar Hamel in $1944 .^{52}$ The painting was sold as lot 370 in the Mauerbach Auction in 1996.

Today it is known that many objects from the Mauerbach stock were not restituted to their former owners, but rather handed over to other claimants or sold in the auction in 1996.

Firstly, as Sophie Lillie and others have stated, no proper research on the objects, property marks, and other hints on the provenance - labels, inscriptions, seals, etc. - was done.

Secondly, a systematic analysis of the sources - Property Cards, protocols, inventories - never happened. On the contrary, lists were produced that made it impossible to retrace from where the items had come to Mauerbach. When looking at the archival material, it is obvious that essential parts of the Mauerbach stock came from Germany and had no relation to Austria in any provenance aspect. But this was not communicated to the Nazi victims and their heirs. The implicit message - not only from the Austrian officials, but also from critics like Andrew Decker - was rather that all Mauerbach objects were related to Austria and its Nazi past. So victims who had lost property outside of Austria during the Third Reich would never have thought to have a look at the lists published in the Wiener Zeitung to find their property and claim it.

Thirdly, the Art and Cultural Assets Settlement Acts offered no satisfying solution for the problem, as can be seen through the small number of restitutions which resulted from them. The items from Hohenfurth and the collection of theatre literature - all in all, about 6,300 objects - should not have been entered as single objects on the published lists. The judges needed more support from provenance experts. If the owner of an object was known, there was no procedure provided to contact this owner or his or her heirs.

For the objects from the Mauerbach stock it is too late: they are no longer under the public administration of the Austrian Republic. But some countries still own assets that are remains from the Third Reich. 21 years after the Mauerbach Auction and 19 years after the Washington Conference Principles on Nazi-Confiscated Art it should be possible to find solutions in a European cooperation.

51 Cf. Sophie Lillie: Was einmal war. Handbuch der enteigneten Kunstsammlungen Wiens. Wien: Czernin Verlag 2003 (= Bibliothek des Raubes VIII), pp. 419, 421.

52 On the file card the name of the art dealer is falsely annotated as "Otto Hamel". 
There is still one final question: How should the art market handle objects that were seized and then restituted - but not to their true former owner - and are now to be sold again. Are these items still Nazi-looted art? 
Open-Access-Publikation im Sinne der CC-Lizenz BY-NC-ND 4.0

(c) 2018, V\&R unipress GmbH, Göttingen

ISBN Print: 9783847107835 - ISBN E-Lib: 9783737007832 


\title{
Michael Wladika
}

\section{Die Beanspruchung von Kunst- und Kulturgegenständen durch die Sammelstellen 1959-1972}

\section{Zusammenfassung}

Die Sammelstellen wurden vom Bundesministerium für Finanzen als ihre oft einzige Quelle nur recht spärlich über in Verwahrung des Bundesdenkmalamtes befindliche Kunstgegenstände unterrichtet und hatten mit dem Manko zu kämpfen, aufgrund der Konzentration auf Liegenschafts- und Betriebsvermögen keine eigene Provenienzforschung durchführen zu können. Der Leiter der Sammelstellen, Georg Weis, war mehrmals überfordert, wie etwa im Fall der Witwe des ehemaligen Reichsaußenministers Joachim von Ribbentrop, die eine Freigabe von in Verwahrung des BDA und des Amtes der Kärntner Landesregierung befindlichen Bilder und Skulpturen erwirken konnte und Weis nur eine Herausgabeverpflichtung für jene Objekte erreichte, die sich in Zukunft als entzogen herausstellen würden. Georg Weis versuchte trotzdem weiter zu verhindern, dass entzogenes Eigentum an den Bund ginge, bzw. strebte einen Vergleich an, der 1969 nach langen Verhandlungen zustande kam. Vor allem der Weg zu diesem Vergleich soll resümiert werden.

Schlagwörter

Sammelstellen, Bundesdenkmalamt

\begin{abstract}
The Claim for Art and Cultural Items by the Collection Points 1959-1972

The Sammelstellen (collection points) were informed only fairly sparsely by the Ministry of Finance about art items in the custody of the Federal Monuments Authority (Bundesdenkmalamt) and were not able to carry out any provenance research of their own due to their focus on real estate and business assets. The head of the collection points, Georg Weis, was out of his depth several times, as in the case of the widow of the former Reich Secretary Joachim von Ribbentrop, who was able to obtain a release of paintings and sculptures from the custody of the Federal Monuments Authority and the Office of the Carinthian Provincial Government, but achieved an obligation of surrender only for those objects which later turned out to be seized property. Georg Weis tried to prevent that looted property from being passed on to the state. He tried to reach a settlement which came about after long negotiations in 1969. This paper describes how this settlement was reached.
\end{abstract}

Keywords

Collection Points, Federal Monuments Authority 
Da der nationalsozialistischen Judenverfolgung ganze Familien zum Opfer gefallen waren, blieb deren Vermögen nach 1945 erblos bzw. unbeansprucht, weil niemand mehr am Leben war, der die Rückstellung des geraubten Vermögens nach den Rückstellungsgesetzen, die Österreich erlassen hatte, hätte fordern können. Schon sehr früh äußerten daher jüdische Organisationen ihre Besorgnis, die Republik Österreich könnte aufgrund des in $\$ 760$ ABGB normierten Heimfallsrechts, wonach erbloses Eigentum an den Staat fällt, genauso wie die ehemaligen „Ariseure“ danach trachten, dieses Vermögen für sich zu behalten. Der Grundgedanke war nun, einen Fonds zu schaffen, der in die Rechte und Pflichten eines geschädigten Eigentümers eintreten sollte, um nach den bestehenden Rückstellungsgesetzen das erblose Vermögen zu beanspruchen und zu sammeln, um es in einem zweiten Schritt an in Österreich lebende Opfer des Nationalsozialismus zu verteilen. ${ }^{1}$ Gerade die Republik Österreich aber, Profiteur nach dem Heimfallsrecht, war deswegen nicht übermäßig erpicht darauf, ein diesbezügliches Gesetz zu erlassen. So blieb es bis zum Abschluss des Staatsvertrages im Mai 1955 bei mehr oder weniger ernst gemeinten Entwürfen sowie unerledigt gebliebenen Regierungsvorlagen. Hauptargument war, dass man vor Ablauf der Rückstellungsfristen nicht sagen könne, wie hoch das erblose und nicht beanspruchte Vermögen sei. Als die Rückstellungsgesetze im Juni 1954 generell verfristet waren, äußerten die Ministerien und Verbände wiederum Bedenken, dass ein Wiederaufleben für diesen Fonds die Bevölkerung und die Wirtschaft beunruhigen könnte. Ganz gewichtig war auch jener Gedanke, der 1953 in den Verhandlungen mit dem Committee for Jewish Claims on Austria zum Tragen kam, als es darum ging, dass Österreich einfach eine Pauschalentschädigung für das erblose und unbeansprucht gebliebene Vermögen zahlen sollte. Die österreichischen Vertreter lehnten diesen Vorschlag mit dem Verweis auf die „Opferrolle“, wonach Österreich das erste Opfer von Hitlers Aggressionspolitik gewesen sei, schlichtweg ab, denn eine derartige Leistung würde einem verschuldensabhängigen Schadenersatz gleichkommen. ${ }^{2}$

Es bedurfte daher erst des österreichischen Staatsvertrages vom Mai 1955, der in Artikel 26 Abs. 2 eine völkerrechtliche Verpflichtung der Republik aussprach, alle Vermögenschaften, Rechte und Interessen, die Personen, Organisationen und Gemeinschaften gehört hatten, die „rassischen“, religiösen oder anderen Verfolgungsmaßnahmen unterworfen gewesen waren, unter seine Kontrolle zu nehmen. Binnen 18 Monaten nach Inkrafttreten des Staatsvertrages sollte dann die Republik Österreich diese Vermögenschaften, Rechte und Interessen einer

1 Zur Gesamtproblematik siehe Margot Werner, Michael Wladika: Die Tätigkeit der Sammelstellen. Wien-München: Oldenbourg 2004 (= Veröffentlichungen der Österreichischen Historikerkommission. Vermögensentzug während der NS-Zeit sowie Rückstellungen und Entschädigungen seit 1945 in Österreich 28).

2 Ebd., S. 15 f. 
Organisation übertragen, damit sie für Hilfe und Unterstützung für Opfer verwendet werden können. ${ }^{3}$

So kam es vor 60 Jahren, am 13. März 1957, zum Erlass des Auffangorganisationengesetzes (AuffOG), mit dem die Sammelstellen geschaffen wurden. ${ }^{4} \mathrm{Da}$ die jüdischen Organisationen von Anfang an eine gesonderte Erfassung des erblosen Vermögens von Jüdinnen und Juden gefordert hatten, das wieder nur jüdischen Opfern zu Gute kommen sollte, kam man überein, zwei Sammelstellen zu schaffen. Dabei sollten nun der Sammelstelle A jene Vermögenschaften, Rechte und Interessen zukommen, die Personen zustanden, die am 31. Dezember 1937 der IKG angehört hatten, während der Sammelstelle B die erblosen Vermögen der anderen NS-Opfer zukamen, also auch der als Juden Verfolgten, die nicht mehr der IKG angehört hatten. ${ }^{5}$

Da diese Vermögenswerte aber nicht pauschal übertragen wurden, wie ursprünglich vorgesehen, mussten die Sammelstellen den mühsamen Weg gehen, jeden einzelnen, durch eigene Recherchen erhobenen Vermögensgegenstand nach den Rückstellungsgesetzen, die für sie wieder mit der gesamten Problematik und den Versäumnissen geöffnet wurden, einzufordern. Hinzu kamen die sogenannten „Billigkeitswerber“: Es waren dies jene geschädigten Personen, die bislang keinen Rückstellungsantrag vor österreichischen Behörden oder Gerichten eingebracht hatten oder hatten einbringen können, weil sie etwa die Frist versäumt hatten. Ihnen stand nun ein Antragsrecht an die Sammelstellen auf Herausgabe ihres Vermögens zu. Falls beispielsweise die Sammelstellen eine Liegenschaft erfolgreich geltend gemacht hatten, mussten sie diese an den Billigkeitswerber abtreten. Sie verlangten für die bisherige Nichtgeltendmachung des Anspruches durch den Billigkeitswerber aber eine „Mühewaltungsentschädigung“, die nur auf den ersten Blick wie eine „Strafzahlung“ aussieht, kam diese doch wiederum den Opfern zugute. ${ }^{6}$

\section{Die Beanspruchung von Mobilien}

Welche Vermögenswerte haben die Sammelstellen nun in erster Linie beansprucht? Im Rahmen eines gemeinsamen Projekts für die Österreichische Historikerkommission haben Margot Werner und der Autor dieses Beitrages eine Datenbank angelegt und aus einem Gesamtbestand von 19.383 Anspruchsakten ein Auswahlsample von 1.157 Fällen getroffen. Das Ergebnis war beeindruckend, denn

3 Ebd., S. 17.

4 BGBl. Nr. 73/1957.

$5 \$ 2$ Abs. 1 AuffOG.

6 Werner, Wladika: Die Tätigkeit der Sammelstellen (Anm. 1), S. 253-271. 
die Vermögensobjekte bestanden zu 77 Prozent aus Liegenschaften, gefolgt von 17 Prozent Geschäfts- und Betriebsvermögen und nur sechs Prozent sonstigen Vermögenswerten wie Mobilien. ${ }^{7}$ Warum dem so war, lässt sich leicht erklären: Schon für den Geschäftsführer der beiden Sammelstellen, Georg Weis (18981992), stand von Anfang an fest, dass der wesentliche Teil des Vermögens, den die Sammelstellen beanspruchen könnten, aus Liegenschaften bestehen würde. Einerseits brachten sie bei der anschließenden Verwertung für die NS-Opfer viel Geld ein, andererseits waren sie noch relativ leicht auszuforschen, wenn dies auch mit enormer Arbeit verbunden war. So durchforstete etwa die Erhebungsabteilung der Sammelstellen von Februar 1959 bis Oktober 1960 zunächst die circa 66.600 Vermögensanmeldungen nach der „Verordnung über die Anmeldung des Vermögens von Juden“"vom 26. April 1938, die in eine eigene Kartothek gebracht wurden, um dann in einem zweiten Schritt die Akten des Oberfinanzpräsidenten durchzusehen, ehe die Arbeit bei den Grundbuchgerichten begann. ${ }^{8}$

Wie sah aber nun die Beanspruchung von jenen sechs Prozent „sonstiger Vermögenswerte“ aus, zu denen auch die Kunst- und Kulturgegenstände zählten - das eigentliche Thema dieses Beitrages: „Schlecht“, lässt sich pauschal urteilen. Die Sammelstellen haben diese Vermögenswerte aufgrund der Konzentration auf Liegenschafts- und Betriebsvermögen vernachlässigt. Mangels Provenienzforschung konnten sie auch keine Beanspruchung in der Weise durchführen, indem sie etwa die Rückstellung von in Museen befindlichen Kunstgegenständen beantragt hätten. Georg Weis hat dies zwar als „Probeballon“ ein paar Mal versucht, blitzte aber bei den Museumsdirektoren sofort ab, die sich auf den Gutglaubenserwerb der Kunstgegenstände nach $\$ 367$ ABGB beriefen. ${ }^{9}$ So mussten die Sammelstellen auch den Billigkeitswerbern, die mit Wünschen nach Recherchen an sie herantraten, mitteilen, dass sie ohne Provenienzforschung vor allem bei Kunstgegenständen, über deren Verbleib nichts bekannt war, nichts unternehmen konnten. Dies geht etwa aus einem Schreiben von Georg Weis an Karl Max Blauhorn vom September 1959 hervor, der auf der Suche nach der großen Kunstsammlung seiner Eltern war. ${ }^{10}$

Es blieb daher oft als einzige Quelle das Bundesministerium für Finanzen (BMF), welches die Sammelstellen aber nur recht spärlich über zumeist in Verwahrung des Bundesdenkmalamtes (BDA) befindliche Kunstgegenstände unterrichtete. Dabei wussten die Sammelstellen dann wiederum nicht, ob diese Objekte entzogen waren oder nicht.

7 Ebd., S. $77 \mathrm{f}$.

8 Ebd., S. 80-85.

9 Ebd., S. 226.

10 Österreichisches Staatsarchiv (ÖStA), Archiv der Republik (AdR), Bundesministerium für Finanzen (BMF), Bestand Sammelstellen, Kt. Liegenschaften/Verkauf, Billigkeitsakt, Zl. 10.028, Georg Weis an Karl Max Blauhorn, 19.09.1959. 
Im Detail werden diese Schwierigkeiten auch nachvollziehbar: So erging am 5. Juni 1959 eine Anfrage von der Abteilung 34 des BMF an die Sammelstellen bezüglich einer Freigabe von in Verwahrung des Bundesdenkmalamtes, Depot Salzburg, und des Amtes der Kärntner Landesregierung befindlichen Bildern und Skulpturen des 1946 als Kriegsverbrecher hingerichteten ehemaligen Reichsaußenministers Joachim von Ribbentrop an seine Witwe. Anhand einer beiliegenden Liste sollten die Sammelstellen wie in all diesen Fällen „ehestens“ bekannt geben, ob sich darunter entzogenes Vermögen befinden würde, da eine Klage drohe. ${ }^{11}$ Georg Weis zeigte sich sichtlich überfordert: Er antwortete, dass auf Grund der bisherigen Erhebungen keine Tatsachen bekannt geworden seien, die auf entzogenes Vermögen hindeuten würden, es möge jedoch Frau Ribbentrop eine Verpflichtung auferlegt werden, jene Bilder oder Skulpturen an die Sammelstellen auszufolgen, die sich in Zukunft als entzogen herausstellen würden. ${ }^{12}$ Diese Maßnahme klang doch eher sehr zahnlos.

Anders lag der Fall, als die Abteilung 33 des BMF die Sammelstellen im Juli 1960 von Erwerbungen der „Verwertungsstelle für jüdisches Umzugsgut der Gestapo (Vugesta)“ für das geplante, aber nie realisierte „Linzer Museum“ Adolf Hitlers verständigte, die vom BDA in der Salzburger Residenz verwahrt werden würden. ${ }^{13}$ Weil es sich dabei offenbar um entzogenes, bisher nicht beanspruchtes ehemaliges jüdisches Eigentum des Deutschen Reiches handelte, beantragte Georg Weis bei der Finanzlandesdirektion (FLD) für Wien, Niederösterreich und dem Burgenland die Erlassung eines Feststellungsbescheides, mit dem den Sammelstellen diese Vugesta-Erwerbungen übertragen worden wären. ${ }^{14} \mathrm{Im}$ Oktober 1960 langte bei der Sammelstelle A eine neuerliche Verständigung der Abteilung 33 ein, dass sich drei weitere, für das „Linzer Museum“ bestimmte Bilder in der Salzburger Residenz befinden würden, die zwar eine Herkunftsbezeichnung aufweisen würden, von denen aber nicht bekannt sei, ob der Erwerb von einem Zweiterwerber oder vom ursprünglichen Eigentümer herrühre. ${ }^{15}$ Während die Sammelstelle A im Juni 1961 lediglich Meldeerhebungen bezüglich der in den Herkunftsbezeichnungen genannten Personen durchführen konnte, ${ }^{16}$ verzögerte sich die Erlassung eines Feststellungsbescheides wegen der Frage der

11 ÖStA, AdR, BMF, Abteilung (Abt.) 34, Zl. 206.003-34/59, Gottfried Klein an Georg Weis, 05. 06. 1959.

12 ÖStA, AdR, BMF, Abt. 34, Zl. 206.003-34/59, Weis an Klein, 02.07.1959.

13 ÖStA, AdR, BMF, Bestand Sammelstellen, Mappe S 9b „Kunstgutgesetz, Korrespondenz mit Ministerien und FLD“, BMF, Abt. 33, Wittermann an die Sammelstellen A und B, 08. 07. 1960.

14 ÖStA, AdR, BMF, Bestand Sammelstellen, Mappe S 9b „Kunstgutgesetz, Korrespondenz mit Ministerien und FLD“, Georg Weis an das BMF, Abt. 33, Wittermann, 28. 07.1960.

15 ÖStA, AdR, BMF, Bestand Sammelstellen, Mappe S 9b „Kunstgutgesetz, Korrespondenz mit Ministerien und FLD“, BMF, Abt. 33, Zl. 350.391-33/60, an Sammelstelle A, 26. 10. 1960.

16 ÖStA, AdR, BMF, Bestand Sammelstellen, Mappe S 9i „Kunstgutgesetz, Erhebungen und Neue Galerie“, Fitzinger an Weigl, 12.06.1961. 
örtlichen Zuständigkeit. Am 21. Juni 1961 erklärte die FLD Salzburg dann plötzlich, dass auf Grund der "Aktenlage alleine“ das Vorliegen einer Vermögensentziehung im Sinne der Rückstellungsgesetze „nicht eindeutig“ angenommen werden könne. ${ }^{17}$ Um die mit 30. Juni 1961 ablaufende Anspruchsfrist zu wahren, hatte die Sammelstelle A schon vorher, am 29. Mai 1961, Rückstellungsanträge nach dem Zweiten Rückstellungsgesetz (RStG) für jedes einzelne Bild bei der FLD Salzburg eingebracht. ${ }^{18}$ Überall dort, wo nach der Herkunftsbezeichnung nur ein Zuname bekannt war, wie im Beispielsfall „Pilzer", mussten in den Anträgen „Behauptungen“, egal ob richtig oder nicht, aufgestellt werden, wie die deutsche Staatsbürgerschaft und der Verfall des Vermögens nach der „Elften Verordnung zum Reichsbürgergesetz ${ }^{\text {“19 }}$ vom 25. November 1941 bzw. die tschechoslowakische Staatsbürgerschaft und der Verfall des Vermögens nach der „Verordnung über den Verlust der Protektoratsangehörigkeit“ vom 2. November 1942. ${ }^{20}$ Weil wiederum der FLD Salzburg hinsichtlich Kunstgegenstände in der betreffenden Abteilung noch kein ähnlicher Fall vorgekommen war, musste sie ständig die Finanzprokuratur um Stellungnahmen ersuchen. ${ }^{21}$ Am 18. Dezember 1961 langte bei der Sammelstelle A schließlich eine Aufforderung der FLD Salzburg ein, binnen zwei Wochen bei allen Anträgen den vollständigen Namen und die vollständige letzte Anschrift des geschädigten Eigentümers bekanntzugeben sowie das Eigentumsrecht nachzuweisen. Darüber hinaus wäre mit einer genauen Darstellung der Entziehung ein Nachweis zu erbringen, dass dieser geschädigte Eigentümer Jude gewesen sei. ${ }^{22}$ Diese Nachweise konnte die Sammelstelle A natürlich nicht erbringen. Erst 1963 zeigte sich die Republik Österreich vergleichsbereit. Am 5. Juni 1963 schloss die Sammelstelle A, wieder für jedes Bild einzeln, Vergleiche, bei einer Ausfolgung die Verwahrungsgebühren von durchschnittlich 1.500 Schilling zu bezahlen. ${ }^{23}$ Die Sammelstelle erwirkte zwar nach drei Jahren die Herausgabe von zehn Gemälden, doch damit war der Fall noch lange nicht abgeschlossen.

Bei den Bildern, die Herkunftsbezeichnungen aufwiesen, hatte die Sammelstelle A im November 1961 Schreiben an jene Personen gerichtet, die in ihrer,

17 ÖStA, AdR, BMF, Bestand Sammelstellen, Mappe S 9b „Kunstgutgesetz, Korrespondenz mit Ministerien und FLD“, BMF, Abt. 33, Zl. 200.122/8-33/61, an Sammelstelle A, 21.06.1961.

18 ÖStA, AdR, BMF, Bestand Sammelstellen, Mappe S 9i „Kunstgutgesetz, Erhebungen und Neue Galerie“, Georg Weis an RA Moser, 10.07.1961.

19 RGBl. I 1941 S. $722 \mathrm{ff}$.

20 ÖStA, AdR, BMF, Bestand Sammelstellen, Kt. Liegenschaften/Verkauf, GZl. 5153.

21 ÖStA, AdR, BMF, Bestand Sammelstellen, Mappe S 9i „Kunstgutgesetz, Erhebungen und Neue Galerie“, RA Moser an Georg Weis, 17.07.1961.

22 ÖStA, AdR, BMF, Bestand Sammelstellen, Kt. Liegenschaften/Verkauf, FLD Salzburg, Zl. 18/ 2-IV-R 1961, an Sammelstelle A, 06.12.1961.

23 Archiv des BDA Wien, Rest. K 24, Mappe 9, „Riepp, Salomon und die Königin von Saba“, Vergleichsausfertigungen vor der FLD Salzburg, 05.06.1963. 
sowie in der Kartei des Hilfsfonds aufschienen. Im Beispielsfall „Pilzer“, bei dem nur der Zuname bekannt war, waren dies alleine zehn Personen. Eine genaue Bezeichnung des Bildes wurde jedoch vermieden und erst in einer zweiten „Runde“ bekanntgegeben, da sich sieben Personen mit dem Zunamen „Pilzer“ gemeldet hatten, die zumindest Bilder besessen hatten oder aber wieder Verwandte namhaft machen konnten, bei denen diese Voraussetzungen auch zutrafen. Letztlich konnte bis Sommer 1963 kein Eigentümer gefunden werden. ${ }^{24}$ Nach dem Rücktransport der Kunstgegenstände auf Kosten der Sammelstellen nach Wien musste erst ein Versteigerungstermin mit dem Dorotheum abgestimmt werden, da sich herausgestellt hatte, dass dies der einzig gangbare Weg einer Verwertung war. ${ }^{25}$ Zuvor mussten noch einige Bilder restauriert werden, um bessere Preise zu erzielen. ${ }^{26}$ Nachdem in der Versteigerung vom Februar 1964 nicht alle Bilder einen Käufer gefunden hatten, musste dann noch eine Auktion im Herbst abgewartet werden, bis der Fall Ende 1964 endgültig abgeschlossen werden konnte. ${ }^{27}$ Die Sammelstellen erzielten schließlich einen Erlös von 731.700 Schilling. ${ }^{28}$ In zwei Fällen waren Billigkeitsanträge innerhalb offener Frist eingebracht worden. Eine Person konnte einen Erbnachweis erbringen, der Erlös wegen der Verzögerungen jedoch nicht mehr an sie ausgefolgt werden, da sie ohne weitere Erben verstorben war. ${ }^{29}$ An die andere Person konnte der Erlös nicht ausgefolgt werden, da sie keinen Erbnachweis erbringen konnte. ${ }^{30}$

In der von Georg Weis am 24. Mai 1966 im Zusammenhang mit dem „Bundesgesetz vom 7. Juli 1966, betreffend Abgeltung von Ansprüchen der ,Sammelstellen' (Sammelstellen-Abgeltungsgesetz)“31 abgegebenen Entfertigungserklärung, gegen den Erhalt von 22,7 Mio. Schilling auf alle Rückstellungsansprüche gegenüber der Republik Österreich zu verzichten, hatte er einen, wie sich später herausstellen sollte, weitsichtigen Vorbehalt gemacht. Demnach bezog sich die Erklärung nicht auf Ansprüche der Sammelstellen „,auf Herausgabe von in Österreich entzogenen Bildern und anderen Kunstgegenständen, die sich in der Obhut des BDA oder der von diesem beauftragten Stellen befinden, soweit sie nicht dem geschädigten Eigentümer rückgestellt werden“. Nachdem der Leiter des jüdischen Dokumentationszentrums in Wien, Simon Wiesenthal,

24 ÖStA, AdR, BMF, Bestand Sammelstellen, Kt. Liegenschaften/Verkauf, GZl. 5153.

25 ÖStA, AdR, BMF, Bestand Sammelstellen, Kt. Liegenschaften/Verkauf, GZl. 5153, Fitzinger an BDA, 02.08. 1963.

26 Archiv des Bundesdenkmalamtes Wien, Rest K 24, Mappe 9 „Riepp, Salomon und die Königin von Saba“, Zl. 1622/64, Restaurierung.

27 Ebd., Fitzinger an BDA, 13.05.1964.

28 ÖStA, AdR, BMF, Bestand Sammelstellen, Mappe S 9b „Kunstgutgesetz, Korrespondenz mit Ministerien und FLD“, Aktenvermerk der Sammelstelle A, 08.10.1965.

29 Ebd.

30 Ebd.

31 BGBl. Nr. 150/1966. 
in einem Zeitungsartikel und einer Pressekonferenz von bis zu 2.000 wertvoller entzogener Bilder sprach, fühlte sich Georg Weis auf Nachfrage vom BMF hinters Licht geführt, welches einmal 273 Kunstgegenstände als „herrenlos“ bezeichnete, die in Verwahrung des BDA stünden, dann wiederum die Zahl mit 967 angab. Das war der Hauptgrund, warum die Sammelstellen gegen den Entwurf zum Ersten Kunst- und Kulturgutbereinigungsgesetz - Stichwort MauerbachBestand - Protest einlegten, der den Verfall der Gegenstände an die Republik Österreich nach Ablauf einer allgemeinen Anmeldefrist für geschädigte Eigentümer vorsah. Auch mit der Abänderung des $\$ 8$ waren die Sammelstellen nicht zufrieden, der ihnen ein subsidiäres Antragsrecht zugestand, weil sich der Staat an erblosem Vermögen bereichern würde. Im Jänner 1967 änderte das BMF $§ 8$ des Entwurfes erneut um, der nunmehr eine Verpflichtung der Anmeldestelle für potentiell Geschädigte vorsah, die Sammelstellen von jedem Eigentumsübergang auf den Bund zu verständigen, die dann ihrerseits einen Antrag auf Übertragung stellen konnten. Da Akteinsicht, Parteistellung und eine Wiedereröffnung der Billigkeitsfristen nicht vorgesehen waren, war auch diese Abänderung für die Sammelstellen nicht geeignet, zu verhindern, dass entzogenes Eigentum an den Bund ginge.

\section{Die Strategien der Sammelstellen gegen das Erste Kunst- und Kulturgutbereinigungsgesetz vom 27. Juni 1969, BGBI. Nr. 294/1969}

Georg Weis verfolgte eine klare Strategie gegen das Erste Kunst- und Kulturgutbereinigungsgesetz, als er seinen Mitarbeitern und Mitarbeiterinnen von der Rechtsabteilung im Herbst 1966 den Auftrag gab, anhand eines Ausstellungskataloges der Neuen Galerie des Kunsthistorischen Museums (KHM) in Wien, der unter 138 Gemälden moderner Malerei auch 24 enthielt, die zwischen 1938 und 1945 erworben worden waren, nun doch deren Provenienz auf jüdische Voreigentümer zu prüfen. ${ }^{32}$ Neben diesen Recherchen, die mangels einer Auskunftspflicht der Museen nach Ablauf der Rückstellungsfristen nur sehr mühsam über noch vorhandene alte Werkskataloge vor sich gehen konnten, ${ }^{33}$ legte er Ordner mit abgewiesenen Rückstellungsverfahren an, die Kunstgegenstände betrafen. Diese zwei Ordner mit Kopien von Rückstellungskommissions-Akten befinden sich noch heute im Bestand Sammelstellen des Österreichischen Staatsarchives und tragen die Bezeichnung „Bilder“. Sie enthalten zumeist nicht

32 ÖStA, AdR, BMF, Bestand Sammelstellen, Mappe S 9i, „Kunstgutgesetz, Erhebungen und Neue Galerie“, Georg Weis an die United Restitution Organisation Berlin, 10.01.1969.

33 Ebd. 
komplette Verfahrensakten wie etwa zu den Fällen Mahler-Werfel, Francesco de Mendelsohn, Wilhelm Freund oder Oskar Bondy.

Georg Weis ließ danach eine Liste von jenen Personen erstellen, von denen Museen Kunstgegenstände erworben hatten. Er überreichte der FLD Wien diese Liste ohne Mitteilung der Hintergründe mit dem Ersuchen, zu erheben, ob Rückstellungsanträge vorliegen würden. Bei positiven Ergebnissen verglich er darauf die Anmeldungen der Museen nach der Vermögensentziehungsanmeldeverordnung. ${ }^{34}$ Weis war auf der richtigen Spur. Seine Absicht bestand darin, einen Nachweis zu erbringen, dass das Kunst- und Kulturgutbereinigungsgesetz nichts anderes als ein großes Ablenkungsmanöver sei: Während man ein großes „Tamtam“ um „zerbrochene Bilderrahmen und Deckerl“ mache, die wertlos seien, befänden sich die wirklich wertvollen Gemälde aus ehemals jüdischem Eigentum längst nicht mehr in „Gewahrsame“ des Bundes, sondern im „Eigentum der Galerien“. Deren Direktoren hätten es unterlassen, eine Anmeldung entzogenen Vermögens zu erstatten und würden sich nunmehr auf den Erwerb von Kunsthändlern berufen, von denen sie gutgläubig erworben hätten. ${ }^{35}$ Als er diese Vorwürfe im Juli 1968 auch an Bundeskanzler Josef Klaus herantrug, ${ }^{36}$ versprach dieser Aufklärung durch das BMF, welches am 25. Februar 1969 einen Bericht über lediglich 39 überprüfte Gemälde vorlegte: Die Prüfung sämtlicher Unterlagen hätte ein „wenig befriedigendes Ergebnis“ erbracht, da „nur in einigen Fällen Hinweise auf Einzelheiten des Erwerbers beziehungsweise auf die Herkunft der Werke gefunden werden konnten“, so ein Vertreter des BMF. ${ }^{37}$ So stammten 25 der überprüften, zwischen 1938 und 1945 erworbenen Gemälde, aus Deutschland und der Schweiz. Von den 14 in Österreich erworbenen Bildern stammten zwölf von Kunsthändlern, die zumeist nicht mehr tätig oder gestorben waren. Bei zwei von einer Privatperson erworbenen Gemälden war ein Rückstellungsverfahren abgewiesen worden. ${ }^{38}$

Weis interpretierte diesen Bericht in einer vertraulichen Mitteilung an seine Rechtsabteilung als „unglaubwürdig“: Es wäre „auffallend“, dass in all jenen Fällen, in denen die Erwerbsakten auf eine nicht-jüdische Quelle zurückzuführen schienen, diese auch Aufzeichnungen über die Herkunft des Bildes enthielten, während ihnen dagegen in allen anderen zahlreichen Fällen nichts zu

34 ÖStA, AdR, BMF, Bestand Sammelstellen, Mappe S 9b, „Kunstgutgesetz, Korrespondenz mit Ministerien und FLD“, Aktenvermerk über eine Unterredung zwischen Herrn Dr. Weis und HR Lippert, 09.11.1966.

35 ÖStA, AdR, BMF, Bestand Sammelstellen, Mappe S 9 h, „Kunstgutgesetz, Diverse Korrespondenz und Stella Klein-Löw“, Georg Weis an Jellinek, 31.01.1967.

36 ÖStA, AdR, BMF, Bestand Sammelstellen, Mappe S 9b, „Kunstgutgesetz, Korrespondenz mit Ministerien und FLD“, Georg Weis an BK Josef Klaus, 12.07.1968.

37 ÖStA, AdR, BMF, Bestand Sammelstellen, Mappe S 9i, „Kunstgutgesetz, Erhebungen und Neue Galerie“, Miklas an Weis, 25.02.1969.

38 Ebd. 
entnehmen wäre. Man könne daher „vermuten, dass die Akten manipuliert“ worden wären, „wenn auch schon kurz nach 1945“, wie er vorsichtigerweise hinzufügte. ${ }^{39}$

Zu dem Zeitpunkt, als er dem „World Jewish Congress“ (WJC) im November 1968 schrieb, dass er bei nur 15 Gemälden einen einwandfreien Beweis von entzogenem jüdischen Eigentum benötigen würde, um Einsicht in die Museumsakten zu erhalten, aus denen „doch ganz wahrscheinlich hervorgehe“, dass den Museumsdirektoren die Provenienz bekannt gewesen sein mussten, war er längst bereit, die Ansprüche der Sammelstellen aus dem Kunst- und Kulturgutbereinigungsgesetz zu vergleichen. Weis war „offenbar nicht mehr in der Lage, in dieser Sache die Interessen der Sammelstellen hinreichend zu vertreten“. ${ }^{40}$

\section{Der Vergleich des BMF mit den Sammelstellen über die Ansprüche aus dem (Ersten) Kunst- und Kulturgutbereinigungsgesetz}

In einer Sitzung des Unterausschusses des Finanz- und Budgetausschusses am 19. Februar 1969, an der auch die Minister Theodor Piffl-Percevic (1911-1994, Unterricht) und Stephan Koren (1919-1988, Finanzen) sowie Kuratoren der Sammelstelle A und der Sammelstelle B teilnahmen, machte Georg Weis erstmals den Vorschlag, auf alle Ansprüche nach dem Kunst- und Kulturgutbereinigungsgesetz gegen eine Summe von zehn Mio. Schilling zu verzichten. Die Republik Österreich sollte jedoch nicht „aus dem Titel der Bilder“, sondern aus den Verpflichtungen nach dem Staatsvertrag bezahlen, denn der Bund hätte das „erblose Gut“ auszuforschen und an die Sammelstellen zu übergeben gehabt, was er nicht getan hätte. Dadurch seien den Sammelstellen Verwaltungskosten von ungefähr 15 Mio. Schilling entstanden, auf welche ursprünglich nur fünf Millionen Schilling gezahlt worden wären. ${ }^{41}$ Der Vertreter des Kuratoriums der Sammelstelle B, Kanonikus Alfred Kostelecky (1920-1994), der wegen seiner guten Kontakte zu ÖVP-Kreisen in dieser Sache mehrmals vorgesprochen hatte, signalisierte jedoch sofort, dass diese Summe zu hoch gegriffen wäre, weswegen wieder eine Eiszeit zwischen den beiden Sammelstellen ausbrach. ${ }^{42}$

Finanzminister Stefan Koren erklärte sich wiederum aus budgetären Gründen

39 Ebd., Weis an Fitzinger, 03.03.1969.

40 ÖStA, AdR, BMF, Bestand Sammelstellen, Mappe S 9h, „Kunstgutgesetz, Diverse Korrespondenz und Stella Klein-Löw“, Weis an Brassloff, 29.11.1968.

41 ÖStA, AdR, BMF, Bestand Sammelstellen, Mappe S 9h, „Kunstgutgesetz, Diverse Korrespondenz und Stella Klein-Löw“, Georg Weis an die Mitglieder des Kuratoriums der Sammelstelle A, 20.02.1969.

42 ÖStA, AdR, BMF, Bestand Sammelstellen, Mappe S 9c, „Korrespondenz mit Kuratorien und Dr. Goldmann“, Georg Weis an Charles Kapralik, 06.03.1969. 
außerstande, diese Summe zu bezahlen, der zusätzlich die Junktimierung mit mittlerweile zwei Gesetzesentwürfen im Wege stand. ${ }^{43}$

Am 11. März 1969 teilte Georg Weis den Mitgliedern des Kuratoriums der Sammelstelle A mit, dass der Finanzminister nach Verhandlungen mit Kanonikus Kostelecky fünf Millionen Schilling „bieten“ würde. „Obwohl ungern“, empfahl er die Annahme. ${ }^{44}$ Nachdem sich die beiden Kuratorien am 20. März 1969 auf diese Summe geeinigt hatten, wurde der Vergleich in einer Sitzung des Unterausschusses am 5. Mai 1969 als geänderter $\$ 8$ in den Gesetzesentwurf des Kunst- und Kulturgutbereinigungsgesetzes mit einem „budgetären Zahlungsziel von acht Monaten nach Inkrafttreten des Gesetzes“ aufgenommen. ${ }^{45}$ Der Paragraph lautete nun: „Zur Abgeltung aller Ansprüche der ,Sammelstellen“ auf Übertragung von Kunst- und Kulturgut, das Personen gehört hat, die durch das NS-Regime verfolgt wurden, ist den ,Sammelstellen' spätestens acht Monaten nach Inkrafttreten dieses Bundegesetzes der Betrag von fünf Millionen Schilling zu überweisen."

Zuvor musste der Geschäftsführer beider Sammelstellen, Georg Weis, am 26. März 1969 eine „Annahmeerklärung“ dieses Anbotes unterschreiben, von dem dieser glaubte, dass es sich gesetzeskonform um einen Verzicht auf die Ansprüche nach dem Kunst- und Kulturgutbereinigungsgesetz handeln würde. Das Ministerium verlangte jedoch eine generelle Verzichtserklärung der Sammelstellen auf Ansprüche „auf entzogenes und nicht beanspruchtes Kunst- und Kulturgut gegenüber dem Bund“, um „zukünftige Streitigkeiten zu vermeiden“, ${ }^{46}$ die Weis nach Ermächtigung durch beide Kuratorien am 2. April 1969 unterzeichnete. ${ }^{47}$

Die Unterzeichnung eines von der Sammelstelle B formulierten Dankesbriefes an Finanzminister Koren lehnte Weis jedoch wegen der verschwiegenen Kunstgegenstände, der Tatsache, dass das Kunst- und Kulturgutbereinigungsgesetz so gefasst wurde, dass die früheren Eigentümer Ansprüche wegen der Bilder in den Museen nicht geltend machen konnten und wegen der Formulierung des Gesetzes, die den Sammelstellen „überflüssige administrative Lasten aufhalste“, ab. ${ }^{48}$

Das „Bundesgesetz über die Bereinigung der Eigentumsverhältnisse des im

43 ÖStA, AdR, BMF, Bestand Sammelstellen, Mappe S 9h, „Kunstgutgesetz, Diverse Korrespondenz und Stella Klein-Löw“, Georg Weis an die Mitglieder des Kuratoriums der Sammelstelle A, 20.02.1969.

44 ÖStA, AdR, BMF, Bestand Sammelstellen, Mappe S 9c, „Korrespondenz mit Kuratorien und Dr. Goldmann“, Georg Weis an Charles Kapralik, 10.03.1969.

45 Ebd., Georg Weis an das Kuratorium der Sammelstelle A, 13.03.1969.

46 ÖStA, AdR, BMF, Bestand Sammelstellen, Mappe S 9b, „Kunstgutgesetz, Korrespondenz mit Ministerien und FLD“, Zl. 68.042/5-16b/69, BMF an Georg Weis, 26.03.1969.

47 ÖStA, AdR, BMF, Bestand Sammelstellen, Mappe S 9b, „Kunstgutgesetz, Korrespondenz mit Ministerien und FLD“, Zl. 68.042/7-16b/69, BMF an Georg Weis, 10.04.1969.

48 ÖStA, AdR, BMF, Bestand Sammelstellen, Mappe S 9c, „Korrespondenz mit Kuratorien und Dr. Goldmann“, Weis an Feldsberg, 01.04.1969. 
Gewahrsam des Bundesdenkmalamtes befindlichen Kunst- und Kulturgutes (Erstes Kunst- und Kulturgutbereinigungsgesetz)“ wurde am 27. Juni 1969 mit dem die Sammelstellen betreffenden $\$ 8$ im Nationalrat beschlossen. ${ }^{49}$ In den ersten Apriltagen des Jahres 1970 wurden fünf Millionen Schilling auf ein Konto der Sammelstellen überwiesen. ${ }^{50}$

\section{Resümee des Vergleichsabschlusses}

Lange sinnierte Georg Weis, ob die Vergleichssumme der Höhe nach angemessen war. Dass es überhaupt zu dieser gekommen war, führte er darauf zurück, dass es ihm „gelungen“ wäre, die Bilder in der Neuen Galerie des Kunsthistorischen Museums „mit zur Debatte zu stellen“. ${ }^{51}$ Entgegen der anlässlich der Beschlussfassung des Gesetzes getätigten Aussage des Abgeordneten Erwin Machunze (1911-1982), die Sammelstellen würden sich über die fünf Mio. Schilling freuen, ${ }^{52}$ betonte Weis, nie ein „Hehl daraus gemacht“ zu haben, mehr als diesen Betrag anzustreben. ${ }^{53}$

Auf der anderen Seite gab er wiederum zu bedenken, dass die fünf Mio. Schilling ,in gar keiner verhältnismäßigen Beziehung zu dem wirklichen oder angegeben Wert" der in einer dem Gesetz angeschlossenen und im Amtsblatt der Wiener Zeitung vom 2. September 1969 veröffentlichten Liste der in Gewahrsam des Bundesdenkmalamtes befindlichen Kunstgegenstände gestanden wären. Selbst wenn diese Gegenstände „1.000 Millionen“ Wert wären, wäre selbstverständlich anzunehmen, dass sich gerade für die wertvolleren Bilder frühere Eigentümer finden würden. Daher wäre es auch durchaus möglich, dass von den nur in Österreich entzogenen Gegenständen gerade einmal Werte von 100.000 Schilling für die Sammelstellen übrig bleiben würden. ${ }^{54}$

Nach $\$ 2$ Abs. 1 des Kunst- und Kulturgutbereinigungsgesetzes musste eine Anmeldung von Personen, die ihr Eigentumsrecht behaupteten, bis spätestens 31. Dezember 1970 erfolgt sein. Obwohl Georg Weis die Liste der Kunstgegenstände in auf Kosten der Sammelstellen erstellten, 5.000 vervielfältigten Exem-

49 BGBl. Nr. 294/1969.

50 ÖStA, AdR, BMF, Bestand Sammelstellen, Mappe S 9b, „Kunstgutgesetz, Korrespondenz mit Ministerien und FLD“, Zl. 31.180-16/70, BMF an die Sammelstelle A, 02.04.1970.

51 ÖStA, AdR, BMF, Bestand Sammelstellen, Mappe S 9c, „Korrespondenz mit Kuratorien und Dr. Goldmann“, Weis an Krell, 03.07.1969.

52 Stenographisches Protokoll der 145. Sitzung des Nationalrates, XI. GP, 27.06. 1969.

53 ÖStA, AdR, BMF, Bestand Sammelstellen, Mappe S 9c, „Korrespondenz mit Kuratorien und Dr. Goldmann“, Georg Weis an die Mitglieder des Kuratoriums der Sammelstelle A und der Sammelstelle B, 18.08.1969.

54 ÖStA, AdR, BMF, Bestand Sammelstellen, Mappe S 9c, „Korrespondenz mit Kuratorien und Dr. Goldmann“, Weis an Krell, 08.09.1969 und Weis an Kapralik, 11.09.1969. 
plaren in den verschiedensten Ländern verbreiten ließ, langten bis zu diesem Datum entgegen allen Erwartungen gerade einmal um die 400 Anmeldungen ${ }^{55}$ ein, die sich auf die eher wertloseren Dinge bezogen. ${ }^{56}$ In einem Bundesgesetz vom 30. Juni $1971^{57}$ wurde die Anmeldefrist daher bis zum 31. Dezember 1972 wieder eröffnet. ${ }^{58}$

Bis zum 31. Dezember 1972 war es für Georg Weis höchst zweifelhaft, ob die fünf Mio. Schilling „ein guter oder ein schlechter Vergleich“ waren, zumal er in einer späteren retrospektiven Betrachtung auch zu verstehen gab, dass durch die Fristverlängerung und einer Behandlung der Anmeldungen ab Jänner 1973 die Sammelstellen bis 1976 hätten existieren müssen. ${ }^{59}$ Doch knapp vor Ende der ersten Anmeldefrist, am 14. Dezember 1970, langte eine Anmeldung der Bundesrepublik Deutschland auf 460 Bilder ein, nachdem ein Herausgabeansuchen von österreichischer Seite abgelehnt worden war. ${ }^{60}$ Da Georg Weis diese Anmeldung als „sehr gut belegt und mit Sicherheit erfolgreich“ qualifizierte, weil diese Bilder dem österreichischen Staat nicht gehören würden, hätten die Sammelstellen für die restlichen „wertlosen Dinge“ fünf Millionen Schilling erhalten, weswegen er diese Mitteilungen als „streng vertraulich“ zu behandeln wünschte. Es hätte sonst der Eindruck entstehen können, die Sammelstellen wären „überzahlt“ worden. ${ }^{61}$

Die Sammelstellen wurden nach Beendigung ihrer Tätigkeit am 10. Mai 1972 aufgelöst. Doch die Prognose von Georg Weis über einen positiven Ausgang der deutschen Anmeldung bestätigte sich nicht. Die Republik Österreich, die während der Zeit aufrechter Rückstellungsfristen zahlreiche Rückstellungsanträge nach dem Ersten Rückstellungsgesetz mit der Begründung abgewiesen hatte,

55 Nach Kurt Haslinger langten bis zum Ende der Frist am 31.12.1972 insgesamt 543 Anmeldungen ein, von denen allerdings 200 wieder zurückgezogen wurden. Folglich beanspruchten 343 insgesamt 1.231 Positionsnummern laut Liste. Kurt Haslinger: Mauerbach und der lange Weg bis zur Auktion: 1969 bis 1996. In: Kunstraub, Kunstbergung und Restitution in Österreich 1938 bis heute. Hg. von Theodor Brückler. Wien-Köln-Weimar: Böhlau 1999, S. 43.

56 ÖStA, AdR, BMF, Bestand Sammelstellen, Mappe S 9h, „Kunstgutgesetz, Diverse Korrespondenz und Stella Klein-Löw“, Weis an Fraenkel, 08.01.1971.

57 BGBl. Nr. 311/1971.

58 ÖStA, AdR, BMF, Bestand Sammelstellen, Mappe S 9c, „Korrespondenz mit Kuratorien und Dr. Goldmann“, Georg Weis an die Mitglieder des Kuratoriums der Sammelstelle A und der Sammelstelle B, 25.08.1971.

59 ÖStA, AdR, BMF, Bestand Sammelstellen, Mappe S 9h, „Kunstgutgesetz, Diverse Korrespondenz und Stella Klein-Löw“, Georg Weis an die Mitglieder des Kuratoriums der Sammelstelle A und der Sammelstelle B, 25.08.1971.

60 ÖStA, AdR, BMF, Bestand Sammelstellen, Mappe S 9e, „Kunstgutgesetz, Bundesschatzministerium“, Ernst Katzenstein, Conference on Jewish Material Claims against Germany, an Georg Weis, 26.07.1972.

61 ÖStA, AdR, BMF, Bestand Sammelstellen, Mappe S 9h, „Kunstgutgesetz, Diverse Korrespondenz und Stella Klein-Löw“, Georg Weis an Simon Wiesenthal, 28.01.1971. 
dass sich die Kunstgegenstände nicht in „Verwaltung“ des Bundesdenkmalamtes im Sinne dieses Gesetzes, sondern lediglich in „Obhut“ oder in einer „treuhändigen Verwahrung“ für einen noch nicht identifizierten ursprünglichen Eigentümer befinden würden, brachte eine Parallelanmeldung zur deutschen Anmeldung ein. Wenn sich die Behauptung im Antrag der BRD als richtig erweisen sollte und es sich um Reichseigentum handle, argumentierte sie nun, dann wäre dieses Eigentum auf Grund des Staatsvertrages 1955 auf die Republik Österreich übergegangen. ${ }^{62}$

Damit war auch ein von Anfang an nicht allzu großes Engagement von deutscher Seite auf ein Minimum reduziert. Ein Vertreter des deutschen Bundesministeriums für Wirtschaft und Finanzen erklärte im Sommer 1972 gegenüber Georg Weis, der auch nach der Auflösung der Sammelstellen wegen einer möglichen Übergabe der Kunstgegenstände an eine andere Organisation weiter verhandelte, die Anmeldung wäre nur „sicherheitshalber“ eingebracht worden, um zukünftige Verhandlungen zu wahren. Er könne den Eigentumsnachweis an den Bildern gar nicht zu 100 Prozent, wie verlangt, erbringen. ${ }^{63}$

Zuletzt führte dieser Vertreter am 9. August 1972 aus, dass „rechtmäßig erworbenes Reichseigentum“ gemäß Artikel $22 \$ 1$ und 11 des österreichischen Staatsvertrages auf Grund des Protokolls der Berliner Konferenz vom 2. August 1945 an Österreich gefallen war. Sowohl am Tage dieser Konferenz hätten sich die Kunstgegenstände in Österreich als auch am Tage des Abschlusses des Staatsvertrages wieder in Österreich befunden. ${ }^{64}$ Am 25. September 1972 schrieb Georg Weis an seinen Verbindungsmann in der BRD, Ernst Katzenstein von der „Conference on Jewish Material Claims against Germany“, in dieser Angelegenheit „nichts weiter zu unternehmen“. ${ }^{65}$

62 ÖStA, AdR, BMF, Bestand Sammelstellen, Mappe S 9e, „Kunstgutgesetz, Bundesschatzministerium“, Georg Weis an Ernst Katzenstein, 12.06.1972.

63 Ebd., Georg Weis an Ernst Katzenstein, 26.07.1972.

64 Ebd., Georg Weis an Ernst Katzenstein, 09.08.1972.

65 Ebd., Ernst Katzenstein an Georg Weis, 02. 10.1972. 


\title{
Alexandra Caruso / Anneliese Schallmeiner
}

\section{Das Bundesdenkmalamt und der Bestand der sogenannten „1960er Jahre Zuweisungen“}

\begin{abstract}
Zusammenfassung
Der angesprochene Bestand konstituiert sich als solcher erst nach dem Urteil zum Vermögensverfall Adolf Hitlers im September 1952. Die der Republik Österreich verfallenen Kunstobjekte lagerten über Jahre in mehreren Depots, die von der Denkmalbehörde verwaltet wurden. Die Deponierungen innerhalb Österreichs und die Schritte bis hin zur treuhänderischen Übernahme und der schlussendlichen Inventarisierung in den Bestand der Bundesmuseen in Wien zogen sich hin bis in das Jahr 1965. Heute steht dieser Bestand im Fokus der Provenienzforschung.
\end{abstract}

Schlagwörter

Bundesdenkmalamt, 1960er Jahre-Zuweisungen, Treuhand

\begin{abstract}
The Federal Monuments Authority (Bundesdenkmalamt, BDA) and the Inventory of the So-Called "Assignments of the 1960s"

The inventory under consideration here was constituted only after the ruling in September 1952 which stripped Adolf Hitler of all financial assets. The objects of art that were forfeited to the Republic of Austria were deposited in different warehouse locations and administered by the Monuments Office. Deposition in Austria and the steps towards the objects being taken into trust and the final creation of inventories in the public museums in Vienna continued until 1965. Today this inventory is a major focus of provenance research.
\end{abstract}

Keywords

Federal Monuments Authority, Assignments of the 1960s, Trusteeship 


\section{Der Bestand in der heutigen Forschung}

Anders als in der Provenienzforschung meist üblich, geht es im nachfolgenden Beitrag nicht vordergründig um die Darstellung des individuellen Wegs einzelner Kunstwerke, sondern um die Provenienz eines Teilbestands, der unter den österreichischen ProvenienzforscherInnen als die sogenannten „1960er JahreZuweisungen" tituliert wird.

„1960er Jahre-Zuweisungen“ deshalb, weil die Kunstobjekte nach komplexen Vorgängen, die sie zu einer Gruppe zusammenfügten, in den 1960er Jahren vier Wiener Bundesmuseen, nämlich dem Kunsthistorischen Museum, der Österreichischen Galerie, der Grafischen Sammlung Albertina und, im Fall eines Objekts, dem Österreichischen Museum für angewandte Kunst in treuhändige Verwahrung und in der Folge zur Inventarisierung übergeben wurden. Die meisten Werke befinden sich auch heute noch in den Museen, einige wurden in den letzten Jahren an die RechtsnachfolgerInnen der ursprünglichen EigentümerInnen zurückgegeben.

Um die Konstituierung des Bestands, so wie er sich 1963 bzw. 1965 präsentierte, nachvollziehen zu können, muss eine Vielzahl an Archivalien vor allem aus dem Archiv des Bundesdenkmalamts und dem Österreichischen Staatsarchiv herangezogen werden.

Die wichtigsten Hinweise finden sich in Materialien zum geplanten „Linzer Kunstmuseum“" ${ }^{\text {, }}$ zum Münchner Central Art Collecting Point ${ }^{2}$, in Akten zum Depot Stift Kremsmünster ${ }^{3}$ und zu jenen des Bundesdenkmalamts in Wien und Salzburg, ${ }^{4}$ zu den Bergungsorten Salzberg Altaussee und Schloss Thürnthal ${ }^{5}$ sowie in Beständen der Ministerien für Unterricht und Finanzen im Österreichischen Staatsarchiv ${ }^{6}$.

Dieser Aufsatz bemüht sich um eine knappe Darstellung des historischen Gerüsts, der Herausformung des Bestands zu dem, was er heute ist, um dessen

1 Bundesdenkmalamt-Archiv (BDA-Archiv), Restitutionsmaterialien (RestMat.), Karton (Kt.) $13-13 / 2$.

2 BDA-Archiv, RestMat., Kt. 11-12/4.

3 BDA-Archiv, RestMat., Kt. 13/3-13/4.

4 BDA-Archiv, RestMat., Kt. 5/1-5/2; Kt. 6-6/1; Kt. 7-7/1.

5 BDA-Archiv, RestMat., Kt. 22-22/5; Kt. 3-3/1.

6 V.a. Österreichisches Staatsarchiv (ÖStA), Archiv der Republik (AdR) 06, Bundesministerium für Finanzen (BMF), Kartei Bundesministerium für Finanzen, Abt. 33; Federführende Abteilung des BMF alle Angelegenheiten Kunstgegenstände betreffend, war die Abteilung 32. Ihr kam im Wesentlichen die Zusammenarbeit mit dem BDA zu; erforderlichenfalls musste sie sich mit den Abteilungen 35 - zuständig für deutsches und ausländisches Eigentum sowie Rückführungen aus Deutschland, 31 - Vermögen der NSDAP und ihrer Gliederungen so wie 32 - Vermögen von Kriegsverbrechern, ins Einvernehmen setzen. ÖStA, AdR 06, BMF, Sonderlegung 10, Adolf Hitler, Vermögensverfall; ÖStA, AdR 02, BMU, 15 B1, Denkmalamt. 
Abgrenzung innerhalb des Gesamtbestands „Führermuseum“ sowie um die Beleuchtung des behördlichen Umgangs mit den Objekten in diesem Konvolut.

\section{Beschreibung des Bestands}

Bei der treuhändigen Übernahme durch die vier Bundesmuseen im Jahr 1963, umfasste der Bestand 98 Positionen mit insgesamt 266 Kunstobjekten und setzte sich aus Gemälden, Grafiken, Skulpturen sowie einem holländischen Spieltisch zusammen. Allen gemeinsam war, dass sie für die Sammlungen des von Adolf Hitler in Linz geplanten Museums (d.h. „Führermuseum“ bzw. „Kunstmuseum Linz") bestimmt gewesen waren.

Die ursprünglichen EigentümerInnen der Kunstwerke waren in den meisten Fällen bekannt, sogar im Fall einiger aus dem Kunsthandel erworbener Objekte. Etliche der ehemaligen EigentümerInnen stellten nach dem Krieg Rückstellungsansprüche.

\section{Analyse des Bestands als ein Teilbestand der Sammlung für das Kunstmuseum in Linz}

Es ergeben sich acht Kategorien, in die die Objekte eingeteilt werden können:

1. Objekte aus dem, gemäß der 11 . Verordnung zum Reichsbürgergesetz ${ }^{7}$, dem Deutschen Reich verfallenen Vermögen. So wurde zum Beispiel das Bild von Johann Gualbert Raffalt, Zwei Ungarn mit zwei Pferden in der Puszta, Öl/ Holz, unentgeltlich dem „Führermuseum“ zugeteilt. Die ehemalige Eigentümerin Wally Kulka (1882-1942) überlebte den Holocaust nicht. ${ }^{8}$ Eine Reihe von Missverständnissen im Zusammenhang mit dem Rückstellungsantrag der ErbInnen führten dazu, dass das Kunstwerk weiterhin in der Masse Vermögensverfall Adolf Hitler verblieb. Im März 2003 empfahl der Beirat gemäß $\$ 3$ des Bundesgesetzes über die Rückgabe von Kunstgegenständen aus den Österreichischen Bundesmuseen und Sammlungen, BGBl. I Nr. 181/ 1998 (Kunstrückgabegesetz) schließlich eine Rückgabe an die RechtsnachfolgerInnen nach Wally Kulka.

7 RGBl. I 1941, S. 722-724.

8 Siehe URL: http://www.provenienzforschung.gv.at/beiratsbeschluesse/Kulka_Wally_200303-11.pdf (abgerufen am 16.06.2017). 
2. Objekte, die von verfolgten Personen an diverse EinkäuferInnen ${ }^{9}$ für das geplante „Führermuseum “ und in der Folge an den „Sonderbeauftragten “10 verkauft wurden. In diesen Fällen kann aufgrund der geleisteten diskriminierenden Abgaben, wie Reichsfluchtsteuer und Judenvermögensabgabe, nicht davon ausgegangen werden, dass der Verkaufserlös den Personen zur Verfügung gestanden hat. ${ }^{11}$

3. Objekte, die an Zahlungsstatt von verfolgungsbedingt bereits emigrierten Personen an deren Rechtsvertreter übergeben und über diese an das „Linzer Kunstmuseum " verkauft wurden. ${ }^{12}$ Anzuführen wäre hier unter anderem das Gemälde von Franz Eybl, Bäuerin am Spinnrad, das im März 1940 durch den Rechtsvertreter von Josef (1883-1944) und Auguste (1885-1975) Blauhorn, Dr. Hans Dechant (1889-1970), an das „Kunstmuseum Linz“ verkauft wurde. Ein zweimaliger Antrag auf Rückstellung nach dem Dritten Rückstellungsgesetz bei der Finanzlandesdirektion für Wien, Niederösterreich und Burgenland sowie bei der Rückstellungskommission beim Landesgericht in Salzburg wurde zurückgewiesen. Schon im Jahr der treuhändigen Übernahme durch die Österreichische Galerie wurde das Gemälde im Zuge eines Tauschverfahrens abgegeben.

$9 \mathrm{Zu}$ den bekannten EinkäuferInnen für das „Führermuseum“ zählten etwa die Münchner Galeristin Maria Almas-Dietrich (1892-1971) und der Kunsthändler Karl Haberstock (1878-1956).

10 Der Sonderauftrag Linz wurde von Hitler im Juni 1939 eingerichtet. Erster Sonderbeauftragter für das Kunstmuseum in Linz war Hans Posse (1879-1942). Ihm folgten nach seinem Tod Hermann Voss (1884-1969) und als Referent des Sonderauftrags Gottfried Reimer (1911-1987) nach.

11 Zur Vorgangsweise hieß es wie folgt: „[...] Soweit Gegenstände beschlagnahmt oder sichergestellt wurden, ohne dass eine Einziehung zu Gunsten des Reiches erfolgt ist, stehen sie weiterhin im Eigentum der Juden. Um sie entsprechend dem Willen des Führers zum Ausbau und zur Vervollständigung der ostmärkischen Kunstsammlungen verwerten zu können, ist es erforderlich, Zwangsentjudungsverfahren einzuleiten. Den Juden müsste - soweit sie ausgewandert sind, durch Bekanntmachung im Reichsanzeiger - gemäss $\$ 6$ der Verordnung über den Einsatz des jüdischen Vermögens vom 3. Dezember 1938 - Reichsgesetzbl. I S 1709 - die Veräusserung der betreffenden Gegenstände an den Beauftragten des Führers, Direktor Posse, gegen einen festgesetzten Schutzpreis aufgegeben werden. Erforderlichenfalls wären zur Durchführung dieser Veräusserungen Treuhänder nach $\$ 2$ der Verordnung vom 3. Dezember 1938 einzusetzen. Der Erlös würde formell dem Juden zustehen, praktisch aber regelmässig zur Deckung der Steuerforderungen des Reiches und zur Bezahlungen sonstiger Schulden dienen. Ein etwaiger Überschuß wäre auf Devisensperrkonto einzuzahlen. [...] “. BDA-Archiv, RestMat., Kt. 8, M. 11, fol. 7, Reichswirtschaftsminister an Reichsminister und Chef der Reichskanzlei, 10.06.1940.

12 Siehe dazu: Anita Stelzl-Gallian: Der Fall Blauhorn. Das Schicksal einer Sammlung. In: ... wesentlich mehr Fälle als angenommen. 10 Jahre Kommission für Provenienzforschung. Hg. von Gabriele Anderl, Christoph Bazil, Eva Blimlinger u. a. Wien-Köln-Weimar: Böhlau 2015 (=Schriftenreihe der Kommission für Provenienzforschung 1), S. 360-374, hier S. 369. 
4. Objekte, die über Vertrauenspersonen an das „Linzer Kunstmuseum“ verkauft wurden. Wie im Fall der Holzskulptur des Meisters von Großlobming, Auferstandener Heiland („Pfenningberger Schmerzensmann“), die vorerst dem Vater von Franz Erlach (1892-1974) bzw. Schwiegervater Helene Erlachs (1897-?) zur Verwahrung übergeben worden war. Später übernahm der Bruder Franz Erlachs, Alois Erlach (1893-1948), die sichergestellte Skulptur und verkaufte sie an den „Sonderauftrag Linz“. Nach 1945 versuchten die ehemaligen Eigentümer in mehreren Rückstellungsverfahren erfolglos das Kunstwerk zurückzuerlangen. Im Dezember 2007 wurde die Rückgabe der Skulptur an die Erben beschlossen. ${ }^{13}$

5. Objekte, die von Personen im Zuge eines Notverkaufs, z. B. zur Absicherung des Lebensunterhalts, verkauft wurden, wobei den Verkäufern der Erlös zur Verfügung stand. Hierzu gibt es im Bestand der „1960er-Zuweisungen“ einige Beispiele. Etwa musste Leopoldine Mannaberg (1903-1996) nach dem Tode ihres Ehemanns, Julius Mannaberg (1860-1941), ab 1941 mehrere „Notverkäufe“ tätigen. Obwohl Mannaberg mit seiner Ehefrau, die als „Arierin“ galt, in einer so genannten „privilegierten Mischehe“ gelebt hatte, war er nach der NS-Gesetzgebung verpflichtet gewesen, sein Vermögen $\mathrm{zu}$ deklarieren. ${ }^{14}$ Seine Witwe musste dann zur Begleichung der noch nicht gänzlich abgegoltenen „Judenvermögensabgabe“ und der für sie und ihre Tochter anfallenden Lebenserhaltungskosten, meist über Vermittlung von Kunsthändlern, u. a. die Zeichnung von Adolf Menzel, Fahnen- oder Laternenträger und Frau mit Kind auf dem Arm an den "Sonderauftrag Linz“ verkaufen. Nach 1945 wurden keine Rückstellungsansprüche geltend gemacht. Im Juli 2014 empfahl der Beirat die Rückgabe des Blattes an die RechtsnachfolgerInnen. ${ }^{15}$

6. Objekte, deren Erlös den VerkäuferInnen ohne Wenn und Aber zur Verfügung stand. Bekanntester Fall ist der von Jaromir Czernin (1908-1966), der sein Gemälde Der Künstler in seinem Atelier von Jan Vermeer ohne erkennbaren Druck an Hitler verkaufte. ${ }^{16}$

7. Objekte, die über Ankäufe aus dem Kunsthandel in den Bestand des geplanten „Führermuseums“ kamen. Dies betraf vier grafische Werke aus der ehemaligen Sammlung Julius Freund (1869-1941), die sich seit September 1933 unter mehreren hundert Werken als Leihgaben in den Museen in

13 Siehe URL: http://www.provenienzforschung.gv.at/beiratsbeschluesse/Erlach_Franz_Hele ne_2007-12-07.pdf (abgerufen am 16.06.2017).

14 Gemäß der „Verordnung über die Anmeldung des Vermögens von Juden“vom 26. April 1938.

15 Siehe URL: http://www.provenienzforschung.gv.at/beiratsbeschluesse/Mannaberg_Julius_ 2014-07-03.pdf (abgerufen am 16.06.2017).

16 Siehe dazu: Die verkaufte Malkunst. Jan Vermeers Gemälde im 20. Jahrhundert. Hg. von Susanne Hehenberger und Monika Löscher. Wien-Köln-Weimar: Böhlau 2013 (= Schriftenreihe der Kommission für Provenienzforschung 4). 
Winterthur und Basel befanden. Etwas später schenkte Freund diese Leihgaben seiner Tochter Gisèle Freund (1908-2000). Im März 1942 wurden ca. 350 Werke bei der Auktion Sammlung Julius Freund. Aus dem Besitz von Frau Dr. G. Freund, Buenos Aires. Gemälde, Aquarelle, Zeichnungen und Druckgraphik in der Galerie Fischer in Luzern angeboten. Darunter befanden sich auch jene, die - angekauft über den „Sonderauftrag“ für das „Linzer Museum " - schließlich in den 1960er Jahren in der Albertina als so genanntes „Verfallsgut" inventarisiert wurden. ${ }^{17}$

8. Objekte, die durch NS-Behörden beschlagnahmt wurden. Die Sammlungen von Louis und Alphonse Rothschild waren wohl die bekanntesten in dieser Kategorie. Trotz groß angelegter Rückstellungen an die Familien Rothschild ab 1947 war der Holländische Spieltisch nach japanischer Art aus der ehemaligen Sammlung von Louis Rothschild übersehen worden und im Zuge der 1960er Jahre-Zuweisungen an das Museum für Angewandte Kunst gelangt, wo er bis zur Rückgabeempfehlung des Kunstrückgabebeirats im Jahr 2009 verblieb. ${ }^{18}$

\section{Depots und Verlagerungen}

Im Gefolge der Machtergreifung durch die Nationalsozialisten in Österreich wurden zahlreiche Kunstsammlungen und Einzelobjekte beschlagnahmt oder sichergestellt. Diese lagerten dann, gemeinsam mit den Ankäufen für das geplante „Linzer Museum“, in dem durch den Kommissarischen Leiter und späteren Ersten Direktor des Kunsthistorische Museums, Fritz Dworschak (1890-1974), verwalteten Zentraldepot in der Neuen Burg sowie in Depots der Denkmalbehörde. Bereits in der Anfangsphase und schon mit der Absicht ein „Führermuseum“ zu installieren, wurden Wiener Ankäufe für Linz nach München in den „Führerbau“ zur Inventarisierung und Deponierung übersandt. Mit fortschreitendem Kriegsverlauf wurden die in Wien lagernden Kunstwerke in das Stift Kremsmünster verbracht, bis sie schließlich im Salzbergwerk von Altaussee, beziehungsweise die großformatigen Gemälde in Schloss Thürnthal bei

17 Der Beirat empfahl gemäß $\$ 3$ des Bundesgesetzes über die Rückgabe von Kunstgegenständen aus den Österreichischen Bundesmuseen und Sammlungen, BGBl. I Nr. 181/1998 i.d.F. BGBl. I Nr. 117/2009, (Kunstrückgabegesetz) im Juni 2016 auf Basis des von Pia Schölnberger verfassten, unveröffentlichten Dossiers die Nichtrückgabe der Blätter: URL http://www.provenienzforschung.gv.at/beiratsbeschluesse/Freund_Julius_2016-06-23.pdf (abgerufen am 16.06.2017).

18 Siehe URL: http://www.provenienzforschung.gv.at/beiratsbeschluesse/Rothschild_Louis_ 2009-09-11.pdf (abgerufen am 16.06.2017). 


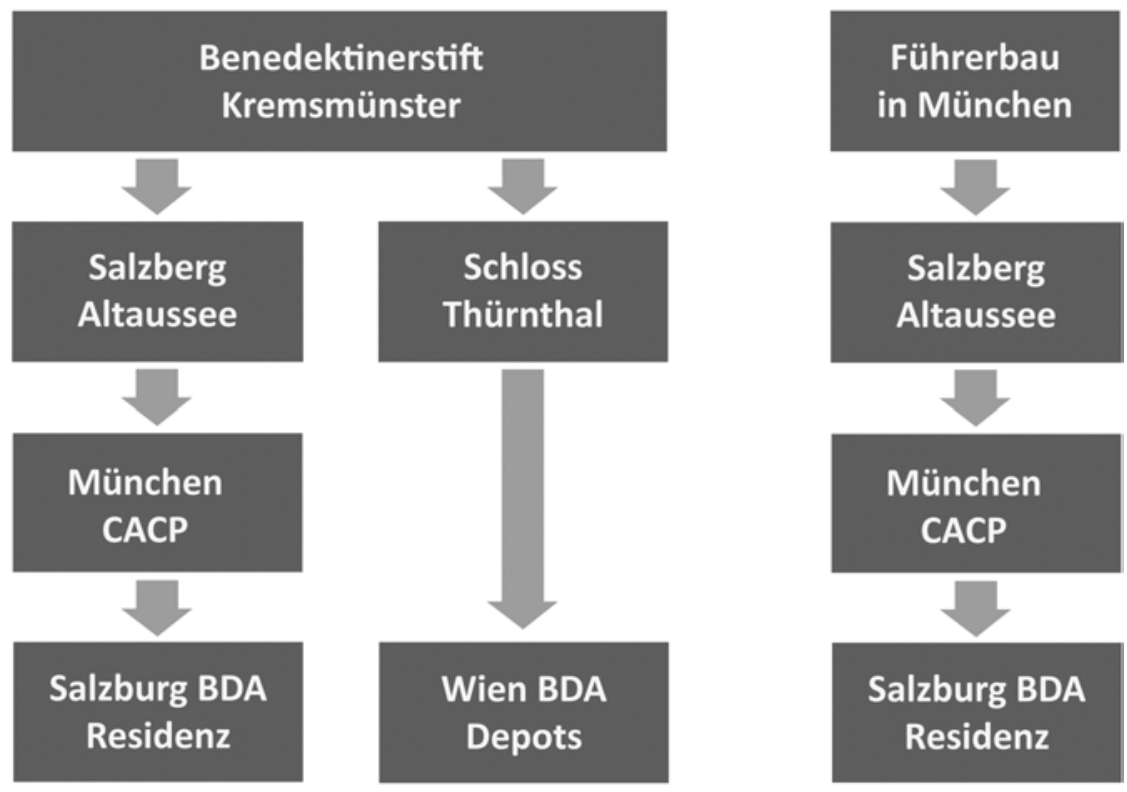

Abb. 1. Verlagerungen der Kunstwerke 1940-1963

Fels am Wagram geborgen wurden. Ab Mitte Mai 1944 wurden auch die Objekte aus dem „Führerbau“ in München nach Altaussee ausgelagert. ${ }^{19}$

Nach Kriegsende wurden diese im Salzbergwerk gelagerten Kunstwerke in dem von der amerikanischen Militärbehörde eingerichteten Central Art Collecting Point in München zusammen getragen. Im Laufe des Jahres 1947 gelangten im Rahmen eines „Sonderauftrags“ mit der Bezeichnung „Rückgabe des enteigneten privaten Kunstbesitzes“ Kulturgüter nach Wien, in die Depots Löwelstraße und Neue Burg. Diese ursprünglich für das „Führermuseum“ bestimmten Kulturgüter, die in Schloss Thürnthal lagerten und von der sowjetischen Besatzungsmacht verwaltet wurden, wurden im Sommer 1948 in Depots der Denkmalbehörde nach Wien verbracht. ${ }^{20}$

Ab 1948 wurde der Großteil des in der amerikanischen Zone befindlichen Teilbestands mit von der US-Militärverwaltung organisierten Transporten ${ }^{21}$ aus

19 Anneliese Schallmeiner: „Die modernen Nibelungen salzen ihre Schätze ein“. Altaussee als Bergungsort des Instituts für Denkmalpflege. In: Bergung von Kulturgut im Nationalsozialismus. Mythen - Hintergründe - Auswirkungen. Hg. von Pia Schölnberger und Sabine Loitfellner. Wien-Köln-Weimar: Böhlau 2015 (= Schriftenreihe der Kommission für Provenienzforschung 5), S.103-127, hier S. 118.

20 BDA-Archiv, RestMat., Kt. 3/1, M. 6, GZl. 5931/1948, Abschrift Schreiben BDA an Prof. Sazanow, Sowjetischer Teil der Interalliierten Kommission in Österreich, 16.07.1948.

21 Obwohl die amerikanischen Transporte schon ab der zweiten Hälfte des Jahres 1945 durchgeführt wurden, kamen die Kunstgüter des zu behandelnden Bestands erst 1948 nach 






der mit dem 16. and 17. amerik. Transport von 15.III.1948 von

Hinchen nach Salzburg ruckgefuhrten Kunstgegenstïnde der Sammlungf

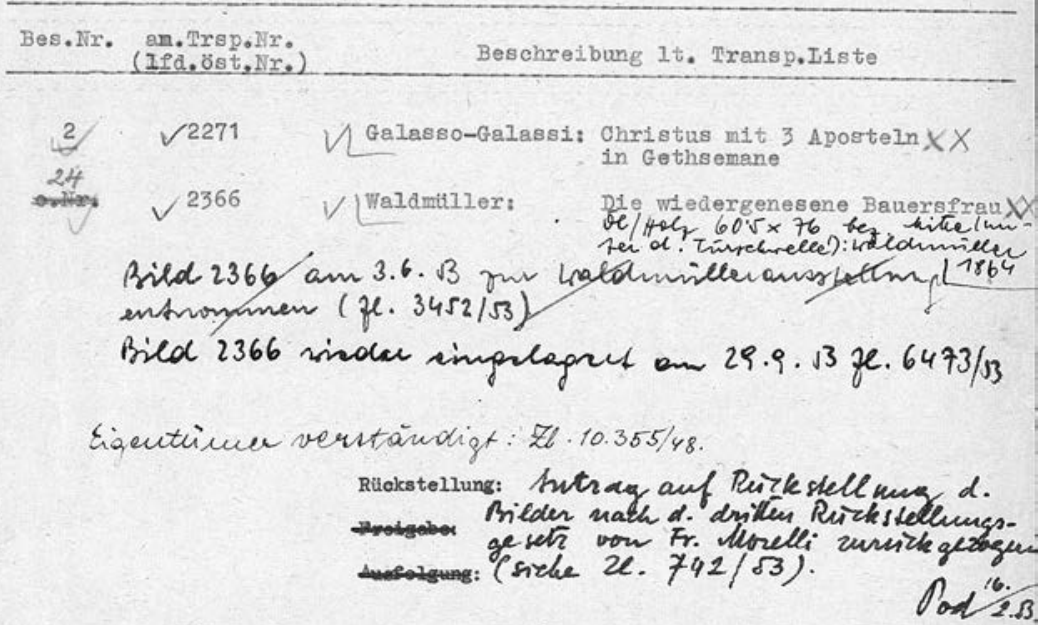

Bei repolnantulle v.15.- 17.5. 1950

rulanden (ince fe. $4951 / 50$ )

Pei depostantiolle am $3^{2} 4.4951$.

rukanden ( rieke fl. 3080/57)

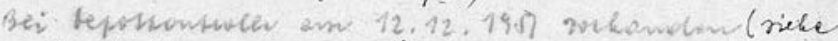

o. $9471 / 51$ )

Bei beputhankolla v. 19.1. B milanden $($ He. 2056/53)

ivale.

$2271 \mathrm{fs}$. He. $6666 / 13$ der Repubeis ìten. 8.4. 3

recfellen mind on BMF rieleyperncl.

Abb. 2: Transportübernahme 1948 mit weiterführenden Kontrollvermerken

Österreich zurück und wurden in dem vom BDA verwalteten Depot in der Salzburger Residenz zwischengelagert. BDA-Archiv, RestMat., Kt. 12/4, M. 2, ohne GZl., fol. 2-67. 
München in ein Depot des Bundesdenkmalamts in der Salzburger Residenz überführt. Die Objekte standen zwar nach wie vor in der Verfügungsgewalt der amerikanischen Militärbehörde, wurden aber von dieser zur Gewährleistung der Rückstellungen dem Bundeministerium für Finanzen in „treuhändige Verwaltung“ übergeben. Die Denkmalbehörde fungierte ab diesem Zeitpunkt als „treuhändiger Verwahrer“. ${ }^{22}$

\section{Juristische Aspekte des Bestands nach dem Krieg: Der Vermögensverfall Adolf Hitler}

Anstoßgebend für die Einleitung des Vermögenverfallsverfahrens gegen Adolf Hitler im Jahr 1952 war, unter anderem, das neuerlich von Jaromir Czernin gegen die Republik Österreich eingeleitete Verfahren zur Rückstellung des Gemäldes von Jan Vermeer, Der Künstler in seinem Atelier. In den vorangegangenen Jahren waren Verfahren in dieser Causa bereits in allen drei Instanzen meritorisch abgewiesen worden. Überdies hatte Czernin bei der Rückstellungskommission Wien einen Rückstellungsantrag gegen das Deutsche Reich eingebracht. ${ }^{23}$ Auf Nachfrage des Bundesministeriums für Finanzen, wie viele Kunstgegenstände aus dem Eigentum Hitlers sich damals in Österreich befänden, berichtete das Bundesdenkmalamt als nachgeordnete Dienststelle des Bundesministerium für Unterricht, dass sich in seiner Verwahrung

„kein Wertgegenstand befindet, der eindeutig als Privateigentum Adolf Hitlers anzusprechen wäre; dieser Bericht gilt aber nicht für jene Objekte, die seinerzeit für das sogenannte Führermuseum in Linz bestimmt waren $[\ldots]^{\text {“. } .24}$

Das Bundesdenkmalamt habe sich bereits seit Jahren vergeblich bemüht, „Beweise ausfindig zu machen, die das ausschließliche und tatsächliche Eigentum Adolf Hitlers an den für das geplante ,Linzer Kunstmuseum' bestimmten Objekten einwandfrei erwiesen hätten " und ersuche daher um die Vorlage derartiger Beweise. ${ }^{25}$ Daraufhin drängte die Finanzprokuratur auf eine beschleunigte Abwicklung des Verfallsverfahrens. Das Gemälde Vermeers sei eindeutig als Privateigentum Hitlers zu betrachten, weshalb eine Passivlegitimation des Deutschen Reichs in diesem Rückstellungsverfahren hinfällig wäre.

22 BDA-Archiv, RestMat., Kt. 11, M. 2, GZl. 330/1948, GZl. 1606/1948.

23 ÖStA, AdR 06, BMF, Sonderlegung 10, Adolf Hitler, Vermögensverfall, GZl. 191.457-32/1952, Aktenvermerk o. D.

24 ÖStA, AdR 06, BMF, Sonderlegung 10, Adolf Hitler, Vermögensverfall, GZl. 191.457-32/1952, BMU an BMF, 05. 04. 1952.

25 BDA-Archiv, RestMat., Kt. 27/1, M. 6, GZl. 6666/1953, BDA an BMF, 20.10.1953. 
„[...] Zwecks Vermeidung weiterer Kuratorskosten und um vor allem zu erreichen, dass die Rep. Österreich in einem eventuell weiteren Rückstellungsverfahren unmittelbar legitimiert wäre und einschreiten könnte, empfiehlt sich ein umgehendes Verfallserkenntnis im Gegenstande $[\ldots]^{\text {“. }}{ }^{26}$

Da Hitler in seiner Funktion als Führer und Reichskanzler des ehemaligen Deutschen Reichs als Kriegsverbrecher im Sinne des Kriegsverbrechergesetzes (KVG) anzusehen war, bestand von Seiten der Republik Österreich Interesse, die im KVG vorgesehene Strafe des Vermögensverfalls in einem selbstständigen Verfahren gemäß $\$ 24$ des Volksgerichts- und Vermögensverfallsgesetzes von 1947 (VvVvG) anzustrengen. ${ }^{27}$ Bereits im Vorfeld der Einleitung des Verfahrens wurde auf Antrag der Staatsanwaltschaft Wien und mit Beschluss der Ratskammer des Landesgerichts für Strafsachen Wien die Beschlagnahme des Vermögens nach Adolf Hitler gemäß $\$ 5$ des $\mathrm{VvVvG}^{28}$ angeordnet. $^{29}$

Am 5. September 1952 wurde dem Antrag der Staatsanwaltschaft Wien auf Verfall des Vermögens zu Gunsten der Republik Österreich ganz allgemein und ohne Spezifizierung einzelner Vermögensbestandteile Folge geleistet. Das Bundesministerium für Finanzen, in dessen Verantwortlichkeit das Vermögen von Kriegsverbrechern fiel, setzte sich mit seiner Ansicht durch, dass alle zum Bestand des geplanten „Linzer Kunstmuseums“ gehörigen Objekte unter den Begriff „Vermögen Adolf Hitler“ einzuordnen und demnach der Republik Österreich verfallen seien.

In weiterer Folge erstellte die Denkmalbehörde eine Auflistung der „verfallenen“ Objekte, die sich in ihrer Verwahrung befanden. Im Oktober 1953 ordnete das Bundesministerium für Finanzen die Sicherstellung der gelisteten Kunstwerke an. ${ }^{30}$ Mit der Übergabe der aus München von den Amerikanern rücktransportierten Kulturgüter war die Auflage verbunden, sie auf Basis der österreichischen Rückstellungsgesetzgebung den bereits zum Zeitpunkt der Freigabe genannten Rückstellungswerbern auszufolgen. Da die meisten Objekte jedoch weiterhin der amerikanischen Jurisdiktion unterlagen, betrachtete die Denkmalbehörde den Umfang der Sicherstellungen als problematisch. ${ }^{31}$

Vor und während des Vermögensverfallsverfahrens gegen Hitler waren für diesen Bestand mehrere Rückstellungsverfahren nach dem Ersten, Zweiten und

26 ÖStA, AdR 06, BMF, Sonderlegung 10, Adolf Hitler, Vermögensverfall, GZl. 191.457-32/1952, Abschrift Schreiben Finanzprokuratur an Bundesministerium für Justiz, 17.07.1952.

27 ÖStA, AdR 06, BMF, Sonderlegung 10, Adolf Hitler, Vermögensverfall, GZl. 191.457-32/1952, Aktenvermerk o. D.

28 StGBl. Nr. 177/1945.

29 BDA-Archiv, RestMat., Kt. 27/1, M. 6, GZl. 2126/1952, Abschrift Beschluss der Ratskammer des Landesgerichts für Strafsachen Wien (Vg 8e Vr 68/1952), 31.03.1952.

30 BDA-Archiv, RestMat., Kt. 27/1, M. 6, GZl. 6666/1953, BMF an BDA, 02.10.1953.

31 BDA-Archiv, RestMat., Kt. 27/1, M. 6, GZl. 6666/1953, BDA an BMF, 20.10.1953. 
Dritten Rückstellungsgesetz anhängig. ${ }^{32}$ Nach dem Vermögensverfall war ein Antrag auf Rückstellung der in das Vermögen der Republik Österreich übergegangenen Objekte jedoch ausschließlich nach den Bestimmungen des Zweiten Rückstellungsgesetzes möglich. Dieses besagte, dass entzogene Vermögen, die nach dem Vermögensverfall in das Eigentum der Republik Österreich übergegangen waren, den vormaligen Eigentümern oder deren Erben wegen Nichtigkeit der seinerzeitigen Vermögensentziehung zurückzustellen seien. ${ }^{33}$ Der durch den Verfall bedingte Wechsel in den Zuständigkeiten sorgte bei Antragstellern und auch bei den behandelnden Behörden für Verwirrung und Verschleppung der Verfahren.

\section{Letzte Zu- und Abgänge}

In der zweiten Hälfte der 1950er Jahre erhielten die „1960er Jahre-Zuweisungen“ im Wesentlichen ihr heutiges Gesicht. Ein Großteil der für das „Linzer Kunstmuseum" bestimmten Kunstwerke österreichischer Provenienz war bereits vor dem Vermögensverfall Hitlers nach geltenden Rückgabegesetzen oder davor noch, im Central Art Collecting Point, im Zuge der territorialen Restitutionen, rückerstattet worden. Eine weitere Tranche war als unbeanspruchtes Vermögen den im Gefolge des österreichischen Staatsvertrags ${ }^{34}$ eingerichteten Sammelstellen übergeben worden. ${ }^{35}$ Im Jahr 1957 kamen wiederum mit Teil V des Überleitungsvertrags, der die Bundesrepublik Deutschland zur Fortführung des Restitutionsprogramms der Alliierten verpflichtete, ${ }^{36}$ ehemals für das „Führermuseum " bestimmte Kunstwerke aus Deutschland nach Österreich, von denen rund 20 in den hier behandelten Bestand einflossen. ${ }^{37}$

32 BGBl. Nr. 156/1946, 27.06.1946; BGBl. Nr. 53/1947, 06.02.1947; BGBl. Nr. 54/1947, 06.02. 1947.

33 Gerhard Sailer: Rückbringung und Rückgabe: 1945-1966. In: Kunstraub, Kunstbergung und Restitution in Österreich 1938 bis heute. Hg. von Theodor Brückler. Wien-Köln-Weimar: Böhlau 1999 (= Studien zu Denkmalschutz und Denkmalpflege 19), S. 31-38.

34 Staatsvertrag betreffend die Wiederherstellung eines unabhängigen und demokratischen Österreich. Siehe: BGBl. Nr. 152/1955 i.d. F. BGBl. III Nr. 179/2002.

35 Ab März 1957 laut BGBl. 73/1957.

36 Bruno Simma, Hans-Peter Folz: Restitution und Entschädigung im Völkerrecht. Die Verpflichtungen der Republik Österreich nach 1945 im Lichte ihrer außerpolitischen Praxis. Wien-München: Oldenbourg 2004 (=Österreichische Historikerkommission 6), S. 140-142.

37 Diese Gegenstände wurden im Depot im Wiener Arsenal, Objekt 15, zwischengelagert. Weitere Objekte, die im Zuge des Überleitungsvertrags nach Österreich zurückkamen, versuchte das BDA in den noch nicht aufgelösten Depots in Wien zwischenzulagern. BDAArchiv, RestMat., Kt. 23/1, M. 21, GZl. 10375/1959. 


\section{Wertbemessung}

Im Februar 1959 wurde das Bundesdenkmalamt erstmals durch das Bundesministerium für Unterricht angehalten, die Schätzwerte der Kunstwerke „für den ausschließlichen internen Gebrauch" festzustellen. Dieses sah sich jedoch außer Stande, „eine auch nur annähernd zuverlässige Schätzung, selbst nicht in weiten Grenzen, zu bewerkstelligen“. Es verfüge über keine Spezialisten, die über die Verhältnisse am Kunstmarkt zumindest soweit informiert seien, dass sie ein „einigermaßen brauchbares Schätzgutachten“ abgeben könnten. Dessen ungeachtet empfahl das Bundesdenkmalamt in einem Nachsatz, die „künstlerisch und geschichtlich interessanten Stücke [...] für eine Zuweisung an Bundes- und Landessammlungen ins Auge zu fassen “. .38

1961 wurde die Denkmalbehörde erneut aufgefordert - diesmal durch das Bundesministerium für Finanzen -, den Wert der einzelnen Kunstwerke für Buchungszwecke festzusetzen. ${ }^{39}$ Mit Hilfe von Sachverständigen wurden Schätzungen durchgeführt, wobei man auch auf Wertbemessungen aus der Kriegszeit zurückgriff. ${ }^{40} \mathrm{Da}$ die meisten Kunstwerke durch einen Erwerbsvorgang, das heißt durch Kauf, in den Bestand des „Führermuseums“ übergegangen waren, bat nun im Februar 1962 die Finanzprokuratur um Mitteilung, wem der bezahlte Preis zugekommen sei - dem Eigentümer oder einer Reichsstelle. Weiters solle festgestellt werden, welche Aufwendungen für die einzelnen Kunstwerke in Hinblick auf Restaurierung, Rahmung, Lagerung etc. seit deren Übernahme durch die Denkmalbehörde getätigt worden seien. Das Bundesdenkmalamt sah sich jedoch, erneut aufgrund von Arbeitskräftemangel und praktischer Schwierigkeiten, nicht in der Lage, derartige Bemessungen und detaillierte Berechnungen durchzuführen. ${ }^{41}$

38 BDA-Archiv, RestMat., Kt. 23/1, M. 21, GZl. 9579/1959, BDA an BMU, 20.11.1959, (GZl. 101.673-5/59).

39 BDA-Archiv, RestMat., Kt. 13/1, M. 10, GZl. 5157/1962, BMF an BDA, 19.05.1962, (GZl. 200.122/6-33(32)/1961).

40 BDA-Archiv, RestMat., Kt. 13/1, M. 10, GZl. 4156/1961.

41 „Es ist äußerst wichtig, dass Kaufpreis und Aufwendungen genau zu jedem einzelnen rückstellungsverfangenen Bild bekannt gegeben werden, weil im Falle der - sehr unwahrscheinlichen - Rückstellung der Gemälde, allenfalls diese Kosten von der Sammelstelle zu begleichen sind.“ BDA-Archiv, RestMat., Kt. 13/1, M.11, GZl. 1870/1962, Finanzprokuratur an BDA, 27.02.1962 (GZl. 8448-6/1962). 


\section{Räumung der Depots und ressortmäßige Übernahme des Bestands durch das Bundesministerium für Unterricht}

Per Erlass vom 20. Juli $1962^{42}$ forderte das Bundesministerium für Finanzen, als treuhändiger Verwalter, das Bundesministerium für Unterricht auf, die „für das Linzer Kunstmuseum Hitlers bestimmt gewesenen Objekte“ ressortmäßig zu übernehmen. $^{43}$

Jahrelang hatte das Amt der Salzburger Landesregierung bereits auf Räumung der beiden Depoträume in der Salzburger Residenz gedrängt. Als diese schließlich für die Salzburger Universität vorgesehen wurden, kam es zu einer endgültigen Befristung mit September $1963 .{ }^{44}$ Aufgrund der Urlaubsplanungen des zuständigen Ministerialrats im Bundesministerium für Unterricht war eine formelle Übernahme des Bestands vom Bundesministerium für Finanzen jedoch nicht mehr zeitgerecht bis September zu bewerkstelligen. ${ }^{45}$ Um das Residenzdepot dennoch fristgerecht zu räumen, entschloss man sich auf Vorschlag des Bundesdenkmalamts, die für die spätere Übernahme ohnedies bereits vorgesehenen Bundesammlungen aufzufordern, die Kunstwerke vorläufig treuhändig zu übernehmen. ${ }^{46}$ So wurde das Bundesdenkmalamt noch vor der formellen ressortmäßigen Übernahme durch das Bundesministerium für Unterricht ermächtigt, die Kunstwerke den erwähnten Sammlungen vorerst in treuhändige Verwahrung zu übergeben. ${ }^{47}$

Am 22. August 1963 fand der Transport der Kunstwerke aus Salzburg statt. Sie wurden kurzfristig im bereits überfüllten Depot Löwelstraße zwischengelagert, einzig Großformate wie das Gemälde von Galasso Galassi, Christus und die drei Aposteln aus dem ehemaligen Besitz Berta Morellis sowie das 1050 x $400 \mathrm{~cm}$ große Gemälde von Hans Makart, Venedig grüßte Katharina Cornaro gingen direkt in die für sie vorgesehenen Museen. ${ }^{48}$ Kurz danach brachte man auch die Objekte aus dem Depot Löwelstraße, dem Arsenal und der Neuen Burg in die

42 BDA-Archiv, RestMat., Kt. 13/1, M. 11, GZl.6376/1962, BMF an BDA, 20.07.1962 (GZl. 208.039/12-33(32)/1962).

43 BDA-Archiv, RestMat., Kt. 12/4, M. 2, GZl. 3846/1963, Pro domo, 05.06.1963.

44 BDA-Archiv, RestMat., Kt. 12/4, M. 2, GZl. 4389/1963, Pro domo, 11.07.1963.

45 Carl Blaha (1923-2002), Abt. 6 des BMU, siehe: BDA-Archiv, RestMat., Kt. 12/4, Mappe 2, GZl. 4389/63, Pro domo 11.07.1963; ÖStA, AdR 02, BMU/15 Kunst, Kt. 177, GZl. 71.681-5/ 1963, BDA an BMU, 05.06.1963.

46 Bereits 1962 hatte das BMF die umfangreichen Bestände Ribbentrop und Bormann zur ressortmäßigen Übernahme durch das BMU freigegeben. Die Alt-Aquarelle aus der Sammlung Bormann waren damals direkt in die Albertina gelangt. ÖStA, AdR 02, BMU/15 Kunst, GZl. 71.681-5/1963; BDA-Archiv, RestMat., Kt. 12/4, M. 2, GZl. 4389/1963, Pro domo, 11.07.1963.

47 ÖStA, AdR 02, BMU/15 Kunst, Kt.177, GZl. 71.681-5/1963; BDA-Archiv, RestMat., Kt. 12/4, M. 2, GZl. 4830/1963, BMU an BDA, 29.06.1963.

48 BDA-Archiv, RestMat., Kt.13/1, M. 10, GZl. 5977/1963, Pro domo, 23.08.1963. 
entsprechenden Häuser. ${ }^{49}$ Zwischen Dezember 1963 und Jänner 1964 fielen die als "Verfallsgut“ in das Eigentum der Republik Österreich übergegangen Kunstgegenstände schließlich auch ressortmäßig vom Bundesministerium für Finanzen an das Bundesministerium für Unterricht. Der für „Buchungszwecke einvernehmlich festgelegte Übergabswert" sämtlicher Kunstgegenstände belief sich auf 1.850 .400 Schilling. ${ }^{50}$

\section{Übernahme in die Inventare der Bundesmuseen}

Am 25. März 1965 erfolgte schließlich die Weisung des Bundesministeriums für Unterricht, die ehemals für das „Linzer Kunstmuseum“ bestimmt gewesenen Objekte, die per Vermögensverfall in das Eigentum der Republik übergegangen und 1963 den Sammlungen des Bundes in treuhändige Verwahrung gegeben worden waren, definitiv in das Inventar des entsprechenden Museums zu übernehmen. ${ }^{51}$

\section{Die Provenienzforschung und die „1960er Jahre-Zuweisungen“}

Der Bestand der „1960er Jahre-Zuweisungen“ interessiert ProvenienzforscherInnen und MuseumskuratorInnen auch heute noch. Dabei spielen die unterschiedlichen Erwerbungsvorgänge durch den NS-Machtapparat sowie formale Fehler bei der Rückstellungswerbung - etwa ein Antrag nach dem falschen Rückstellungsgesetz - oder nicht konsequent betriebener Rückstellungsbemühungen durch die Behörden nach dem Krieg eine Rolle. ${ }^{52}$ Auch ist der gesetzliche Blickwinkel auf Bedenklichkeit oder Unbedenklichkeit von Erwerbsvorgängen zeitbedingten Veränderungen unterworfen. Die heutige Provenienzforschung hat bereits mehrere Fälle aufgegriffen, die im Zuge der Rückstellungsgesetzgebung der Nachkriegsjahre (einschließlich der Fristverlängerungen) negativ beschieden worden waren. ${ }^{53}$ Von diesen hat der Kunstrückgabebeirat bis heute sechs zur Rückgabe an die ErbInnen bzw. deren RechtsnachfolgerInnen empfohlen. ${ }^{54}$

49 BDA-Archiv, RestMat., Kt.13/1, M. 11, GZl. 5831/1963.

50 ÖStA, AdR 02, BMU/15 Museen, GZl. 110.129-6/1963, Ressortübergabe von Kunstgegenständen an das BMU.

51 BDA-Archiv, RestMat., Kt.13/1, M. 11, GZl. 2397/1965, Abschrift BMU an Ersten Direktor KHM, 25.03.1965, 52.535-II/2/1965.

52 Wie im Fall eines Gemäldes von Eduard von Grützner (1846-1925) aus dem ehemaligen Eigentum Serena Lederers (1867-1943). Siehe URL: http://www.provenienzforschung.gv.at/ beiratsbeschluesse/Lederer_August_Serena_2012-03-02.pdf (abgerufen am 22.06.2017).

53 Es handelt sich um zehn Fälle.

54 Die Empfehlungen an die zuständige Bundesministerin / den zuständigen Bundesminister 


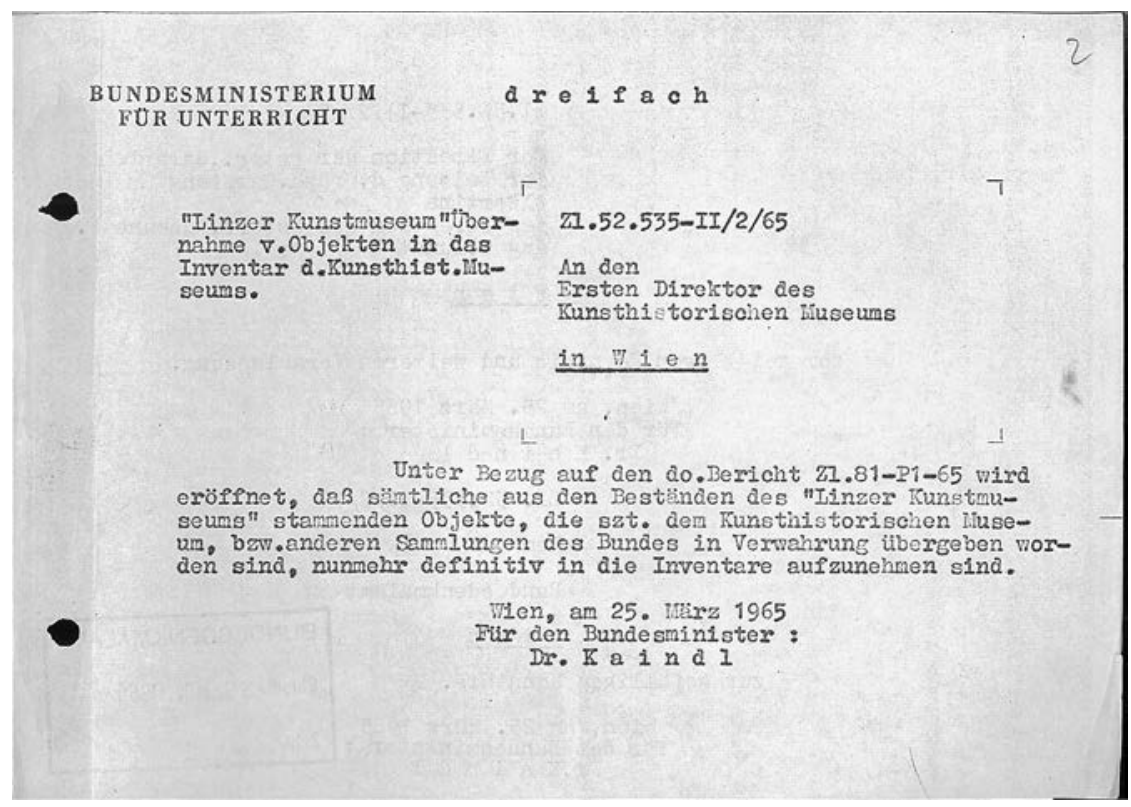

Abb. 3: Übernahme von Objekten des ehemaligen Linzer Kunstmuseums

Wie man etwa der Homepage der Österreichischen Galerie Belvedere entnehmen kann, hat die Provenienzforschung der latenten Brisanz des Bestands Rechnung getragen und führt die einzelnen Werke mit dem Namen des ursprünglichen Eigentümers, dem Erwerb durch den „Sonderauftrag Linz“ respektive für das „Linzer Museum“ und den Inventarzugang aufgrund der Übergabe durch das Bundesdenkmalamt an. ${ }^{55}$ Bereits im Jahr 2000 wurden von der Provenienzforschung an der Albertina jene Werke, die in Folge des Vermögensverfalls Adolf Hitler in das Eigentum der Republik Österreich übergegangen waren und sich seit 1963 im Sammlungsbestand befinden, in einem Gesamtdossier erfasst. Sukzessive wird heute die Herkunftsgeschichte der im Dossier angeführten Objekte von den ProvenienzforscherInnen untersucht und in Einzelfalldossiers dargestellt. In gleicher Weise verfahren auch die ProvenienzforscherInnen im Belvedere, im Kunsthistorischen Museum und im Museum für Angewandte Kunst. Es ist davon auszugehen, dass auch in Zukunft einzelne Kunstwerke aus dem Bestand der „1960er Jahre-Zuweisungen“ die Provenienzforschung beschäftigen werden.

erfolgten meist nach $\S 1$, Z. 2 des Kunstrückgabegesetzes BGBl. I Nr. 181/1998 i.d. F. BGBl. I Nr. 117/2009, der besagt, dass betroffene Objekte zwar rechtmäßig in das Eigentum des Bundes gelangt sind, zuvor aber Gegenstand eines nichtigen Rechtsgeschäftes gewesen waren.

55 Siehe URL: http://digital.belvedere.at/ (abgerufen am 25.04.2017). 
Open-Access-Publikation im Sinne der CC-Lizenz BY-NC-ND 4.0

(c) 2018, V\&R unipress GmbH, Göttingen

ISBN Print: 9783847107835 - ISBN E-Lib: 9783737007832 


\title{
Jana Kocourek
}

\section{"Offene Vermögensfragen“ - von der Suche nach sogenannten Schlossbergungsbeständen in der SLUB Dresden}

\section{Zusammenfassung}

Am 1. Dezember 2014 endete für Museen, Archive und Bibliotheken im Osten Deutschlands die zwanzigjährige Nießbrauchsfrist für Kulturgüter, die im Zuge der Bodenreform 1945/1946 enteignet worden waren. Nach der deutschen Wiedervereinigung regelt das Entschädigungsund Ausgleichleistungsgesetz (EALG) vom 27. September 1994 die Rückgabe beweglicher Sachen. Bis 1995 konnten Enteignete und ihre Erben eine Restitution bei den zuständigen Ämtern beantragen. In der Folge sahen sich Einrichtungen des Freistaates Sachsen mit zahlreichen Restitutionsbegehren konfrontiert. Ein Projekt ermöglichte der Sächsischen Landesbibliothek - Staats- und Universitätsbibliothek Dresden (SLUB) die systematische Bestandsrecherche in sämtlichen Zugängen der Jahre $1945 \mathrm{ff}$. Im Ergebnis konnte Eigentum des ehemaligen sächsischen Königshauses, aber auch weitere Bestände aus den sogenannten Schlossbergungen dokumentiert, restituiert und teilweise angekauft werden.

Schlagwörter

Provenienzforschung, NS-Raubgut, Schlossbergungen, Bodenreform, Sachsen, DDRUnrecht

\begin{abstract}
"Unsettled Property Issues" - About the Research for So-Called Castle Salvaging in the Saxon State and University Library Dresden

On December 1, 2014 the twenty-year usufructuary period for cultural property, which had been expropriated in the course of the land reform from 1945, ended for the museums, archives and libraries in East Germany, e.g. more than 170 Saxon aristocratic libraries. After the German reunification, the compensation and equalization law (EALG) of September 27, 1994 has regulated the return of movable property. Until 1995, the defendants and their heirs could apply for restitution to the relevant authorities. As a result, several institutions of the Free State of Saxony were confronted with numerous requests for restitution. A project enabled the SLUB to carry out a systematic inventory search in all the accessions of the year 1945 and later years. As a result, the property of the former Saxon royal house as well as further holdings from the so-called Schlossbergungen could be documented, returned and partly purchased.
\end{abstract}

Keywords

Provenance Research, Nazi-looted Art, Saxony 
Der 1. Dezember 2014 war für Museen, Archive und Bibliotheken Deutschlands in den sogenannten neuen Bundesländern, ein bedeutendes Datum, endete doch eine zwanzigjährige Nießbrauchsfrist für im Zuge der Bodenreform enteignete Kulturgüter. „Wettiner greifen wieder nach Sachsens Schätzen“, titelte die Dresdner Morgenpost am 28. Oktober 2013; „Schloss Burgk und die Angst vorm Adel“, überschrieb die Leipziger Volkszeitung am 16. Januar 2014 einen Artikel zu den Hintergründen „ungeklärter Vermögensfragen“ aus der Zeit der sowjetischen Besatzung nach dem Zweiten Weltkrieg. Die gesetzlichen Grundlagen der legitimierten Rückforderungen bedürfen eines Exkurses, haben und hatten sie doch für die betroffenen Sammlungen erhebliche Auswirkungen.

Noch vor der deutschen Wiedervereinigung, am 15. Juni 1990, hieß es in der „Gemeinsamen Erklärung“ damals noch beider deutscher Regierungen: „Die Enteignungen auf besatzungsrechtlicher bzw. besatzungshoheitlicher Grundlage (1945 bis 1949) sind nicht mehr rückgängig zu machen. “' Die abschließende Entscheidung jedoch werde ein künftiges gesamtdeutsches Parlament zu treffen haben. Dieses verabschiedete im September 1994 das Entschädigungs- und Ausgleichsleistungsgesetz (EALG) ${ }^{2}$. Das „die vom Vermögensgesetz noch offen gelassene Entschädigung für Enteignungen in der DDR ebenso regelt wie die vom Einigungsvertrag dem gesamtdeutschen Gesetzgeber vorbehaltene Ausgleichsleistung für Enteignungen auf besatzungsrechtlicher bzw. besatzungshoheitlicher Grundlage“.3 Berührt werden vom EALG mehrere Gesetze oder Änderungsgesetze, wichtig im hier zu besprechenden Kontext sind drei: Erstens das „NS-Verfolgtenentschädigungsgesetz“ (NS-VEntschG) für Vermögensverluste zwischen 1933 und 1945, zweitens das „Gesetz über staatliche Ausgleichsleistungen für Enteignungen auf besatzungsrechtlicher oder besatzungshoheitlicher Grundlage, die nicht mehr rückgängig gemacht werden können“ (Ausgleichsleistungsgesetz - AusglLeistG), die Jahre 1945 bis 1949 betreffend, sowie drittens das „Gesetz über die Entschädigung nach dem Gesetz zur Regelung offener Vermögensfragen“ (EntschG) für die Jahre der DDR, 1949 bis 1990. Die Formulierung „Enteignungen, [...] die nicht mehr rückgängig gemacht werden

1 Gemeinsame Erklärung der Regierungen der Bundesrepublik Deutschland und der Deutschen Demokratischen Republik zur Regelung offener Vermögensfragen (Anlage III des Vertrages zwischen der Bundesrepublik Deutschland und der Deutschen Demokratischen Republik über die Herstellung der Einheit Deutschlands - Einigungsvertrag) vom 15. Juni 1990 (BGBl. 1990 II S. 1237). URL: https://www.gesetze-im-internet.de/reggerkl_einigvtr/BJNR2123709 90.html (abgerufen am 01.05.2017).

2 „Gesetz über die Entschädigung nach dem Gesetz zur Regelung offener Vermögensfragen und über staatliche Ausgleichsleistungen für Enteignungen auf besatzungsrechtlicher oder besatzungshoheitlicher Grundlage“.

3 Bundesamt für zentrale Dienste und offene Vermögensfragen: Allgemeine Ausführungen zum EALG vom 27. September 1994. URL: http://www.badv.bund.de/DE/OffeneVermoegensfragen/ Entschaedigungsrecht/AllgemeineAusfuehrungen/start.html (abgerufen am 01.05.2017). 
können“ im Gesetzestitel des AusglLeistG trifft jedoch nach $\$ 5$ auf „bewegliche“ Sachen - wie dies beispielsweise Kunstwerke und Bücher sind - nicht zu. Absatz 2 des AusglLeistG lautet: „Zur Ausstellung für die Öffentlichkeit bestimmtes Kulturgut bleibt für die Dauer von 20 Jahren unentgeltlich den Zwecken der Nutzung seitens der Öffentlichkeit oder der Forschung gewidmet (unentgeltlicher öffentlicher Nießbrauch). “4

Im Lauf der folgenden 20 Jahre - von 1994 bis zum Stichtag am 1. Dezember 2014 - erreichten die öffentlichen Sammlungen auf dem Gebiet der ehemaligen sowjetischen Besatzungszone zahlreiche Restitutionsersuchen; allein die Sächsische Landesbibliothek - Staats- und Universitätsbibliothek Dresden (SLUB) zählte weit über 100 Anfragen, u.a. aus den jeweils zuständigen Ämtern zur Regelung offener Vermögensfragen. Dass die sächsischen Museen, Archive und Bibliotheken zum Stichtag 2014 die bedeutendsten Fälle abgeschlossen, d.h. recherchiert, dokumentiert und restituiert hatten, lag an einer vom Freistaat Sachsen geförderten, mehrere Institutionen übergreifenden Provenienzrecherche. Am Beispiel der SLUB sollen nachfolgend Methoden und Ergebnisse der Suche nach Adels- oder Schlossbibliotheken, die im Zuge der Bodenreform auf sowjetischem Besatzungsgebiet in den Jahren 1945/1946 enteignet wurden, vorgestellt werden.

Als das Gründungsdatum der heutigen SLUB gilt das Jahr 1556. In der zweiten Hälfte des 16. Jahrhunderts waren die Bücher Teil der Kunstkammer des sächsischen Kurfürsten August (reg. 1553-1586) und dienten wohl zunächst ausschließlich seinem persönlichen wissenschaftlichen Gebrauch. Insbesondere im 18. Jahrhundert durch den Erwerb so herausragender Sammlungen wie der des Grafen Heinrich von Bünau (1697-1762) und der des verstorbenen Premierministers Heinrich von Brühl (1700-1763) bedeutend angewachsen, stand der Bibliothek mit dem Umzug in das Japanische Palais ab 1786 ein großzügiges und repräsentatives Gebäude zur Verfügung. Seit 1788 nannte sie sich Kurfürstliche Öffentliche Bibliothek und ab 1806, nachdem Sachsen Königreich geworden war, Königliche Öffentliche Bibliothek. ${ }^{5}$ Bis zum Jahr 1945 beherbergte das nahe der Elbe gelegene Japanische Palais die zu den Staatlichen Sammlungen für Kunst und Wissenschaft gehörende Institution. Während des Krieges, beginnend ab dem Jahr 1942, lagerte man die wertvollen Bestände in umliegende Schlösser und Herrenhäuser aus. Die kostbarsten Objekte wurden in Bankfilialen in Zittau, Bautzen und Schirgiswalde verbracht bzw. im Tiefkeller des Japanischen Palais in

$4 \S 5$ Abs. 2 AusglLeistG. URL: http://www.gesetze-im-internet.de/ausglleistg/_5.html (abgerufen am 01.05.2017).

5 Vgl. Von der Liberey zur Bibliothek. 440 Jahre Sächsische Landesbibliothek. Hg. von Lothar Koch u. a. Dresden: Sandstein 1996. 
luft- und wasserdichten Stahlbehältnissen sichergestellt. ${ }^{6}$ Die Bombardierungen Dresdens am 13./14. Februar, vor allem aber der Tagesangriff vom 2. März 1945, zerstörten das Gebäude nahezu und eindringendes (Grund-)Wasser schädigte die dort gelagerten Bestände aufs Schwerste. Mehrere Mitarbeiter kamen ums Leben, während sie versuchten, das Gebäude und die Bücher zu retten, etwa 200.000 Bände verbrannten in jenen Tagen (siehe Abbildung 1).

Weitere 200.000 Bände wurden in den Folgemonaten von der sowjetischen Trophäenkommission nach Russland verbracht. ${ }^{7}$ Mit einem Kasernengebäude im Norden der Stadt war nach einigen Umzügen schließlich 1947 ein Behelfsquartier gefunden, das - der Natur von Provisorien entsprechend - über 50 Jahre Bestand haben sollte. Die seit der Revolution von 1918 nicht mehr Königliche, sondern Sächsische Landesbibliothek (SLB) fusionierte 1996 mit der Bibliothek der Technischen Universität, die ihrerseits auf eine Reihe Vorgänger zurückblickt. Erst 2003 bezog die Bibliothek einen Neubau auf dem Campus der Universität. Doch Gegenstand folgender Ausführungen ist die SLB im Jahr 1945 und den folgenden - die Aufnahme adliger Büchersammlungen in den Nachkriegsjahren und die Suche nach ihnen in den Nachwendejahren.

Die Etablierung eines gebildeten Adels in Sachsen spiegelt sich wider im Entstehen teilweise bedeutender Sammlungen und Bibliotheken in den mehr als tausend Schlössern und Herrenhäusern seit dem 18. Jahrhundert. Von den zahlreichen Beispielen, über die zu berichten wäre, können nur einige hier genannt werden: Vom 16. Jahrhundert bis 1945 befand sich das Schloss Siebeneichen, idyllisch oberhalb der Elbe bei Meißen gelegen, im Besitz der Familie von Miltitz. Deren Mitglieder, vielfach in Diensten des sächsischen Hofes, sahen sich stets der Förderung der Künste und Wissenschaften verpflichtet. Das Schloss verfügte über eine herausragende Bibliothek, deren Anfänge im 18. Jahrhundert liegen. Ebenfalls sehr lange Zeit, seit dem 17. Jahrhundert, befand sich das Schloss Wildenfels im Erzgebirge im Besitz der Grafen zu Solms-Wildenfels. Auch hier stand bis zum Jahr 1945 eine umfangreiche Büchersammlung. In der Lausitz, im Osten Sachsens, verfügte die Herrschaft der Grafen von SchallRiaucour auf Schloss Gaußig seit dem 18. Jahrhundert über eine typische, sehr repräsentative Adelsbibliothek. In der Nähe der Stadt Leipzig wiederum bewohnte die Familie der Freiherren von Friesen seit dem 16. Jahrhundert das Schloss Rötha mit einer bedeutenden Bibliothek, die unter anderem die Shakespeare-Sammlung des sächsischen Hofmarschalls Hermann von Friesen (1802-1882) beinhaltete (siehe Abbildung 2).

6 Vgl. Akten im Hauptstaatsarchiv Dresden (HStA), 10701, Staatskanzlei, Nr. 320/7, Bl. 54 und Bll. 98-100.

7 Vgl. Die Trophäenkommissionen der Roten Armee. Eine Dokumentensammlung zur Verschleppung von Büchern aus deutschen Bibliotheken. Hg. von Klaus-Dieter Lehmann, Ingo Kolasa. Frankfurt am Main: Klostermann 1996 (= ZfBB, Sonderhefte 64). 


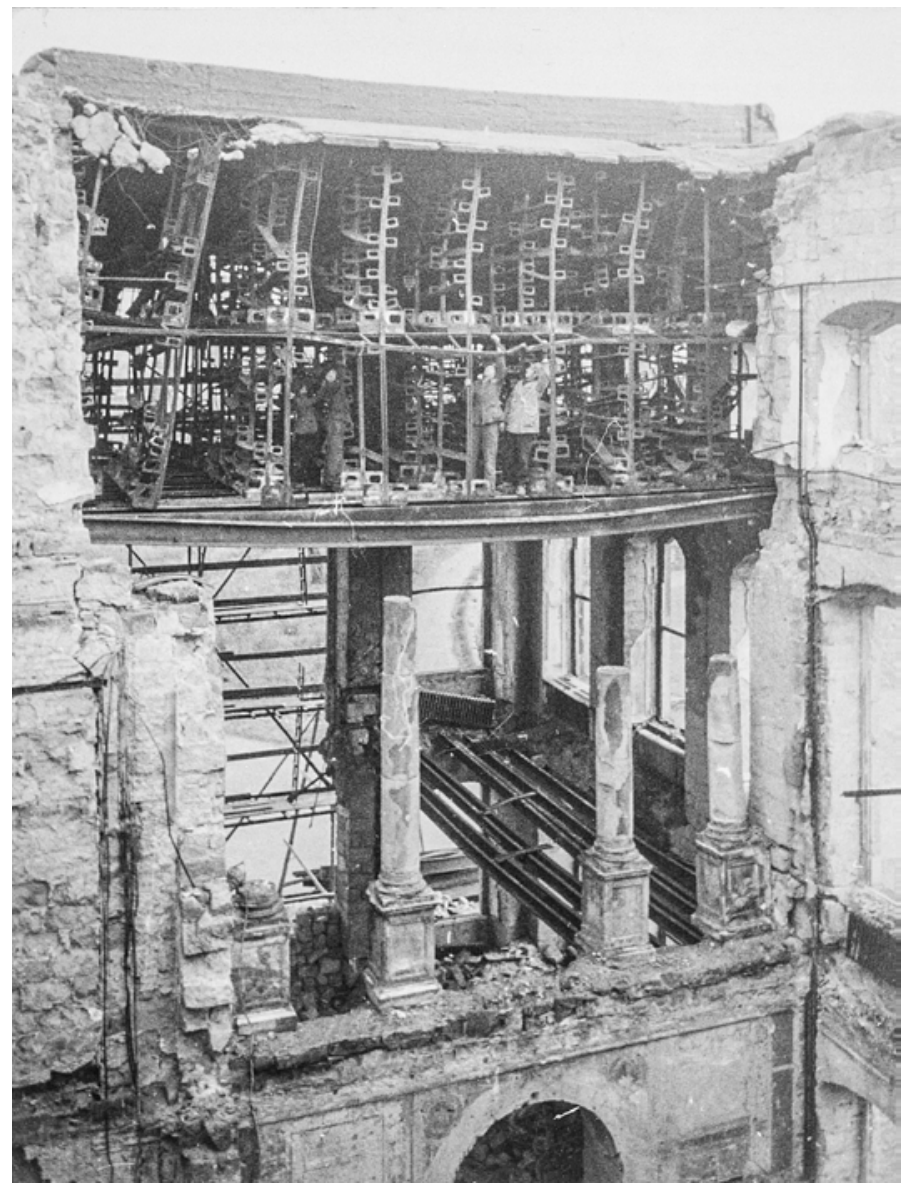

Abb. 1: Die Ruine des Japanischen Palais 1945. Elbflügel mit Regalflächen der Sächsischen Landesbibliothek

Das Röthaer Schloss steht exemplarisch für das Schicksal sächsischer Adelssitze: Nach der Enteignung der Familie von Friesen im Jahr 1945 diente das Gebäude in der Nachkriegszeit als Wohnraum für Flüchtlinge und Vertriebene und als Kindergarten. Da es nie baulich instand gesetzt werden konnte, verfiel es zusehends und wurde 1969 gesprengt, worauf das Gelände anschließend der örtlichen kollektivierten Landwirtschaft zur Verfügung gestellt wurde. ${ }^{8}$

Viele Sammlungen wären aufzuzählen, die nach Kriegsende und unter der sowjetischen Besatzung das gleiche Schicksal teilen: Jahrhundertelange Herr-

8 Vgl. Jana Kocourek: Sächsische Adelsbibliotheken in der SLUB. Die „Freiherrlich von Friesen'sche Schlossbibliothek zu Rötha“. In: BIS - Das Magazin der Bibliotheken in Sachsen 2017, 10(1), S. $17 \mathrm{f}$. 


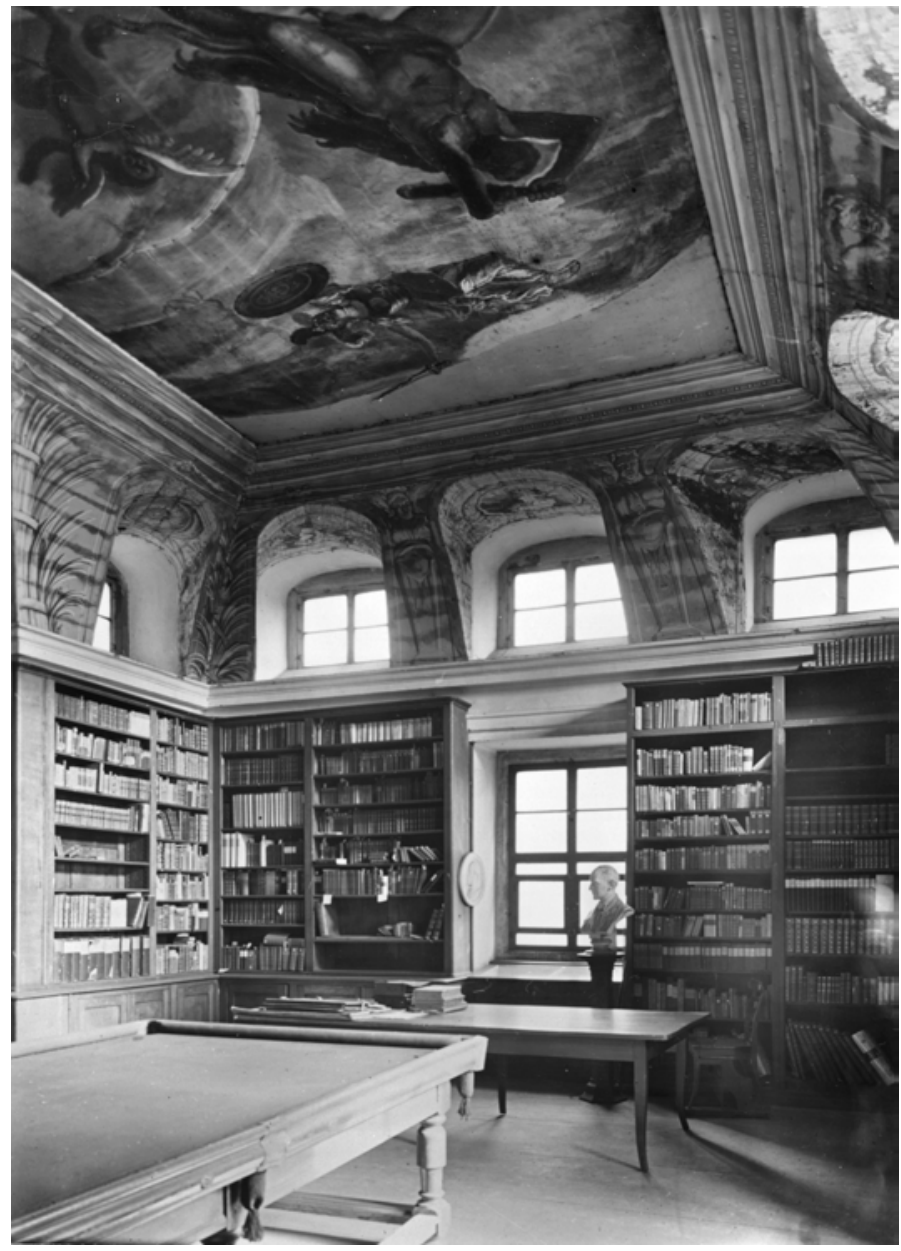

Abb. 2: Schlossbibliothek Rötha, um 1935

schaft und oft ebenso lang währende Sammeltätigkeit endeten mit der Auflösung des Grundbesitzes im Zuge der Bodenreform und mit der völligen Verstreuung der Bestände. Grundlage der Liquidierung von mehr als 1.000 Gütern in Sachsen war die im September 1945 von der Landesverwaltung erlassene „Verordnung über die Durchführung der landwirtschaftlichen Bodenreform“: Wie Artikel 1, Absatz 2 der Verordnung zu entnehmen ist, wurde entschädigungslos enteignet der Grundbesitz sowohl von Kriegsverbrechern und Kriegsschuldigen, Naziführern und „aktiven Verfechtern der Nazipartei [...] sowie den führenden Personen des Hitlerstaates“ sowie der „gesamte feudal-junkerliche Boden und der Großgrundbesitz mit über 100 Hektar mit allen Bauten, lebendem und totem 
Inventar [...]. “ Die Maßnahme betraf demnach nicht nur Grund und Boden, sondern auch persönlichen Besitz wie Möbel, Hausrat und Kunstwerke. Auf Anordnung des Ministeriums für Volksbildung waren die Landräte dazu aufgerufen, alle „Schlösser, Herrensitze und Güter, in denen sich Privatbibliotheken befinden oder befunden haben zu melden“. 9

In Sachsen waren mehr als 170 Privatbibliotheken zwischen Herbst 1945 und Sommer 1946 „herrenlos“ geworden. Dieser Zeit entstammt der Begriff „Schlossbergung“, der aus Sicht der seinerzeit beteiligten Museums- und Bibliotheksmitarbeiter keineswegs nur euphemistisch gemeint war. ${ }^{10}$ Ein im August 1946 erlassener Befehl der sowjetischen Militäradministration schloss die Rückkehr der Enteigneten aus, erlaubte ihnen nicht einmal das „Betreten jenes Kreises, in dem sich der Besitz befand“. ${ }^{11}$ Es gibt jedoch Berichte über Versuche der Grundbesitzer, wenigstens wichtige Familienerbstücke zu retten, teilweise in direktem Widerstand, ${ }^{12}$ teilweise in Versuchen, mit den staatlichen Organen zu kooperieren. Die Sicherung der zunächst verlassenen Herrenhäuser und ihres Inventars, z. B. der Buchbestände, oblag den Landratsämtern und Bürgermeistern. ${ }^{13}$ Diese hatten weder geeignete Räume noch Personal, um den Schutz der Kulturgüter zu gewährleisten, wie zahlreiche Berichte über Plünderungen und Zerstörungen, aber auch willkürliche Verteilungen auf lokale Institutionen belegen. ${ }^{14}$ Zwar bemühte sich die beim sächsischen Ministerium für Volksbildung angesiedelte Kommission, der neben Mitarbeiterinnen und Mitarbeitern der

9 Christine Simmich: Die Bedeutung der antifaschistisch-demokratischen Reformen für die Sächsische Landesbibliothek Dresden in ihrer Funktion als regionales Bestandszentrum gesellschaftswissenschaftlicher Literatur. Masch. Diplomarbeit am Institut für Bibliothekswissenschaft und wissenschaftliche Information der Humboldt-Universität zu Berlin. Berlin 1983, S. 15, die ein Rundschreiben zitiert, das im Bibliotheksarchiv der SLUB vorliegt.

10 Vgl. Thomas Rudert, Gilbert Lupfer: Die so genannte „Schlossbergung“ als Teil der Bodenreform 1945/46. In: Museumskunde 73 (2008), Heft 1, S. 57-64, hier S. 57.

11 Wolfgang Frühauf: Schlossbibliotheken in der SLUB und ihre gegenwärtige Restituierung. In: Tradition und Herausforderung. Aus der Arbeit der Sächsischen Landesbibliothek zwischen 1960 und 1990. Hg. von Jürgen Hering. Dresden: SLUB 2000 (= Schriftenreihe der Sächsischen Landesbibliothek - Staats- und Universitätsbibliothek Dresden 1), S. 127-137, hier S. 128.

12 Anna Miksch: Die Sicherung und Nutzung kultureller Werte der ehemaligen Herrensitze des Großgrundbesitzes in Sachsen (Herbst 1945 bis Ende 1949). Ein Beitrag zum Problemkreis des kulturellen Erbes in der antifaschistisch-demokratischen Umwälzung. Masch. Diss. KarlMarx-Universität Leipzig 1979, z. B. S. 37, S. 120, S. 128-130.

13 Vgl. Miksch: Sicherung und Nutzung (Anm. 12), S. 141; Christian Alschner: Die Bearbeitung der wissenschaftlichen Altbestände. Eine vergleichende Darstellung der Sächsischen Landesbibliothek Dresden und der Universitäts- und Landesbibliothek Halle. Masch. Hausarbeit Dresden 1962, S. 2.

14 Vgl. bspw. zur Standesherrschaft Königsbrück: Frühauf: Schlossbibliotheken (Anm. 11), S. 128; Miksch: Sicherung und Nutzung (Anm. 12), z. B. S. 143, S. 153, S. 167 f. S. 179, S. 168, S. 189ff.; Simmich: Bedeutung der antifaschistisch-demokratischen Reformen (Anm. 9), S. 15 . 
großen Museen unter anderem die Direktoren der SLB Dresden und der UB Leipzig angehörten, nach Kräften, die häufig nicht angemessen gelagerten Bibliotheksbestände zeitnah zu sichten und zu sichern, doch fehlende Transportmöglichkeiten erschwerten dieses Vorhaben, wie zahlreiche Unterlagen im hauseigenen Archiv der SLUB belegen. ${ }^{15}$ Aus diesen Gründen wurde eine erste Auswahl an Beständen häufig direkt vor Ort vorgenommen und die Sammlungen bei dieser Gelegenheit bereits auseinander gerissen. Es war geplant, die wissenschaftliche Literatur in ein zentrales Buchbergungslager zu überführen, das für Sachsen im Jahr 1946 wegen ihrer enormen Bestandsverluste unter dem provisorischen - Dach der SLB eingerichtet wurde. Dieses Buchbergungslager existierte bis 1948, danach wurden die verteilten Buchbestände amtlich zum Besitz der jeweiligen Bibliotheken und Institutionen erklärt, die die Bücher aufgenommen hatten, folglich auch der SLB. Bis in die 1980er Jahre hinein wurden diese - wobei allerdings im Laufe der Zeit das Wissen um ihre Provenienz weitestgehend verloren gegangen war - inventarisiert, vielfach aber auch ausgeschieden. Die dafür zuständige „Dienststelle für Altbestände“ nannten die Mitarbeiter bis in die 1980er Jahre noch „das Schloss“ ${ }^{16}$

Keine der Adelsbibliotheken überdauerte als geschlossene Sammlung. In der SLB kamen zum einen nur Teilbestände an, zum anderen wurden laut Statistik in den Jahren 1948 bis 1961 von den Altbeständen nur 46 Prozent aufgenommen, 49 Prozent Dubletten anderen Bibliotheken überwiesen und ca. vier Prozent makuliert. ${ }^{17}$ Die übernommenen Schlossbibliotheken ähnelten sich häufig in ihrer Zusammensetzung, so dass mit der Abgabe der zahlreich anfallenden Dubletten die Sammlungszusammenhänge komplett zerstört wurden. Die Doppelstücke kamen an die zunächst in Gotha, später in Berlin angesiedelte Zentralstelle für wissenschaftliche Altbestände (ZwA), die die Weiterverteilung an wissenschaftliche Bibliotheken übernahm. ${ }^{18}$ Außerdem standen seit den 1970er Jahren die Bibliotheken unter enormem Druck, zur Devisenbeschaffung beizutragen und besonders wertvolle Dublettenbestände, etwa Inkunabeln bzw. seltene Drucke im Allgemeinen, z. B. über das Zentralantiquariat in Leipzig in das westeuropäische Ausland zu veräußern. ${ }^{19}$ Umgangen wurde dies an der SLB, indem mehrere Tausend Dublettenbände nicht inventarisiert wurden, womit sie

15 Vgl. Miksch: Sicherung und Nutzung (Anm. 12), z. B. S. 184.

16 Christine Simmich: Ein Schloß in der Sächsischen Landesbibliothek. In: SLB-Kurier 2 (1988), Heft 3, S. 4-5.

17 Vgl. Alschner: Bearbeitung wissenschaftlicher Altbestände (Anm. 13), S. 12.

18 Vgl. Andreas Mälck: Zum Wirken der Zentralstelle für wissenschaftliche Altbestände in Vergangenheit und Gegenwart. In: ZfB 103 (1989), S. 537-545.

19 Vgl. dazu Björn Biester: Deutsch-deutsche Büchergeschäfte 1945 bis 1989. Anmerkungen zur Rolle des Antiquariatsbuchhandels. In: 95. Deutscher Bibliothekartag Dresden 2006. Netzwerk Bibliothek. Frankfurt am Main: Klostermann 2007, S. 249-257. 
praktisch nicht existierten und dem staatlichen Zugriff entzogen waren. Die vorangegangenen Ausführungen mögen einen Eindruck von der Ausgangslage zum Zeitpunkt der Wiedervereinigung Deutschlands vermittelt haben.

Konfrontiert mit den Forderungen des ehemaligen sächsischen Königshauses, dem Haus Wettin Albertinischer Linie (A.L.), hatte der Freistaat Sachsen ein umfangreiches Provenienzforschungsprojekt (Daphne) aufgelegt, angesiedelt bei den Staatlichen Kunstsammlungen Dresden. Ein Teilprojekt ermöglichte der SLUB die systematische Bestandsrecherche in sämtlichen Zugängen der Jahre 1945 bis 1990. Zwar hatte die SLUB langjährige Erfahrungen mit Restitutionen, denn bereits in den frühen 1990ern gab es Rückgaben jüdischen Eigentums ${ }^{20}$ und vereinzelte, anlassbezogene Recherchen nach den sogenannten Schlossbergungen waren sporadisch durchgeführt worden, allerdings war man sich immer bewusst, dass diese Maßnahmen nicht sämtliches Schlossbergungsgut ermitteln konnten. Eine ganze Reihe „offener Vermögensfragen“ waren aufzuklären. Dem Projekt zur systematischen Recherche blieb mit dem Beginn im Jahre 2009 bis zum Stichtag 2014 und angesichts der zu bewältigenden Menge sehr wenig Zeit für die Aufbereitung der Vorgänge, gleichzeitig jedoch barg es die Möglichkeit, umfassend Daten zu erheben. Ziel war es, vor Ablauf der Nießbrauchsfrist Klarheit über die Herkunft der Bestände und Rechtssicherheit zu erlangen.

Weil Bücher per se Massenware und keine Unikate sind, schien das systematische Vorgehen zielführender als die Titelsuche anhand von Suchlisten. Wegen der Aufstellung der Bestände nach Numerus currens seit 1927 ließ sich das Recherchekorpus deutlich abgrenzen. Ausgangspunkt der Recherchen war im ersten Schritt immer das Objekt und die Spuren, die seine Vorbesitzer in ihm hinterlassen haben: Evidenzen wie Exlibris, Stempel, Autogramme, Widmungen, vielfach auch nur Initialen oder Signaturen. Deshalb bedeutete systematische Recherche tatsächlich das In-die-Hand-Nehmen und Durchsehen von etwa 360.000 Drucken, Handschriften und Karten - in einem Zeitraum von etwa drei Jahren. Sämtliche Provenienzen wurden in einer separaten Datenbank erfasst, weil die SLUB im Südwestverbund keine Exemplare pflegt und das lokale Katalogsystem $\mathrm{zu}$ wenig geeignete Felder für Provenienzinformationen bietet. Lange wurde hier nach einer Lösung gesucht, inzwischen werden die Provenienzsätze aus der Datenbank an die lokalen Exemplardaten angespielt und im SLUB-Katalog gezeigt. Von Beginn an stand fest, dass ein Nutzen der Daten zur Auswertung und Dokumentation nur dann gewährleistet sein kann, wenn die Erfassung standardisiert erfolgt. Nach dem sogenannten Weimarer Modell er-

20 Frank Aurich: Rückgaben jüdischen Eigentums aus der Sächsischen Landesbibliothek Staats- und Universitätsbibliothek. In: Jüdischer Buchbesitz als Raubgut. Zweites Hannoversches Symposium. Hg. von Regine Dehnel. Frankfurt am Main: Klostermann 2006, S. 357-360. 
folgte die Erfassung mit dem Vokabular des Thesaurus der Provenienzbegriffe TPRO. $^{21}$ Teilweise bis zu 14 Hilfskräfte arbeiteten im fast fußballfeldgroßen Magazin der SLUB.

Daneben wurde eine Bilddatenbank geführt, die zunächst als Hilfe für die Erfasser gedacht war und der Qualitätssicherung dienen sollte. Die wichtigsten Provenienzmerkmale wurden fotografiert und mit einem eindeutigen Identifier versehen. Vom Arbeitsinstrument entwickelte sich diese Datenbank zu einem großartigen Datenschatz. Neben den Aufnahmen von den Provenienzmerkmalen fasst sie sämtliche Informationen zu den betreffenden Personen und Körperschaften zusammen. Die Recherchen zielen auf die Identifizierung der Vorbesitzer, um für diese individualisierte Personen- oder Körperschaftsnormdatensätze in der Gemeinsamen Normdatei (GND) anzulegen und sie damit für die kooperative Provenienzforschung, aber auch weiterführende wissenschaftliche Fragestellungen nachnutzbar zu machen.

Sicher identifizierte Provenienzmerkmale, die mit einem GND-Satz verknüpft sind, werden sukzessive in der Bilddatenbank der Deutschen Fotothek veröffentlicht. ${ }^{22}$ Von den über 15.000 Bildern, die während der Projektlaufzeit von 2009 bis 2013 entstanden, sind aktuell 4.900 online recherchierbar und mit einem Permalink referenzierbar (siehe Abbildung 3). ${ }^{23}$

Bis zum 1. Dezember 2014 konnten auf diese Weise alle größeren, also mehr als 1.000 Bände umfassenden „offenen Vermögensfragen“ im Hinblick auf Bodenreformbestände abgeschlossen werden. Die erhobenen Daten bildeten die Grundlage teilweise umfangreicher Dossiers, mit denen sowohl die SLUB selbst als auch die jeweiligen Ämter zur Regelung offener Vermögensfragen in Verhandlungen mit Eigentümern und ihren Erben oder Anwälten treten konnten. Im Ergebnis wurde u. a. ein Vergleich mit dem ehemaligen sächsischen Königshaus geschlossen, aber auch weitere Bestände aus den sogenannten Schlossbergungen dokumentiert, restituiert und teilweise angekauft, darunter die oben genannten Bibliotheken der Schlösser Siebeneichen und Wildenfels. Für die ermittelten Reste der Freiherrlich von Friesen'schen Schlossbibliothek zu Rötha ist ebenfalls eine einvernehmliche Lösung gefunden worden: Die Eigentumsrechte der 2014 an die Familie restituierten Bücher gingen auf die Stiftungen der Sparkasse

21 T-PRO Thesaurus der Provenienzbegriffe, erarbeitet 2003 bis 2009 von Dr. Jürgen Weber (HAAB Weimar), ab 2009 gemeinsam mit Michaela Scheibe, Staatsbibliothek zu Berlin Preußischer Kulturbesitz. URL: https://provenienz.gbv.de/T-PRO_Thesaurus_der_Proveni enzbegriffe (abgerufen am 01.05.2017).

22 Vgl. die Serie „Provenienzmerkmale SLUB-Bestand“ in der Deutschen Fotothek. URL: http://www.deutschefotothek.de/ (abgerufen am 01.05.2017).

23 Jana Kocourek: Provenienzmerkmale der SLUB online. Über 4.000 Exlibris, Stempel, Etiketten und Autogramme zum Nachschlagen. In: BIS - Das Magazin der Bibliotheken in Sachsen 8(2015)2, S. 94-96. 


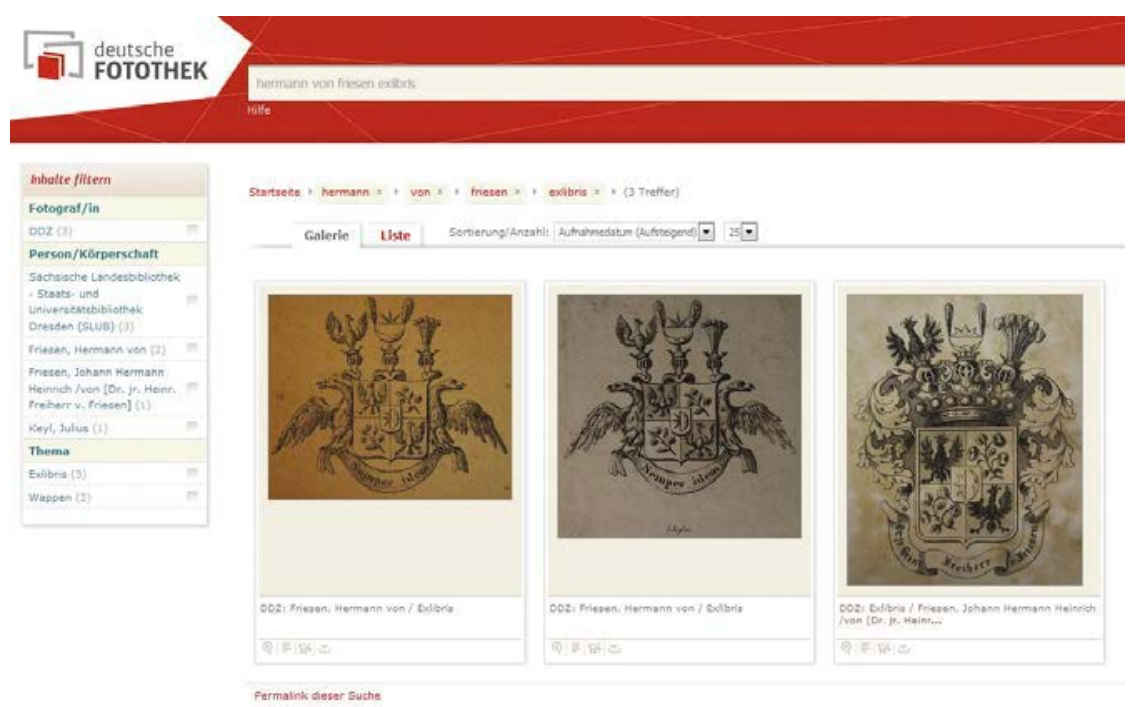

Abb. 3: Beispiele typischer Exlibris der Röthaer Bibliothek in der Bilddatenbank der Deutschen Fotothek

Leipzig über. Vorerst werden die in der SLUB, aber auch andernorts aufgefundenen Bücher und Handschriften Röthaer Provenienz als Depositum in der SLUB verbleiben und stehen damit weiterhin der Forschung zur Verfügung. Ein von der Stiftung gefördertes und an der SLUB angesiedeltes Projekt sieht die virtuelle Rekonstruktion der Schlossbibliothek Rötha vor - auf der Basis der erhobenen Provenienzdaten.

Die Suche nach den Schlossbergungsbeständen brachte nicht nur Reste verstreuter Adelsbibliotheken an das Licht. Obgleich die systematische Bestandsrecherche erst bei den Zugängen des Jahres 1945 und der folgenden ansetzte, fanden sich in den Nachkriegserwerbungen zahlreiche Hinweise auf NS-Raubgut. Dieser Befund, basierend auf gewachsener Bestandskenntnis, war der Anlass eines Provenienzprojekts zur Suche nach NS-verfolgungsbedingt entzogenem Kulturgut in den Zugängen zwischen 1933 und 1945. Die systematische Recherche konnte über 18 Monate, von Oktober 2011 bis März 2013, mit Förderung der damaligen Arbeitsstelle für Provenienzforschung (AfP), jetzt Deutschem Zentrum Kulturgutverluste, durchgeführt werden. Etwa 1.000 Exemplare mit Raubgutverdacht oder gar der Sicherheit, dass es sich um Raubgut handelt, wurden ermittelt und der „Lost Art“-Datenbank gemeldet.

Der Fall Steinthal/Vollmann illustriert anschaulich, wie die Formen des Kulturgutraubes sich verschränken. Im November 2011 restituierte die SLUB 115 Bücher an die Erben der Familie Steinthal - sowohl aus dem Besitz des Ehepaares Max (1850-1940) und Fanny Steinthal (1866-1941) sowie dem ihrer Kinder 
stammend. Die in Berlin ansässige Familie besaß eine bedeutende Kunst-, der Sohn Erich (1890-1963) eine herausragende Büchersammlung. Er und seine Schwester Eva (1892-1993) waren von den Eltern, die in Berlin auf die Ausreise wartend verstorben waren, zu den Erben bestimmt worden - wie auch Evas ehemaliger "arischer“ Ehemann Richard Vollmann. Dieser hatte wegen der Bombengefahr in Berlin die Sammlungen der Familie Steinthal in sein Haus nach Dresden verbracht. Nach seiner „Republikflucht“ zu Beginn der 1950er Jahre wurde sein Besitz eingezogen. Die Bücher gelangten nach verschiedenen Stationen in städtischen Einrichtungen im Jahr 1971 über den Rat der Stadt Dresden an die damalige Sächsische Landesbibliothek. ${ }^{24}$

\section{Fazit und Ausblick}

Die systematische Bestandsdurchsicht der Zugangsjahre 1933 bis $1990 \mathrm{im}$ Rahmen von Provenienzforschungsprojekten versetzt die SLUB Dresden in die Lage, NS-verfolgungsbedingt entzogene Bestände und unrechtmäßige Erwerbungen im Zuge der Bodenreform oder DDR-Unrechts zu identifizieren und zu dokumentieren. Die sehr umfangreichen Daten erlauben es auch, sollte sich beispielsweise eine Provenienz durch neuere Forschungen als unrechtmäßiger Besitz herausstellen, eine schnelle Bearbeitung und Restitution des entsprechenden Falls vorzunehmen.

Ein weiteres Projekt zur Suche nach NS-verfolgungsbedingt entzogenem Kulturgut widmet sich - dank der Förderung durch das Deutsche Zentrum Kulturgutverluste - seit September 2017 der Recherche nach sogenanntem sekundärem NS-Raubgut, den mehreren Hundert Verdachtsfällen in den Zugängen nach 1945. Die Komplexität der Verlustumstände, mit denen die SLUB, aber nicht nur sie, konfrontiert ist, vermag das letzte Beispiel anzudeuten: In den Beständen eines Endes der 1940er Jahre enteigneten - entnazifizierten - Gärtnereibesitzers aus dem Umland fielen anlässlich einer Stolpersteinverlegung und eher zufällig vorgenommenen Recherchen Bücher der Familie Kaps auf. William (Willi) Ernst Kaps (1872-1943) war der Sohn des Dresdner Klavier- und Flügelfabrikanten Ernst Kaps. Seine Frau Berta entstammte der seit 1884/1885 in Dresden ansässigen jüdischen Familie Beck. Bertas Mutter Aurelie Beck, geb. Simon ( $\left.{ }^{\star} 1856\right)$, kam am 8. September 1942 in Theresienstadt ums Leben, wie auch Bertas Bruder, der Arzt Guido Beck $\left({ }^{\star} 1879\right)$. William Ernst Kaps besaß eine bibliophile Sammlung. Nach dem Tod ihres nichtjüdischen Ehemanns William Ernst im August 1943 hatte seine jüdische Witwe Berta keinen beschränkten

24 Vgl. Website zum NS-Raubgut an der SLUB Dresden. URL: https://nsraubgut.slub-dresden. de/aktuelles/ (abgerufen am 01.05.2017). 
Schutz mehr und wurde am 19. Oktober 1943 nach Auschwitz deportiert. Unter der Nummer 32456/1943 ist ihr Tod am 1. November 1943 im Sterbebuch Auschwitz dokumentiert. Die Bibliothek des Ehepaars wurde von der Gestapo beschlagnahmt. Offensichtlich hatte der Gärtnereibesitzer Zugang zu diesen beschlagnahmten Beständen. Ob sich in seinem Besitz weiteres NS-Raubgut befand, ist Gegenstand der Recherchen des aktuellen Projekts, das in so vielen Fällen wie möglich zu einer fairen und gerechten Lösung im Sinne der Washingtoner Erklärung führen soll.

Ausgehend von der Suche nach Beständen im Rahmen der Klärung offener Vermögensfragen im Zusammenhang mit der Bodenreform war die SLUB mit verschiedenen kritischen Bestandsgruppen konfrontiert: NS-verfolgungsbedingt entzogenen Kulturgütern - in den Zugängen vor und nach 1945, Enteignungen im Zuge der Bodenreform auf besatzungsrechtlicher Grundlage sowie in der DDR unrechtmäßig entzogenem Besitz. Hinzu kommen, aber dies ist nicht Thema dieses Beitrages, eigene Kriegsverluste der SLUB, jene Bestände also, die infolge des Zweiten Weltkrieges verstreut worden sind. Es bleiben viele offene Aufgaben: Mit dem Zusammengehen zweier starker deutscher Bibliotheksverbünde, des Südwestdeutschen (SWB), dem die sächsischen Bibliotheken angehören, und dem Gemeinsamen Bibliotheksverbund (GBV), kann die SLUB ihre Provenienzen zukünftig verbundweit verzeichnen. ${ }^{25}$ Dies ist umso wichtiger, als wir um die Verstreuung der Bestände wissen. Provenienzforschung ist und bleibt eine kooperative Aufgabe, die zentrale Nachweisinstrumente benötigt - sowohl für die historisch-biographischen Forschungsdaten als auch die exemplarbezogenen. $^{26}$

25 Michaela Scheibe: Standards in der Provenienzerschließung - Bericht aus der Arbeitsgemeinschaft Handschriften und Alte Drucke in der Sektion IV des dbv, UAG Provenienzforschung und Provenienzerschließung. In: ZfBB 61 (2014) 6, S. 367-375.

26 Annette Gerlach, Peter Prölß: Forschungs-Verbunddatenbank „Provenienzforschung“. In: Bibliotheksdienst 46 (2012), Heft 1, S. 15-21. 
Open-Access-Publikation im Sinne der CC-Lizenz BY-NC-ND 4.0

(c) 2018, V\&R unipress GmbH, Göttingen

ISBN Print: 9783847107835 - ISBN E-Lib: 9783737007832 


\title{
Christian George
}

\section{Bücher als „Danaergeschenk “' . Nachkriegszugänge der UB Mainz durch die französische Militärregierung}

\author{
Zusammenfassung \\ 1946 wurde die Universität Mainz auf Initiative der französischen Besatzungsmacht \\ gegründet. Diese besondere Situation brachte die UB Mainz in die positive Lage, von \\ zahlreichen Zuwendungen der Militärregierung zu profitieren. Andererseits erwiesen \\ sich viele dieser Zugänge als Danaergeschenke, da sich darunter u. a. NS-Raubgut oder \\ Bücher mit unklarem Besitzstatus befanden. Seit mehreren Jahren ist die UB Mainz \\ bestrebt, problematische Bestände zu identifizieren und unklare Rechtssituationen zu \\ bereinigen. Die verschiedenen rechtlichen Implikationen von Bücherzuweisungen \\ durch die Besatzungsmacht werden am Beispiel der Bibliothek von Reichsleiter Martin \\ Bormann sowie von Buchbeständen der Kunsthistorischen Forschungsstätte Paris und \\ der Familie Sabatini verdeutlicht. \\ Schlagwörter \\ Universität Mainz, NS-Raubgut, Martin Bormann, Familie Sabatini, Militärregierung
}

\begin{abstract}
Books as Greek Gifts. Donations by the French Military Government after World War II at Mainz University Library

In 1946 Mainz University was founded by the French occupying powers. This special situation enabled the university library to benefit from donations by the French. Since looted books or books with dubious property rights were often included in these donations, a lot of them turned out to be Greek gifts. Mainz University Library has been trying for several years to identify problematic book collections and to clarify the status of dubious books. Different challenges and solutions for these matters will be illustrated by the examples of the Sabatini library, the library of Reichsleiter Martin Bormann and the collections of the Kunsthistorische Forschungsstätte Paris.
\end{abstract}

1 Die Metapher vom Danaergeschenk greift auf einen von Thomas Jahn 2006 im Hinblick auf von der Gestapo an Bibliotheken übereignete „arisierte“ Buchbestände verwendeten Begriff zurück. Siehe dazu: Thomas Jahn: Die Recherche nach NS-Raubgut aus jüdischem Vorbesitz in der Bayerischen Staatsbibliothek München. In: Die Suche nach NS-Raubgut in Bibliotheken. Recherchestand, Probleme, Lösungswege. Hg. von Bernd Reifenberg. Marburg: Universitätsbibliothek Marburg 2006 (= Schriften der Universitätsbibliothek Marburg 126), S. 13-32, hier S. 29. 
Keywords

Mainz University, Looted Books, Martin Bormann, Sabatini Family, Occupying Powers

„Am 6.4.47 ist ein LKW mit 2000 in Freiburg [Anm.: von den französischen Besatzungsbehörden] beschlagnahmten Büchern hier eingetroffen, ein Verzeichnis lag nicht bei, die früheren Eigentümer sind unbekannt [...].“2 Dieses Zitat aus einem Brief des damaligen Mainzer UB-Direktors Walter Menn (1890-1967) an das Rektorat beschreibt anschaulich eine typische Erwerbungssituation der Nachkriegszeit an der UB Mainz. Reguläre Erwerbungen durch Kauf waren auf Grund des zusammengebrochenen Büchermarktes vor der Währungsreform nur in Ausnahmefällen zu realisieren. Die Schenkung war dagegen der Normalfall. Unter den verschiedenen Geschenkgebern der Jahre 1946 bis 1949 nehmen die französischen Besatzungsbehörden eine besondere Rolle ein. Nicht nur, weil sie einen Großteil der in den Zugangsbüchern verzeichneten Schenkungen übereigneten, ${ }^{3}$ sondern weil es sich bei den auf Betreiben französischer Stellen an die UB gelangten Titel vielfach um Bücher mit zweifelhafter Provenienz handelt, die aus heutiger Sicht zu den Beständen zählen, die im Verdacht stehen, NS-verfolgungsbedingt entzogenes Kulturgut ${ }^{4} \mathrm{zu}^{\mathrm{u}}$ enthalten.

Bei der Provenienzforschung in Bibliotheksbeständen hat sich seit längerem die Einsicht durchgesetzt, dass es nicht nur die Jahre 1933 bis 1945 (bzw. in Österreich 1938 bis 1945) sind, die auf problematische Zugänge hin untersucht werden müssen, sondern auch Erwerbungen aus der Besatzungszeit kritisch in den Blick zu nehmen sind. ${ }^{5}$ Es ist heute unstrittig, dass das Thema NS-Raubgutforschung „für jede einzelne Bibliothek, die über Buchbestände mit Erscheinungsjahr vor 1945 verfügt, relevant ist“ ${ }^{~}{ }^{6}$ Gerade in der Nachkriegszeit

2 Universitätsarchiv [UA] Mainz, Best. 7 Nr. 31, Walter Menn an das Rektorat, 25.04.1947.

3 Unter den 30.000 Büchern, die die UB im WS 1946/47 erhielt, stammten fast 13.000 von der französischen Militärregierung. Siehe dazu: Corine Defrance: La politique culturelle de la France sur la rive gauche du Rhin 1945-1955. Strasbourg: Presses Universitaires de Strasbourg 1994, S. 72.

4 Diesen Terminus verwendet die Erklärung der Bundesregierung, der Länder und der kommunalen Spitzenverbände zur Auffindung und zur Rückgabe NS-verfolgungsbedingt entzogenen Kulturgutes insbesondere aus jüdischem Besitz aus dem Jahr 1999. URL: http://www. kmk.org/fileadmin/Dateien/veroeffentlichungen_beschluesse/1999/1999_12_09-Auffindung -Rueckgabe-Kulturgutes.pdf (abgerufen am 12.06.2017). Im Folgenden wird der Begriff "(NS-)Raubgut" synonym verwendet.

5 Bruno Bauer, Christina Köstner-Pemsel und Markus Stumpf: Einleitung. In: NS-Provenienzforschung an österreichischen Bibliotheken. Anspruch und Wirklichkeit. Hg. von Bruno Bauer, Christina Köstner-Pemsel und Markus Stumpf. Graz-Feldkirch: W. Neugebauer Verlag 2011, S. 16-22, hier S. 18.

6 Ebd., S. 16. 
sind viele Erwerbungen „tendenziell als problematisch anzusehen“. ${ }^{7}$ Aus Sicht der UB Mainz ist zu ergänzen, dass sich insbesondere die Zuweisungen der französischen Militärregierung als problematisch erwiesen haben. ${ }^{8}$ Somit stellt sich auch für die erst 1946 gegründete UB Mainz die Frage nach der Herkunft ihrer älteren Bestände.

Die UB Mainz ist heute mit über 3,4 Mio. Printmedien ${ }^{9}$ der zentrale Informationsversorger der Johannes Gutenberg-Universität Mainz mit ihren ca. 32.000 Studierenden und über 500 Professorinnen und Professoren. Die Anfänge dieser heute größten wissenschaftlichen Bibliothek in Rheinland-Pfalz kann man sich jedoch nicht bescheiden genug vorstellen. ${ }^{10}$ Als erste Universitätsgründung in Deutschland nach dem Ende des Zweiten Weltkriegs konnte die Universität Mainz auf keine Vorgängerinstitution zurückgreifen und musste vollständig neu aufgebaut werden. ${ }^{11}$ Ideell bemühte man sich zwar, an die von 1477 bis 1798 bestehende alte Mainzer Universität anzuknüpfen. Sowohl die französischen Gründer als auch die Universität selbst sprachen daher 1946 von der Wiedergründung der Universität. Dennoch bleiben die Traditionsbrücken über die rund 150 Jahre andauernde universitätslose Zeit in Mainz hinweg sehr schmal. ${ }^{12}$ Gerade im Hinblick auf die Universitätsbibliothek hätte sich jedoch ein Anknüpfungspunkt geboten, da die Buchbestände der alten Mainzer Universität

7 Ebd., S. 18.

8 Auch die Bayerische Staatsbibliothek München hat bereits 2006 Abgaben von durch die Besatzungsbehörden konfiszierten Beständen aus ehemaligem NS-Besitz als einen der Hauptwege identifiziert, auf welchen arisierte Bestände in die Bibliothek gekommen sind. Vgl. dazu Jahn: Recherche nach NS-Raubgut (Anm. 1), S. 20.

9 UB in Zahlen. Jahresübersicht 2016. URL: https://www.ub.uni-mainz.de/ub-in-zahlen/ (abgerufen am 12.06.2017).

10 Zur Anfangszeit der UB Mainz vgl. Jahresbericht der Universitätsbibliothek Mainz 1946; Walter Menn: Die Universitätsbibliothek Mainz im Aufbau. In: Mitteilungen für den Buchhandel in der französisch besetzten Zone 4 (1949), H. 6, S. 113-115; Helmut Mathy: Die erste Landesuniversität von Rheinland-Pfalz. Studien und Essays zu ihrer Entstehungsphase. Mainz: Johannes Gutenberg-Universität 1997 (Schriften der Johannes Gutenberg-Universität Mainz 8), S. 195. Eine ausführliche Geschichte der UB Mainz wird zurzeit von Christian König bearbeitet und erscheint voraussichtlich noch 2018.

11 Zur Gründungsgeschichte der Johannes Gutenberg-Universität durch die französische Militärregierung vgl. insbesondere Defrance: La politique culturelle (Anm. 3); Corine Defrance: Die Franzosen und die Wiedereröffnung der Mainzer Universität 1945-1949. In: Kulturpolitik im besetzten Deutschland 1945-1959. Hg. von Gabriel Clemens. Stuttgart: Franz Steiner Verlag 1994, S. 117-130; Helmut Mathy (Bearb.): Die Wiedereröffnung der Mainzer Universität 1945/46. Dokumente, Berichte, Aufzeichnungen, Erinnerungen. Mainz: Vereinigung der Freunde der Universität 1966.

12 Vgl. dazu Leo Just: Von der alten zur neuen Universität. In: Miscellanea Moguntina. Vorträge der Mainzer Universitätswoche vom 13.-20.5.1962. Wiesbaden: Franz Steiner Verlag 1964 (= Beiträge zur Geschichte der Universität Mainz 6), S. 1-11 und Helmut Mathy: Die Universität Mainz 1477-1977. Mainz: Verlag Dr. Hanns Krach 1977. 
nach deren Auflösung von der Mainzer Stadtbibliothek übernommen wurden. ${ }^{13}$ So war es naheliegend, dass sich nach Eröffnung der Universität 1946 zunächst eine enge Kooperation mit der Stadtbibliothek etablierte. Die Stadtbibliothek ersetzte gleichsam die noch im Aufbau befindliche Universitätsbibliothek. Anfangs standen sogar Pläne im Raum, beide Bibliotheken unter der Leitung des Direktors der Stadtbibliothek zusammenzuführen, die sich jedoch mit der Einstellung von Walter Menn als erstem UB-Direktor im Sommer 1946 zerschlugen. Dieser stand damit 1946 vor der Aufgabe, unter den widrigen Umständen der Nachkriegszeit und ohne auf bestehende Strukturen oder vorhandene Buchbestände zurückgreifen zu können, möglichst schnell eine Universitätsbibliothek aufzubauen, um Lehre und Forschung an der neuen Universität zu unterstützen.

Die französische Militärregierung sah in der Gründung der Johannes Gutenberg-Universität vor allem die Chance, die angestrebte Re-éducation, Demokratisierung, Entnazifizierung und Entmilitarisierung der deutschen Bevölkerung voranzutreiben. Diese Möglichkeit schien bei der Neugründung einer Universität eher gegeben, als bei den bestehenden Universitäten der französischen Zone, Tübingen und Freiburg, die durch das noch aus der NS-Zeit vorhandene Lehrpersonal ein deutliches Beharrungsvermögen gegenüber den Demokratisierungsversuchen der Besatzungsbehörden zeigten. ${ }^{14}$ Um dieses Ziel zu erreichen, förderte die französische Militärregierung, allen voran die Kulturabteilung, die Direction de l'Éducation publique unter General Raimond Schmittlein (1904-1974), „ihre“ Universität Mainz nach Kräften. Sie stellten Baumaterial und Arbeitskräfte ebenso zur Verfügung wie Bücher und Zeitschriften für die aufzubauende Universitätsbibliothek. Dabei übereigneten sie der UB Mainz aus bibliothekarischer Sicht wahllos Buchbestände, die ihnen in die Hände fielen. ${ }^{15}$ Der übergeordnete Zweck, der Aufbau von Universität und Universitätsbibliothek, hatte dabei höhere Priorität als Überlegungen hinsichtlich möglicher ungeklärter Eigentumsverhältnisse der Bücher oder der Rechtmäßigkeit solcher Übereignungen. Vielfach wurde solche Unklarheiten bewusst in Kauf genommen und der UB Mainz Bücher gleichsam „treuhänderisch“ - ohne dass dieser Begriff in den zeitgenössischen Quellen zu finden

13 Vgl. dazu Annelen Ottermann: „La Bibliothèque de Mayence est mise à la disposition de la commune." Geburtsjahre einer Bibliothek. In: 200 Jahre Stadtbibliothek Mainz. Hg. von Annelen Ottermann, Stephan Fliedner. Wiesbaden: Harrassowitz 2005 (= Veröffentlichungen der Bibliotheken der Stadt Mainz 52), S. 31-34.

14 Defrance: La politique culturelle (Anm. 3); Defrance: Die Franzosen und die Wiedereröffnung (Anm. 11).

15 Rektor Schmidt bemerkte in einem Schreiben an die Militärregierung am 16.06.1946, dass der Erfolg der Beschlagnahmen für die UB nur gering veranschlagt werden könne, da kaum wissenschaftliche Werke erfasst würden. UA Mainz, Best. 7 Nr. 31. 
wäre - oder als Leihgabe übergeben. Im Zuge der Besatzungszeit sind somit in Mainz vielfach irreguläre oder zumindest fragwürdige Zugänge in den Bestand gelangt, wie im Folgenden an Hand von vier Beispielen erläutert werden soll.

\section{Beispiel 1: Die Bibliothek von Reichsleiter Bormann}

Als Bibliothekdirektor Walter Menn im Sommer 1946 seinen Dienst antrat, fand er einen Buchbestand von rund 3.000 Titeln vor, darunter 80 Kisten mit der beschlagnahmten Bibliothek des ehemaligen Leiters der Parteikanzlei der NSDAP und engem Vertrauten Hitlers Martin Bormann (1900-1945), Teile der Bibliothek des Rassentheoretikers und Wegbereiters der nationalsozialistischen Rassenideologie Hans F. K. Günther (1881-1968), verschiedene Schwarzwälder Kriegsbüchereien und eine Bücherspende der Schweizer Zeitschrift Weltwoche. ${ }^{16}$ Genaue Zahlen über die einzelnen Bestände liegen nicht vor. Laut einer erhaltenen Liste des Buchbestands der Bibliothek Günther, handelt es sich dabei nur um rund fünfzig Titel, ${ }^{17}$ während nach einer aktuellen Erhebung mindestens 1.550 Bände zur Bormann-Bibliothek zu rechnen sind. Legt man die von Menn genannte Gesamtzahl zu Grunde, machte die Bormann-Bibliothek mehr als die Hälfte des Anfangsbestands aus, mit der die UB Mainz ihren Betrieb aufgenommen hat. Unklar ist, wie die Bücher nach Mainz kamen und wo sie den französischen Militärbehörden in die Hände gefallen sind. Die Studie von Charlet Flauaus legt jedoch überzeugend die Vermutung nahe, dass die Bücher ursprünglich in den Anwesen Bormanns in Pullach und auf dem Obersalzberg aufgestellt waren und gegen Kriegsende in eine Villa Bormanns im Schwarzwald evakuiert und dort von französischem Militär beschlagnahmt wurden. Für diese These sprechen u.a. die Schwarzwälder Kriegsbüchereien, deren Bestände ebenfalls zum Anfangsbestand der UB Mainz gehörten. ${ }^{18}$

Dadurch, dass die beschlagnahmten Bücher aus der Bormann-Bibliothek der Universität zur Verfügung gestellt wurden, bevor die UB Mainz eingerichtet worden war, wurden die Bücher je nach Thema von einzelnen Universitätsinstituten vereinnahmt und den jeweiligen Institutsbibliotheken zugewiesen. Die Bücher wurden so über viele Standorte auf dem Universitätscampus verstreut. Allein die Tatsachen, dass die Bücher durchweg einen eindeutig erkennbaren Stempel trugen - vielfach auch die eigenhändige Unterschrift Bormanns - und dass die Universitätsbibliothek bis 1949 ein zentrales Zugangsbuch führte, in

16 Menn, Universitätsbibliothek im Aufbau (Anm. 10), S. 113.

17 UA Mainz, Best. 83 Nr. 3.

18 Charlet Flauaus: Der NS-Funktionär und seine private Bibliothek. Die Bücher des Reichsleiters Martin Bormann in der Universitätsbibliothek Mainz, unveröffentlichte Magisterarbeit, Universität Mainz 2014 (UA Mainz, S 6 Nr. 212). 
dem auch die Bestände der Institutsbibliotheken rückwirkend aufgenommen wurden, ermöglichen es noch heute, den Bestand der Bormann-Bibliothek zu rekonstruieren. Eine in den Akten vorhandene Liste gibt weitere Hinweise, ${ }^{19}$ lässt aber auf Grund unpräziser bibliographischer Angaben häufig keinen eindeutigen Schluss auf das genaue Exemplar zu. Nachdem Anfang der 2000er Jahre ein erster Versuch unternommen wurde, einen Überblick über den noch vorhandenen Gesamtbestand der Bormann-Bibliothek zu bekommen, konnte 2014 in einem zweiten Anlauf unter Zuhilfenahme der Zugangsbücher der Bestand auf 1.223 eindeutig zu identifizierende Titel mit 1.549 Bänden bestimmt werden. ${ }^{20}$ Der inhaltliche Schwerpunkt liegt dabei auf Werken zur Kirchengeschichte, insbesondere zum Jesuitenorden, politischen und historischen Werken sowie auf NS-Literatur. ${ }^{21}$

Die Quellenlage zur Bormann-Bibliothek erschwert allerdings die Provenienzrecherche und die Ermittlung von möglicherweise im Bestand enthaltenem NS-Raubgut. Sind schon die genauen Umstände des Zugangs der Bücher an der UB Mainz ungeklärt, so lassen sich die Erwerbungen der einzelnen Bände durch Bormann selbst nur an Hand der vereinzelt in den Büchern enthaltenen Exlibris, Buchhändleretiketten oder sonstiger Vorbesitzspuren ermitteln. Die umfangreiche Untersuchung von Einzelfällen durch Charlet Flauaus ergab keinen einzigen Fall, der eindeutig oder auch nur mit hoher Wahrscheinlichkeit als Raubgut einzustufen wäre. ${ }^{22} 23$ Titel wurden von ihr jedoch als "verdächtig“ eingestuft und näher untersucht. Verdachtsmomente waren dabei u.a. die Exlibris von Personen oder Familien, deren Bibliotheken nachweislich enteignet wurden oder unter Zwang verkauft werden mussten. Ein eindeutiger Raubgutfall konnte dabei jedoch nicht nachgewiesen werden. Obwohl Bormann in den Kulturgutraub der Nationalsozialisten verwickelt und zeitweilig für den Aufbau der „Führerbibliothek“ in Linz verantwortlich war, ließen sich keine Hinweise darauf finden, dass Bormann privat im großen Stil vom NS-Bücherraub profitiert hätte. ${ }^{23}$

Aktuell wird die geschlossene Aufstellung der Bormann-Bibliothek im RaraMagazin der Zentralbibliothek unter Zusammenführung der dezentralen Bestände aus den Instituts- und Bereichsbibliotheken umgesetzt.

19 UA Mainz, Best. 86 Nr. 68, Inventaire des Livres du Reichsleiter Bormann.

20 Es ist davon auszugehen, dass durch den Verlust von Eigentumsstempeln auf Grund von Bindearbeiten nicht mehr alle Bände eindeutig zu bestimmen sind und die ursprüngliche Bandzahl der Bormann-Bibliothek daher größer gewesen ist.

21 Flauaus, NS-Funktionär (Anm. 18), S. 28.

22 Ebd., S. 48.

23 Ebd., S. 56. 


\section{Beispiel 2: Regionale Beschlagnahmungen}

Um den Aufbau ihrer Universitätsgründung voranzutreiben, wiesen die Besatzungsbehörden der UB Mainz nicht nur Buchbestände zu, die ihnen als vermeintlich herrenlos in die Hände gefallen waren. Sie bemühten sich darüber hinaus um die aktive Beschaffung von Büchern. Auf der Grundlage des Gesetzes Nr. 52, ${ }^{24}$ das den Umgang mit dem Vermögen ehemaliger Nationalsozialisten regelte, beschlagnahmte die französische Militärregierung vielfach Bibliotheken von ehemaligen Parteimitgliedern und übereignete sie der UB Mainz. ${ }^{25}$ Den Vorschlag, die Universitätsbibliothek durch Bestände privater wissenschaftlicher Bibliotheken von Personen, die im Rahmen des Entnazifizierungsverfahrens als belastet eingestuft worden waren, zu ergänzen, hatte General Schmittlein bereits in einem Memorandum zur Gründung der Universität Mainz im Februar 1946 formuliert. ${ }^{26}$ Dort hieß es, die Universität Mainz sollte vorrangig bei der Zuteilung beschlagnahmter Bibliotheken berücksichtigt werden. So trafen seit dem Frühjahr 1946 regelmäßig Bücherlieferungen der französischen Behörden in Mainz ein.

Die UB Mainz sah diese Übereignungen von Anfang an kritisch. Schon im Sommer 1946 wandte sich Gründungsrektor Josef Schmid (1898-1978) an den städtischen Vertreter der französischen Militärregierung und warnte davor, dass die Beschlagnahmungen eine erhebliche Missbilligung in der Bevölkerung hervorriefen und zudem nur einen geringen Mehrwert für den Aufbau der UB Mainz böten, da sie wenig wissenschaftliche und mehr belletristische Literatur enthielten. Zudem bestünde „die Gefahr, dass diese Massnahme [sic!] dem Humanitätsbestreben der Universität empfindlich schadet“. ${ }^{27}$ Offenbar befürchtete Rektor Schmid einen erheblichen Ansehensverlust der jungen Universität in der Bevölkerung.

Auch blieb die Eigentumsfrage der zugewiesenen Bücher zunächst ungeklärt. Den betroffenen Personen waren von den Militärbehörden widersprüchliche

24 Gesetz Nr. 52 über die Sperre und Kontrolle von Vermögen vom 14.07.1945, abgedruckt in Journal officiel du Commandement en Chef Français en Allemagne 2 (1946/47) Nr. 59, S. 586-588 und Anordnung Nr. 1 zur Durchführung des Gesetzes Nr. 52, abgedruckt ebd. S. 589-593.

25 Wie aus einem Schreiben des Bürgermeisters der Stadt Bühl und des Landrates des Kreises Alzey an die Universität vom 07.09.1946 bzw. 07.06.1946 (in UA Mainz, Best. 7 Nr. 31) hervorgeht, ging diese Entscheidung auf einen Befehl von General Émile Laffon (1907-1957) zurück und wurde den deutschen Behörden offen mitgeteilt.

26 Raimond Schmittlein: Memorandum über die Gründe einer Neugründung der Universität Mainz vom 25. Februar 1946. In: Quellen zur Geschichte von Rheinland-Pfalz während der französischen Besatzung März 1945 bis August 1949. Mainz: v. Hase und Koehler 1985, S. 151-160, hier S. 156.

27 UA Mainz, Best. 7 Nr. 31, Rektor Schmid an Oberst Delobel, 16.06.1946. 
Angaben über die Form der Beschlagnahmung gemacht worden. Die Bücher wurden als Leihgabe oder als Spende bezeichnet, zum Teil den Betroffenen eine Entschädigung in Aussicht gestellt. ${ }^{28}$ Wiederholt fragte die Universität daher bei den zuständigen französischen Stellen nach, wie es um die Eigentumsverhältnisse der beschlagnahmten Bücher bestellt sei. Erst nach mehreren Rückfragen erhielt die Universität im Februar 1947 die Auskunft, die Bücher seien der UB Mainz „d'une manière définitive“ zugewiesen und jeder Anspruch eines Vorbesitzers sei zurückzuweisen. ${ }^{29}$ Da die Bücher größtenteils für die Zwecke der UB unbrauchbar waren - und vielleicht auch weil Direktor Menn Zweifel an der Dauerhaftigkeit dieser Entscheidung hegte - wurden die Bücher dennoch im Magazin der UB separat aufgestellt und vorerst nicht in den Bestand eingearbeitet. $^{30}$

Mit zunehmender Dauer des Entnazifizierungsverfahrens wurden viele ehemals als belastet eingestufte Personen nun als Mitläufer bewertet und die Sperrung ihrer Vermögen oder vorübergehende Amtsenthebungen und Berufsverbote aufgehoben. ${ }^{31}$ Nachdem dadurch der ursprüngliche Grund für die Beschlagnahmung ihrer Bibliotheken weggefallen war, wandten sich einige der Betroffenen an die Universität Mainz und forderten die beschlagnahmten Bücher zurück. ${ }^{32}$ Erst im Spätsommer 1949 gelang es der UB, die Genehmigung für die Rückgabe der Bücher bei der Militärregierung zu erwirken. Mit Ausnahme des nationalsozialistischen Schriftguts sollten nun alle beschlagnahmten Bücher an die Vorbesitzer zurückgegeben, für bereits eingestellte Titel eine angemessene Entschädigung gezahlt werden. ${ }^{33}$ Die Rückgabe der Bücher erwies sich als mühsam und langwierig. Vielfach konnten die Vorbesitzer nicht mehr ermittelt werden, da bei der Beschlagnahmung keine Listen angefertigt wurden oder Listen bzw. Bücher beim Transport verlorengegangen oder durcheinandergeraten waren. Mehrere Jahre dauerte das Bemühen der UB Mainz um die Rückgabe der beschlagnahmten Bücher. 1958 fand sich noch ein Restbestand von 6.000 bis 7.000 Büchern in den Kellern der UB, der nicht zugeordnet oder zu-

28 UA Mainz, Best. 1 Nr. 101.

29 „Aucune réclamation émanant des anciens propriétaires de ces livres ne doit être prise par vous en considération. La dévolution définitive de ces livres sera prononcée ultérieurement", UA Mainz, Best. 86 Nr. 68, der Delegierte des Distrikts Rheinhessen an Rektor August Reatz, 11.02.1947.

30 UA Mainz, Best. 86 Nr. 68, UB Mainz an Anton Melzenbach in Cochem, 26.09.1949.

31 Zur Entnazifizierung in Rheinland-Pfalz vgl. Rainer Möhler: Entnazifizierung in RheinlandPfalz und im Saarland unter Französischer Besatzung von 1945 bis 1952. Mainz: v. Hase \& Koehler 1992.

32 Vgl. exemplarisch dafür ein Schreiben des Oberbürgermeisters von Konstanz an die Délégation du District du Hesse-Rhénane vom 22.09.1948. In: UA Mainz, Best. 86 Nr. 68.

33 UA Mainz, Best. 86 Nr. 68, UB Mainz an Prof. Erwin Pfeiffer in Offenburg, 12.12.1951. 
rückgegeben werden konnte. ${ }^{34}$ Nur etwa die Hälfte der von der Militärregierung zugewiesenen Bücher konnte auf diese Weise letztlich restituiert werden. ${ }^{35}$

Rückblickend formulierte Direktor Menn, die Zuwendungen der Militärregierung haben „viel Verdruss und Mühe“ gebracht. ${ }^{36}$ Gleichzeitig erzielten die Bücherzuweisungen nicht den von General Schmittlein erhofften positiven Effekt für den Aufbau der UB Mainz, da - wie Menn es formulierte - nicht „mit wahllos eingebrachten Haufen von Büchern [...] eine brauchbare Bibliothek“ geschaffen werden könne. ${ }^{37}$ Zudem ergaben sich aus den in der Nachkriegszeit einem starken Wandel unterliegenden Rechtsvorstellungen zunächst Unsicherheiten, spätestens aber ab 1948 berechtigte Zweifel an der Dauerhaftigkeit der Eigentumsübertragungen, die schließlich ab $1949 \mathrm{zu}$ arbeitsaufwändigen Rückgabeverfahren führten.

\section{Beispiel 3: Die Bibliothek der Familie Sabatini}

In einem Fall, der der UB Mainz unrühmliche Aufmerksamkeit bescherte, wurde NS-Raubgut den Vorbesitzern zurückerstattet. Es handelt sich um die Bücher der Familie Sabatini aus Pescocostanzo in Umbrien/Italien. Diese waren der Familie im Winter 1943/44 von der Wehrmacht geraubt worden und vermutlich im Schwarzwald den französischen Militärbehörden in die Hände gefallen, die wiederum die Bücher an die UB Mainz weitergaben. Darunter befanden sich auch wertvolle Bücher und Autographen aus dem 16. bis 19. Jahrhundert. ${ }^{38}$ Bereits 1947 stieß ein Werkstudent auf Besitzvermerke der Familie Sabatini in den von den französischen Stellen übereigneten Beständen, machte die Familie ausfindig und sandte ihr - ohne UB-Direktor Menn zu informieren - vier Bände

34 UA Mainz, Best. 86 Nr. 68, Kurator Eichholz an das Ministerium für Finanzen und Wiederaufbau, 17.04.1958.

35 Jahresbericht der Universitätsbibliothek Mainz 1951.

36 Ebd.

37 UA Mainz Best. 86 Nr. 11, Walter Menn: Bericht über das erste Vierteljahr der Universitätsbibliothek Mainz.

38 Die genaue Herkunft der Bücher ist unbekannt. Es gibt keine schriftlichen Dokumente über die Zuweisung der Bibliothek, UA Mainz Best. 86 Nr. 53; Bericht von Günter Richter zur Rückgabe der Bibliothek Sabatini. Eine tendenziöse Darstellung der Geschichte der SabatiniBibliothek findet sich bei Anja Oehlers: Die Bücher der Sabatinis. In: Die Zeit, Hamburg, 21.06.1991, URL: http://www.zeit.de/1991/26/die-buecher-der-sabatinis (abgerufen am 12.06.2017); vgl. auch Detlev Franz: Die Bücher der Sabatini. 46 Jahre nach Kriegsende gibt die Uni eine geklaute Bibliothek zurück. In: Unipress 272 (1991), S. 3; Veronica Albrink: Von Büchern, Depots und Bibliotheken. Zur Restitutionsgeschichte nach 1945. In: Die Suche nach NS-Raubgut in Bibliotheken. Recherchestand, Probleme, Lösungswege. Hg. von Bernd Reifenberg. Marburg: Universitätsbibliothek Marburg 2006 (= Schriften der Universitätsbibliothek Marburg 126), S. 110-149, hier S. 127. 
zu. $^{39}$ Nachdem Direktor Menn einige Jahre später von Gottfried Opitz (1904-1976), Geschäftsführer der Monumenta Germaniae Historica, auf die Bestände der Familie Sabatini angesprochen wurde, teilte Menn nach Rücksprache mit dem damaligen Werkstudenten mit, es seien keine weiteren Bücher mit dem Besitzvermerk Sabatini vorhanden. Allerdings ergaben die Nachforschungen Menns, dass die Bücher offenbar zunächst an das von Mussolini begründete propagandistische Filminstitut LUCE ${ }^{40}$ gegangen waren. ${ }^{41}$ Da die Anfrage in die Zeit fiel, in der die UB Mainz damit beschäftigt war, die Eigentümer der von der französischen Militärregierung in ihrer Besatzungszone beschlagnahmten Bücher zu ermitteln und die entsprechenden Bücher zurückzugeben, schloss Menn: „Wir hoffen, dass wir das auch mit diesem italienischen Bestand tun können, sobald wir feststellen können, wer der Eigentümer dieser Bücher war. “42 Obwohl Menn wegen der Rückgabe der Bücher angeblich bereits mit der italienischen Botschaft in Bonn in Verbindung stand, ${ }^{43}$ erfolgte keine Kontaktaufnahme mit der Familie Sabatini, die sich zu der Zeit intensiv um die Auffindung ihrer Bibliothek bemühte.

Mitte der 1960er Jahre stieß ein Mitarbeiter der UB erneut auf die Bestände und setzte sich für deren Rückgabe ein, ohne bei der damaligen Bibliotheksleitung Gehör zu finden. Erst ein Wechsel der Leitung Ende der 1980er Jahre führte zu einem ernsthaften Bemühen um die Rückgabe der Bücher. Das Bestreben der UB wurde jedoch durch einen im Juni 1991 in der Wochenzeitung Die Zeit erschienenen Artikel zu den Büchern der Sabatinis konterkariert, der die vermeintlich ablehnende Haltung der UB in den Vordergrund rückte. ${ }^{44} \mathrm{Im}$ Sommer 1991 wurden schließlich 1.253 Bücher aus der geraubten Bibliothek an zwei angereiste Mitglieder der Familie zurückgegeben - 45 Jahre nachdem die UB Mainz erstmals auf den Bestand aufmerksam gemacht worden war.

Das Beispiel der Sabatini-Bibliothek unterstreicht deutlich, dass erst in jüngster Vergangenheit eine Sensibilität für die Raubgutthematik entstanden ist. Der überspitzten Einschätzung von Jürgen Weber, nach 1945 habe die Integration der geraubten Sammlungen Priorität vor der Provenienzklärung und Restitution gehabt, ${ }^{45}$ ist mit Blick auf dieses Beispiel sicher zuzustimmen.

39 UA Mainz Best. 86 Nr. 3, Walter Menn an Gottfried Opitz, 31.12.1953.

40 Das Akronym LUCE steht für L'Unione Cinematografica Educativa.

41 UA Mainz Best. 86 Nr. 53, Walter Menn an Peter Karstedt, 09.11.1953.

42 UA Mainz Best. 86 Nr. 3, Walter Menn an Gottfried Opitz, 31.12.1953.

43 UA Mainz Best. 86 Nr. 53, Walter Menn an Peter Karstedt, 09.11.1953.

44 Oehlers: Bücher der Sabatinis (Anm. 38); vgl. dazu die Perspektive der UB im Bericht von Günter Richter zur Rückgabe der Bibliothek Sabatini. In: UA Mainz Best. 86 Nr. 53.

45 Jürgen Weber: Sammeln als Konsum. Die Verwaltung von NS-Raubgut in deutschen Bibliotheken. In: NS-Raubgut in Museen, Bibliotheken und Archiven. Viertes Hannoversches Symposium. Hg. von Regine Dehnel. Frankfurt: Klostermann 2012 (= Zeitschrift für Bibliothekswesen und Bibliographie, Sonderband 108), S. 33-40, hier S. 33. 


\section{Beispiel 4: Die Bibliothek der Kunsthistorischen Forschungsstätte Paris}

Den Grundstock der Bibliothek des Kunsthistorischen Instituts der Universität Mainz bildeten Bücher der ehemaligen Kunsthistorischen Forschungsstätte Paris, die dem Institut 1946 von der französischen Militärregierung zugewiesen wurden. ${ }^{46}$ Die Forschungsstätte war 1942 im besetzten Frankreich als Abteilung des Deutschen Instituts Paris gegründet worden. ${ }^{47}$ Die Leitung übernahm Dr. habil. Hermann Bunjes (1911-1945), ein Schüler des Bonner Kunsthistorikers Alfred Stange (1894-1968). ${ }^{48}$ Schnell baute Bunjes eine umfangreiche deutschund französischsprachige Fachbibliothek auf, die am Ende gut 6.000 Bände umfasste. Im Sommer 1944 stellte die Kunsthistorische Forschungsstätte ihren Betrieb ein. Die Bibliothek wurde nach Schloss Bürresheim und ins Kloster Himmerod in der Eifel ausgelagert. ${ }^{49}$ Beide Auslagerungsstätten befanden sich in der französisch besetzten Zone. Das Kunsthistorische Institut Mainz bemühte sich intensiv darum, die Buchbestände für die Institutsbibliothek zu erhalten. Eine Schlüsselrolle spielte dabei offenbar der Kunsthistoriker Heinrich Gerhard

46 Friedrich Gerke: Das Kunstgeschichtliche Institut der Johannes Gutenberg-Universität Mainz 1946-1955. In: Jahrbuch der Vereinigung „Freunde der Universität Mainz“ 1955, S. 58-71, hier S. 65; Nikola Doll: „[...] das beste Kunsthistorische Institut Grossdeutschlands“. Das Kunsthistorische Institut der Rheinischen Friedrich-Wilhelms-Universität Bonn im Nationalsozialismus. In: Kunstgeschichte im Nationalsozialismus. Beiträge zur Geschichte einer Wissenschaft zwischen 1930 und 1950. Hg. von Nikola Doll, Christian Fuhrmeister und Michael H. Sprenger. Weimar: Verl. und Datenbank für Geisteswissenschaften 2005, S. 49-60, hier S. 55; Listen der Bücher befindet sich in UA Mainz Best. 45 Nr. 210 und Best. 86 Nr. 50.

47 Nikola Doll: Politisierung des Geistes. Der Kunsthistoriker Alfred Stange und die Bonner Kunstgeschichte im Kontext nationalsozialistischer Expansionspolitik. In: Griff nach dem Westen. Die „Westforschung“ der völkisch-nationalen Wissenschaften zum nordwesteuropäischen Raum (1919-1960). Hg. von Burkhard Dietz, Helmut Gabel und Ulrich Tiedau. Münster-New York-München-Berlin: Waxmann 2003 (= Studien zur Geschichte und Kultur Nordwesteuropas 6), S. 979-1015, hier S. 1005; Eckard Michels: Das Deutsche Institut in Paris 1940-1944. Ein Beitrag zu den deutsch-französischen Kulturbeziehungen und zur auswärtigen Kulturpolitik des Dritten Reiches. Stuttgart: Dr. Franz Steiner Verlag 1993, S. 92-94.

48 Frank Rutger Hausmann: „Auch im Krieg schweigen die Musen nicht“. Die Deutschen Wissenschaftlichen Institute im Zweiten Weltkrieg. Göttingen: Vandenhoeck und Ruprecht 2001 (= Veröffentlichungen des MPI für Geschichte 169), S. 101; Christina Kott: „Den Schaden in Grenzen halten...“. Deutsche Kunsthistoriker und Denkmalpfleger als Kunstverwalter im besetzten Frankreich 1940-1944. In: Kunstgeschichte im „Dritten Reich“. Theorien, Methoden, Praktiken. Hg. von Ruth Heftrig, Olaf Peters und Barbara Schellewald. Berlin: Akademie Verlag 2008 (= Schriften zur modernen Kunsthistoriographie 1), S. 362-392, hier S. 372; Anja Heuss: Kunst- und Kulturgutraub. Eine vergleichende Studie zur Besatzungspolitik der Nationalsozialisten in Frankreich und der Sowjetunion. Heidelberg: Winter 2000, S. 80-82.

49 Doll: Politisierung (Anm. 47), S. 1006, Fußnote 110. 
Franz (1916-2006), der bereits als Mitarbeiter Bunjes' in Paris tätig gewesen war und seit 1946 als Privatdozent an der Universität Mainz lehrte. ${ }^{50}$ Die Übergabe des ersten Teils - vermutlich der ursprünglich nach Schloss Bürresheim ausgelagerten, zum Zeitpunkt der Übergabe in Schloss Gondorf befindlichen Bestände - erfolgte Ende $1946 .{ }^{51}$ Bis zur Übergabe der im Kloster Himmerod lagernden Beständen bedurfte es noch über ein Jahr zäher Verhandlungen. Nachdem der UB Mainz im Herbst 1947 mitgeteilt wurde, dass sie den zweiten Teil der Bibliothek auf Grund des Widerstands französischer Stellen nicht erhalten könne, ${ }^{52}$ führte erst die persönliche Intervention des französischen Bezirksdelegierten von Trier dazu, dass die Bücher 1948 schließlich doch nach Mainz kamen. ${ }^{53}$

Die Bücher wurden der Universität zunächst ausdrücklich nur leihweise überlassen. ${ }^{54}$ Und auch bei der zweiten Lieferung wurde 1948 explizit darauf hingewiesen, dass es bei der Übergabe des Bestandes nicht um eine Besitzübertragung handle. ${ }^{55}$ Die Eigentumsverhältnisse konnten erst nach langen Verhandlungen geklärt werden. Erst 1951 informierte General Schmittlein Kanzler Fritz Eichholz (1902-1994) darüber, dass die Bücher als Eigentum der Universität zu betrachten seien und fügte hinzu: „Es ist selbstverständlich, dass jeder Anspruch der Erben des ehemaligen Besitzers oder irgendeiner anderen Instanz nur die Auslieferung der fraglichen Bibliothek an die französische Regierung zur Folge haben könnte. ${ }^{\text {" } 56}$

Der rund 3.000 Bände umfassende Buchbestand enthält vorwiegend kunstgeschichtliche Werke in deutscher und französischer Sprache sowie eine große

50 Sabine Arend: Studien zur deutschen kunsthistorischen „Ostforschung“ im Nationalsozialismus. Die Kunsthistorischen Institute an den (Reichs-) Universitäten Breslau und Posen und ihre Protagonisten im Spannungsfeld von Wissenschaft und Politik, phil. Diss., Humboldt-Universität Berlin 2009, S. 80-82. URL: http://edoc.hu-berlin.de/dissertationen/ arend-sabine-2009-07-15/PDF/arend.pdf (abgerufen am 12.06.2017).

51 Rektor Schmid informierte den Leiter des Kunsthistorischen Instituts, Prof. Gerke, am 04.09. 1946 darüber, dass „nach langen Verhandlungen“ nun die entsprechende Genehmigung erfolgt sei und die Bestände auf Schloss Gondorf abgeholt werden könnten. UA Mainz, Best. 1 Nr. 101; vgl. auch Hans-Jürgen Imiela: Zur Chronik des Kunstgeschichtlichen Instituts. In: Tradition und Gegenwart. Studien und Quellen zur Geschichte der Universität Mainz. Mit besonderer Berücksichtigung der Philosophischen Fakultät. 2. Teil. 2. Halbband: Institute der Philosophischen Fakultät 1946-1972. Wiesbaden: Dr. Franz Steiner Verlag 1981 (= Beiträge zur Geschichte der Universität Mainz 11,2,2), S. 25-32, hier S. 26.

52 UA Mainz Best. 102 Nr. 1, Aktenvermerk über eine entsprechende Mitteilung des französischen Stadtkommandanten Delobel, 03.10.1947.

53 UA Mainz Best. 1 Nr. 101, Kanzler Eichholz an Prof. Gerke, 15.12.1947.

54 UA Mainz Best. 7 Nr. 32, Vermerk von Rektor Schmid für Prof. Gerke, 04. 09. 1946; Imiela: Chronik (Anm. 51), S. 26.

55 UA Mainz Best. 1 Nr. 101, Amt für kontrollierte Vermögen an die Universität Mainz, 12.11. 1948.

56 UA Mainz Best. 1 Nr. 101, General Schmittlein an Kanzler Eichholz, 23.01.1951. 
Anzahl an Auktionskatalogen. Der enge Kontakt von Hermann Bunjes zum Einsatzstab Reichsleiter Rosenberg und zu Hermann Göring (1893-1946) ${ }^{57}$ legt den Verdacht nahe, dass die Bestände NS-Raubgut enthalten könnten. Dieser Verdacht ließ sich 2015 in einer ersten Vorstudie erhärten, so dass aktuell im Rahmen eines vom Deutschen Zentrum Kulturgutverluste geförderten Projekts der Bestand auf seine Provenienz hin untersucht wird. ${ }^{58}$

Bemerkenswert ist in diesem Zusammenhang, dass einer der ab 1946 führenden Mitarbeiter und spätere Direktor der UB Mainz, Hermann Fuchs (1896-1970), während der deutschen Besetzung Frankreichs im Zweiten Weltkrieg als Leiter des Referats Bibliotheksschutz in Paris tätig war und in diesem Rahmen für die Reichstauschstelle große Mengen ausländischer Literatur ankaufte. $^{59}$ Eine Verbindung zwischen Fuchs und der kunsthistorischen Forschungsstätte ist aus den Mainzer Akten nicht nachweisbar. Angesichts des überschaubaren Personenkreises des wissenschaftlichen Personals im besetzten Paris ist es zumindest aber sehr wahrscheinlich, dass Bunjes und Fuchs einander kannten.

Eine Sensibilität für die mögliche Unrechtmäßigkeit von Bucherwerbungen unter den Bedingungen eines Besatzungsregimes war an der UB Mainz in der Nachkriegszeit nicht vorhanden oder wurde dem Wunsch nach dem schnellen Aufbau einer funktionsfähigen Institutsbibliothek untergeordnet. Bemerkenswerterweise war, obwohl es sich um mutmaßliche irreguläre Erwerbungen in Frankreich handelte, auch von französischer Seite kein Bestreben erkennbar, die Erwerbungsumstände oder die Vorbesitzer zu ermitteln oder gar einzelne Bände $\mathrm{zu}$ restituieren.

Diese vier Beispiele beleuchten jeweils unterschiedliche Facetten irregulärer Zugänge, die jeweils durch die französischen Militärbehörden an die UB Mainz gelangten. Während im Fall der Bibliotheken Bormann und Sabatini die Eigentumsverhältnisse in der Nachkriegszeit nie thematisiert wurden und sowohl die UB als auch die Militärregierung stillschweigend davon ausgingen, dass die Bücher in den Besitz der UB Mainz übergegangen sind - selbst dann noch als der Raubgutkontext der Sabatini-Bibliothek evident wurde -, wurde die Frage des

57 Michels, Das Deutsche Institut (Anm. 47), S. 93.

58 Der Mainzer Bibliotheksbestand der Kunsthistorischen Forschungsstätte Paris (KHF). URL: https://www.kulturgutverluste.de/Content/03_Forschungsfoerderung/Projekt/Universitaets bibliothek-Mainz/Projekt1.html (abgerufen am 12.06.2017).

59 Cornelia Briel: „Fortschaffung eurer Bücher wird vorbereitet - stop - örtliche Verhandlungen zwecklos“. Zu den Bücherlagern der Reichstauschstelle 1943 bis 1946. In: NSRaubgut in Museen, Bibliotheken und Archiven. Viertes Hannoversches Symposium. Hg. von Regine Dehnel. Frankfurt: Klostermann 2012 (= Zeitschrift für Bibliothekswesen und Bibliographie, Sonderband 108), S. 133-145, hier S. 137, 145; Cornelia Briel: Beschlagnahmt, erpresst, erbeutet. NS-Raubgut, Reichstauschstelle und Preußische Staatsbibliothek zwischen 1933 und 1945. Berlin: Akademie Verlag 2013, S. 128-130 und 254-256. 
Besitzes bei den von Privatpersonen beschlagnahmten Beständen und der Bibliothek der Kunsthistorischen Forschungsstätte Paris intensiv diskutiert. In beiden Fällen jedoch unter sehr unterschiedlichen Vorzeichen. Während die UB Mainz auf Grund der widersprüchlichen Angaben hinsichtlich der Privatbibliotheken auf Klärung drängte - sehr bald mit dem Ziel, die unwillkommenen Bestände zurückgeben zu können -, war sie bei den Beständen der Kunsthistorischen Forschungsstätte eher daran interessiert, eine definitive Besitzeinweisung zu erhalten, um etwaige Besitzansprüche Dritter abwehren zu können. Zwar erklärte Menn 1953, alle von den französischen Behörden zugewiesenen Bücher würden nur in Verwahr genommen und zurückgegeben, soweit die Eigentümer ermittelt werden können ${ }^{60}$ und suggerierte damit eine treuhänderische Verwaltung der Bestände. Ein ernsthaftes Bemühen um die Rückgabe ist jedoch nur bei den Büchern aus regionalen Beschlagnahmungen erkennbar - im augenfälligen Gegensatz zur Bibliothek der Familie Sabatini, deren unrechtmäßige Erwerbung seit 1947 bekannt war. Während die UB Mainz im ersteren Fall aus mehreren Gründen - etwa der für den Bibliotheksaufbau geringe Wert der Bestände, die befürchtete Rufschädigung der UB und nicht zuletzt die räumliche Nähe der Geschädigten, die zum Teil persönlich bzw. brieflich in Mainz vorstellig wurden - daran interessiert war, sich für eine zügige Rückführung einzusetzen, so bestand im Fall der Sabatini-Bibliothek zumindest in den Nachkriegsjahren kein Unrechtsbewusstsein, das der Provenienzforschung einen Vorrang vor den Bestrebungen des Bestandsaufbaus eingeräumt hätte.

Nur die Sabatini-Bibliothek hat sich bislang eindeutig als Beutegut erwiesen, so dass die Bestände restituiert werden konnten. Dagegen wird im Fall der Bibliotheken von Martin Bormann und der Kunsthistorischen Forschungsstätte Paris bislang nur der Verdacht auf möglicherweise enthaltenes Raubgut nahegelegt. Hinsichtlich der Bormann-Bibliothek haben sich diese Verdachtsmomente trotz intensiver Forschungen nicht bestätigen lassen. Für die Kunsthistorische Forschungsstätte Paris läuft ein entsprechendes Provenienzforschungsprojekt, das näheren Aufschluss über die Thematik geben wird. Für die Nachkriegszeit insgesamt lässt sich an Hand der Beispiele dagegen konstatieren, dass dem Ziel, eine funktionsfähige Bibliothek für die neugegründete Universität Mainz aufzubauen, sowohl von deutscher als auch von französischer Seite alle Bedenken hinsichtlich möglicher Ansprüche von Vorbesitzern untergeordnet wurden. Deutlich wird an diesen Beispielen auch, dass die Provenienzforschung bei Erwerbungen der Nachkriegszeit häufig vor größere Schwierigkeiten gestellt ist. Zugänge aus der Nachkriegszeit sind in der Regel über Umwege, nach Evakuierung und Auslagerung, Auffindung durch die Besatzungsbehörden, Zwischenlagerung etc. an die Bibliotheken gekommen. Die

60 UA Mainz Best. 86 Nr. 3, Walter Menn an Gottfried Opitz, 31.12.1953. 
Übergaben wurden vielfach nicht dokumentiert bzw. eventuell vorhandene Dokumentationen gingen verloren, so dass sich die Vorbesitzer in vielen Fällen nicht mehr ermitteln lassen. Die Sekundärerwerbung über die Besatzungsbehörden bedeutet häufig einen zusätzlich erforderlichen Schritt in der Provenienzermittlung, der diese mitunter nicht unwesentlich erschwert.

Hinzu kommen die besonderen Rechtsverhältnisse der Nachkriegszeit. Die langwierigen Verhandlungen und die vielfach erst auf mehrfache Nachfrage erfolgte Klarstellung der Besitzverhältnisse verdeutlichen, dass die Rechtsgrundlage vieler Erwerbungen zunächst unklar und - wie sich nach dem Ende der Besatzungszeit zeigte - auch sehr kurzlebig war. Viele Regelungen aus der Besatzungszeit wurden nach deren Ende von deutschen Behörden aufgehoben oder geändert. Die Zeit der Besetzung bot so ein ideales Umfeld für irreguläre Zugänge. Einmal mehr wird deutlich, dass die Provenienzrecherche auch für nach 1945 gegründete Bibliotheken ein relevantes Thema ist. 
Open-Access-Publikation im Sinne der CC-Lizenz BY-NC-ND 4.0

(c) 2018, V\&R unipress GmbH, Göttingen

ISBN Print: 9783847107835 - ISBN E-Lib: 9783737007832 


\title{
Michal Bušek
}

\section{Provenance Research in the Book Collection of the Jewish Museum in Prague}

\author{
Zusammenfassung \\ Provenienzforschung im Buchbestand des Jüdischen Museums Prag \\ Im Jahr 2001 begann die Provenienzforschung am Jüdischen Museum Prag mit dem \\ Projekt „Identifizierung der Vorbesitzer von Büchern“. Die physische Durchsicht des \\ gesamten Buchbestandes konnte um den Jahreswechsel 2015/2016 abgeschlossen werden. \\ Das Projekt wurde durch die historischen Umstände angeregt, aufgrund deren viele Bü- \\ cher während des oder nach dem Zweiten Weltkrieg in die Bestände des Jüdischen $\mathrm{Mu}-$ \\ seums eingingen, die zuvor einen anderen Besitzer hatten. Die Methode, der Prozess und \\ die Schwierigkeiten und Herausforderungen des Projekts sollen dargestellt werden. Jeder \\ Vorbesitzer wurde dokumentiert und in eine eigene Datenbank aufgenommen. Ab- \\ schließend geht der Beitrag auch auf die Restitutionspolitik des Jüdischen Museums Prag \\ und die Schwierigkeiten und Probleme, die im Laufe der Restitutionen entstehen können, \\ ein. \\ Schlagwörter \\ Jüdisches Museum Prag, Provenienzforschung, Buchbestand, jüdische Bibliotheken, \\ Ghetto Theresienstadt, Restitution
}

\begin{abstract}
This paper introduces the project of the Jewish Museum in Prague (JMP) "Identifying the Books' Original Owners", which started in 2001. The physical examination of the museum's entire book collection finished at the end of 2015. The project started due the fact that the museum's book collection contains books originally owned by other owners than JMP that became part of the library holdings during and after the WWII. It will introduce the project's methodology, the difficulties and challenges which arose during the research and the database of the original owners. It will also focus on the restitution policy of the Jewish Museum in Prague.
\end{abstract}

Keywords

The Jewish Museum in Prague, Provenance Research, Book Collection, Jewish Libraries, Ghetto Theresienstadt, Restitution 
The holdings of the library in the Jewish Museum in Prague (JMP) have been shaped by historical events, particularly World War II and the postwar situation in the Czech Republic. The museum was established in 1906 and its task was to collect, preserve, and exhibit ceremonial artifacts, historical documents, manuscripts, and rare prints originating from Prague or other cities in the Czech lands. ${ }^{1}$ There was no separate library in the pre-war museum and books formed one collection together with archival documents - so called written material. The museum operated until the Nazi occupation in 1939, at which time the collections were placed under the administration of the Jewish community. The Jewish community was trying to find a way to preserve and rescue various Jewish items from 153 communities in Bohemia and Moravia. In spring of 1942 community members formed a plan to establish the Jewish Central Museum, where the holdings of the Czech Jewish museums and of the communities could be collected. After lengthy negotiations with the Nazis, the project was approved and the Central Museum began to operate on September 3, 1942. The pre-war museum's collection became part of the Jewish Central Museum, in which thousands of other items started to arrive. For each item incorporated into the Central Museum collections a card in the catalogue was created containing details about the item, the date of recording, the mark of the cataloger, the inventory number and what is most important for our current research - the location from where the item came. This so called German Catalogue can help us find out when and from where some of the books came and whether they stayed in the museum after the war. It must be stressed that the location from where the boxes came to the museum was very often just a collection place where the property of a community had been gathered and not the actual original provenance location. The Jewish Central Museum's library holdings comprised of mostly traditional literature from Jewish communities like rabbinic writings, prayer books, fiction and nonfiction books by Jewish authors.

The property of the deported individuals was placed under the administration of the Treuhandstelle - an organization established in 1941 to take care of confiscated property. ${ }^{2}$ It happened sometimes that the rare or significant books from the Treuhandstelle's storages were taken and transferred to the Jewish Central Museum. Another group of books added to the Jewish Central Museum was comprised of the donations from individual collectors who tried to protect their libraries against confiscation. At the end of the WW II around 46,000 items,

1 For more details see: Magda Veselská: Defying the Beast. The Jewish Museum in Prague 1906-1940. Prague: Jewish Museum in Prague 2006 and Magda Veselská: Archa Paměti. Cesta pražského židovského muzea pohnutým 20. stoletím. Praha: Academia/Židovské muzeum v Praze 2012.

2 For more details about the Treuhandstelle see: Helena Krejčová: Židé v protektorátu. Hlášení Židovské náboženské obce v roce 1942. Praha: Ústav pro soudobé dějiny AV ČR 1997. 


\section{German Catalogue}

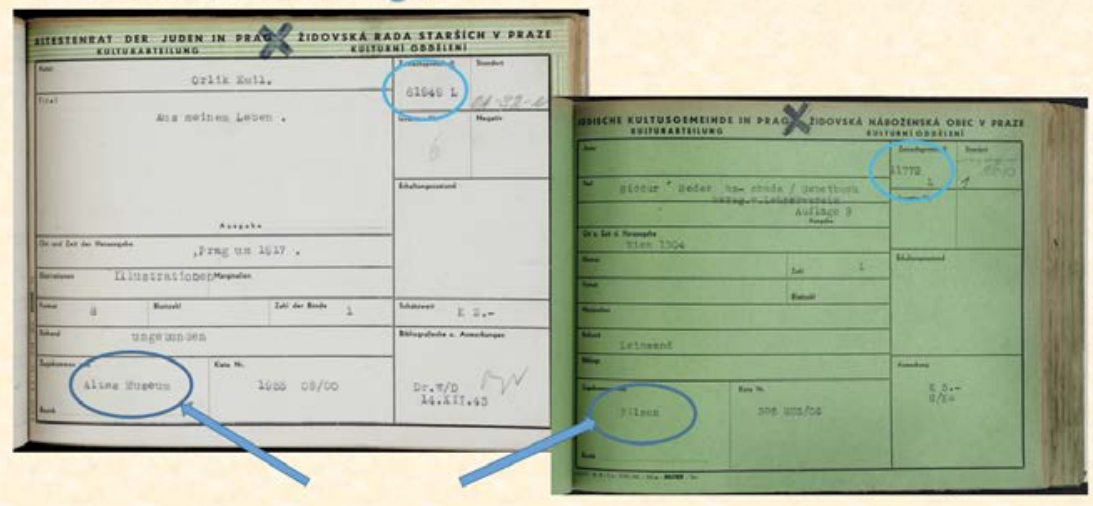

Abb. 1

including counted books and music scores, were processed in the Jewish Central Museum.

After World War II the JMP was renewed. One of the necessities of the renewed museum was to cope with the accumulated objects which had flowed into the museum during the War, and in case of books mainly after the war as will be documented further.

The biggest transfer of books took place in the museum in the years 1945-1950. Firstly books from the Zentralbücherei of Ghetto Theresienstadt, approximately 100,000 books, and parts of the collection of Hebrew books - the so called Hebrew Library - were transferred to the museum in 1945. The Zentralbücherei Theresienstadt was founded in 1942 and contained approximately 250,000 books. ${ }^{3}$ The collection included books confiscated from deportees, but the largest number of books came from abolished German-Jewish organizations, Masonic lodges, or rabbinic seminaries in Berlin and Budapest, and other institutions. The Hebrew Library was non-public and stayed apart from the Zentralbücherei Theresienstadt. This collection of Hebrew prints had been stolen by the Nazis from various mostly Jewish institutions and organizations in occupied parts of Europe was sorted by Talmudkommando in the Ghetto Theresienstadt. The aim was to create a representative library of Hebraica and Judaica. The libraries of the Jewish community in Berlin, Vienna and Budapest or the Jewish theological seminary in Wroclaw, the Rabbinic seminary in Berlin and others are

3 About The Central Library of the Terezín Ghetto see: Michal Bušek: "Hope is on the next page". 100 Years of the Library of the Jewish Museum in Prague. Prague: Jewish Museum in Prague 2007, pp. 36-45. 
considered as the most significant parts of this collection. The Talmudkommando under the lead of Otto Muneles (1894-1967), later the first post-war librarian of the JMP, together with 20 rabbis, 15 theologians, history researchers and librarians had catalogued 28,245 books of roughly 60,000 volumes. We know about the work of the Talmudkommando from postwar report of Otto Muneles ${ }^{4}$ who mentioned that the call-number series began with Jc 3001 with the highest number Jc 19.225. The group processed 28,245 books under 16,225 call numbers. The differences in the numbers proving the presence of duplicates, which were assigned the same call numbers. The books were given the label Jc (apparently, a shortening for Judaica) with the number, written in the books and yellow labels pasted on the spines of the books. For each book a catalogue card was prepared with a brief bibliographical entry. This card catalogue from Ghetto Theresienstad is now kept in our museum and is available on-line on the library website. The duplicities of the books were to be given to the Jewish National and University Library in Jerusalem or sold. ${ }^{5}$

\section{Card catalogue - Hebrew Library (Terezín)}
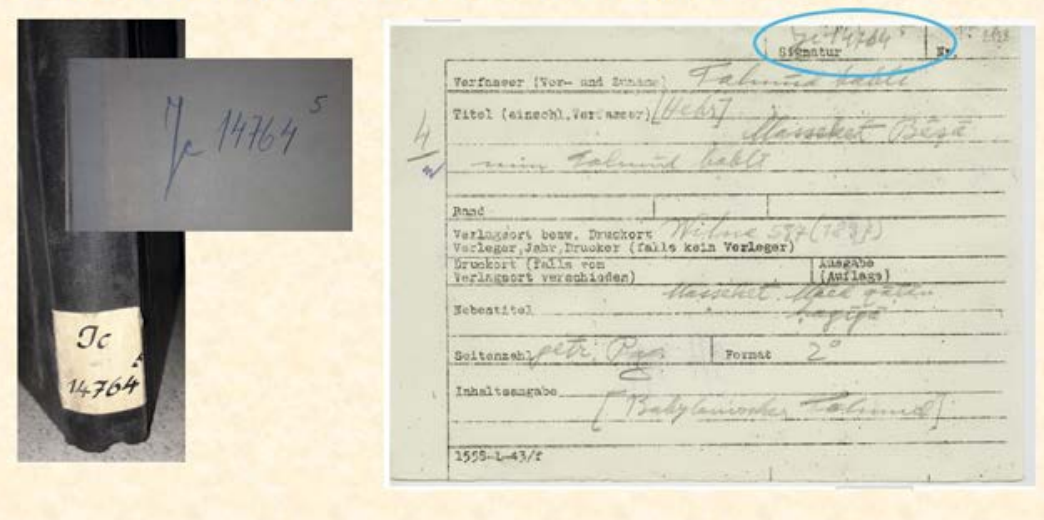

Abb. 2

Otto Muneles chose alone which books should become part of the museum's collection after the war. The main goal was to rescue and keep selected books and make them accessible for the public and researchers and try to renew and continue the pre-war scholarly tradition. We know from memories of the mu-

4 Archive of Shoa History Department, Jewish Museum in Prague, Archive collection Terezín 321d, Bericht über die "Bucherfassungs"- Arbeiten, gez. Dr. Muneles.

5 At the end not all of the books were transferred to Jerusalem. Some others were sold. But the archive material does not give detail information. See also: Andrea Jelínková: Books in the Terezín ghetto and their post-war fate. In: Judaica Bohemiae XLVII-1 (2012), pp. 85-107. 
seum's staff ${ }^{6}$ that Otto Muneles was very often searching and looking for Hebrew books in all Prague to get them for the museum. ${ }^{7}$

In 1946 the library of the pre-war Prague Jewish community was brought back to Prague, to the Jewish Museum. This library had been confiscated by the Nazis in 1939 and taken away from Prague then. ${ }^{8}$ Part of this library is missing as are the rarest prints. The books from the pre-war Prague Jewish community can be identified by the stamps of the library as well as by the call numbers in the books. The catalogue of this library survived the war and it is also kept in the JMP.

The next group of books which arrived in JMP in the year 1947 consisted of selected Hebraica and Judaica books from various castles in North Bohemia. The Nazis had originally collected stolen libraries and archives from occupied Europe in Berlin but they decided to move them out of the city in late 1944 when bomb attacks intensified. Four Castles were chosen in North Bohemia - Mimonn, Houska, Nový Falkenburk and Nový Berštejn. ${ }^{9}$

The reports about book transfers to the museum give us unfortunately only a little knowledge about the number of books or crates which came to the museum. Here we do not have any lists or any other specifications about the books and their original provenance.

Nevertheless not all the books which came to the museum stayed there. Between 1945 and 1950 some of the library holdings (mostly prayer books) were loaned temporarily to approximately 52 reestablished Jewish communities in the Czech Republic. Parts of the collection were donated to The American Jewish Joint Distribution Committee (34,900 books), United Nations Relief and Rehabilitation Administration (65,115 books), and the National and University Library in Jerusalem (40,000 books), while other books were subject to restitution claims. Again it must be stated that we do not have any lists of restituted books, we only know the numbers of books returned in the restitution claims. The selection of the books for the restitution was made often at random without paying attention to the provenance. The priority was to satisfy the restitution claim. ${ }^{10}$

In 1950, the JMP and its collections came under the administration of the

6 Interview with Jiřina Hlaváčová, carried out May 31, 2006, tapes held by author.

7 For more about Otto Muneles see: Michal Bušek: "Hope is on the next page". 100 Years of the Library of the Jewish Museum in Prague. Prague: Jewish Museum in Prague 2007, pp. 60-70.

8 About the history of Prague's Community Library see: Michal Bušek: "Hope is on the next page". 100 Years of the Library of the Jewish Museum in Prague. Prague: Jewish Museum in Prague 2007, pp. 18-23.

9 For further details about this topic I recommend the precise work by Patricia Kennedy Grimsted.

10 More about the Jewish Museum in Prague in years 1945-1950 see: Hana Volavková. Zpráva o činnosti Státního židovského musea za léta 1945-1950. Sestavená na základě měsíčních výročních zpráv. Praha: Státní židovské muzeum 1950. 
state. 90,000 books were donated to the National Recovery Foundation (an institution operating between 1945-1951, which provided temporary management and distribution of confiscated enemy property).

More than 190,000 books were transferred to the JMP in the years 1945-1950. But 158,132 of them were restituted, sold or handed over to the renewed Jewish communities or other Jewish organizations in the same period. ${ }^{11}$

During the communist regime only duplicates were sold or given away. However, those books were not the holdings of the JMP but only held in trust. ${ }^{12}$

It is obvious that for clarifying which books from the museum's library belonged to the museum and which have other original owners, the museum had to look at every book. Only in 2001 the research could start.

Pairs of library employees have worked in shifts, checking the collections even while the library was fully operational. The work has been carried out in the storage facilities, where there are optimal conditions for books but not so much for humans. The project was organized to allow one staff member checking a book itself while the other was typing the information in a special database. Because of the time-consuming character of the project, only the most essential information have been put into the records -inventory number, call number and the owner's original record. This information included the abbreviation of an institution's or individual's name, type of record like bookplates, card, stamp, written entry, signature, binding, etc. and the text of stamps or cards containing the full name of the institution or, in case of individuals, address or other relevant details. Some of the records of individuals contain also dates and years of the record or places of living, which can help us to identify the original owners better. Quite frequently very similar names have been found and without any other details it is not possible to identify the rightful owner. In the future a complete database entry might also contain bibliographical information of the book. Of course it is not always possible to fill in all the details completely, especially if the title page is missing. ${ }^{13}$

During the research owner records in different languages have been found - in addition to Czech and German, there was also Hebrew, Yiddish, Latin, Italian,

11 About the post war book-transfer in The Jewish Museum in Prague see: Andrea Braunová: Origin of the book collection of the library of the Jewish Museum in Prague. In: Judaica Bohemiae XXXVI (2000), pp. 160-172.

12 More about the books collection's history see: Michal Bušek: Identifying owners of books held by the Jewish Museum in Prague. In: Vitalizing memory. International perspectives on provenance research. Washington, DC: American Association of Museums 2005 [the essays in this publication originally were presented at the International Provenance Research Colloquium, Nov. 15-16, 2004, Washington, D.C.; the colloquium was organized by the American Association of Museums], pp. 138-142.

13 During the research more than 95.000 books have been reviewed of which more than 49.000 books contain owner's record. In 823 books the former owner could not be identified. 


\section{Web presentation - Restituted book}

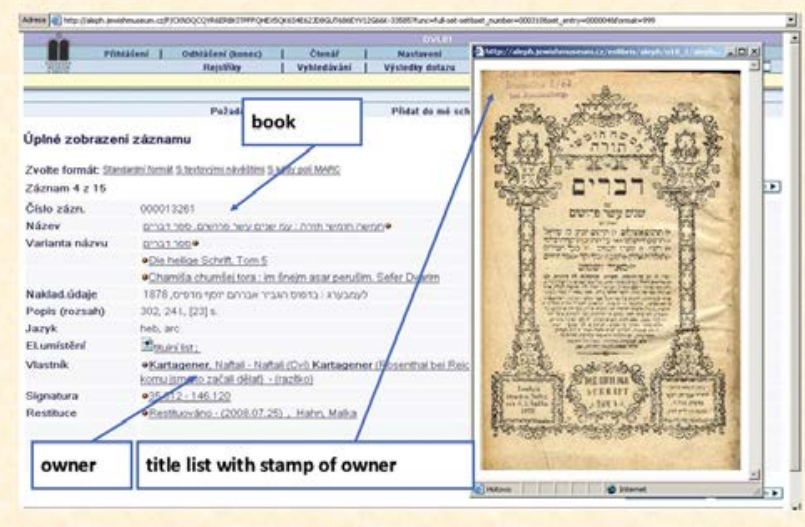

Abb. 3

Russian, Hungarian and English. The biggest problem was reading and decoding owner records, especially handwritten names and signatures. The owner's name that was unreadable stayed unidentified but remarks were put into the database with every detail which we were able to discover. Overall we have been unable to identify 823 owner records. It was impossible to determine some of the Hebrew and Yiddish names too. Since the transcription of the names in the database is done by library staff it is possible that the real form of the names was different. And of course today the spelling of a name can be different too. Another problem was the appearance of more than one owner recorded in a single book. As it is not possible to determine the chronology of each notation, all the information found was entered in the database. In case the book belonged to an institution or an organization as well as to an individual owner the JMP's approach was not mentioning the names of individuals in our database. Here only an organization name was entered into the record. ${ }^{14}$

The first phase of the provenance research was carried out between May 2001 and October 2003. During this phase 80,527 books were examined, of which nearly 34,000 contain owner records - of individuals or institutions. The project continued with the second phase, which was launched in May 2008 at the depository, where books that were shipped to the museum from the Ghetto Theresienstadt after WW II were kept. These are some of the books

14 More about the project see: Michal Bušek: Restitution in the Jewish Museum in Prague in the case of Naftali Zvi Kartagener. In: Holocaust era assets. Ed. by Jiři Schneider, Jakub Klepal, Irena Kalhousova [Conference Proceedings, Prague June 26-30, 2009]. Prague: Forum 2000 Foundation 2012, pp. 1104-1110. 
catalogued by the Talmudkommando. Research was undertaken in a similar way as in the first phase, but this time once a week rather than every day. During the second phase, which we finished at the end of 2015, we checked 18,731 books, of which 14,998 contain owner record, mostly organizations and institutions, rarely individuals.

A database of owners was originally created by using Microsoft Access. After long considerations, however, it was decided that this database was unsuitable for the requirements of future website presentations. The possibility of using the Aleph electronic library system, which is currently in use at the JMP, was then offered. This system is used by many libraries and it is easy to search and share information about books using its online database. We intend to make use of this service in the future and when entering information about books a record regarding the ownership will be included. The entries can be supplemented by illustrations, which we plan to add to owners' records (scans of stamps, signatures and bookplates, etc.) but also to the actual books (title pages). The records of the books from Ghetto Theresienstadt - the so called Hebrew Library, will contain the picture of a card from the original card catalogue. The database is fully searchable by owner's name and place and it is possible to display a list of books containing a specified owner's mark. This database will be accessed online on museum's website as part of our library catalogue.

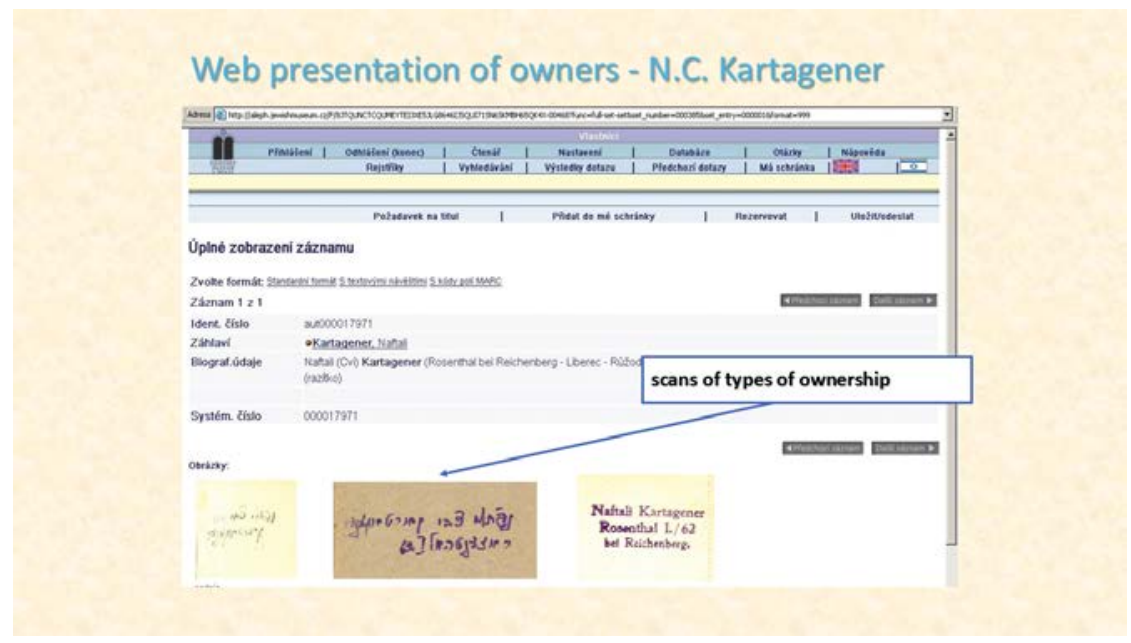

Abb. 4

Some of the books contain ownership records which have been damaged, cut out or blackened. These owners have been entered into our database, too, as unidentified, but in few cases we have been able to identify the original owner in some way, mostly on the basis of similarity to legible stamps. 
From the beginning of the provenance research there is one case of some of the identified books having been returned to the original owners or their heirs on the basis of the museum's database. The individuals (not the institution) are those who can put forward a restitution claim, provided if they meet the Terms for the filing of claims for the restitution of books from the library collection of the Jewish Museum in Prague which were unlawfully seized from natural persons during the period of Nazi occupation, which came into effect in July 2007. The full wording of the terms is available on the museum's website. ${ }^{15}$

It was only after the adoption of the terms that the JMP could register a claim from the descendants of Mr. Kartagener for the return of books originally owned by Mr. Kartagener. The heirs first contacted the museum in the 1990s, when Mr. Kartagener's daughter asked the library staff whether there were books belonging to her father in the museum's book collection.

The JMP has responded to questions concerning the presence of the books belonging to specific persons in our collections on the basis of information from the database. If such books are found, a report is drawn up for the Restitution Commission, which, subsequent to the request being approved, recommends if the restitution claim can be registered and presents it further to the administrative and supervisory boards for approval. The claim is positively evaluated if the terms are met. In the Kartagener case, the restitution claim was for five books and was registered on June 7,2007. After a period of one year, the terms were met and Mr. Kartagener's books were handed over to his heirs in September 2008. The list of restituted books is available on the museum's website. ${ }^{16}$

In connection with the aforementioned case, I would like to touch upon the most basic and most frequent problems that occur when restitution claims are dealt with. It is always beneficial if there is more detailed information about the person in question available like the places of residence or the signature of the owner. A clear restitution claim is not even proved by just coinciding name, if no further accurate information is known. In order to prevent a breach of the property rights of another owner, JMP cannot restitute such a book if it is not certain that the individual in question can be demonstrably identified. This is why the JMP prefers to keep such a book in its collection with an awareness that it cannot be proved who the original owner is. As expressed in the title of the the conference proceedings JMP accepts and holds these books "in trust". The same procedure applies to books whose last owner cannot be reliably determined. In many books, the names of two or more people are included in an

15 URL: http://www.jewishmuseum.cz/en/collection-research/provenance-research-restitution/ museum-s-restitution-policy/ (accessed on June 14, 2017).

16 URL: http://www.jewishmuseum.cz/en/collection-research/provenance-research-restitution/ what-has-been-restituted/ (accessed on June 14, 2017). 
owner record. In such instances, the museum is not able to determine the name of the very last owner from whom the book was confiscated. Such an instance occurred in Kartagener's case when there was identified a total of 13 books where N.C. Kartagener appeared as an owner. It was possible to clearly identify Mr. Kartagener's ownership of only five of these books. The name of another owner appeared in other books, which is why they remained in the museum's collection and have not been restituted.

It brings us to a very important question: What to do with the books of which original owner cannot be determined? What to do with the books that we know the original owner of but there is no one to whom we could return the books? The JMP as a Jewish cultural and educational institution prefers to keep these books in the museum's collection and to continue to provide the books for the use and study in our library with consciousness that these books belonged to other owners before WW II. 


\title{
Provenance Research in the National Library of the Czech Republic
}

\section{Zusammenfassung}

Provenienzforschung an der Tschechischen Nationalbibliothek

Während des Zweiten Weltkrieges wurden viele Bücher ins Sudentenland gebracht. Nach dem Krieg wurde die Tschechische Nationalbibliothek mit der Aufsicht dieser Bücher betraut. Leider wurde dabei wenig Augenmerk auf die Bibliotheksbestände gelegt, die auf diese ungewöhnliche Weise in die Bibliothek kamen. Abgesehen von den jüdischen Beständen interessierte sich niemand für die Struktur, die Vorbesitzer oder ihre historische Bedeutung. Erst mit dem Start des Projekts „Books Discovered Once Again“ (Bücher wiederentdeckt) im Januar 2015 begann die Auseinandersetzung mit diesem Bestand. Viele dieser Sammlungen waren nicht einmal katalogisiert. Seit 2015 wurden von Forschern und Bibliothekaren an der Tschechischen Nationalbibliothek nahezu 15.900 Bücher durchgesehen und über 2.000 Vorbesitzer identifiziert. Wer diese Vorbesitzer waren, was ihr Hintergrund war und wie sie ihre Buchsammlungen verloren haben, wird in diesem Beitrag behandelt. In einem zweiten Teil wird auf die Schwierigkeiten hingewiesen, die eine intensivere internationale Zusammenarbeit mit sich bringt, wie etwa ein gemeinsames Katalogisierungssystem von Provenienzmerkmalen oder die Etablierung eines internationalen Zentrums für Provenienzforschung, das die Verbindung der Institute und Universitäten herstellen könnte, die sich mit diesem Thema beschäftigen.

Schlagwörter

Nationalbibliothek Prag, Projekt „Books Discovered Once Again“, Provenienzforschung

\begin{abstract}
During World War II many books were transferred to the Sudetenland. After the war, the National Library in Prague was given the task of looking after them. Unfortunately little interest was devoted to the library documents which were acquired by the library in this non-standard way. With the exception of Judaic documents, almost no one cared about their structure, original ownership or their importance for history since the project "Books Discovered Once Again" was launched in January 2015. Until that time, most of these collections had never been properly catalogued. Since 2015 the library researchers and librarians have processed almost 15,900 books and identified more than 2,000 former owners. Who were they? What was their background? Why did they lose their book collections? - These are all questions that will be one part of the paper. Another part
\end{abstract}


concerns the challenges facing more intensive international cooperation such as the special system of shared cataloguing of the book collections with noticeable provenance signs or the establishment of the International Centre for Provenance Research, which could become the connecting link among the cultural heritage institutions and universities dealing with this topic.

Keywords

National Library Prague, project “Books Discovered Once Again”, Provenance Research

The National Library of the Czech Republic is the largest and oldest library in the Czech Republic. Its modern history dates back to 1777, when the original university library was declared the Imperial and Royal Public and University Library by decree of the Empress Maria Theresa. Since the establishment of the independent Czechoslovak Republic in 1918, it changed its structure and its name several times until attaining its present form. ${ }^{1}$

The National Library's book collections are general and include foreign literature. The number of volumes stored there exceeds seven million. Besides the regular collections, it also has a collection called the Reserve Collection, which is the main topic of this article. There is still considerable uncertainty about its composition, structure and scale. According to the estimates of librarians, the Reserve Collection comprises about 600,000 volumes of domestic and foreign production, including periodicals. Despite the value of its content, the library's former attitude to this collection can be described as restrained, to say the least, as evidenced by the following anonymous words: “...sets of minor material of Masonic origin that does not even belong to a university library ${ }^{2}$ (minor material). The cost of processing it would far exceed its value and it would burden the department. Actually, the value of these items is rather problematic for the library." ${ }^{3}$ Let us look briefly at the history of the Reserve Collection and the reasons for the negative attitude towards it, which fortunately began to change rapidly after the revolution in 1989 .

The Reserve Collection of the National Library of the Czech Republic gradually came into existence between 1918 and 1938, consisting mostly of duplicates and multiple copies. Shortly after the end of World War II, confiscated material was added that the library was to manage. The common denominator of all material contained in the Reserve Collection today is that it is missing any

1 In the post-war years, we are talking about the National and University Library of the Czechoslovak Republic. As the name suggest, internally the library was divided into the national and university sections.

2 The library refered to is the National and University Library.

3 Hand written note left in one of the books of Masonic provenance, found during cataloguing in 2015. 
accompanying documentation, and that no lists of these books were provided when they came to the library.

The second fundamental problem of the Reserve Collection is that the items it contains have been stored in the library depositories. The historic building of the Clementinum, which remains the seat of the National Library to this day, was reconstructed in the 1920s and 1930s to provide storage capacity for the expansion of the library holdings until the year 2000. The information explosion that was to come, and the subsequent expansion of library collections, which resulted in a desperate shortage of storage capacity, could not have been predicted at the time of the reconstruction. ${ }^{4}$ Moreover, future historic developments could not have been predicted, either, and neither could the fact that the library would be, as a result, augmented after World War II by confiscated books. Since the 1950s the library has used several temporary repositories. As they have never been places designed for this purpose, we can only doubt the suitability of the conditions there for storing books. These included, for example, the repository at Houska Castle, Postoloprty, the repository in Nučice, and lastly the still-used depository in Neratovice. In addition to the environmental conditions of these buildings, their inaccessibility from Prague was also problematic in the past. Items from the Reserve Collection were stored in all these repositories, including the Clementinum. The entire collection was moved to Neratovice only after the construction of the Central Depository in Hostivař $\check{r}^{5}$ in the 1990s. To give an idea of this, it included, for example, 6,272 metres of books from Postoloprty, and 3,319 metres from the Clementinum. ${ }^{6}$ It must be said that the issue of storage space in the National Library has not been resolved satisfactorily to this day.

Attempts to process the Reserve Collection have been made several times in the past. For example, in the 1960s and 1970s, the philologist Bohuslav Havránek (1893-1978) started working on it together with volunteers. However, subsequent numerous relocations disrupted the structure of the Reserve Collection, and although some of the catalogue cards survived, it is difficult to tell the period from which they come from the catalogue numbers.

The complex processing of the non-Bohemican part of the Reserve Collection according to all valid library standards started only with the project "Books Discovered Once Again". The project was 80 \% funded from EEA (European

4 National Library of the Czech Republic: annual report. Prague: National Library of the Czech Republic 1992, p. 27.

5 The central depository in Hostivař was put into operation in 1996; an extensive reconstruction and expansion took place in 2014, after the definitive failure of plans for the construction of a new purpose-built building of the National Library.

6 Jana Kohoutová: Rezervní fondy se stěhují do Neratovic [Reserve fonds is moving to Neratovice]. In: Information bulletin of the National Library of the Czech Republic, year 7, issue 17 (1996), pp. 17-18. 
Economic Area) and Norwegian funds, and implemented in cooperation with a Norwegian partner, Stiftelsen Arkivet. ${ }^{7}$ The initial duration of the project was from January 2015 to April 2016. During this period the basic goals of the project were accomplished - the processing of 12,000 volumes from the Reserve Collection, the legal analysis of the documents deposited in the Reserve Collection, historical research into the confiscation of books in post-war Czechoslovakia and the relation of this issue to the Reserve Collection, a virtual and physical exhibition - and finally the organization of two final seminars. The project was extended until the end of April 2017, with the aim of deepening the information base by means of the project website ${ }^{8}$ and other historical research. In the course of 2016, BFB initiatives ${ }^{9}$ and follow-ups began, within which a team of authors compiled the profiles of selected original owners ${ }^{10}$ and launched a promotional video $^{11}$ and an educational programme ${ }^{12}$, which was presented during the Norwegian-Czech lecture tour in early 2017. As is clear from the above, the aim of the project was not to conduct detailed provenance research, but to process and catalogue these books and to make them accessible to the public. In terms of methodological rules of provenance research, we can talk about a systematic survey of the collection, while more detailed research into original ownership has so far been only an added value of the project. Although the internal database of original owners of books stored in the Reserve Collection is constantly being updated, its more detailed processing and publishing is dependent on the financial support of the projects.

From the approximately 16,000 volumes processed so far, it is clear that the structure of the Reserve Collection faithfully mirrors the link between the National Library, the development of Czechoslovak librarianship and post-war history. The library records two thousand original owners of these books, who used over 2,500 marks of provenance (stamps and exlibris). In brief, we can say that these were:

a) books that came to the library in a standard manner (donations, bequests, purchase of estates, books discarded and withdrawn from other libraries),

7 See URL: http://www.stiftelsen-arkivet.no/ (accessed on November 30, 2017).

8 See URL: www.knihyznovunalezene.eu (accessed on July 21, 2017).

9 EEA Funds for the bilateral cooperation.

10 Osudy psané knihami: původní vlastníci knih uložených v rezervních fondech NK ČR [Fortunes written by books: Original owners of books stored in the Reserve Collection of the National Library of the Czech Republic]. Ed. by Marcela Strouhalová. Prague: National Library of the Czech Republic 2016.

11 See URL: https://www.youtube.com/watch?v=I3gYPX4ZQSc (accessed on July 21, 2017).

12 See URL: http://www.gjenoppdagedeboker.no/cs/domovska-stranka/ (accessed on July 21, 2017). 
b) books that came to the library in the post-war period after being confiscated according to the Decrees of the President of the Republic, ${ }^{13}$

c) books that came to the library after the Communists took power in 1948 .

The most discussed part of the Reserve Collection remains the books of foreign provenance. I believe that the history of the library of the Security Service (Sicherheitsdienst), which subsequently became the library of the Reichssicherheitshauptamt (Reich Main Security Office) has already been satisfactorily analysed ${ }^{14}$ in the literature, therefore we include only basic facts here: in August 1943, after heavy raids on Berlin, an order was issued to evacuate the materials held by the Amt VII to safer places. Under the codes Brabant II, Burgund I-IV and Sigfried, parts of libraries were transferred to several castles in the Sudetenland and Silesia-Wölfelsdorf (Wilkanów), Niemes (Mimoň), Hauska (Houska), Neu Peerstein (Nový Berštejn), Neufalkenburg (Nový Falkenburk), and Theresienstadt (Terezín). ${ }^{15}$ During May and June, various persons and committees in Czechoslovakia were appointed to secure monuments in the Sudetenland area. Gradually, reports began to come to the Provincial Office in Prague, the Ministry of Education and the Ministry of Foreign Affairs that a large number of foreign books had been found in the four Sudeten castles (Houska, Nový Berštejn, Nový Falkenburk and Mimoň). Not much was yet known about their origin. They were said to have belonged to "something like Amt VII". ${ }^{16}$ It was only during the summer of 1945 that the National Library was assigned the task of securing all book material. ${ }^{17}$ There is no document dated immediately after the liberation of Czechoslovakia that would give us the exact number of books stored in the

13 Form of post-war legislation.

14 Compare: Dov Schidorsky: The Library of the Reich Security Main Office and Its Looted Jewish Book Collections. In: Libraries and the Cultural Record, Vol. 42, No. 1, 2007 and Patricia Kennedy Grimsted: Sudeten Crossroads for Europe's Displaced Books: The Mysterious Twilight of the RSHA Amt VII Library and the Fate of a Million Victims of War. In: Restitutions of confiscated art works. Wish or reality? Documentation, identification and restitution of cultural property to the victims of World War II: Contributions from the international scientific conference in Liberec (24-26 October 2007). Prague: Documentation Centre for Property Transfers of Cultural Assets of WW11 Victims at the Institute of Contemporary History, Academy of Sciences of the Czech Republic. Ed. by Mečislav Borák. Šenov u Ostravy: Tilia 2008, pp. 120-177.

15 Lecture by Helge Bjørn Horrisland at the 3rd workshop of "Books Discovered Once Again" Kristiansand, Norway in November 2015, comp. Patricia Kennedy-Grimsted, quoted document, pp. 142-145. P. Grimsted lists the Schlesiersee castle among evacuation places for Masonic libraries.

16 Marcela Strouhalová: Knihy znovu nalezené: konfiskované knihy po druhé světové válce ve správě NK ČR [Books Discovered Once Again: Books confiscated after WWII held by the National Library of the Czech Republic]. Prague: National Library of the Czech Republic 2016, p. 37.

17 Ibid. p. 33. 
castles. Moreover, the count is complicated by the fact that, after the liberation, the Nový Falkenburk castle was occupied by the Red Army, which, according to the statement of the librarian Miroslav Patava, took away some of the Russian books, so that only part of the library from Kiev was found at the castle. Patava's description of the books' storage will not help us much with the numbers:

"In Mimoň, $3000 \mathrm{~m}$ of shelves, 250 crates, $240 \mathrm{~m}^{3}$ of freely stacked books, approximately two freight cars; at Nový Falkenburk, 310 shelves units $1.30 \mathrm{~m}$ wide, $911 \mathrm{~m}$ of shelves, 80 crates, $28 \mathrm{~m}^{3}$ freely stacked; in Houska, 530 metres of shelves, 500 crates and $100 \mathrm{~m}^{3}$ of freely stacked books. ${ }^{18}$

The news from Nový Berštejn castle followed on July 18, 1945, with the statement that it was not possible to determine the real situation, saying that the attic was the worst: "all the boxes of books and documents were broken and the contents were scattered across the ground." ${ }^{\prime 19}$ Although the Ministry of Foreign Affairs was informed that there were a large number of foreign books, it was not possible to determine the precise number, because all of the storage places were "found in the greatest disarray from SS sorting, so parts of one library can be found in several, quite distant castles." 20

The sorting-out of books in these castles began mostly in the autumn of 1945 . Poor accessibility caused by the coming of winter delayed the work at Houska Castle to the following spring. The sorting was mainly about dividing foreign property from property of demonstrably Czech origin (e.g., books with bookplates of the Emmaus monastery). Unmarked material was categorized by language. In his inspection report, the National Library's director, Josef Beckka (1894-1955), remarks that:

"The working conditions of officials on the ground were very poor, and required not only expertise, but also considerable physical exertion and great discomfort; especially on the first three Sundays, they suffered from exposure to the cold [...] only later, improvements were made when more suitable accommodation was obtained, and at least some fuel, and the German auxiliary forces were assigned the hardest work (carrying books, unpacking boxes, etc.)". ${ }^{21}$

18 Národní archiv [National Archive], Zemský úřad Praha [Regional Office - Department for Foundations, the Church and Education], box no. 802, Report of July 16, 1945 on examination and securement of libraries evacuated from Germany and from Prague to the Česká Lípa region.

19 Národní archiv, Zemský úrad Praha, box no. 802, Report of 18/7 on securement works in the yard of Medna near Mimoň.

20 Ibid. letter of August 2, 1945, file.no. 841, from the Secretariat for registering and rescuing historical documents and artistic and natural monuments, addressed to the Ministry of Foreign Affairs.

21 Národní archiv, Ministerstvo školství a kultury [Ministry of Eduacation and Culture], box. no 597, sign. 4Fb1, Report on visitaion of securement storages dated November 8, 1945. 
At Mimoň Castle, a member of staff fell ill with severe pneumonia, for which she had to be hospitalized. ${ }^{22}$

The reports of Josef Bečka about the completion of the work at the castles give us at last the number of books stored there: Mimoň: 256,626 volumes; Nový Falkenburk: 93,624 volumes; Nový Berštejn: 59,927 volumes; and Houska: 98,356 volumes. Altogether, there were more than half a million books. ${ }^{23}$ In most cases, fewer than half of the books were without marks of ownership. The books were ready to be transported to Prague. ${ }^{24}$

Books of foreign provenance - at least the books that came from Allied states were prepared for restitution proceedings. We have information about books being returned to France, Belgium, the Netherlands, Italy, Yugoslavia, Poland and Switzerland ${ }^{25}$. The books that came from the German Reich were taken to Prague. These included libraries of at least a hundred Masonic lodges from all over Germany, and they have been processed since 2015 as part of the "Books Discovered Once Again" project. ${ }^{26}$ While cataloguing these books it was found that there were also several items in this sub-collection which do not meet the criteria defining property of the German Reich. Probably the best-documented example is the Norwegian Masonic lodge Den Norske Store Landsloge. The 19 pressmarks currently found in the National Library's Reserve Fonds are, in the authors' view, examples of human error, especially considering the time pressure and inhospitable working conditions described above. The transcript of the stamp of this library: "D. N. St. Landsloges Bibliothek" must inevitably lead to confusion with the property of the German Reich.

22 Marcela Strouhalová: Knihy (Fn. 16), p. 40.

23 Národní archiv, Zemský úřad Praha, box no. 801, letter by the National and University Library of May 4, 1946, ref.no. 92/46, on matters concerning castle libraries, and Archiv Národní Knihovny [National Library Archives], Národní a universitní knihovna [National and University Library], box no. B3/6, copy of National and University Library, ref.no. 1695/ 46, addressed to the Ministry of Education, on the completion of sorting work at Houska castle, dated September 23, 1946.

24 We do not know whether the books without a mark of provenance were included in the "life" collection or the Reserve Collection, and unfortunately we will most likely never find out.

25 Národní archiv, Ministerstvo kultury [Ministry of Culture], box no. 11, inv.no. 101, Appendix to MZV 263.336/55-VS/3 of August 30, 1951.

26 Unfortunately, archival sources remain silent about this sub-collection. It is only stated that it was found at these castles. No relevant documents give significant information on whether it was secured as German property for reparations. It is not clear, therefore, whether the Decree of the Ministry of Interior no. 1710-30/4-46-89-Vb/3 of July 12, 1946, on identifying the property to be confiscated for reparations, was observed. Under this decree, the property of the following was to be included in the Czechoslovak Republic's share of war reparations: the German Reich public administration and public enterprises of the German Reich; private companies with their seat in Germany; legal entities that after September 29, 1938 had their legal seat in Germany; and natural persons of German nationality with Reich citizenship as of September 29, 1938. 
The second group of confiscated items in the Reserve Collection is of domestic origin; it is primarily the property of persons designated in the post-war period as enemies of the republic.

Already during the war, as the exiled Czechoslovak politicians dealt with the question of how new political and economic conditions should be organized, an important question arose in connection with the punishment of war criminals. All these negotiations were going on practically continuously from 1940. One of the results of the post-war talks was the programme statement by the government, known as the Košice government programme. One article in the programme concerned the national administration of property owned or managed by citizens of hostile states - especially German and Hungarian citizens in the Czechoslovak Republic who actively helped the invasion and occupation of Czechoslovakia, and those who betrayed the nation by actively supporting the occupiers. At the same time, a request was made in the Košice government programme for the return of property that was taken from its original owners during the occupation due to national, political and racial persecution. Lastly, a demand was issued for the confiscation of property in the sense of implementing new land reform. ${ }^{27}$ This government programme was fulfilled, but gradually views on the punishment of enemies and economic recovery were radicalized, eventually resulting in more extensive property interventions.

In May 1945, the first Decree of the President of the Republic on the property rights of citizens was passed. Decree No. 5/1945 Coll., On the Invalidity of Some Transactions Involving Property Rights from the Time of the Loss of Freedom, and Concerning the National Administration of the Property of Germans, Hungarians, Traitors, Collaborators and Certain Organizations, meant a significant restriction on the exercise of property rights. The property was put under national administration; its original owner remained only a formal owner, with no possibility to use the property or otherwise dispose of it. ${ }^{28}$ The national administration seized the property of persons with an unreliable attitude to the state considered to be persons of German or Hungarian nationality, persons conducting activities against state sovereignty, autonomy, integrity, the democraticrepublican form of the state and the security and defence of the Czechoslovak Republic, as well as persons who instigated and deliberately supported the German and Hungarian occupiers in any way. Corporate bodies whose man-

27 Dokumenty k ústavnímu vývoji Československa II/A (1945-1948) [Documents on the Constitutional Development of Czechoslovakia]. Ed. by Ján Gronský. Prague: Karolinum 2002, document 2, Program nové československé vlády Národní fronty Čechů a Slováků (Košický vládní program [The Košice Government Programme]), pp. 19-20.

28 Jan Kuklík: Znárodněné Československo [Nationalised Czechoslovakia]: od znárodnění k privatizaci - státní zásahy do vlastnických a dalších majetkových práv v Československu a jinde v Evropě, Prague: Auditorium 2010, p. 144. 
agement had deliberately and intentionally served the German or Hungarian war leadership or fascist and Nazi purposes were considered unreliable. Nationality was the decisive point here, as each person who professed one of these nationalities was considered a German or Hungarian. Thus, anyone who was listed as being of German or Hungarian nationality in the census from 1929, or who had become a member of a political party or group or unit of those nationalities, fell under the authority of this decree. Subsequently, in June 1945, came out Decree No. 12/1945 Coll., On the Confiscation and Cxpedited Division of the Agricultural Property of Germans, Hungarians, and Traitors and Enemies of the Czech and Slovak Nations. It pertained to practically the same parts of the population as Decree No. 5/1945 Coll. Although the decree "confiscates agricultural property for the purposes of land reform", we must bear in mind that this agricultural property included all the livestock and equipment belonging to this land. Under this decree, 496 castles and mansions were seized, including their movables, among which there were also castle libraries. ${ }^{29}$

Ultimately, however, the greatest interference with property rights came with Decree No. 108/1945 of October 25, 1945, On the Confiscation of Enemy Property and on the National Recovery Funds. Under this decree, all immovable and moveable property, including property rights, were to be confiscated without compensation for the Czechoslovak Republic. It hit the largest section of the population, as it included property of the German Reich, the Kingdom of Hungary, the National Socialist German Workers' Party, Hungarian political parties and all associated organizations; natural persons of German or Hungarian nationality, with the exception of those who could prove that they remained faithful to the Czechoslovak Republic and had not acted against the Czech and Slovak nations or that they had actively participated in the struggle for liberation or suffered under Nazi and fascist terror; physical persons who carried out an activity aimed against the State's sovereignty, autonomy, integrity, democratic-republican political system or the security and defence of the Czechoslovak Republic; persons who incited such activities or tried to seduce other persons to them; persons who intentionally supported German or Hungarian occupiers in any way; persons who supported Germanization or Hungarianization in the territory of the Czechoslovak Republic, or who were hostile to the Czechoslovak Republic or to the Czech or Slovak nations, as well as persons who tolerated such activities in those managing their property or business. In March 1946 came a more detailed list of subjects falling under Decree No. 108/1945 Coll. ${ }^{30}$ In the case of the German Reich and the Hungarian Kingdom, it was

29 Kristina Uhlíková: Národní kulturní komise 1947-1951 [National Cultural Committee 1947-1951]. Prague: Artefactum 2004, p. 23.

30 Konfiskace, správa a převod nepřátelského majetku: zákony, vyhlášky, směrnice a pokyny 
property usually referred to as state-owned - companies, institutions, funds, state administration property and all its components, German Reich railways, military assets, and more. It is clear from this enumeration that in many cases these were institutions whose confiscated property would include a library (administrative library for work purposes). In addition, there were a large number of other organizations, businesses and departments which derived their existence from the political parties (in the case of Germany, this could be only the Nazi Party), and their subordinate units and components, such as NS-Deutscher Ärztebund, NS-Lehrerbund, Deutsches Frauenwerk, Reichsstand des deutschen Handwerks, Reichsarbeitsdienst and others. Even from this list, it is clear that these were organizations which, by their nature, had to have their own libraries. To put it simply, according to this decree all valuable German and Hungarian property was to be seized for Czechoslovakia - about one million flats, villas and farmhouses were confiscated with all their internal equipment, including libraries. The National Recovery Fund was established to carry out tasks related to the interim administration of confiscated property and its division.

On 11 May 1945 the National Committee in Prague and the Czech National Council established a Secretariat for registering and rescuing historical documents and artistic and natural monuments. The main task of the Secretariat was to secure the monuments in the borderland, which were naturally at greatest risk. Radio appeals were repeatedly made to citizens not to recklessly destroy the property of the Germans and to report any threat to it. Although the Secretariat did not have the necessary executive apparatus, during its short existence it managed to establish contacts with leading experts (museologists, archivists, conservators) as well as teachers from the border regions who were familiar with local circumstances. ${ }^{31}$ The Secretariat's activities did not last long; at its meeting on 13 June, 1945, after roughly one month of operation, it was stated that its activity was practically finished because it had neither the material resources nor the necessary personnel. ${ }^{32}$ The original powers of the Secretariat were divided, according to the nature of their subjects, among different institutions. The Secretariat continued to gather information about unsecured cultural property and hand it over to the designated institutions. The National Library was charged with securing libraries, as has already been said in the section devoted to books

[Confiscation, administration and transfer of enemy property], Prague, Legal Department of the Settlement Office and the National Recovery Fund 1947, Decree of the Ministry of Interior no. 1799-27/3-46-82-Vb/3 of 31 March 1946, Decree no. 108/45 Coll., On Confiscation of Enemy Property and National Recovery Funds - directive to $\$ \$ 1$ and 2, pp. 51-69.

31 Uhlíková: Národní kulturní komise (Fn. 29), pp. 20-21.

32 Národní archiv, Zemský úřad Praha, box no. 802, Minutes of meeting of the archival, museum, library and heritage sections of the Cultural Committee of the Regional National Committee in Prague on June 13, 1945. 
of foreign provenance. The books were to be stored in secure rooms, preferably in the National Committee's buildings. The chairman of the Local National Committee was responsible for the condition and completeness of the material secured, but the disposition of all assets was up to the National University Library. ${ }^{33}$ The Moravian University Library in Brno functioned similarly for the territory of Moravia and Silesia.

In practice, securement usually meant that Czech institutions (which were, however, sparse in this area) were approached with an offer to select the books that they wanted to keep. Sometimes books were sent automatically to these institutions, while German ones were sent to the Clementinum in Prague. ${ }^{34}$ The situation was further complicated by the fact that often even the local national committees, which had executive powers in the early post-war years, did not know where basic institutions like museums or libraries were located in the relevant area. By the end of 1945, books from 178 places outside Prague had been transferred to the National Library, but it soon turned out there would be no available space in the Clementinum for them. Local National Committees were thus asked to send lists of these books to the library if possible, but to keep the books in a safe place for the time being, or send them to the library by post or by train. In total, 1,540 cases were handled in this way - i. e. books were reported from 1,540 places in the Czech Republic. ${ }^{35}$

More than twelve million volumes went through the National Library during the period under review. This number includes the property confiscated from emigrants after 1948. The exact number of books selected for the National Library is not clear, as they were distributed among various collection. In addition, the library distributed books to other institutions. In practice, some of the books were processed first, and afterwards scientific institutions were asked to send their representatives to the library to choose books and take them away. The institutions then sent lists of the selected books back to the National Library. According to reports, the books were distributed to the faculty and departmental libraries of individual universities in Prague, Brno and Olomouc, and to study libraries, museums and other scientific institutions. ${ }^{36}$ There is no record of how many books from those twelve million sorted volumes remain in the National Library. Kamil Groh (1914-2010), the manager of confiscation department, stated that 1.8 million volumes were brought to the Clementinum. He estimated

33 Ibid. box no. 796, memorandum from the National and University Library of August 9, 1945 addressed to the cultural officer of the Regional National Committee in Prague, Vilém Kremer.

34 Comp. e.g. Ibid, box no. 802, a report drafted [by securement committee] on June 20, 1945 on the securement of the library and museum in Hostinné.

35 Archiv Národní knihovny, Výroční zprávy [Annual Reports], Annual Report 1945, p. 22.

36 Marcela Strouhalová: Knihy (Fn. 16), pp. 47-48. 
that $60 \%$ were Czech-language books, and $40 \%$ were foreign literature. However, of this property only the scientific literature was categorized, and the remainder was handed over to Orbis ${ }^{37}$, which won the National Recovery Fund's tender for the sale of books abroad.

Another, though not so large, group of Reserve Collection books were originally the property of the Church. Although we cannot talk about the core component of the Reserve Collection in this chapter, we can very well illustrate the attitude of the administration and cultural institutions to property of professional and cultural value after 1948; that is, after the Communists came to power.

On the night of April 13 to April 14, 1950, the first stage of operation "K" was carried out, the carefully planned dissolution of male monasteries. The plan itself was adopted by the Bureau of the Central Committee of the Communist Party of Czechoslovakia on January 20,1950, which initially envisaged a multi-stage operation in which, firstly, the monasteries in towns, and later, the monasteries in the countryside, were to be liquidated. Initially, a legislative ban on the activities of some "dangerous" orders was also considered, but there was great concern that such actions could lead to allegations of unlawfulness. Finally, it was decided that the monasteries would be taken over in two stages. ${ }^{38}$

The first stage hit members of the most numerous orders, namely the Salesians of Don Bosco, the Redemptorists, the Jesuits, the Franciscans, the Premonstratensians, the Congregation Fratrum Consolatorum de Gethsemani and the Teutonic knights. In the second stage, which took place fourteen days later on April 28 members of the remaining orders were taken to "concentration" monasteries. All superior generals, abbots and reactionary friars were taken to the Želiv Monastery, which was adapted as an internment camp with a strict regime. Overall, operation " $K$ " hit 219 monasteries and 2,376 monks, of whom 2,201 were "concentrated" and 175 were interned. The buildings were taken over

37 The nationalised publishing house managed by the Ministry of Information, also in charge of the trade with foreign literature.

38 Vojtěch Vlček, Likvidace mužských řádů v českých zemích $\mathrm{v} 50$. letech $\mathrm{v}$ středoevropském kontextu, aneb Ochráníme vás před hněvem pracujícího lidu [Dissolution of male religious orders in the Czech lands in the 1950s in the context of Central Europe], in: Securitas imperii, 2011, volume. 19/2, pp. 144-157, here p. 147. Before operation "K" started, it needed to be justified. Several senior representatives of religious orders were arrested and submitted to weeks-long interrogations to get them to admit to treason and espionage. Between March 31 and April 4 there was a political trial with ten members of religious orders, among them Josef Urválek and Karel Čížek. They were accused of espionage for the Vatican, the possession of weapons, preparing a political coup and disruption of collectivisation and the socialist education of young people. There were four death penalties and one life sentence, and the others were sentenced to 132 years of imprisonment. This was one of the first show-trials in Communist Czechoslovakia; compare ibid. p. 148. 
by the army and the Ministry of the Interior..$^{39}$ In the emptied monastic buildings, a large amount of the inventory remained, including works of art and numerous libraries.

To carry out the collection of books and art objects from monasteries, technical groups of two kinds were established - one for the collection of books and one for the collection of art objects. Groups of the first kind were run by the National Library. The person responsible for the gathering of books was František Horák (1911-1983). The second kind were established under the Museum of Applied Arts and the National Gallery. Valuable books were to be taken to the National Library, while others were to be taken over by Regional National Committees for regional study libraries. ${ }^{40}$ Only 19 libraries were to remain in place, while others were to be gradually transported. ${ }^{41}$ The National Library took over 300,000 volumes; the State University Library in Brno, 112,000 ; the University Library in Olomouc, 300,000; the Zdeněk Nejedlý State Research Library (SRL) in České Budějovice, 87,000; the SRL in Plzeň, 135,000; the SRL in Liberec, 50,000; the Zdeněk Nejedlý SRL in Hradec Králové, 30,000; the SRL in Opava, 53,000. A total of 150,000 volumes were transported to the Strahov Library (Premonstratensian abbey). In total, the number was over 1.2 million books. ${ }^{42}$ However, the source document does not indicate whether this is the number of books that the libraries absorbed into their collections, or the total number of books sorted by them within their area of competence. Securing monastic libraries was not without its difficulties. The Emmaus Monastery in Prague was visited by staff of the National Library and the National Museum in Prague at the end of June 1950. The books were concentrated in two rooms in the central part of the monastery. The representative of the monastery was asked by František Horák to close the rooms and leave everything as it was until the books had been collected for transportation to the relevant institutions. The Emmaus Monastery was listed as the last of the Prague Monastery buildings in terms of urgency, so the clean-out was not to begin until the end of August 1950. However, when the staff arrived on 30 August they found that the building had already been handed over to the Ministry of Health to become the State University Hospital. In the two rooms which were supposed to remain secure and

39 Ibid. p. 150.

40 Národní archiv, Státní úřad pro věci církevní [State Office for Matters of the Church], box no. 5, Record of the meeting of the committee of the State Office for Matters of the Church with the Deputy Prime Minister on August 31, 1950.

41 Ibid. draft of the Meeting of the Cultural Committee of State Office for the Matters of the Church at the Cabinet of Prime Minister Fierlinger (31.08.1950). However, the list of collected valuables on which the report to the Prime Minister is based gives the number of libraries as 18.

42 Ibid. List of valuables collected for the purposes of the report to Prime Minister, p. 2. 
inaccessible, the tables and bookshelves had disappeared, and the books were thrown on the ground. Among the rubbish was found, for example, an old print of Pavel Stránský's "Respublica Bohemiae", the Amsterdam edition of 1634. The complaint about this treatment said, quite rightfully, that the books were treated "in a way that is unworthy of civilized society." ${ }^{43}$ These books did not become part of the National Library holdings, but were kept in the Reserve Collection. After the Revolution of 1989, it was decided to include these books in restitutions.

In late 1992 and in the first half of 1993, the National Library negotiated with the representatives of the Assembly of Superior-Generals the technical and organizational conditions for the gradual sorting and return of books in the Reserve Collection that originally belonged to the Church. A procedure was established, including the method of selecting books and handing them over to the appropriate orders and bishoprics. Because there were no records about books belonging to the Church in the Reserve Collection, it was necessary to examine each publication individually. As evidence of ownership, the National Library acknowledged mark of ownership, stamp, ex-libris, supralibros or other sign of provenance (binding, etc.).

The official handover was finished in 1995, but it is clear from a provenance survey that sorting was not performed without errors. ${ }^{44}$

A large portion of the books so-far processed are, for example, school library books, many from office libraries, and so on. For this group, we can assume that the books came to the National Library in the standard way, no longer needed for the purposes of these institutions. The Reserve Collection also contains a number of items belonging to important figures of Czechoslovak science of the period of the First Republic (1918-1938). This cannot be explained by any other way than by purchases of estates, bequeathment and donations to the National Library.

Summarizing the above, the structure of the Reserve Collection today looks roughly as follows:

Confiscated items of foreign provenance:

- property of the German authorities (SD/RSHA Office Libraries),

- property of private German individuals ${ }^{45}$ (e.g. Heinz Schwiertz, Bernhard Brandt, Mimi von Mallmann),

43 Ibid. Complaint of August 31, 1950 by the National and University Library addressed to the State Office for the Matters of the Church, concerning the Benedictine monastery library in the Emmaus Monastery.

44 Ibid. pp. 36-37.

45 For people who are not publicly known, it is very difficult to determine whether they were Germans, living in the Reich, whose property was confiscated by the Nazi administration and 
- property of leading figures of the Nazi administrative apparatus ${ }^{46}$ (e.g. Gregor Schwartz-Bostunitsch, Horst Böhme),

- property of German (Reich) libraries (e.g. Kaiser-Wilhelm-Akademie, Military Library in Toruń, Berlin City Archives),

- property of German organisations, associations and societies persecuted by the Nazis (e.g. Masonic lodges, Theosophical Society in Leipzig),

- property of other than German provenance (e.g. Masonic lodges in Norway, Belgium or Austria, Theosophical Society in Brussels).

Confiscated items of domestic provenance:

- property of the German authorities established in the Protectorate of Bohemia and Moravia (e.g. the offices of the Gestapo, the Office of the Reich Protector, the district and provincial courts, the Supreme Court);

- property of private German individuals (e.g. Felix and Sonja Haas),

- property of German public libraries (e.g. the library and reading room in Teplice, the municipal library in Chomutov, the library in Rumburk)

- property of German school libraries (e.g. the German Grammar School in the New Town in Prague, the State Realgymnasium in Prague II, the Realgymnasium in Děčín, a number of institutions of the German University in Prague)

- property of German associations (e.g. the Association of German Jurists in Prague, the reading and rhetorical club of German students in Prague, the association of friends in České Budějovice).

Other origin $=$ non-confiscated property $^{47}$

- property of Czechoslovak administration libraries (e.g. the municipal office in Písnice near Prague, the Governor's Office in Prague, the Supreme Court and many ministries),

- property of Czechoslovak school libraries (e.g. e.g. the Academic Grammar School in Prague, the grammar school in Žitná street, the Girls' Lyceum at St. Ursuline in Prague, university institutes)

then brought to the territory of the Protectorate, or citizens of Czechoslovakia whose property was confiscated by Presidential Decree.

46 This category is on the border of both foreign and domestic provenance, despite the fact that it concerned Germans whose activities were, in a certain way, connected with the Protectorate.

47 The cataloguing of, for example, aristocratic libraries, is problematic - there are only fragments of the Thun-Hohenstein library, which was sold in auctions in the early $20^{\text {th }}$ century. We can only imagine how these books got into the Reserve Collection. They might have been purchased by the National and University Library, or they might have been purchased for the library by the Ministry of Education. 
- property of Czechoslovak enterprises (e.g. Landesbank, Petschek \& Co., ČKD, Škoda),

- property of Czechoslovak associations (e.g. the Merkur merchant association in Prague, the Union of Lawyers in Prague, the Zemgor association, the Women's Manufacturing Society),

- the property of private persons (e.g. Václav Brtník, František Pražák, František V. Schwarz),

- property of religious institutions (e.g. the Děcín Jesuits, the Liberec Capuchins, the Redemptorists in Prague).

Up to now, more than 16,000 books have been processed from the Reserve Collection of the National Library. This is about five per cent of the total amount of foreign (non-Bohemian) books stored there. A further survey of the collection may improve what is known about former owners. The historical research in the Czech and foreign archives continues. However, the main problem of processing the Reserve Collection is a lack of finances. The books are being processed within the framework of projects. Currently the survey continues within the project IN-PROVE (Building an Integrated Environment for Research, Protection, Research and Evidence of Modern Library Collections). 


\title{
Johana Prouzová
}

\section{Die Sammlung „Pollák“ in den Prager Museen}

\section{Zusammenfassung}

Die Sammlung des Kaufmanns Josef Pollák, der in Auschwitz ermordet wurde, überstand die NS-Okkupation nur fragmentarisch. Im Jahre 1939 übergab er dem Kunstgewerbemuseum in Prag 957 Kunstgegenstände und eine nicht genau beschriebene Menge von Grafiken als Leihgabe. Kurz danach wurden 37 Bilder und Plastiken durch eine ähnliche Vereinbarung in die Nationalgalerie in Prag überstellt. Zwanzig dieser Gemälde verkaufte die Gestapo im Juni 1942. In seinem letzten Willen vererbte Pollák dem Kunstgewerbemuseum und der Nationalgalerie die ausgesuchten Gegenstände. Den Rest erbten seine Mutter, die das Ghetto Theresienstadt überlebte, und seine Halbschwester. Die Forscher aus dem Prager Documentation Centre for Property Transfers of the Cultural Assets of WW II Victims arbeiten an der Zusammenstellung eines ausführlichen Katalogs der ganzen Sammlung.

Schlagwörter

Provenienzforschung, NS-Raubkunst, jüdische Deposita, Nationalgalerie in Prag, Kunstgewerbemuseum in Prag

\begin{abstract}
The Pollák Collection in Museums of Prague

The art collection of the entrepreneur Josef Pollák, who was murdered in Auschwitz, survived the Nazi Occupation only in fragments. In 1939, Pollák gave the Museum of Decorative Arts in Prague a loan of 957 objects and many insufficiently described graphic sheets. Soon afterwards, 37 paintings, drawings and sculptures were also given as a loan to the National Gallery in Prague. Twenty paintings of them were sold by Gestapo in June 1942. According to Pollák's last will, the museum and the gallery inherited the selected works of art. The rest went to his mother, who had survived the Theresienstadt ghetto, and his half-sister. The researchers from the Documentation Centre for Property Transfers of the Cultural Assets of WW II Victims of Prague have been working on a detailed catalogue of the whole collection.
\end{abstract}

Keywords

Provenance Research, Looted Art, Jewish Deposits, National Gallery in Prague, Museum of Decorative Arts, Prague 


\section{Josef Polláks Leben und seine Familie}

Die Forscher aus dem Prager Documentation Centre for Property Transfers of the Cultural Assets of WW II Victims ${ }^{1}$ haben sich mit den Kunstgegenständen aus dem Nachlass des Prager Großhändlers Josef Pollák, der in Auschwitz ermordet worden ist, schon im Jahre 2008 beschäftigt. Die Teile seiner Bilder- und Porzellansammlung befinden sich heute teilweise in der Nationalgalerie in Prag und im Kunstgewerbemuseum in Prag. In der Publikation Návraty Paměti (2008) ${ }^{2}$ beschreiben die Autoren neben dem Depositum Pollák auch andere Schicksale der jüdischen Bestände im Kunstgewerbemuseum und ihrer ursprünglichen Eigentümer. Seither wurden neue Artefakte identifiziert und die gesamten $\mathrm{Ob}$ jekte der Sammlung Pollák soll in einer neuen Publikation veröffentlicht werden.

Josef Pollák (1896-1944) stammte aus einer wohlhabenden Familie. Sein Vater Antonín (oder auch Anton) Pollák (1858-1938) war ein k. u. k. Kommerzienrat, Großhändler und Inhaber der Firma Joachim Wedeles Sohn, Prag I. ${ }^{3}$, die mit Textil handelte. ${ }^{4}$ Daneben war er noch als Zensor des Bankamtes des Finanzministeriums tätig. ${ }^{5}$ Im Jahre 1892 heiratete Antonín Pollák in der Synagoge in der damaligen Prager Vorstadt Karolinenthal Kamila Wagner (1868-1951). Die ganze Familie wohnte danach in der Kollárgasse 276, ebenfalls in Karolinenthal. Josef Pollák hatte eine ältere Schwester namens Zdenka (1894-1933), die später den Juristen Alfred Gintz heiratete. ${ }^{6}$ Es wurden ihnen zwei Töchter geboren. Die

1 Es wurde im Jahre 2012 aufgrund des Gesetzes 212/2000 Sb. als gemeinnützige Gesellschaft gegründet, die von dem Kulturministerium der Tschechischen Republik finanziert ist. Seine Forscher beschäftigen sich hauptsächlich mit der Provenienzforschung, Publikationstätigkeit oder Veranstaltung der internationalen Konferenzen. Siehe auch: URL: www.cdmp.cz (abgerufen am 20.07.2017).

2 Helena Krejčová, Mario Vlček: Návraty paměti. Deponáty židovského majetku v Uměleckoprůmyslovém museu v Praze [Memories Returned: Jewish Property at the Museum of Decorative Arts, Prague]. 1. vydání. Šenov u Ostravy: Centrum pro dokumentaci majetkových převodů kulturních statků obětí II. světové války při Ústavu pro soudobé dějiny Akademie Věd České republiky, Tilia 2008. Für die englische Übersetzung der einführenden Studie, sowie weiterer Teile des Buches siehe Anmerkung 27.

3 Národní archiv České republiky v Praze (NA) [Nationalarchiv der Tschechischen Republik], fond Policejní ŕeditelství Praha II - evidence obyvatelstva (PŘ II - EO) [Fond Polizeidirektion Prag II - Verzeichnis der Bürger], Antonín Pollák.

4 NA, fond Policejní ředitelství Praha II - všeobecná spisovna - 1931-1940 (PŘ 1931-1940) [Fond Polizeidirektion II - allgemeine Registratur - 1931-1940], Sg. P 2566/3, Karton 9753, Antonín Pollák, Antrag des Magistrats der Hauptstadt Prag für die Bescheinigung der Polizeidirektion in Prag, ob Antonín Pollák den Handel mit Textil betreiben kann, 07.02.1900.

5 NA, PŘ 1931-1940, Sg. P 2566/3, Karton 9753, Antonín Pollák, Antrag auf die Ausstellung eines Reisepasses, 02.07.1923.

6 Archiv hlavního města Prahy (AHMP) [Archiv der Hauptstadt Prag], fond Magistrát hlavního města Prahy I. (MHMP I.) [Fond Magistrat der Hauptstadt Prag I.], Referát IV. popisní, Soupis pražského obyvatelstva 1830-1949, Pražané (1911) 1920-1949 [Referat IV., Verzeichnis der Prager Bürger 1830-1949, die Prager (1911) 1920-1949], Antonín Pollák. 
Familie Gintz - außer Zdenka Gintz, die früh gestorben war - wurde im Juni 1942 in das Ghetto Theresienstadt deportiert, wo die jüngere Tochter nach acht Monaten starb. Ihren Vater und ihre Schwester deportierte man im Jahre 1943 nach Auschwitz, keiner der beiden überlebte. ${ }^{7}$

Josef Pollák arbeitete schon als 25-Jähriger in der Firma seines Vaters Joachim Wedeles Sohn und reiste deswegen häufig dienstlich nach Deutschland. ${ }^{8}$ Im Jahre 1922 lebte Pollák in Pichelsberg bei Spandau und beabsichtigte, an der hiesigen Handelshochschule zu studieren, womit er die Verlängerung seines Reisepasses begründete. ${ }^{9}$ Es gibt keine weitere Information über sein Studium und seinen Berliner Aufenthalt in der Dokumentation der Prager Polizeidirektion, aber es ist sicher, dass er weiter für die Firma seines Vaters arbeitete - so reiste er z. B. im Jahre 1924 in einer dienstlichen Angelegenheit nach England. ${ }^{10}$

Nach zwei gescheiterten Ehen heiratete Josef Pollák im November 1939 Veronika Michálková (geb. $1916^{11}$ ). Ein Jahr später wurde ihnen Tochter Zdenka geboren, die man wahrscheinlich nach Polláks verstorbener Schwester benannte. Das Kind starb jedoch im Dezember $1941 .^{12}$ Es ist anzumerken, dass die Ehe mehr als ein Jahr vor diesem tragischen Ereignis im Juli 1940 geschieden worden war, vermutlich aufgrund der Gesetze des NS-Regimes, welche sogenannte Mischehen auflösten. Die Scheidung wurde nämlich nach dem Krieg rückgängig gemacht. $^{13}$

Seinen Führerschein musste Josef Pollák im Februar 1941 dem Verkehrsamt zurückgeben. ${ }^{14}$ Von der Meldung eines Wachmannes im April desselben Jahres wegen des beschädigten Daches des Hauses in der Teyngasse 632 war festzustellen, dass der frühere Eigentümer ein Jude gewesen ist, und dass das Gebäude

7 Miroslav Kárný, kol.: Terezínská pamětní kniha [Theresienstädter Gedenkbuch]. 1. vydání. Praha: Nadace Terezínská iniciativa v nakladatelství Melantrich 1995, Bd. I, S. 628.

8 NA, PR 1941-1950, Sg. P 2735/2, Karton 8882, Josef Pollák, die Firma Joachim Wedeles Sohn bestätigt, dass Josef Pollák in ihrem Betrieb angestellt sei und dass er dienstlich nach Berlin und Hamburg reisen müsse, 30.01.1921.

9 NA, PŘ 1941-1950, Sg. P 2735/2, Karton 8882, Josef Pollák, Konsulat der Tschechoslowakischen Republik in Berlin an die Polizeidirektion in Prag, 03.11.1922.

10 NA, PŘ 1941-1950, Sg. P 2735/2, Karton 8882, Josef Pollák, die Firma Joachim Wedeles Sohn bestätigt der Polizeidirektion in Prag, Passstelle, dass Josef Pollák dienstlich nach England reisen müsse, 05.05.1924.

11 Das Todesdatum konnte bisher in keiner der zu Verfügung stehenden Quellen gefunden werden. Zwar ist anzunehmen, dass Veronika Michálková mittlerweile verstorben ist, aber da die Autorin in keinem der relevanten Archive das Todesdatum hat finden können, bleibt dieser Sachverhalt ungeklärt.

12 Památník Terezín, Registrační listiny [Gedenkstätte Theresienstadt, Liste der Mitglieder der Jüdischen Kultusgemeinde Prag].

13 AHMP, MHMP I., Referát IV. popisní, Soupis pražského obyvatelstva 1830-1949, Pražané (1911) 1920-1949, Josef Pollák.

14 NA, PŘ 1941-1950, Sg. P 2735/2, Karton 8882, Josef Pollák, Josef Pollák and das Verkehrsamt der Polizeidirektion in Prag, 04.02.1941. 
der „Verwaltung- und Verwertungsstelle des Auswanderungsfonds für Böhmen u. Mähren " gehörte. ${ }^{15}$ Neben diesem Gebäude wurde der Familie Pollák auch das Haus in der Kollárgasse 276, die Häuser 16a und 93 und die Bauparzelle 131 in Rostok bei Prag weggenommen. Ein Viertel des Grundbesitzes in Rostok hatte Josef Pollák gehört, die Hälfte seinen Nichten Gintz und ein Viertel seiner Mutter Kamila Polláková. Die Häuser in der Kollárgasse und Teyngasse hatten Kamila und Josef Pollák je zur Hälfte besessen. Alle diese Gebäude und Grundstücke wurden nach dem Krieg an Kamila Polláková restituiert. ${ }^{16}$

Im April 1941 vernahm man Josef Pollák in der Polizeidirektion in Prag, weil sein Personalausweis nicht mit dem Buchstaben „“" gekennzeichnet war. Der Polizeibeamte beschrieb ihn als Juden und ehemaligen Händler und fügte hinzu, dass Pollák geschieden worden sei, eine einjährige Tochter habe und derzeit ohne Einkommen aus seinem Kapital lebe. ${ }^{17}$

Josef Pollák wurde am 30. Januar 1942 in das Ghetto Theresienstadt mit dem Transport V deportiert, Kamila Polláková folgte ihm am 13. Juli 1942 mit dem Transport AAq. Sie überlebte den Holocaust in Theresienstadt ${ }^{18}$, ihr Sohn wurde aber am 28. September 1944 nach Auschwitz deportiert, wo er starb. ${ }^{19}$

Nach ihrer Rückkehr aus dem Ghetto lebte Kamila Polláková in ihrem Haus in der Kollárgasse nicht alleine, sondern mit ihrer Tochter Aloisie WagnerováHudcová (geb. $1919^{20}$ ). Sie war höchstwahrscheinlich ein uneheliches Kind, denn Wagnerová war der Mädchenname von Kamila Polláková. Die offiziell angeführten Eltern von Aloisie Wagnerová-Hudcová waren Hynek Hudec und Marie, geborene Vajnerová, trotzdem wurde in ihrer Anmeldung des Daueraufenthalts im November 1949 gezeichnet, dass sie bei ihrer „Mutter - Polláková“ wohnte. ${ }^{21}$ Kamila Polláková ist im September 1951 gestorben ${ }^{22}$ und hinterließ der Tochter vermutlich ihr ganzes Vermögen.

15 NA, PŘ 1941-1950, Sg. P 2735/2, Karton 8882, Josef Pollák, das Polizeikommissariat Altstadt an die Polizeidirektion in Prag, 08.04. 1941. Der Auswanderungsfond offiziel verwaltete das sämtliche beschlagnahmte jüdische Vermögen.

16 NA, Ministerstvo financí II Praha (1945-1968) (MF) [Finanzministerium II Prag (1945-1968)], Restituce [Restitutionen], Kamila Polláková, Bescheide des Ministeriums für Arbeitsschutz und soziale Fürsorge: über das Haus in der Kollárgasse vom 13.12.1945, über den Grundbesitz in Rostok vom 25.01.1946 und über das Haus in der Teyngasse, auch vom 25.01.1946.

17 NA, PŘ 1941-1950, Sg. P 2735/2, Karton 8882, Josef Pollák, das Protokoll von der Vernehmung Polláks, 21.04.1941.

18 Miroslav Kárný, kol.: Terezínská pamětní kniha (Anm. 7). Bd. II, S. 774.

19 Miroslav Kárný, kol.: Terezínská pamětní kniha (Anm. 7). Bd. I, S. 323.

$20 \mathrm{Ihr}$ Todesdatum konnte bisher von der Autorin nicht festgestellt werden.

21 NA, PŘ II - EO, Sg. W, Aloisie Wagnerová-Hudcová.

22 NA, PŘ II - EO, Sg. P, Kamila Polláková. 


\section{Sammlung Pollák in der Nationalgalerie in Prag}

Im Zuge einer mündlichen Vereinbarung zwischen der damaligen Nationalgalerie in Prag und Josef Pollák vom 6. März 1939 wurden $37^{23}$ Gemälde, Plastiken und gerahmte Zeichnungen aus der privaten Sammlung Pollák der Galerie als Leihgabe überstellt. ${ }^{24}$ Es ging um Ölgemälde tschechischer, flämischer, niederländischer, österreichischer, deutscher und anderer Meister, Zeichnungen und Aquarelle tschechischer Meister und zwei Plastiken. ${ }^{25}$

Mit den zunehmenden antijüdischen Maßnahmen war die Pflicht verbunden, nicht-arisches Vermögen den NS-Behörden zu melden. Deshalb wandte sich die Direktion der Galerie am 28. Juli 1939 an Josef Pollák, um nachzufragen, ob seine Sammlung ordnungsgemäß angemeldet worden war. Nach einem Jahr wiederholte die Direktion ihre Anfrage mit einer Mahnung und Pollák bestätigte, dass er die Kunstgegenstände angemeldet hatte. ${ }^{26}$

Mitte des Jahres 1943 schaltete sich die Treuhandstelle beim Ältestenrat der Juden $^{27}$ in der Sache der Sammlung Pollák ein und ersuchte die Direktion um die Mitteilung, unter welchen Bedingungen Pollák die Sammlung der Galerie überlassen hatte. Bemerkenswert ist, dass die Treuhandstelle die Transportnummer Polláks V 481 als ihr Zeichen benutzte. ${ }^{28}$ Die Direktion der Galerie antwortete, dass sie am 12. Mai 1939 als Leihgabe in Verwaltung 37 Kunstwerke übernommen hatte, und fügte ihre Liste hinzu. Die Direktion führte weiter an:

23 Es kann von 39 Kunstwerken ausgegangen werden, drei Zeichnungen von Aleš waren in einem Rahmen.

24 Národní galerie (NG) [Nationalgalerie], fond ANG 1945-1958, sk. 231/8 (odkazy věcné) [Fond Archiv der Nationalgalerie 1945-1958, Gruppe 231/8 (materielle Nachlässe)], Josef Pollák, Pollák bestätigt der Galerie die mündliche Vereinbarung, 03.05.1939.

25 NG, ANG 1945-1958, sk. 231/8 (odkazy věcné), Josef Pollák, Direktion der Galerie an Josef Pollák, „Verzeichnis von Bildern, Plastiken und Zeichnungen, die laut Vereinbarung vom 6. März [...] am 12. Mai 1939 als Leihgabe in Verwaltung übernommen wurde“, 12.05. 1939.

26 NG, ANG 1945-1958, sk. 231/8 (odkazy věcné), Josef Pollák, Direktion der Galerie an Josef Pollák, 28.07.1939 und 11.10.1940, Josef Pollák an die Direktion, 11.10.1940.

27 Die Treuhandstelle wurde von der Prager Zentralstelle für jüdische Auswanderung im Oktober 1941 als die größte Abteilung der Jüdischen Kultusgemeinde in Prag errichtet und verwaltete sämtliches Vermögen der deportierten Personen. Der offizielle Inhaber dieses Besitzes war jedoch der Auswanderungsfond. Vgl. Helena Krejčová, Mario Vlček: Memories Returned: Jewish Property at the Museum of Decorative Arts, Prague. (Translation of the opening pages, introductory study and editorial notes from the Czech-language publication Návraty paměti). 1. edition. Šenov u Ostravy: Documentation Centre for Property Transfers of Cultural Assets of World War II Victims, Institute for Contemporary History, Academy of Sciences of the Czech Republic, Tilia Publishers 2008, S. 21-46.

28 NG, ANG 1945-1958, sk. 231/8 (odkazy věcné), Josef Pollák, Treuhandstelle beim Ältestenrat der Juden an die Direktion der Nationalgalerie Prag, 15.06.1943. 
„Von diesen Kunstwerken wurden von der Geheimen Staatspolizei durch Herrn Studienrat Baudisch unter gutachtlicher Heranziehung des Herrn Univ. Prof. Dr. K. M. Swoboda ausgeschieden und von der Geheimen Staatspolizei zu Verkaufszwecken übernommen: [Es folgte die Liste von 20 Gemälden.] Der Rest wurde der [...] Galerie in Prag als Reichseigentum zur Verwaltung und Aufbewahrung übergeben und zwar sind es folgende Kunstwerke: [die Liste von 17 Kunstwerken.] “29

Jindřich Baudisch war seit 1939 bei der Gestapo als Sachverständiger tätig und entschied, welche von den beschlagnahmten Kunstwerken verkauft werden sollten und welche für Museen bestimmt waren. In der damaligen Nationalgalerie begegnete er oft Prof. Karl Maria Swoboda (1889-1977), der als Getreuer des Staatssekretärs Karl Hermann Frank in den Museumssachen die Kunstgegenstände aus dem beschlagnahmten Besitz für die Museen im Protektorat aussuchte. ${ }^{30}$ Im Jahre 1944 wurde Swoboda zu dem sogenannten Verwalter des reichseigenen Kunstgutes bei der Böhmisch-Mährischen Landesgalerie (d.h. der damaligen Nationalgalerie) ernannt. Im Unterschied zu Baudisch war er berechtigt, die Lager der Treuhandstelle zu besuchen und von hier die Kunstwerke in der Galerie, aber z. B. auch im Kunstgewerbemuseum in Prag als Reichseigentum zu deponieren. ${ }^{31}$

Swoboda wurde in Prag geboren, studierte in Wien und zählte zu den Vertretern der so genannten Wiener kunsthistorischen Schule. Im Jahre 1934 berief man ihn nach Prag, um das Prager Kunsthistorische Institut der Deutschen Universität zu leiten. In diesem Jahr wurde er auch zum Professor ernannt und er wirkte als Vorstand des Instituts bis $1945 .{ }^{32}$ Danach wurde er kurz verhaftet, aber die tschechischen Kollegen bezeugten sein unbescholtenes Verhalten und setzten sich für seine frühe Freilassung ein. Swoboda soll nicht nur zahlreiche Kunstwerke für den tschechischen Staat in den Galerien und Museen gesichert haben. Er hatte wahrscheinlich enge Kontakte mit dem Amt des Reichsprotektors, was ihm ermöglichte, sich für manche Persönlichkeiten aus dem tschechischen Kulturbereich einzusetzen, die zum Tode verurteilt worden waren. Swoboda war

29 NG, ANG 1945-1958, sk. 231/8 (odkazy věcné), Josef Pollák, Direktion der Galerie an die Treuhandstelle, 29.06.1943.

30 Helena Krejčová, Otomar L. Krejča: Jindřich Baudisch a konfiskace uměleckých děl v protektorátu [Jindřich Baudisch und die Konfiskation von Kunstwerken im Protektorat]. 1. vydání. Praha: Centrum pro dokumentaci majetkových převodů kulturních statků obětí druhé světové války, Tilia, Ústav pro soudobé dějiny AV ČR 2007, S. $49 \mathrm{f}$.

31 Vladimír Drbohlav, Vanda Drbohlavová, Helena Krejčová, Johana Prouzová und Mario Vlček: The Fate of Wartime Depostits: Museum of Decorative Arts, Prague, Vol 2. 1. edition. Prague: Documentation Centre for Property Transfers of Cultural Assets of World War II Victims, p. b. o. 2015, S. 8 f.

32 Sigrid Canz: Karl Maria Swoboda (1889-1977) Kunsthistoriker. Wissenschaftler zwischen Wien und Prag. In: Monika Gettler, Alena Mišková: Prager Professoren 1938-1948: Zwischen Wissenschaft und Politik. 1. Auflage. Essen: Klartext Verlag 2001 (= Veröffentlichungen zur Kultur und Geschichte im östlichen Europa 17), S. 175-190, hier S. 175-178. 
nie NSDAP-Mitglied. Im Jahre 1946 wurde er zum ordentlichen Professor am Wiener Kunsthistorischen Institut ernannt. ${ }^{33}$ Die Rolle Swobodas und ähnlicher Persönlichkeiten in der Maschinerie des größten Kunstraubes im 20. Jahrhundert bleibt bis heute umstritten.

Die 20 Ölgemälde aus der Sammlung Pollák, die Baudisch und Swoboda zum Verkauf bestimmten, waren vorwiegend die Kunstwerke niederländischer Meister, eine Landschaft von einem flämischen Maler (der Liste von der Übergabe der Kunstgegenstände Polláks der Galerie nach ging es um Teniers ${ }^{34}$ ), zwei Porträts von Franz von Lenbach (1836-1904), vier Werke der böhmischen Maler Emil Orlik (1870-1932), Václav Brožík (1851-1901) und Jaroslav Čermák (1830-1878) und ein Gemälde von William Collins (1788-1847). Nach dem Krieg wurde von diesen Werken nur die Ruhe auf der Flucht nach Ägypten des niederländischen Malers Pieter van Avont (1600-1652) gefunden.

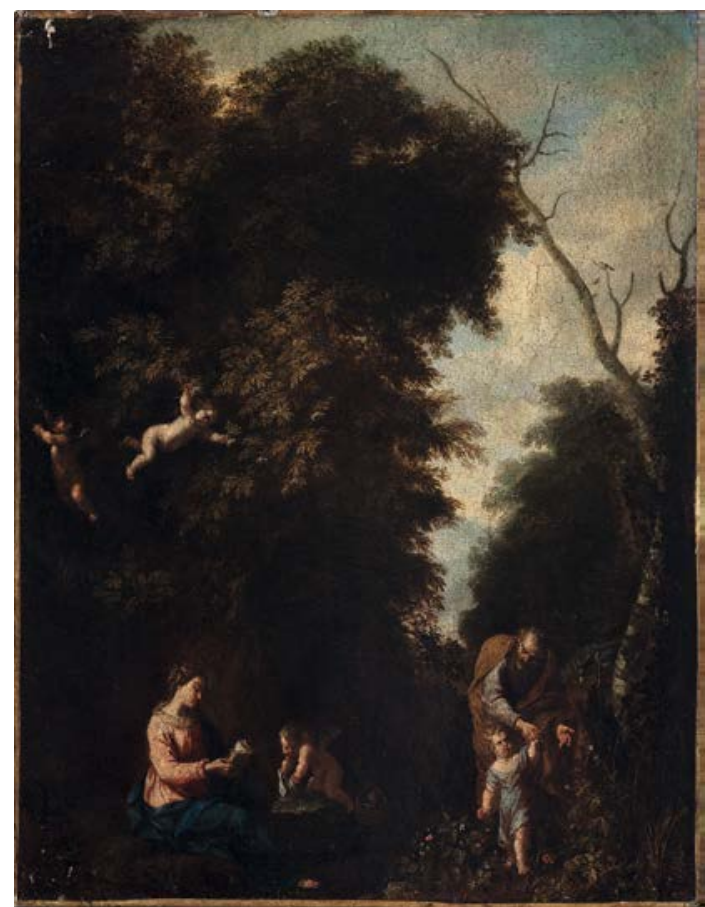

Abb. 1: Niederländischer Meister aus der 1. Hälfte des 17. Jahrh. (Pieter van Avont), Ruhe auf der Flucht nach Ägypten, Öl auf Leinwand, 42,5 x $33 \mathrm{~cm}^{35}$

33 Ebd., S. 186-189.

34 In der Liste (Anm. 25) wurde nicht angegeben, ob David Teniers der Ältere (1582-1649) oder der Jüngere (1610-1690) gemeint war.

35 Dieses Werk wurde im Jahre 1942 mit 19 anderen Gemälden durch die Gestapo verkauft und 
Josef Pollák verfasste seinen letzten Willen nur vier Tage vor seinem Transport nach Theresienstadt. Der Mutter Kamila Polláková vererbte er die Geldsumme in der Höhe des Sechstels seines Nachlasses. Als Erbin wurde seine Halbschwester Aloisie Hudcová eingesetzt. Dem Kunstgewerbemuseum in Prag, dem Museum der Hauptstadt Prag und der Nationalgalerie hinterließ Pollák diejenigen Kunstgegenstände, die sich diese Institutionen zum Zweck der Dauerausstellung aussuchen würden. ${ }^{36}$

Ende November 1947 wandte sich die Direktion der Galerie an die so genannte Nationalverwaltung, die das durch die Treuhandstelle und Gestapo beschlagnahmte Vermögen übernommen hatte und die Restitutionsanträge der ursprünglichen Eigentümer oder ihrer Erben erledigte. ${ }^{37}$ Die Direktion der Galerie ersuchte die Nationalverwaltung um eine Auskunft über die im Jahre 1942 verkauften Gemälde, wies auf den letzten Willen Josef Polláks hin und beanspruchte alle verbliebenen Kunstwerke aus seinem Depositum in der Galerie. Die Nationalverwaltung sollte deshalb den Erben keine der Kunstgegenstände herausgeben. ${ }^{38}$ Darauf antwortete die Nationalverwaltung, dass ihre Nachforschung nach den verkauften Gemälden bis jetzt erfolglos gewesen war. In der Sache der übrigen Kunstwerke beabsichtigte sie, auf den Beschluss des Gerichts zu warten. ${ }^{39}$

Da sich auch die Gemälde und Zeichnungen Polláks in dem Kunstgewerbemuseum in Prag befanden, waren die Vertreter der Nationalgalerie bei der Verhandlung über diese anwesend und sie suchten 69 Kunstwerke für die Galerie aus. Dazu sollte noch die Galerie eine Menge von Grafiken auswählen. Das Kunstgewerbemuseum beanspruchte 53 Miniaturen und Zeichnungen und das Museum der Hauptstadt Prag drei Bilder und elf Porzellanfiguren.$^{40}$ Einen Tag später legte Aloisie Hudcová dem Notar und den Vertretern der Galerie und des Kunstgewerbemuseums 27 Fotografien der Kunstwerke vor, von denen Kamila

als das einzige von diesen nach dem Krieg wiedergefunden. Heute befindet es sich in der Nationalgalerie.

36 NG, ANG 1945-1958, sk. 231/8 (odkazy věcné), Josef Pollák, der letzte Wille Josef Polláks, 26.01.1942.

37 Mehr zur Tätigkeit der Nationalverwaltung vgl. Helena Krejčová: Post-War Restitutions in Czechoslovakia and Related Problems. In: Restitution of Confiscated Art Works - Wish or Reality? Documentation, identification and restitution of cultural property of the victims of World War II; Proceedings of the international academic conference held in Liberec on 24-26 October, 2007. Ed. by Mečislav Borák. 1. edition. Šenov u Ostravy: Documentation Centre for Property Transfers of Cultural Assets of World War II Victims, Institute for Contemporary History, Academy of Sciences of the Czech Republic, Tilia Publishers 2008, S. 43-63.

38 NG, ANG 1945-1958, sk. 231/8 (odkazy věcné), Josef Pollák, Direktor der Nationalgalerie an die Nationalverwaltung, 25.11.1947.

39 NG, ANG 1945-1958, sk. 231/8 (odkazy věcné), Josef Pollák, Nationalverwaltung an die Direktion der Nationalgalerie, 08.01.1948.

40 NG, ANG 1945-1958, sk. 231/8 (odkazy věcné), Josef Pollák, Protokoll verfasst von dem Notariat als Gerichtskommissariat in Prag - Karolinenthal, 07.01.1948. 
Polláková einige als Besitz ihres Mannes Antonín Pollák bezeichnete. Antonín Pollák habe die Kunstgegenstände seinem Sohn angeblich nicht übergeben. ${ }^{41}$

Bei der Verhandlung über den Nachlass Polláks in der Nationalgalerie legte Aloisie Hudcová die Erklärung von Kamila Polláková vor, dass sie alle in der Galerie verbliebenen Kunstwerke beanspruche. ${ }^{42}$ Nun begannen die beiden Seiten zu lizitieren. Die Galerie beharrte auf den 17 Kunstwerken, die die Direktion der Galerie, den eigenen Worten nach, vor dem Verkauf durch die Gestapo gerettet hatte, und auf den 25 Gemälden und Zeichnungen von den früher im Kunstgewerbemuseum 69 ausgesuchten Werken. ${ }^{43}$

Der Streit wurde erst durch die Verhandlung über den Nachlass Polláks vom 24. Juni 1948 beendet. Aloisie Hudcová entschied sich, den Verlust von Kamila Polláková wettzumachen. An Polláková wurden offiziell diejenigen Gegenstände restituiert, die ihr eigener Besitz gewesen sein sollen, und sie gab der Galerie einige von diesen zurück. Als Ersatz dieses Verlustes konnte Polláková aus dem Erbe von Hudcová beliebige Kunstgegenstände in dem Wert von denen der Galerie zurückgegebenen Gegenstände aussuchen.

Dem Beschluss des Finanzministeriums vom 27. August 1949 nach restituierte Kamila Polláková 15 Ölgemälde, zwei Plastiken und acht Zeichnungen, von denen zwei Zeichnungen und sieben Ölgemälde dem Depositum Pollák in dem Kunstgewerbemuseum und der Rest dem Depositum in der Galerie angehört hatten. Die Galerie bekam von den restituierten Kunstwerken zehn Gemälde, zwei Plastiken und drei Zeichnungen und diese wurden von Aloisie Hudcová, der Inhaberin ersetzt. In dem Beschluss sind zwei Fehler zu finden: Josef Pollák sei angeblich im Jahre 1945 in Theresienstadt gestorben. Vielleicht wurde dies mit dem Datum 28. März 1945 verwechselt, als Pollák für tot erklärt wurde. ${ }^{44}$ Der zweite Fehler betraf die Kunstwerke für die Nationalgalerie, die in dem Beschluss unterstrichen waren, aber im Falle eines Ölbilds wurde dies vergessen. ${ }^{45}$ Dieses Gemälde musste die Direktion der Galerie später vor der Auktion retten, weil es versehentlich an Frau Chmelíková, früher Hudcová herausgegeben wurde. Auch wenn es so aussieht, entstand dieses Missverständnis nicht aufgrund des Fehlers in dem Beschluss, sondern weil man das Gemälde einfach zusammen mit dem

41 NG, ANG 1945-1958, sk. 231/8 (odkazy věcné), Josef Pollák, Protokoll verfasst von dem Notariat als Gerichtskommissariat in Prag - Karolinenthal, 08.01.1948.

42 NG, ANG 1945-1958, sk. 231/8 (odkazy věcné), Josef Pollák, Protokoll verfasst von dem Notariat als Gerichtskommissariat in Prag - Karolinenthal, 19.01.1948.

43 NG, ANG 1945-1958, sk. 231/8 (odkazy věcné), Josef Pollák, Direktion der Nationalgalerie an Judr. Jan Sakař, den Rechtsanwalt von Kamila Polláková, 14.04.1948.

44 AHMP, MHMP I., Referát IV. popisní, Soupis pražského obyvatelstva 1830-1949, Pražané (1911) 1920-1949, Josef Pollák.

45 NG, ANG 1945-1958, sk. 231/8 (odkazy věcné), Josef Pollák, Beschluss des Finanzministeriums über die Restitution der Bilder von Kamila Polláková, 27.08.1949. 
zweiten Bild desselben Malers und der gleichen Größe der Erbin ausgab. ${ }^{46}$ Eine aktuelle Bestandsaufnahme der Kunstwerke Pollák in der Nationalgalerie wird in dem vorbereiteten Katalog des Documentation Centre beschrieben.

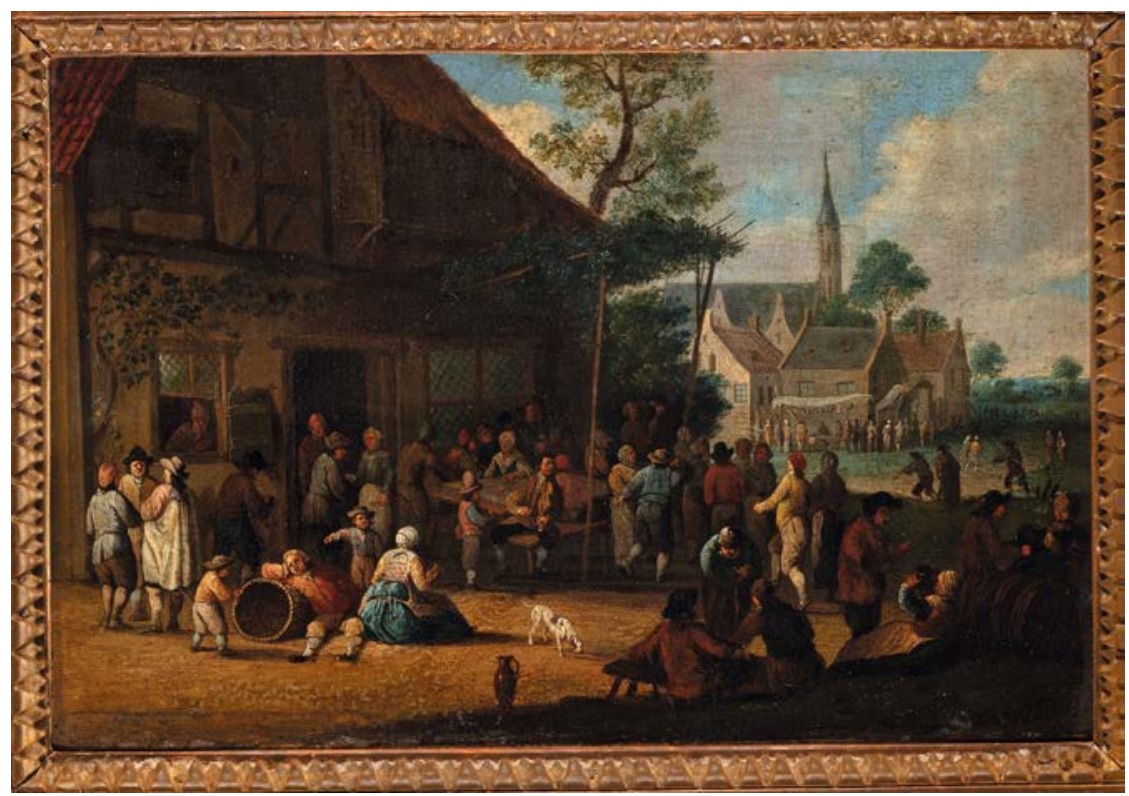

Abb. 2: Franz de Paula Ferg (1689-1740), Kleinstadtmarkt (Dorfszene I), Öl auf Kupfer, 16,8 x $25,2 \mathrm{~cm}^{47}$

\section{Sammlung Pollák in dem Kunstgewerbemuseum in Prag}

Zu den Kunstgegenständen Josef Polláks, die im Kunstgewerbemuseum deponiert wurden, sind wenige archivarische Daten erhalten geblieben, und dies ganz im Unterschied zu den Gemälden Polláks in der Nationalgalerie. Das Protokoll der mündlichen Vereinbarung zwischen der Direktion des Museums und Josef Pollák vom März 1939 ähnelte demjenigen aus der Nationalgalerie: er überstellte dem Museum als Leihgabe in die Verwaltung seiner kunstgewerblichen Sammlungen, besonders Porzellan, Miniaturen und andere Gegenstände der beige-

46 NG, ANG 1945-1958, sk. 231/8 (odkazy věcné), Josef Pollák, die Direktion der NG ersucht das Auktionshaus Obchod klenoty, hodinami a starožitnostmi, n. p. um das Herausnehmen des Werks aus der Auktion, 10.11.1952.

47 Dieses Gemälde aus dem Depositum Pollák musste die Nationalgalerie vor der Auktion retten, weil es versehentlich der Erbin herausgegeben wurde. 
fügten Liste nach. ${ }^{48}$ Die Originalliste der Sammlung ist verloren gegangen. Das Depositum wurde unter der Nummer 1164 registriert. Das erste und einzige vollständige, allerdings nicht datierte Verzeichnis stellte man vermutlich während der Okkupation zusammen, weil es mit dieser Überschrift versehen wurde: Sammlung des Juden Josef Pollák. ${ }^{49}$ Die Liste hat 41 Seiten, auf denen 957 Gegenstände detailliert beschrieben werden, und sie ist in mehrere Teile gegliedert: Miniaturen (1-26; 67-182), Pfeifen (27-66), gemalte Porzellanplastiken (183-268), Porzellanfiguren (269-486), Porzellangefäße (487-626), weiße Biskuit-Büsten (627-749) und Bilder (750-957). Unter der letzten Nummer 958 befinden sich die folgenden Gegenstände: 1.289 Zeichnungen und Aquarelle und zwei Hefte, 1.001 Kupferstiche, 87 Lithografien, 162 Xylografien, 60 Reproduktionen und moderne Grafiken, 13 japanische Leporellos und 102 japanische Farbenholzschnitte. Jeder Gegenstand, von dem letzten Konvolut der Grafiken abgesehen, wurde einfach beschrieben, es folgte die Technik der Herstellung, die Signatur, Porzellanmarke, Autor und Größe. Man kennzeichnete 231 Gegenstände mit roter Farbe und fügte die Notiz „übernommen von Kamila Polláková, 1948“ hinzu. Die bisherige Forschung hat allerdings ergeben, dass zumindest acht Gegenstände, die rot verzeichnet wurden, sich heute in dem Kunstgewerbemuseum befinden, sodass diese Notizen nicht als ausschlaggebend angesehen werden können. ${ }^{50}$

Ein weiteres Dokument ist die vierseitige, auf Tschechisch verfasste Liste der 112 Objekte, die die Vertreter des Kunstgewerbemuseums am 9. Januar 1948 für das Museum aus dem Nachlass Josef Polláks aussuchten. ${ }^{51}$ Die ursprüngliche Nummerierung aus der Liste Sammlung des Juden Josef Pollák ist erhalten geblieben, neu ist der Schätzwert in tschechoslowakischen Kronen. Zehn Stücke haben keine Nummer, weil es sich um die japanischen und chinesischen Grafiken handelt, die der letzten Grafiksammlung unter der Nummer 958 angehören. Diese Leporellos und Farbenholzschnitte befinden sich heute in der Nationalgalerie, weil das Museum in den 1960er Jahren der Galerie angegliedert war und viele Asiatika der Galerie überstellt wurden. ${ }^{52}$

48 Uměleckoprůmyslové museum v Praze (UPM) [Kunstgewerbemuseum in Prag], 1164 S, J. Pollák: Praha X, Kollárova 13, Josef Pollák an die Direktion des Kunstgewerbemuseums in Prag, 09.03. 1939. Diese beigefügte Liste befindet sich nicht mehr in den Dokumenten.

49 UPM, 1164 S, J. Pollák: Praha X, Kollárova 13, Sammlung des Juden Josef Pollák.

50 Die Forscher aus dem Documentation Centre identifizierten die Gegenstände aufgrund der Beschreibung und anderer Zeichen, die später angeführt werden.

51 UPM, 1164 S, J. Pollák: Praha X, Kollárova 13, Verzeichnis der Kunstgegenstände, die am 09.01.1948 für das Kunstgewerbemuseum in Prag aus dem Nachlass des Herrn Josef Pollák ausgesucht wurden.

52 UPM, Př́růstková kniha č. 27 [das Zuwachsbuch Nr. 27], die Inventarnummern 32992-32939 vom 30.05.1952 wurden durch den Auftrag 2884/61 des Direktors aus dem Inventar entfernt und an die Nationalgalerie - Orient übergeben. 


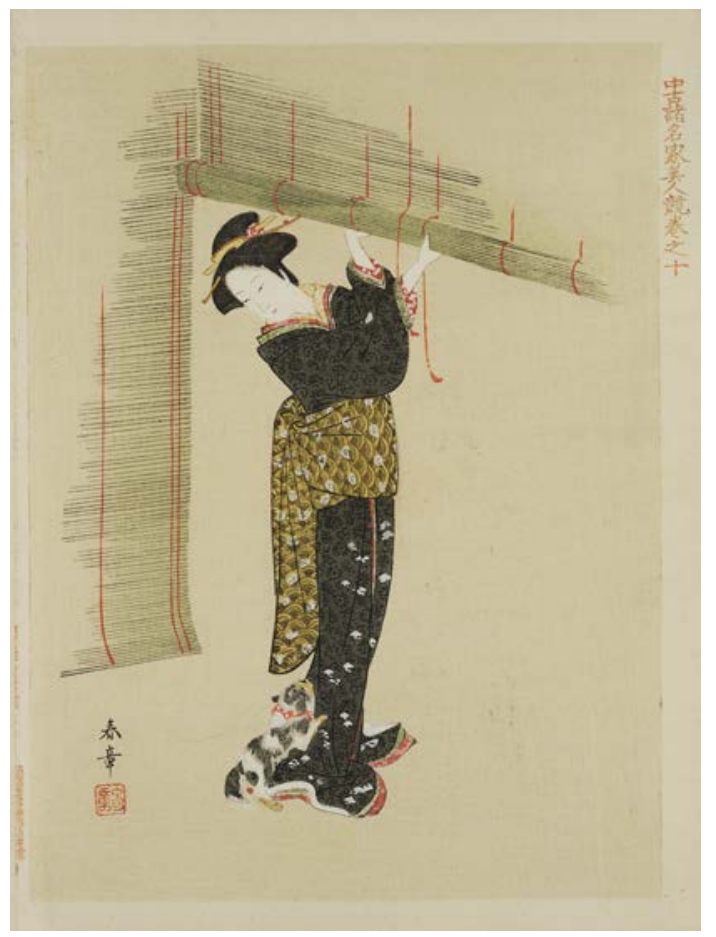

Abb. 3: Japanisches Leporello in hellblauem Brokat mit zwölf handkolorierten Holzdrucken, nach den verschiedenen japanischen Meistern. Sog. Kyoto Drucke, Japanerinnen in verschiedenen Kostümen bei Arbeit und Spielen darstellend. Papier, Brokat, Japan, Ende des 19. Jahrhunderts, $18,5 \times 25 \mathrm{~cm}^{53}$

In der Publikation Návraty paméti wurden im Kunstgewerbemuseum insgesamt 108 Kunstgegenstände Polláks identifiziert: 55 Porzellanplastiken, 25 Büsten, 21 Miniaturen, fünf Porzellanpfeifen, zwei Tassen mit Untertassen und eine Uhr (Porzellan, II. Rokoko). ${ }^{54}$ Wenn man diese Angaben mit dem Vermerk über das Resultat der physischen Inventur aus dem Jahre 1962 vergleicht, wirkt diese Inventur wenigstens komisch. Das ganze Depositum soll angeblich außer sechs Objekten zurückgegeben worden sein. ${ }^{55}$

Die Möglichkeiten der Identifizierung des Depositums sind unterschiedlich. Zuerst ist die Provenienz der Gegenstände in den Zuwachsbüchern des Museums zu finden. ${ }^{56}$ Zweitens erscheint auf vielen Gegenständen die Nummer des

53 Im Jahre 1961 aus dem Kunstgewerbemuseum der Nationalgalerie überstellt.

54 Helena Krejčová, Mario Vlček: Návraty paměti (Anm. 2), S. 168-222.

55 UPM, 1164 S, J. Pollák: Praha X, Kollárova 13, Vermerk über Resultat der physischen Inventur vom 31.12.1962.

56 Die größte Menge der Objekte wurde in dem Zuwachsbuch Nr. 27 (Přírůstková kniha č. 27, 32251-33000, 26.01.1952-18.06.1952) inventarisiert. 
Depositums 1164. Schon in der ersten Welle der Forschung wurde noch eine interessante Kennzeichnung der Sammlung entdeckt: Viele Gegenstände verzeichnete man mit einem kleinen Stempel, der den Davidstern zeigt, wobei dies wahrscheinlich von Josef Pollák selbst gemacht wurde.

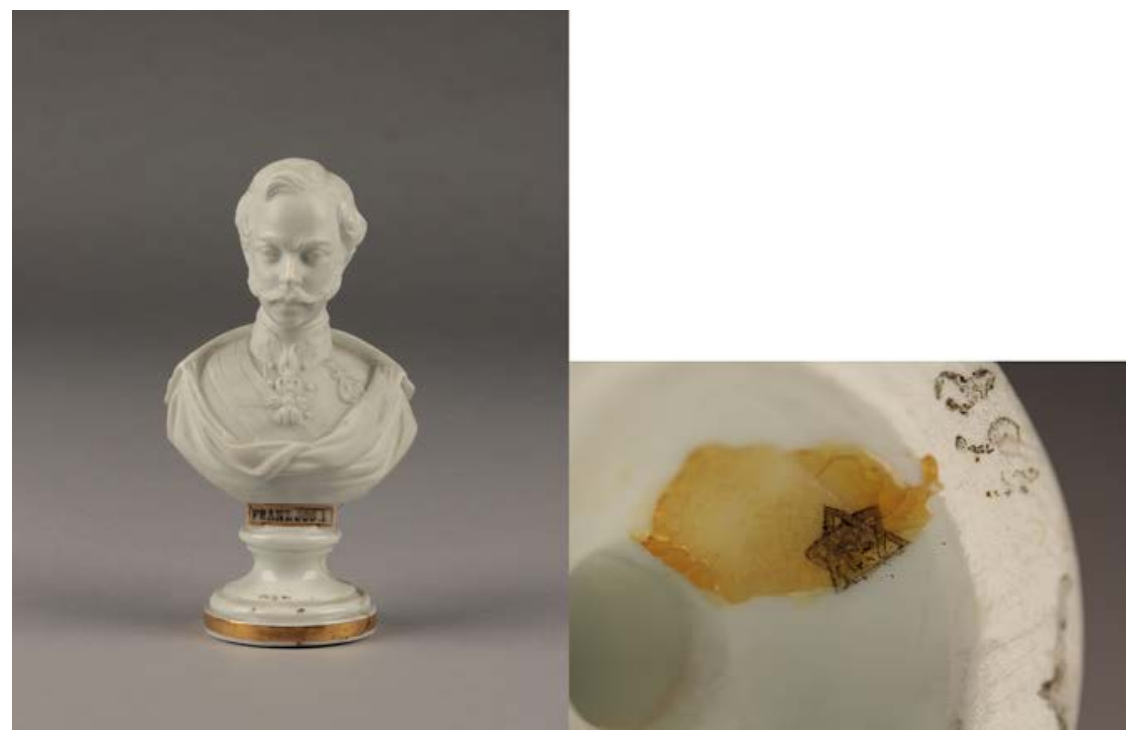

Abb. 4 und 5: Franz Joseph I., 13,5 $\mathrm{cm}^{57}$

In dem Zuwachsbuch wurde angeführt, dass man die Büste bei dem Antiquitätenhändler in Prag II, Mikulandská Straße gekauft hatte. ${ }^{58}$ Es ist jedoch klar, dass sie aus dem Besitz Polláks stammt, weil sich der kleine Davidstern auf der Rückseite befindet.

Die Anführung der falschen Provenienz kann zwei Gründe haben. Entweder war es die Initiative eines Mitglieds der Einkaufskommission, das sich auf diese Weise auf unlauterem Wege Geld dazu verdiente und einen noch nicht inventarisierten Gegenstand aus der Sammlung Pollák für den eingekauften ausgab. Die zweite Begründung ist nicht sehr wahrscheinlich, aber dennoch möglich: Kamila Polláková übernahm den Gegenstand und sie oder ihre Tochter verkauften ihn an den Antiquitätenhändler, von welchem ihn das Museum zurückgewann. Einen sehr ähnlichen Fall der falschen Provenienz stellt die Miniatur von dem böhmischen Miniaturmaler Quast dar.

57 UPM, 1164 S, J. Pollák: Praha X, Kollárova 13, „Sammlung des Juden Josef Pollák“, S. 28, Nr. 719. Die angeführte Beschreibung der Büste stammt aus der Liste „Sammlung des Juden Josef Pollák“. Diese Büste wurde zudem in der Liste rot verzeichnet, was vermuten lässt, dass das Museum den Gegenstand an Kamila Polláková im Jahre 1948 übergab.

58 Př́růstková kniha č. 28, Inventarnummer 33408, 15.10.1952. 


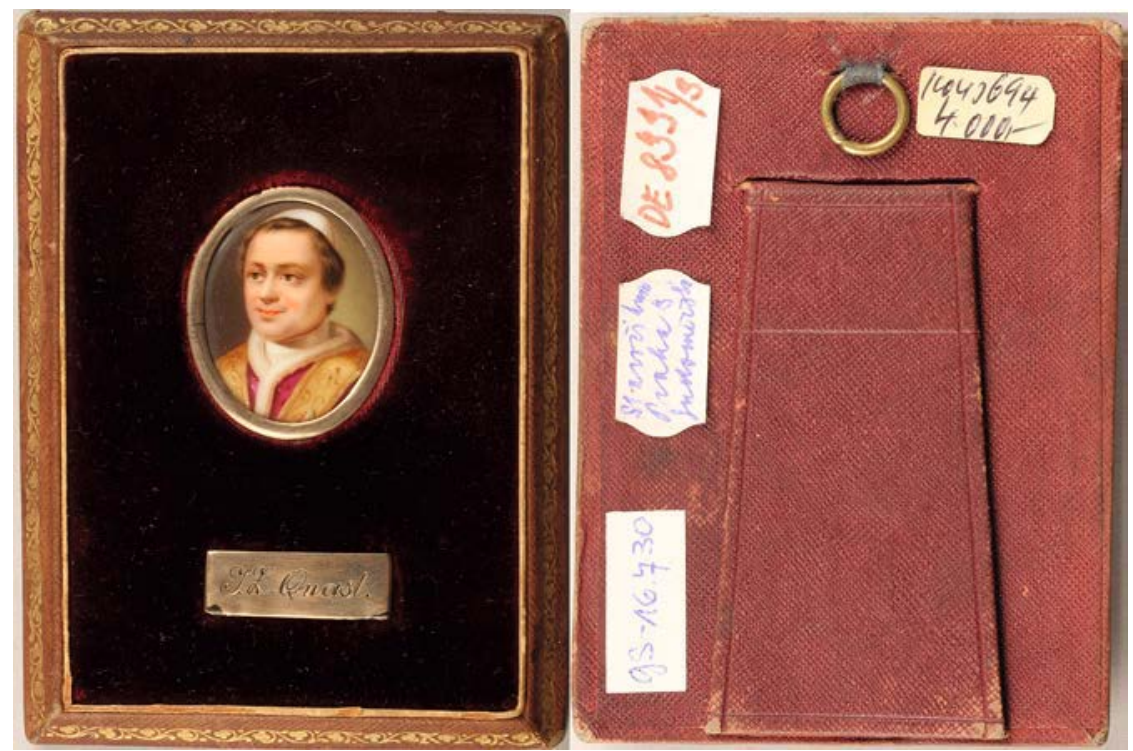

Abb. 6 und 7 : Büste des Papstes Pius IX. in Samtrahmen mit Schild: J. Z. Quast, Oval, Johann Zacharias Quast (1814-1891), 7 × $9 \mathrm{~cm}^{59}$

Auch wenn die Identifizierung der Kunstwerke aufgrund der bloßen Beschreibung fast unmöglich und manchen Kunsthistorikern nach auch dilettantisch erscheinen mag, gibt es in den Fällen mit der fehlerhaft bestimmten Provenienz keine andere Möglichkeit. Selbstverständlich kann man nicht z.B. die Porzellanfiguren derart identifizieren, aber wenn es sich um eine Zeichnung mit Signatur und genauen Maßangaben handelt, hat man eine sichere Methode zur Hand.

Wenn man das Schicksal des Depositums Pollák in der Nationalgalerie mit dem im Kunstgewerbemuseum vergleicht, kann es derart wirken, dass die Galerie mit den Gegenständen besser als das Kunstgewerbemuseum umging. Andererseits hängt der Umgang natürlich auch mit der Größe des Depositums und mit dem Wert der Einzelstücke zusammen. Ein ausführlicherer Katalog, wo die Unterschiede noch deutlicher gekennzeichnet werden, wird derzeit von den Forschern des Prager Documentation Centre zusammengestellt.

59 UPM, 1164 S, J. Pollák: Praha X, Kollárova 13, Sammlung des Juden Josef Pollák, S. 5, Nr. 127. Diese Miniatur wurde den Zetteln auf der Rückseite nach bei dem Antiquitätenhändler in Prag 3., Sudoměřská Straße für 4.000 Kronen gekauft, aber die Beschreibung und der kaum sichtbare Davidstern deuten mit ziemlicher Sicherheit darauf hin, dass es sich um den ehemaligen Besitz Polláks handelt. 


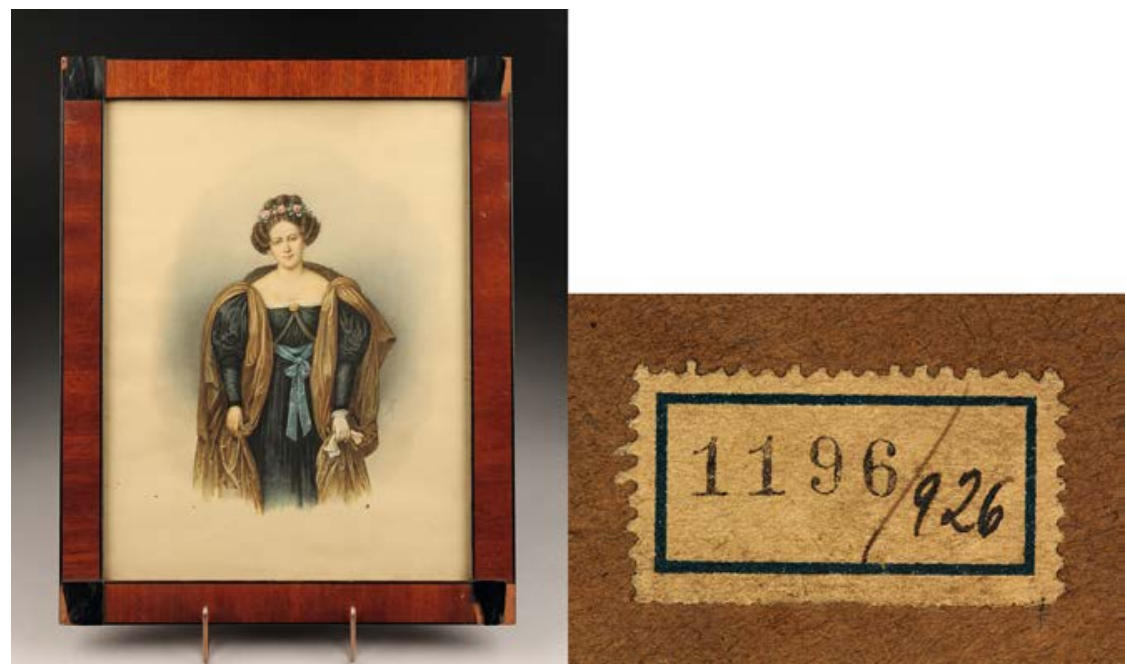

Abb. 8 und 9: Bildnis einer älteren Dame in breitem Mantel auf Papier, brauner Holzrahmen, Rechteck, sign. A. Bock ${ }^{60}, 30 \times 37 \mathrm{~cm}^{61}$

60 Wahrscheinlich Adolf Bock (1890-1968).

61 UPM, 1164 S, J. Pollák: Praha X, Kollárova 13, Sammlung des Juden Josef Pollák, S. 8, Nr. 180. Der Gegenstand wurde hier irrtümlich unter dem Depositum 1196 vermerkt, wobei er in Wirklichkeit in das Depositum 1164 gehört; in diesem Falle wurde das Bild aufgrund der Beschreibung, der Signatur und der Maßangaben identifiziert. 
Open-Access-Publikation im Sinne der CC-Lizenz BY-NC-ND 4.0

(c) 2018, V\&R unipress GmbH, Göttingen

ISBN Print: 9783847107835 - ISBN E-Lib: 9783737007832 


\title{
"Treuhänderische“ Übergaben von Kunstwerken an die Österreichische Galerie im Kontext der aktuellen Provenienzforschung
}

\section{Zusammenfassung}

Abgesehen von der Übernahme von 45 Kunstwerken als bedingtes Eigentum aus dem sogenannten Mauerbach-Bestand wurden der Österreichischen Galerie ab 1961 Werke aus dem ehemaligen Besitz von NS-Funktionären wie Martin Bormann und Joachim von Ribbentrop zur Übernahme in das Museums-Inventar zugewiesen. Im Fokus der aktuellen Provenienzforschung stehen jene 70 Objekte, die 1963 aus den Beständen des Sonderauftrag Linz in treuhändige Verwahrung und 1965 auch definitiv inventarisch übernommen wurden. Anhand exemplarischer Fälle von erfolgten Restitutionen auf der Grundlage des Kunstrückgabegesetzes soll der problematische Umgang mit der Herkunft dieser Kunstwerke in der Nachkriegszeit thematisiert werden.

Schlagwörter

Mauerbach-Bestand, Sonderauftrag Linz, 1963, Österreichische Galerie Belvedere

\begin{abstract}
"Fiduciary" Transfers of Works of Art to the Austrian Gallery Belvedere in the Context of Current Provenance Research

Apart from the transfer of 45 works of art as conditional property from the so-called Mauerbach inventory, works from the former holdings of NS-functionaries such as Martin Bormann and Joachim von Ribbentrop were entrusted to the Austrian Gallery Belvedere and allocated to the museum's inventory. Those 70 objects, which were at first transferred from the inventory of the Sonderauftrag Linz to fiduciary deposit in 1963 and were definitely inventoried in the year 1965, are at the focus of current provenance research. Based on exemplary cases of effected restitutions according to the Art Restitution Law the problematic dealing with the provenance of these works of art in the Post-War Era will be discussed.
\end{abstract}

Keywords

Mauerbach Stock, Sonderauftrag Linz, 1963, Austrian Gallery Belvedere 
Symptomatisch für den problematischen Umgang mit entzogenem Kunst- und Kulturgut in der Nachkriegszeit erscheint der folgende Aktenvermerk des Unterrichtsministeriums vom Jänner 1948 zur Rückstellungscausa Mahler-Werfel ${ }^{1}$ :

„Rechtsanwalt Dr. Hein hat als Vertreter der Frau Alma Mahler Werfel am 19. d. M. bei Min. Rat Dr. Wisoko vorgesprochen und darauf aufmerksam gemacht, dass infolge der Stellungnahme der Finanzprokuratur in dem Rückstellungsverfahren, in der die Frage des deutschen Eigentums in Österreich eine wesentliche Rolle spielt, die Gefahr besteht, dass die amerikanische Besatzungsmacht die ganze Frage aufrollt und eine neuerliche Überprüfung aller Erwerbungen der öffentlichen Museen während der Zeit von 1938-1945 angeordnet wird und dadurch den Museen und darüber hinaus der Republik Österreich schwere Nachteile erwachsen könnten. “2

Im Sinne einer Aufarbeitung von Versäumnissen und Fehlentwicklungen in der Restitutionspolitik in Österreich nach 1945 erfolgt seit 1998 an den österreichischen Bundesmuseen eine umfassende Untersuchung sämtlicher Erwerbungen, die während der NS-Zeit und nach dem Zweiten Weltkrieg getätigt wurden. ${ }^{3}$

Dabei erweist sich erneut das besondere Ausmaß und die Planmäßigkeit des NS-Kunstraubes in Österreich bzw. die unsensible und unreflektierte Haltung in der Erwerbungspolitik der Museen auch nach 1945. Fragen nach der Provenienz der Kunstwerke und damit die Möglichkeit eines vorangegangenen Vermögensentzuges blieben meist vollständig ausgeblendet.

Wie sehr dies auch jene Kunstwerke betrifft, die ab den 1960er Jahren zunächst treuhänderisch der Österreichischen Galerie übergeben wurden, soll im Folgenden ausgeführt werden.

Bereits 1961 waren 13 Kunstwerke „aus dem Besitz des ehem. Reichsleiters

1 Zur Rückstellungscausa Mahler-Werfel siehe Michael Wladika: „Ersuche ich daher, ... in keiner Weise Frau Alma Mahler-Werfel entgegenzukommen." Alma Mahler-Werfel im Rechtsstreit mit der Republik Österreich. In: Enteignete Kunst. Raub und Rückgabe. Österreich von 1938 bis heute. Hg. von Verena Pawlowsky, Harald Wendelin. Wien: Mandelbaum Verlag 2006, S. 79-103. Am 09.05.2007 wurde Edvard Munchs Gemälde Sommerabend am Strand aus dem Bestand der Österreichischen Galerie Belvedere entsprechend der Empfehlung des Kunstrückgabebeirates vom 08.11.2006 an die Enkeltochter Alma Mahler-Werfels restituiert. Siehe URL: http://www.provenienzforschung.gv.at/beiratsbeschluesse/MahlerWerfel_Alma_2006-11-08.pdf (abgerufen am 27.07.2017).

2 Österreichisches Staatsarchiv (ÖStA), Archiv der Republik (AdR), Bundesministerium für Unterricht (BMU), Sammelmappe 141, Alma Mahler-Werfel, Aktenvermerk des Unterrichtsministeriums, 22.01.1948.

3 Siehe dazu Ingo Zechner: Zweifelhaftes Eigentum. Fußnoten zur Kunstrestitution in Österreich. In: NS-Kunstraub in Österreich und die Folgen. Hg. von Gabriele Anderl, Alexandra Caruso. Innsbruck: Studienverlag 2005, S. 235-246; ... wesentlich mehr Fälle als angenommen. 10 Jahre Kommission für Provenienzforschung. Hg. von Gabriele Anderl, Christoph Bazil, Eva Blimlinger, Oliver Kühschelm, Monika Mayer, Anita Stelzl-Gallian und Leonhard Weidinger. Wien-Köln-Weimar: Böhlau Verlag 2008 (=Schriftenreihe der Kommission für Provenienzforschung 1). 
Martin Bormann [...] der Österreichischen Galerie zur Übernahme in das do. Inventar zugewiesen ${ }^{\text {“4 }}$ worden. ${ }^{5}$ Die 1951 in Bad Gastein sichergestellten Gemälde u.a. von Friedrich Loos und Rudolf von Alt waren nach dem Vermögensverfall an die Republik Österreich im Jahr 1953 vom Bundesdenkmalamt im Depot der Salzburger Residenz in Verwahrung genommen worden. ${ }^{6}$

Ab 1964 erfolgten Zuweisungen von insgesamt 84 Objekten aus der Sammlung des ehemaligen Reichsaußenministers Joachim von Ribbentrop. ${ }^{7}$ Nach der Einlagerung der Bestände der Sammlung Ribbentrop 1949 bei der Property Control Salzburg war es 1951 zum Vermögensverfall nach dem Zweiten Rückstellungsgesetz gekommen. Die Provenienz eines Großteils dieser Werke kann im Sinne des Kunstrückgabegesetzes als unbedenklich bewertet werden: etliche waren auf der Großen Deutschen Kunstausstellung in München gezeigt worden und waren im Sinne einer NS-Kunstförderung von dem bzw. für den führenden Funktionär erworben worden.

Im Fokus der Provenienzforschung stehen jene 70 Kunstwerke, die 1963 aus den Beständen des Sonderauftrag Linz in treuhändige Verwahrung des Museums gelangten und 1965 definitiv inventarisch übernommen wurden. Anhand exemplarischer Fälle von erfolgten Restitutionen auf der Grundlage des Kunstrückgabegesetzes soll der problematische Umgang mit der Herkunft dieser Kunstwerke in der Nachkriegszeit thematisiert werden. Sophie Lillie hat in Bezug auf den sogenannten Mauerbach-Bestand ${ }^{8}$ zu Recht auf „das eklatante Versäumnis [hingewiesen], Objekte trotz Wissens um deren Herkunft nicht an die

4 Archiv der Österreichischen Galerie Belvedere (ÖG), Zl. 760/1961, Unterrichtsministerium an die Leitung der Österreichischen Galerie, 11.10.1961.

5 Archiv der ÖG, Zl. 760/1961, Übernahmebestätigung der Österreichischen Galerie, 03.11. 1961.

6 Siehe dazu Archiv des Bundesdenkmalamtes (BDA), Restitutionsmaterialien, Karton 31/3, Martin Bormann. Ein Gemälde aus dem Bormann-Bestand, Rudolf von Alts Prager Veitsdom, war bereits 1955 an die ehemalige Eigentümerin Elsa Gall restituiert worden. Siehe dazu Archiv der ÖG, Zl. 525/1955.

7 Die in Verwahrung des Bundesdenkmalamtes in der Residenz in Salzburg untergebrachten Kunstwerke wurden auf der Grundlage eines im August 1961 von Hans Aurenhammer, Kustos der Österreichischen Galerie, erstellten Aufteilungsvorschlags 1964 an die Albertina, das Kunsthistorische Museum und die Österreichische Galerie zugewiesen. Siehe dazu Archiv der ÖG, Zl. 604/1961. Der Großteil der an das Kunsthistorische Museum zugeteilten Werke wurde 1987 der Österreichischen Galerie übergeben. Siehe dazu Archiv des BDA, Restitutionsmaterialien, Karton 44/1, Joachim von Ribbentrop.

8 Der sogenannte Mauerbach-Bestand ist benannt nach dem Aufbewahrungsort in der ehemaligen Kartause Mauerbach in Niederösterreich. Es handelt sich dabei um jenen Restbestand an Kunstobjekten, der von den Alliierten im Jahr 1955 mit der Auflage, die rechtmäßigen EigentümerInnen zu suchen und Rückstellungen zu tätigen, an die Republik Österreich übertragen wurde. 
rechtmäßigen EigentümerInnen zu restituieren“. ${ }^{9}$ Dies betrifft, wie ausgeführt werden soll, auch Zuweisungen der 1960er Jahre.

Die durchgeführten Rückgaben seit 1999 sind Beleg für einen Paradigmenwechsel in der österreichischen Restitutionspolitik. Zum einen ist man von der kollektiven Entschädigung ab den 1960er Jahren ${ }^{10}$ zur individuellen Restitution der Nachkriegszeit zurückgekehrt. Zum anderen wurde erstmals die aktive Rückgabe zum Prinzip gemacht und auf jegliche Antragsfrist verzichtet. Darüber hinaus sind rückgestellte Kunstwerke im Kunstrückgabegesetz ausdrücklich vom Ausfuhrverbot ausgenommen. ${ }^{11}$

Für etliche der 1963 in treuhändige Verwahrung der Österreichischen Galerie übernommenen Objekte wurden bereits Rückgabeempfehlungen des Beirates ausgesprochen. Als Beispiele genannt seien eine Ungarische Landschaft Johann Gualbert Raffalts aus der Sammlung Wally Kulka ${ }^{12}$, vier Apothekenladenschilder Ferdinand Georg Waldmüllers aus der Sammlung Hermann Eissler ${ }^{13}$ und vier Teile eines Deckengemäldes Allegorien der Künste von Eduard Grützner aus der Sammlung Ludwig Mayer ${ }^{14}$. Auf weitere Kunstwerke von Eduard Grützner, Edmund Hellmer und Carl Markó aus den Sammlungen Winter, Lederer und Kraus soll in kurzen Fallstudien detailliert eingegangen werden.

1974, 1980 bzw. 1984 wurden insgesamt 45 Kunstwerke durch das Bundesministerium für Finanzen an die Österreichische Galerie als bedingtes Eigentum übergeben: auf der Grundlage des Ersten Kunst- und Kulturgutbereinigungsgesetz hatte sich das Museum zur Übernahme der im Eigentum des Bundes stehenden Objekte „bereiterklärt“ ${ }^{15}$

Das ergänzende Angebot des Präsidenten des Vereins der Museumsfreunde, Max Allmayer-Beck, „eventuell dringend gebrauchte Objekte aus dieser Masse

9 Sophie Lillie: „Herrenlos“? Die ungeklärte Akte Mauerbach. In: Recollecting. Raub und Restitution. Hg. von Alexandra Reininghaus. Wien: Passagen Verlag 2008, S. 217.

10 Zur Tätigkeit der für erblos gebliebene Vermögenswerte zuständigen Sammelstellen siehe den Beitrag von Michael Wladika in diesem Band.

11 Siehe dazu Eva Blimlinger, Clemens Jabloner: Die Regelung der Kunstrückgabe in Österreich. In: Verantwortung wahrnehmen. NS-Raubkunst - Eine Herausforderung an Museen, Bibliotheken und Archive. Hg. von der Koordinierungsstelle für Kulturgutverluste. Magdeburg: Koordinierungsstelle für Kulturgutverluste 2009 (= Veröffentlichungen der Koordinierungsstelle für Kulturgutverluste 7), S. 203-223.

12 Siehe URL: http://www.provenienzforschung.gv.at/beiratsbeschluesse/Kulka_Wally_200303-11.pdf (abgerufen am 27.07.2017).

13 Siehe URL: http://www.provenienzforschung.gv.at/beiratsbeschluesse/Eissler_Hermann_ 2009-06-24.pdf (abgerufen am 27.07.2017).

14 Siehe URL: http://www.provenienzforschung.gv.at/beiratsbeschluesse/Mayer_Ludwig_ 2009-11-20.pdf (abgerufen am 27.07.2017).

15 Archiv der ÖG, Zl. 618/1974, 719/1979, 895/1984. 
mit Mitteln des Vereins der Museumsfreunde anzuschaffen ", ${ }^{16}$ wurde durch die Direktion der Österreichischen Galerie, „sei es aus Sach-, sei es aus Qualitätsgründen " abgelehnt. ${ }^{17}$

Die aus dem Gewahrsam des Bundesdenkmalamtes übernommenen Werke wurden im handschriftlichen Museumsinventar mit einer M-Signatur $[M=$ Mauerbach] eingetragen. Abgesehen von vier Gemälden u.a. von Josef Danhauser und Ferdinand Georg Waldmüller, die an die ehemaligen EigentümerInnen bzw. deren RechtsnachfolgerInnen restituiert wurden, verblieben die Kunstwerke bis zur Mauerbach-Benefizauktion 1996 in der Verwahrung des Museums. ${ }^{18}$

Im August 1984 wurde das Portrait einer Lesenden Dame im türkischen Kostüm von Friedrich Amerling von der Österreichischen Galerie übernommen und im Inventarbuch unter der Nummer M 6427 eingetragen.

Das Gemälde war mit zwei weiteren Bildern aus der sichergestellten Wiener Kunstsammlung von Wilhelm Freund ${ }^{19}$ im Juni 1939 in das Verzeichnis der national wertvollen Kunstwerke eingetragen worden und verblieb in Verwahrung der Zentralstelle für Denkmalschutz. 1969 wurden Amerlings Lesende Dame in türkischem Kostüm und ein Damenbildnis Hans Canons im Amtsblatt der Wiener Zeitung laut Erstem Kunst- und Kulturgutbereinigungsgesetz als „herrenloses Gut“ publiziert. Im Oktober 1996 erfolgte die Versteigerung von Amerlings Gemälde Die Morgenländerin anlässlich der Mauerbach-Benefizauktion. Dass die Herkunft des Bildes bekannt war, beweist der Eintrag im Auktionskatalog: unter Provenienz ist der Vater Wilhelm Freunds, „Dr. Richard Freund, Vienna“, angeführt. ${ }^{20}$

Sophie Lillie stellte fest, dass dies für die ErbInnen nach Wilhelm Freund „und die vielen KunstsammlerInnen, deren Eigentum der Österreichische Staat wider besseren Wissens zur Versteigerung freigab, [...] einer zweiten Enteignung

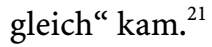

Die Österreichische Galerie hatte 1939/40 zwei Gemälde von Anselm

16 Archiv der ÖG, Zl. 618/1974, Max Vladimir Allmayer-Beck, Verein der Museumsfreunde, an Direktor Hans Aurenhammer, 21.05.1974.

17 Archiv der ÖG, Zl. 618/1974, Hubert Adolph, Kustos der Österreichischen Galerie, an Max Vladimir Allmayer-Beck, Verein der Museumsfreunde, 05.07.1974.

18 Siehe dazu Otto Fritscher: Kontroversen um den „Mauerbach-Schatz“. Die Restitutionsverfahren von 1969 bis 1986. Wien: new academic press 2012.

19 Zur Sammlung Freund siehe Sophie Lillie: Was einmal war. Handbuch der enteigneten Kunstsammlungen Wiens. Wien: Czernin Verlag 2003, S. 368-371; Monika Mayer: Wilhelm Freund. In: Recollecting. Raub und Restitution. Hg. von Alexandra Reininghaus. Wien: Passagen Verlag 2008, S. 239; Monika Mayer: Wilhelm Freund. Unpubliziertes Dossier der Kommission für Provenienzforschung. Wien 2002.

20 Katalog Mauerbach Benefit Sale on Behalf of the Federation of Jewish Communities of Austria. Christie's Wien 1996, S. 230, Lot 517.

21 Lillie: „Herrenlos“? (Anm. 9), S. 220. 


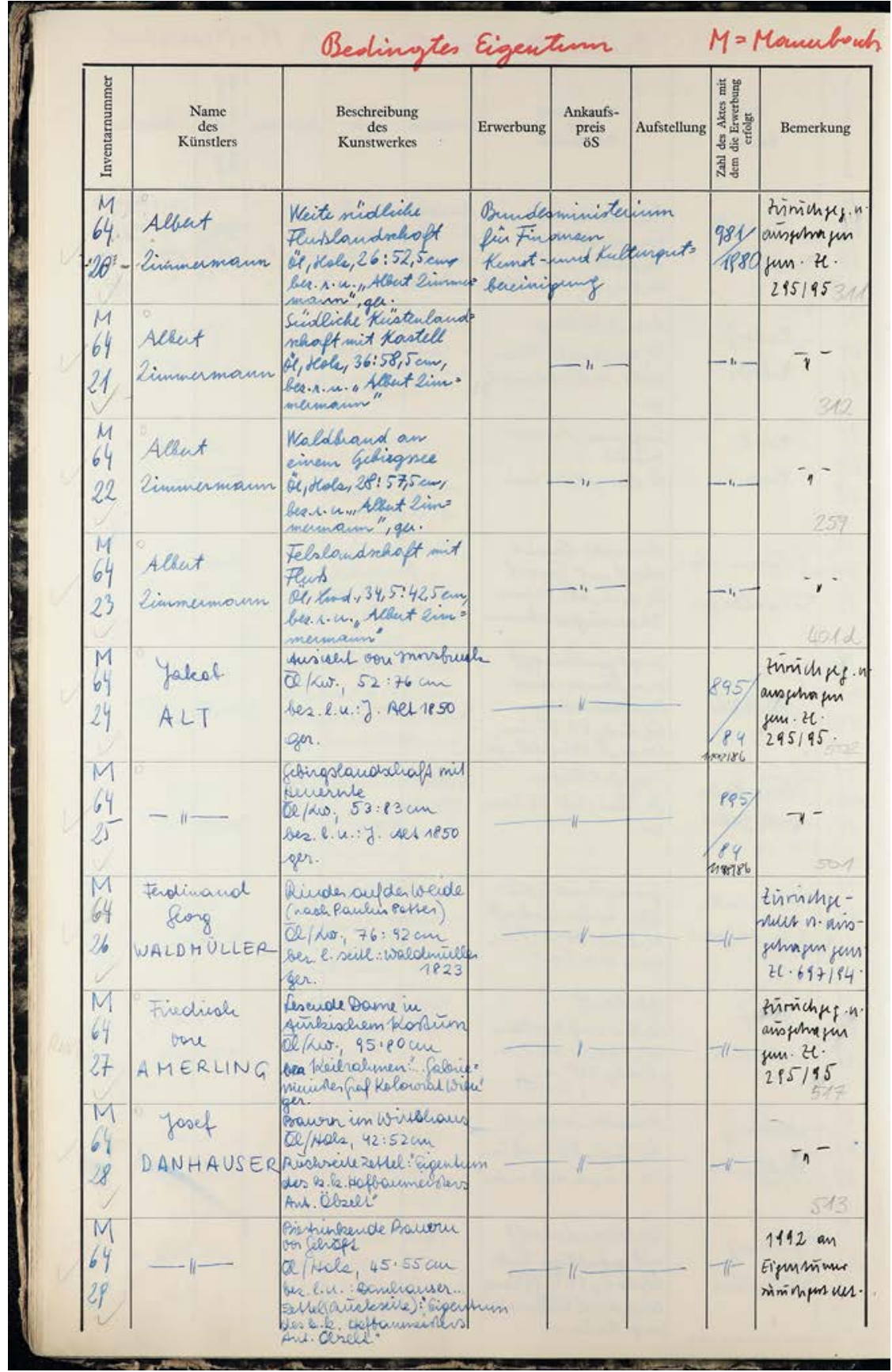

Abb. 1: Inventarbuch der Österreichischen Galerie Belvedere, Wien 
Feuerbach und Anton Romako von Wilhelm Freund, der sich mit „der Entgegennahme der Zahlung auf Sperrkonto einverstanden erklärt ${ }^{\text {“22 }}$, erworben. Betont wurde seitens der Direktion „die ungewöhnliche Gelegenheit, ein Hauptwerk Feuerbachs im Marktwerte von 80.000 RM um wenig mehr als die Hälfte der Summe für den staatlichen Besitz zu sichern" ${ }^{23}$

1946 wurden im Zuge der Vermögensentziehungs-Anmeldungsverordnung die beiden in der NS-Zeit erworbenen Gemälde als „entzogenes“ Vermögen „aus der Sammlung des Herrn Wilhelm FREUND, dzt. London“ deklariert; „ein Rückstellungsanspruch [sei] bisher nicht erhoben “ worden. ${ }^{24}$ Auch die Finanzlandesdirektion stellte in Erwiderung eines Memorandums der Sammelstellen im Jänner 1969 fest, dass es sich bei Anselm Feuerbachs Gemälde Medea „um das einzige von den in Rede stehenden Kunstwerken [handelt], das einwandfrei als entzogenes Vermögen anzusehen wäre. ${ }^{25}$ Es sollte allerdings bis Oktober 2002 dauern, bis der Beirat die Empfehlung zur Rückgabe der beiden Bilder von Feuerbach und Romako aus dem Bestand der Österreichischen Galerie an die RechtsnachfolgerInnen nach Wilhelm Freund aussprach. ${ }^{26}$

Evident ist, dass der österreichische Staat, repräsentiert durch die Musealbzw. Denkmalamtsbürokratie, mögliche Restitutionen von entzogenem Vermögen nicht als Bring-, sondern als Holschuld erachtete. Dies beweist auch der Umgang mit in der NS-Zeit enteigneten oder zwangsweise veräußerten Objekten aus dem Bestand des Sonderauftrag Linz, ${ }^{27}$ die als Verfallsgut beansprucht und 1963 diversen Bundesmuseen zugewiesen wurden. Nach der Auffindung der Kunstwerke in diversen Bergungsorten in Österreich wurden diese 1945 in den Central Collecting Point in München überführt. Ab den späten 1940er Jahren beanspruchte das Denkmalamt die Rückführung dieser Objekte mit der Auflage, deren Restitution an die rechtmäßigen VorbesitzerInnen abzuwickeln. ${ }^{28}$

Es entbehrt nicht einer gewissen Ironie, dass das Denkmalamt als zuständige Behörde sowohl für die Sicherstellung bzw. Enteignung von Kunstwerken ab

22 Archiv der ÖG, Zl. 583/1939, Direktion der Österreichischen Galerie an die Devisenstelle, 11.05.1939.

23 Archiv der ÖG, Zl. 311/1939, Direktion der Österreichischen Galerie an das Ministerium für innere und kulturelle Angelegenheiten, 02.05.1939.

24 Archiv der ÖG, Zl. 318/1946, Fritz Novotny, Interimistischer Direktor der Österreichischen Galerie, an das Magistratische Bezirksamt für den I. Bezirk, 03.12.1946.

25 BMF-GZ 68.042/-16b/69, Finanzlandesdirektion für Wien, Niederösterreich und Burgenland an das Finanzministerium, 29.01.1969; eine Kopie wurde von Dr. Theodor Brückler, ehemaliger Archivar des Bundesdenkmalamts, zur Verfügung gestellt.

26 Siehe URL: http://www.provenienzforschung.gv.at/beiratsbeschluesse/Freund_Wilhelm_ 2002-10-30.pdf (abgerufen am 27.07.2017).

27 Zum Sonderauftrag Linz siehe Birgit Schwarz: Hitlers Museum. Die Fotoalben Gemäldegalerie Linz: Dokumente zum „Führermuseum“. Wien-Köln-Weimar: Böhlau Verlag 2004.

28 Siehe dazu die Beiträge von Alexandra Caruso und Anneliese Schallmeiner bzw. von Leonhard Weidinger in diesem Band. 
1938 als auch für deren Rückstellung nach 1945 mitverantwortlich war und im Archiv entsprechende Aktenbestände vorhanden waren und sind, die eine Identifizierung bzw. Zuordnung ermöglicht hätten.

Rechtsgrundlage für die sogenannten 1963er Zuweisungen war ein Erlass des Unterrichtsministeriums vom 29. Juni 1963 der

„das Bundesdenkmalamt ermächtigt eine Anzahl von Kunstgegenständen, welche seinerzeit für das von Hitler geplante Linzer Kunstmuseum bestimmt waren und in nächster Zeit dem Bundesministerium für Unterricht für die zuständigen Sammlungen des Bundes ressortmäßig übergeben werden, bereits jetzt diesen Sammlungen in treuhändige Verwahrung zu übergeben [...]. Die derzeit in Salzburg eingelagerten Kunstgegenstände werden dann im Laufe des Monats August nach Wien gebracht und können sodann von der do. Sammlung übernommen werden. ${ }^{\text {“29 }}$

Im März 1965 wurden die betroffenen Museen informiert,

„daß sämtliche aus den Beständen des ,Linzer Kunstmuseums` stammenden Objekte, die szt. dem Kunsthistorischen Museum, bzw. anderen Sammlungen des Bundes in Verwahrung übergeben worden sind, nunmehr definitiv in die Inventare aufzunehmen sind“. ${ }^{30}$

Im September 1963 wurde das Gemälde In der Klosterbibliothek von Eduard Grützner aus dem Depot des Bundesdenkmalamtes im Wiener Arsenal an das Kunsthistorische Museum zur treuhändigen Verwahrung übergeben. ${ }^{31}$

Elisabeth Bachofen-Echt hatte das aus der Kunstsammlung ihrer Eltern August und Serena Lederer stammende Bild unter dem Druck der nationalsozialistischen Repression um 1940 „als Treuhänderin“ an die Galerie Sanct Lucas in Wien veräußert. ${ }^{32}$ Trotz eines Feststellungsbescheides der Reichsstelle für Sippenforschung, sie sei eine illegitime Tochter des Malers Gustav Klimt, blieb ihr Vermögen nicht vor den Zugriffen des NS-Regimes geschützt. Aufgrund ihrer prekären finanziellen Situation war Elisabeth Bachofen-Echt gezwungen, vom „Verkauf ihrer Habseligkeiten (zu) leben“. ${ }^{33}$ Im März 1942 wurde der Grützner für den Sonderauftrag Linz um 16.500 RM angekauft.

29 Archiv der ÖG, Zl. 702/1963, Bundesdenkmalamt an die Direktion der Österreichischen Galerie, 17.07.1963.

30 Archiv der ÖG, Zl. 466/1965, Bundesministerium für Unterricht an die Direktion des Kunsthistorischen Museums, 25.03.1965.

31 Archiv des BDA, Restitutionsmaterialien, Karton 31, Bachofen-Echt, Zl. 7308/1963, Aktenvermerk Dr. Otto Demus, 07.11.1963.

32 Zu Elisabeth Bachofen-Echt, née Lederer, siehe Lillie: Was einmal war (Anm. 19), S. 144-148; Monika Mayer: Elisabeth Bachofen-Echt. Unpubliziertes Dossier der Kommission für Provenienzforschung. Wien 2012.

33 Elisabeth Bachofen-Echt, Budapest, an Erich Lederer, Genf, 24.07.1940; eine Kopie aus Privatbesitz wurde von Sophie Lillie zur Verfügung gestellt. 


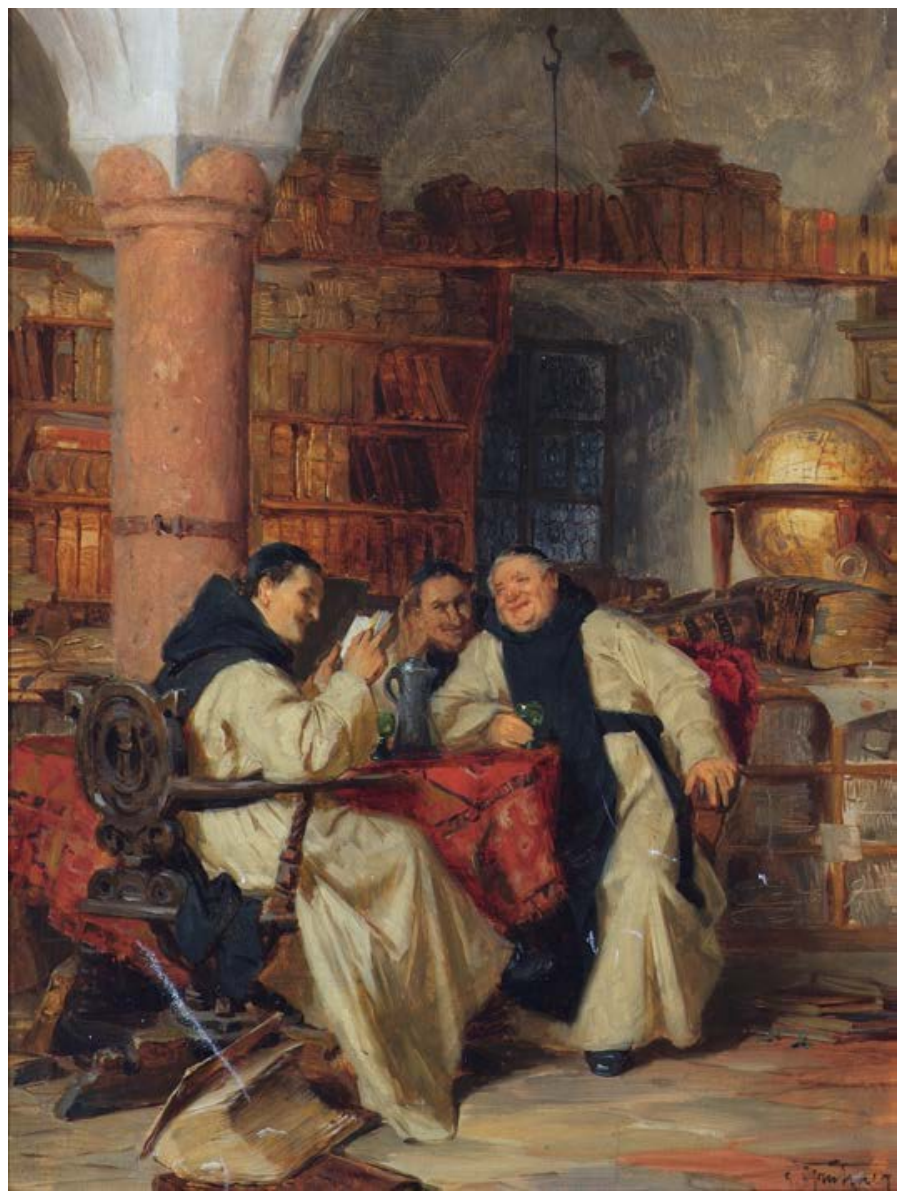

Abb. 2: Eduard Grützner, In der Klosterbibliothek, Österreichische Galerie Belvedere, Wien

Im Entscheid des Bundesamtes für äußere Restitution in Bad Homburg auf Rückführung des Gemäldes an die Republik Österreich vom März 1958 wird wie folgt ausgeführt:

„Die Antragstellerin [Republik Österreich, Anm.] beansprucht Restitution der vorbezeichneten Skizze. Sie stamme aus dem früheren Besitz der Familie Bachofen-Lederer, Wien und habe sich bereits vor dem 12. März 1938 in Österreich befunden. Etwa um das Jahr 1940 habe Frau Lisl Bachofen-Lederer die Skizze verkauft und sich hierbei der Galerie St. Lucas, Wien, als Vermittlerin bedient. Die Antragstellerin hat eine eidesstattliche Erklärung des Herrn Wolfgang Bachofen-Echt vom 23. Januar 1958 [...], worin dieser bestätigt, daß [...] seine Ehefrau sie etwa um das Jahr 1940 verkauft habe. ${ }^{\text {(34 }}$

34 Archiv des BDA, Restitutionsmaterialien, Karton 31, Bachofen-Echt, Zl. 2468/1958, Ausfer- 
In dem Aktenvermerk anlässlich der Einvernahme von Wolfgang Bachofen-Echt im Bundesdenkmalamt im Jänner 1958 zur Identifizierung des Grützner-Gemäldes wird festgehalten,

„dass im Falle einer Rückstellung des gegenständlichen Gemäldes dieselbe an Herrn Erich Lederer, Genf, Hotel Bristol, durchzuführen wäre, da das Bild durch die verstorbene Frau Bachofen-Lederer nach Angabe von Baron Bachofen-Echt [...] nur treuhändig im Auftrage ihrer Eltern verkauft worden sei“ ${ }^{35}$

Der Präsident des Bundesdenkmalamtes, Dr. Otto Demus, informierte in der Folge Wolfgang Bachofen-Echt über die erfolgte Rückführung des Grützner Gemäldes nach Österreich, das sich in der Verwahrung des Bundesdenkmalamtes befand. Demus wies darauf hin, dass die Erben einen Rückstellungsanspruch bei der Finanzlandesdirektion für Wien, Niederösterreich und das Burgenland einbringen könnten. ${ }^{36}$ Offen bleiben muss, warum nicht Erich Lederer, als Rechtsnachfolger nach August und Serena Lederer, über die Möglichkeit eines Rückstellungsantrages offiziell in Kenntnis gesetzt wurde. Dies ist umso bemerkenswerter als Erich Lederer im Zusammenhang mit der angedachten Ausfuhr des Beethovenfrieses von Gustav Klimt seit den späten 1940er Jahren in engem Kontakt mit der Denkmalbehörde stand.

Mit Beschluss des Beirates vom 2. März 2012 wurde die Rückgabe des Grützner-Gemäldes an die RechtsnachfolgerInnen nach Serena Lederer empfohlen, 54 Jahre nach der erfolgten äußeren Restitution der Ölskizze an die Republik Österreich. ${ }^{37}$

Bereits 2002 hatte der Beirat eine Rückgabeempfehlung für mehrere Kunstwerke aus der Sammlung von Gottlieb und Mathilde Kraus ${ }^{38}$ ausgesprochen. ${ }^{39}$ Ein Seestück Carl Markós und eine Landschaft August Pettenkofens aus dem Bestand der Österreichischen Galerie wurden 2004 an die RechtsnachfolgerInnen ausgefolgt.

Ein Referent des Denkmalamtes hatte im Jänner 1923 die Wohnung Kraus in der Wiener Wohllebengasse besichtigt und die Sammlung mit „guten Bildern

tigung des Entscheides des Bundesamtes für äußere Restitution in Bad Homburg, 13.03. 1958.

35 Archiv des BDA, Restitutionsmaterialien, Karton 31, Bachofen-Echt, Zl. 571/1958, Aktenvermerk Erika Doberer, 23.01.1958.

36 Archiv des BDA, Restitutionsmaterialien, Karton 31, Bachofen-Echt, Zl. 571/1958, Briefkonzept Otto Demus, 10.12.1958.

37 Siehe URL: http://www.provenienzforschung.gv.at/beiratsbeschluesse/Lederer_August_Se rena_2012-03-02.pdf (abgerufen am 27.07.2017).

38 Zur Sammlung Kraus siehe Lillie: Was einmal war (Anm. 19), S. 596-600. Monika Mayer: Gottlieb und Mathilde Kraus. Unpubliziertes Dossier der Kommission für Provenienzforschung. Wien 2001.

39 Siehe URL: http://www.provenienzforschung.gv.at/beiratsbeschluesse/Kraus_Gottlieb_ 2002-06-19.pdf (abgerufen am 27.07.2017). 


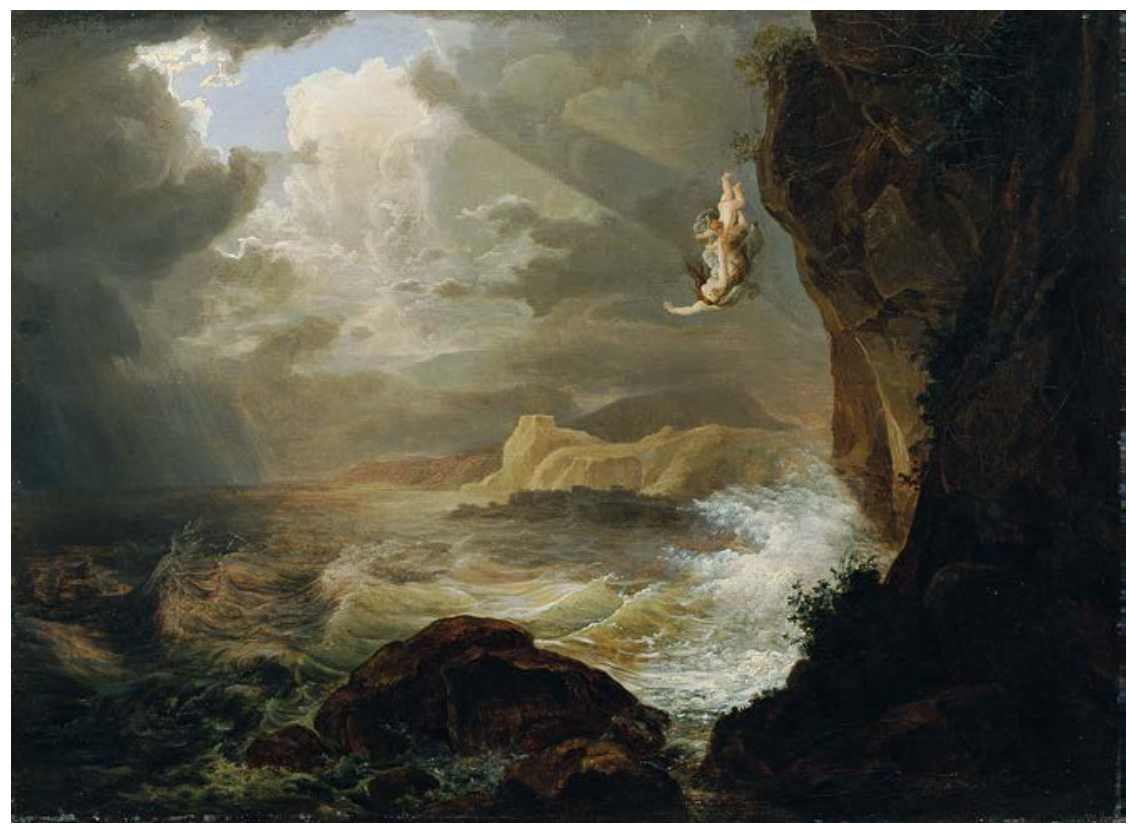

Abb. 3: Carl Markó d. Ä., Seestück, 2004 Rückgabe an die Rechtsnachfolger nach Gottlieb Kraus

von Pettenkofen, Gauermann, Waldmüller, J. Emil Schindler, Ribarz, Rudolf Alt, Marko und modernen Malern" zur öffentlichen Besichtigung gewidmet. ${ }^{40}$ Das gesamte Vermögen des aus rassistischen Gründen aus Österreich vertriebenen Ehepaares Kraus wurde 1941 mit Verfügung der Gestapo beschlagnahmt; darunter auch die bei der Spedition Kirchner \& Co. deponierte Kunstsammlung. Im Dezember 1941 übernahm das Institut für Denkmalpflege aus dem Speditionslager zwölf Kunstwerke zur fotografischen Dokumentation. Auf der im Archiv des Bundesdenkmalamtes überlieferten Übernahmeliste findet sich der Vermerk: „Beschlagnahmungen der Vugesta Wien, I. Bauernmarkt“ ${ }^{41}$

In der Folge erwarb die Österreichische Galerie laut Schreiben an die Vugesta $^{42}$ vom 14. April 1942 das Gemälde Zigeunergespann von Pettenkofen um 3.000 RM. ${ }^{43}$ Auch dieses Werk wurde 1946 von der Direktion des Museums nach

40 Archiv des BDA, Zl. 66/1923, Wohnungsanforderungsakten, Gottlieb Kraus.

41 Archiv des BDA, Restitutionsmaterialien, Karton 8/1, Kraus, Liste der aus dem Lager Kirchner übernommenen Bilder, 19.12.1941.

42 Die Vugesta war die Verwaltungsstelle für jüdisches Umzugsgut der Gestapo.

43 Archiv der ÖG, Zl. 134/1942, Abschrift der Rechnung der Vugesta an die Österreichische Galerie, 14.04.1942. 
der Vermögensentziehungsanmeldungsverordnung mit dem Vermerk „Unbekannter Eigentümer" und als Ankauf von der Vugesta angemeldet. ${ }^{44}$

Das für das Führermuseum in Linz bestimmt gewesene Gemälde von Markó befand sich unter den 1948 von US-Behörden von München nach Salzburg ins Residenzdepot rückgeführten Kunstgegenständen unbekannter Eigentümer aus Vugesta-Erwerbungen. Als eines jener der Republik Österreich verfallenen Kunstwerke wurde Markós Seestück 1963 von der Österreichischen Galerie in treuhändige Verwahrung übernommen. Ein Blick nicht nur in die Aktenbestände des Denkmalamtes, sondern auch auf die Bildrückseiten hätte bereits in der Nachkriegszeit eine Zuordnung zur Sammlung Kraus ermöglicht. Auf der Rückseite befanden sich Klebeetiketten der Spedition Kirchner \& Co. mit der Chiffre 590 als Namenskürzel, das aufgrund von Dokumenten der Familie Kraus zugeordnet werden kann.

Offen bleiben muss, warum das in den Akten als „Vugesta-Erwerbung“ geführte Seestück von Carl Markó als angeblich erb- bzw. herrenloses Kunstwerk nicht an die Sammelstellen gelangte.

Für drei weitere aus der Sammlung Kraus stammende Werke von Rudolf von Alt, Emil Jakob Schindler und Ferdinand Georg Waldmüller, die 1942 über die Vugesta an den Sonderauftrag Linz verkauft wurden, hatte die Sammelstelle A 1961 einen Rückstellungsantrag gestellt. Das Bundesdenkmalamt stellte dazu fest, dass „nach Ansicht der Finanzprokuratur eine Erledigung zu erwarten [ist], nach welcher die erwähnten Bestände möglicherweise der Sammelstelle zu übergeben sein werden. “45

Die Familie Kraus hatte bereits im Jahr 1947 ihren Wiener Rechtsanwalt beauftragt „alle Schritte zu unternehmen, welche notwendig sein mögen, um unser unbewegliches oder bewegliches Vermögen in Österreich wiederzuerlangen “ ${ }^{46}$ 1956 wurde ein Antrag auf Rückstellung von drei Bildern aus der Sammlung Kraus an die Neue Galerie in Graz gerichtet und dabei explizit als entzogenes Vermögen auch August Pettenkofens Zigeunerwagen am Wasser angeführt, das 1942 von der Österreichischen Galerie erworben worden war.

Als abschließende Fallstudie einer 1963er Überweisung soll Edmund Hellmers Beethovenbüste aus der Sammlung von Josefine Winter ${ }^{47}$ vorgestellt

44 Archiv der ÖG, Zl. 318/1946, Dr. Fritz Novotny, Interimistischer Direktor der Österreichischen Galerie, an das Magistratische Bezirksamt für den I. Bezirk, 03.12.1946 (Anm. 24).

45 Archiv des BDA, Restitutionsmaterialien, Karton 13,1, Mappe 10, Zl. 5.157/1962, undatierter Entwurf des Schreibens des Bundesdenkmalamtes an das Bundesministerium für Finanzen.

46 ÖStA, AdR, Bundesministerium für Finanzen (BMF), Akten der Finanzlandesdirektion für Wien, Niederösterreich und Burgenland (FLD), Gottlieb Kraus, Zl. 19.633.

47 Zur Sammlung Winter siehe Lillie: Was einmal war (Anm. 19), S. 1298-1330; Monika Mayer: Josefine Winter. Unpubliziertes Dossier der Kommission für Provenienzforschung. Wien 2004. 
werden. Die von Josef von Winter vom Künstler selbst erworbene Büste befand sich 1938 nachweisbar im Besitz seiner Witwe Josefine Winter. 1943 wurde diese durch den Sonderauftrag Linz von Michael Auner um 15.000 RM erworben. Josefine Winter war als Jüdin der NS-Verfolgung ausgesetzt und kam 1943 nach der Deportation nach Theresienstadt ums Leben.

Aus den Ausfolgeansuchen von Marianne Nechansky, der Tochter Josefine Winters, an das Bundesdenkmalamt ab 1947 bzw. aus dem Antrag von Konrad Nechansky auf Herausgabe der Beethovenbüste nach dem Zweiten Kunst- und Kulturgutbereinigungsgesetz im Jahr 1986 ist die Eigentumsübertragung in der NS-Zeit nicht rekonstruierbar.

Wie dem Schreiben der Finanzlandesdirektion an Konrad Nechansky vom 10. April 1987 zu entnehmen ist, kam Nechansky „dem Ersuchen der Prüfstelle, die zur Klärung des Sachverhaltes erforderlichen ergänzenden Angaben zu machen sowie Beweismittel vorzulegen, nicht nach" ${ }^{48}$

Grundsätzlich lässt sich eine Entziehung der Büste durch die NS-Bürokratie nach dem März 1938 quellenmäßig nicht belegen; auszugehen ist von einem verfolgungsbedingten Verkauf der Büste an Michael Auner durch Josefine Winter oder deren Angehörige, die als „Juden“ den rassistischen Repressionen des NS-Regimes ausgesetzt und zur Leistung der Judenvermögensabgabe gezwungen waren.

Der Beirat führte entsprechend in seinem Beschluss vom 16. März 2005 aus, dass eine derartige Kaufvereinbarung laut Nichtigkeitsgesetz aus dem Jahr 1946 ein nichtiges Rechtsgeschäft sei. „Es ist somit mit Sicherheit davon auszugehen, dass der in Rede stehende Kunstgegenstand rückzustellen gewesen wäre. “49

Zum Schluss kommend sei noch einmal Sophie Lillie zitiert:

„Die Kunst- und Kulturgüterbereinigungsgesetze erfolgten zu spät, zu schleppend und nur unter Druck ausländischer Presse; sie waren gekennzeichnet von halbherzigen Recherchen, dem bewussten Negieren berechtigter Rückstellungsansprüche und einer tiefliegenden Missachtung der Opfer der NS-Verfolgung. ${ }^{\text {“50 }}$

Die auf Grundlage des Kunstrückgabegesetzes durchgeführten Recherchen müssen als später, aber umso wichtigerer Versuch einer längst fälligen Aufarbeitung jahrzehntelanger Versäumnisse gewertet werden, die auch eine Hinterfragung der institutionellen Sammlungspolitik und die Involvierung von $\mathrm{Mu}$ seumsexperten impliziert. Dank der Provenienzforschung gelang in zahlreichen Fällen eine Rückgabe von in der NS-Zeit entzogenen Kunstwerken.

48 ÖStA, AdR, BMF, FLD, Akten zum 2. Kunst- und Kulturgutbereinigungsgesetz, Zl. 10242, Finanzlandesdirektion an Konrad Nechansky, 10.04.1987.

49 Siehe URL: http://www.provenienzforschung.gv.at/beiratsbeschluesse/Winter_Josefine_ 2005-03-16.pdf (abgerufen am 27.07.2017).

50 Lillie: „Herrenlos“? (Anm. 9), S. 221. 
Open-Access-Publikation im Sinne der CC-Lizenz BY-NC-ND 4.0

(c) 2018, V\&R unipress GmbH, Göttingen

ISBN Print: 9783847107835 - ISBN E-Lib: 9783737007832 


\title{
Die „1963er Zuweisungen“ an das Kunsthistorische Museum. Einige Fallbeispiele
}

\begin{abstract}
Zusammenfassung
Von 26 Kunstgegenständen, die ursprünglich für den „Sonderauftrag Linz“ bestimmt gewesen waren und die 1963 durch das Österreichische Ministerium für Unterricht überwiesen wurden, befinden sich heute neun Gemälde und vier Kunstkammer-Objekte im Inventarbestand des Kunsthistorischen Museums. Anhand ausgewählter Fallstudien soll der Stand der Provenienzforschung gezeigt und auf VorbesitzerInnen, Quellenlage und Problematiken verwiesen werden. Breiten Raum nimmt dabei der Bremer Kunsthändler Johannes Jantzen ein, der für die Bremer Werkschau tätig war, aber auch andere Akteure wie der Kunsthändler Ferdinand Spany, die Galerie L.T. Neumann und die Galerie St. Lucas werden kurz vorgestellt. Mit einem Bild aus der Sammlung Eissler wird schließlich ein Fall beleuchtet, der vor dem Kunstrückgabebeirat behandelt wurde.
\end{abstract}

Schlagwörter

Kunsthistorisches Museum, „Sonderauftrag Linz“, staatliche Zuweisung 1963

\begin{abstract}
The State Assignments in 1963 to the Kunsthistorisches Museum. Some Case Studies In the year 1963, the Austrian Ministry of Education passed 26 works of art, which originally had been purchased from the "Sonderauftrag Linz", to the Kunsthistorisches Museum. Nowadays there are nine paintings and four Art Chamber objects in the inventory of the museum. On the basis of selected case studies, the state of provenance research as well as previous owners, sources and specific problems will be shown. The Bremen art dealer Johannes Jantzen, who worked for the Bremer Werkschau, will be the focus of this paper, but other art dealers, such as Ferdinand Spany, the gallery L.T. Neumann and the gallery St. Lucas will also be presented. Finally, the case of a painting from the Eissler Collection will be introduced, which has already been discussed before the advisory board.
\end{abstract}

Keywords

Kunsthistorisches Museum, ,Sonderauftrag Linz', State Assignment 1963 
Wie in mehreren Beiträgen in diesem Band dargestellt, ermächtigte das Bundesministerium für Unterricht mit Erlass vom 29. Juni 1963 das Bundesdenkmalamt, Kunstgegenstände, die für „das von Hitler geplante ,Führermuseum in Linz' bestimmt waren“, den Sammlungen des Bundes zur treuhändigen Verwahrung zu übergeben, da zwei Depoträume geräumt werden mussten. ${ }^{1}$ Wie Birgit Schwarz in ihren Forschungsarbeiten über den NS-Kunstraub und die Museumspolitik zeigt, erwarb der „Sonderauftrag Linz“ nicht nur für das geplante „Linzer Museum“, sondern auch für kleinere Museen. ${ }^{2}$ Anhand der Fallbeispiele aus dem Kunsthistorischen Museum, die in diesem Beitrag vorgestellt werden, wird dies deutlich, da diese Kunstwerke oft nicht von besonders herausragender Qualität waren. Heute werden sie zum allergrößten Teil im Zentraldepot verwahrt, ein einziges Bild, nämlich die Hl. Maria Magdalena von Nicolas Régnier, wird in der Dauerausstellung gezeigt.

Im Jahr 1963 kamen so insgesamt 22 Bilder, drei Bronzefiguren und ein Terrakottarelief aus den verschiedenen Depots in die Gemäldegalerie bzw. an die Sammlung für Plastik und Kunstgewerbe des Kunsthistorischen Museums. Zwei Jahre später wurden mit Ministeriumserlass diese Objekte auch inventarisch übernommen. ${ }^{3}$ Heute befinden sich 13 Kunstgegenstände im Inventarbestand des Kunsthistorischen Museums, alle anderen wurden 1986 an das Belvedere abgegeben. ${ }^{4} \mathrm{Zu}$ zwei Objekten gibt es keine Hinweise auf die Vorprovenienz. Dies betrifft das Gemälde mit der Inventarnummer GG 9574 (siehe Abbildung 1). In der Museumsdatenbank wird es als Das Jüngste Gericht, Zuschreibung Holländisch geführt. ${ }^{5}$ In der Datenbank „Sammlung des Sonderauftrages Linz“ des Deutschen Historischen Museums („Linz-Datenbank“) wird es unter diesem Titel jedenfalls nicht gelistet, ob es ident mit jenem Gemälde ist, das in der Datenbank als Die Verdammnis eingetragen ist ${ }^{6}$, kann erst durch eine Rückseitenautopsie verifiziert werden.

1 Vgl. Kunsthistorisches Museum (KHM) Archiv, 9/VI/Gal/1963, Tripp/BDA an die Direktion des KHM, 17.07.1963. Vgl. auch den Beitrag von Alexandra Caruso und Anneliese Schallmeiner in diesem Band.

2 Birgit Schwarz: Hitlers Museum. Die Fotoalben Gemäldegalerie Linz. Dokumente zum „Führermuseum“. Wien-Köln-Weimar: Böhlau 2004.

3 Vgl. KHM-Archiv, 41/GG/98, Schütz an die Generaldirektion, 09.07.1998. Vgl. auch die Inventareinträge zu GG 9565-GG 9580. Der Vermerk, dass die Bilder für das geplante „Führermuseum" in Linz bestimmt gewesen seien, ist wohl zu kurz gegriffen.

4 Vgl. KHM-Archiv, 21b/GG/1986.

5 Eintrag in der Museumsdatenbank TMS (The Museum System) zu GG 9574.

6 Abfrage „Die Verdammnis“. URL: https://www.dhm.de/datenbank/linzdb/ (abgerufen am 04.05.2017). 


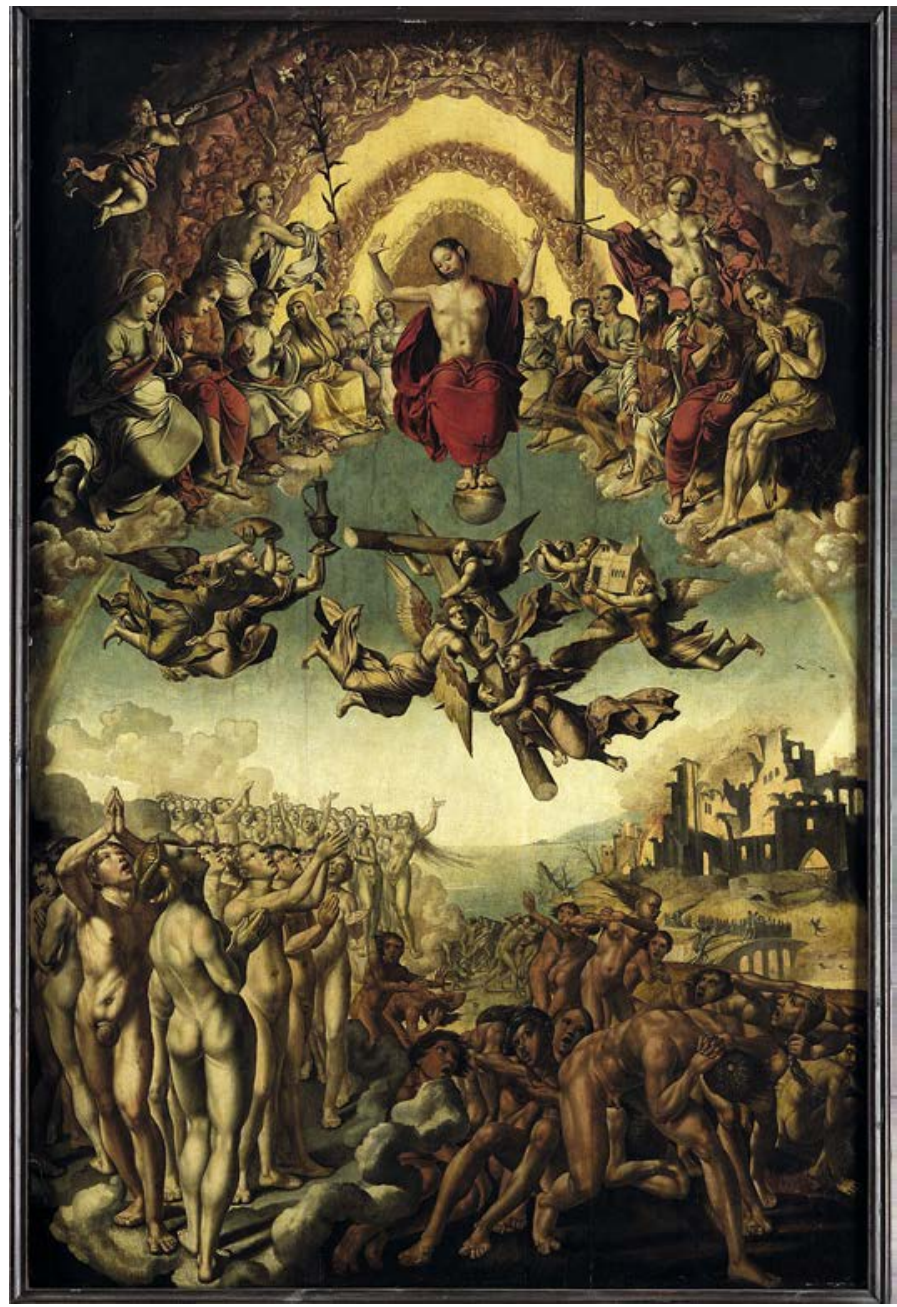

Abb. 1: Jüngstes Gericht, niederländisch, um 1530/50

Zur Bronzestatuette Stehende weibliche Figur, die unter der Inventarnummer KK 10012 geführt wird, sind keine weiteren Hinweise bekannt (siehe Abbildung 2). In der Linz-Datenbank konnte bisher kein passender Eintrag gefunden werden. ${ }^{7}$

7 TMS Eintrag zu KK 10011. 


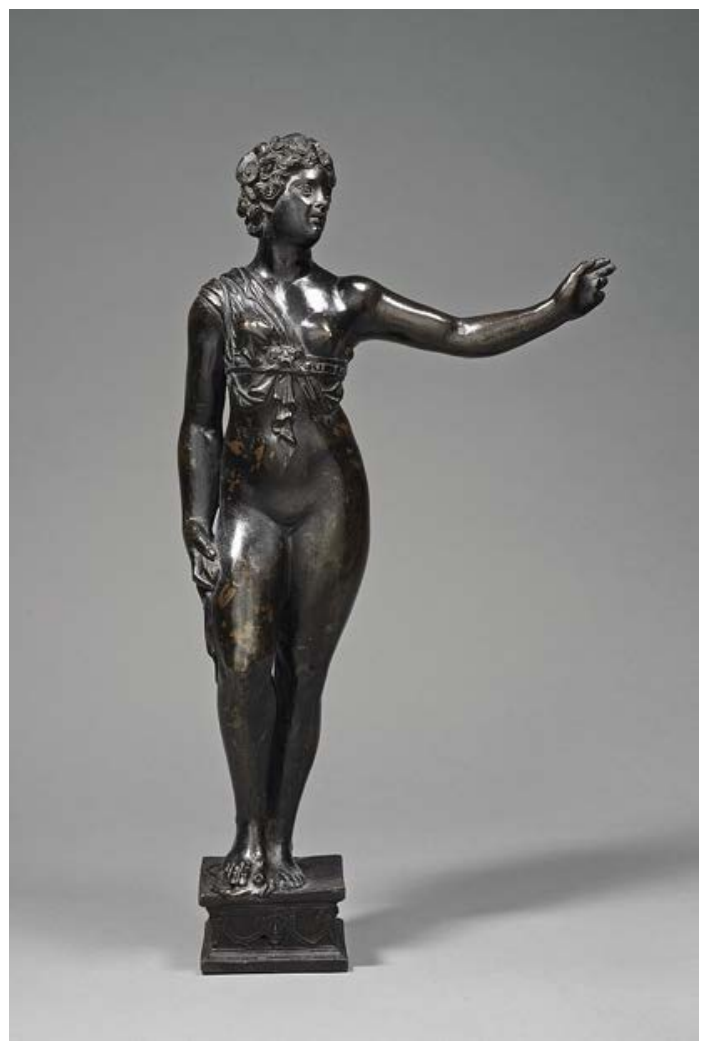

Abb. 2: Stehende weibliche Figur, italienisch, um 1560

\section{Zu den Objekten mit der Vorprovenienz Johannes Jantzen}

Insgesamt fünf Objekte, die über Vermittlung des Bremer Kunsthändlers Johannes Jantzen (1887-1972) vom „Sonderauftrag Linz“ erworben wurden, befinden sich heute im Inventarbestand des Kunsthistorischen Museums. ${ }^{8} \mathrm{Er}$ war Vertreter der Bremer Werkschau, die vom Kaufmann Ludwig Roselius (1874-1943), Erfinder des entkoffeinierten Kaffees HAG (Handels-Aktien-Gesellschaft), gegründet wurde. ${ }^{9}$ Tochterunternehmen der Bremer Werkschau war

8 Vgl. Silke Reuther, Museum für Kunst und Gewerbe Hamburg, Dossier Provenienz Dr. Johannes Jantzen (1887-1972). Ich danke an dieser Stelle Silke Reuther für die vielen Hinweise und Gespräche zu Johannes Jantzen.

9 Vgl. URL: http://www.boettcherstrasse.de/ und URL: http://www.museen-boettcherstrasse. de/museen/ludwig-roselius-museum/ (abgerufen am 29.06.2017). Vgl. auch Nicola Vetter: Ludwig Roselius. Ein Pionier der deutschen Öffentlichkeitsarbeit. Bremen: Hauschild 2002. 
die Alte Deutsche Kunst GesmbH (ADK), 1940 kam die Abteilung Galerie für alte Kunst dazu. Sie sollte vorrangig mit Gemälden, Zeichnungen und Großplastiken handeln, die $A D K$ hingegen mit Kunstgewerbe, Kleinplastiken und Möbeln. Beide Betriebe wurden im Spätsommer 1944 durch einen Bescheid der Reichskammer der bildenden Künste geschlossen ${ }^{10}$, allerdings wurde die Stilllegung nur wenige Wochen später - nach dem erfolgten Hinweis der Zusammenarbeit mit dem „Sonderbeauftragten des Führers"11 - wieder aufgehoben. ${ }^{12}$

Der in Shanghai in eine hanseatische Kaufmannsfamilie geborene Johannes Jantzen studierte Rechtswissenschaften in München, Freiburg, Lausanne und Leipzig. ${ }^{13} 1911$ promovierte er mit einer Doktorarbeit über Die freiwillige Veräußerungen von Schiffen und Schiffsparten ${ }^{14}$, ab 1911 war er in Bremen gemeldet. ${ }^{15}$ Gemäß Eintrag im Adressbuch von 1922/23 war Jantzen am Bremer Landgericht als Richter der 1. und 3. Strafkammer tätig ${ }^{16}$, im Adressbuch von 1926 war er aber nicht mehr unter den tätigen Richtern angeführt ${ }^{17}$ und der Eintrag für die Rembrandtstrasse 4, wo er bis zu seinem Umzug nach Wien im Jahr 1941 lebte, lautete von nun an „Richter a.D““. ${ }^{18}$ Während der NS-Zeit übte Johannes Jantzen laut eigenen Angaben den Anwaltsberuf nicht aus, da er - wie er 1947 Edwin C. Rae (1911-2002) ${ }^{19}$, dem Leiter der Monuments, Fine Arts and Archives Section der US-Armee mitteilte - nichts mit den nationalsozialistischen Gerichten zu tun haben wollte. ${ }^{20}$ Allerdings suchte er im September 1933 um

10 Vgl. Archiv Böttcherstraße, Korrespondenz Puvogel-Jantzen, Nolting-Hauff an Jantzen, 02.09.1944 und 04.09.1944.

11 Vgl. Archiv Böttcherstraße, Korrespondenz Puvogel-Jantzen, unbekannt an die Reichskammer der bildenden Künste, 02.09.1944.

12 Vgl. Archiv Böttcherstraße, Korrespondenz Puvogel-Jantzen, Puvogel an Jantzen, 21.09. 1944.

13 Vgl. Archiv der Universität Leipzig, Quaestur 040477, freundliche Mitteilung von Petra Hesse, weitergeleitet von Silke Reuther.

14 Vgl. Archiv der Universität Leipzig, Promotionsverzeichnis der Juristischen Fakultät, UAL_JurFak_01_02_Bd_03_0178, freundliche Mitteilung von Petra Hesse, weitergeleitet von Silke Reuther.

15 Vgl. Adressbuch Bremen 1911. URL: http://brema.suub.uni-bremen.de/periodical/page view/708078 (abgerufen am 02.05.2015).

16 Vgl. Adressbuch Bremen 1922/23. URL: http://brema.suub.uni-bremen.de/periodical/page view/510063 (abgerufen am 29.06.2017).

17 Vgl. Adressbuch Bremen 1926. URL: http://brema.suub.uni-bremen.de/periodical/page view/725831 und http://brema.suub.uni-bremen.de/periodical/pageview/72623 (abgerufen am 29.06.2017).

18 Vgl. Adressbuch Bremen 1941. URL: http://brema.suub.uni-bremen.de/periodical/page view/733054 (abgerufen am 29.06.2017).

19 Vgl. Iris Lauterbach: Der Central Collecting Point in München. Kunstschutz, Restitution, Neubeginn. München-Berlin: Deutscher Kunstverlag 2015, S. 28-30, S. 62f. (= Veröffentlichungen des Zentralinstituts für Kunstgeschichte in München 34).

20 Vgl. URL: www.fold3.com/image/\#269944706, Jantzen an Rae, 25.07.1947 (abgerufen am 07.07.2015). 
seine (Wieder-)Zulassung als Rechtsanwalt für die bremischen Gerichte und das Hanseatische Oberlandesgericht in Hamburg bei der Hanseatischen Anwaltskammer an. ${ }^{21}$ Jantzen selbst besaß eine Privatsammlung, „deren Inhalt neben Objekten der Kleinkunst aus verschiedenen Epochen und Kulturkreisen auch eine bedeutende Kollektion von Glaskunstwerken umfasste". ${ }^{22}$ Die Wiener Wohnung in der Wohllebengasse 9 in Wien 4, wo er ab 4. Juli 1941 gemeldet war $^{23}$, verwendete Jantzen als Büro bzw. Empfangsraum für die Kuratoren der Museen und andere größere KundInnen. ${ }^{24}$ Wien wurde wohl deswegen gewählt, weil zu diesem Zeitpunkt der Kunsthandel noch einigermaßen intakt war. Jantzen hatte Kontakte u.a. mit dem Wiener Kunsthändler Hermann Voigt $^{25}$, dem Hamburger Kunsthändler Kurt Köster ${ }^{26}$, dem Wiener Kunstverlag Wolfrum ${ }^{27}$ sowie dem Wiener Kunsthändler Otto Schatzker (1885-1959), der ursprünglich Mitglied der Israelitischen Kultusgemeinde in Wien gewesen war, aber 1941 vom Reichssippenamt zum „Arier“ erklärt wurde. ${ }^{28}$

Bis Anfang 1945 gab es eine Reihe von Geschäften mit dem „Sonderauftrag Linz", von denen Jantzen unmittelbar profitierte, da er mit einem Drittel am Gewinn der Bremer Kunsthandelsfirmen beteiligt war, wie Silke Reuther konstatiert. ${ }^{29}$ Im Bundesarchiv in Koblenz finden sich zahlreiche Belege über den Handel, den Jantzen anfänglich mit Hans Posse (1879-1942), dem Sonderbeauftragten für das geplante „Linzer Museum“, sowie dessen Nachfolger

21 Vgl. Staatsarchiv Bremen (StaB) 3-A.5.bJ.Nr. 3, Jantzen an Hanseatische Handelskammer, 04.09.1933, zit. n. Reuther: Dossier (Anm. 8), S. 7.

22 Herbert Haupt: Der Kunsthändler Johannes G. Jantzen. In: Recollecting. Raub und Restitution. Hg. von Alexandra Reininghaus. Wien: Passagen 2009, S. 313.

23 Vgl. Wiener Stadt- und Landesarchiv (WStLA), Meldeanfrage Johannes Jantzen.

24 Vgl. Archiv Böttcherstraße, Korrespondenz Puvogel-Jantzen, Jantzen an Puvogel, 25.02. 1945.

25 Vgl. Archiv Böttcherstraße, Korrespondenz Puvogel-Jantzen, Jantzen an Puvogel, 20.05. 1944. Vgl. URL: http://www.bva.bund.de/DE/Themen/Kunstverwaltung/Provenienzfor schung_Kunstbesitz/Provenienzdokumentation/Daten/7000_7999/7490.html (abgerufen am 03.05.2018).

26 Vgl. URL: http://www.bva.bund.de/DE/Themen/Kunstverwaltung/Provenienzforschung Kunstbesitz/Provenienzdokumentation/Daten/7000_7999/7490.html (abgerufen am 03.05.2018).

27 Vgl. Archiv Böttcherstraße, Korrespondenz Puvogel-Jantzen, Puvogel an Jantzen, 03.11. 1944.

28 Vgl. Archiv Böttcherstraße, Korrespondenz Puvogel-Jantzen, Jantzen an Puvogel, 30.07. 1944. Vgl. Gabriele Anderl: „Am Wiener Platz“: Schlaglichter auf die Rolle des Wiener Kunsthandels während der NS-Zeit. In: NS-Kunstraub in Österreich und die Folgen. Hg. von Gabriele Anderl, Alexandra Caruso. Innsbruck-Wien-Bozen: Studienverlag 2005, S. 171211, hier S. 172, S. 182-184. URL: http://derstandard.at/1216918696497/Entartete-Kunstge schaefte (abgerufen am 29.06.2017).

29 Vgl. Reuther: Dossier (Anm. 8), S. 17, sowie Rainer Stamm: Geschäfte laut Auftrag. Die Bremer Verbindung: Neues zu Hitlers Museum in Linz. In: FAZ, 01.09.2004. Laut Stamm lassen sich 33 Altmeistergemälde in den Geschäftsunterlagen der Galerie für Alte Kunst aufzeigen, die Jantzen verkaufte. 
Hermann Voss (1884-1969) trieb. ${ }^{30}$ Laut Reuther lieferte Jantzen sowohl aus dem Warenbestand der beiden Bremer Kunsthandelsfirmen als auch aus seiner privaten Sammlung. ${ }^{31}$ Der bereits genannte Edwin C. Rae wollte im Juni 1947 von Jantzen binnen zehn Tagen wissen, wie viele und welche Kunstgegenstände er oder seine MitarbeiterInnen an den Sonderbeauftragten für das Kunstmuseum Linz verkauft hatten, auch woher diese kamen. ${ }^{32}$ Dieser nannte drei Objekte, die er als Privatperson an den Sonderbeauftragten für Linz verkauft hatte. Tatsächlich waren es jedoch wesentlich mehr. ${ }^{33}$ Diese Verkäufe hätten - so Jantzen nichts zu tun „with the sales of the firms for which I held powers of attorney“. ${ }^{34}$ Weiters schrieb er: „The policy was a) not to buy pieces which were jewish property b) not to acquire pieces in occupied countries. During the occupation not one person, on behalf of the said companies, has ever been in France, Belgium, Holland, Denmark etc. ${ }^{\text {"35 }}$ Auch in einem späteren Brief an Dr. Erika Zeise (1914-2005) $)^{36}$, Mitarbeiterin am Collecting Point München, betonte Jantzen, dass die Firma sich „grundsätzlich nicht mit jüdischem Eigentum oder solchen

30 Vgl. Bundesarchiv (BA) Koblenz, B 323/154: Linz Rechnungen Zahlungsanweisungen; B 323/ 136: Erwerbungen Linz; B 323/98 Band 1: Zettelkartei der an Linz gelieferten Kunstgegenstände; B 323/149 Sonderauftrag Linz, Erwerbungen 1940-45; B 323/153: Sonderauftrag Linz, Rechnungen über Ankäufe aus dem Kunsthandel 1944; B 323/155 Sonderauftrag Linz, Rechnungen; B 323/173 Sonderauftrag Linz, Rechnungen 1. Teil, 1944; B 323/174 Sonderauftrag Linz, Rechnungen 2. Teil, 1944-45; B 323/583 Rechnungen, Korrespondenzen 1940-1944.

31 Vgl. Reuther: Dossier (Anm. 8), S. 18.

32 Vgl. Bundesdenkmalamt (BDA)-Archiv, Restitutionsmaterialien, K. 38, Personenmappe (PM) Jantzen, Johannes, S. 6, Rae an Jantzen, 10.06.1947.

33 Wenn nur die Objekte gezählt werden, die Jantzen eigenständig verkaufte, finden sich noch folgende Kunstgegenstände: Rechnung vom 01.04.1944 über 63.900 RM (Lenbach, Frauenbildnis, Studienblatt mit vier Figuren von Scheffer von Leonhardhoff, Aquarell von Brunner und Handzeichnung, wohl englisch 18. Jahrhundert), Rechnung vom 17.04.1944 (zwei Pastelle von Rotari Hirte und Hirtin, Rahl Bildnis Emma Gripenberger), Rechnung vom 14.05.1944 (Elfenbeinminiatur von Sharpe Porträt einer jungen Dame), Rechnung vom 09. 10.1944 (Grisaille Miniatur von De Gault Drei Kinderköpfe), Rechnung vom 14.10.1944 (Miniatur auf Papier, Bildnis eines älteren Herrn), Rechnung vom 21.10.1944 (63 Blatt Graphiken). Vgl. BA Koblenz, B 323/98, Nr. 64; B 323/149, Nr. 22, S. 103; B 323/153, S. 82, Nr. 435 und S. 85, Nr. 454; B 323/154, Nr. 92, S. 498; B 323/155, Nr. 21, S. 121.

34 Siehe URL: https://www.fold3.com/image/269944701, Jantzen an Rae, 25.07.1947 (abgerufen am 29.06.2017).

35 Siehe URL: https://www.fold3.com/image/269944706, Jantzen an Rae, 25.07.1947 (abgerufen am 29.06.2017).

36 Erika Zeise wurde am 25.11.1913 als Erika Maria Lengnick in Ostpreußen geboren und studierte zunächst Philosophie in Rostock, ehe sie sich der Kunstgeschichte zuwandte, URL: http://matrikel.uni-rostock.de/id/300000623 (abgerufen am 29.06.2017). 1942 promovierte sie mit einer Arbeit über die Ikonographie der Marienkrönung in Marburg bei Richard Hamann, die 1944 im Druck erschien, URL: http://www.researchgate.net/researcher/ 22354975_Erika_Lengnick (abgerufen am 29.06.2017). 2006 wurde sie gemeinsam mit ihrem Mann posthum als „Gerechte unter den Völkern“ ausgezeichnet, URL: http://www.tagesspie gel.de/berlin/verfolgte-vor-den-nazis-versteckt/751156.html (abgerufen am 29.06.2017). 
aus den besetzten Gebieten befaßte“, womit aber im Einzelnen gehandelt wurde, könne er sich nicht erinnern, da sämtliche Geschäftsunterlagen nach Bremen geschickt wurden. ${ }^{37}$ Was Jantzen vergessen hatte anzugeben, waren die Auktionen Budge in Berlin 1937, auf denen er steigerte, obwohl es sich um die Zwangsauflösung einer jüdischen Sammlung handelte. ${ }^{38}$

Von den fünf Kunstgegenständen, die von Johannes Jantzen an den „Sonderauftrag"verkauft wurden, sollen nun zwei Objekte näher vorgestellt werden. Die Bronzefigur Reitender Krieger (ohne Pferd) (s. Abbildung 3) ist in der Kunstkammer unter der Nummer KK 10010 inventarisiert. Jantzen bot Voss im Juni 1944 die Statuette zum Preis von 25.000 RM an. ${ }^{39}$ Nach kurzem Zögern stimmte ein Mitarbeiter von Voss Anfang Juli dem Kauf zu ${ }^{40}$ Zur Vorprovenienz gab Jantzen 1947 gegenüber der amerikanischen Militärregierung in Bayern an, dass er das Stück vom Wiener Kunsthändler Oskar Hamel (1889-1946) gekauft hatte. ${ }^{41}$ Dieser war bereits seit 9. Mai 1933 Mitglied der NSDAP (MitgliedsNr. 1,614.098), suchte 1938 um Aufnahme in die Reichskammer der bildenden Künste $\mathrm{an}^{42}$ und war seit 1940 gerichtlich beeideter Sachverständiger. ${ }^{43}$ Von 1943 bis 1945 war er als Experte der Kunstabteilung im Dorotheum tätig. ${ }^{44} \mathrm{Ge}$ schäftsbücher seiner Kunsthandlung dürften nicht mehr vorhanden sein, wenn man den Aussagen seiner Lebensgefährtin Lilly Nehammer-Prinz Glauben schenken kann. ${ }^{45}$ Weitere Unterlagen, die Auskunft über die Provenienz geben

37 Vgl. Archiv Böttcherstraße, Korrespondenz Puvogel-Jantzen, Jantzen an Zeise, 27.04.1951.

38 Vgl. Reuther: Dossier (Anm. 8), S. 17 sowie die Beiratsempfehlung vom 08.10.2013, URL: http://www.provenienzforschung.gv.at/wp-content/uploads/2014/04/Budge_Emma_201310-08.pdf (abgerufen am 29.06.2017).

39 Vgl. BA Koblenz, B323/136, Ankäufe für den „Sonderauftrag Linz“ aus dem deutschen und österreichischen Kunsthandel und Privatbesitz. Bd. 8: 1940-1945, Nr. 202, Jantzen an Voss, 26.06.1944.

40 Vgl. BA Koblenz, B323/136, Ankäufe für den „Sonderauftrag Linz“ aus dem deutschen und österreichischen Kunsthandel und Privatbesitz. Bd. 8: 1940-1945, Nr. 200, Oertel an Jantzen, 04.07.1944.

41 Im Wiener Adressbuch Lehmann von 1942, Branchenverzeichnis, findet sich unter Antiquitäten „Nehammer-Hamel. Antike Möbel und Holzplastiken. VIII/65, Piaristengasse 11“, als Kunsthandlung „Oskar Hamel. Alte Kunst. VIII/65, Piaristengasse 11“.

42 Im Archiv der Berufsvereinigung der bildenden Künstler Österreichs in Schönbrunn gibt es keinen Akt zu Oskar Hamel. Laut E-Mail-Auskunft des Archivars Karl Novak vom 04. 12.2012 könnte sich sein Akt eventuell unter jenen 800 Akten von belasteten Parteimitgliedern befinden, die 1945 von der Staatspolizei konfisziert und nicht mehr an die Berufsvereinigung der bildenden Künstler Österreichs zurückgegeben wurden.

43 Vgl. Österreichisches Staatsarchiv (ÖStA), Archiv der Republik (AdR), Zivilakten der NSZeit (ZNsZ), BMI/Gauakten, Nr. 3770, Oskar Hamel.

44 Vgl. Esther Tisa Francini: Liechtenstein und der Internationale Kunstmarkt 1933-1945. Sammlungen und ihre Provenienzen im Spannungsfeld von Flucht, Raub und Restitution. Zürich: Chronos 2005 (= Veröffentlichungen der Unabhängigen Historikerkommission Liechtenstein Zweiter Weltkrieg 4), S. 182-188.

45 Vgl. BDA-Archiv, Restitutionsmaterialien, K. 42, PM Nehammer-Prinz, Lilli (Oskar Hamel). 
könnten, sind derzeit nicht bekannt. ${ }^{46}$ Hamel trat als Zwischenhändler und Nutznießer von Arisierungen in Erscheinung, sodass der Verdacht auf Raubkunst nicht ausgeschlossen werden kann. ${ }^{47}$

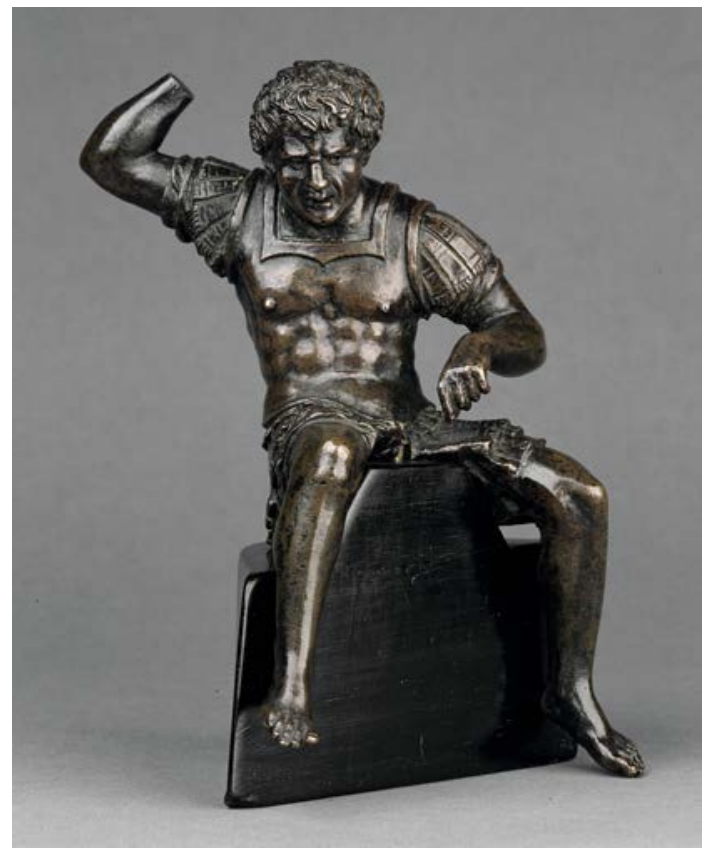

Abb. 3: Reitender Krieger (ohne Pferd), oberitalienisch, 1. Hälfte 16. Jahrhundert

Ein Früchtestillleben wurde unter GG 9568 in der Gemäldegalerie inventarisiert (s. Abbildung 4). Im Bestand des Bundesarchivs Koblenz findet sich ein Hinweis, dass ein Neapolitanisches Barockstillleben - es dürfte sich hierbei um das Früchtestillleben handeln - gemeinsam mit dem Gemälde Kinderkopf von Joseph Aigner um zusammen 28.000 RM im Oktober 1944 für das „Linzer Museum" angekauft wurde. ${ }^{48}$ Auf einer Karteikarte findet sich weiters die von der Bremer Werkschau vergebene Nummer $138 .{ }^{49}$ Unter dieser Nummer findet

46 Keine Hinweise fanden sich in diesen Beständen: WStLA, Vermögensentziehungsanmeldeverordnung (VEAV) Zl. 618, 1. Bezirk, Oskar Hamel.

47 Vgl. die Restitutionsberichte des Wienmuseums aus den Jahren 2002 und 2005. Siehe URL: http://www.wienmuseum.at/fileadmin/user_upload/PDFs/Restitutionsbericht_2002.pdf und URL: http://www.wienmuseum.at/fileadmin/user_upload/PDFs/Restitutionsbericht_2005. pdf (abgerufen am 29.06.2017).

48 Vgl. BA Koblenz, B 323/155, S. 16.

49 Vgl. BA Koblenz, B323/98, Kleine Kartei, „Auszüge aus Akten, die dem Central Collecting Point München zur Verfügung standen, den Ankauf von Objekten, die für den Sonderauftrag Linz erworben wurden, betreffend“. 


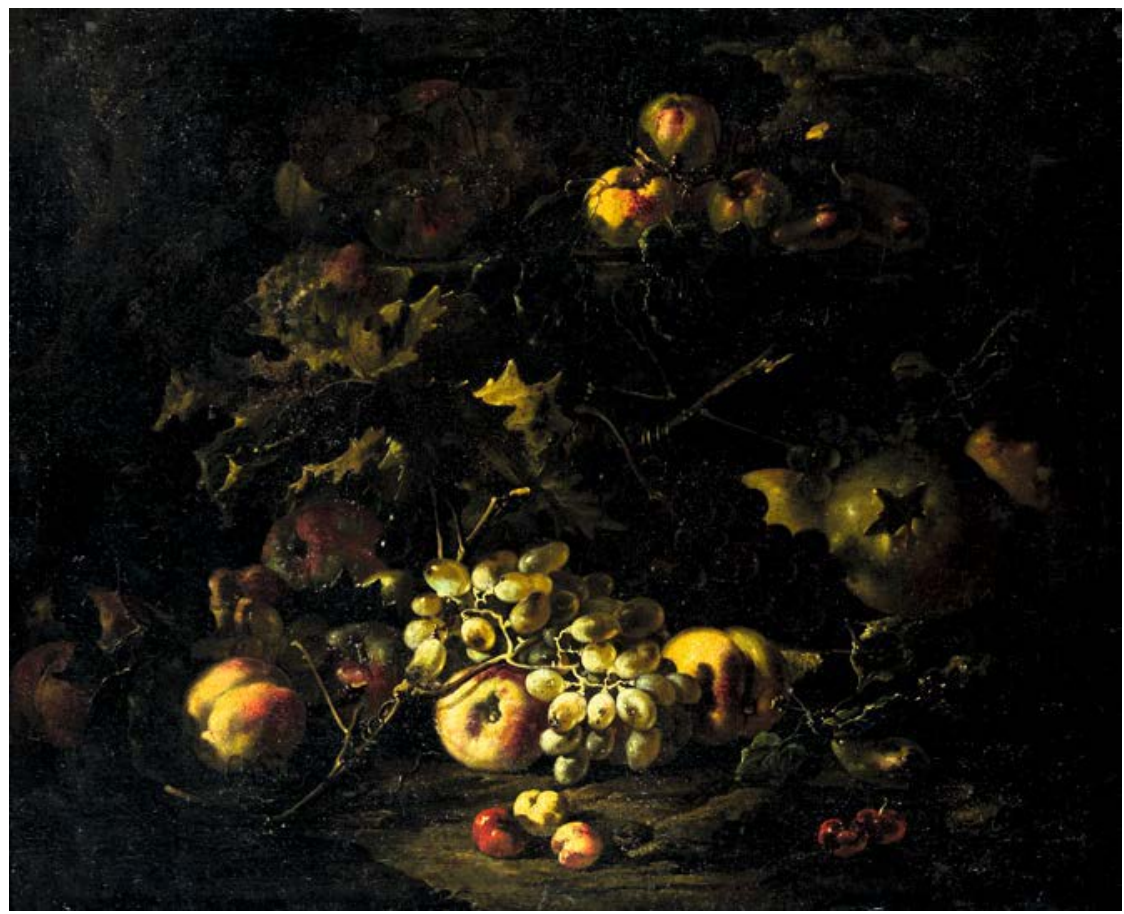

Abb. 4: Früchtestillleben, römisch, Ende 17. Jahrhundert

man im Journal Bremer Werkschau GmbH und Johannes Jantzen GbR 1940-61 den Eintrag, dass das Bild am 21. Oktober 1944 von „Gem. Abels“ - gemeint ist die Gemäldegalerie Abels ${ }^{50}$ in Köln - angekauft und noch am selben Tag an Voss weiterverkauft wurde. ${ }^{51}$ In der Linz-Datenbank scheint als Vorbesitzer des Gemäldes mit der Münchner Nummer 2085 allerdings ein Miller-Aichholz auf. $^{52} \mathrm{Ob}$ dieser ident mit August Miller-Aichholz ist, der ein Geschäft am Hohen Markt hatte und in - zumindest geplanten - Geschäftsbedingungen mit Jantzen stand ${ }^{53}$, oder einem anderen Vertreter der Familie, wie etwa Romedio Miller-Aichholz, der eine Galerie am Lobkowitzplatz besaß, kann derzeit nicht

50 Vgl. URL: http://www.galerie20.smb.museum/kunsthandel/K1.html (abgerufen am 13.06. 2017). Vgl. auch Daniela Wilmes: Wettbewerb um die Moderne: Zur Geschichte des Kunsthandels in Köln nach 1945. Berlin: Akademie Verlag 2012 (=Schriften zur modernen Kunsthistoriographie).

51 Vgl. Archiv Böttcherstraße, „Journal Bremer Werkschau GmbH und Johannes Jantzen GbR 1940-61“, S. 43.

52 Abfrage „Früchtestillleben“. URL: http://www.dhm.de/datenbank/linzdb/ (abgerufen am 22.06.2017).

53 Vgl. Archiv Böttcherstraße. Korrespondenz Puvogel-Jantzen, Jantzen an Puvogel, 28.12. 1942. 
gesagt werden. ${ }^{54}$ Im Wiener Adressbuch Lehmann ist der Name MillerAichholz im Jahr 1942 insgesamt siebenmal verzeichnet. ${ }^{55}$ In der umfangreichen Personenmappe August Miller-Aichholz im Archiv des Bundesdenkmalamtes wird jedenfalls ein Früchtestillleben nicht genannt. ${ }^{56}$ Auch hier werden weitere Recherchen folgen.

\section{Nicolas Régnier, HI. Maria Magdalena}

Nun aber zu dem einzigen Gemälde, das in der permanenten Ausstellung der Gemäldegalerie gezeigt wird und unter GG 9569 inventarisiert ist (s. Abbildung 5). Im März 1944 verkaufte Ferdinand Spany (1897-1983), Wien 3, Landstraßer Hauptstraße 58, das damals noch als Büssende Magdalena bezeichnete Gemälde über das Dorotheum an Voss. ${ }^{57}$ Laut der Linz-Datenbank wurde das Bild damals Pompeo Girolamo Batoni zugeschrieben. ${ }^{58} \mathrm{Zu}$ Spany sei hier aus dem Eintrag im Lexikon der österreichischen Provenienzforschung zitiert:

„Ab 1921 war er Geschäftsführer im Kunstauktionshaus Leo Schidlof, bis er sich vier Jahre später als Kunst- und Antiquitätenhändler selbstständig machte. Im Zusammenhang mit dem versuchten Verkauf eines vermeintlichen Rubens-Gemäldes wurde er 1927 festgenommen und vier Jahre später wegen Veruntreuung und Betruges zu zehn Monaten Haft verurteilt; es folgten weitere Verurteilungen wegen leichter Körperverletzung, Ehrenbeleidigung und Veruntreuung. Nach dem „Anschluss“ 1938 war Spany [...] [für die] „Abwicklungsstelle für die Liquidierung und Arisierung des Uhren- und Juwelenfaches" tätig. [...] Wegen der Verleitung zum Missbrauch der Amtsgewalt bzw. wegen Beihilfe zu selbiger wurde Spany [...] zu mehreren Monaten Freiheitsstrafe verurteilt. Aufgrund seiner zahlreichen Vorstrafen blieb Spany, der seit 1938 Parteianwärter war, letztlich die Mitgliedschaft in der NSDAP und der Reichskammer für bildende Künste verwehrt. [...] Trotz einer Reihe weiterer anhängiger Gerichtsverfahren in der Nachkriegszeit war Spany nach 1945 erfolgreich als Kunst- und Antiquitätenhändler tätig. [...] 1974 erhielt Spany auf Antrag der Akademie der bildenden Künste den Berufstitel Professor und 1983 das Goldene Ehrenzeichen der Republik

54 Vgl. auch Oliver Kühschelm: Vom glanzvollen Aufstieg bis zur „Tragödie alten Reichtums“. Familien- und Firmenstruktur im Haus Miller-Aichholz. In: Bürgerliche Familien. Lebenswege im 19. und 20. Jahrhundert. Hg. von Hannes Stekl. Wien-Köln-Weimar: Böhlau 2000, (= Bürgertum in der Habsburgermonarchie 8), S. 109-167.

55 Siehe URL: http://www.digital.wienbibliothek.at/wbrobv/periodical/zoom/265564 (abgerufen am 22.06.2017).

56 BDA-Archiv, Restitutionsmaterialien, K. 41, PM Miller Aichholz.

57 Vgl. BDA-Archiv, K. 13, M. 4, Linzer Bestände: Nachweis österreichischer Herkunft. Schreiben Dorotheum an das Staatliche Denkmalamt, 16.01.1945, fol. 6.

58 Abfrage „Büßende Magdalena“, URL: http://www.dhm.de/datenbank/linzdb/ (abgerufen am 19.04.2017). 
Österreich u.a. für die Schenkung wertvoller Kunstwerke an die Österreichische Galerie und seinen Forschungen zum Paneuropa-Gedanken verliehen. ${ }^{\text {“59 }}$

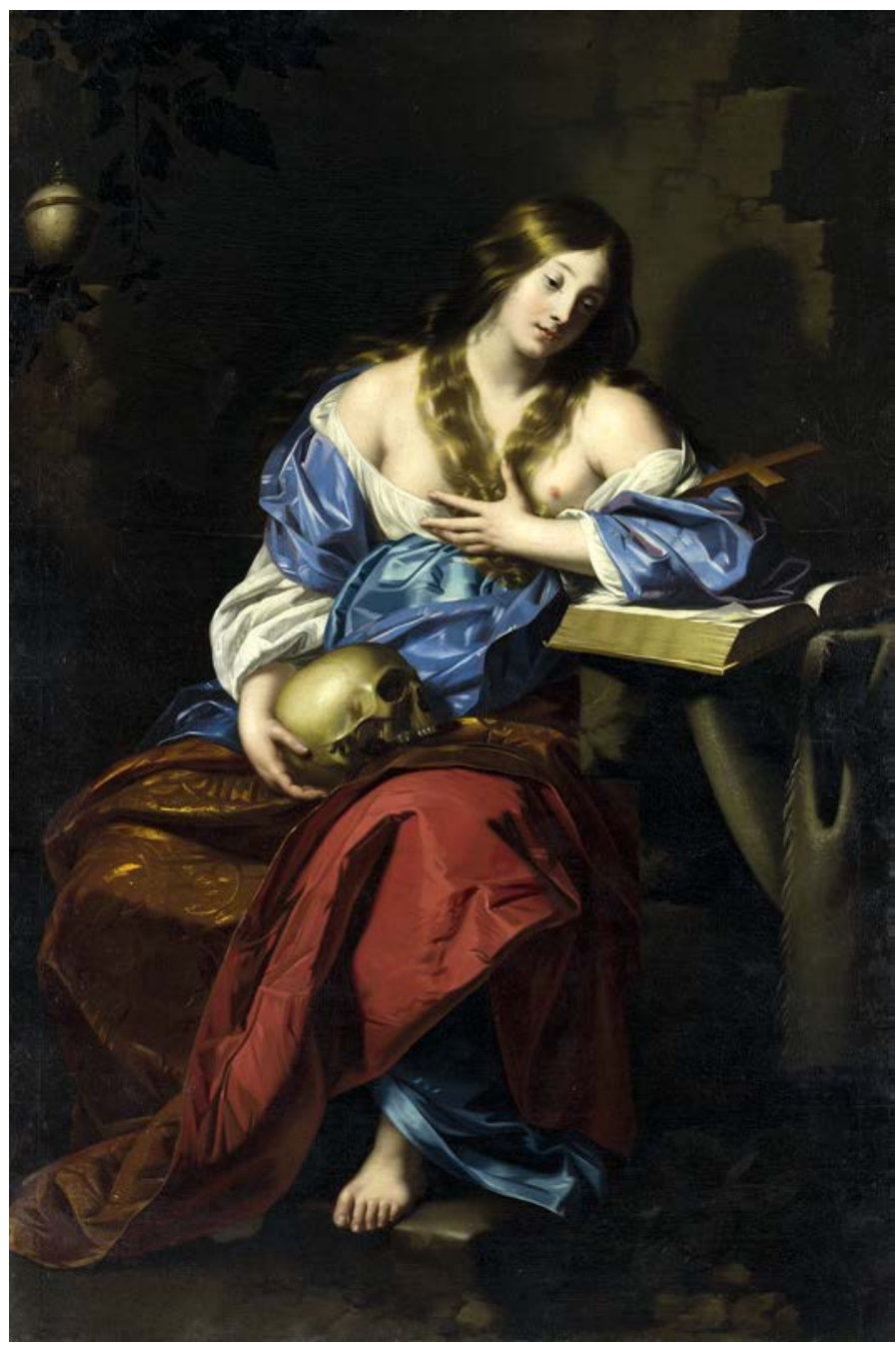

Abb. 5: Hl. Maria Magdalena, Nicolas Régnier, um 1655/1660

59 Vgl. Eintrag zu Ferdinand Spany im Lexikon österreichischer Provenienzforschung (LÖPF), erstellt von Konstantin Ferihumer. URL: http://www.lexikon-provenienzforschung.org/ spany-ferdinand (abgerufen am 02.05.2018). 
Spany gab im Dezember 1947 beim Bundesdenkmalamt an, dass er das Gemälde von Alois Hornig (1896-1976) ${ }^{60}$, Wien 3, Weyrgasse 8, gekauft hatte, dieser hatte es 1939 wiederum von der Galerie Falkenberg, Wien 2, Praterstraße 15, erworben. ${ }^{61}$ Grete Falkenberg (1892-1965) ${ }^{62}$ bestätigte, dass sie das Gemälde 1939 gekauft habe und dass es auch 1938 in österreichischem Besitz war. Den Namen des Vorbesitzers gibt sie allerdings nicht preis und das Bundesdenkmalamt gab sich mit der Antwort zufrieden. ${ }^{63}$ Ihr Ehemann, der Maler und Restaurator Karl Falkenberg (1885-1956) ${ }^{64}$, war Professor am Konservatorium für Musik und darstellende Kunst und war als Schätzmeister für die Vermögensverkehrsstelle tätig. ${ }^{65}$ Eine Spur führt zum Bilder- und Antiquitätenhandel von Max (1866-1943) und Johanna Löbl (1865-1942) in Baden bei Wien. Deren Enkelin Helga Fries klagte nach 1945 Karl und Grete Falkenberg auf eine Restschuld in der Höhe von 1.600 RM aus dem erzwungenen Verkauf von Antiquitäten und Möbeln. Die Kunsthandlung Falkenberg hätte die Bezahlung „beharrlich verweigert“, wie die Enkelin zu Protokoll gab und sprach von einer „Arisierung der übelsten Sorte“. ${ }^{66}$ In der Vermögensanmeldung von Max Löbl vom 12. Juli 1938 gab er einen Bilder- und Antiquitätenhandel an, zum Betriebsvermögen gehörten: „Stockuhren, Bilder, Gläser, alte Wohnungseinrichtungsgegenstände, Luster, Nippes, Porzellan, Broschen, Figuren und anderes“. Eine detaillierte Auflistung liegt leider nicht bei. ${ }^{67}$ Das Ehepaar Löbl wurde im Juni 1942 nach Theresienstadt deportiert und dort ermordet. ${ }^{68}$ An dem Bild wurde im Jänner 2012 eine Rückseitenautopsie vorgenommen. Das Gemälde wurde in Saal IV abgehängt, die Rückseitenverschalung entfernt. Weder auf der Leinwand, die laut Einschätzung des Restaurators im 19. Jahrhundert oder noch früher doubliert

60 Siehe URL: https://www.friedhoefewien.at/grabsuche?submitHidden=true\& name=Alois + Hornig\& friedhof $=-1 \& \mathrm{jdb} \_$von $=\& \mathrm{jdb} \_$bis $=\&$ historischerGrab $=$ false\& latitudeWGS84 $\mathrm{y}=$ \&longitudeWGS84_x $=($ abgerufen am 29.5.2017).

61 Vgl. BDA-Archiv, Restitutionsmaterialien, K. 47, PM Spany, Ferdinand, Spany an das BDA, 02.12 .1947$.

62 Vgl. URL: https://www.friedhoefewien.at/grabsuche?submitHidden $=$ true\& name=Alois+ Hornig\& friedhof $=-1 \& \mathrm{jdb} \_$von $=\& \mathrm{jdb} \_$bis $=$\& historischerGrab $=$false \&latitudeWGS84_y $=$ \&longitudeWGS84_x $=($ abgerufen am 29.05.2017) .

63 Vgl. BDA-Archiv, Restitutionsmaterialien, K. 47, PM Spany, Ferdinand, Falkenberg an das BDA, 12.12.1947.

64 Vgl. URL: https://www.friedhoefewien.at/grabsuche? submitHidden $=$ true \& name=Alois + Hornig\& friedhof $=-1 \&$ jdb_von $=\&$ jdb_bis $=\&$ historischerGrab $=$ false \& latitudeWGS84_y $=\&$ longitudeWGS84_x $=$ (abgerufen am 29.05.2017).

65 Vgl. Eintrag zu Richard Leitner im LÖPF, erstellt von Pia Schölnberger, URL: http://www.lexi kon-provenienzforschung.org/richard-leitner (abgerufen am 02.05.2018).

66 Vgl. WStlA, VEAV Grete Falkenberg, 2./20. Bezirk, Zl. 1629.

67 Vgl. Niederösterreichisches Landesarchiv (NÖLA), Vermögensanmeldung (VA), 44874, Max Löbl, 12.07.1938.

68 Dokumentationsarchiv des österreichischen Widerstandes (DÖW): Shoah-Opfer, Personensuche. URL: http://www.doew.at/personensuche (abgerufen am 23.05.2017). 
wurde, noch auf dem Keilrahmen befanden sich Aufkleber, Stempel, Beschriftungen oder sonstige Hinweise auf etwaige VorbesitzerInnen.

\section{Galasso Galassi, Christus mit drei Aposteln in Gethsemane}

Ein einziges Bild aus den „1963er Zuweisungen“ wurde bisher vor dem Kunstrückgabebeirat behandelt und soll hier kurz dargestellt werden (s. Abbildung 6). Im März 2005 erhielt das Kunsthistorische Museum ein Schreiben der Rechtsanwaltskanzlei Schink \& Studzinski. Gemeinsam mit dem Rechtsanwalt David J. Rowland, New York, vertrat sie die Erbin nach Dr. Hermann Eissler (1860-1953) und ersuchte - unter Bezugnahme auf die Publikation von Sophie Lillie $^{69}$ - um Auskunft, ob sich das Gemälde von Galasso Galassi noch im Museum befände. ${ }^{70}$ In weiterer Folge wurde ein Dossier erstellt, das am 24. Juni 2009 dem Kunstrückgabebeirat vorgelegt wurde. Der Industrielle Hermann Eissler, der eine bedeutende und auch international bekannte Kunstsammlung besaß, war tschechoslowakischer Staatsbürger und galt in der NS-Diktion als Jude, seine Ehefrau Hortense Eissler (1895-1983) als „Arierin“. Ihm gelang die Flucht über Ungarn und die Schweiz nach Nizza. Offensichtlich im Zusammenhang mit der Sicherung des Vermögens beantragte Hortense Eissler die Aufhebung der Ehe. 1951 heirateten Hermann und Hortense Eissler erneut in Nizza. Seine uneheliche Tochter Berta Morelli (1893-1975), die nach den Nürnberger Gesetzen als „Mischling ersten Grades“ galt, heiratete im November 1938 den ungarischen Staatsbürger, Rechtsanwalt Dr. Karl Morelli, der zugleich Rechtsvertreter ihres Vaters war. Hermann Eissler schenkte Christus mit drei Aposteln in Gethsemane seiner Tochter Berta bereits 1917 anlässlich ihrer ersten Eheschließung mit dem Geschäftsmann Paul Munk. Das Gemälde wurde nach längeren Verhandlungen u. a. forderte der Anwalt Morellis eine Erhöhung des Kaufpreises - von Hans Posse im Oktober 1940 für den „Sonderauftrag“ erworben. Die Kaufsumme wurde z.T. in Devisen ausbezahlt. Der Kunstrückgabebeirat sprach keine Rückgabeempfehlung aus und begründete dies damit, dass „die vorliegenden Unterlagen jedoch nicht den Schluss zu [lassen], dass die Veräußerungen unter dem Druck einer Verfolgung stattfanden, sondern legen eher nahe, dass Berta Morelli die Gemälde freiwillig und unabhängig von einer Verfolgungssituation veräußerte“. Danach kam es noch zu Verhandlungen zwischen Posse und Morelli über weitere Verkäufe, die aber ergebnislos abgebrochen wurden, als sie sich

69 Vgl. Sophie Lillie: Was einmal war. Handbuch der enteigneten Kunstsammlungen Wiens. Wien: Czernin Verlag 2003, S. 322-329.

70 Vgl. KHM-Archiv, 24/GG/2005, Schreiben der Rechtsanwaltskanzlei Schink \& Studzinski, 04.03.2005. 
nicht mit den Bedingungen Posses einverstanden erklärte, was für sie ohne Konsequenzen blieb. ${ }^{71}$

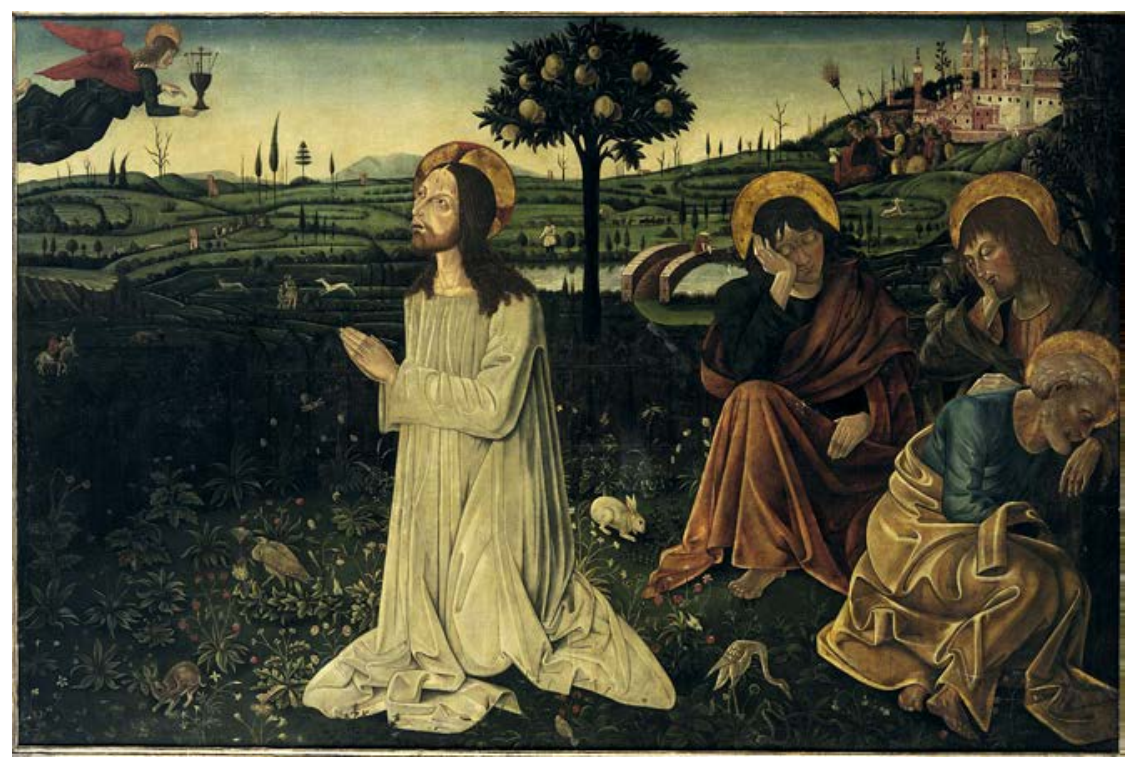

Abb. 6: Christus mit drei Aposteln in Gethsemane, Galasso Galassi, 2. Hälfte 15. Jahrhundert

\section{Giuseppe Recco, Knabe mit Krebsen}

In der Linz-Datenbank wird als Einlieferer des Gemäldes (s. Abbildung 7) die Galerie L.T. Neumann genannt. ${ }^{72}$ Die Galerie wurde 1883 gegründet, hatte ihre Adresse in Wien am Kohlmarkt 11 und am Michaelerplatz 4 und war auf Gemälde und Grafik, Alte und Neue Meister spezialisiert. Inhaber war der ursprünglich aus Deutschland kommende August Eymer (1894-1987). Er trat 1919 der Galerie bei und war seit 1944 Alleininhaber des Kunsthandelsunternehmens, seine Frau war für finanzielle Belange und im Verkauf tätig. Eymer war zudem gerichtlich beeidigter Schätzmeister und Sachverständiger, unterhielt gute Beziehungen zu Museen und war Funktionär der Reichskammer der bildenden Künste in Wien. ${ }^{73}$ Nach 1945 war er Vorstandsmitglied der

71 Vgl. URL: http://www.provenienzforschung.gv.at/empfehlungen-des-beirats/beschluesse/ beschluesse-alphabetish/?decisions-letter=E (abgerufen am 27.04.2017).

72 Abfrage „Recco“, URL: https://www.dhm.de/datenbank/linzdb/ (abgerufen am 04. 05.2017).

73 Vgl. Eintrag zu Eymer im LÖPF, erstellt von Pia Schölnberger. URL: http://www.lexikon-pro venienzforschung.org/eymer-august-friedrich (abgerufen am 02.05.2018). 


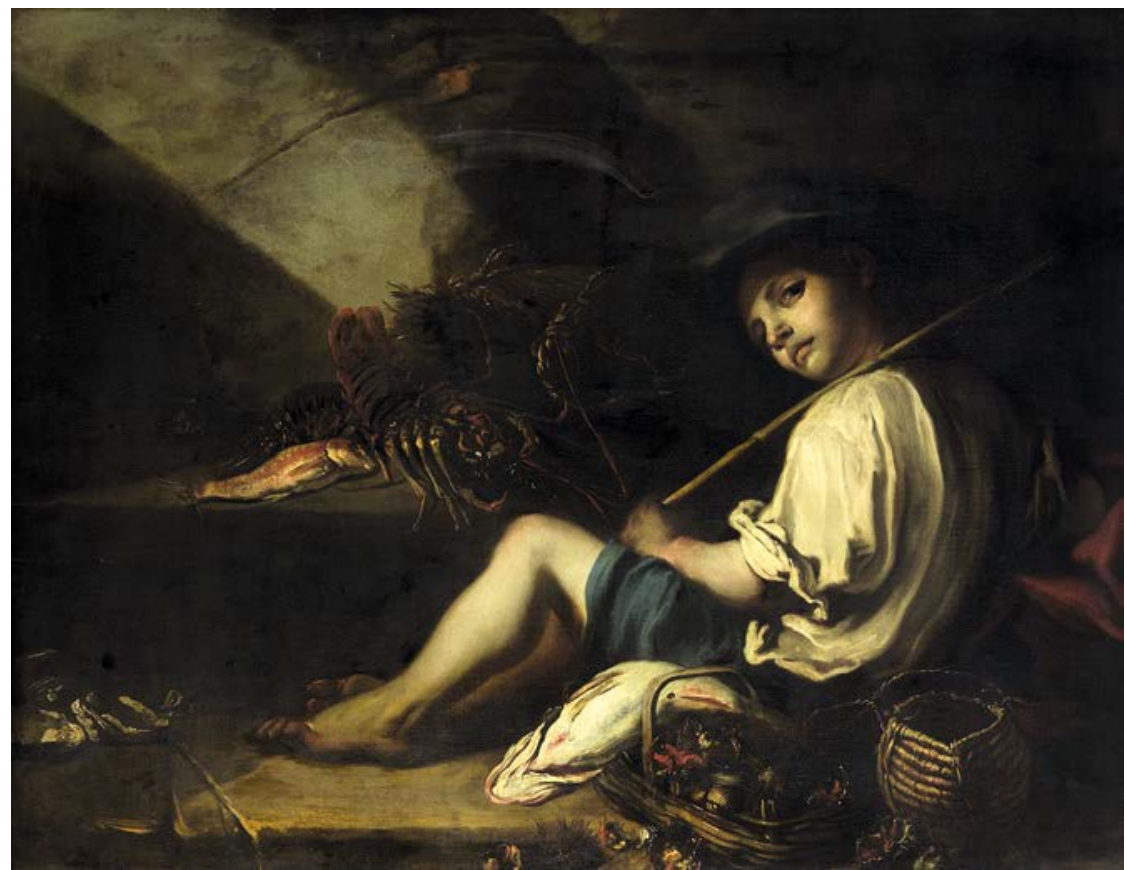

Abb. 7: Knabe mit Krebsen, Giuseppe Recco, 2. Hälfte 17. Jahrhundert

Wiener Kunsthändler. ${ }^{74}$ Auf der Seite des Bundesamtes für zentrale Dienste und offene Vermögensfragen wird im Zusammenhang mit einem Bild von Gabriel Cornelius Ritter von Max die Kunsthandlung Neumann genannt. Wissenschaftliche Untersuchungen lägen noch keine vor, allerdings scheine die Kunsthandlung häufig als „Zwischenhändler“ von Kunstwerken aus ehemals jüdischem Eigentum auf. Von einem Nachlass der Kunsthandlung sei nichts bekannt. ${ }^{75}$ In der Tat scheint im Archiv des Bundesdenkmalamtes der Name Neumann in einer ganzen Reihe von Linz-Erwerbungen auf, allerdings wird das besagte Gemälde von Recco nicht explizit genannt. ${ }^{76}$ Der Knabe mit Krebsen wurde im September 1944 um 9.200 RM an den Sonderauftrag verkauft. ${ }^{77}$ In den im Bundesarchiv Koblenz überlieferten Unterlagen sind zwar Aussagen Eymers

74 Vgl. Alte und Moderne Kunst XIX (1974 / Heft 136 und 137), S. 75, in: URL: http://hauspubli kationen.mak.at/viewer/image/1368763708151_0001/81/LOG_0019/ (abgerufen am 10.04. 2017).

75 Vgl. http://www.bva.bund.de/DE/Themen/Kunstverwaltung/Provenienzforschung_Kunst besitz/Provenienzdokumentation/Daten/3000_3999/3724.html (abgerufen am 03.05.2018).

76 Vgl. BDA-Archiv, K. 13, M. 5b, Eymer (Inhaber Galerie Neumann).

77 Vgl. BA Koblenz, BA 323/140, Ankäufe für den Sonderauftrag Linz aus dem deutschen und österreichischen Kunsthandel und Privatbesitz. Bd. 12: 1940-1945, XXa, 6, Rechnung der Galerie L.T. Neumann, 26.09.1944. 
aus dem April 1949 überliefert, aber auch hier wird das Gemälde nicht erwähnt. Eymer beteuerte, wie so viele andere KunsthändlerInnen, die mit dem „Sonderauftrag Linz“ zu tun hatten, dass bei ihm kein Bild unter Druck oder Zwang verkauft worden wäre und dass es sich um eine ganz legale Geschäftsabwicklung gehandelt habe. ${ }^{78}$ Auch hier konnte der Name des Vorbesitzers bislang nicht eruiert werden.

\section{Joris van Son, Frühstücksstillleben mit Hummer}

Dieses Gemälde (s. Abbildung 8) wurde im Februar 1944 von der Galerie St. Lucas an den Sonderbeauftragten Voss verkauft ${ }^{79}$ und im Juli 1944 nach München in den Führerbau verbracht ${ }^{80}$. Die Galerie, die auf Alte Meister und Gemälde des 19. Jahrhunderts spezialisiert war, war eine der bedeutendsten Kunsthandlungen in Wien. So erwarb die Gemäldegalerie des Kunsthistorischen Museums im Zeitraum von 1923 bis 1945 rund 20 Bilder $^{81}$, für die Jahre von 1938 bis 1945 sind unter anderem der Verkauf von 26 Werken, darunter Gemälde von Rudolf von Alt, Waldmüller, Grützner und Makart, an den „Sonderauftrag Linz“ belegbar. $^{82}$ Die Kunsthandlung wurde 1919 unter der Bezeichnung „Galerie St. Lukas Herzig \& Loibl Gesellschaft m.b.H. in Liquidation“ von Karl Herzig und Anton Johann Loibl mit Sitz im Palais Pallavicini in Wien 1, Josefsplatz 5, gegründet. Unter den mehrmaligen Gesellschafterwechsel Mitte der 1920er Jahre ist der Einstieg von Adolf Fritz Mondschein (1894-1968) als Gesellschafter der Kunsthandlung im April 1925 erwähnenswert, 1930 wurde er alleinig vertretungsbefugter Gesellschafter. 1938 verlor er diese Position - in der NS-Diktion galt er als Jude - und er musste 1939 nach New York emigrieren. Dort änderte er seinen Namen in Fredrick Mont und war weiterhin im Kunsthandel tätig. Er verkaufte hauptsächlich Gemälde Alter Meister an amerikanische Museen und war auch als Vermittler für den Prinzen von Liechtenstein für den Verkauf von Werken aus dessen Sammlung tätig. Er starb als amerikanischer Staatsbürger 1968 in New York. ${ }^{83}$ Die Galerie St. Lucas übernahm kurz nach dem „Anschluss“ Robert Herzig, der Sohn des Firmengründers, der sie bis 1974 führte. ${ }^{84}$

78 Vgl. BA Koblenz, B323/332, Sonderauftrag Linz. Kunsthändler-Aussagen, Band 2, Aussage des Mitbesitzers A. Eymer am 22.04.1949, fol.112.

79 Vgl. BDA-Archiv, K. 13, M. 4, Linzer Bestände: Nachweis österreichischer Herkunft. Dorotheum an das Denkmalamt, 16.01.1946.

80 Vgl. BDA-Archiv, K.13, M.5a, Liste von Bildern des Sonderauftrages Linz, die nach München Führerbau am 05.07.1944 gelangten.

$81 \mathrm{Vgl}$. TMS Einträge zu Galerie St. Lucas.

82 Vgl. den Eintrag zur Galerie St. Lucas im LÖPF, erstellt von René Schober. URL: http://www.le xikon-provenienzforschung.org/sanct-lucas-galerie (abgerufen am 02.05.2018).

$83 \mathrm{Vgl}$. URL: https://www.dorotheum.com/auktionen/aktuelle-auktionen/kataloge/list-lots-de 
In den im Bundesarchiv Koblenz überlieferten Nachkriegsrecherchen wird ein Hummerstillleben nicht besprochen. ${ }^{85}$ Auch aus den Überlieferungen im Archiv des Bundesdenkmalamtes geht nicht hervor, wer der Vorbesitzer war. Eine diesbezügliche Anfrage an die Galerie, die sich heute noch im Palais Pallavicini befindet, im Dezember 2015 verlief ergebnislos. ${ }^{86}$

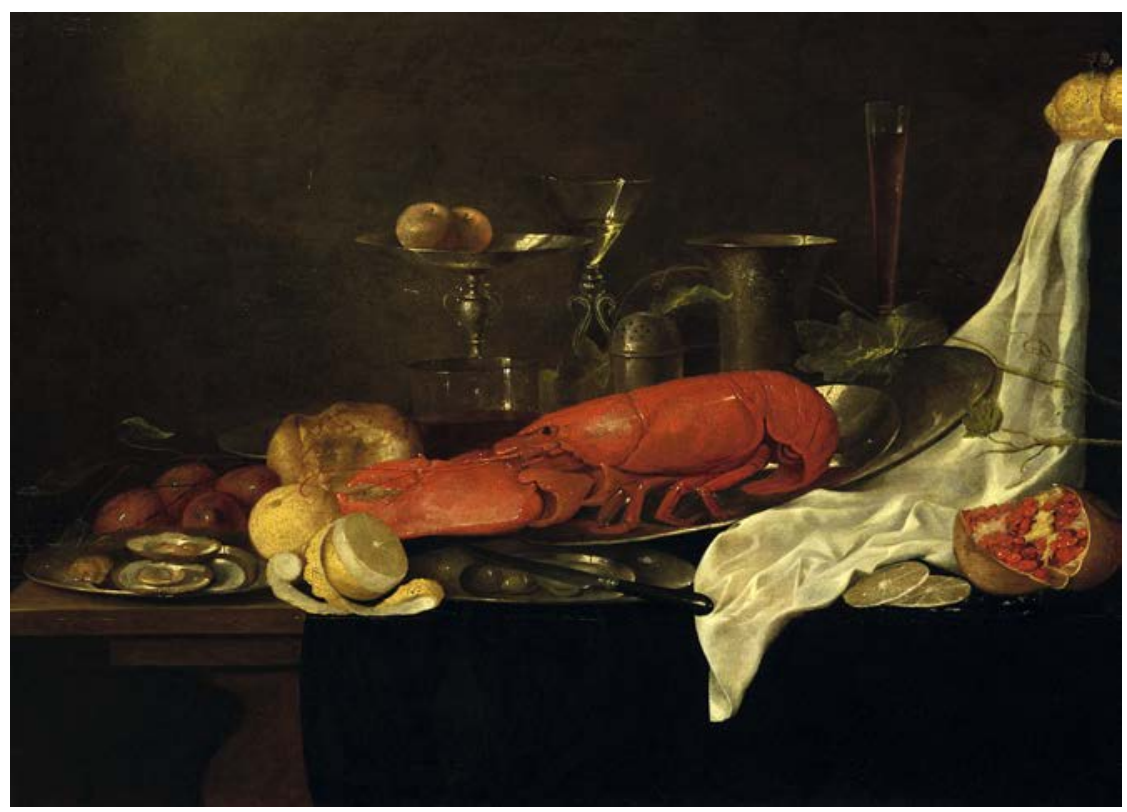

Abb. 8: Frühstücksstillleben mit Hummer, Joris van Son, 2. Drittel 17. Jahrhundert

\section{Fazit}

Will man die „1963er Zuweisungen“ an das Kunsthistorische Museum zusammenfassen, lässt sich folgendes sagen: Oft fehlende bzw. widersprüchliche Provenienzangaben, sich ändernde Zuschreibungen, kaum oder gar keine überlieferte Aktenlage der involvierten KunsthändlerInnen gestalten die Nachforschungen nicht immer einfach. Selbst in dem Fall der Bremer Werkschau, wo das Firmenarchiv noch vorhanden ist und im Krieg kaum Verluste erlitten hatte,

tail/auktion/10313-alte-meister/lotID/597/lot/1585038-francesco-salviati.html (abgerufen am 14.06.2017).

84 Vgl. Schober, Galerie St. Lucas (Anm. 82).

85 Vgl. BA Koblenz, B323/332, Sonderauftrag Linz. Kunsthändler-Aussagen, Band 2, fol. 145-150.

86 E-Mail Auskunft von Eva Metelka, 23.12.2015. 
lassen sich nur spärliche Angaben zu den VorbesitzerInnen finden. Eine Rückseitenautopsie aller genannten Bilder wird derzeit dank der Unterstützung der MitarbeiterInnen der Gemäldegalerie und des Fotoateliers vorgenommen, um weitere Rückschlüsse ziehen zu können. Aber auch dies wird nicht immer zu dem gewünschten Ergebnis führen, wie man anhand der Hl. Maria Magdalena feststellen musste. Trotz mehrerer Kunsthandlungen, durch die das Gemälde ging, lassen sich auf der Rückseite keinerlei Stempel, Etiketten oder ähnliche Hinweise finden. 
Open-Access-Publikation im Sinne der CC-Lizenz BY-NC-ND 4.0

(c) 2018, V\&R unipress GmbH, Göttingen

ISBN Print: 9783847107835 - ISBN E-Lib: 9783737007832 


\title{
"[...] ich kann Sie versichern, daß ich Ihnen das Paket mit den biogr. Schriften mit dem größten Vergnügen aufhebe“. "Treuhänderisch“ übernommene Sammlungen in der Wienbibliothek
}

\author{
Zusammenfassung \\ Unter den in der Wienbibliothek im Rahmen ihrer Provenienzforschung als „bedenklich“ \\ identifizierten Objekten nehmen zwei relativ umfangreiche Bestände einen wichtigen \\ Platz ein: die Sammlung der Schwestern Helene (1861-1942) und Elise Richter \\ (1865-1943) sowie die Bibliothek von Michael Holzmann (1860-1930). Beide kamen \\ nach dem Kriegsende als „herrenloses Gut“ an die Stadt Wien. In beiden Fällen war die \\ Wienbibliothek erst aufgrund der systematischen Untersuchung der Nachkriegskorre- \\ spondenz auf die „treuhänderischen“ Übernahmen gestoßen, waren beide Sammlungen \\ doch viel später inventarisiert worden. Der Beitrag befasst sich mit der Geschichte der \\ beiden Bestände, ihres Vorbesitzers bzw. ihrer Vorbesitzerinnen, der Übernahme und der \\ weiteren Behandlung des Materials, aber auch mit aus diesen Fällen zu ziehenden Kon- \\ sequenzen für die Provenienzforschung. \\ Schlagwörter \\ Bedenkliche Erwerbung, Provenienzforschung, treuhänderische Übernahme, Wien- \\ bibliothek im Rathaus, herrenloses Gut, Michael Holzmann, Elise Richter, Helene Richter
}

\begin{abstract}
s
„[...] ich kann Sie versichern, daß ich Ihnen das Paket mit den biogr. Schriften mit dem größten Vergnügen aufhebe“. Questionable Acquisitions in the Vienna City Library Among those objects in the Vienna City Library identified as "dubious" within the scope of its provenance research two relatively extensive holdings take an important place: the collection of the sisters Helene (1861-1942) and Elise Richter (1865-1943) as well as the library of Michael Holzmann (1860-1930). Both came after the end of World War II to the city of Vienna as heirless property. In both cases the Vienna City Library found the fiduciary takeovers only on account of the systematic investigation of the post-war correspondence, because both collections were accessed much later. The contribution deals with the history of both holdings, their previous owners, the takeover and the further treatment of the objects, but also with conclusions to be drawn from these cases for the provenance research.

Keywords

Questionable Acquisition, Provenance Research, Fiduciary Takeover, Vienna City Library, Heirless Property, Michael Holzmann, Elise Richter, Helene Richter
\end{abstract}




\section{Provenienzforschung in der Wienbibliothek}

Mit den Beschlüssen des Gemeinderates vom 29. April 1999 und vom 29. April 2011 hat sich die Stadt Wien verpflichtet, jene Kunst- und Kulturgegenstände aus den Museen, Bibliotheken, Archiven, Sammlungen und sonstigen Beständen der Stadt, die von Verfolgten des Nationalsozialismus stammen, unentgeltlich an die ursprünglichen Eigentümer oder deren Rechtsnachfolger zu übereignen. Diese Beschlüsse sind analog zu den Rechtsnormen zur Rückgabe von Kunst- und Kulturgegenständen auf Bundesebene zu sehen. Die Wienbibliothek im Rathaus hat 1999 mit einer intensiven Provenienzforschung begonnen und nahm dabei in der österreichischen Bibliotheksszene gemeinsam mit der Österreichischen Nationalbibliothek eine Pionierfunktion ein.

2.855 einzelinventarisierte Objekte (davon 180 Bücher und Zeitschriftenbände, 2.198 Handschriften der Literatur, 206 Handschriften der Musik und 271 Musikdrucke) sowie 24 nicht erschlossene Archivboxen wurden bisher an die Rechtsnachfolger der ursprünglichen Eigentümerinnen und Eigentümer restituiert, wobei der überwiegende Teil wieder angekauft oder der Bibliothek zum Geschenk gemacht wurde. In bisher sieben Fällen - der achte dürfte demnächst dazukommen - steht zwar die Restitution fest, die Suche nach Erbinnen und Erben konnte bisher aber nicht erfolgreich abgeschlossen werden. ${ }^{1}$

2.011 Objekte, darunter auch solche mit Provenienzvermerken von Personen, die als Jüdinnen und Juden im Sinne der Nürnberger Rassegesetze möglicherweise (aber nicht erwiesen!) durch Dritte geschädigt wurden, sind in der KunstDatenbank des Nationalfonds verzeichnet. ${ }^{2}$ Sämtliche Provenienzvermerke, darunter auch die große Masse hauseigener Sammlungsvermerke, können im Online-Katalog der Wienbibliothek recherchiert werden.

Unter den zahlreichen Sammlungen und Einzelobjekten, die die Wienbibliothek im Rahmen ihrer Provenienzforschung als „bedenklich“ identifiziert hat, nehmen zwei relativ umfangreiche Bestände einen wichtigen Platz ein: die Sammlung Richter und die Sammlung Holzmann. Beide kamen erst nach dem Kriegsende als „herrenloses Gut“ an die damalige Stadtbibliothek, die sie treuhänderisch übernahm: die Sammlung Holzmann mit 218 Druckschriften, 203 Handschriften sowie einer halben Archivbox mit nicht einzelkatalogisierten Autographen einerseits, die Sammlung Richter, bestehend aus 1.866 Handschriften, andererseits.

1 Die Berichte des amtsführenden Stadtrats zur Provenienzforschung der Stadt Wien sind auf der Site Wienbibliothek im Rathaus: Restitutionsberichte. URL: http://www.wienbibliothek. at/ueber-uns/provenienzforschung/restitutionsberichte (abgerufen am 12.06.2017), abrufbar.

2 Nationalfonds der Republik Österreich für Opfer des Nationalsozialismus: Kunstdatenbank. URL: https://www.kunstdatenbank.at/startseite.html (abgerufen am 12.06.2017). 


\section{Die Sammlung Holzmann}

Der Germanist und Lexikograph Michael Holzmann wurde am 21. Juni 1860 in Slawathen, Mähren (Slavetin, Tschechische Republik), geboren und starb am 20. Oktober 1930 in Wien. Nach Studien an den Universitäten Wien, Berlin und Lemberg, die er mit der Promotion zum Dr. phil. abschloss, trat er 1891 in den Dienst der Universitätsbibliothek Wien, aus der er 1922 als Oberstaatsbibliothekar mit dem Titel Hofrat ausschied. Gemeinsam mit Hanns Bohatta schuf er lexikalische Standardwerke wie das siebenbändige Deutsche Anonymen-Lexikon (1902-1928) oder das Deutsche Pseudonymen-Lexikon (1906), die ihm internationale Achtung sicherten. ${ }^{3}$ Seit 1916 war er mit Charlotte Planer verheiratet, mit der er in Wien 9, Alserbachstraße 2, lebte. ${ }^{4}$ Aus der Ehe gingen keine Kinder hervor. Ab Mai 1940 war Charlotte Holzmann in Wien 9, Servitengasse 22/9, gemeldet und wurde am 20. Juni 1942 nach Theresienstadt deportiert. Von dort kam sie noch im September des gleichen Jahres nach Treblinka; ab diesem Zeitpunkt gibt es keine Nachrichten mehr über sie. ${ }^{5}$

Im Mai 1945 schrieb Julia Meissl in ihrer Eigenschaft als provisorische Treuhänderin der „Althan-Garage“ in Wien-Alsergrund an das Amt für Kultur und Volksbildung, dass an diesem Ort wertvolles Schrifttum und Bücher aus dem Besitz des verstorbenen Gelehrten Prof. Holzmann aufbewahrt würden. Die Materialien wären von Charlotte Holzmann vor ihrer Deportation der Garagenbesitzerin Philomena Klinz - sie wird im Schreiben als „Pg.“ (also als Mitglied der NSDAP) bezeichnet - zur Aufbewahrung übergeben worden. Diese hatte seitdem die Sammlung aufbewahrt. Die Treuhänderin der Garage bat nun um rasche Abholung der Bestände wegen ständiger Plünderungsgefahr. ${ }^{6}$

Wie in einem Aktenvermerk festgehalten wurde, handelte es sich bei dieser Bibliothek um einen Bestand von „6 normalen Fächerkasten voll“, vor allem um ältere und neuere bibliographische, biographische und literaturgeschichtliche Werke. Hervorgehoben wurden Drucke des frühen und mittleren 19. Jahrhunderts, etwa Literatur von Ludwig Börne, der Literarische Zodiacus oder die norddeutsche Zeitschrift Der Freihafen. Es waren auch „Briefe u.dgl.“ vorhanden, wobei es sich um den privaten Briefwechsel von Michael Holzmann han-

3 Vgl. Österreichisches Biographisches Lexikon 1815-1950. Band 2: Glae-Hüb. Wien: Verlag der Österreichischen Akademie der Wissenschaften 1959, S. 411.

4 Vgl. Das Jahrbuch der Wiener Gesellschaft. Biographische Beiträge zur Wiener Zeitgeschichte. Hg. von Franz Planer. Jg. 1929. Wien: Selbstverlag 1929, S. 268.

5 Dokumentationsarchiv des österreichischen Widerstandes (DÖW): Shoah-Opfer, Personensuche. URL: http://www.doew.at/personensuche (abgerufen am 12.06.2017).

6 Wienbibliothek im Rathaus (WBR), Hausarchiv (HA), Julia Meissl an das Amt für Kultur und Volksbildung, 18.05.1945 (MA 9-126/45). 


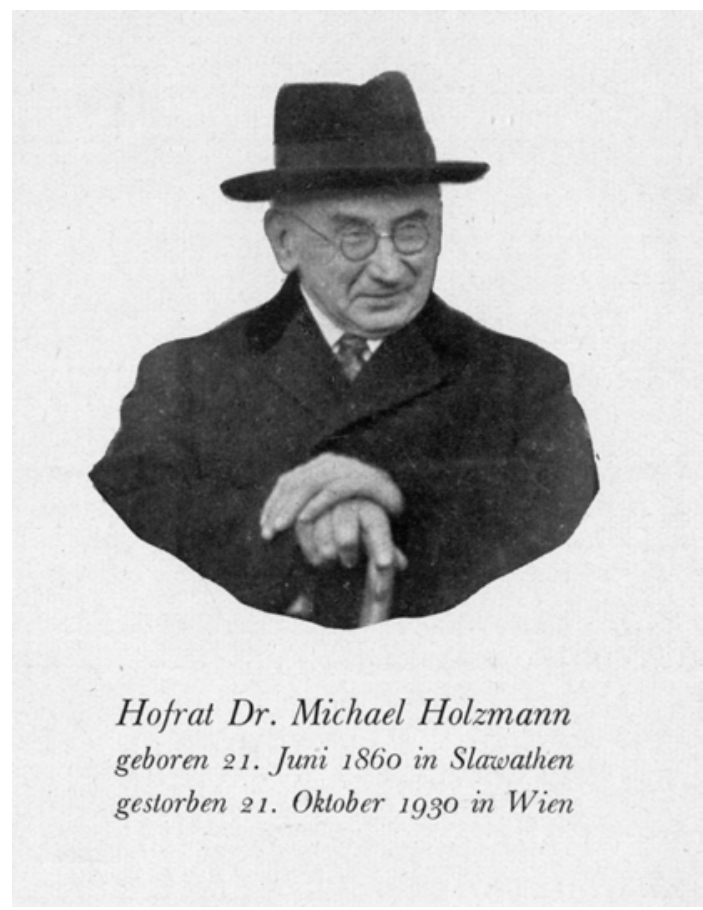

Abb. 1: In memoriam Dr. Michael Holzmann. Nachruf in der Zeitschrift für die Geschichte der Juden in der Tschechoslowakei 1 (1930/1931), H. 1/2

delte. Julia Meissl übernahm die Verantwortung für den Transport in die Volkshalle des Rathauses, der am 2. Juni 1945 in zwei Fuhren erfolgte. ${ }^{7}$

Mit dem Rechtsanwalt Oskar Löwenthal wurde vereinbart, die treuhändige Verwahrung durch die Stadtbibliothek in Form eines Briefes an ihn festzulegen. Die Direktion der Bibliothek bestätigte daher am 6. Juni 1945 in einem Schreiben an den Anwalt, dass sich die Bücherbestände nun ,in der treuhändigen Verwahrung der Wiener Stadtbibliothek bis zu einer weiteren Verfügung der vorgesetzten Dienststelle" befänden. ${ }^{8}$

Im Jänner 1950 wandte sich die Stadtbibliothek erneut an Rechtsanwalt Löwenthal und erinnerte ihn an die seinerzeitige Übernahme der Sammlung in die treuhändige Verwaltung. Der Rechtsanwalt wurde nunmehr gebeten, Verwandte der Witwe Holzmann und deren Adressen bekannt zu geben. ${ }^{9}$ Der Kanzleinachfolger des mittlerweile verstorbenen Löwenthal konnte keine An-

7 WBR, HA, Aktenvermerk von Karl Alfred Pfob, 05.06. 1945 (MA 9-126/45).

8 WBR, HA, Stadtbibliothek an Rechtsanwalt Oskar Löwenthal, 06.06.1945 (MA 9-126/45).

9 WBR, HA, Stadtbibliothek an Rechtsanwalt Oskar Löwenthal, 20.01.1950 (MA 9-40/50). 
gaben machen, ebensowenig Philomena Klinz, die ebenfalls angeschrieben wurde. $^{10}$

Nachdem der Bestand Holzmann vorerst im Depot verwahrt worden war, begann die Bibliothek 1965 mit der Inventarisierung der Objekte unter dem Stichwort „Donum“. Akten zu diesem Entschluss gibt es nicht. Fazit: In den ersten Nachkriegsjahren war sich die damalige Stadtbibliothek der Treuhandschaft bewusst und versuchte zumindest punktuell, Informationen über mögliche Erben einzuholen. Warum sie sich den Bestand nach 20 Jahren stillschweigend einverleibte, muss leider offen bleiben.

\section{Die Sammlung Elise und Helene Richter}

Etwas anders verlief der Erwerb der Sammlung Richter, der 1947 von der Stadt Wien übernommen wurde.

Elise und Helene Richter waren die Töchter von Maximilian Richter, Chefarzt der Wiener Südbahn. Elise, geboren am 2. März 1865 in Wien, studierte an der Universität Wien Romanistik, allgemeine Sprachwissenschaft, klassische Philologie und Germanistik. 1901 erste weibliche Promovierende im Bereich der Wiener Romanistik, habilitierte sie sich 1907 als erste Frau an der Universität Wien; 1921 wurde sie außerordentliche Professorin. Sie beschäftigte sich wissenschaftlich vor allem mit Semantik und Phonetik. Außerdem gehörte sie zu den Mitbegründerinnen des Verbandes der akademischen Frauen Österreichs, dessen Vorsitzende sie zeitweise war. ${ }^{11}$

Helene Richter, geboren am 4. August 1861 in Wien, bildete sich mittels autodidaktischer Studien sowie Vorlesungen an der Universität Wien (als Gasthörerin) weiter. Nach anfänglichen literarischen Versuchen wandte sie sich hauptsächlich der wissenschaftlichen Publizistik zu, vor allem auf dem Gebiet der englischen Literatur. Ihren Ruf als Anglistin begründete sie mit einer Geschichte der englischen Romantik; darüber hinaus wurde sie mit ihren Forschungen zu Shakespeare und durch Monographien bedeutender englischer Schriftsteller bekannt. Auf Grund ihrer wissenschaftlichen Leistung wurde sie 1931 Dr. h.c. der Universitäten Heidelberg und Erlangen. Die beiden unverheirateten Schwestern lebten gemeinsam; ihre Wohnung in Wien 19, Weimarer Straße 83, war durch Jahre hindurch ein Treffpunkt bedeutender Wiener Wissenschaftler und Künstler. ${ }^{12}$

10 WBR, HA, Rechtsanwalt Alexander Burian bzw. Philomena Klinz an die Wiener Stadtbibliothek, 24.01. bzw. 15.04.1950 (MA 9-40/50).

11 Vgl. Österreichisches Biographisches Lexikon 1815-1950. Band 9: Rázus Martin-Savić Žarko. Wien: Österreichische Akademie der Wissenschaften 1988, S. $123 \mathrm{f}$.

12 Ebd., S. 127. 
1938 wurde Elise Richter „aus rassischen Gründen“ ihrer universitären Funktion enthoben. Die Möglichkeiten der beiden Damen, ihren Lebensunterhalt zu bestreiten, sank von Jahr zu Jahr. So wurde Elise seitens der Universität Wien eine Alterspension verweigert, die Möglichkeit des freien Publizierens blieb auf das Ausland beschränkt. Seit 1939 von der Delogierung bedroht, mussten die beide Wissenschaftlerinnen im März 1942 in das Jüdische Altersheim Seegasse umziehen, wobei sie unter anderem ihre umfangreiche Bibliothek in der Weimarer Straße zurückließen. Der größte Teil der Romanica, Anglistica, Korrespondenzen, Autographen und Theatermappen wurden im April 1942 „sichergestellt" und in die Nationalbibliothek verbracht. Von dort wurde das meiste nach Köln versandt - die Schwestern waren auf Grund ihrer prekären finanziellen Situation schon länger mit der dortigen Universität in Verhandlungen gestanden. ${ }^{13}$

Am 9. Oktober 1942 wurden die Schwestern aus dem jüdischen Altersheim in der Seegasse 9 (Wien-Alsergrund) nach Theresienstadt deportiert. Helene Richter starb bereits im folgenden Monat (8. November 1942), die jüngere Elise einige Monate später (21. Juni 1943). ${ }^{14}$ Erst im Dezember 1972 wurden die beiden Wissenschaftlerinnen auch offiziell für tot erklärt. Anlass für die Einleitung des Verfahrens war die Löschung des für die beiden noch immer auf die Adresse Weimarer Straße 83 eingetragenen Wohnrechts. ${ }^{15}$

Bereits im Jahr 1941 hatte sich eine ehemalige Studentin von Elise Richter, Christine Rohr, bereit erklärt, einen Teil der Autographe und Briefe in Verwahrung zu übernehmen und in ihrem Haus zu verwahren. Auch zu ihr kurze biographische Angaben: Sie war die Tochter des königlich-ungarischen Feldmarschalls Franz Joseph Rohr Baron von Denta und wurde 1892 in Rodaun geboren. Sie studierte Romanistik an der Universität Wien und promovierte 1918. Im November 1919 wurde sie als erste Frau in den akademischen Bibliotheksdienst der Österreichischen Nationalbibliothek aufgenommen und führte das Referat für Romanistik. 1951 krankheitshalber in den vorzeitigen Ruhestand versetzt, starb sie 1961 in Wien. ${ }^{16}$

13 Da diese Fakten für den in der Wienbibliothek verwahrten Bestand nicht direkt relevant sind, hier nur als kurze Zusammenfassung wiedergegeben; ausführlich vgl. Thierry Elsen, Robert Tanzmeister: In Sachen Elise und Helene Richter. Die Chronologie eines „Bibliotheksverkaufs“. In: Geraubte Bücher. Die Österreichische Nationalbibliothek stellt sich ihrer NS-Vergangenheit. Hg. von Murray G. Hall, Christina Köstner und Margot Werner. Wien: Österreichische Nationalbibliothek 2004, S. 128-138 bzw. Christiane Hoffrath: Bücherspuren. Das Schicksal von Elise und Helene Richter und ihrer Bibliothek im „Dritten Reich“. 2. erg. Aufl. Köln-WeimarWien: Böhlau 2010 (= Schriften der Universitäts- und Stadtbibliothek Köln 19).

14 DÖW: Shoah-Opfer, Personensuche (Anm. 5).

15 Hoffrath: Bücherspuren (Anm. 13), S. 89.

16 Edith Stumpf-Fischer: Christine Rohr. In: BiografiA. Lexikon österreichischer Frauen. Hg. von Ilse Korotin. Band 3: P-Z. Wien-Köln-Weimar: Böhlau 2016, S. $2730 \mathrm{f}$. 
Im März 1941 schrieb Rohr an Elise Richter :

„Es tut mir jetzt leid, daß ich damals, als wir von Ihren biogr. Aufzeichnungen sprachen, nicht weiter darauf eingegangen bin, wie gerne ich Ihnen die Sachen in Verwahrung nehmen würde. [...] ich kann Sie versichern, daß ich Ihnen das Paket mit den biogr. Schriften mit dem größten Vergnügen aufhebe und daß ich froh bin, wenn ich Ihnen in diesen schweren Zeiten wenigstens damit behilflich sein kann! [...] Ich kann also ohne weiteres diese Schriften die ja doch ganz unpolitisch sind, in Verwahrung nehmen, es können da für mich keinerlei Unannehmlichkeiten erwachsen. “17

Die Bibliothekarin übernahm vorerst nur einige wenige Manuskripte; erst die Androhung der Beschlagnahme der Sammlung dürfte Richter dazu bewogen haben, einen größeren Teil an ihre ehemalige Studentin abzugeben. ${ }^{18}$

1947 übergab Christine Rohr den von ihr verwahrten Bestand den Städtischen Sammlungen (heute: Wien Museum). Da es sich ausschließlich um handschriftliches Material handelt („ein Konvolut von Tagebüchern und Korrespondenz aus dem Nachlaß von Dr. Elise Richter, Univ.-Prof. Wien geb. 1865, und Helene Richter, Schriftstellerin Wien geb. 1861“ [sic!]), wurde es im Mai 1947 an die Handschriftensammlung der Wiener Stadtbibliothek abgetreten. ${ }^{19}$ Offenbar hatte die loyale und hilfsbereite Bibliothekarin noch einige Zeit nach Kriegsende auf ein Lebenszeichen von Elise und Helene Richter gewartet, bevor sie sich entschloss, den Bestand einer öffentlichen Institution zu übergeben. Der Eingangsakt im Wien Museum ist leider nicht erhalten geblieben. ${ }^{20}$

Der Nachlassteil in der Handschriftensammlung der Wienbibliothek umfasst 1.868 Inventarnummern, bestehend aus der persönlichen Korrespondenz der Schwestern, Notizkalendern, Tagebüchern sowie anderen Lebensdokumenten. ${ }^{21}$ Die Erwerbung wurde im Mai 1947 in das Zuwachsprotokoll der Handschriftensammlung eingetragen. Die Einzelobjekte wurden zu einem kleinen Teil in den Jahren 1950-1952 katalogisiert, deren überwiegende Mehrheit erst um die Jahrtausendwende. Auch hier gibt es keinen Aktenvermerk oder Ähnliches, warum diese nun als Besitz der Bibliothek angesehen wurden.

17 WBR, Handschriftensammlung, Christine Rohr an Elise Richter, 07.03. 1941 (H.I.N. 232611).

18 Thierry Elsen, Robert Tanzmeister: In Sachen Elise und Helene Richter (Anm. 13), S. 136.

19 WBR, HA, Aktenvermerk der Städtischen Sammlungen an die Stadtbibliothek, 09. 05.1947 (MA 9-335/1947). Es handelt sich bei diesem aus zwei Sätzen bestehenden Dokument um das einzige Aktenstück zur Erwerbung.

20 Auskunft von Michael Wladika, Wien Museum, April 2017.

21 Eintrag im Inventar der Handschriftensammlung unter H.I.N. 108532-108680, 114195, 114315-114450, 223403-223413, 231808-231940 sowie 231943-233378. 


\section{Wren, $t^{2} / \pi \operatorname{lit} 48$}

\section{Hohwrearte traw Puferton!}

\& tut mir jeft leid, dars it damals, als vis $2 a$ Them tiogs. Anfzeidengeen Praccen, nidit veitis daranf eingegangen tini vic perne is o

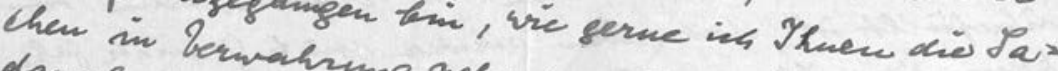
damals mint Ios walle nuid jent mus einige folrangen mol hake Jemen chadene

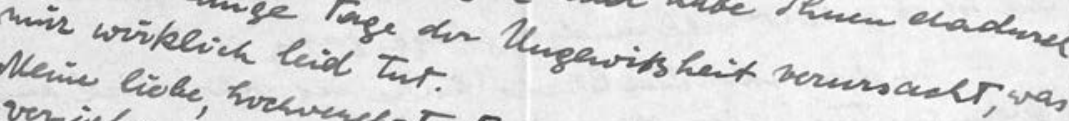
Nenic livbe, hacuesent

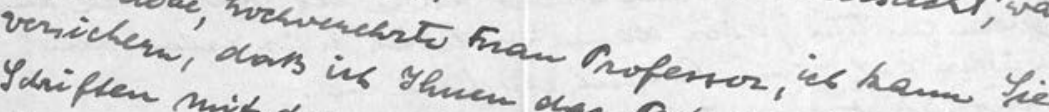

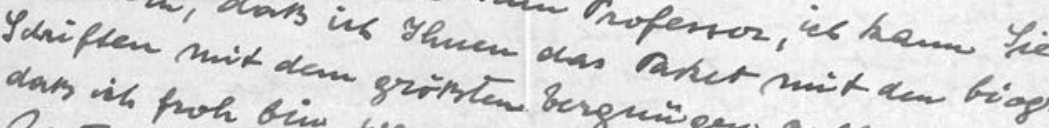

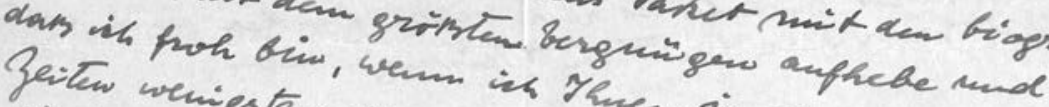

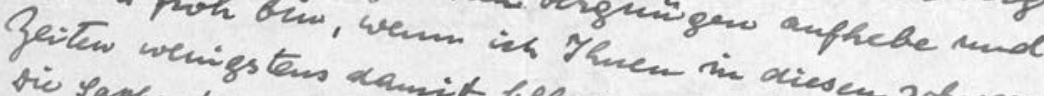

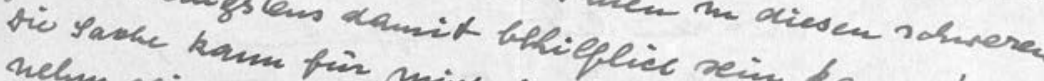

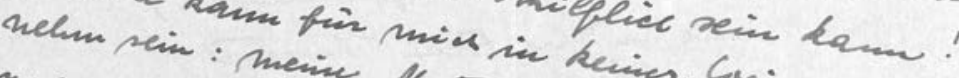
nomerer Jeans in Mutar it Mnes brice mange =

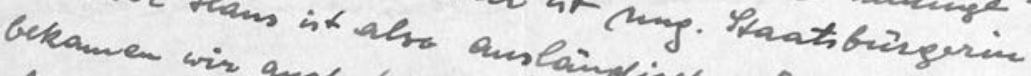

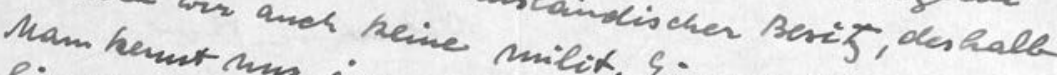

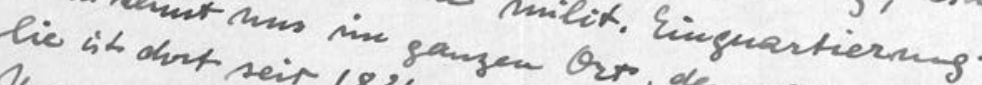

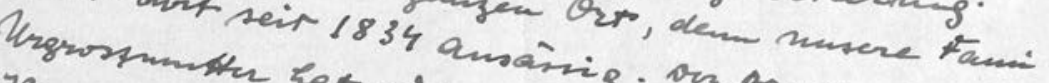
Hous gelant, Lat sie der $g$. Th bunder meives

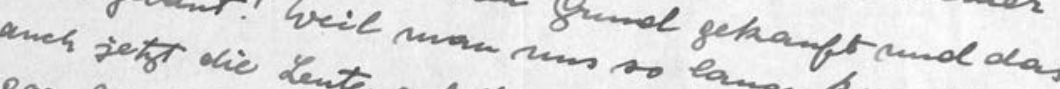

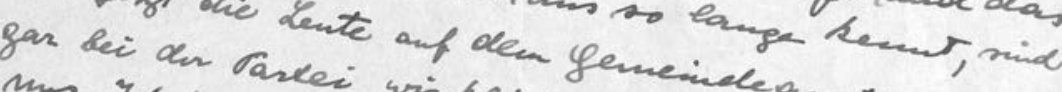

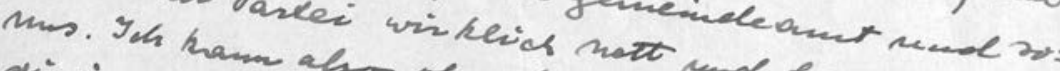

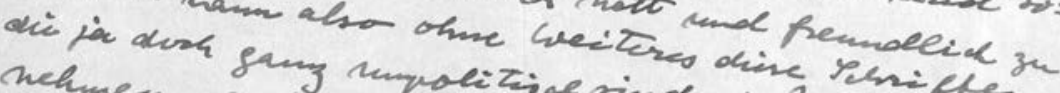

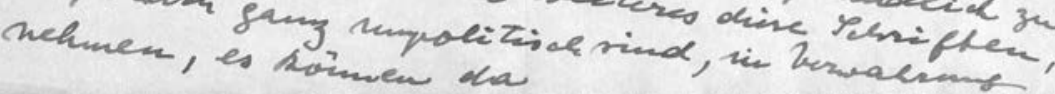

Abb. 2: Brief von Christine Rohr an Elise Richter, 07.03.1941 


\section{Konsequenzen für die Provenienzforschung}

Aus diesen beiden Fällen kann man für die praktische Provenienzforschung einige Lehren ziehen. Es stellte sich heraus, dass sich Provenienzforschung nicht auf Erwerbungen in der NS-Zeit (bis zur Befreiung Wiens im April 1945) und nicht auf Inventarbücher und Erwerbungsakten beschränkt. Auch andere Aktenkategorien können Hinweise auf geraubte Kunst- und Kulturgüter vermitteln. Es bedarf daher der Durchsicht der „normalen“ Korrespondenz zumindest in der Zeit bis 1950. Ohne diese wären die beiden von der Stadtbibliothek treuhänderisch übernommenen Sammlungen zumindest nicht so rasch gefunden worden.

Im Laufe der Jahre hat sich auch gezeigt, dass Einträge in Inventarbüchern wie „Donum“, „Alter Bestand“, ein Hinweis auf Raubkunst sein können, aber es nicht zwingend so sein muss. Näheres Hinschauen ist aber jedenfalls geboten!

Manchmal bedarf es auch externer Hinweise, um eine Sammlung oder ein Einzelobjekt als „bedenkliche Erwerbung“ zu identifizieren. Das können etwa auch Zeitschriftenartikel aus den 1970er Jahren oder periphere Anmerkungen in Gerichtsakten sein, wie es etwa beim jüngsten „Fund“ in der Wienbibliothek der Fall war. 
Open-Access-Publikation im Sinne der CC-Lizenz BY-NC-ND 4.0

(c) 2018, V\&R unipress GmbH, Göttingen

ISBN Print: 9783847107835 - ISBN E-Lib: 9783737007832 


\title{
„Den neuen Mietern zur treuhändigen Verwahrung übergeben“. Die Räumungen von „Judenwohnungen“ im Auftrag von „Vugesta“ und „Zentralstelle“
}

\begin{abstract}
Zusammenfassung
In einem perfiden System der erzwungenen Kollaboration wurde das Wohnungsreferat der Israelitischen Kultusgemeinde Wien durch verschiedene nationalsozialistische Behörden in einen Prozess der Delogierung und „Umsiedlung“ eingebunden, der schließlich in der Vertreibung und Vernichtung endete. Im Auftrag der „Zentralstelle für jüdische Auswanderung in Wien " erstellte sie Adress-, Haus- und Wohnungslisten, die zunächst zur Kontrolle der jüdischen Bevölkerung, später zu ihrer Deportation dienten. Das zurückgelassene Inventar wurde unter Aufsicht von Schätzmeistern und Antiquitätenhändlern von der „Verwaltungsstelle für jüdisches Umzugsgut der Geheimen Staatspolizei“ bei den Räumungen beschlagnahmt, in diverse Möbellager verbracht und wiederverkauft.

Schlagwörter

Vugesta, Zentralstelle für jüdische Auswandung in Wien, Registrierung der jüdischen Bevölkerung Wiens, Delogierung von jüdischen Mieterinnen und Mietern, Sammelwohnungen, Deportation, Zwangsarbeit bei Wohnungsräumungen, Hauslisten im Archiv der Israelitischen Kultusgemeinde Wien
\end{abstract}

\footnotetext{
Abstract

"Den neuen Mietern zur treuhändigen Verwahrung übergeben". Evictions of So-Called "Judenwohnungen" (Jews' Apartments) by Order of "Vugesta" and "Central Office" National Socialist authorities forced the housing office of the Jewish Community of Vienna to collaborate in a process that started with eviction and relocation and ended in the expulsion and murder of Austrian Jews. By order of the "Central Office of Jewish Emigration in Vienna", it compiled address lists, which served at first as a means of control and later on to facilitate the organisation of the deportations. Under Gestapo supervision, antiquarian booksellers and treasurers seized property and furnishings in vacated apartments, brought it to depots and resold it.

Keywords

Vugesta, Central Office of the Jewish Emigration of Vienna, Registry of the Jewish Community of Vienna, Eviction of Jewish Tenants, Collection Flats, Deportation, Forced Labour, House lists in the Archive of the Jewish Community of Vienna
} 
In einem perfiden System der erzwungenen Kollaboration wurde das Wohnungsreferat der Israelitischen Kultusgemeinde Wien durch verschiedene nationalsozialistische Behörden in einen Delogierungs-, Umsiedlungs- und Vertreibungsprozess eingebunden, der schließlich in der Vernichtung endete. Für die „Zentralstelle für jüdische Auswanderung“, einer Dienststelle des Sicherheitsdienstes der SS, der Abteilung B des Haupternährungsamtes sowie den Polizeipräsidenten in Wien, erstellte sie Haus-, Wohnungs- und Adresslisten, die zunächst zur Kontrolle der jüdischen Bevölkerung, später zur Zusammenstellung der Deportationstransporte dienten. ${ }^{1}$

Nach der Volkszählung vom Mai 1939, bei der historisch einmalig nicht nur die Religionszugehörigkeit, sondern auch die Abstammung nach den „Nürnberger Gesetzen“ abgefragt wurde, startete im September desselben Jahres eine zusätzliche „Sonderzählung der Juden“ in Wien, wo durch den erzwungenen Zuzug aus den anderen Bundesländern 97 Prozent der jüdischen Bevölkerung Österreichs wohnten. ${ }^{2}$

Die Menschen hatten sich mit ihren Meldezetteln bei der IKG einzufinden und etwaige Adressänderungen bekannt zu geben. Das Jüdische Nachrichtenblatt berichtete:

„Es wird darauf aufmerksam gemacht, daß in Hinkunft die Inanspruchnahme der IKG in Angelegenheiten der Auswanderung, der Fürsorge und in allen sonstigen Fragen nur auf Grund dieser Anmeldungen möglich sein wird, so daß das Nichtbeachten dieser Aufforderung schwerwiegende Folgen nach sich ziehen kann. ${ }^{“ 3}$

„Nichtglaubensjuden“ wurden auf ähnliche Weise von der „Aktion Gildemeester“, der späteren „Auswanderungshilfsorganisation für nicht mosaische Juden“, erfasst. Mit den erhobenen Daten erstellte die „Zentralstelle“ ihre so genannte „Judenkartei“.. Die verpflichtende Annahme der zusätzlichen Vornamen „Sara“ und „Israel“ ab Jänner 1939, die in den Kennkarten eingetragen und bei der Polizeibehörde angezeigt werden musste, ermöglichte die Anlage weiterer Adressverzeichnisse.

Ab November 1939 wurden Lebensmittelkarten von einer eigenen Abteilung des Haupternährungsamtes der Gemeindeverwaltung Wien mit einem „J“ ver-

1 Siehe: Jonny Moser: Österreich. In: Dimension des Völkermords. Die Zahl der jüdischen Opfer des Nationalsozialismus. Hg. von Wolfgang Benz. 1. Aufl. München: Oldenbourg 1991, S. 66-85, hier S. 68, bzw. Doron Rabinovici: Instanzen der Ohnmacht. Wien 1938-1945. Der Weg zum Judenrat. 1. Aufl. Frankfurt/Main: Jüdischer Verlag 2000, S. 213-222.

2 Gudrun Exner, Peter Schimany: Amtliche Statistik und Judenverfolgung. Die Volkszählung von 1939 in Österreich und die Erfassung der österreichischen Juden. In: Geschichte und Gesellschaft 32 (2006), S. 93-118, hier S. 99 bzw. S. 108.

3 Jüdisches Nachrichtenblatt, Ausgabe Wien, Nr. 71/72, 08.09.1939. Zit. in: Exner, Schimany: Amtliche Statistik (Anm. 2), S. 108.

4 Deren Verbleib nach 1945 ist unbekannt. 
sehen. Die Eröffnung der zentralen Lebensmittelkartenstelle für Jüdinnen und Juden in der Taborstraße ein Jahr später diente den nationalsozialistischen Machthabern wiederum als weiterer Vorwand, die IKG mit der Erstellung und kontinuierlichen Aktualisierung von Listen zu beauftragen. An Hauswarte und NS-Blockleiter wurden Formulare ausgeschickt, in denen die jüdischen Hausbewohnerinnen und -bewohner sowie ihr eventueller Arbeitgeber eingetragen werden mussten. ${ }^{5}$ Wer sich nicht registrieren ließ, konnte sich keine Bezugsscheine besorgen - diese waren nicht übertragbar - und sich folglich nicht mit den ohnehin für Jüdinnen und Juden beschränkten und rationierten Nahrungsmitteln versorgen.

Kenn- und Lebensmittelkarten, Identität und aktuelle Wohnadresse waren auf diese Weise in einem Personenstandsnachweis miteinander verknüpft. Die jüdische Bevölkerung wurde somit auf verschiedene Weise erfasst, ihre persönlichen Daten und die gewonnenen Informationen an Verwaltung und Partei weitergegeben. Die NS-Behörden hatten damit drei menschliche Grundbedürfnisse erfasst - Nahrung, Wohnen, Arbeit - und die jüdische Bevölkerung unter lückenlose Kontrolle gebracht. Das Statistische Amt der Stadt Wien berichtete am 10. Februar 1941 an den Bürgermeister:

„In der Judenkartenstelle II., Taborstraße 24a wurde der neueste Stand der Besetzung von Wohnungen durch Juden in Wien wie folgt festgestellt: [...] 12.624. Im Wohnungsamt konnte festgestellt werden, dass mit dem Stichtag vom 11. Jänner laufenden Jahres 10.926 Judenwohnungen in Wien noch vorhanden waren. Die Differenz zwischen dem Ergebnis der Zählung der Judenkartenstelle und dieser Auskunft kann möglicherweise darin liegen, dass in der Judenkartenstelle Untermieter nicht gesondert ausgewiesen sind und ein geringer Teil von Juden in minderwertigen Ubikationen untergebracht ist, die für die Erfassung durch das Wohnungsamt nicht in Betracht kommen. ${ }^{\text {"6 }}$

Ab diesem Zeitpunkt dienten die Hauslisten der „Zentralstelle“ als Datenabgleich und Aktualisierung ihrer „Judenkartei“ für die Zusammenstellung der großen Deportationen, die unmittelbar danach einsetzten. Durch die erzwungene Emigration, Delogierungen und Umsiedlungen innerhalb des Stadtgebietes drohten fehlerhafte Adresslisten für die NS-Behörden schließlich zum Problem zu werden. ${ }^{7}$

5 Siehe: Archiv der IKG Wien, Wohnungsangelegenheiten, Judenwohnungen, bezirksweise geordnet (aufgrund der Hauslisten von Oktober 1942 erarbeitete Aufstellung), A/W 435.

6 Zit. in: Exner, Schimany: Amtliche Statistik (Anm. 2), S. 115.

7 Aktenvermerk über die Besprechung im Büro des Obergebietsführers Müller betreffend die Evakuierung der Juden aus Wien, 12.02.1941. Zit. in: Widerstand und Verfolgung in Wien 1934-1945. Hg. von Dokumentationsarchiv des österreichischen Widerstandes. Band 3, 1. Aufl. Wien: Österreichischer Bundesverlag 1984, S. 290 f. 
In der Spalte „Übersiedlungen“ wurde, falls die Parteien nicht innerhalb Wiens umzogen, jeweils mit rotem oder blauem Buntstift in römischen Zahlen die Nummern der Transporte in den Tod eingetragen. Die im Archiv der IKG Wien vorhandenen Listen von 1941/42 bzw. 1943 sind nach den 26 Bezirken Groß-Wiens und innerhalb derselben alphabetisch nach Straßennamen geordnet, wobei einzelne Bezirke leider fehlen. ${ }^{8}$ Aufgrund des enormen Umfangs von mehr als zehntausend Seiten ist diese für die Erforschung der Zwangsumsiedlung und Deportation der jüdischen Bevölkerung Österreichs äußerst wertvolle Quelle wissenschaftlich noch nicht bearbeitet.

\section{Die „Eingerückten“}

Des Weiteren befinden sich im Bestand „Wohnungsangelegenheiten“ des Archivs der IKG Wien unter anderem Unterlagen über die sogenannten „Aushebungen, Verzeichnisse und Aufstellungen betreffend zur Abwanderung bestimmte, in den Wohnungen belassene, eingerückte oder zur Aufnahme in eine geschlossene Anstalt bestimmte Personen“ bzw. „eine vollständige oder teilweise Räumung von Wohnungen“ von April bis Juli 1942.

Während die Deportierten im Frühjahr 1941 noch schriftlich aufgefordert worden waren, sich in einem der Sammellager zu melden, leisteten viele Betroffene im Herbst 1941 der Einberufung keine Folge mehr, nachdem Rückkehrer der bereits im Oktober 1939 nach Nisko am San Verschleppten von den Zuständen in den Ghettos im „Generalgouvernement“ berichtet hatten und Nachrichten über Massenerschießungen durch die Einsatzgruppen durchgesickert waren. $^{9}$

Die „Aushebungen“ nahmen daher in der Folge sogenannte Rechercheure, Angestellte der IKG vor, die durch ihre Tätigkeit vorerst ihr eigenes prekäres Überleben sicherten und in ihren Wohnungen verbleiben durften. Die „Eingerückten“ waren diejenigen, die als letzte Station vor der Deportation in eines der Sammellager in der Malzgasse, der Kleinen Sperlgasse oder der Castellezgasse im zweiten Wiener Gemeindebezirk verbracht wurden. Die euphemistisch so genannte „Abwanderung“ führte zu diesem Zeitpunkt, als die Grenzen des

8 Die Hauslisten sind im Archiv der IKG Wien entweder als Buch gebunden im Original, zumeist aber als Digitalisat, (A/VIE/IKG/II/BEV/WOHN/1-12), diejenigen aus den „Central Archives for the History of the Jewish People“ in Jerusalem als Mikrofilm einsehbar (A/W 436,1-16 bzw. A/W 437,1-3).

9 Gabriele Anderl, Dirk Rupnow: Die Zentralstelle für jüdische Auswanderung als Beraubungsinstitution. Wien-München: Oldenbourg 2004 (= Nationalsozialistische Institutionen des Vermögensentzuges 1; Veröffentlichungen der Österreichischen Historikerkommission 20/1), S. 29. 
Großdeutschen Reichs für jüdische Flüchtlinge seit geraumer Zeit geschlossen waren, nach Minsk, Izbica, Sobibor, Theresienstadt und Auschwitz. ${ }^{10}$

Die Dokumente beinhalten neben den genauen Adressen inklusive der Türnummern und der Anzahl der „eingerückten“ Personen auch Spalten mit den Überschriften „belassen“, „Altersheim“ und „Spital“. Diese Blätter dürften allerdings nur der Vorbereitung oder der Statistik gedient haben, für die Durchführung der „Aushebungen“ selbst folgten „Dienstzettel“. Jeweils drei „Rechercheure“ wurden einer Wohnung zugewiesen, die gesamte „Aktion“ überwachte ein „Gruppenführer“. Die Spalte „Übersiedlungen“ enthält das Datum der „Aushebungen“ und die Transportnummer. Unter „Anmerkungen“ bzw. „Entscheidungen“ ist festgehalten, ob es sich bei den einzelnen Wohnparteien um Angestellte der IKG oder der „Auswanderungshilfsorganisation für nichtmosaische Juden in der Ostmark“, um „privilegierte“ oder „nicht-privilegierte Mischehen" handelte, die vorerst in ihren Wohnungen verbleiben durften. Ansonsten wurde lediglich auf Jüdinnen und Juden ausländischer Staatsbürgerschaft - sofern diese mit den „Achsenmächten“ verbündet oder neutral waren Rücksicht genommen.

Nach erfolgtem Abtransport meldeten die „Erhebungsbeamten“ der IKG die zurückgebliebenen Mieterinnen und Mieter - meist in (Zwangs-)Arbeit, „Mischlinge“ oder „Mischehen“, - versperrten die Zimmer und berichteten, ob die Wohnungen noch möbliert oder tatsächlich unbewohnt waren. Die Vollzugsmeldung lautete in diesem Fall: „alle Personen im Lager“. In die leergeräumten Zimmer wurden von der Abteilung „Judenumsiedlung“ des Wiener Wohnungsamtes in den darauffolgenden Tagen neue Untermieterinnen und -mieter zugewiesen. ${ }^{11}$

Aber nicht alle Betroffenen ließen sich ohne weiteres abführen. In einer handschriftlichen Notiz zur Kleinen Stadtgutgasse 3, Tür 16/III, verzeichnete beispielsweise Rechercheur Ing. Kuhn am 21. April 1942 sieben Personen als „abwesend“, drei als „abgängig“ und drei Wohnungen bereits als „leer“. Entweder waren die Bewohnerinnen und Bewohner per Zufall nicht zu Hause oder offensichtlich rechtzeitig geflüchtet bzw. untergetaucht. Auf dem Blatt befindet sich zudem eine handgezeichnete Skizze der Wohnung mit einem genauen Eintrag der Türen und Fenster. Für die nicht aufgefundenen Personen bat in einem folgenden Schreiben die „Zentralstelle“, die die „dringende Recherche“

10 Jonny Moser: Demographie der jüdischen Bevölkerung Österreichs. Wien: 1999 (=Schriftenreihe des Dokumentationsarchivs des österreichischen Widerstandes zur Geschichte der NS-Gewaltverbrechen 5), S. $80 \mathrm{f}$.

11 Siehe Philipp Mettauer: „Das ewige Übersiedeln“. Die Wiener Sammelwohnungen 1939-1942. In: In die Häuser schauen. Aspekte jüdischen Wohnens vom Mittelalter bis ins 20. Jahrhundert. Juden in Mitteleuropa. Hg. von Institut für jüdische Geschichte Österreichs. St. Pölten: Institut für Jüdische Geschichte Österreichs 2016, S. 48-55. 
angeordnet hatte, um Mitteilung, „ob der/die Genannte tatsächlich ausgewandert ist". 12

\section{„Ungeheure Mengen an Wertgegenständen“}

Sofern sich die Betroffenen oder deren Angehörigen nicht mehr selbst darum kümmern konnten, führte die IKG in Abstimmung mit der Abteilung „Judenumsiedlung“ des Wiener Wohnungsamts auch Wohnungsräumungen durch. In einem Brief im April 1939 berichtete beispielsweise Stella Leist ihrer Schwester Trude Wang, die bereits nach Santa Fe, der Hauptstadt der gleichnamigen Provinz in Argentinien emigriert war, was die Delogierungen für ältere Menschen bedeuten konnte:

„Mit der Mama ist es ein Kreuz. Wir haben bei den Eltern von Rudis Frau ein Zimmer gemietet, da aber die Eltern nicht Wirtschaft führen, muss Mama zur Jenny essen gehen. Bei Jenny ist aber gar kein Platz, nach dem Essen müsste sie wieder in ihr Zimmer in den 4ten Stock und das ist nicht möglich. So wird sie zur Tante Stephanie gehen. [...] Die Wohnung in der Custozzagasse ist bereits per 1.V. vermietet, wir sind oft dort, um alle Sachen wegzubringen. Einen Teil bekommt die Jenny, den anderen die Kultusgemeinde und der Tandler." ${ }^{“ 13}$

Die IKG übernahm aber nicht nur Möbel und Alltagsgegenstände, sondern stellte für die „Zentralstelle“ beispielsweise auch die technischen Apparaturen „abgegangener Kranken- bzw. Zahnbehandler" sicher. ${ }^{14}$ Wertvolles Inventar wurde von Schätzmeistern im Auftrage der „Verwaltungsstelle für jüdisches Umzugsgut der Geheimen Staatspolizei“ („Vugesta“) beschlagnahmt, in diverse Möbellager in der Krummbaumgasse 8, der Ferdinandstraße 25, Rotensterngasse 11 oder Mohapelgasse 3 verbracht und an Partei- und Volksgenossen wiederverkauft.

Die im September 1940 gegründete „Vugesta“ stellte dabei, wie die beim „Ministerium für Arbeit und Wirtschaft“ angesiedelte „Vermögensverkehrsstelle“, ein „ostmärkisches“ Spezifikum als „Arisierungsbehörde“ dar. Während im „Altreich“ die Beschlagnahme und Verwertung des jüdischen „Umzugsguts“ sowie der zurückgebliebenen Wohnungseinrichtungen meist direkt durch Staatspolizei und Finanzverwaltung erfolgte, wurde in Wien eine eigene, dazwischen geschaltete Stelle geschaffen, die zwar formal der Gestapo unterstand,

12 Archiv der IKG Wien, Wohnungsangelegenheiten, Berichte, Mitteilungen und Durchführungsbestätigungen über Wohnungsräumungen, A/W 434.

13 Institut für jüdische Geschichte Österreichs, Lebenserinnerungen österreichischer Jüdinnen und Juden; Stella Leist (Wien) an Trude Wang (St. Fe), 21.04.1939; Siehe weiterführend: Philipp Mettauer: Erzwungene Emigration nach Argentinien. Österreichisch-jüdische Lebensgeschichten. Münster: Aschendorff 2010.

14 Archiv der IKG Wien, (Anm. 12), A/W 434. 
de facto aber in der Speditionsbranche angesiedelt war und in ihrem Agieren stark privatwirtschaftliche Züge entwickelte. ${ }^{15}$

Ihre erste Aufgabe war die Abwicklung der Liquidierung der durch unterschiedliche Ursachen liegengebliebenen und bei Speditionen eingelagerten sogenannten „Lifts“, so der zeitgenössische Terminus für Übersiedlungscontainer nach Übersee, „reichsflüchtiger Juden“, die vom „Reichssicherheitshauptamt“ beschlagnahmt worden waren. Von der Gestapo bestellte, externe Gutachter schätzten die Warenwerte und trafen die Vorbereitungen für den Verkauf.

Von den insgesamt 20 Schätzmeistern der „Vugesta“ war Bernhard Witke (1896-1970) ${ }^{16}$ wohl einer der Bekanntesten. Als SA-Sturmführer und Möbeltischler hatte er die Kunst- und Antiquitätenhandlung Rudolf Berger \& Sohn in Wien-Mariahilf „arisiert“, um dann als beeideter Sachverständiger in das florierende Geschäft der Hehlerei von jüdischen Wohnungseinrichtungen einzusteigen. Sein Kompagnon, Michael Oberhuber (geb. 1878), der seit Jahrzehnten Angestellter der Firma gewesen war, berichtete bei seiner Einvernahme im Volksgerichtsprozess, der 1945 gegen die beiden geführt wurde: „Die Anlieferungen erfolgten von der Vugesta mittels Kraftwagen und zwar wöchentlich in drei bis vier Fuhren. Meist wurden Möbel gebracht, aber auch Glaswaren in Körben und Kisten, Porzellan, Teppiche und anderes. Auch Silberwaren und Bilder wurden gebracht. “17

Witke wiederum sagte aus, dass anfangs die Kultusgemeinde die Räumungen durchgeführt und das Mobiliar in ihren Magazinen, „die zweifellos auch so groß waren als die meinen, “ untergebracht und verkauft habe. „Erst später erhielt ich diese Liste nicht mehr von der Kultusgemeinde, sondern von der Gestapo selbst. “18

15 Siehe: Sabine Loitfellner: Die Rolle der „Verwaltungsstelle für jüdisches Umzugsgut der Geheimen Staatspolizei“ (Vugesta) im NS-Kunstraub. In: NS-Kunstraub in Österreich und die Folgen. Hg. von Gabriele Anderl, Alexandra Caruso. Innsbruck-Wien-Bozen: Studienverlag 2005, S. 110-120.

16 Die Schreibweise in historischen Quellen und Fachliteratur variiert zwischen Witke und Wittke.

17 Gabriele Anderl: Die Stunde der Ariseure. In: DerStandard.at, 02. 10.2009. URL: www.der standard.at/1254310468078/Restitution-Die-Stunde-der-Ariseure (abgerufen am 16.06. 2017), bzw. United States Holocaust Memorial Museum Archives, Wiener Prozesse wegen NS-Verbrechen, Volksgerichtsverfahren gegen Bernhard Witke, Michael Oberhuber u. a., LG Wien Vg 2 d Vr 2331/45 bzw. LG Wien 8e Vr 761/55. URL: https://collections.ushmm.org/fin dingaids/RG-17.003M_01_fnd_de.pdf (abgerufen am 16.06.2017). Witke wurde zu dreieinhalb Jahren Haft verurteilt, Oberhuber freigesprochen, das Verfahren gegen den GestapoMitarbeiter Anton Grimm 1955 eingestellt.

18 Beschuldigtenvernehmung Bernhard Witke, 29.11.1946. Zit. in: Gabriele Anderl, Edith Blaschitz, Sabine Loitfellner, Mirjam Triendl und Niko Wahl: „Arisierung“ von Mobilien. Veröffentlichungen der Österreichischen Historikerkommission. Vermögensentzug während der NS-Zeit sowie Rückstellungen und Entschädigungen seit 1945 in Österreich. Band 15. Wien-München: Oldenbourg 2004, S. 141. 
Ab 1942 leitete Witke gemeinsam mit Gestapo-Inspektor Anton Grimm die „Möbelverwertungsstelle Krummbaumgasse“, in der die zurückgelassenen Einrichtungs- und Gebrauchsgegenstände aus den Wohnungen der in die Ghettos, Konzentrations- und Vernichtungslager Deportierten veräußert wurden. Die Schätzmeister waren dabei an die Weisungen der Staatspolizei gebunden und arbeiteten eng mit der „Zentralstelle“ zusammen. Karl Ebner, zunächst Leiter des "Judenreferats“, danach stellvertretender Chef der Gestapo in Wien, stellte in einem Schreiben an den Reichsstatthalter in Niederdonau folgendes fest:

„Pg. Witke ist seit 19.8.1940 ununterbrochen für meine Dienststelle tätig. In seinen Aufgabenkreis fiel die Freimachung der Wohnungen und Unterkünfte von 48.500 Juden, die ich aus meinem Dienstbereich (Wien-Niederdonau) nach den Ostgebieten evakuiert habe. Er hat sich besondere Verdienste dadurch erworben, dass er in einer derart kurzen Frist die Wohnungen freimachte, wie es bisher im gesamten Reichsgebiet nicht erfolgte. [...] Ich habe ihn immer wieder gebeten, für mich weiter zu arbeiten, da es mir nicht verantwortlich erschien, die Unterkünfte dem Wohnungsmarkt länger als unbedingt notwendig vorzuenthalten. [...] Ich darf noch bemerken, dass er sich auch beachtliche Verdienste bei der Zustandebringung [sic] von wertvollen Kunstschätzen erworben hat, die sonst dem Reich verloren gegangen wären. ${ }^{\text {“19 }}$

Nach Angaben eines weiteren „Vugesta“-Schätzmeisters, Leopold Berka (geb. 1890), hätte Witke das Mobiliar der in das Protektorat, Grimm hingegen das der in die restlichen besetzten Gebiete Zwangsverschickten zur Verwertung übernommen. ${ }^{20}$ Diese Aussage dürfte allerdings keine allgemeine Gültigkeit besitzen, erhielt Witke doch beispielsweise im Juli 1942 - in diesem Monat gingen vier Transporte nach Theresienstadt - lediglich eine Wohnungseinrichtung bestehend aus „1 Kasten, 1 Ottoman, 1 Küchenkredenz, 1 Bett, 1 Inrusabett, 1 Küchentisch, 2 Sessel, 1 Stockerl, 1 P. Gummistiefel“ ${ }^{\text {“ }}$ abgeliefert. ${ }^{21}$ Später übernahm er zudem zurückgelassene Gegenstände der im Transport 47e am 24. Februar 1944 nach Auschwitz Deportierten. ${ }^{22}$

Im Laufe des Jahres 1943 brachten Witke und Oberhuber schließlich ihr Raubgut vor den alliierten Bombenangriffen nach Niederösterreich in Sicherheit. Sie nutzten dabei das Pfarrhaus in Purgstall an der Erlauf und für „die wertvolleren Sachen (Tabernakel [!], Fauteuils, Kommoden, Vitrinen, Schreib-

19 Schreiben von Karl Ebner an den Reichsstatthalter in Niederdonau, 05.07.1943, Beweismaterial im Verfahren gegen Bernhard Witke, Vg 2 d Vr 2331/45, Bl. 54. Zit. in: Anderl: „Arisierung" (Anm. 18), S. 145.

20 Dieter Hecht, Eleonore Lappin-Eppel, Michaela Raggam-Blesch: Topographie der Shoah. Gedächtnisorte des zerstörten jüdischen Wien. Wien: Mandelbaum 2015, S. 377-379.

21 Archiv der IKG Wien, Monatsbericht der Amtsdirektion, 01.-31.07.1942, A/W 434.

22 Anderl: „Arisierung“(Anm. 18), S. 144. 
kommoden etc.)“ den Pfarrhof von Stadt Haag, Bezirk Amstetten. ${ }^{23}$ „Ungeheure Mengen an Wertgegenständen“ waren nach Kriegsende noch vorhanden, „welche die Depots [...] eng aneinander gepfercht, nahezu bis zur Decke füllten“, so der Sachverständige 1947 an das Volksgericht Wien. „Man muss dieses Depot in Haag gesehen haben, um sich hievon eine richtige Vorstellung machen zu können. “24

\section{„Willi, du arbeitest bei mir!“}

$\mathrm{Zu}$ den Räumungs- und Lagerarbeiten wurden jüdische Zwangsarbeiter und Zwangsarbeiterinnen herangezogen. Ein Zeitzeuge, der 1934 in Wien geborene Harry Merl, erzählt im lebensgeschichtlichen Interview seinem Enkel über das Überleben während des NS-Regimes:

„Im März 1942 mussten wir erneut unser Zimmer verlassen und übersiedelten in eine Sammelwohnung am Nestroyplatz, daneben war die Ruine vom Tempel. Wir teilten uns eine Wohnung mit dem Ehepaar Kandl und einem Alexander Grün. Eine Hausliste vom Nestroyplatz aus dem Jahr 1943 zeigt, dass zu diesem Zeitpunkt alle Juden entweder für die Vugesta oder Rudolf Schneeweiss arbeiteten. Bei meinen Eltern, sowie bei Karl und Marie Kandl, die den Krieg überlebten, findet sich unter ,In Arbeit für' der Eintrag ,Möbelverwertungsstelle Schätzmeister Wittke ““. ${ }^{\text {(25 }}$

Genannter Rudolf Schneeweiss war in mehreren gewinnbringenden Geschäftsfeldern tätig. Einerseits hatte er im August 1938 die Lebensmittelhandlung Alfred Schweiger \& Co. am Viktor Adler Markt „arisiert“ und belieferte von dort die in der Castellez- und Sperlgasse eingerichteten Sammellager sowie die Deportationstransporte selbst mit Lebensmitteln - wobei die Ausgaben der IKG in Rechnung gestellt wurden. Andererseits handelte er als „Vugesta“-Funktionär in seinem Magazin am Bacherplatz mit Bildern aus den leergeräumten Wohnungen, wie ein Nachbar der Merls am Nestroyplatz, Ing. Fritz Kraus, 1947 als Zeuge im Volksgerichtsprozess bestätigte. ${ }^{26}$

23 Polizeiliche Niederschrift mit Michael Oberhuber, 27.09.1945, Verfahren gegen Bernhard Witke, Bl. 73. Zit. in: Anderl: „Arisierung“ (Anm. 18), S. 139.

24 Bericht des gerichtlich beeideten Sachverständigen und Schätzmeisters Julius Werthner an das Volksgericht Wien, 22.07.1947, Verfahren gegen Bernhard Witke, Bl. 17. Zit. in: Anderl, "Arisierung" (Anm. 18), S. 159.

25 Pascal Merl: Harry Israel Merl - Persönliche Erinnerungen eines jüdischen Kindes im historischen Kontext der Zeit des Nationalsozialismus in Wien. Bachelorarbeit an der Pädagogischen Hochschule Oberösterreich, Linz 2014, S. 17-20. Ein Gruppenfoto, aufgenommen in dieser Sammelwohnung, ist abgedruckt in: Mettauer: „Das ewige Übersiedeln“ (Anm. 11), S. 48.

26 Vierter Bericht des amtsführenden Stadtrates für Kultur und Wissenschaft über die gemäß dem Gemeinderatsbeschluss vom 29. April 1999 erfolgte Übereignung von Kunst- und 
Nach Zwangseinsätzen in den Arbeitslagern in Eisenerz und Traunkirchen war Wilhelm Merl, von Beruf Goldschmied, im Mai 1941 nach Wien zurückgekehrt, wo er sich zunächst als Hilfsarbeiter der IKG und schließlich als Möbelpacker verdingte. Harry Merl erzählt im Interview:

„Witke sah meinen Vater am Bahnhof und da er durch seinen kräftigen Körperbau
auffiel, rief er ihm zu: ,Willi, du arbeitest bei mir!‘ [...] Es ist nicht klar, warum er
meinen Vater beim Namen kannte. Vermutlich wurde der Kontakt über Karl Ebner
hergestellt. Wir waren geschützt, da meine Eltern als Zwangsarbeiter gebraucht und
von der Deportation verschont wurden. Diese Arbeiten waren unter Juden dement-
sprechend begehrt. Der Schutz galt solange, bis man nicht mehr gebraucht wurde.
Meine Eltern arbeiteten beide bei der Vugesta bis zu Beginn des Jahres 1945. Es muss für
sie als Juden demütigend gewesen sein, Wohnungen von deportierten Juden auszu-
räumen, aber sie äußerten sich nie dazu. Während sie arbeiteten, war ich oft alleine in
unserem Zimmer. Mir wurde eine Thermoskanne mit Tee und etwas zu Essen dage-
lassen. ${ }^{\text {(27 }}$

Mutter Sabine Merl war als Buchhalterin zunächst der Lebensmittelhandlung Schneeweiss, danach ebenfalls der Möbelverwertungsstelle Krummbaumgasse zugeteilt und ,arbeitete bei künstlichem Licht manchmal bis in die Nacht hinein an einem Berg Rechnungen. [...] Die anderen, die in unserer Wohnung gewohnt haben, sind auch deportiert worden. Der Tod war immer irgendwie da. ${ }^{\text {"28 }}$

Nachdem die Wohnungsräumungen im Laufe der Zeit immer seltener geworden waren und die Gefahr „,abgeholt zu werden“ bedrohlicher wurde, versuchte Sabine Merl ihren durch die Zwangsarbeit entstandenen Kontakt zu einem leitenden NS-Funktionär zu nutzen und für das prekäre Überleben der Familie Vorsorge zu treffen.

„Da war bei der Gestapo ein Mann namens Rixinger, ${ }^{29}$ der hat dann begonnen, mit der Ambivalenz zu spielen. Das heißt, er hat sich irgendwie sichern wollen, dass er auch sagen kann, er hat Juden geholfen. Der hat zu meiner Mutter gesagt, sie soll jeden Tag anrufen, ob auch wir deportiert werden. Da hat's das Codewort gegeben: ,Werden die Kohlen schon geliefert?' Und wenn die Kohlen geliefert werden, dann ist es Zeit zum Untertauchen. ${ }^{\text {“30 }} \mathrm{Zu}$ Beginn des Jahres 1945 bezogen die Merls dann tatsächlich ihr Versteck in einem Kohlenkeller in der Odeongasse, wo sie die Befreiung durch die Rote Armee erlebten.

Kulturgegenständen aus den Sammlungen der Museen der Stadt Wien sowie der Wiener Stadt- und Landesbibliothek. Hg. von Stadt Wien. 10.11.2003, S. 87 bzw. 99 f.

27 Merl: Erinnerungen (Anm. 25), S. 19.

28 Interview mit Harry Merl am 29.05.2015 in Gramastetten, Kamera Wolfgang Gasser, Interviewer Philipp Mettauer. Kopie am Institut für jüdische Geschichte Österreichs.

29 Der Referatsleiter für „Judenangelegenheiten“ bei der Gestapo Johann Rixinger (geb. 1897).

30 Interview Merl (Anm. 28). 


\section{„An Herrn Schätzmeister Grimm abgeliefert“}

Seit dem Beginn der großen Deportationen im ersten Halbjahr 1941 wurden von der Israelitischen Kultusgemeinde im Auftrag der „Vugesta“ täglich Wohnungen geräumt, worüber vom Wohnungsreferat, der Rechnungskontrolle und dem Recherchebüro ausführlich Buch geführt wurde. Listen und Übernahmebestätigungen der geräumten Adressen aus diesem Zeitraum haben sich teilweise im Archiv der IKG erhalten. Die Erhebungsbeamten bestätigten darin entweder den Abtransport der Einrichtungen in die Möbellager oder die Übergabe der „fremden Gegenstände in treuhändige Verwahrung“ an die neuen - zwangsübersiedelten - Wohnparteien.

Im Wochenbericht der Amtsdirektion von 27. April bis 2. Mai 1942 heißt es beispielsweise:

„16 Wohnungen mit 26 Wohnräumen für 58 Personen geräumt. 11 Wohnungen wurden geöffnet und die darin befindlichen Einrichtungsgegenstände den neuen Mietern zur treuhändigen Verwahrung übergeben. 13 Wohnungen wurden geöffnet und die darin befindlichen Einrichtungsgegenstände den Eigentümern übergeben. 40 Wohnungen wurden insgesamt freigemacht. ${ }^{\text {(33 }}$

Die Liste der von der IKG übernommenen Objekte beinhaltet unter anderem Betten, Matratzen, Nachtkästchen, Kästen, Sessel, Tische, Fauteuils, Teppiche, Bücher, Bilder, Koffer, Wäsche und Kleider. Im Monatsbericht vom Juli 1942 findet sich schließlich eine minutiöse Aufstellung der Wertgegenstände, die „an Herrn Schätzmeister Grimm abgeliefert “ und unter dessen Beisein übernommen wurden:

„1 zusammenlegbares Opernglas, 1 Keksdose, 1 antikes Elfenbeinstück auf grünem Samt montiert, 7 Mikroskopgläser, 1 Kassette mit Stück 5 Messern (möglicherweise Silber), 1 Mappe Bilder, 1 Bronzefigur (Herkules), Stück 10 Münzen mit Inschrift ,Victoria regina', Jahreszahl verschieden, 1 kleiner Kerzenleuchter für 9 Kerzen, ${ }^{32} 1$ Bild von Elminger, 1 Lorgnon, 1 türkische Brosche, 1 Halskette (möglicherweise Bernstein) mit Schließe (möglicherweise Gold), 2 Perservorleger (klein), beschädigt, 1 japanischer Ring, 26 Stück diverses Besteck, 2 Tassen, 1 Serviettenring, 1 Granatenarmband, 1 Streichholzbehälter, 2 Goldwaagen, diverses Uhrenmaterial, 1 Stock mit Silbergriff, 1 Kassette mit diversen Anleihepapieren, alte Banknoten“33

und vieles mehr.

Wie aus einer handschriftlichen Aufzeichnung auf einer Empfangsbestätigung vom 6. Juli 1942 hervorgeht, stammten diese Dinge aus der „Sicherstellung

31 Archiv der IKG Wien, Wohnungsangelegenheiten, Wochenbericht der Amtsdirektion, 27.04.-02.05.1942, A/W 434.

32 Offensichtlich ein Chanukkaleuchter.

33 Archiv der IKG Wien, Monatsbericht der Amtsdirektion, 01.-31.07.1942, A/W 434. 
der Wohnung Wien II., Ferdinandstraße 13, Tür 6“. Sie gehörten demnach den neun bzw. 13 Bewohnerinnen und Bewohnern, die entweder kurz zuvor oder innerhalb der darauffolgenden Wochen über Theresienstadt nach Auschwitz deportiert wurden oder noch vor Ort „verstarben“.

Eine von ihnen war Helene Kanitz, die am 22. Oktober 1884 als Henie Lopater in Tarnopol geboren wurde. Seit 1908 war sie mit ihrem Ehemann Leopold in der Esterhazygasse 25, Tür 11, gemeldet. Dieser beging am 6. Juli 1938 Selbstmord durch Kopfschuss und wurde am Zentralfriedhof beerdigt. Daraufhin musste Helene Kanitz innerhalb Wiens mehrmals ihren Wohnsitz aufgeben, knapp zwei Wochen nach dem Tod ihres Mannes wechselte sie im gleichen Haus auf Tür 16. Seit Dezember 1939 als verwitwet in der Hofmühlgasse 25, Tür 10, gemeldet, zog sie im November 1940 in den 19. Bezirk in das Altersheim der Kultusgemeinde, Hohe Warte 32. Schließlich übersiedelte sie im Juli 1942 an ihre letzte Wohnadresse in die Ferdinandstraße 13, 1. Stiege, 1. Stock, Tür 6, in eine sogenannte „Alterswohngemeinschaft", wo sie mit ihrer Mutter Chuwe Lopater ein Zimmer bewohnte. Am 10. September 1942 wurden beide nach Theresienstadt deportiert, Helene Kanitz am 9. Oktober 1944 alleine weiter nach Auschwitz transportiert, das sie nicht überlebte. ${ }^{34}$

Am Beispiel ihres Lebensweges werden die Auswirkungen der Zwangsübersiedlungen besonders deutlich: in den Meldeunterlagen ist sie zunächst noch berufstätig als Wirtschafterin und Küchenleiterin verzeichnet, dann nur mehr "privat“, bis sie schließlich zum „Pflegling“ wurde. In der „Wohngemeinschaft“ blieb sie gerade zwei Monate bis zur Deportation. An der Ecke Gumpendorferstraße/Esterhazygasse im 6. Bezirk ist für sie und ihren Mann eine Erinnerungstafel angebracht. ${ }^{35}$

Die wenigen erhaltenen Korrespondenzen im Konvolut des IKG-Wohnungsreferats ermöglichen es, das Schicksal von Personen nachzuzeichnen, die kurz vor der Deportation standen und ihr letztes Hab und Gut zurücklassen mussten. So schrieb beispielsweise Margarethe „Sara“ Broda, Große Pfarrgasse 4/11, an Josefine Keil, Darwingasse 13/28, am 10. Mai 1942:

„Auf Ihren Wunsch bestätige ich Ihnen, daß das in meinem Wohnraum befindliche Lotterbett mit 3 Matratzen Ihr ausschließliches Eigentum darstellt, da ich es Ihnen im Jahre 1938 vor meiner damals bevorstehenden, dann aber gescheiterten Ausreise richtig ins Eigentum übergeben habe. Ich habe das bezeichnete Bett, welches Sie mir dann zur Benützung geliehen haben, in ordnungsgemäßen Zustand gehalten und hinterlaße [sic] zuhause einen Zettel, aus welchem sich Ihr klares Eigentum ergibt.“

34 Archiv der IKG Wien, Hauslisten mit Deportationsvermerken 1942, A/W 436,2; Wiener Adressbuch. Lehmanns Wohnungsanzeiger, 1. Aufl. Wien: Verlag August Scherl Nachfolger 1940; Wiener Stadt- und Landesarchiv, Meldekartei 1941-1947, Signatur: 2.5.1.4.K4.

35 Erinnern für die Zukunft. Ein Projekt zum Gedächtnis an die Mariahilfer Opfer des NSTerrors. URL: http://www.erinnern-fuer-die-zukunft.at (abgerufen am 03.03.2017). 
Drei Wochen nach diesem Schreiben, am 2. Juni 1942, wurde Margarethe Broda nach Maly Trostinec deportiert und dort ermordet, am 4. Juni wurde auf dem Blatt Papier handschriftlich vermerkt: „Bei Räumung der Wohnung ausfolgen. “36

Jüdinnen und Juden mit einem „arischen“ Partner hatten eine relativ größere Freiheit, was die Wahl des Wohnortes betraf. Manche übersiedelten „freiwillig“ in andere Bezirke, andere wurden von der „Auswanderungshilfsorganisation für nichtmosaische Juden “ in Sammelwohnungen eingewiesen. Deren Leiter, Erich Fasal (1909-1943), bat am 9. Mai 1942 den bei der IKG für die Räumungen zuständigen Robert Prochnik (1915-1977), Assistent des Amtsleiters und engster Mitarbeiter Benjamin Murmelsteins: „Wir bitten Sie, nachstehende Wohnungen dringend vom Mobiliar zu räumen, da wir Parteien mit unaufschiebbaren Räumungsterminen bereits zugewiesen haben, die teilweise die Wohnung bereits bezogen haben. ${ }^{\text {(37 }}$

In einem weiteren Schreiben am 13. Mai 1942 berichtete Fasal an den Leiter der technischen Abteilung Wilhelm Bienenfeld: „Wir erhielten von der Zentralstelle für jüdische Auswanderung den Auftrag, die Wohnung II. Grosse Stadtgutgasse 16/11 zu räumen. [...]. Gleichzeitig erhielten wir die Genehmigung zur gewaltsamen Oeffnung der Wohnung. Bei Oeffnung derselben wurde festgestellt, dass das Mobiliar Eigentum der IKG ist, da die Wohnungsinhaberinnen sich in der Grossen Mohrengasse ${ }^{38}$ befinden. Wir ersuchen die sofortige Räumung zu veranlassen, da diese Wohnung dringendst mit Mischehepaaren besiedelt werden muss. Anbei der Schlüssel zu dem Vorhangschloss, welches wir angebracht haben. Wir ersuchen nochmals um sofortige Räumung und Rückgabe der Schlüssel an uns. “ ${ }^{\text {39 }}$

Nach dem Ende der großen Transporte im Oktober 1942 lebten von den ursprünglich 206.000 Jüdinnen und Juden nach den „Nürnberger Rassengesetzen“ noch rund 8.000, in Zwangsarbeit oder "Mischehe“, in der Stadt. Die „Zentralstelle für jüdische Auswanderung“ wurde im März 1943 aufgelöst, da ihre Aufgaben als erledigt betrachtet wurden. Bis zur Befreiung vom Nationalsozialismus übernahm ihre Agenden die mit ihr zuvor in „Judenangelegenheiten“ im ständigen Kompetenzkonflikt gelegene Geheime Staatspolizei.

36 Archiv der IKG Wien, Wohnungsräumungen, Bestätigungen über die Übernahme von Gegenständen der Wohnparteien 1942, A/W 439.

37 Archiv der IKG Wien (Anm. 12).

38 Das Notstandsheim der IKG.

39 Archiv der IKG Wien (Anm. 12). 
Open-Access-Publikation im Sinne der CC-Lizenz BY-NC-ND 4.0

(c) 2018, V\&R unipress GmbH, Göttingen

ISBN Print: 9783847107835 - ISBN E-Lib: 9783737007832 


\title{
Johannes Gramlich
}

\section{Kunstwerke aus NS-Besitz auf dem Weg in die Bayerischen Staatsgemäldesammlungen - Amerikanische Restitutionspolitik und bayerische Treuhänderschaft}

\begin{abstract}
Zusammenfassung
Der Aufsatz untersucht den Umgang mit Kunst- und Kulturgütern in der amerikanischen Besatzungszone nach dem Zweiten Weltkrieg. Mit der Nachkriegsgeschichte des NSKunstraubs widmet er sich einem Thema, das die Forschung bislang vernachlässigt hat. Im Fokus des Aufsatzes stehen zunächst die alliierte Restitutionspolitik und -praxis. Als die (äußere) Rückerstattung von NS-Raubkunst weit vorangeschritten war, übergaben die Amerikaner 1948/49 die verbliebenen Objekte im Central Collecting Point in die Verwahrung des Bayerischen Ministerpräsidenten - ohne ihren Einfluss gänzlich aufzugeben. Welche Aufgaben und Pflichten mit der Treuhänderschaft verbunden waren und wie der Ministerpräsident diesen nachgekommen ist, erörtert der Text abschließend.
\end{abstract}

Schlagwörter

Bayerische Staatsgemäldesammlung, Treuhandschaft

\begin{abstract}
The Transfer of Works of Art of Former Nazi Property to the Land of Bavaria and the Bavarian State Painting Collections - Legal Foundations and Individual Responsibility The essay analyses the way art and cultural objects were handled in the American occupation zone after the Second World War. With the post-war history of Nazi art looting, it addresses a topic that provenance research has so far neglected. Initially, the focus of the article is on the Allied Restitution Policy and Practice. When the (external) restitution of Nazi looted art was well established, the Americans handed over the remaining objects in the Central Collecting Point into the custody of the Bavarian Prime Minister in 1948-49 - although by no means giving up their influence entirely. Thus, the essay further clarifies the tasks and duties connected with this transfer, and how the Prime Minister fulfilled these obligations.
\end{abstract}

Keywords

Bavarian State Painting Collections, Trusteeship 


\section{Einleitung}

In den Bestand der Bayerischen Staatsgemäldesammlungen gelangten nach dem Ende des Zweiten Weltkriegs rund 900 Kunstgegenstände, die zuvor hochrangigen Funktionären und Organisationen der NSDAP gehört hatten - darunter Werke aus den ehemaligen (Privat-)Sammlungen von Adolf Hitler, Hermann Göring, Martin Bormann, Heinrich Hoffmann und Hans Frank. ${ }^{1}$ Auf Basis alliierter Direktiven konnte sich der Freistaat Bayern diese Objekte vor allem in den 1950er und 1960er Jahren zu Eigentum übertragen. Zuvor hatten sie sich seit 1948/49 für einige Jahre in Treuhänderschaft des bayerischen Ministerpräsidenten und der bundesdeutschen Treuhandverwaltung für Kulturgut befunden. Diesem Teilaspekt der Nachkriegsgeschichte des „NS-Kunstraubes“2 - der treuhänderischen Übernahme und Verwahrung von Kunst- und Kulturgütern widmete die Wiener Universitätsbibliothek im Mai 2017 eine international ausgerichtete Tagung.

Die Bayerischen Staatsgemäldesammlungen konnten dort Ergebnisse des laufenden Forschungsprojekts „Überweisungen aus Staatsbesitz“ vorstellen, das sie 2012/13 zu ihren Kunstgegenständen aus früherem NS-Besitz aufgelegt haben. Dieses Projekt verfolgt zwei Ziele: Erstens recherchiert und dokumentiert es sukzessive die Provenienzen der fraglichen Kunstwerke so lückenlos wie möglich. ${ }^{3}$ Über die objektbezogenen Recherchen hinaus rekonstruiert, analysiert und verschriftlicht das Projekt zweitens den Gesamtprozess des mehrstufigen Vermögenstransfers von den Nationalsozialisten über die Alliierten zum Freistaat Bayern und zu den Bayerischen Staatsgemäldesammlungen - zu dem auch die zwischenzeitlichen Treuhänderschaften zählen. Das Forschungsvorhaben untersucht die rechtlichen und politischen Grundlagen, auf deren Basis NS-Vermögen auf den Freistaat Bayern übergehen konnte. Auch mit Blick auf die Restitutionspolitik der Nachkriegszeit fragt es zudem nach Verantwortlichkeiten, Handlungsspielräumen und Motiven der alliierten und deutschen Stellen,

$1 \mathrm{Zu}$ ihren Kunstwerken aus der Sammlung Hermann Görings publizierten die Bayerischen Staatsgemäldesammlungen bereits 2004 erste Forschungsergebnisse in Buchform, vgl.: Ilse von zur Mühlen: Die Kunstsammlung Hermann Görings. Ein Provenienzbericht der Bayerischen Staatsgemäldesammlungen. München: Bayerische Staatsgemäldesammlungen 2004.

2 Die Begriffe „NS-Kunstraub“ bzw. „NS-Raubkunst“ sind für das Phänomen, das sie bezeichnen sollen, unscharf. Insbesondere umfasst dieser sogenannte Raub nicht nur den direkten Entzug von Kunst- und Kulturobjekten durch den NS-Staat und die NSDAP - schon hier sind verschiedene Methoden und (schein-)legalisierende Grundlagen zu differenzieren -, sondern auch alle anderen Arten von Vermögenstransfers, die dem Druck der Verhältnisse geschuldet waren, vgl. hierzu u. a.: Berthold Unfried: Vergangenes Unrecht. Entschädigung in einer globalen Perspektive. Göttingen: Wallstein 2014, S. 276.

3 Mit Stand Juli 2017 sind rund die Hälfte der gut 900 Objekte bearbeitet worden; 273 Werke sind auf lostart.de gemeldet, vier Werke konnten restituiert werden. 
die mit diesen Vorgängen befasst waren. Dabei ist auch das Fortbestehen personeller Konstellationen und Loyalitäten im Anschluss an die NS-Herrschaft zu fokussieren, wenn etwa die Familien vormaliger NS-Funktionäre zum Teil selbstbewusst und erfolgreich die Rückgabe hochwertiger Objekte von VertreterInnen des Freistaats Bayern durchsetzen konnten. Als Teil einer Rezeptionsgeschichte des Nationalsozialismus wird das Forschungsprojekt überdies den Umgang der bayerischen Staatsregierung und der nachgeordneten Bayerischen Staatsgemäldesammlungen mit diesem Kunstbestand bis heute skizzieren und damit seine eigene Vorgeschichte erhellen.

Der vorliegende Aufsatz orientiert sich am Vortrag der Wiener Tagung und wird demgemäß Aspekte der treuhänderischen Verwahrung von Kunstwerken in der amerikanischen Besatzungszone bzw. im Freistaat Bayern in den Blick nehmen. Da die treuhänderische Übergabe dieser Objekte an den bayerischen Ministerpräsidenten eng mit der Restitutionsthematik verknüpft war - die amerikanischen Alliierten wollten möglichst nur Kunstwerke übergeben, deren Herkunft geklärt war -, wird der Aufsatz zunächst auf die alliierten Restitutionsregeln und ihre Umsetzung eingehen. Darauf aufbauend thematisiert er die Aufgaben und Pflichten, die mit der Treuhänderschaft verbunden waren - und wie der Ministerpräsident diesen nachgekommen ist.

\section{Forschung}

Indem das Forschungsprojekt der Bayerischen Staatsgemäldesammlungen Vermögenskontinuitäten im Übergang vom NS-Staat zur Bundesrepublik Deutschland in den Blick nimmt, bearbeitet es ein Desiderat der Geschichtswissenschaft. Diese hat sich bislang kaum mit dem Verbleib und der Behandlung des Vermögens von NSDAP und Deutschem Reich nach 1945 beschäftigt. ${ }^{4}$ Da das Projekt allein Kunst- und Kulturgegenstände fokussiert, ist es überdies nicht nur im Zusammenhang mit der zeithistorischen Forschung zu Kontinuitäten und Brüchen um $1945 \mathrm{zu}$ sehen. Es steht auch im politisch-moralischen Kontext der zeitgenössischen Provenienzforschung, die seit der Washingtoner Erklärung von 1998 in zunehmendem Maße an Museen, Bibliotheken und außeruniversitären Forschungsinstituten betrieben wird. ${ }^{5}$ Ihr primäres Ziel ist es, NS-verfolgungsbedingte Verluste von Kunst- und Kulturgütern aufzuklären, um deren Rück-

4 Allein an der Forschungsstelle für Zeitgeschichte in Hamburg entsteht aktuell eine vergleichbare Regionalstudie, die den Umgang mit Vermögenswerten aus NS-Besitz nach Kriegsende für die britische Besatzungszone und den Stadtstaat Hamburg untersucht.

5 In der Washingtoner Erklärung vom 03.12.1998 verpflichteten sich 44 Staaten, die Identifizierung und Restitution von NS-verfolgungsbedingt entzogenen Kunst- und Kulturgütern voranzutreiben. 
erstattung an die rechtmäßigen Eigentümer oder deren Erben zu ermöglichen. ${ }^{6}$ Dazu recherchiert die Provenienzforschung einerseits objektbezogen zu den Eigentumsverhältnissen einzelner Kunstwerke. Andererseits widmet sie sich Kunsthändlern, Sammlern und dem Kunstmarkt der NS-Zeit, um - quasi vom Allgemeinen zum Besonderen - Vermögensverschiebungen identifizieren, rekonstruieren und bewerten zu können.

Nicht systematisch untersucht hat die Provenienzforschung bislang hingegen die Restitutionspolitik und -praxis der frühen Nachkriegszeit - ihr Fokus liegt überwiegend auf den Jahren 1933 bis 1945. Damit hat sie einen wichtigen Teil ihrer eigenen Geschichte vernachlässigt. ${ }^{7}$ Die Geschichtswissenschaft wiederum beschäftigt sich seit den 1990er Jahren verstärkt mit der sogenannten Wiedergutmachung nach 1945, fühlt sich dabei aber kaum und schon gar nicht im Detail für Kunstobjekte zuständig. Allein die Rechtswissenschaften haben sich der Nachkriegsrestitution von Kunst- und Kulturgütern bisher ausführlicher gewidmet; sie konzentrieren sich allerdings auf die juristischen Rahmenbedingungen, nicht auf die Praxis der Rückerstattung. ${ }^{8}$ So ist die Kritik des Historikers Berthold Unfried an der Provenienzforschung weiterhin gültig:

„Eine Forschung im eigentlichen Sinn, die über die zweckorientierte Recherche von Einzelfällen hinausgeht, indem sie etwa Logiken der Restitution und Entschädigung von Kunstobjekten in der Nachkriegszeit zu ihrem Forschungsgegenstand machen würde, gibt es bis heute nicht. “"

Insbesondere mit Blick auf die Bedeutung, die der Provenienzforschung als wichtiger vergangenheitspolitischer Aufgabe in Medien, Politik und Wissen-

6 Inzwischen weitet sich der Untersuchungsgegenstand aus, indem bspw. vermehrt auch Vermögensverschiebungen der Kolonialzeit fokussiert werden. Darüber hinaus lässt sich eine Ausweitung der Fragestellung beobachten, womit Untersuchungen zur Translokation von Kunst- und Kulturgütern für zunehmend breitere wissenschaftliche Erkenntnisinteressen fruchtbar gemacht werden; vgl. u. a. das umfangreiche Forschungsprojekt „Translocations“, das jüngst am Institut für Kunstwissenschaft und Historische Urbanistik der TU Berlin initiiert worden ist, vgl.: URL: http://www.kuk.tu-berlin.de/menue/forschung/einzelne_for schungsprojekte/translocations (abgerufen am 04.01.2018).

7 Lediglich zu einzelnen Institutionen und Sammlungen liegen entsprechende Beiträge vor. $\mathrm{Zu}$ den wenigen grundlegenden Untersuchungen, die sich mit diesem Themenfeld beschäftigen, gehört Iris Lauterbach: Der Central Collecting Point in München. Kunstschutz, Restitution, Neubeginn. Berlin-München: Deutscher Kunstverlag 2015.

8 Vgl. u. a.: Michael Anton: Rechtshandbuch Kulturgüterschutz und Kunstrestitutionsrecht, 3 Bde. Berlin-New York: De Gruyter 2010; Thomas Armbruster: Rückerstattung der NaziBeute. Die Suche, Bergung und Restitution von Kulturgütern durch die westlichen Alliierten nach dem Zweiten Weltkrieg. Berlin: De Gruyter 2008 (= Schriften zum Kulturgüterschutz); Sabine Rudolph: Restitution von Kunstwerken aus jüdischem Besitz. Dingliche Herausgabeansprüche nach deutschem Recht. Berlin: De Gruyter 2007 (= Schriften zum Kulturgüterschutz).

9 Unfried: Unrecht (Anm. 2), S. 458. 
schaft seit der Jahrtausendwende beigemessen wird, ist dieser Zustand erstaunlich und beklagenswert, weil er in der Debatte um „NS-Raubkunst“ und Restitution zu Ungenauigkeiten und Unsachlichkeiten führt. Welcher Stellenwert der Rückerstattung von Kunstgegenständen in der Nachkriegszeit zukam, welche Anstrengungen von alliierten und deutschen Verantwortlichen unternommen und welche Nachlässigkeiten von ihnen begangen worden sind, ist nicht fundiert zu benennen. ${ }^{10}$ Auch welche Maßstäbe damals in der Praxis angelegt worden sind, um einen Transfer von Kunst- und Kulturgütern als „NSverfolgungsbedingt“ ${ }^{\text {“ }} \mathrm{zu}$ klassifizieren, ist noch nicht systematisch erforscht worden. Weder die zeitgenössische Provenienzforschung noch die öffentlichen Debatten um einen adäquaten Umgang mit potentiell kontaminierten Kunstsammlungen konnten und können sich folglich auf solche Kenntnisse beziehen. Die Tagung in Wien und der vorliegende Sammelband leisten für die Forschung zum Umgang mit Kunst- und Kulturgütern in der Nachkriegszeit folglich wichtige Pionierarbeit.

\section{Vor der treuhänderischen Übergabe: Die (amerikanischen) Alliierten und die Restitution von Kunst- und Kulturgütern}

\section{Alliierte Restitutionsregeln}

Ab dem Jahr 1943 intensivierten die Alliierten ihre Planungen, was mit Deutschland und Europa nach dem Krieg geschehen solle, welche Maßnahmen einzuleiten seien und wie sie ihre Politik und Einflusssphären zu organisieren und zu koordinieren gedachten. Neben zentralen Forderungen wie der Entmilitarisierung und Entnazifizierung Deutschlands spielte auch die Restitution von Vermögenswerten in den alliierten Überlegungen früh eine wichtige Rolle. Dies schlug sich zuerst in der Londoner Erklärung vom 5. Januar 1943 nieder, in der sich 18 alliierte Staaten das Recht vorbehielten, Vermögenstransfers für nichtig zu erachten und rückabzuwickeln, die im Zuge der deutschen Expansion ab 1938/39 zustande gekommen waren. In dieser Erklärung, die nach Kriegsende als wichtiger Bezugspunkt für entsprechende Regelungen fungierte, waren bereits

10 Die Rolle jüdischer Organisationen bei der Rückerstattung von Kunst- und Kulturgütern ist hingegen insbesondere im bibliothekarischen Bereich verschiedentlich untersucht worden, vgl. u. a.: Elisabeth Gallas: „Das Leichenhaus der Bücher“. Kulturrestitution und jüdisches Geschichtsdenken nach 1945. 2. Aufl. Göttingen: Vandenhoeck \& Ruprecht 2016; Dov Schidorsky: The Salvaging of Jewish Books in Europe after the Holocaust. In: Jüdischer Buchbesitz als Raubgut. Zweites Hannoversches Symposium. Hg. von Regine Dehnel. Frankfurt/Main: Vittorio Klostermann 2006 (= Zeitschrift für Bibliothekswesen und Bibliographie Sonderheft 88), S. 197-212. 
drei wichtige Merkmale der alliierten Rückerstattungspraxis nach 1945 angelegt: Erstens bezog sich die Erklärung explizit auf die von Deutschland besetzten und annektierten Gebiete und Länder Europas, nicht auf Eigentumsverschiebungen innerhalb des Deutschen Reichs (in den Grenzen von 1937). In der Nachkriegszeit bestätigte sich, dass die Alliierten dieser sogenannten äußeren Restitution eine größere Bedeutung beimaßen als inneren Rückerstattungen. Dies lag unter anderem daran, dass sich die äußeren Restitutionen direkt auf materielle Kriegsverluste vieler alliierter Staaten und Staatsbürger bezogen. Außerdem waren sie im Gegensatz zu inneren Restitutionen durch bestehendes Völkerrecht legitimiert. Zweitens war die Londoner Erklärung nicht ganz eindeutig formuliert und ließ Interpretationsspielraum. Wenngleich sie die alliierten Bemühungen dokumentiert, eine einheitliche Restitutionspraxis zu entwickeln, deutete sie damit gleichfalls an, dass dies nach dem Krieg ein schwieriges Unterfangen werden sollte. Letztlich konnten sich die Alliierten nicht auf eine gemeinsame und einheitliche Vorgehensweise einigen, weil die nationalen Interessen dafür zu unterschiedlich waren. ${ }^{11}$ Außerdem konnten Kritik und Ablehnung insbesondere von deutscher Seite an den inhaltlichen Unschärfen der Erklärung anknüpfen. Drittens offenbart bereits die Entstehungsgeschichte der Londoner Erklärung, dass die Alliierten dem Umgang mit Kunstwerken eine besondere Bedeutung beimaßen. So war die Erklärung in nicht unbedeutendem Maße dadurch motiviert, dass Frankreich und andere alliierte Mächte durch den massiven Zugriff Deutschlands auf Kunst- und Kulturgüter während des Zweiten Weltkriegs den Verlust ihrer kulturellen Identität fürchteten. ${ }^{12}$ Auch wenn die Amerikaner selbst keine Kunstwerke verloren hatten, erkannten sie die Bedeutung dieser Angelegenheit für die betroffenen Staaten und agierten entsprechend - nicht zuletzt, weil in ihrer Zone mehr Kunstwerke sichergestellt wurden als in den anderen Besatzungszonen zusammen. ${ }^{13}$ So folgte die amerikanische Militärregierung bei der äußeren Restitution einem strengen Ansatz, der für Kunstgegenstände weitreichender war als für andere Vermögensarten. Grund-

11 Vgl. u.a.: Armbruster: Rückerstattung (Anm. 8), S. 222, 265, 278, 381-402; Wolfgang Fiedler: Die Alliierte (Londoner) Erklärung vom 05.01.1943: Inhalt, Auslegung und Rechtsnatur in der Diskussion der Nachkriegsjahre. In: Private Law in the International Arena. From National Conflict Rules Towards Harmonization and Unification. Hg. von Jürgen Basedow u. a. Den Haag: T.M.C. Asser Press 2000, 197-218. Der Alliierte Kontrollrat, das höchste gemeinsame Gremium der vier Siegermächte, brachte nach dem Krieg zwar verschiedene Proklamationen, Definitionen und Richtlinien zur Restitution von Vermögenswerten hervor, die letztlich aber nicht in eine gemeinsame Vorgehensweise mündeten.

12 Armbruster: Rückerstattung (Anm. 8), S. 215.

13 „The restitution of looted works of art to their rightful owner nations now has a high priority as a military necessity." Headquarters, US Forces, European Theater to Commanding General Eastern Military District, 23.08.1945. In: National Archives and Records Administration (NARA), RG 260, M1946, Roll 4 (via fold3.com). 
sätzlich mussten alle Vermögensobjekte, die während der deutschen Aggression in einem besetzten oder annektierten Land konfisziert und ins Deutsche Reich gebracht worden waren, an die entsprechenden Staaten rückerstattet werden. Betroffen waren zudem - und dies war umstritten, da völkerrechtlich nicht gedeckt - sämtliche Kunstwerke, die während der deutschen Besatzung infolge von Privatgeschäften nach Deutschland gelangt waren, unabhängig davon, ob diese Transaktionen freiwillig oder unter Druck bzw. Zwang zustande gekommen waren. ${ }^{14}$ Diese Vorgabe war wichtig und folgenreich, da der Kunsthandel in Westeuropa im Zuge der deutschen Expansion floriert hatte. Etliche deutsche HändlerInnen, SammlerInnen und Museen hatten diese Märkte genutzt und waren auch aufgrund ihrer Währungsvorteile bereit gewesen, große Summen auszugeben; durch ihre Nachfrage und Zahlungsbereitschaft waren die Preise für Kunstwerke während des Zweiten Weltkriegs immens gestiegen, was neben der deutschen Verfolgungspolitik dazu beigetragen hatte, dass Kunst- und Kulturgüter in verstärktem Maße zirkulierten. ${ }^{15}$

Die weitreichenden Bestimmungen zur äußeren Restitution von Kunstwerken gab die amerikanische Militärregierung in Form von Verwaltungsvorschriften in den Military Government Regulations Title 18 vor, die sie ab Dezember 1945 in mehreren Fassungen vorlegte. ${ }^{16}$ Die Regulations richteten sich an die Monuments, Fine Arts, and Archives Section (MFAA) der US-Armee, die in der amerikanischen Besatzungszone insgesamt für den Umgang mit Kunstwerken zuständig war. ${ }^{17}$ Für die äußere Restitution anderer Vermögensarten galten die

14 Von deutscher Seite wurde gegen die weitreichenden Bestimmungen vielfach protestiert, u. a. vom Verein Bayerischer Kunst- und Antiquitätenhändler in München; selbst dem amerikanischen Kunstschutzoffizier Samson Lane Faison, Jr., der den Central Collecting Point in München 1950/51 leitete, gingen die Regelungen zu weit, vgl.: Bundesarchiv (BArch) Koblenz, B 323/326, fol. 185-188; NARA, RG 260, M1946, Roll 4 (via fold3.com).

15 Dies konstatierten bereits die alliierten Untersuchungsberichte in der Nachkriegszeit, die in verschiedenen Studien aufgegriffen worden sind, vgl. u. a.: Lynn H. Nicholas: Der Raub der Europa. Das Schicksal europäischer Kunstwerke im Dritten Reich. München: Kindler 1995. Der Ökonom Kim Oosterlinck bestätigt diesen Befund, vgl. u. a.: Art as a Wartime Investment. Conspicuous Consumption and Discretion. In: The Economic Journal 127 (2017), S. 2665-2701; ders., Jeroen Euwe: Art Price Economics in the Netherlands during World War II. In: Journal for Art Market Studies 1 (2017), H. 1, S. 47-67.

16 Office of Military Government for Germany, United States (OMGUS), Military Government Regulations Title 18: Monuments, Fine Arts and Archives (Entwurf vom 10.12.1945, genehmigt am 22.12.1945, ergänzt und korrigiert am 10.01.1946). In: NARA, RG 260, M1947, Roll 28 (via fold3.com).

17 Die amerikanischen und britischen Streitkräfte unterhielten zunächst unter gemeinsamem Befehl, nach Kriegsende im Rahmen ihrer Militärregierungen eine MFAA, in der Kunstexperten aus verschiedenen Ländern zusammenarbeiteten. 
Military Government Regulations Title 19, die mit Blick auf freie Verkäufe milder ausfielen. ${ }^{18}$

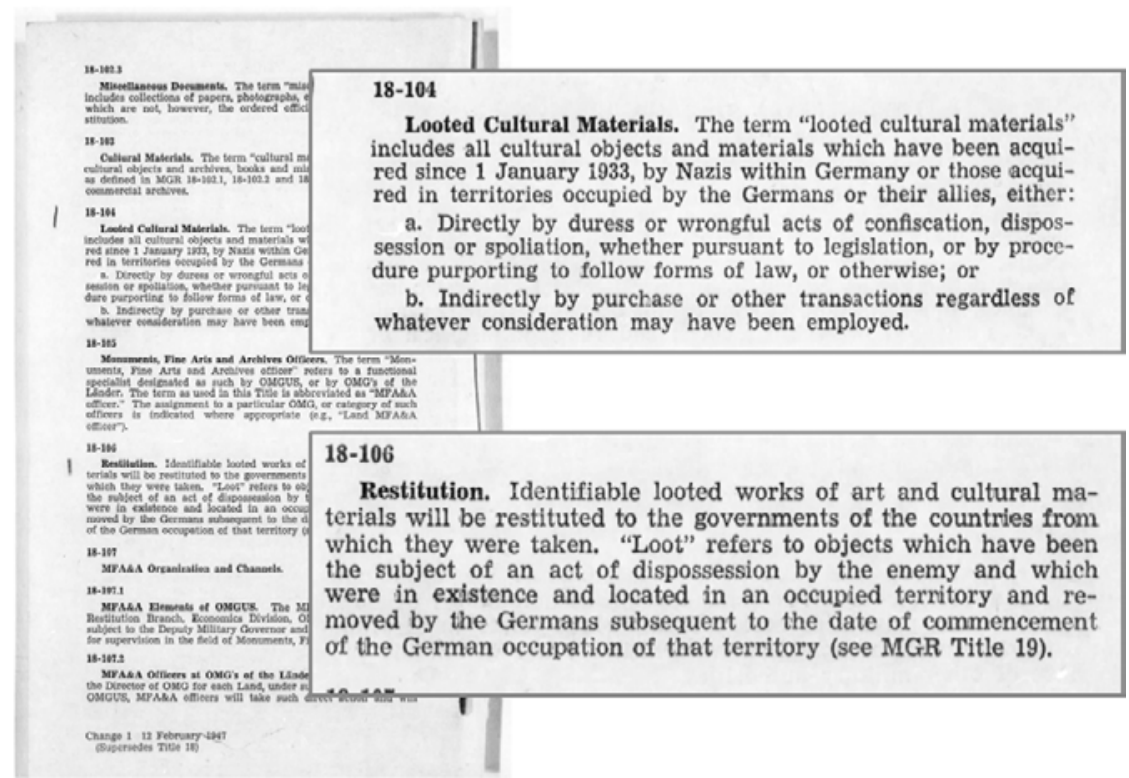

Abb. 1: Auszug aus den Military Government Regulations Title 18 in der Fassung vom 12.02. 1947; beide Definitionen unterscheiden sich nur unwesentlich vom Wortlaut der vorhergehenden Fassungen (NARA, RG 260, M1946, Roll 8)

Die Definitionen zeigen, dass die Amerikaner grundsätzlich auch den Transfer von Kunstwerken innerhalb des Deutschen Reichs ab 1933 als „looted“ klassifizieren konnten; den Begriff „restitution“ bezogen sie allerdings ausdrücklich auf Objekte, die sich vormals in den besetzten Gebieten befunden hatten (Abb. 1). Noch 1946 mahnte die amerikanische Militärregierung, dass für die sogenannte innere Restitution eine eigene gesetzliche Regelung zu schaffen sei auch weil sie hierfür nicht auf das Völkerrecht zurückgreifen konnte. ${ }^{19}$ Am 10. November 1947 erließ sie schließlich als erste westalliierte Macht ein entsprechendes Gesetz. Demnach konnten Verfolgte des NS-Regimes die Rückerstattung von Vermögenswerten beantragen, die ihnen ,aus Gründen der Rasse, Religion, Nationalität, Weltanschauung oder politischer Gegnerschaft gegen den Nationalsozialismus" innerhalb des Deutschen Reichs entzogen worden waren. ${ }^{20}$

18 Vgl.: Ordenberg Bock von Wülfingen: Restitution von Kunstwerken (Vortragsskript), 20.4. 1949. In: BArch B 323/326, fol. 469-472.

19 OMGUS, Office of Political Affairs and Restitution Branch, Economics Division (Memorandum), 11.03.1946, in: NARA, RG 260, M1946, Roll 4 (via fold3.com).

20 OMGUS, Gesetz Nr. 59, Rückerstattung feststellbarer Vermögensgegenstände. In: Amtsblatt 
Neben direkten Enteignungen durch Staat und Partei - die Bundesländer agierten hierbei als Rechtsnachfolger - konnten auch private Rechtsgeschäfte betroffen sein. Anders als bei der äußeren Restitution von Kunst- und Kulturgütern war die Rückabwicklung von Privatgeschäften in diesem Gesetz hingegen an Bedingungen gebunden, die aber relativ weit gefasst waren - vereinfacht und allgemein formuliert musste erkennbar sein, dass eine Transaktion dem Druck der Verhältnisse geschuldet war. ${ }^{21}$ Anträge auf innere Rückerstattung, die innerhalb einer kurzen Frist bis zum 31. Dezember 1948 eingereicht werden mussten, waren vor deutsche Behörden und Gerichten zu verhandeln. ${ }^{22}$ Die Hoheit über äußere Rückerstattungen behielten hingegen die Amerikaner inne, bis sie diese für nahezu abgeschlossen hielten - letztlich bis zum Ende der Besatzung 1955. ${ }^{23}$ Anschließend war die Bundesrepublik Deutschland noch bis 1956 verpflichtet, entsprechende Anträge entgegenzunehmen und zu bearbeiten. ${ }^{24}$ Für innere Restitutionen erließ die Bundesregierung ebenfalls auf Druck der Westalliierten 1957 ein eigenes Gesetz, das sich allerdings nicht mehr auf Privatgeschäfte bezog. ${ }^{25}$

\section{Restitutionspraxis der amerikanischen Alliierten}

Zum Schutz und zur konservatorischen Behandlung, vor allem aber zur Restitution von Kunstwerken richtete die amerikanische Militärregierung verschiedene Sammelstellen, die sogenannten Central Collecting Points (CCP), ein. Die

der Militärregierung Deutschland, Amerikanisches Kontrollgebiet (Ausgabe G, 10.11.1947), S. 1-25.

21 Wenn ein Veräußerer jüdischer Herkunft eine Vermögensübertragung anfocht, die nach dem Erlass der Nürnberger Gesetze am 15.09.1935 stattgefunden hatte, musste der Erwerber belegen, dass das Geschäft auch ohne die Herrschaft des Nationalsozialismus getätigt worden wäre. Für Vermögensübertragungen, die vorher vollzogen worden waren, war der Spielraum etwas größer, vgl. allgemein zur Restitutionspolitik der Nachkriegszeit u. a.: Jürgen Lillteicher: Raub, Recht und Restitution. Die Rückerstattung jüdischen Eigentums in der frühen Bundesrepublik. Göttingen: Wallstein 2007.

22 Ein Board of Review, das unter Kontrolle der amerikanischen Militärregierung stand, konnte allerdings bei Streitfällen in letzter Instanz eingreifen, vgl. Artikel 69 des amerikanischen Rückerstattungsgesetzes (Anm. 20).

23 Im Besatzungsstatut der alliierten Westmächte, das von 1949 bis 1955 in Kraft war, behielten sich diese unter anderem das Recht vor, die Kontrolle über Restitutionen auszuüben. Obwohl es zwischenzeitlich anderslautende Überlegungen gab, machte die amerikanische Militärregierung hiervon bis zur Aufhebung des Statuts Gebrauch, vgl.: Amtsblatt der Hohen Alliierten Kommission in Deutschland (Nr. 1, 23.09.1949), S. 13-15.

24 Vgl. den 5. Teil des Vertrages zur Regelung aus Krieg und Besatzung entstandener Fragen („Überleitungsvertrag“), BGBl. II 1955, S. 405.

25 Bundesgesetz zur Regelung der rückerstattungsrechtlichen Geldverbindlichkeiten des Deutschen Reichs und gleichgestellter Rechtsträger vom 19.07.1957, BGBl. I S. 734. 
MFAA zog darin ab Juni 1945 Kunst- und Kulturgüter aus über 600 Bergungsdepots in ihrer Besatzungszone (auch aus Österreich) zusammen. ${ }^{26}$ Rund 80 Prozent der Kunstgegenstände, die die Alliierten insgesamt in Depots im Deutschen Reich auffanden, sollen in der amerikanischen Zone gelegen haben. ${ }^{27}$ Dies hatte unter anderem damit zu tun, dass München, Nürnberg und der Obersalzberg zentrale Orte für die NSDAP und ihre Funktionäre gewesen waren, so dass etliche Sammlungen aus diesem Umfeld in Bayern geborgen worden waren. Auch die Bestände aus dem amerikanisch besetzten Altaussee in Österreich, darunter die Sammlung für das geplante „Führermuseum“ in Linz, wurden in den größten CCP nach München gebracht. ${ }^{28}$ Neben Beständen aus dem direkten Umfeld von NSDAP und NS-Staat fanden sich in den Depots auch Kunstwerke von PrivatsammlerInnen, KunsthändlerInnen und Museen. Die Alliierten suchten zudem verdächtige Akteure der Kunstwelt gezielt auf, um ihren Kunstbesitz sicherzustellen und in die CCPs zu überführen. ${ }^{29}$ Gesetzliche Grundlage für dieses Vorgehen war das Gesetz Nr. 52 zur Sperre und Kontrolle von Vermögen vom 18. September 1944, das es der Militärregierung ermöglichte, Vermögenswerte des NS-Staats und der NSDAP, aber auch von inhaftierten und verdächtigen Personen $\mathrm{zu}$ beschlagnahmen und $\mathrm{zu}$ verwalten. ${ }^{30}$ Außerdem mussten KunstbesitzerInnen, die potentiell restitutionspflichtige Objekte beherbergten, diese unaufgefordert bei alliierten bzw. deutschen Stellen melden.

Zur Identifizierung von „NS-Raubkunst“ in den CCPs konnte die MFAA unter anderem auf die Untersuchungsergebnisse der Art Looting Investigation Unit zurückgreifen, einer geheimdienstlichen Einheit des amerikanischen Militärs. ${ }^{31}$ Die MFAA recherchierte aber auch auf eigene Faust; sie dokumentierte und inspizierte die Objekte, sie wertete schriftliche Unterlagen aus und sie befragte ExpertInnen und ProtagonistInnen des internationalen Kunsthandels, deren

26 Vgl. O. A.: Bericht über den Central Collecting Point München, 28. 02. 1951, BArch B 323/325, fol. $314 \mathrm{f}$.

27 Lauterbach: Central Collecting Point (Anm. 7), S. 26.

$28 \mathrm{Vgl.} \mathrm{zu}$ den Beständen des CCP München die Online-Datenbank unter URL: www.dhm.de/da tenbank/ccp/ (abgerufen am 04.01.2018).

29 So erging es zum Beispiel Hildebrand Gurlitt, der allerdings einen (großen) Teil seines Kunstbesitzes vor den Alliierten verstecken konnte, vgl.: BArch N 1826/44 u. 45; Meike Hoffmann, Nicola Kuhn: Hitlers Kunsthändler. Hildebrand Gurlitt 1895-1956. Die Biographie. München: Beck 2016, S. 233-240, 271-274.

30 Dieses Gesetz wurde noch vor der Zonenteilung 1944 vom Supreme Headquarters, Allied Expeditionary Force (SHAEF) erlassen und später in den drei Westzonen aufrechterhalten, vgl.: Amtsblatt der Militärregierung Deutschland, Amerikanische Zone (Ausgabe A, 01.06. 1946), S. 24-26.

31 Die Art Looting Investigation Unit fertigte Untersuchungsberichte zu verschiedenen Protagonisten des nationalsozialistischen Kunstraubes an, eine Synthese findet sich in ihrem „Final Report“ vom 01.05.1946. In: NARA, RG 239, M1782 (via fold3.com). 


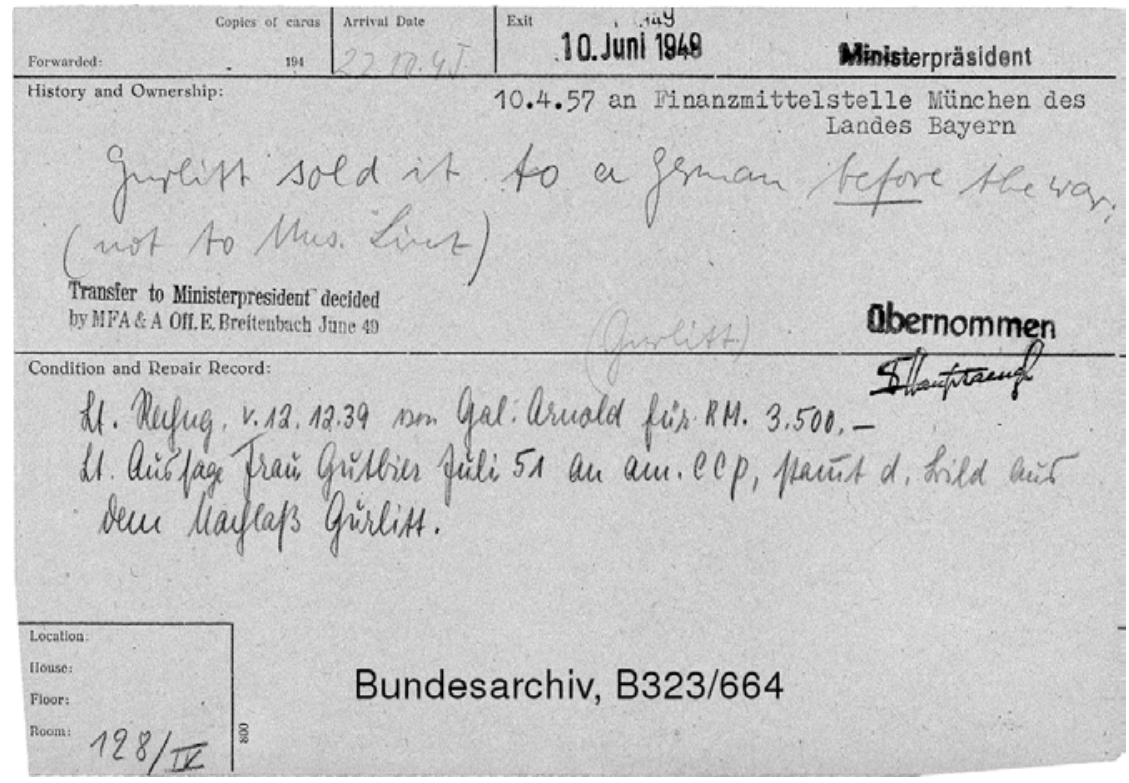

Abb. 2: Auf den Property Cards (hier aus dem Münchner CCP, Nr. 11205, Rückseite) wurden alle Informationen zu einem Objekt festgehalten. Der Hinweis „before the war“ ist auf vielen dieser Karten $\mathrm{zu}$ finden; er besagt, dass sich das Objekt schon vor Kriegsbeginn in Deutschland befunden haben soll und somit nicht der äußeren Rückerstattung unterlag

Aussagen allerdings mit Vorsicht zu genießen waren. ${ }^{32}$ Ab August 1945 trafen im Münchner CCP zudem VertreterInnen ausländischer Staaten ein, die Ansprüche auf äußere Restitutionen geltend machten und bei der Identifizierung der entsprechenden Werke halfen. ${ }^{33}$ Noch im selben Jahr und bevor die offiziellen Regularien erlassen worden waren, gingen die ersten Transporte zurück in die Herkunftsländer. ${ }^{34}$ So zeigte sich auch in der Praxis, dass der äußeren Restitution von Kunst- und Kulturgütern große Bedeutung zukam. Sie wurde insgesamt zügig vorangetrieben, auch weil die notwendigen Recherchen verhältnismäßig einfach waren. Es war nicht notwendig, detailliert die Umstände eines Vermö-

32 Vgl. u. a.: S. Lane Faison an Eberhard Hanfstaengl, 11.06. 1951. In: NARA, RG 260, M1946, Roll 4 (via fold3.com).

33 Lauterbach: Central Collecting Point (Anm. 7), S. 93-96.

34 Aufgrund der Bedeutung, die die Amerikaner der äußeren Restitution von Kunstwerken beimaßen, nahmen sie schon im Sommer 1945 erste Rückerstattungen besonders hochwertiger Objekte „ad interim“ vor - in jener Zeit glaubten sie noch an eine interalliierte Lösung. Auf Geheiß von Präsident Harry S. Truman und Oberbefehlshaber Dwight D. Eisenhower begannen sie aber schon kurze Zeit später damit, die äußere Restitution von Kunstgegenständen unilateral voranzutreiben, vgl.: Craig Hugh Smith: Repatriation of Art from the Collecting Point in Munich after World War II. Background and Beginnings. Maarssen-Den Haag: SDU Publishers 1988, S. 57. 
genstransfers zu rekonstruieren, um feststellen zu können, ob dieser dem Druck der Verhältnisse geschuldet war. Es reichte der Nachweis aus, dass sich ein Objekt vormals in einem der von Deutschland besetzten oder annektierten Gebiete befunden hatte. Bei der inneren Rückerstattung verließ man sich hingegen vor allem auf das entsprechende Gesetz vom November 1947, nach dem Restitutionsberechtigte und -pflichtige Anträge bzw. Meldungen zu machen, deutsche Behörden und Gerichte darüber zu entscheiden hatten. ${ }^{35}$

\section{Die treuhänderische Übergabe von Kunstwerken an den bayerischen Ministerpräsidenten}

Der amerikanischen Militärregierung war grundsätzlich daran gelegen, ihre Besatzungsmacht möglichst indirekt auszuüben und sich auf Anleitung und Überwachung deutscher Stellen zu konzentrieren - dies entlastete sie personell wie finanziell und stärkte gleichzeitig ihr Ansehen und ihre Legitimität in der deutschen Bevölkerung. Vor allem aber sollte Deutschland, zumindest der Teil, den die Amerikaner verantworteten, zu einem selbstständigen Teil eines starken demokratischen Europas werden, was auch im Zusammenhang mit dem zunehmenden Ost-West-Konflikt zu sehen ist. Nachdem der amerikanische Militärgouverneur 1947 neue Richtlinien aus Washington erhalten hatte, intensivierte er diesen Kurs. Die demokratische Selbstverwaltung und die Delegation von Verantwortlichkeiten an deutsche Regierungsstellen waren zu beschleunigen. ${ }^{36}$

Bereits 1946 hatte die amerikanische Militärregierung die Kontrolle über sichergestellte Vermögenswerte in Verantwortung des Freistaats Bayern übergeben, der hierfür das Bayerische Landesamt für Vermögensverwaltung und Wiedergutmachung gründete. ${ }^{37}$ Eine Sonderrolle spielten erneut Kunst- und Kulturgüter, die von der Übergabe ausgeschlossen waren. Die CCPs sollten unter amerikanischer Kontrolle verbleiben, bis die Restitution der sichergestellten Kunstwerke weitgehend abgeschlossen war. Obwohl dieser Prozess zügig und

35 Auch mit Blick auf die innere Rückerstattung wurden proaktive Recherchen im CCP durchgeführt; die bisher ausgewerteten Quellen legen aber die Vermutung nahe, dass die äußere Restitution wesentlich engagierter vorangetrieben wurde, vgl.: Eberhard Hanfstaengl an bayerisches Kultusministerium, 07.05.1951; Bernhard Hoffmann, Memorandum, 23.05. 1950, Bayerische Staatsgemäldesammlungen (BStGS), Registratur, 20/5c, Nr. 576.

36 Vgl. grundlegend zur alliierten Besatzungspolitik: Deutschland unter alliierter Besatzung 1945-1949/55. Hg. von Wolfgang Benz. Berlin: Akademie Verlag 1999; ders.: Potsdam 1945. Besatzungsherrschaft und Neuaufbau im Vier-Zonen-Deutschland. 4. Aufl. München: dtv 2005.

37 Tobias Winstel: Verhandelte Gerechtigkeit. Rückerstattung und Entschädigung für jüdische NS-Opfer in Bayern und Westdeutschland. München: Oldenbourg 2006, S. 21. 
reibungslos angelaufen war, musste die MFAA bald feststellen, dass die verbleibenden Objekte zunehmend schwierig zu identifizieren und zuzuordnen waren. ${ }^{38}$ Die Schließung der CCPs und die Übergabe der Restbestände in Treuhänderschaft des bayerischen Ministerpräsidenten musste mehrfach verschoben werden. Murray D. Van Wagoner (1898-1986), „Land Director“ der Militärregierung für Bayern, vollzog sie schließlich mit einem Brief an den Ministerpräsidenten Hans Ehard (1887-1980) im August 1948, endgültig erst im Laufe des Jahres $1949 .{ }^{39}$ Ehard delegierte die Treuhänderschaft an das bayerische Kultusministerium, das sie an den Generaldirektor der Bayerischen Staatsgemäldesammlungen, Eberhard Hanfstaengl (1886-1973), weiterleitete. Bis zu diesem Zeitpunkt hatten die Amerikaner gut 30.000 Posten an Kunst- und Kulturgütern, die rund 250.000 Einzelobjekte umfasst haben sollen, an zwölf Staaten restituiert. ${ }^{40} 10.600$ Inventarnummern, die im Münchner CCP verblieben waren, wurden dem Ministerpräsidenten überantwortet. Dabei handelte es sich um Werke aus öffentlichem und privatem Besitz in Deutschland sowie um Objekte, die vormals dem Deutschen Reich, dem Land Preußen, der NSDAP und hohen Parteifunktionären gehört hatten. Gegenstände, die zum Zeitpunkt der Übergabe als restitutionspflichtig oder restitutionsverdächtig identifiziert waren, verblieben unter amerikanischer Kontrolle. Kunstgegenstände, die sich nach der Übergabe als restitutionspflichtig erweisen sollten, musste der Ministerpräsident der Besatzungsmacht aushändigen. ${ }^{41}$

„Land Director" Van Wagoner hatte mit dem, was in deutscher Übersetzung als „Treuhänderschaft" bezeichnet wird, „the custody and the care, control and maintenance" der übergebenen Kunst- und Kulturgüter gemeint. ${ }^{42}$ Welche Rechte und Pflichten der bayerische Ministerpräsident damit konkret übernommen hatte, blieb allerdings lange Zeit im Detail unklar. Schon anderthalb Monate nach der Übergabe fühlte sich Van Wagoner bemüßigt, seine Angaben zu präzisieren, da die Instruktionen nicht ausreichend klar gewesen seien und zu Missinterpretationen geführt haben. ${ }^{43}$ Dennoch holten die deutschen Verant-

38 Vgl. u. a.: MFAA, Memorandum, 20.06. 1947, NARA, RG 260, M1946, Roll 1 (via fold3.com).

39 Murray D. Van Wagoner an Hans Ehard, 03.08.1948, BArch B 323/356, fol. 499-501. Die treuhänderische Übergabe verzögerte sich, weil der amerikanische Direktor des CCP in jener Zeit zurücktrat, vgl.: O. A.: Bericht über den Central Collecting Point München, 28. 02. 1951, BArch B 323/325, fol. 314f.

40 Vgl. entsprechende Auflistungen in: BArch B 323/325, fol. 284-287. Die äußeren Restitutionen schufen mit der Zeit neue Probleme, da sie an Staaten erfolgten, die die Objekte sofern diese nicht in öffentlichem Eigentum standen - hernach nicht immer mit demselben Engagement an die berechtigten Privatpersonen weitergaben.

41 Murray D. Van Wagoner an Hans Ehard, 03. 08. 1948, BArch B 323/356, fol. 499-501; Bernhard Hoffmann, Bericht, 26.10.1949; ders., Tätigkeitsbericht der Treuhandverwaltung von Kulturgut München, 01.10.1962, BArch B 323/325 u. 762.

42 Murray D. Van Wagoner an Hans Ehard, 03.08. 1948, BArch B 323/356, fol. 499-501.

43 Murray D. Van Wagoner an Hans Ehard, 21.09. 1948, BArch B 323/356, fol. $497 \mathrm{f}$. 
wortlichen noch knapp drei Jahre später ein juristisches Gutachten ein, um weiterhin bestehende Unklarheiten zu beseitigen. In diesem Gutachten heißt es:

„Es wird dem Bayerischen Minister-Präsidenten dadurch nur die Verantwortung für die Erhaltung, nicht aber für die Verfügung und Restitution dieses Kunstgutes übertragen; es handelt sich nur um eine Änderung in der, custody', nicht in der,jurisdiction`. Diese Unterscheidung ist typisch amerikanisch. ${ }^{* 44}$

Tatsächlich blieben die Amerikaner mit ihrer Hohen Kommission, die 1949 auf die Militärregierung gefolgt war, bis 1955 für Restitutionen verantwortlich. Ihr Ziel, die Herkunft sämtlicher Objekte vor Beginn der bayerischen Treuhänderschaft zu klären, hatten sie nicht zur Gänze erreichen können. Daher kam auch dem bayerischen Treuhänder auf diesem Gebiet Verantwortung zu. Zumindest ein Teil des Bestandes war ihm mit der Bedingung übergeben worden, die Provenienzrecherchen hierzu fortzusetzen (Abb. 3). Von den rund 900 Objekten, die später in den Bestand der Bayerischen Staatsgemäldesammlungen gelangten, betraf diese Aufforderung knapp 50 Werke. $^{45}$

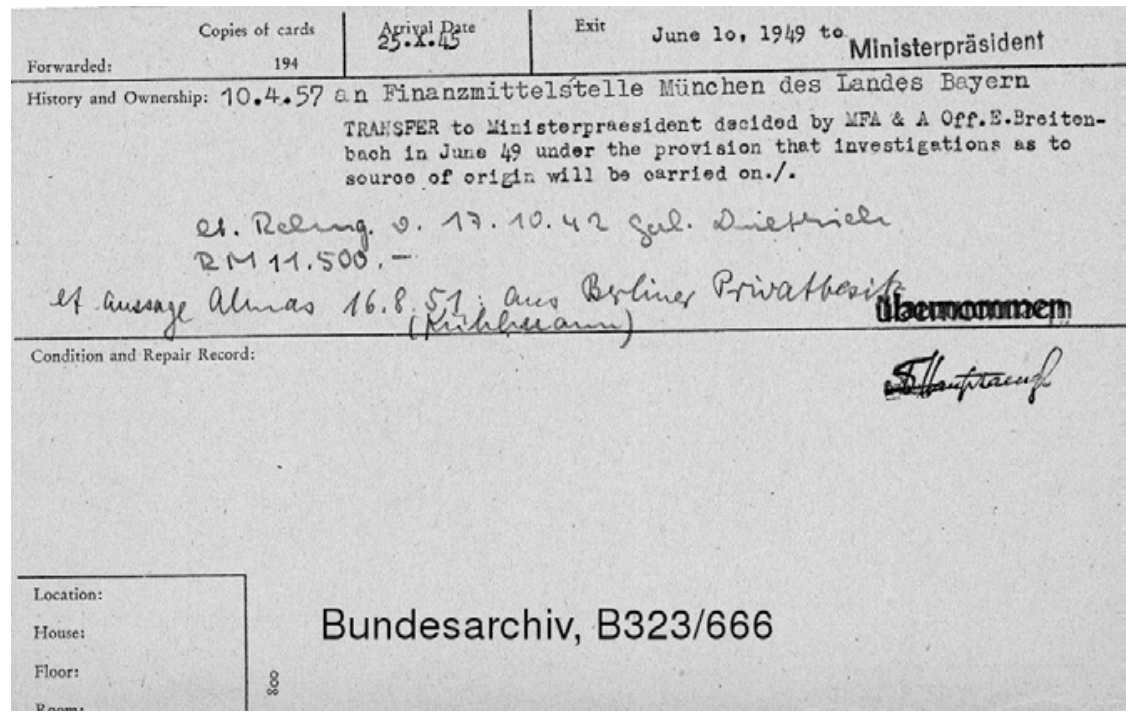

Abb. 3: Auf den Property Cards wurde die treuhänderische Übergabe an den bayerischen Ministerpräsidenten vermerkt - nur teilweise mit der Aufforderung ,that investigations as to source of origin will be carried on“ (hier aus dem Münchner CCP, Nr. 12373, Rückseite)

44 Friedrich-August von der Heydte, Gutachten, 04.04.1951, BArch B 323/326, fol. 248-251 (248).

45 Diese Aufforderung ist auf den entsprechenden Inventarkarten (Property Cards) notiert, die zu jedem Objekt im CCP erstellt wurden (siehe Abb. 3). 
Ob Bayern tatsächlich nur die Objekte überprüfen sollte, deren Property Cards mit einem entsprechenden Vermerk versehen waren, ist noch nicht eindeutig zu beantworten. Die bisher ausgewerteten Quellen deuten darauf hin, sind in ihren Aussagen aber nicht immer eindeutig. ${ }^{46}$ Erkennbar ist, dass die Motivation der bayerischen Verantwortungsträger, die Restitution aktiv voranzutreiben, nicht grenzenlos gewesen ist. Mit Blick auf die weitreichenden Bestimmungen zur äußeren Rückerstattung umtrieb sie ohnehin schon das Gefühl, dass etliche Kunstwerke unbegründet und unrechtmäßig ans Ausland abgegeben worden seien. ${ }^{47}$ Aufgrund des großen Engagements der Militärregierung in dieser Sache hielten sie weitere Untersuchungen zudem für wenig aussichtsreich. Eberhard Hanfstaengl zumindest bemerkte, dass die vorhandenen Unterlagen vom CCP bereits ausgewertet worden seien und „nunmehr nur noch sekundäre Hilfsmittel zur Verfügung stehen. Diese sind weit weniger ausgiebig und erfolgsversprechend“. ${ }^{48}$ Wie umfassend und gewissenhaft Hanfstaengl und seine Mitarbeiter der Aufgabe nachkamen, die Recherche zu einigen der überantworteten Objekte fortzusetzen, ist noch nicht klar ersichtlich. ${ }^{49}$ In jedem Fall zeigte sich der Hohe Kommissar der USA für Deutschland, John McCloy (1895-1989), im August 1950 zwar zufrieden mit der Zusammenarbeit und dem guten Willen des Treuhänders. „Ich bin jedoch in Sorge über die Langsamkeit, mit der die Prüfung des noch nicht identifizierten Materials vor sich geht. ${ }^{\text {"50 }}$ So sandte McCloy für gut ein Jahr mit S. Lane Faison einen eigenen Mitarbeiter nach München, der die Bearbeitung hunderter Objekte beschleunigt vorantreiben sollte, die aus österreichischen Depots stammten - darunter vor allem Kunstwerke, die für Hitlers „Führermuseum“ in Linz vorgesehen waren.

46 Bernhard Hoffmann, der vor und nach der treuhänderischen Übergabe im CCP arbeitete, ging 1949 davon aus, dass nur die Objekte vom bayerischen Treuhänder weiter auf ihre Herkunft zu untersuchen waren, deren Property Card eine entsprechende Aufforderung zeigte; dies betraf nur knapp 50 der 900 Objekte aus ehemaligem NS-Besitz, die später in den Bestand der Bayerischen Staatsgemäldesammlungen gelangen sollten, vgl.: Bericht vom 26. 10. 1949, BArch B 323/325, fol. 129-133 (130f.). Eberhard Hanfstaengl schrieb am 14.09.1950 allerdings an das bayerische Kultusministerium von der „neuerdings von amerikanischer Seite aufgestellte[n] Forderung, für jedes Objekt den Herkunftsnachweis möglichst bis 1933 zu erbringen“, BArch B 323/356, fol. 481f. (482).

47 Stellvertretend für vergleichbare Äußerungen: „Diese Bestimmungen [der Military Government Regulations 18] sind für die Deutschen nicht nur hart[,] sondern auch in einem erheblichen Masse diskriminierend, da bei allen Kunsterwerbungen unterstellt wird, dass ein ,loot' vorliegt." Memorandum zum Schreiben des Amerikanischen Hohen Kommissars McCloy vom April 1951, BArch 323/326, fol. 216-220 (217).

48 Eberhard Hanfstaengl an bayerisches Kultusministerium, 14.09.1950, BArch B 323/356, fol. $481 \mathrm{f}$.

49 Während der Treuhänderschaft des bayerischen Ministerpräsidenten von 1949 bis 1952 wurden insgesamt 201 Objekte nach außen und 27 Objekte nach innen restituiert, vgl.: BArch $323 / 325$, fol. 4 .

50 John McCloy an Hans Ehard, 03. 08. 1950 (Übersetzung), BArch B 323/356, fol. 486. 
Im Februar 1952 übernahm die bundesdeutsche Treuhandverwaltung von Kulturgut, die dem Auswärtigen Amt zugeordnet war, vom bayerischen Ministerpräsidenten die Verwaltung des Restbestands. Das neu geschaffene Bundesamt für äußere Restitutionen zeichnete nach Aufhebung des alliierten Besatzungsstatuts 1955 für ausstehende Rückerstattungen verantwortlich. Die bayerische Staatsregierung hatte allerdings auch nach Abschluss ihrer Treuhänderschaft noch lange Zeit mit ihrer Nachfolgerin zu tun. Direktiven des Alliierten Kontrollrats hatten schon 1947/48 vorgesehen, dass Vermögenswerte von Organisationen und Funktionären der NSDAP, die nicht restitutionspflichtig waren, an das Bundesland übereignet werden sollten, in dem sie sich bei Kriegsende befanden. ${ }^{51}$ Der Freistaat Bayern hatte folglich Aussicht auf beachtliche Kunstwerte, die er während seiner Treuhänderschaft aber noch nicht zu Eigentum übernehmen konnte, eben weil ihm nur die „custody“, nicht die „jurisdiction“ übertragen worden war. Darüber hinaus sahen die amerikanischen Alliierten zur Übereignung von Kunstwerken aus NS-Besitz restriktive Sonderbestimmungen vor. ${ }^{52}$ Erst die Treuhandverwaltung von Kulturgut konnte entsprechende Objekte freigeben, wenn keine Anträge auf äußere oder innere Restitution $\mathrm{zu}$ ihnen vorlagen. Gleichwohl sollte die Übereignung von Kunstgegenständen aus NS-Besitz an den Freistaat Bayern keineswegs reibungslos verlaufen. Um ihre Ansprüche organisieren und durchsetzen zu könnten, mussten sich die bayerischen Behörden intensiv und kontrovers mit der Bundesrepublik auseinandersetzen. Dabei war der Freistaat Bayern allerdings imstande, mehr Engagement zu zeigen, als bei den Provenienzrecherchen und Kunstrestitutionen in der Nachkriegszeit, wenngleich ihm - wie der Aufsatz umrissen hat - für diese Aufgaben auch nur eine begrenzte Verantwortung zugekommen war.

51 Direktiven Nr. 50 u. 57 des Alliierten Kontrollrats (29.04.1947/15.01.1948). In: Amtsblatt des Kontrollrats in Deutschland, Nr. 15, S. 275-278 u. Nr. 18, S. 302-307.

52 Die amerikanische Hohe Kommission gab Vermögen der NSDAP und ihrer Mitglieder zum 15.12.1949 aus der Vermögenskontrolle nach Militärregierungsgesetz Nr. 52 frei. Ausgenommen waren restitutionspflichtige und künstlerische Vermögensgegenstände, vgl.: Amt des Amerikanischen Hohen Kommissars für Deutschland an Hans Ehard, 13.01. 1950, BArch B 323/356. 


\title{
"In Trusteeship" or "Guilty Secret"? The "Rudolf von Alt Aktion" 1938, the "Collection" of Martin Bormann and the "Fiduciary" Transfers of "Former Nazi Property" to the Bavarian State after $1945^{1}$
}

\author{
Zusammenfassung \\ „Treuhänderschaft“ oder „Strafbares Geheimnis“? Die „Rudolf von Alt-Aktion“ 1938, die \\ Sammlung Bormann und der treuhänderische Transfer von ehemaligem Nazi-Eigentum \\ an den Bayerischen Staat nach 1945 \\ Ziel eines Kooperationsprojekts zwischen der Staatlichen Graphischen Sammlung (SGSM) \\ und dem Zentralinstitut für Kunstgeschichte (ZI) in München war die Provenienz von 617 \\ Zeichnungen und Aquarellen von Rudolf von Alt (1812-1905) und seinem Vater Jakob Alt \\ (1789-1872) zu untersuchen. Als Teil eines Konvoluts von einst über 800 Blättern, die der \\ Reichsleiter der NSDAP und spätere „Stellvertreter des Führers“ Martin Bormann \\ (1900-1945) nach dem „Anschluss“ Österreichs im März 1938 durch Mittelsmänner zu- \\ sammentragen ließ, wurde der Bestand in den 1950er Jahren als „ehemaliger NSDAP- \\ Besitz" an die SGSM überwiesen. Die "Alt-Aktion“ ist ein dringendes Forschungsdesi- \\ derat, da einige Werke eindeutig als Raubkunst identifiziert werden konnten.
}

Schlagwörter:

Martin Bormann, Rudolf von Alt, Aquarelle, ehemaliger NSDAP-Besitz, Central Collecting Point (CCP) München, Plünderung, Restitutionskomitee, Äußere Restitution (an Österreich)

\begin{abstract}
The cooperation project between the Staatliche Graphische Sammlung (SGSM) and the Zentralinstitut für Kunstgeschichte (ZI) in Munich tried to investigate the provenance of 617 drawings and watercolours by Rudolf von Alt (1812-1905) and his father Jakob Alt (1789-1872). Originally part of more than 800 works, they were purchased through intermediaries on behalf of the "Reichsleiter" of the NSDAP and later "Deputy" of Hitler, Martin Bormann (1900-1945), directly after the "Anschluss" of Austria in March 1938. They were finally handed over to the SGSM as "former Nazi party assets" in the 1950s. The

1 Translations by Carol Carl and Meike Hopp. A comprehensive essay with a catalog and detailed appendices was published in 2015. See Meike Hopp: "Weiß gar nicht, wo sie alle hingerathen sind". Der Münchner Bestand der Werke Rudolf von Alts und die "Sammlung Bormann" Eine Herausforderung für die Provenienzforschung. In: Andreas Strobl, ed.: Rudolf von Alt. “... genial, lebhaft, natürlich und wahr”. Der Münchner Bestand und seine Provenienz. Berlin: Deutscher Kunstverlag 2015, pp. 146-190.
\end{abstract}


urgent need for further research on the so-called Alt Aktion becomes obvious, as some of the works have clearly been identified as Nazi-looted art.

Keywords

Martin Bormann, Rudolf von Alt, Watercolours, Former Nazi Property, Central Collecting Point (CCP) Munich, Plundering, Restitution Committee, External Restitution (to Austria)

In August 1902, on the occasion of the $90^{\text {th }}$ birthday of the Austrian painter Rudolf von Alt (1812-1905), a reporter visited the painter in his summer resort in Goisern, Upper Austria. Fascinated, the journalist described the conversation with Alt, whom he had met at work and who had eagerly continued to paint throughout the interview. Finally, Alt summarized: "How many pictures have I painted in my life? They must run into thousands. I don't know where they have all ended up. I've never kept a record of them. Have always had to work."2

In 1905, now at the ripe old age of 92, Rudolf von Alt worked on what was to be his last watercolour - a view of his studio. He died in March of the same year, leaving behind this unfinished artwork. ${ }^{3}$ The place in the picture where the artist had intended to include himself remained a white spot.

This final work was acquired at an unknown time after the death of the painter by the Jewish industrialist, writer and painter Stephan Mautner from Vienna (1877-1944). Up to 1930, Mautner had been deputy general director of his father's enterprise, Textilwerke Mautner A.G. and, from 1925 onwards, owner of the company Textilfabrik Marienthal. After the "Anschluss" in March 1938, the extensive properties of the family were forced into liquidation and placed under provisional administration. ${ }^{4}$ On July 15, 1938 Mautner had to declare his assets an obligation that applied to all Jews. He informed the authorities that shortly after the "Anschluss" he had sold four of the pictures from his collection. ${ }^{5}$ On November 3, 1938 Stephan Mautner registered himself and his family as having moved to Budapest. In 1944, he and his wife Elsa (1884-1944) were deported to an assembly camp in the centre of Budapest and from there taken to the death camp of Auschwitz after July of the same year. There, both of them were mur-

2 Quote after Walter Koschatzky: Rudolf von Alt. 2. erw. Aufl. Wien-Köln-Weimar: Böhlau 2001, pp. 352-353 (Original source: Th. Thomas: Ein Besuch bei Rudolf von Alt. In: Neue Freie Presse Wien, 24.08.1902, p. 7). The total oeuvre was estimated by Koschatzky at about 5,000 works.

3 Cf. Ludwig Hevesi: Rudolf von Alt. Sein Leben und sein Werk. Wien: Ed. k. k. Ministerium für Kultus und Unterricht 1911, p. 144.

4 Sophie Lillie: Was einmal war. Handbuch der enteigneten Kunstsammlungen Wiens. Wien: Czernin 2003, pp. 748-753.

5 Österreichisches Staatsarchiv (ÖStA), Archiv der Republik (AdR), Vermögensverkehrsstelle (VVSt), Vermögensanmeldung (VA) 7327 Stephan Mautner; Bundesdenkmalamt Wien (BDA) Archiv, Personenmappen, K 41/1, Karl F. Mautner. Cf. Lillie: Was einmal war (Fn. 4), pp. 748-753, here p. 750. 
dered. The couple's three children, who had managed to emigrate in time, survived the Holocaust. ${ }^{6}$

As it later turned out, the pictures sold by Mautner in 1938 must have been four watercolours by Rudolf von $\mathrm{Alt}^{7}$ - among them the unfinished View of His Studio, which in the same year was passed on to the "Collection" of Nazi party "Reichsleiter" Martin Bormann (1900-1945) and therefor registered under the inventory number $\mathrm{J} 1638$ as an Interior of a Salon. ${ }^{8}$ According to an invoice of the Kunstgewerbliche Werkstätten Karl Pfefferle in Munich, a watercolour named Interior (of the Artist's Last Work) was fitted with a new passe-partout and frame on behest of Bormann in December 1938. ${ }^{9}$ Along with Hitler's collection for the "Führermuseum", the watercolour was stored in a repository in the salt mines of Altaussee in Austria during war time, to protect it from air raid. At the end of the war, the Allies seized the works of art from the Nazi depots. ${ }^{10}$ On October 29, 1945 the picture was handed over to the so called Central Collecting Point (CCP) in Munich now given the title Interior with Large Cupboard. ${ }^{11}$ Stephan Mautner's youngest son Karl F. Mautner (1915-2002), captain in the US army and liaison officer in Berlin, launched investigations into his father's art collection in January 1947 and approached the CCP in Munich with a detailed search request, including a description of the final watercolour:

"Watercolour 'Studio of the Artist', with an unfinished spot in the centre, where the artist intended to insert his self-portrait. It is the last picture painted by Alt, acquired by Mr. Stephan Mautner at the artist's memorial exhibition in Vienna in 1904 or 1905. I Possible Size: $55 \times 77 \mathrm{~cm}^{\text {". }}{ }^{12}$

6 Cf. Wolfgang Hafer: Die anderen Mautners: Das Schicksal einer jüdischen Unternehmerfamilie. Berlin: Hentrich und Hentrich 2014.

7 Apparently, the director of the Österreichische Galerie had passed them on to Bormann's intermediary in April 1938. Österreichische Galerie (ÖG) Archiv, 1938 Z. 227, NSDAP München Schulte-Strathaus. Aquarelle von Alt aus Privatbesitz, Delivery note, 04.04.1938.

8 For a list of the "Bormann Collection"(reconstructed in the postwar period) see Bundesarchiv (BArch) Koblenz, Bestand der Treuhandverwaltung von Kulturgut, B 323/13, Sammlung Bormann.

9 Invoices for restoration works and framings have been preserved in Bayerisches Hauptstaatsarchiv (BayHStA), NSDAP Bauakten, 11673, Bildankäufe 1936-1944.

10 Altogether more than 700 sheets by Rudolf von Alt and his father from the "collection" of Bormann were stored in repositories in Altaussee, in Berchtesgaden, in the monastery of Höglwörth and in his villa in Pullach near Munich.

11 Hopp: Sammlung Bormann (Ill. 2) (Fn. 1), pp. 146-190, here p. 149. The "Property Cards" of the CCP Munich can be researched via a database of the German Historical Museum, URL: http://www.dhm.de/datenbank/ccp (accessed on 17.12.2017). On the history of the CCP Munich see, most recently, Iris Lauterbach: Der Central Collecting Point in München. Kunstschutz, Restitution, Neubeginn. München/Berlin: Deutscher Kunstverlag 2015.

12 BDA Wien Archiv, Personenmappen, K 41/1, Karl F. Mautner, Letter by Karl F. Mautner, 02. 02. 1947. 


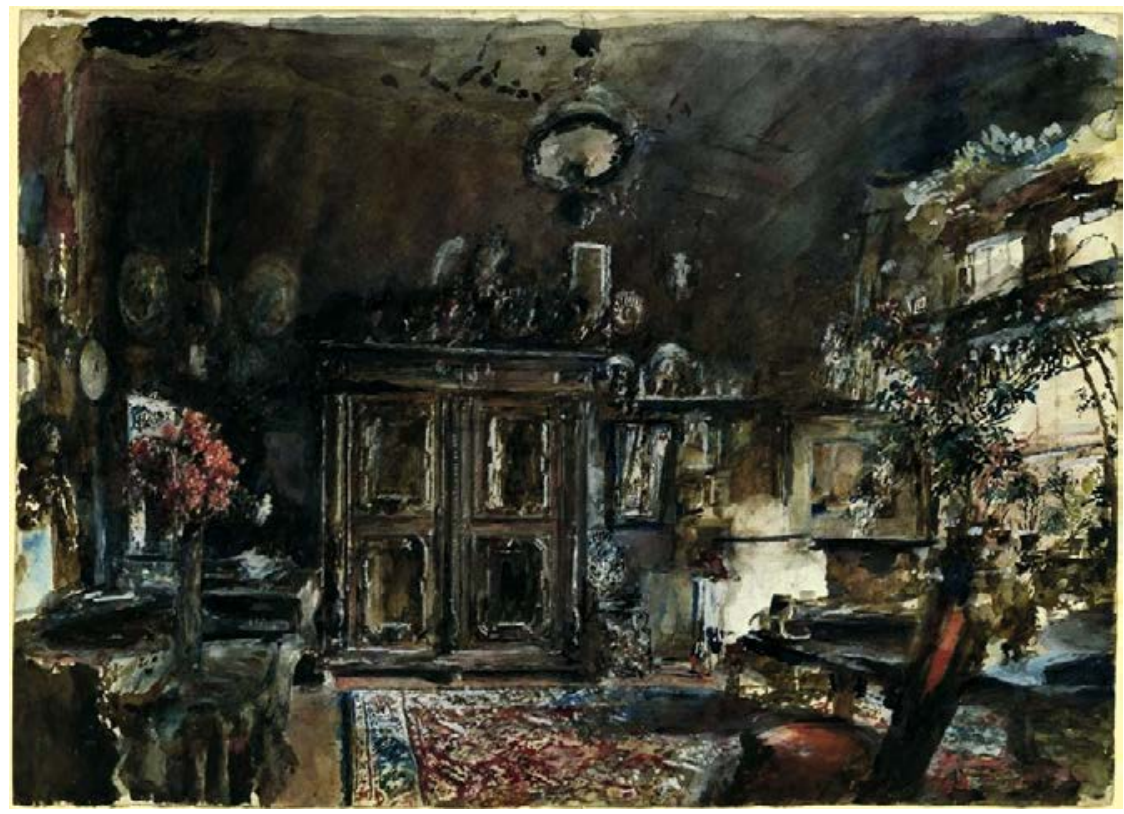

Abb. 1: Rudolf von Alt, Das Arbeitszimmer des Künstlers, 1905, watercolour, 56.3 x $77.8 \mathrm{~cm}$, SGSM Inv.-No. $45625 \mathrm{Z}$

However, Mautner, who in late 1947 had even paid the CCP in Munich a personal visit, was disinformed and led to believe that no picture with that motif could be found. ${ }^{13}$ Cruelly unaware of how very close he was to the watercolour from his father's collection, he left Munich 'empty-handed'.

On December 10, 1956 the watercolour was transferred to the Minister President of the Free State of Bavaria and subsequently to the Staatliche Graphische Sammlung München (State Graphic Collection, SGSM) together with 600 other watercolours and drawings by Rudolf von Alt and his father Jakob Alt (1789-1872).

In December 1980, Mautner approached the SGSM with a written search request:

“On my last visit to Austria I received a delightful book on Rudolf von Alt by the privy councilor Walter Koschatzky. To my surprise, I found in it a reproduction of the artist's final watercolour - a view of his studio with an empty spot in it, where he had intended to insert his self-portrait. This painting [...] was in the possession of my family up to 1938. [...] In 1946 [sic] I tried [...] to find out from the collecting point in Munich, the $\mathrm{CCP}$, if the Alt pictures had been found. Their answer, I remember well, was distinctly

13 Ibid. Report by Robert S. Leonard, Monuments, Fine Arts, and Archives Section (MFA\&A), 12.03.1947. 
uninformative. [...] I'm not obsessed with the return of the artworks, but do believe, that the circumstances will also be of interest to you and [...] help to complete the history of the picture of the studio." ${ }^{14}$

Despite this comprehensive letter from Karl F. Mautner, the SGSM never seemed to doubt its right to the ownership of the work in the following years. When a research project started in 2011, however, it was possible for the first time to examine the issue of ownership in depth and successively reconstruct the history of the watercolour, so that it was finally restituted to the heirs of Karl F. Mautner in $2016 .^{15}$

\section{The Alt Estate}

But how is it that all these watercolours and drawings ended up in Munich? Rudolf von Alt spent the final years of his life almost exclusively at home in the care of his daughter Louise (Aloisia Gabriele, 1859-1941). When he died on March 12, 1905 he left behind few cash assets. The same year his family arranged to have a major portion of the artworks in his estate auctioned. ${ }^{16}$ At the auction, which took place at the H. O. Miethke gallery in Vienna from February 12 to 16, 1906 , some 432 works of the artist were sold, all of which had received an official estate stamp. ${ }^{17}$ A significant number of Alt's works - in particular his drawings remained in family ownership. The art critic Ludwig Hevesi (1843-1910) described this mixed assortment of works in 1906 as "a quite enormous portfolio, more than a span high [...] hors d'œuvres and entremets of a richly laden table." 18

Following the sudden death of her fiancé in January 1905 and shortly after the death of her father, Louise von Alt - a trained pianist - was without a regular income. Although she received a pension from the city of Vienna she suffered increasingly from financial worries, especially during the inflation after the First

14 Staatliche Graphische Sammlung München (SGSM), Altregistratur, Karl F. Mautner to the SGSM, 12.11.1980. Mautner refers to the first edition of the catalog raisonné by Walter Koschatzky: Rudolf von Alt. Salzburg: Residenz Verlag 1975.

15 The project was funded by the then Berlin-based Arbeitsstelle für Provenienzforschung (Office for Provenance Research), Stiftung Preussischer Kulturbesitz (Prussian Cultural Heritage Foundation) from 2011 to 2013. The results have been presented to the public in an exhibition in Munich in 2015, see Hopp: Sammlung Bormann (Fn. 1), pp. 146-190.

16 Koschatzky: Rudolf von Alt, p. 332.

17 See Galerie H. O. Miethke, ed.: Öffentliche Versteigerung des künstlerischen Nachlasses von Rudolf von Alt am 12. Februar 1906. Wien: Miethke 1906, p. 16.

18 Ludwig Hevesi: Der Nachlaß Rudolf von Alt’s. In: Pester Lloyd, 53 (1906), 36, p. 2. 
World War. ${ }^{19}$ As a consequence, Louise von Alt was repeatedly forced to sell items from her father's estate or pass them on to the city of Vienna. ${ }^{20}$ In May 1938, she sold a particularly large assortment of her father's works to the "Reichsamtsleiter" at the "Braunes Haus" in Munich, Ernst Schulte Strathaus (1891-1968). He was sent to Vienna at the behest of "Reichsleiter" Martin Bormann to "purchase" paintings and watercolours by Rudolf von Alt shortly after the "Anschluss" of Austria in March 1938. On June 3, 1938 Schulte Strathaus applied to the Federal Monuments Authority Austria in Vienna for permission to export " 4 boxes and 1 folder with pictures" plus a "framed oil painting" by Rudolf von Alt. ${ }^{21}$ In fact, this shipment contained more than 400 drawings from Alt's estate, most of which today are held by the SGSM.

\section{Reception of Rudolf von Alt in the "Third Reich"}

But why was Bormann - who was not considered a passionate art collector ${ }^{22}$ - so interested in the works of Rudolf von Alt? It was due not least of all to Hitler's personal preferences that $19^{\text {th }}$-century art - and in particular works of German Romanticism - became increasingly the focus of the art trade and private collectors from 1933 onwards. ${ }^{23}$ With the "Anschluss" of Austria in March 1938 it was above all two Austrian painters of the Biedermeier period who aroused the interest of the new clientele of collectors from the "Third Reich", Ferdinand Georg Waldmüller (1793-1865) and Rudolf von Alt. Just how deeply rooted Hitler's personal interest in Alt was, is evident from a report in the newspaper Deutsche Allgemeine Zeitung on an exhibition at the Albertina in Vienna in July 1938:

"In the great row of famous names there are some whom we Reich Germans do not know well. [...]. Among those not recognized sufficiently, one master in particular [...]: Rudolf von Alt. [...] When one observes Rudolf v. Alt's works [...], one can literally feel

19 Cf. ÖStA, Allgemeines Verwaltungsarchiv (AVA), Ministerium für Kultus und Unterricht (KUM), Nr. 21117/1905.

20 Koschatzky: Rudolf von Alt, pp. 350-351.

21 For a list of export applications for artworks by Rudolf von Alt between 1938 and 1945 see Hopp: Sammlung Bormann (Fn. 1), pp. 146-190, appendix pp. 275-280.

22 See, for instance, Birgit Schwarz: Geniewahn. Hitler und die Kunst. Wien, Köln, Weimar: Böhlau 2009, pp. 246-247; Volker Koop: Martin Bormann. Hitlers Vollstrecker. Wien-KölnWeimar: Böhlau 2012, pp. 33-43.

23 Most recently, Christian Fuhrmeister and Meike Hopp: Der Handel mit Kunst des 19. Jahrhunderts in München 1937-1945. Überlegungen zu einem Forschungsfeld. In: Kunsthändler, Sammler, Stifter. Günther Franke als Vermittler moderner Kunst in München 1923-1976. Ed. by Felix Billeter. Berlin-Boston: De Gruyter 2017 (Schriften der Forschungsstelle "Entartete Kunst" 11), pp. 163-186. 
the spark that must have sprung from him to the artistic soul of the sixteen-year-old Adolf Hitler. Adolf Hitler came to Vienna in the same year that Alt died [...]. He felt inspired to become an artist like him [...]. There appears to be a brilliant, inner relationship between the young architecture student Adolf Hitler and the venerable artist who had just passed away - and whose art is at its finest when related to architecture."24

\section{The "Rudolf von Alt Aktion" $1938^{25}$}

Shortly after the "Anschluss" of Austria on March 13, 1938, the then "Reichsamtsleiter" Ernst Schulte Strathaus was sent to Vienna to acquire works by Rudolf von Alt:

"Hitler asked me to seek out Alt pictures when I reached Vienna. [...]. So off I went to Vienna. My search for watercolours, drawings and paintings by Alt was so successful that I managed to assemble some 180 works. When word passed around, as it always does in Vienna, that I was interested in Alt, the offers just poured in. [...] I bought mainly from dealers, but also from private collectors. The prices were generally very high [...] Whether Jews were among the private collectors [...] I don't know. At least I wasn't aware of that. Any talk of me putting the sellers under 'pressure' is futile."26

His 1949 statement that he did not know Jewish collectors were among the owners of Alt's watercolours is absurd. In fact, on May 20, 1938, he had received from Josef Bürckel (1895-1944), "Reich Commissioner for the reunification of Austria and the Reich", the right of first refusal "for acquisitions from confiscated collections", formerly Jewish ones. ${ }^{27}$ This had enabled Schulte Strathaus to gather an enormous number of the artist's watercolours shortly after the "Anschluss".

The Director of the Austrian Gallery in the Belvedere Palace, Bruno Grimschitz (1892-1964), promised to support him in acquiring Alt watercolours from private collectors. ${ }^{28}$ So, too, did Heinrich Leporini (1875-1964) and Otto Benesch (1896-1964), custodians of the Albertina Museum, who on June 30, 1938 had reached an agreement with Schulte Strathaus on how to divide the watercolours

24 Staatliche Museen zu Berlin (SMB), Zentralarchiv (ZA), Künstlerdokumentation: Alt, Rudolf von, Zeitungsausschnitte. Unknown: Rudolf von Alt. Ein Wiener Meister der Aquarellmalerei. In: Deutsche Allgemeine Zeitung, 27.07.1938.

25 Christian M. Nebehay was the first to introduce this term, see ibid.: Das Glück auf dieser Welt. Erinnerungen. Wien: Brandstätter 1995, pp. 62-65, here p. 63.

26 BArch Koblenz, B 323/12, Fol. 71. Affidavit by Ernst Schulte Strathaus, 28.04.1949.

27 Österreichische Nationalbibliothek (ÖNB) Wien, Handschriftensammlung, Sign. Cod. ser. n. 52335; IV/V. Circular letter, 11.06.1938.

28 ÖG Archiv, 1938 Z. 227, NSDAP München Schulte-Strathaus. Aquarelle von Alt aus Privatbesitz. 
and paintings from the collection of the Jewish brewery owner and patron Moriz Kuffner (1854-1939). It was decided that "some parts of the Kuffner collection are to be acquired by the Führer and Reich Chancellor, other parts by the Albertina, while the remaining works should be made available to Mr. Kuffner for transfer abroad". ${ }^{29}$ Some of the works taken from the Kuffner collection under this agreement are still missing to this day. ${ }^{30}$

\section{The Trade with Alt's Watercolours}

Even though Schulte Strathaus' had been able to obtain special permission to purchase and export Alt's works, the Austrian authorities nevertheless regarded this 'exodus' of artworks with great scepticism. Evidently, the Central Office for the Protection of Monuments together with the Head of the Reich Chamber of Fine Arts in Vienna, Leopold Blauensteiner (1880-1947), had tried to prevent the export of the watercolours and drawings on several occasions. ${ }^{31}$ The "Rudolf von Alt Aktion" had aroused covetous feelings in Austrian institutions. Practically all of Alt's works, for which export applications had been submitted, were "banned from being exported". ${ }^{32}$ This meant the owners of the pictures - predominantly Jewish emigrés - had been forced to leave the artworks behind them in Austria. It was permissible to sell them, but only in Austria or - in special cases - to the socalled "Altreich" ("Old Reich", i.e. Germany). However, this action had failed to take into account the consequence that it played into the hands of agents and dealers acting in the interests of the party.

It had been well known for some time on the Munich art market that highly solvent buyers were showing considerable interest in Alt's works. Three of the most important purchasers of works of art for the "Bormann Collection" were the Munich art dealers Adolf Weinmüller (1886-1958), Eugen Brüschwiler (1889-1967) and Maria Almas Dietrich (1892-1971)..$^{33}$ As part of their activities

29 ÖStA, AdR, Unterricht allgemein (U-Allg.), Fasz. 3161, 1938/23722. On the fate of the Kuffner family and its collection see Lillie: Was einmal war (Fn. 4), pp. 615-621.

30 Bundesdenkmalamt Wien, ed.: Verzeichnisse gesuchter Kunstwerke aus österreichischem Besitz. [Unknown], p. 10.

31 Eva Frodl-Kraft: Gefährdetes Erbe. Österreichs Denkmalschutz und -pflege 1918-1945. Wien, Köln, Weimar: Böhlau 1997, p. 192. Eva Frodl-Kraft even speaks of a "power struggle of the party authorities". Cf. Wiener Stadt- und Landesarchiv (WStLA), Landesgericht für Strafsachen Wien (LG St), Volksgericht (Vg), Vr, 404/45, Strafsache Leopold Blauensteiner. Records by Marcell Kammerer, 03.06.1945.

32 Hopp: Sammlung Bormann (Fn. 1), here appendix pp. 275-280.

33 See invoices to Bormann in BayHStA, NSDAP-Bauakten 11614, 11673 and 11631. For Weinmüller cf. Meike Hopp: Kunsthandel im Nationalsozialismus. Adolf Weinmüller in München und Wien. Wien-Köln-Weimar: Böhlau 2012, pp. 215-221. 
for Hitler's planned but never realized "Führermuseum" in particular the latter two enjoyed substantial privileges. ${ }^{34}$ It is known that Brüschwiler bought at least 22 of Alt's works for Bormann, so it is reasonable to assume he must have acquired some of them in Vienna. Since there is no known record of an estate related to his art dealing business known yet, the precise origin of most of the works he sold remains unclear, with the exception of Rudolf von Alt's The Old North Railway Station, Vienna from the murdered Jewish collector Valerie Heissfeld (1876-1942). This very work was sold to Bormann by Brüschwiler in July 1942 and that was restituted by the SGSM to her heirs in 2011. It is equally difficult to reconstruct the purchase of Alt's works by the art dealer Maria Almas Dietrich, as hardly any detailed receipts are available for scrutiny. ${ }^{35}$ However, according to testimonies in Austrian restitution material she knew how to exert extortionate pressure on private collectors. One of them, Richard Stein, reported in April 1956:

"At the end of April 1938, the owner of the art gallery 'Almas' Brienner Strasse 55 in Munich, visited me and announced that the paintings belonging to me were to be confiscated. She explained she was in a position to buy my possessions and, as a consequence, confiscation could be averted." ${ }^{36}$

\section{The "Bormann Collection" - A complex term}

All of Rudolf von Alt's works acquired for the "Bormann's Collection" were removed from their original mounting either whilst still in Vienna or directly after their delivery to Munich. They were partially restored, and fitted with new passe-partouts and new frames. ${ }^{37}$ The loss of the original passe-partouts explains why the watercolours bear hardly any concrete traces of provenance such as labels on the reverse side of the picture or inscriptions, stamps or other marks. It is most likely that during this process the sketch books, too, were taken apart and

34 Bundesarchiv (BArch) Berlin, NS 6/73, fol. 32-34. Report to the "Reichsleiter" Bormann, 05.05 .1943$.

35 Cf. et al. Salzburger Landessammlungen 1939-1955. Ed. by Roswitha Juffinger, Gerhard Plasser, Toni Stoss. Salzburg: Residenzgalerie Salzburg 2007, pp. 111-112; Sophia Barth: Maria AlmasDietrich. Bachelorarbeit. München: Ludwig-Maximilians-Universität 2014. URL: https://epub. ub.uni-muenchen.de/41206/8/Barth_Sophia_Maria\%20Almas-Dietrich.pdf (accessed on May 1, 2018); Hopp: Sammlung Bormann (Fn. 1), pp. 170-172.

36 BDA Wien Archiv, Personenmappen, K 47/1, Stein, Richard. Copy of a statement by Stein, 28.04. 1956.

37 Hopp: Sammlung Bormann (Fn. 1), pp. 146-190, appendix on restoration and framings of artworks by Alt between 1938 and 1945, p. 281. 
disassembled into individual sheets. ${ }^{38}$ Additionally, custom-made cassettes were produced to store the watercolours and drawings.

However, terms like the "Bormann Collection" or "inventory" are problematic, as they create an impression of a self-contained collection. But according to a report by the Trustee Administration for Cultural Property Munich (Treuhandverwaltung von Kulturgut, founded in 1951), the "Bormann Collection" was made up of different lots, namely both private acquisitions for his villa on the "Reichssiedlung Rudolf Heß" in Pullach and the transactions he ordered as fiduciary of the Nazi party concerning the interior fittings of the Obersalzberg and party buildings in Berlin, Munich or Bayreuth. ${ }^{39}$ Official purchases were recorded on file cards, and from 1939 onwards, all the items registered for Obersalzberg were given round yellow labels with a 'J-number', some of which are still on the back of some watercolours today.

It is unlikely the light-sensitive watercolours were intended to serve solely as décor. The amount of effort and expense alone of restoring and conserving them would negate this purpose. It cannot be entirely ruled out that parts of it were destined for the "Führermuseum".

At the end of the 1950s, the Trustee Administration for Cultural Property compiled from diverse file cards, lists, photographs and invoices an "inventory list" of the art items acquired on behalf of Bormann. ${ }^{40}$ They included 816 watercolours and drawings by Rudolf von Alt and his father Jakob Alt. Today one can assume that more than 100 fell victim to theft and plundering. ${ }^{41}$ Bormann must obviously have kept at least part of the "collection" in his "chief-of-staff villa" in Pullach near Munich. In a press release on March 1952 it was reported that the federal police in Salzburg had succeeded in seizing 14 valuable paintings, from the possession of a certain Johann Lafenthaler, among them three works by Rudolf von Alt. ${ }^{42}$ Until 1945, Lafenthaler had been Reichsleiter Martin Bormann's caretaker in his villa in Pullach near Munich. After evacuation on April 26, 1945 and before the US army invasion of Pullach on April 30, the "Bormann residential estate" was plundered by the local

38 See Christiane Schachtner: Kosmos auf Papier. Künstlerische Praxis und Werkprozesse bei Rudolf von Alt. In: Strobl: Rudolf von Alt (Fn. 1), pp. 72-110.

39 Records and reports on the reconstruction of the "Bormann Collection" in BArch Koblenz, B 323/12-14. Here especially B323/355, fol. 1956. Report Dr. Hoffmann, 23.11.1956.

40 Ibid.

41 Hopp: Sammlung Bormann (Fn. 1), pp. 146-190, with appendix on the Watercolours whose whereabouts are unknown, pp. 293-299.

42 BDA Wien Archiv, Personenmappen, K 31/3 Bormann, Martin (Vermögensverfall). Newspaper clipping Unknown: Bormanns Sohn soll als Zeuge aussagen. Wem gehören die Ölgemälde aus der Bormann-Villa? In: Die Presse, 19.11.1952. 


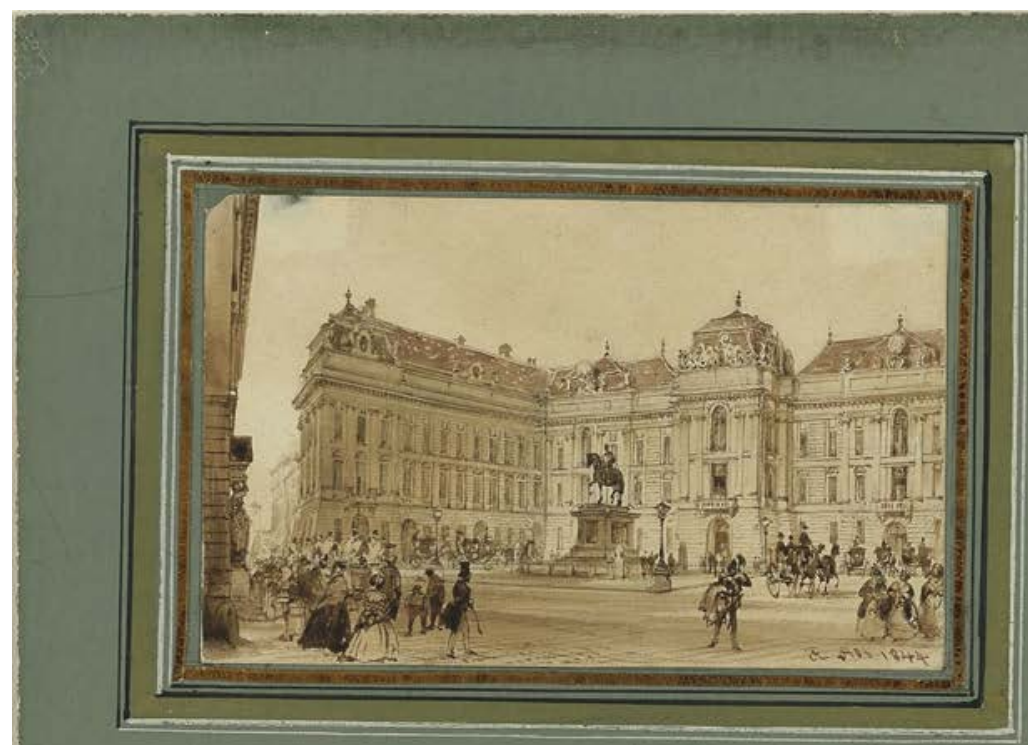

\section{$3 \varepsilon V \&$}

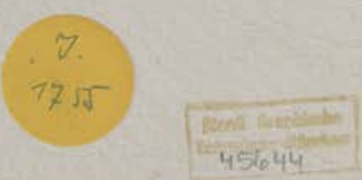

Abb. 2 und 3: Rudolf von Alt, Hofbibliothek in Wien, 1844, watercolour, 7.3 x 11.4 cm, SGSM Inv.No. $45644 \mathrm{Z}$. On the reverse a yellow label with an inventory number 
population and practically everything was taken. ${ }^{43}$ It is still not known today, how many artworks were lost on this occasion. ${ }^{44}$

\section{Returned to Austria}

More than 700 watercolours and drawings by Alt from the "Bormann Collection" had been recovered by the Allies and handed over to the Central Collecting Point (CCP) Munich. In August 1948, the Austrian Federal Monuments Authority in Vienna assigned Elisabeth Gasselseder (1903-1969) to go to Munich to collect and identify art items belonging to Austrians that had been stored in the CCP. ${ }^{45}$ Only a few days after her arrival she had already managed to identify the first Alt watercolours from Viennese art collections amongst the more than 45,000 property cards registered.

However, initial euphoria over the progress of identifying works soon dissipated. Yet her predecessor at the CCP, Johannes Morawitz, had commented in March 1948 in his records that:

"the work here in Munich is very laborious and not at all gratifying; there are disputes going on the whole time [...], yet every now and then something does turn up. For instance, 15 folders containing Alt drawings and watercolours were suddenly discovered amongst stocks of Pinakothek works and nobody knew [...] anything about them". ${ }^{46}$

Gasselseder's entries on her work on an "Alt file" also reflect increasing displeasure with the conditions at the CCP. In November 1948, she noted curtly that "the inspection of the Alt watercolours has been thwarted" and on March 11, 1949 Gasselseder wrote to the Federal Monuments Authority in Vienna and

43 Cf. Bodo Hechelhammer, Susanne Meinl: Geheimobjekt Pullach. Von der NS-Mustersiedlung zur Zentrale des BND. Berlin: Christoph Links Verlag 2014, pp. 117-118.

44 It cannot be ruled out that more watercolours and drawings have been kept in Bormann's bunker on the Obersalzberg, or in his villa bought in 1943 (from expropriated Jewish possession) in the municipality of Schluchsee in the Black Forest, where also Bormann's private library was to be sent. This library was later seized by the French military administration and transferred to the newly founded University Library Mainz in 1946. Cf. Charlet Flauaus: Der NS-Funktionär und seine private Bibliothek. Die Bücher des Reichsleiters Martin Bormann in der Universitätsbibliothek Mainz (unpublished MA-Thesis, Johannes Gutenberg-Universität Mainz). Mainz 2014. See also the article of Christian George in this volume. Furthermore, it would be conceivable that his wife Gerda Bormann (1909-1946) took some pictures with her while fleeing to South Tyrol with her children in April 1945.

45 See BDA Wien Archiv, Personenakten (PA), Gasselseder, Elisabeth.

46 BDA Wien Archiv, K 11 M 2, Fol. 76. Progress Report, 06.09.1948. 
complained that for days she had been repeatedly prevented from inspecting the original watercolours. ${ }^{47}$

But without comparison with the originals it was impossible to establish whether the Alt watercolours sought through identification from lists were correct or not. And to be able to identify the pictures successfully - and ultimately restitute them - Gasselseder needed descriptions that were as exact as possible from their former owners. But this was often no longer feasible as the owners had not survived the Holocaust, and only in rare cases were their heirs in a position to provide details. And so it was that only a few owners were able to provide such detailed information on their artworks as the Viennese art collector Leopoldine Mannaberg, who had even personally drawn a picture of the watercolour Lake Traunsee with Traunstein that she and her husband had been "forced to sell to an assignee of Hitler in June 1938" for a so-called "Munich Search File Index". ${ }^{48}$ However, it transpired that it is not identical to the work kept at the CCP, now held by the SGSM. ${ }^{49}$

One of Gasselseder's core tasks in the CCP Munich was - together with her German colleague Dr. Renate Haars- to draw up a "list of categories" that would pave the way for the restitution of artworks to Austria. Particularly in view of the imminent dissolution of the CCP, which was to take effect on November 30, 1949 negotiations over the "remaining stock" of items at the CPP - those being works whose owners could no longer be traced - became increasingly intense. ${ }^{50}$ Therefore, the "Austrian" objects were supposed to be separated according to the state of their origin. The assignments of art works under "category II: material whose Austrian origin is properly documented" provoked fierce conflicts, because this category listed objects that would be eligible for restitution to Austria. Hence the individual objects were constantly moved between the categories. ${ }^{51}$

Another conflict of interests was caused by the classification of objects in "category I: works of Austrian artists - without stamp of the Federal Monuments Office Vienna". The Austrian side laid claim to all the objects retrieved by the Allied forces from Austrian repositories and additionally - arguing on formal and 'moral' grounds - to those works by Austrian artists which illegitimately had been sent to the German Reich: "A particular example of this are Alt's works [...] for which normally export would never have been granted". ${ }^{52}$ in the evaluation in

47 See Gasselseder's reports in BDA Wien Archiv, K 11 M 1, Fol 4-5 and K 11 M 3, Fol. 59-67 and 71-72.

48 BDA Wien Archiv, Münchner Suchkartei. File Card with enclosed drawing by Leopoldine Mannaberg. I would like to thank Anneliese Schallmeiner for this reference.

49 Cf. Hopp: Sammlung Bormann (Fn. 1), pp. 146-190, here (ill. 25-27), pp. 178-179.

50 See, for instance, Lauterbach: Central Collecting Point (Fn. 11), pp. 151-159.

51 See Correspondence in BDA Wien Archiv, K 11 M 1 und K 23 M 2.

52 BDA Wien Archiv K 11 M 2, Fol. 158. Report “München - Art Collecting Center”, 13. 02.1948. 
particular of Schulte Strathaus' 'purchases' of Alt's watercolours in Vienna in 1938 - for which permission to export had indeed been given -quickly developed into a major political issue at the CCP in Munich, especially as there was no longer any documentation to indicate who the former owners were.

For this reason a special committee was set up at the beginning of 1951 to deal with the "Austrian Question". ${ }^{53}$ It had the task of examining whether the CCP should return without exception all the objects that had been sent from Austrian repositories to Munich and were among the unidentified "remaining stock" of the CCP. This led to rumours of "Austria's insane claims" in the German media in the summer of $1951 .^{54}$ They reported about "mysterious crates" ready for transport and consequently "finally removed from German control". There were also references to an "indigestible "Linzer Torte" and the "demolition of German art possessions" ${ }^{\prime \prime 5}$, while the Austrian press retaliated with wild speculation as well. $^{56}$

At the beginning of 1952, a total of 967 artworks from the stocks of the CCP in Munich were returned to Austria in order to redeem the obligations "which the American authorities in Germany committed against American authorities in Austria on transfer of these items to Munich." ${ }^{17}$ Among those artworks were 86 works by Rudolf von Alt and his father which had previously been in the "Bormann Collection" ${ }^{58}$ It is hardly comprehensible, however, according to which criteria these objects were selected for 'restitution'. Concerning Rudolf von Alt's watercolours, delivered to Austria on January 18, 1952, one at least could gain at least the impression that they had been chosen totally at random. ${ }^{59}$

The items were handed over "in trust" to Austrian Museums or stored in the Carthusian monastery of Mauerbach. ${ }^{60}$ By implementing the measures stipulated in the two "Artistic and Cultural Assets Settlement Acts" of 1969 and 1986 the Republic of Austria has indeed succeeded in restituting part of these watercolours. In 1996, the rest of Alt's works were sold in the so called Mauerbach Auction - a move which has been severely criticised, as many of the items that

53 See, for instance, BArch Koblenz, B 323/332, fol. 2-4 and B323/465, here especially fol. 82-87. As well as BayHStA, Kultusministerium (MK), 51499.

54 Unknown: Der Streit um Hitlers Kunstsammlung. In: Die Presse, 01.09.1951, p. 3.

55 Staatsarchiv München (StAM), Bezirksfinanzdirektion III (BFD III), 124 and BArch Koblenz, B 323/332. Newspaper clippings from Süddeutsche Zeitung, 13.08. 1951; Münchner Merkur, 30.08.1951; Quick München, 16.09.1951.

56 Th. P. M.: 4000 Alt-Aquarelle in Hitlers Kunstsammlung. In: Die Presse, 18.09.1951, p. 5.

57 BArch Koblenz, B323/332, fol. 233. Here Unknown: Stellungnahme der Regierung. In: Münchner Merkur, 12.01.1952.

58 For detailed lists of watercolours and drawings by Jakob Alt and Rudolf von Alt that were shipped to Austria in 1952 and have later been restituted or sold at "Mauerbach Auction" in 1996, see Hopp: Sammlung Bormann (Fn. 1), pp. 146-190, here appendices pp. 282-292.

59 Hopp: Sammlung Bormann (Fn. 1), (note 231), p. 180.

60 For the "fiduciary" transfers to the museums, see the article by Monika Mayer in this volume. 
were auctioned off could have been restituted if their origin had been carefully examined. $^{61}$

\section{Transfer to the Free State of Bavaria}

By December 1956, the remaining 617 works by Jakob and Rudolf von Alt from "the estate of former Reichsleiter Martin Bormann" were formally transferred from the Trustee Administration for Cultural Property in Munich to the "safekeeping of the Bayerische Staatsgemäldesammlungen (Bavarian State Painting Collections)". ${ }^{62}$ In fact, as far back as September 1948, the artworks in the safekeeping of the CCP had first been transferred to the Bavarian Minister President and were to be kept by the state of Bavaria until such a time as a future central government of Germany could legally dispose of them as it wished. These artworks "in trusteeship" awakened covetous motives, as a memo in one of the files of the Ministry of Culture shows. In it, back in September 1948, State Commissioner Philipp Auerbach (1906-1952) had ordered the head of the Allied Monuments Fine Arts \& Archives Section to clarify the legal status of former NS assets, arguing that in view of the damage incurred by the so-called Degenerate Art Campaign, " $t$ ] he German museums have suffered harm in a similar way to those persecuted politically and racially on their level. Reparations to them should therefore be a primary concern of the government bodies responsible." ${ }^{63}$

Dr. Eberhard Hanfstaengl (1886-1973), General Director of the Bavarian State Painting Collections since 1945, was delegated the task of resolving this issue. He was appointed head of a Restitution Committee, which was screated for this purpose and, in 1951, assigned to the Federal German government as the Munich-based Treuhandverwaltung von Kulturgut (TvK). In February 1952, this body handed over a part of the artworks from the trusteeship of the Bavarian State to the German government for administration. These works included the collection of the "Führermuseum" in Austrian Linz, which was never accom-

61 Christie's, ed.: Mauerbach Benefit Sale. Items Seized by the Nazis to be Sold for the Benefit of the Victims of the Holocaust (Sale 5638). Wien: Christie's 1996, Lot 244-251 and 503-509. Cf. Sophie Lillie: "Herrenlos"? Die ungeklärte Akte Mauerbach. In: Recollecting, Raub und Restitution. Ed. by Alexandra Reininghaus. Wien: Passagen Verlag 2009, pp. 211-223; Otto Fritscher: Kontroversen um den "Mauerbach-Schatz". Die Restitutionsverfahren von 1969 bis 1986. Wien: New academic press 2012. See also the contribution of Leonhard Weidinger in this volume.

62 Protocols and transcripts of the several deeds of transfer are kept in numerous archives, e.g. BayHStA, MK 74106; StAM, BFD III, 237/11-12, 1241 and 1244; BArch Koblenz B323/354 and 355.

63 BayHStA, MK 51498, Übertragung von Kunstwerken (Restitution) u. Einziehung von Kunstwerken. Personal note by Dr. H. K. Röthel, 15.09.1948. 


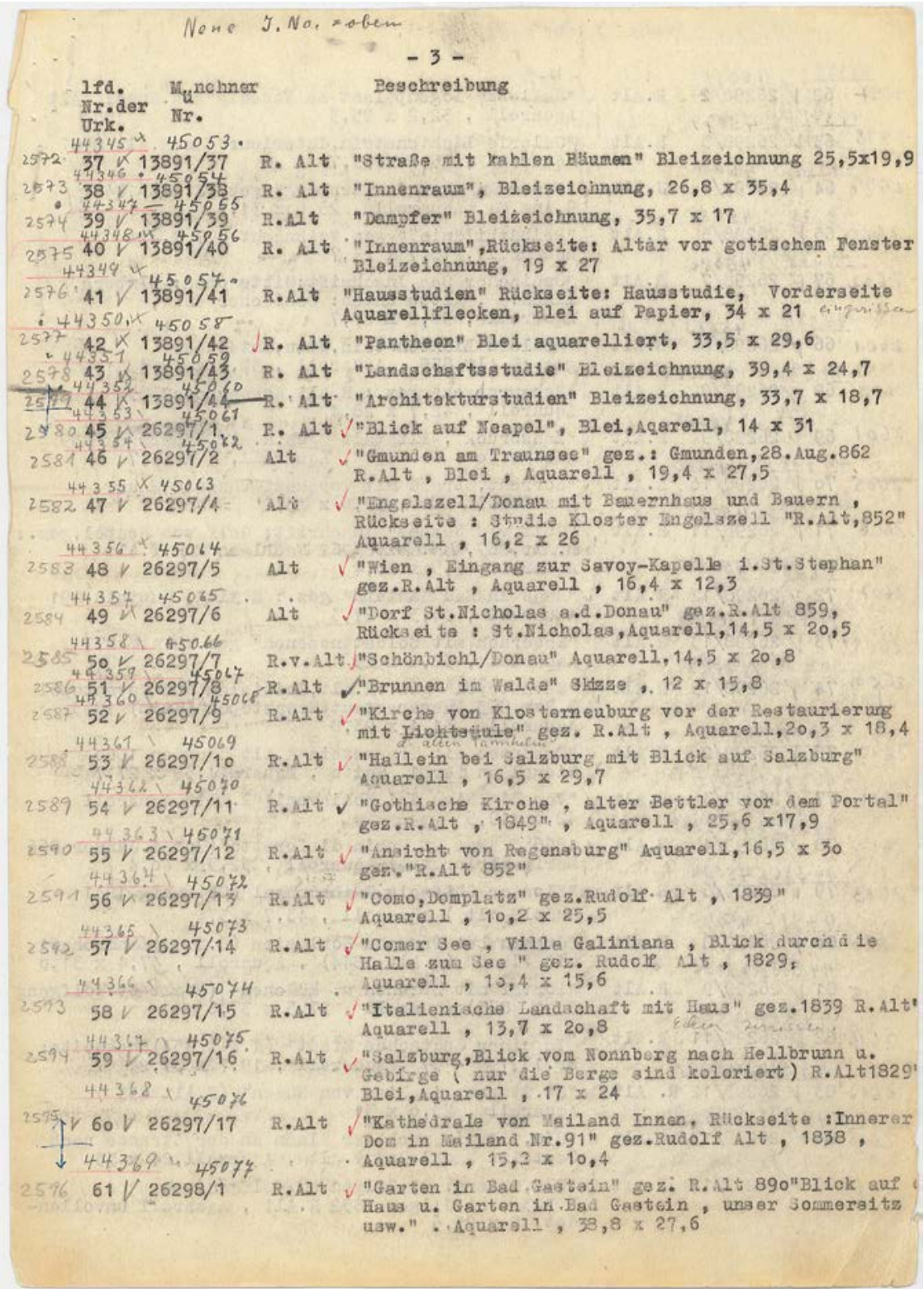

Abb. 4: Excerpt of the deed of transfer No. 2019/V dated April 20, 1953. Transcript, SGSM Altregistratur 
plished. However, the assets of the former NSDAP, its affiliates, organizations and many functionaries of the NS state were not transferred to Federal Germany's central government but passed into the ownership of the Bavarian State in accordance with directives 50 and 57 of the Control Council. ${ }^{64}$ These assets included the works of Rudolf von Alt from the "Bormann Collection". The 26-page protocol of April 20, 1953 and a further protocol of December 10, 1956 regulated the transfer to the SGSM. ${ }^{65}$

\section{Conclusion}

For the first time in 2011, the stock of works by Rudolf von Alt, which have not been exhibted in public since the 1950s, could be examined in depth. It is hard to avoid the suspicion that the origin of the almost 200 watercolours and over 400 drawings were kept hidden away as a guilty secret for a long time in Munich but also in Austria. The results of the research project illustrate how complex the history of the origin of the watercolours and drawings has been and just how difficult research in individual cases can be. Today we can turn Rudolf von Alt's statement around. Whereas in 1902 the artist had hardly any idea where his works had "ended up", today we hardly know where all of them came from. But precisely this demonstrates that it is our indispensable duty to not just scrutinize stocks like the one from the former "Bormann Collection", that were once handed over to public in trusteeship, but to document the - partly ambivalent outcomes and findings and to present them to the public.

64 On the transfers of the "former Nazi party assets" cf. et al. StAM, BFD III, 1241. In 2013, the Bayerische Staatsgemäldesammlungen established a research project on this issue, see the article by Johannes Gramlich in this volume.

65 Cf. et al. StAM, BFD III, 237/11-12, 1241 and 1244. 
Open-Access-Publikation im Sinne der CC-Lizenz BY-NC-ND 4.0

(c) 2018, V\&R unipress GmbH, Göttingen

ISBN Print: 9783847107835 - ISBN E-Lib: 9783737007832 


\title{
Stephan Kellner
}

\section{Abgabe der Alliierten: Die Bibliothek der NS-Ordensburg Sonthofen in der Bayerischen Staatsbibliothek}

\section{Zusammenfassung}

Die Bayerische Staatsbibliothek (BSB) sucht seit 2003 systematisch in ihren Beständen nach NS-Raubgut. Seit 2013 wird sie dabei vom Deutschen Zentrum Kulturgutverluste gefördert. In der Nachkriegszeit kamen Bestände verschiedener ehemaliger NS-Einrichtungen als Zuweisungen der Alliierten in das Haus; der größte war die Bibliothek der ehemaligen NS-Ordensburg Sonthofen mit 40.000 Bänden. Diese Einrichtung hatte als Ausbildungsstätte für künftige NS-Kader fungiert. Da die BSB erhebliche Kriegsverluste erlitten hatte, war dieser Zugang willkommen, um die Bestände wieder aufzubauen. Mit Bekanntwerden der Übernahme der Sonthofen-Bibliothek erhoben verschiedene Personen und Einrichtungen Ansprüche auf Teile der Bestände. Diese Forderungen wurden von der BSB geprüft, wo sie berechtigt waren, wurde ihnen rasch stattgegeben.

Schlagwörter

Bayerische Staatsbibliothek, Treuhand, NS-Ordensburg Sonthofen

\begin{abstract}
Allies' Transfer: The Library of the NS-Ordensburg Sonthofen in the Bavarian State Library

Since 2003, the Bavarian State Library (BSB) has been systematically searching for records of Nazi crimes. Since 2013, it has been sponsored by the German Center for Cultural Heritage. In the post-war period, stocks of various former Nazi institutions came into the house as assignments of the Allies. The largest was the library of the former NS-Ordensburg Sonthofen with 40,000 volumes. This institution had served as a training center for future NS cadres. Since the BSB had suffered considerable losses of war, this accession was welcome to rebuild the holdings. With the acquisition of the Sonthofen library, various persons and institutions claimed parts of the collection. These claims were examined by the BSB; where they were justified, they were quickly granted.
\end{abstract}

Keywords

Bavarian State Library, Trusteeship, NS-Ordensburg Sonthofen 
Seit 2003 sucht die Bayerische Staatsbibliothek systematisch in ihren Beständen nach NS-Raubgut. Bis zur Jahresmitte 2013 geschah dies ausschließlich in Eigeninitiative und mit der Unterstützung von Volunteers. Von Juni 2013 bis Mai 2016 konnte mit Förderung der Stiftung Deutsches Zentrum Kulturgutverluste (bis Anfang 2015 Arbeitsstelle für Provenienzforschung) das Projekt „NSRaubgutforschung an der Bayerischen Staatsbibliothek: Zugänge zwischen 1933 und 1945" durchgeführt und erfolgreich abgeschlossen werden. Der Bestandszuwachs in der NS-Zeit wurde dabei weitestgehend erschlossen, zahlreiche Provenienzen recherchiert und eine Reihe von Restitutionen durchgeführt. ${ }^{1}$

In der Schlussphase dieses Projekts wurden umfängliche Stichproben in jenen Beständen vorgenommen, die in der Nachkriegszeit die Bayerische Staatsbibliothek erreicht hatten. Sie konnten belegen, dass auch hier Raubgut vorhanden ist. Das aktuelle Projekt, wiederum gefördert durch das Zentrum, ermöglicht nun die systematische Sichtung dieser Zugänge. Es handelt sich dabei um kleinere Buchbestände ehemaliger nationalsozialistischer Einrichtungen wie dem Reichsinstitut für die Geschichte des neuen Deutschlands, dem Hauptarchiv der NSDAP oder der Zeitgeschichtlichen Sammlung Rehse; der Umfang beträgt ca. 1.500 Titel. Schwerpunkt ist jedoch ein ca. 36.000 Titel umfassender Fonds aus der Bibliothek der früheren NS-Ordensburg Sonthofen. Alle diese Buchbestände wurden nach Kriegsende zunächst von der amerikanischen Militärregierung beschlagnahmt, später dann der Staatsbibliothek übergeben.

\section{Ordensburgen als Teil des NS-Erziehungssystems}

Schon bald nach der nationalsozialistischen Machtergreifung 1933 entwickelten sich erste Pläne zur Errichtung eigener Stätten zur Schulung des Nachwuchses der NSDAP. ${ }^{2}$ Rasch begann man in Vogelsang (Eifel) und in Krössinsee (Pommern) mit dem Bau der Reichsschulungsburgen - die Bezeichnung Ordensburg etablierte sich erst etwas später. Ende 1934 nahm man auf einem Hochplateau oberhalb der Gemeinde Sonthofen im Allgäu ebenfalls die Errichtung einer

1 Vgl. dazu etwa: Stephan Kellner, Susanne Wanninger: NS-Raubgut auf der Spur. Provenienzforschung an der Bayerischen Staatsbibliothek. In: Bibliotheksmagazin 9 (2014), H. 2, S. 58-61.

2 Dazu allgemein Franz Albert Heinen: NS-Ordensburgen. Vogelsang, Sonthofen, Krössinsee. Berlin: Ch. Links Verlag 2011; Klaus Ring, Stefan Wunsch: Bestimmung: Herrenmensch. NSOrdensburg zwischen Faszination und Verbrechen. Dresden: Sandstein Verlag 2016. Zu Sonthofen Helmut Happel: N.S. Ordensburg Sonthofen. Nutzung und Bedeutung für Sonthofen - einst und jetzt. Immenstadt: Eberl Medien Verlag 2011; Gerhard Klein: Die NSOrdensburg Sonthofen 1933-1945. In: Weltanschauliche Erziehung in Ordensburgen des Nationalsozialismus. Zur Geschichte und Zukunft der Ordensburg Vogelsang. Hg. von Paul Ciupke, Franz-Josef Jelich. Essen: Klartext Verlag 2006, S. 65-84. 
Schulungsburg in Angriff. In mehreren Bauabschnitten entstanden dort weitläufige Gebäude in der Anmutung mittelalterlicher Burgen. Das sich erst allmählich herausbildende Schulungskonzept sah eine dreijährige Ausbildung von Ordensjunkern vor, die bei der Aufnahme etwa 25 Jahre alt sein und sich bereits in der Partei bewährt haben sollten. Für jede der Burgen war ein Aufenthalt von einem Jahr vorgesehen; in Krössinsee fand eine Art Grundausbildung statt, in Vogelsang sollte die nationalsozialistische Ideologie vertieft vermittelt werden, in Sonthofen die „Erziehung zu Mut und Entschlusskraft beim Berg- und Wintersport im Allgäu“ im Mittelpunkt stehen. Aufgabe der vierten Ordensburg Marienburg (Ostpreußen), die nicht über das Planungsstadium hinauskam, wäre die Vorbereitung auf Führungsaufgaben in Militär, Verwaltung und Diplomatie gewesen. ${ }^{3}$ Die Absolventen dieser Ausbildung sollten schließlich in eine Parteiuniversität eintreten, die „Hohe Schule der NSDAP“. Das riesige Gebäude dafür sollte am Nordostufer des Chiemsees erbaut werden - auch hier blieb es bei der Planung. ${ }^{4}$ Getragen wurde dieses Konzept von Robert Ley (1890-1945), dem Reichsorganisationsleiter der NSDAP und Leiter der Deutschen Arbeitsfront. Es stand in Konkurrenz zu den Nationalpolitischen Erziehungsanstalten, die zunächst dem Reichserziehungsministerium, später dann der SS unterstanden.

An den Ordensburgen gelang es de facto nicht, für die Ausbildung einen durchgängigen systematischen Lehrgang zu erstellen. ${ }^{5}$ Es gab Hauptfächer wie Rassenlehre und Geschichte, in denen Lehrer oder externe Dozenten Vorträge hielten, die von den Junkern vor- und nachzubereiten waren. „Kaleidoskopartig wurde Wissen vermittelt. “6

Ergänzt und unterfüttert wurde dieses Konzept durch die 1937 gegründeten Adolf-Hitler-Schulen. ${ }^{7}$ Dies waren Ausleseschulen, die als Vorbereitung für die Ordensburgen gedacht waren. Die Schüler traten nach einem mehrstufigen Ausleseverfahren mit zwölf Jahren ein und erhielten nach sechsjähriger Internatsschulzeit - sie wurde nach Kriegsbeginn auf fünf Jahre reduziert - ein dem Abitur gleichwertiges Diplom. Gestartet wurde 1937 reichsweit mit zehn Schulen. Da deren Bau jedoch nicht vorankam, wurden bald sämtliche Zöglinge der Adolf-Hitler-Schulen in Sonthofen untergebracht, das so zum Hauptstandort wurde und bis 1941 auf 1.450 Schüler anwuchs. Akute Platzprobleme führten

3 Vgl. Heinen: NS-Ordensburgen (Anm. 2), S. 80, Zitat ebd.

4 Heinen: NS-Ordensburgen (Anm. 2), S. $158 \mathrm{f}$.

5 Vgl. dazu Harald Scholtz: Die „NS-Ordensburgen“. In: Vierteljahrshefte für Zeitgeschichte 15 (1967), S. 269-298, hier S. 282.

6 Vgl. Scholtz: Die „NS-Ordensburgen“ (Anm. 5), S. 284.

7 Vgl. Stefan Wunsch: Führer von übermorgen? Die Adolf-Hitler-Schulen und das Erziehungsprojekt ,Herrenmensch'. In: Ring, Wunsch: Bestimmung: Herrenmensch (Anm. 2), S. $210-228$. 


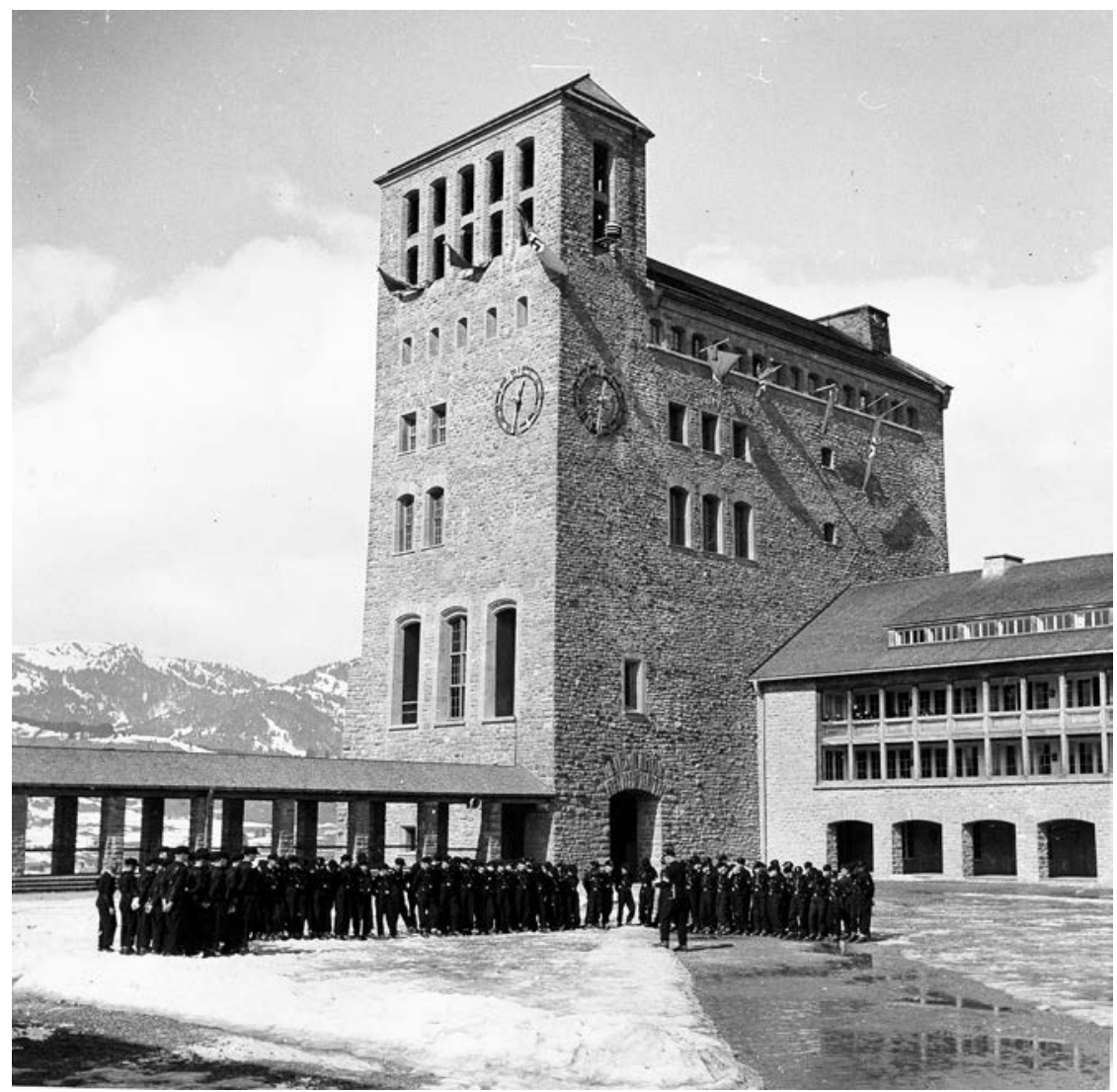

Abb. 1: Heinrich Hoffmann: Ordensburg Sonthofen, 23.03.1942. Abschlussprüfung der AdolfHitler-Schule

schließlich 1942 dazu, dass die Schüler auf sechs weitere provisorische Standorte verteilt wurden. Erziehungsziel war die Heranbildung von

„NS-Führerpersönlichkeiten [...], die über eine fundierte weltanschauliche, schulische sowie musische Bildung verfügen sollten; sportlich und vormilitärisch gedrillt, selbstgewiss, verantwortungsbewusst und willensstark, im engen Rahmen des Nationalsozialismus eigenständig denkend und zugleich gehorsam [...]“. ${ }^{8}$

Der Unterricht fand in Uniform statt, er bestand aus den am Gymnasium üblichen Fächern, ergänzt um „Blick in die Welt“, bei dem auch ausländische Zeitungen rezipiert wurden, Waffenkunde und den sehr wichtigen Sport.

8 Zitat nach Ring, Wunsch: Bestimmung: Herrenmensch (Anm. 2), S. 213. Zum Schulalltag in Sonthofen vgl. Happel: N.S. Ordensburg Sonthofen (Anm. 2), S. 55-80. 
Sonthofen hatte also mehrere Funktionen. ${ }^{9}$ Zwar spielte dort die eigentliche Ausbildung der Junker im Gegensatz zu den anderen beiden Ordensburgen nur eine geringe Rolle, für diese Klientel war Sonthofen vor allem als Standort und Ausgangspunkt für sportliche Aktivitäten interessant. Doch im Gefüge der Adolf-Hitler-Schulen nahm Sonthofen eine zentrale Stellung ein. Zudem fand dort ein umfangreicher Tagungs- und Lehrgangsbetrieb statt. Schließlich wurde in Sonthofen 1937 ein Erzieherseminar eingerichtet, das bis 1945 dort verblieb. Mit der eigenständigen Ausbildung von Lehrern wollten sich die Adolf-HitlerSchulen von staatlichen Seminaren abkoppeln und ihre Pädagogen nach eigenen Vorstellungen formen.

Die Errichtung der Ordensburgen wurde begleitet vom Aufbau umfangreicher Büchersammlungen. Ihnen kam im nationalsozialistischen Bibliothekswesen eine hohe Bedeutung zu; für Dr. Joachim Petzold (geb. 1910), den Leiter der Hauptstelle Büchereiwesen im Hauptschulungsamt der NSDAP, bildeten sie „ihrer Anlage und ihrer Bestimmung nach die wesentlichsten Büchereien der NSDAP“. ${ }^{10}$ Dementsprechend sollten sie in eigenen Bauten untergebracht werden, die „das Modernste darstellen werden, was es auf diesem Gebiet gibt, [...]. Sie bewegen sich in Größenordnungen, die für unsere größten deutschen Bibliotheken zutreffen [...]. “11 Geplant war ein ausdifferenziertes System: Neben einer Hauptbücherei mit Lesesaal sollten wissenschaftliche Institutsbibliotheken eingerichtet werden, deren Bestand sich an den Arbeitsschwerpunkten der jeweiligen Ordensburg orientierte. Hinzu kamen als Handbibliotheken gedachte kleinere Kameradschaftsbüchereien mit der wesentlichen Literatur zum Lehrplan. Darüber hinaus waren Volks- bzw. Werksbüchereien mit Unterhaltungsliteratur für die Angestellten der Ordensburgen vorgesehen. Insgesamt dachte man auch in diesem Bereich in den megalomanen Maßstäben des Nationalsozialismus:

„Daß neben allen diesen Einrichtungen auch Zeitschriften- und Musikalienabteilungen bzw. -säle, Bildstellen, Photo- und Kartenabteilungen, Burgarchive, Buchbindereien mit Spritzkammern [Anm. d. Verf.: für Dekorelemente?] und allem sonstigen Material vorhanden sind, soll nur erwähnt werden. ${ }^{\text {“12 }}$

9 Vgl. Klein: Ordensburg Sonthofen (Anm. 2), S. 67, 78, 82 f.

10 Joachim Petzold: Das Büchereiwesen der NSDAP, unter besonderer Berücksichtigung der wissenschaftlichen und Spezialbibliotheken. In: Zentralblatt für Bibliothekswesen 55 (1928), S. 524-533, hier S. 525.

11 Ebd., S. 526.

12 Ebd., S. 527. 


\section{Bibliotheken in der Ordensburg Sonthofen}

Sonthofen zeigt, dass diese Pläne durchaus umgesetzt wurden; mit großem Aufwand und Energie baute man dort rasch mehrere Büchersammlungen unterschiedlicher Größe und Ausrichtung auf. So verfügte die Erzieher-Akademie über eine eigene Bibliothek, die auch wissenschaftlichen Ansprüchen genügte. Daneben gab es noch eine kleinere Sammlung für die Ordensjunker und eine Bibliothek für die Adolf-Hitler-Schulen. Schließlich wird im Mai 1941 eine Werksbücherei von 850 Bänden erwähnt, die für die zahlreichen Angestellten der Burg zur Verfügung stand. Die dort vorhandene Unterhaltungsliteratur wurde offensichtlich lebhaft nachgefragt. ${ }^{13}$ Zur Betreuung der Bibliotheken scheint beträchtliches Personal vorhanden gewesen zu sein, 1943 werden für die AdolfHitler-Schulen 24 Bibliotheksmitarbeiter genannt; sie kümmerten sich auch um eine großangelegte Presseausschnittsammlung. ${ }^{14}$

Dass die Bibliothek auch einen Gegenpol zum militärisch durchgetakteten Drill des Schulalltags bilden konnte, zeigen die Erinnerungen des Filmschauspielers Hardy Krüger, der von 1941 bis 1944 Schüler in Sonthofen war:

„Alles geschah, abgesehen von den Unterrichtsstunden, im Kommandoton und Gleichschritt. Das hat mich schon damals gestört. Aber dazwischen gab es immer wieder schöne Momente. Etwa wenn mir der feinsinnige Bibliothekar die Augen für die klassische deutsche Literatur öffnete, mir manchmal sogar aus dem Giftschrank ein verbotenes Buch zusteckte. Da verging die Zeit wie im Fluge, und ich hatte Mühe, um neun Uhr abends den Appell zum Zapfenstreich rechtzeitig zu erreichen. ${ }^{\text {“15 }}$

Ein Aufenthalt in der Bibliothek brachte sogar mit sich, dass sich für Krüger die Welt des Films öffnete und er seine Talente entfalten konnte:

„Aber auch meinen späteren Beruf habe ich durch die Adolf-Hitler-Schule kennengelernt. Durch Sonthofen bin ich Filmschauspieler geworden. Wie ich schon sagte, war eines Tages der später berühmte Alfred Weidenmann auf mich aufmerksam geworden. Er überraschte mich in der Bibliothek und fragte mich, ob ich zum Film wolle. Ich meinte, ich wolle lieber Bücher schreiben. Das wiederum fand Weidenmann erstaunlich. Am nächsten Tag bekam ich einen Marschbefehl und eine Fahrkarte und fuhr mit gemischten Gefühlen nach Berlin-Babelsberg zur Ufa. ${ }^{\text {"16 }}$

13 Hildegund Humbert: Aus der Bibliothek. In: Der Burgbrief 2 (1941), H. 3, S. 20. Dort wird auch von einem nicht näher definierten Gesamtbestand von 10.000 Bänden gesprochen.

14 Nach Klein: Ordensburg Sonthofen (Anm. 2), S. 83.

15 Hardy Krüger: Von der Ordensburg nach Babelsberg. In: „Wir waren Hitlers Eliteschüler.“ Ehemalige Zöglinge der NS-Ausleseschulen brechen ihr Schweigen. Hg. von Johannes Leeb. 2. Aufl. Hamburg: Rasch und Röhring 1998, S. 49-56, hier S. 53.

16 Ebd., S. 53. 
Über die Größe der Bibliotheken in Sonthofen gibt es unterschiedliche Angaben. ${ }^{17}$ Ein gutes Bild von den Dimensionen gibt uns ein Schreiben der Diplombibliothekarin Hildegund Humbert (geb. 1909), ${ }^{18}$ die von 1939 bis zum Kriegsende dort tätig gewesen war und von daher über die Verhältnisse sehr gut Bescheid wusste. Humbert hatte sich 1951 an Gustav Hofmann (1909-1982), den Generaldirektor der Bayerischen Staatsbibliothek gewandt, nachdem sie aus der Presse erfahren hatte, dass das Haus einen Teilbestand aus Sonthofen übernommen hatte.

Zum Zeitpunkt ihrer Inhaftierung durch das französische Militär im Juni 1945

„bestanden noch

1. vollzählig die Bibliothek der Erzieher-Akademie der Adolf-Hitler-Schulen mit ca. 100.000 Bänden

2. nicht mehr ganz vollständig die Bibliothek der Ordensburg So.[nthofen] mit ca. 36.000 Bänden (die national-sozialistischen Bücher und Zeitschriften sowie eine Gewerkschaftsbücherei, die 1.200 Bände umfaßte, waren vorher aussortiert worden)

3. Restbestände von 3 Büchereien der Adolf-Hitler-Schulen, die vorher wohl über 20.000 Bände umfaßt hatten

4. Unterrichtsbücher der Adolf-Hitler-Schulen in zahlreichen Exemplaren (Zahl war mir nicht bekannt)

17 Vgl. Klein: Ordensburg Sonthofen (Anm. 2), S. 83, insbesondere Anm. 65.

18 Die Biographie von Hildegund Humbert war nur in Teilen zu ermitteln. Sie wurde am 11. Mai 1909 in Berlin geboren, s. Deutscher Büchereiverband in Zusammenarbeit mit dem Verein der Bibliothekare an öffentlichen Büchereien: Handbuch der öffentlichen Büchereien 1955. Berlin: Deutscher Büchereiverband 1955, S. 185. Sie war die Enkelin des deutsch-französischen Romanisten und Molière-Forschers Claas Hugo Humbert (1830-1904). Nach ihrer Ausbildung wechselte sie zum 01.05.1939 von der Bibliothek der Technischen Hochschule Stuttgart an die Bibliothek der Ordensburg Sonthofen, vgl. die Kurzmeldung in: Die Bücherei. Zeitschrift der Reichsstelle für das Büchereiwesen 6 (1939), S. 326. Über ihren Lebensweg nach 1945 ist, abgesehen von den hier aufgeführten Briefen an die Bayerische Staatsbibliothek sowie zwei im Recherchesystem Kalliope nachgewiesenen Briefen des Bibliothekars Erwin Ackerknecht (1880-1960) an Humbert aus den Jahren 1946 bzw. 1947 wenig bekannt, s. Deutsches Literaturarchiv Marbach / Handschriftenabteilung, Nachlaß A: Ackerknecht, Akquisitionsnummern 60.1853/1 und 60.1853/2. 1955 war Humbert an der Universitätsbibliothek Münster tätig, vgl. Handbuch der öffentlichen Büchereien 1955, S. 185. Im Jahre 1999 wurde Hildegund Humbert, zu dieser Zeit wohnhaft in Osnabrück, gemeinsam mit ihrer Schwester Waltraud Helmert (geb. Humbert) noch als Verwahrerin eines Teilnachlasses ihres Großvaters Claas Hugo Humbert genannt, vgl. Wolbert G.C. Smidt: „La France et l'Allemagne sont faites pour s'aimer". Ein unbekannter Brief Victor Hugos aus dem Jahre 1878. In: Francia 25 (1998), H. 3, S. 163-166, hier S. 163, Anm. 2. Allerdings wird sie bei einer ausführlichen Auflistung der Teilnachlaßbesitzer Claas Hugo Humberts von 1997 desselben Autors nicht aufgeführt, siehe Wolbert G. C. Smidt: Claas Hugo Humbert. In: Biographisches Lexikon für Ostfriesland, Band 2. Hg. von Martin Tielke. Aurich: Ostfriesische Landschaft 1997, S. 175177. Das Sterbejahr ist deswegen nicht genau festzulegen. 
5. Reste von privaten Bibliotheken der Erzieher, Stammführer und dort wohnenden Evakuierten. “19

Humbert kommt zu dem Schluss: „Da nach der Zeitschrift ,Heute‘ etwa 250.000 Bände nach München gekommen sein sollen, müßte tatsächlich der gesamte

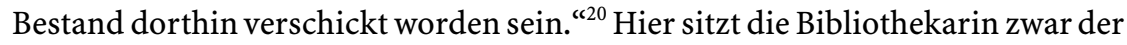
falschen Zahlenangabe in der Publikation auf, doch sie bestätigt damit, dass der Umfang der Sonthofener Sammlungen tatsächlich eine Viertelmillion Bände betragen haben muss. Die Hintergründe von Humberts Schreiben bleiben dabei unklar. Sie selbst nennt als ihre Motivation die Fürsorge für den früher von ihr betreuten Bestand.

\section{Bücher aus Sonthofen in der Bayerischen Staatsbibliothek}

Die Staatsbibliothek hatte bereits Ende März 1946 zahlreiche Werke aus Sonthofen erhalten:

„Die Bücher aus der Ordensburg Sonthofen (160 Kisten) wurden in der vergangenen Woche durch eine Mannschaft der Bayerischen Staatsbibliothek in deren Gebäude gebracht. Die Auspackung der Kisten wird morgen (Freitag) beendet; die ca. 25.000 Bände sind dann schon morgen [...] aufgestellt und werden dort auf Wunsch von Professor Stürmann (Militärregierung) nach Besitzervermerken geordnet, was geraume Zeit erfordern wird. “21

Offensichtlich bestand ein Bewusstsein dafür, dass diese Bücher auch Raubgut enthalten könnten. Der Bibliothek war also direkt aus Sonthofen eine Teilmenge zur Verwahrung übergeben worden, doch sollte es noch bis ins Jahr 1948 hinein dauern, bis sie darüber verfügen durfte.

Sie hatte großes Interesse an diesem Bestand, denn sie hatte bei insgesamt vier Luftangriffen während des Zweiten Weltkriegs insgesamt etwa eine halbe Million Bücher verloren, 20 Prozent ihres Gesamtbestandes. Außerdem war das Gebäude zu sechs Siebtel zerstört worden. ${ }^{22}$ Am schwersten wog der Verlust von

19 Bayerisches Hauptstaatsarchiv (BayHStA), Bestand Generaldirektion der Bayerischen Staatlichen Bibliotheken 1649 (Generaldirektion Bayer. Staatl. Bibliotheken 1649), Hildegund Humbert an Gustav Hofmann, 10.04.1951.

20 BayHStA, Generaldirektion Bayer. Staatl. Bibliotheken 1649, Hildegund Humbert an Gustav Hofmann, 10.04.1951.

21 BayHStA, Generaldirektion Bayer. Staatl. Bibliotheken 1649, Franz Wenninger an den Bayerischen Staatsminister für Unterricht und Kultus, 11.04.1946. Das Schreiben ist irrtümlich auf den 01.04. datiert. Bei Prof. Stürmann handelt es sich wahrscheinlich um Prof. Douwe Stuurman (1910-1991), der als Teil der Library of Congress Mission to Europe unterwegs war; vgl. Anm. 38.

22 Vgl. dazu Hans Halm: Die Schicksale der Bayerischen Staatsbibliothek während des Zweiten 
mehr als 400.000 Bänden beim ersten Luftangriff vom 9. auf den 10. März 1943, danach waren die Bestände ausgelagert worden.

Nachdem die Übernahme bekannt geworden war, meldeten sich auch andere Einrichtungen, die selbst Bedarf an Literatur hatten; so übermittelte man 1948 leihweise 112 Bände an die Bibliothèque française und der Bayerischen Sportakademie acht Bände, drei Jahre später überließ die Staatsbibliothek der benachbarten Universitätsbibliothek München 138 Bände.

Daneben traten verschiedene Interessenten mit vermeintlichen oder tatsächlichen Forderungen auf. Hartnäckig versuchte etwa 1948/49 Eberhard Lietzau, ${ }^{23}$ ein ehemaliger Buchhändler, der Sonthofen beliefert hatte, nicht bezahlte Forderungen in Höhe von etwa 30.000 Reichsmark bei der Staatsbibliothek geltend zu machen, was diese unter anderem mit dem Hinweis ablehnte, „daß ein großer Teil von der [Sonthofener] Bibliothek überhaupt nicht in die Bayerische Staatsbibliothek gelangt ist “. ${ }^{24}$

Mit dem Althistoriker Dr. Heinz-Eberhard Giesecke (1913-1991) ${ }^{25}$ meldete sich im August 1948 ein ehemaliger Dozent der Erzieher-Akademie in Sonthofen, der 1943 seine private Büchersammlung „in drei Kisten in einem Abstellraum der Bibliothek des Erzieherseminars der Adolf-Hitler-Schulen “26 deponiert hatte. Die Staatsbibliothek ermittelte 45 Werke mit seinem Exlibris und suchte auch noch gezielt nach mehreren Titeln, die Giesecke genannt hatte. Weitergehenden Wünschen verweigerte sie sich jedoch, stellte schließlich die Bücher mit einer zweimonatigen Frist zur Abholung bereit, eine Zusendung wurde abge-

Weltkriegs. In: Beiträge zur Geschichte der Bayerischen Staatsbibliothek. Hg. von Rupert Hacker. München: K.G. Saur 2000 (= Bayerische Staatsbibliothek Schriftenreihe 1), S. 309-314. Dazu jetzt auch Susanne Wanninger: „Herr Hitler, ich erkläre meine Bereitwilligkeit zur Mitarbeit.“ Rudolf Buttmann (1885-1947). Politiker und Bibliothekar zwischen bürgerlicher Tradition und Nationalsozialismus. Wiesbaden: Harrassowitz Verlag 2014 (= Beiträge zum Buch- und Bibliothekswesen 59), S. 462-470. Siehe auch der Bericht von Hans Halm: Die Katastrophe. In: „Darf ich Ihnen meinen Wunschzettel mitteilen?“. Die Bayerische Staatsbibliothek in der Literatur. Hg. von Waldemar Fromm und Stephan Kellner. München: Allitera-Verlag 2014 (= Bayerische Staatsbibliothek Schriftenreihe 5), S. 90-93.

23 Die Lebensdaten von Eberhard Lietzau waren leider nicht zu ermitteln.

24 BayHStA, Generaldirektion Bayer. Staatl. Bibliotheken 1649, Bayerische Staatsbibliothek an Ernst Wulf, 15.01.1949.

25 Giesecke wurde am 27.12.1913 in Dresden geboren, siehe die Angabe in Verkehrsblatt 14 (1960), Amtl. Teil, Heft 3, S.60q. Seine deutlich im nationalsozialistischen Sinne verfasste Dissertation bei Wilhelm Weber (1882-1948), Professor an der Friedrich-Wilhelms-Universität Berlin (heute Humboldt-Universität) veröffentlichte er 1939 bereits als Dozent auf der Ordensburg Sonthofen, siehe Heinz-Eberhard Giesecke: Die Ostgermanen und der Arianismus. Leipzig und Berlin: B.G. Teubner-Verlag 1939, insbesondere S. V. Nach dem Zweiten Weltkrieg betrieb Giesecke eine Fleischwarengroßhandlung in Celle. Erst ab 1984 veröffentlichte er wieder regelmäßig Beiträge im Bereich der Alten Geschichte. Sein genaues Sterbedatum konnte nicht ermittelt werden.

26 BayHStA, Generaldirektion Bayer. Staatl. Bibliotheken 1649, Heinz-Eberhard Giesecke an die Bayerische Staatsbibliothek, 28.08.1949. 
lehnt. Zudem musste Giesecke eine eidesstattliche Erklärung vorlegen, dass die Bücher tatsächlich noch sein Besitz waren. Giesecke hatte in der SS eine höhere Position bekleidet, was in der Bibliothek bekannt war. ${ }^{27}$

Der Verdacht auf Raubgut bestätigte sich bei der Durchsicht. Rasch reagierte man auf einen Rückerstattungsanspruch des Österreichischen Gewerkschaftsbundes von 1949 und sonderte einen Bestand von etwa 1.000 Bänden aus, die aus österreichischen Gewerkschaftsbibliotheken stammten. Nach einem Besuch eines Vertreters der Wiener Arbeiterkammer wurden schließlich Mitte Oktober 1950 zehn Kisten der Spedition Wetsch übergeben; Empfänger war die Arbeiterkammer Graz.

Auch verschiedene Freimaurerlogen kamen auf die Staatsbibliothek zu, so etwa der Vertreter der bayerischen „Großloge zur Sonne“. Zwar gingen die Freimaurer von etwa 26.000 Bänden aus Freimaurerbibliotheken im Sonthofener Bestand aus, was viel $\mathrm{zu}$ hoch gegriffen war, doch einigte man sich auf eine tranchenweise Rückgabe von Beständen, wie sie bei der laufenden Katalogisierung und Einarbeitung des Bestandes anfielen. Als Anlaufpunkt kristallisierte sich die Bibliothek des Freimaurermuseums in Bayreuth heraus, dorthin wurden etwa im Juni 195222 Titel übergeben. ${ }^{28}$

Noch während die aus Sonthofen stammenden Bücher sukzessive in den Bestand der Staatsbibliothek integriert und die Dubletten weitergegeben wurden, begann ein neues Kapitel.

\section{Einmal Washington und zurück - die Rolle der Library of Congress}

„Auch ist die Bibliothek der Ordensburg Sonthofen erst nach wiederholten Plünderungen nach Kriegsende, nach Beschlagnahmung eines Teiles der Bibliothek durch die Amerikaner und unter Ausscheidung eines großen Bestandes pädagogisch wichtiger Literatur in die Bayerische Staatsbibliothek gelangt. Diese hat also nur einen Teilbestand erhalten, der auch in sich lückenhaft ist, wofür die Vielzahl unvollständiger mehrbändiger Werke Zeugnis ablegt. “29

27 Details zu seiner Laufbahn in der SS sowie zur wahrscheinlich erfolgten Entnazifizierung Gieseckes konnten bislang nicht ermittelt werden.

28 BayHStA, Generaldirektion Bayer. Staatl. Bibliotheken 1649, Bernhard Beyer von der Freimaurerloge Quatuor Coronati an die Bayerische Staatsbibliothek, 07.06.1952.

29 BayHStA, Generaldirektion Bayer. Staatl. Bibliotheken 1649, Bayerische Staatsbibliothek an das Staatsministerium für Unterricht und Kultus, 04.01.1950. Unklar bleibt, was mit „pädagogisch wichtiger Literatur" exakt gemeint ist, wahrscheinlich hat man sich darunter Werke mit eindeutig nationalsozialistischer Ausrichtung vorzustellen, wie sie nach dem Befehl Nr. 4 des Alliierten Kontrollrats vom 13. Mai 1946 aus den öffentlichen Bibliotheken zu entfernen waren, siehe auch Anmerkung 33. 
Tatsächlich gingen die Bestände von Sonthofen nach dem Krieg weite Wege. Eine Spur führt nach Frankreich, in die Bibliothèque de documentation internationale contemporaine (BDIC) in Nanterre bei Paris. Diese 1918 gegründete Einrichtung, zu der auch ein Archiv und ein Museum gehören, ist die einzige Sammlung in Frankreich, die umfassend Bestände zur europäischen Zeitgeschichte besitzt. Sie hatte bei Kriegsende starke Verluste erlitten. Um diese zu kompensieren, übergaben die Alliierten größere Bestände von Drucken an die BDIC. Dieses Material, insgesamt handelte es sich dabei um etwa 10.000 Bände, stammte zum Großteil aus verschiedenen nationalsozialistischen Einrichtungen. Darunter befanden sich auch gut 2.000 Bände aus der Ordensburg Sonthofen, verschiedenen Adolf-Hitler-Schulen und der Deutschen Arbeitsfront, neben NS-Literatur auch Sachbücher, Belletristik und Reiseliteratur. Mit eingeschlossen waren auch etwa 1.800 Bände aus Büchereien der Arbeiterbewegung, die nach der Aufhebung der Gewerkschaften und der SPD dort geplündert worden waren und anschließend nach Sonthofen gekommen waren. Erst zu Beginn der 1980er Jahre konnte die BDIC dieses Material katalogisieren und beschäftigte sich dabei auch mit den Provenienzen. ${ }^{30}$ Es ist anzunehmen, dass es sich hier nicht um einen Einzelfall gehandelt hat, die Alliierten dürften auch anderen Einrichtungen Bücher aus Sonthofen zur Verfügung gestellt haben.

Schon in der Endphase des Zweiten Weltkriegs setzte ein großer Wettlauf um die Aktenbestände des Dritten Reichs ein. Zahlreiche, auch konkurrierende Dienststellen der Alliierten suchten nach ausgelagerten Beständen, etwa des Auswärtigen Amtes. ${ }^{31}$ Doch auch an Druckschriften, die während der Naziherrschaft publiziert worden waren, bestand ein lebhaftes Interesse. Unmittelbar nach Kriegsende begann die Library of Congress Mission to Europe. ${ }^{32}$ Sie verfolgte mehrere Zwecke: Im Verbund mit 113 US-Forschungsbibliotheken sollte sie die durch die Kriegsjahre entstandenen Lücken in den Beständen durch Ankäufe in Deutschland und Europa schließen. Gleichzeitig galt es, die abgebrochenen Beziehungen zu Verlagen und Buchhändlern wieder aufzunehmen, um künftige Lieferungen zu gewährleisten. Außerdem oblag ihr, die Bereinigung der Bestände öffentlicher Bibliotheken von NS-Literatur zu überwachen, wie sie im Befehl Nr. 4 des Alliierten Kontrollrats vom 13. Mai 1946 festgelegt worden

30 Michel Leiberich: Les séquestres. In: Lettre de l'association des Amis de la BDIC et du Musée 2 (1982), H. 3/4, S. 4-6. Für Hinweise und Material danke ich herzlich Dominique Bouchery (BDIC), der zu diesem Thema einen Vortrag veröffentlichen wird.

31 Aufgearbeitet sind diese Prozesse bei Astrid M. Eckert: Kampf um die Akten. Die Westalliierten und die Rückgabe von deutschem Archivgut nach dem Zweiten Weltkrieg. Stuttgart: Franz Steiner Verlag 2004 (= Transatlantische historische Studien 20).

32 Dazu Eckert: Kampf um die Akten (Anm. 31), S. 209 f. 
war. ${ }^{33}$ Nicht zuletzt machte man es sich in großer Voraussicht zur Aufgabe, umfassend Materialien der nationalsozialistischen Herrschaft zu sammeln:

„We are convinced that the Nazi regime and ideology will be examined repeatedly by historians as one of the great phenomena of our time, and the psychological elements are so prominent that for those studies every possible resource and ample detail are certain to be required. ${ }^{\text {(34 }}$

Doch die Library of Congress Mission entwickelte sich auch allgemein zum Ansprechpartner in Bibliotheksfragen: „Die Armee sah in der Bibliotheksdelegation bald eine geeignete Stelle, überschüssiges Material abzuwälzen, ein Eindruck, den die Kongreßbibliothek gerne beförderte. ${ }^{\text {“35 }}$ Der engagierte Forscher und Publizist Kenneth D. Alford formuliert es prägnanter: „In other words, the Library of Congress was to grab whatever it could get its hands on and ship it to

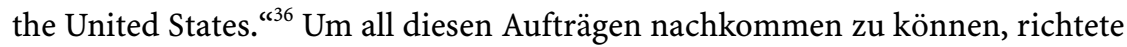
die Library of Congress Mission Büros in Berlin, Frankfurt, München und Wiesbaden ein; insgesamt waren etwa 26 Bibliothekare beschäftigt. Möglicherweise ist der Abtransport von 1.200 Kisten mit Büchern aus Sonthofen und ihre Verschiffung nach Washington im Sommer 1946 auch auf die Tätigkeit der Mission zurückzuführen. ${ }^{37}$ Insgesamt jedoch war das weit ausgreifende Vorgehen der Bibliothekare nicht unumstritten und hatte rasch zu Kritik aus amerikanischen Kollegenkreisen geführt. ${ }^{38}$

Das Unternehmen verlief durchaus erfolgreich: Es konnten 141 nationalsozialistische Sammlungen für die Library of Congress übernommen werden,

33 Der entsprechende Befehl ist jetzt abgedruckt bei Stephan Kellner: Nationalsozialistisches Schrifttum in der Bayerischen Staatsbibliothek nach 1945 und heute. Das Pflichtexemplar als Politikum. In: Zeitschrift für Bibliothekswesen und Bibliographie 64 (2017), S. 158-165, hier S. 160.

34 Paul Vanderbilt: Prints and Photographs of Nazi Origin. In: Quarterly Journal of Current Acquisitions 6 (1949), H. 4, S. 21-27, hier S. 27.

35 Vgl. Eckert: Kampf um die Akten (Anm. 31), S. 210.

36 Kenneth Alford: Nazi plunder. Great treasure stories of World War II. [New York]: Da Capo Press 2003, S. 207.

37 Dies berichtet zumindest die ehemalige Bibliothekarin der Ordensburg. Weiter konnte sie über einen an der Aktion Beteiligten in Erfahrung bringen: „Es sollen Bücher von 4 verschiedenen Universitätsbibliotheken darunter gewesen sein, außerdem eine wertvolle Privatbibliothek eines Professors mit Originalhandschriften Friedrichs des Großen. “ BayHStA, Generaldirektion Bayer. Staatl. Bibliotheken 1649, Hildegund Humbert an Gustav Hofmann, 10.04.1951.

38 Nach Eckert: Kampf um die Akten (Anm. 31), S. 217. Die Library of Congress versuchte, ihr Vorgehen zu rechtfertigen; vgl. etwa Reuben Peiss: European Wartime acquistions and the Library of Congress Mission. In: Library Journal 71 (1946), H. 12, S. 863-876, 911. Hinzu kommen Darstellungen wie Vanderbilt: Prints and Photographs (Anm. 34) sowie Douwe Stuurman: The Nazi Collection: A Preliminary Note. In: Quarterly Journal of Current Acquisitions 6 (1948), H. 1, S. 21-22, die sich freilich nur auf die nationalsozialistischen Kollektionen beziehen. 
die an den unterschiedlichsten Orten aufgefunden worden waren. Sie bestanden aus Büchern, Zeitungen und Zeitschriften, Fotos, Karten, Plakaten und Flugblättern. Insgesamt handelte es sich um etwa anderthalb Millionen $\mathrm{Ob}$ jekte, für deren Verschiffung man mehr als 7.500 Kisten benötigte. ${ }^{39}$ Unter ihnen befand sich etwa Hitlers private Bibliothek, Fotosammlungen von Nazigrößen wie Hermann Göring mit 18.500 Aufnahmen in 47 Bänden oder des Münchner Gauleiters Adolf Wagner mit 7.000 Aufnahmen in 23 Bänden, Archive der NSDAP, die umfangreiche Zeitgeschichtliche Sammlung Rehse zur Geschichte der NSDAP oder auch eine Fotodokumentation des italienischen Feldzugs in Äthiopien 1935/36. ${ }^{40}$

Darüber hinaus hatte die Library of Congress 819.022 Bände an Literatur sowie über eine Million Zeitschriften gesammelt, bei denen es sich nicht um nationalsozialistisches Schriftgut handelte. ${ }^{41}$ Möglicherweise fielen hierunter auch jene Sonthofener Bestände, die von den US-Alliierten 1946 beschlagnahmt worden waren. Prinzipiell achtete man bei diesen Übernahmen darauf, dass kein Raubgut dabei war und vermittelte entsprechende Funde an die zuständigen Stellen, etwa die Central Collecting Points. Trotzdem kam es zu Fehlentscheidungen, die man jedoch rasch korrigierte: Bereits 1948/49 etwa wurde die Weltkriegsbücherei Stuttgart oder Material, das aus Gewerkschaftsbibliotheken geplündert und dann NS-Bibliotheken einverleibt worden war, wieder an die Eigentümer oder Rechtsnachfolger zurückgegeben. ${ }^{42}$

Schon bald nach der Gründung der Bundesrepublik Deutschland kam es durch den Bundestag und die Bundesregierung, aber auch von Seiten der Fachwissenschaft zu ersten Rückgabeforderungen des beschlagnahmten und abtransportierten Aktenmaterials an die Amerikaner und Briten. Während dieser Punkt für die deutsche Seite „zu einer Frage des nationalen Prestiges“ ${ }^{\text {“43 }}$ wurde, nahm sie auf der Seite der Alliierten eine deutlich niedrigere Priorität ein. Erste Aktenbestände wurden bereits 1950 zurückgegeben, doch waren mehrfache lange Verhandlungsrunden nötig, bis die diplomatischen und Militärakten bis Ende der 1960er Jahre wieder überwiegend zurückgekehrt und der Obhut von Behörden wie dem Bundesarchiv übergeben worden waren. ${ }^{44}$

Die Rückkehr von Bibliotheksbeständen aus der Library of Congress ist wohl im Zusammenhang mit den Bemühungen zu sehen, ähnlich wie beim Archivgut

39 Nach Alford: Nazi plunder (Anm. 36), S. 207 ff. Wesentliche Teile der gesammelten Objekte befinden sich bis heute im Besitz der Library of Congress.

40 Vgl. Stuurman: Nazi Collection (Anm. 38) und Vanderbilt: Prints and Photographs (Anm. 34).

41 Nach Alford: Nazi plunder (Anm. 36), S. 207.

42 Vgl. Eckert: Kampf um die Akten (Anm. 31), S. 210.

43 Ebd., S. 457.

44 Ausführlich dargestellt bei Eckert: Kampf um die Akten (Anm. 31), S. $260 \mathrm{ff}$. 
deutsche Bestände wieder zurückzugeben. ${ }^{45}$ Anfang 1963 boten die USA die Rückführung von Bibliotheksbeständen an. Insgesamt scheint es sich um bei den infrage kommenden Beständen um eine halbe Million Bücher gehandelt zu haben. Daraufhin wurde zunächst Dr. Friedel, ein Angehöriger der Bundeswehr, für vier Wochen abgeordnet, um das Material in Washington zu sichten und nach Relevanz zu trennen. Bereits in dieser frühen Phase versuchte die Staatsbibliothek, ihre Belange indirekt zur Sprache zu bringen. ${ }^{46}$

Am 20. November 1963 landeten die Bücher in Bremerhaven. Es handelte sich um etwa 200.000 bis 250.000 Bände, die in 1.050 Kisten sehr unterschiedlicher Größe per Schiff über den Atlantik transportiert worden waren. 300 Kisten aus ehemaligen Wehrmachtsbüchereien wurden sogleich von der Bundeswehr übernommen und abtransportiert. Einige probeweise geöffnete Kisten offenbarten ein großes Chaos; bis auf die Zeitgeschichtliche Sammlung Rehse, die geschlossen verpackt war, lagen „Bücher, Archivgut und ungebundenes Zeitschriftenmaterial völlig ungeordnet durcheinander“ ${ }^{47}$ In einer Besprechung Ende Januar 1964 zwischen Vertretern des Bundesverteidigungsministeriums, des Bundesarchivs, der Stiftung Preußischer Kulturbesitz, des Landes Baden-Württemberg, der Bayerischen Staatsbibliothek sowie des Landes Bremen als treuhänderischem Verwahrer und dessen Staatsbibliothek wurde das weitere Vorgehen festgelegt. Zunächst sollten die Bestände rasch geordnet und katalogisiert werden, dazu ordneten die Bayerische Staatsbibliothek, das Bundesarchiv, die Stiftung und das Verteidigungsministerium jeweils einen Beamten des gehobenen Dienstes für mehrere Monate ab. Inhaltlich kristallisierte sich als Leitlinie heraus: Es galt das Provenienzprinzip, d.h. sofern feststellbar, war die frühere Bibliotheksheimat Indikator für die Verteilung der Bestände. Die Bayerische Staatsbibliothek erklärte sich bereit, das ungebundene Zeitschriftenmaterial zu übernehmen und Mehrfachexemplare später weiterzuleiten. Eine besondere Gruppe bildeten ältere Judaica ohne Besitzvermerke. Die Entscheidung darüber wurde zunächst zurückgestellt, doch in der Folge konnte die Bayerische Staatsbibliothek mit dem Hinweis

45 Das Folgende nach Bayerisches Hauptstaatsarchiv München (BayHStA), Bestand Generaldirektion der Bayerischen Staatlichen Bibliotheken (Generaldirektion Bayer. Staatl. Bibliotheken 1332). Der Akt ist betitelt: Rückführung von Büchern aus den USA. Er enthält Korrespondenz aus den Jahren 1963 bis 1967.

46 Informell erfuhr Gustav Hofmann von Edgar Breitenbach (1903-1977), einem in die USA emigrierten deutschen Bibliothekar und Kunsthistoriker, der nach dem Krieg am Central Collecting Point in München, später dann in der Library of Congress tätig war: „Dr. Friedel, der seit etwa zwei Wochen bei uns ist und die Büchermassen, wohl $\frac{1}{2}$ Mill.[ion]!, nach ehemal. zivilen und militärischen Besitzern trennt, macht einen sehr angenehmen und vernünftigen Eindruck. Er ist durchaus bereit, mit Ihnen über die Überlassung der bayer. Heimatslit.[eratur] zu verhandeln." BayHStA, Generaldirektion Bayer. Staatl. Bibliotheken 1332, Edgar Breitenbach an Gustav Hofmann, 15.09.1963.

47 BayHStA, Generaldirektion Bayer. Staatl. Bibliotheken 1332, Heinrich Middendorf an Generaldirektion der Bayerischen Staatlichen Bibliotheken, 31.01.1964. 
auf ihre große Sammlung an Judaica erreichen, dass diese Bestände ihr zugewiesen wurden. Die Sammlung Rehse hatte das Innenministerium bereits vorab dem Bundesarchiv Koblenz übergeben.

Schon Anfang Februar 1964 meldete sich auch die bayerische Archivverwaltung und bat die Bayerische Staatsbibliothek, ihre Belange mit zu wahren, also ein Auge auf aus Bayern stammende Akten früherer NSDAP-Dienststellen zu haben. Bereits am 18. Februar 1964 nahm Oberamtmann Wilhelm Wimmer von der Staatsbibliothek seine Tätigkeit in Bremerhaven auf. Die Durchsicht ging gut voran, Anfang März konnten erste Kisten mit Büchern nach München abtransportiert werden. Anfang April war die Durchsicht abgeschlossen; ${ }^{48}$ von den nach dem Abtransport der Bundeswehr in Bremerhaven noch vorhandenen 771 Kisten erhielt das Bundesarchiv 359, die Bayerische Staatsbibliothek 264, die Stiftung Preußischer Kulturbesitz 75, die Bundeswehr nochmals 54, die Württembergische Landesbibliothek in Stuttgart 14, außerdem gingen vier Kisten an die Stadt- und Universitätsbibliothek Frankfurt am Main und eine an die Universitätsbibliothek Münster. Der Anteil der Bayerische Staatsbibliothek betrug aufgestellt 500 laufende Meter; sie erhielt die ungestempelten Drucke, außerdem alle Drucke mit bayerischen oder österreichischen Provenienzen, vor allem von NS-Organisationen. Dazu gehörte auch ein erheblicher, leider nicht quantifizierter Anteil aus der Ordensburg Sonthofen.

„Außerdem sind Teile der Privatbibliotheken von Himmler, Rosenberg, Schwarz und Troost dabei. [...] Die Arbeit war ziemlich schmutzig, doch mitunter auch interessant. Die Zusammenarbeit lief kollegial ab. Zusammenfassend kann gesagt werden, dass sich viele zeitgeschichtlichen [!] Dokumente in der Masse befinden und dass nicht wenige darunter Seltenheitswert besitzen. ${ }^{* 49}$

Doch die Verteilung war damit noch nicht abgeschlossen. In allen Berichten bayerischer Vertreter war stets mit Bedauern darauf hingewiesen worden, dass die Sammlung Rehse an das Bundesarchiv gekommen war. Dieses Vorgehen widersprach nicht nur der sonst geübten Praxis der Verteilung nach dem Provenienzprinzip. Auch wenn der wertvollste Teil dieser Sammlung in der Library of Congress geblieben war, so war doch klar, dass es sich um zentrales zeitgeschichtliches Material handelte, insbesondere zur Geschichte der NSDAP. Der deutsche Fotograf und Kunstverleger Friedrich Rehse (1870-1952) hatte seine

48 BayHStA, Generaldirektion Bayer. Staatl. Bibliotheken 1332, E. Seidt an Generaldirektion der Bayerischen Staatlichen Bibliotheken, 06.04.1964. Die Stückzahlen differieren in den verschiedenen Berichten leicht, hier werden die Angaben aus der Endabrechnung wiedergegeben, die der Vertreter des Bremer Senators für das Bildungswesen am 28.08.1964 aufgestellt hatte. Für die Kosten hatten nämlich die übernehmenden Einrichtungen je nach ihrem Anteil aufzukommen.

49 BayHStA, Generaldirektion Bayer. Staatl. Bibliotheken 1332, E. Seidt an Generaldirektion der Bayerischen Staatlichen Bibliotheken, 06.04.1964. 
voluminöse Sammlung zunächst ab etwa 1914 als Privatmann aufgebaut, seit 1929 bildete sie einen Teil des Zentralarchivs der NSDAP. Sie war seit 1938 in der Münchner Residenz weitläufig aufgestellt gewesen. Schließlich konnte bei einem Treffen in größerer Runde, das am 4. Februar 1965 im Bundesarchiv Koblenz stattfand, ein Kompromiss in Sachen Rehse erreicht werden: Die umfangreiche Zeitungsausschnittsammlung wurde komplett an Bayern übergeben, von den 6.000 bis 8.000 Plakaten sollten Doppel- und Mehrfachexemplare an den Freistaat gegeben werden, Einzelstücke jedoch nur, wenn sie von bayerischen Behörden publiziert wurden. ${ }^{50}$ Die Bayerische Staatsbibliothek verzichtete dabei auf die Herausgabe von Druckschriften, sie hatte jedoch bereits mehrere Lieferungen des Bundesarchivs erhalten. Dieser dem Freistaat übergebene Teil der Sammlung Rehse wird heute in der Abteilung V des Hauptstaatsarchivs in München verwahrt. Ebenso ein Dorn im bayerischen Auge war, dass die Bibliothek des bayerischen Armeemuseums zu den Wehrkreisbibliotheken abgewandert war, auch hier konnte schließlich die Herausgabe erreicht werden.

Folgt man den in der Bayerische Staatsbibliothek erhaltenen Zugangsbüchern, so dauerte es bis ins Jahr 1968, bis diese Abgabe aus der Library of Congress komplett katalogisiert war. Die Listen zeigen allerdings auch, dass man zu diesem Zeitpunkt nur noch relativ wenig in den Bestand aufnahm, viel wurde in Münchner Antiquariate wie Wölfle oder an andere Einrichtungen wie die Universitätsbibliothek Erlangen abgegeben.

\section{Vorläufiger Befund}

Die bisherigen Befunde aus dem zu Sonthofen laufenden Projekt NS-Raubgutforschung basieren auf der Durchsicht von etwa einem Viertel des Bestandes. Sie zeigen Sonthofen als universal angelegte Bibliothek, die zeitgenössische Lehr- und Sachbücher ebenso umfasste wie ausländische Literatur, politische und religiöse Schriften. Auch Belletristisches aus dem klassischen Kanon der Literatur findet sich, ebenso historische und kunstgeschichtliche Werke. Von den Besitzstempeln her lassen sich folgende Einzelbestände in Sonthofen unterscheiden: Die „Erzieher-Akademie der Adolf-Hitler-Schulen“, zu der separat eine „Erzieher-AnwärterBücherei“ existierte. An Beständen der Ordensburg Sonthofen werden neben der Hauptbücherei das „Seminar Deutsche Arbeit“ sowie das „Seminar Völkische Behauptung“ genannt. Von den Adolf-Hitler-Schulen finden sich Besitznachweise der Schulen Brandenburg, Chiemsee, Mecklenburg und Westmark; eigens ausgewiesen ist das Fach „Blick in die Welt“. Des Weiteren fanden sich Stempel der

50 BayHStA, Generaldirektion Bayer. Staatl. Bibliotheken 1332, Hermann Fellerer an die Generaldirektion der Bayerischen Staatlichen Bibliotheken, 09.02.1965. 
„Akademie für Deutsches Recht, München“ sowie der SS-Organisation Ahnenerbe, hier auch von der Dienststelle Niederlande. Diese Provenienzen können freilich auch bei Auslagerungen während des Krieges oder späteren Umschichtungen des Bestandes mit dazu gekommen sein.

Der Beitrag hat versucht, erste Informationen zu den Büchersammlungen der NS-Ordensburg Sonthofen zu geben, einem umfangreichen Bestand, über den bislang wenig bekannt ist. ${ }^{51}$ Das ist sicher auch auf die Zersplitterung der Sammlung in der Nachkriegszeit zurückzuführen. Abgesehen von den Beständen, die sich in der Library of Congress befinden, dem in München verwahrten Material und jenen Bänden in der BDIC in Nanterre dürften weitere kleinere oder größere Teilbestände in anderen Bibliotheken zu finden sein, auch im Antiquariatsbuchhandel tauchen immer wieder Werke mit Sonthofen-Provenienz auf. Die Restitutionen der Bayerischen Staatsbibliothek in der Nachkriegszeit und die aktuellen Funde an Raubgut durch das laufende Projekt verweisen auf eine Gemeinsamkeit all dieser Teilbestände, nämlich die in Gewalt und Unrecht liegenden Ursprünge dieser in wenigen Jahren aufgebauten Büchersammlung. Vor allem diese Geschichte ist noch nicht zu Ende geschrieben. ${ }^{52}$

Für freundliche Hinweise danke ich Dominique Bouchery, Bibliothèque de documentation internationale contemporaine, Nanterre sowie Birgit A. Schulte, Fachinformationszentrum der Bundeswehr, Bonn. Für vielfältige Unterstützung danke ich herzlich meinem Kollegen Friedrich Ulf Röhrer-Ertl.

51 Besser erforscht ist die Bibliothek der Ordensburg Vogelsang; vgl. Michael Schröders: „Teile der wertvollen wissenschaftlichen Bibliothek“. Die Universitätsbibliotheken Köln und Bonn und die Bibliothek der ehemaligen NS-Ordensburg Vogelsang. In: Geschichte in Köln 54 (2007), S. 219-240; Michael Schröders: Die Bibliothek der ehemaligen NS-Ordensburg Vogelsang 1944-1947. Fragen zu einem verloren geglaubten Bestand. In: Weltanschauliche Erziehung in Ordensburgen des Nationalsozialismus. Zur Geschichte und Zukunft der Ordensburg Vogelsang. Hg. von Paul Ciupke, Franz-Josef Jelich. Essen: Klartext Verlag 2006, S. 127-140.

52 Was die Geschichte der Bibliothek der Ordensburg Sonthofen betrifft, so fehlt es noch an Grundlagenforschung. Neben den Unterlagen und Hinweisen in bayerischen Archiven (insbesondere im Staatsarchiv Augsburg, das die Überlieferung der Ordensburg verwahrt, sowie im Bayerischen Hauptstaatsarchiv) und im Bundesarchiv sind vor allem noch intensive Forschungen an US-amerikanischen Einrichtungen wie der Library of Congress und den National Archives in Washington, D.C. nötig, möglicherweise auch in den Beständen der Präsidentenbibliotheken von Harry S. Truman (1884-1972) in Independence, Missouri und Dwight D. Eisenhower (1890-1969) in Abilene, Kansas. Auch ehemalige NS-Dokumente, die heute in Archiven der russischen Föderation verwahrt werden, wären zu überprüfen. Auf diese Weise könnte die Rolle der Sonthofener Bibliothek im größeren Geflecht der parteieigenen NS-Bibliothekspolitik erforscht und diese in den Gesamtkontext der deutschen Bibliotheksgeschichte 1933 bis 1945 gestellt werden. 
Open-Access-Publikation im Sinne der CC-Lizenz BY-NC-ND 4.0

(c) 2018, V\&R unipress GmbH, Göttingen

ISBN Print: 9783847107835 - ISBN E-Lib: 9783737007832 


\title{
Julia Stepnowska / Kamil Zeidler
}

\section{The Case of Polish Museums Holding Cultural Objects "in Trust" after WWII}

\section{Zusammenfassung}

Die treuhänderische Verwahrung von Kulturgütern an polnischen Museen nach dem Zweiten Weltkrieg

Während des Zweiten Weltkriegs waren Kunstobjekte ständig der Gefahr des Raubs bzw. der Zerstörung ausgesetzt. Eine gängige Lösung die Objekte zu schützen, war die treuhänderische Übergabe an ein Museum. Heutzutage betrifft daher die Kunstrestitution auch Fälle, in denen Erben sich an Museen wenden, um ihr Erbe zurückzuerhalten, was im Fall von Vorkriegseigentum unvermeidbar problematisch wird. Bei staatlichen Sammlungen wurden die öffentlich gezeigten Objekte auch zu kulturellem Allgemeingut. Daher werfen die Restitutionen im Zusammenhang mit nationalen Kulturgütern nicht nur die Frage nach dem rechtmäßigen Besitzer auf, sondern auch die komplexe Frage nach einer gemeinsamen kulturellen Identität. Viele Restitutionsfälle im Nachkriegspolen fallen in diese Kategorie, sie es direkt oder indirekt. Museen beziehen dabei oft den Standpunkt, dass diese Sammlungsbestände mittlerweile zu Gemeingut wurden und deshalb öffentlich zugänglich bleiben sollen. Auch hatten gerade Museumsmitarbeiter, als eine der wenigen Akteure, die Möglichkeit Kulturgüter in dieser Periode zu beschützen. Demgegenüber stehen die Probleme der Provenienz bei geraubter oder direkt ins Museum eingebrachter Kunst, die vor, während oder nach dem Zweiten Weltkrieg an die Institution kam, was einen weiteren zu beachtenden Kontext bildet. Der Beitrag zielt darauf ab beide Positionen zu beschreiben und präsentiert entsprechende Fälle.

\section{Schlagwörter}

Kulturelles Erbe, Kulturbesitz, Museum, Provenienz, Zweiter Weltkrieg

\begin{abstract}
During WWII, cultural objects were in constant danger of being looted or destroyed. One of the most common solutions for securing goods was to transfer them to museums that would then hold them "in trust." Nowadays, cultural property restitution includes the case when heirs turn to museums to restore their heritage - however, in the case of pre-war ownership, the issue becomes inevitably ambiguous. In the case of state collections, objects at public display begin to constitute a common cultural good in time. The case of restitution when a national cultural heritage item is involved seems to touch upon not only the issue of rightful ownership but also the case of common cultural identity - it is therefore
\end{abstract}


much more complex. In the case of post-war Poland, many restitution cases fall within that category, either directly or indirectly. Museums tend to base their arguments on the fact that their collections have somewhat gained the status of a common good, therefore need to be held at public display. Museum workers, as very few other people, had the possibility to secure a good in the given period. However, the difficulty to track the provenance of property looted or directly transferred to museums during, before, and after the war provides an additional context to be discussed. The following paper aims at researching the rights of both parties and presents case studies reflecting the perspectives in question.

Keywords

Cultural Heritage, Cultural Property, Museums, Provenance, WWII

\section{Art Loot and Expropriation Timeline}

The history of Polish cultural property has always been a struggle. The Swedish Invasion of the $17^{\text {th }}$ century is one of the major events that affected the state of Polish collections. ${ }^{1}$ Looted heritage is still displayed in Swedish museums and galleries and the measures leading to their restitution by the government of Poland should be strengthened if there are arguments and grounds for it. The times of the Partition in the $19^{\text {th }}$ century and WWI constitute vital markers in the context of expropriation and the loss of cultural identity. The most intense period related to art looting in Poland is undoubtedly the period of WWII. Nazi-German troops and art historians had planned the looting even before the armed conflict started. The process of transferring property was well-planned and systematic, therefore it is also the period when, paradoxically, the looting was well-documented. Subsequently, the Soviet Army plundered the territory of Poland impulsively, however. Therefore the documentation issues deepen in this case. The communist regime in Poland was responsible for the mass appropriation of each and every property, including cultural objects. Yet it was in the 1950s that many of looted pieces of art returned to Poland from abroad thanks to the restitution activity of the Polish government.

1 See: Grabieże szwedzkie w Polsce (1). Przyczyny, charakterystyka i skutki. In: Cenne, Bezcenne, Utracone 2005/3, and literature listed there; available online: URL: http://old.nimoz. $\mathrm{pl} / \mathrm{pl} /$ wydawnictwa/wydawnictwa-nimoz/cenne-bezcenne-utracone-1/cenne-bezcenne-utra cone-archiwum/2005-1/nr-32005/grabieze-szwedzkie-w-polsce-1-przyczyny-charakterysty ka-i-skutki (accessed on December 15, 2017). 


\section{Art Restitution Law Timeline (Polish Context)}

Despite the fact that many consider the restitution of goods looted by the Swedish troops in the 17th century as no longer viable, in fact it could have been possible according to the Treaty of Oliva of $1660^{2}$ which granted the restoration of some objects from all looted heritage. What is more, as the Memoir of Lord Castlereagh (1769-1822) to Allied Ministers of $1815^{3}$ shows, restitution is possible despite any political changes that might have resulted in border changes. The Hague Convention of $1907^{4}$ prohibits the pillage of places, seizure, and damage of works of art. The Treaty of Riga $(1921)^{5}$ coined the term of substitute restitutions that has been recommended by the appropriating parties in the case of Polish heritage that is located in Russia or Ukraine as a result of political changes. The 1992 Polish-Russian Treaty of Friendly and Neighbourly Cooperation $^{6}$ acted as a means of disclosing losses located in Russia and facilitating their return. Despite the scope of legal sources in this domain, the process of restoring Polish cultural property from abroad is obstructed by a variety of factors from political and economic to purely practical e.g. the difficulties in identifying the objects to be returned.

The exact number of museums existing before WWII is still unknown. The number varies from one source to another (130-300), it is also worth to mention the case of museums in the areas of pre-war Poland was subject to political changes, such as the west of Ukraine. The database for looted cultural property managed by the government of Poland presents only a fraction of looted items mostly due to the fact that only 10,000 out of 60,000 items listed in the database were photographed. ${ }^{7}$ The number of looted cultural items is estimated to be around 500,000, however it is noteworthy that many museums still have not identified all of the property missing since 1939. Moreover, the number in

2 Peace of Oliva, 3 May 1660; available at the Central Archives of Historical Records in Warsaw (AGAD).

3 A.F. Vrdoljak: International Law, Museums and the Return of Cultural Objects. New York: Cambridge University Press 2008, p. 19.

4 Laws and Customs of War on Land (Hague, IV) Convention signed at The Hague October 18, 1907, available online URL: https://www.loc.gov/law/help/us-treaties/bevans/m-ust0000010631.pdf (accessed on December 15, 2017).

5 Traktat pokoju między Polską a Rosją i Ukrainą podpisany w Rydze dnia 18 marca 1921 roku, Dz.U. $1921 \mathrm{nr} 49$ poz. 300 [Treaty of Peace Between Poland, Russia and the Ukraine, signed at Riga, March 18, 1921, Journal of Laws from 1921, No. 49, item 300].

6 Traktat między Rzecząpospolitą Polską a Federacją Rosyjską o przyjaznej i dobrosąsiedzkiej współpracy, sporządzony w Moskwie dnia 22 maja 1992 r., Dz.U. 1993 nr 61 poz. 291. [Treaty on Friendly and Good Neighbourly Relations between the Republic of Poland and the Russian Federation, done at Moscow on May 22, 1992, Journal of Laws from 1993, No. 61, item 291].

7 It is noteworthy that, according to the Washington Conference Principles on Nazi-Confiscated Art, "Efforts should be made to establish a central registry of such information." 
question does not include private property destroyed during WWII. Moreover, there is a question concerning the property moved from the parts of pre-war Germany that became part of Poland after WWII. Therefore, it is impossible to count how many objects were destroyed and stolen during the war, e.g. burnt in Warsaw during the Warsaw Uprising in 1944. What is more, it is impossible to establish the value of Polish losses. Therefore, these numbers above show us only the scale, but they are not precise.

The documentation issues influencing the difficulties in restoring artworks and other cultural property items affect the process of identifying the rightful owner. The fact that many works have been repainted, restored or renamed could prove to have a more severe impact than the mere fact that the documents related to the provenance of a given work ceased to exist.

Determining ownership is not only a question of provenance or legal grounds but also the fragile issue of moral or emotional obligation. The authors have distinguished three types of works that could be subject to restitution. Objects that were looted as a result of an armed conflict, transferred by the owner to an individual or an entity as a means of securing the good, and abandoned by the owners fleeing from the place of residence. Museum workers and art historians have played a key role in securing or restoring all listed types of works, therefore, under the condition that there is no evident owner, the good could be treated as belonging to the one responsible for securing the good e.g. professor Karol Estreicher (1906-1984), one of the most prominent Polish art historians, responsible for securing and returning looted cultural property (including Da Vinci's Lady with an Ermine) to Poland during and after WWII, who created the first catalogue of looted art in Poland: Cultural losses of Poland. Index of Polish Cultural Losses during the German Occupation, 1939-1944, London 1944 and contributed to restitution initiatives in the follwing years.

\section{Case Study}

The case of private property transferred to a public collection is subject to a dubious interpretation. On the one hand, it could have been the only way of securing a good, on the other, as a result, a given work becomes a part of common heritage, therefore its return affects the public interest. In the instance presented in this study, the original owner is private yet grants public accessibility. The Last Judgement (1467-1471) by Hans Memling (1433/ 40-1494), now exhibited in the National Museum in Gdańsk, Poland, was first looted by a privateer, brought to Gdańsk and donated to St. Mary's 
Church. ${ }^{8}$ In 1807 the piece was looted by the French from the church and moved to Paris. Then, in 1815, it was looted by the Prussian Army and moved to Berlin. The work was returned to Gdańsk in 1817 only to be moved away by the German troops in 1945 and then found and taken over by the Red Army. The altar was moved to the Hermitage Museum and finally returned to Gdańsk in 1956, however, to the National Museum, not to the church. ${ }^{9}$ The piece used to be exhibited in St. Mary's Church in Gdańsk, therefore, there is a vital question where it should be presented today? The arguments behind placing the triptych in a museum after the war could relate to the political context of the (communist) period of its restitution and the will to weaken the religious bonds related to heritage; however, there are counter arguments, especially in the face of the changes after 1989.

\section{Restitution Arguments}

The question of rightful ownership in this case is not only a matter of provenance analysis or legal grounds but primarily an assessment of the social and religious value of the work in question. According to the mentioned Riga Treaty, when a work is an integral part of a world collection, it should not be disintegrated. However, the conditions under which a work is considered to be of such nature are not clearly distinguished by any legal source. Another condition to be revised is the fact that the religious value of this particular work of art is not reflected by the character of the museum as a state, secular venue. On the other hand, the museum could prove to provide better preservation of the work as well as availability to the public. However, as the church is also a public venue, the latter argument could be disregarded. There are many more arguments concerning restiutions, which can be raised by the participants of the restitution discourse. ${ }^{10}$ And that is why they are not only very complex, but also fascinating.

8 See: B. Możejko: “Peter von Danzig”. Dzieje wielkiej karaweli, 1462-1475. Gdansk: Gdansk University Press 2014.

9 Cf. I. Szmelter: Results of multi-criteria research on the Last Judgment from the National Museum in Gdańsk. New attribution of the triptych to Rogier van der Weyden and Hans Memling. In: Muzealnictwo, 2014/55, pp. 37-59.

10 See: Kamil Zeidler: Restitution of Cultural Property: Hard Case, Theory of Argumentation. Philosophy of Law. Gdańsk-Warsaw: Gdańsk University Press - Wolters Kluwer 2016, pp. 131-202. 
Open-Access-Publikation im Sinne der CC-Lizenz BY-NC-ND 4.0

(c) 2018, V\&R unipress GmbH, Göttingen

ISBN Print: 9783847107835 - ISBN E-Lib: 9783737007832 


\title{
Nawojka Cieślińska-Lobkowicz
}

\section{Umgang mit dem sogenannten „postjüdischen“ Kulturgut in Polen von 1945 bis heute}

\section{Zusammenfassung}

90 Prozent der 3,5 Millionen zählenden Vorkriegsbevölkerung der polnischen Juden wurde in der Shoa ermordet. Ihnen entzogenes Kulturgut wurde größtenteils zerstört oder von der Wehrmacht, der SS und der Polizei, Besatzungsämtern und individuellen deutschen Plünderern aus dem Lande verschleppt. Ein Teil der Kunstwerke und Bücher aus jüdischem Besitz wurde jedoch auch von Polen auf unterschiedlichen Wegen übernommen. Die Autorin analysiert die politische und juristische Praxis des kommunistischen Polens (1945-1989), die zur Nationalisierung des in öffentlichen Sammlungen und Bibliotheken angekommenen geraubten jüdischen Kulturguts und zu der damit verbundenen Missachtung dessen Provenienz geführt hatte. Nach dem Fall des Kommunismus wurde diese rechtliche und politische Einstellung offiziell nie in Frage gestellt; auch nicht nach der Washingtoner Konferenz von 1998 mit ihren „Principles on Nazi-Confiscated Art“, die Polen unterzeichnet hat.

\section{Schlagwörter}

NS-Raubkunst der polnischen Juden, Nationalisierung des jüdischen Vermögens in Nachkriegspolen, Gesetzgebung der staatlichen Übernahme der jüdischen Kulturgüter in Nachkriegspolen, Washington Principles on Confiscated Art, Provenienzforschung in Polen, Geschichtspolitik Polens, Jüdische Kulturgüter in öffentlichen Sammlungen Polens, Żydowski Instytut Historyczny - Jüdisches Historisches Institut, Warschau, Ralf Immergluck, Bruno Schulz

\begin{abstract}
Dealing with So-called "Post-Jewish" Cultural Property in Poland from 1945 until the Present Days

$90 \%$ of the 3.5 Millions of Jewish people from Poland before WW II were murdered in the Shoah. Their looted cultural property was mostly destroyed or displaced by Wehrmacht, SS and German Police, Nazi occupation authorities and individual German looters. But a part of the art objects and books from Jewish property was also taken by Poland in different ways. In the paper the political and legal practices of communist Poland (1945-1989) which have driven a nationalisation of looted Jewish cultural property will be analysed. Provenance was ignored with this incorporation into the holdings of public collections and libraries. After the fall of communism this legal and political attitude was never revoked
\end{abstract}


officially, even after Washington conference in 1998 and the "Principles on Nazi-Confiscated Art", which were signed by Poland.

Keywords

Provenance Research, Poland, Washington Principles on Confiscated Art, Ralf Immergluck, Żydowski Instytut Historyczny, Looted Art, Polish Jews, Bruno Schulz

\section{Zerstörung, Raub, Ausfuhr der jüdischen Kulturgüter aus dem besetzten Polen ${ }^{\top}$}

Mehr als drei Millionen polnische Juden wurden in der Shoa ermordet. Das ihnen entzogene Kulturgut wurde von den NS-Besatzern größtenteils zerstört oder „verwertet“. Bücher wurden verbrannt oder geschreddert, Judaika aus Silber und anderen Metallen eingeschmolzen. Die künstlerisch oder materiell wertvollen Objekte wurden zumeist aus dem Lande ins Reich verschleppt und teilweise auch nach Österreich gebracht: viele korrumpierte NS-Funktionäre im Generalgouvernement waren Österreicher, wie z.B. der Gouverneur der Distrikte Krakau und Galizien Otto Gustav Wächter (1901-1949), der SS-Polizeiführer des Distrikts Lublin Odilo Globocnik (1904-1945) - der die „Aktion Reinhardt" organisiert hatte, in der mehr als zwei Millionen polnische Juden vernichtet wurden - oder einer der größten NS-Kunstdiebe Kajetan Mühlmann (1898-1958), der von Göring Anfang Oktober 1939 zum Sonderbeauftragten für den Schutz und die Sicherung von Kunstwerken in den besetzten Ostgebieten ernannt wurde.

\section{Restbestand der jüdischen Kulturgüter in Polen}

Ein Restbestand der Kunstwerke, Judaica und weitere tausende Bücher aus jüdischem - privatem und öffentlichem - Besitz ist jedoch in Polen geblieben. Zunächst handelte es sich um Sammlungen, die kurz vor oder nach dem 1. September 1939 von einigen polnisch-jüdischen Kunstsammlern und Eigen-

1 Mehr über das Schicksal der Judaika und anderer Kulturgüter, die den polnischen Juden gehörten, findet man in der deutschen bzw. englischen Fassung meiner Artikel: Judaika in Polen. Herkunft, Schicksal, Status. In: Osteueropa, April 2011 Heft 4, S. 85-132; The History of Judaica and Judaica Collections in Poland Before, During and After the Second World War: An Overview. In: Neglected Witnesses. The Fate of Jewish Ceremonial Objects during the Second World War and After. Hg. von Julie-Marthe Cohen, Felicitas Heimann-Jelinek. Institute of Art and Law, Crickadarn, Jewish Historical Museum Amsterdam 2011, S. 129-182; Dealing with Jewish Cultural Property in Post-War Poland. In: Art. Antiquity and Law, June 2009 Vol. XIX, Iss 2, pp. 143-166. 
tümern von Privatbibliotheken in öffentlichen Museen und Büchereien zur Sicherheit unter Decknamen deponiert worden waren. Später haben die NS-Besatzer diese (für die Öffentlichkeit geschlossenen) Einrichtungen häufig als Lagerräume für die „museumswürdigen“ Objekte und wertvollen Bibliotheken benutzt, die in „herrenlosen“ oder von den jüdischen Einwohnern „ausgeräumten" Wohnungen und Ghettos beschlagnahmt worden waren. In den ersten Nachkriegsjahren kamen von verfolgten Eigentümern bzw. von geflüchteten NSPlünderern verlassene Kulturgüter dazu, die polnische Kunsthistoriker und Bibliothekare aber auch Vertreter des Zentralkomitees der Juden in Polen (Centralny Komitet Żydów w Polsce, gegründet 1944, CKŻwP) quer durch das Land gesucht und gefunden haben. $\mathrm{Zu}$ diesem Restbestand gehörte schließlich das im „Altreich“ oder in anderen besetzten Ländern Europas den Juden geraubte Kulturgut, das in den Osten des Reiches (vor allem nach Niederschlesien) seit 1943 mit anderen deutschen Sammlungen zur Sicherung verlagert worden war. Dort wurde es teilweise von der Roten Armee gefunden und in die UdSSR verschoben, teilweise haben es jedoch die Polen übernommen.

\section{Grundsätzliches Versprechen und bindende Nachkriegsgesetze}

Im Gründungsmanifest Nachkriegspolens vom 22. Juli 1944 wurde „den rechtmäßigen [Anm. privaten, institutionellen und konfessionellen] Eigentümern die Rückgabe des von den Deutschen geraubten Eigentums“ versprochen (einzige Ausnahme bildeten die Großindustriellen und Grundbesitzer). „Den bestialisch verfolgten Juden wurde der Wiederaufbau ihrer Existenz und rechtliche und faktische Gleichstellung “ zugesichert. ${ }^{2}$

Die danach erlassenen Dekrete - zwei im Jahr 1945 und das endgültige am 8. März 1946 - über das herrenlose, verlassene und ehemals deutsche Vermögen haben keinen Zweifel daran gelassen, dass die politische Realität und die davon ausgehende Praxis mit dem zitierten Dokument und seinen Versprechen nichts zu tun hatten. ${ }^{3}$

Das Hauptziel dieser Vorschriften - die vor allem auf Immobilien gemünzt waren - war nicht die Rückerstattung des Eigentums an die rechtmäßigen Ei-

2 Manifest Polskiego Komitetu Wyzwolenia Narodowego, Lublin 22.04.1944, siehe: URL: https://pl.wikisource.org/wiki/Manifest_Polskiego_Komitetu_Wyzwolenia_Narodowego (abgerufen am 04.05.2018).

3 Dekret z dnia 2 marca 1945 r. o majątkach opuszczonych i porzuconych, Dziennik Ustaw [D.U.] $1945 \mathrm{Nr}$ poz. 45; Ustawa z dnia 6 maja $1945 \mathrm{r}$ o majątkach opuszczonych i porzuconych, D.U. $1945 \mathrm{Nr} 17$ poz. 97; Dekret zndnia 8 marca 1946 r. o majątkach opuszczonych i poniemieckich D.U. $1946 \mathrm{Nr} 13$ poz. 87; alle sind auf der Seite URL: http://isap.sejm.gov.pl (abgerufen am 20.05.2017) zu finden. 
gentümer oder an ihre Erben, sondern die Klärung, welches Vermögen bereits zu Staatseigentum erklärt worden sei und welches für einige Zeit noch treuhänderisch vom Staat verwaltet sein sollte. Die in einem eigenen Artikel vorgesehene Rückerstattung des privaten Besitzes bildete eher eine Ausnahme und war durch viele Bedingungen beschränkt.

Entsprechend der genannten Gesetze wurde das ehemals deutsche - genannt postdeutsche - Vermögen automatisch (per Gesetz) verstaatlicht. Das verlassene Vermögen sollte hingegen - wenn es Immobilien betraf - erst nach zehn Jahren verstaatlicht werden. Im Falle beweglicher Güter war die rechtliche Übernahme schon nach fünf, manchmal sogar nach nur drei Jahren endgültig.

\section{Jüdisches oder postjüdisches Kulturgut}

Das im Dekret vom 8. März 1946 als verlassen bezeichnete Vermögen wurde im sprachlichen Gemeingebrauch analog zu postdeutsch als postjüdisch bezeichnet und war faktisch als Synonym für das ehemals jüdische Vermögen zu verstehen.

Dabei möchte ich auf einen markanten Unterschied aufmerksam machen: Schon damals wurde nicht über das jüdische, sondern über das postjüdische Hab und Gut gesprochen. Jüdisch hätte bedeutet, dass es Eigentümer oder deren Erben gibt, man also auf sie warten bzw. nach ihnen suchen müsste. Der Begriff postjüdisch beinhaltete die implizite Voraussetzung, dass keine Eigentümer bzw. keine Erben zu erwarten waren, da diese höchstwahrscheinlich alle von den Deutschen ermordet worden waren.

Der Gesetzgeber hat weder den Begriff postjüdisch noch ethnische, konfessionelle oder rassistische Kriterien verwendet, weil es im Widerspruch zum Manifest vom 22. Juli 1944 gestanden wäre. ${ }^{4}$ Dennoch stand im Zentrum seines Interesses offenkundig ein für immer verlassenes Vermögen, das nach einigen Jahren der treuhänderischen Verwaltung dem polnischen Staat zukommen sollte.

Auch wenn die nicht-jüdische polnische Bevölkerung viele Opfer verzeichnete, war die Wahrscheinlichkeit, keine Familien bzw. keine Erben zu hinterlassen nur bei jüdischen polnischen Opfern sehr hoch. Hinzu kam, dass ausschließlich die jüdischen, so tragisch dezimierten Überlebenden nach 1945/1946 massenhaft legal oder illegal aus dem befreiten Polen auswanderten. Damit war es für die kommunistische Regierung noch einfacher, ihr Vermögen weiter als „verlassenes Vermögen“ zu behandeln. Es war daher kein Zufall, dass gerade im

4 Nur im Falle des ehemaligen deutschen Vermögens hat der Gesetzgeber eine Ausnahme für die damaligen deutschen Staatsbürger polnischer oder anderer von den Deutschen verfolgten Nationalität vorgesehen. 
März 1946 ein anderes Dekret erlassen wurde, mit dem die Ausfuhr von Kunstwerken und anderer Kulturgüter ins Ausland verboten wurde. ${ }^{5}$

\section{Staatliche Übernahme des Kulturguts aus dem Vorkriegsbestand der jüdischen Kultusgemeinden}

Die von der Centralna Żydowska Komisja Historyczna (Zentralen Jüdischen Historischen Kommission), das bereits im Sommer 1944 vom CKŻwP gegründet worden war, im Lande geretteten und in Museen und Bibliotheken identifizierten Reste der jüdischen Kulturgüter - Thorah-Rollen, Judaica und Bücher aus jüdischen Sammlungen - wurden grundsätzlich dem Żydowski Instytut Historyczny (Jüdisches Historisches Institut, $\dot{Z} I H$ ) in Warschau übertragen. ${ }^{6}$ Aus den von Ministerialkommissionen entdeckten NS-Repositorien in Niederschlesien landeten im ŻIH nicht nur gefundene Teile der Judaistischen Bibliothek der Großen Synagoge in Warschau, sondern auch Teile der Bibliothek des Breslauer Jüdisch-Theologischen Seminars, Bücher und rituelle Gegenstände der Berliner und der Wiener Gemeinde, sogar die von der SS in Thessaloniki geraubten Synagogalia.

Das ŻIH wurde von nicht-konfessionellen Organisationen der polnischen Juden und vom polnischen Staat als ein Verwahrer des polnischen Judentums und seines historischen und kulturellen Erbes betrachtet. In den ŻIH-Sammlungen gibt es deswegen auch viele Objekte, die im Laufe der Zeit von Privatpersonen geschenkt oder erworben wurden. Am häufigsten waren das nichtjüdische Polen, die gewöhnlich keine überzeugende Provenienz der Objekte nennen konnten oder wollten. 1948/1949 wurde auch das ŻIH mitsamt seinen Sammlungen verstaatlicht. Es übernahm kurz darauf die Kunstsammlung des sofort nach dem Krieg reaktivierten und 1949 wieder aufgelösten Żydowskie Towarzystwo Krzewienia Sztuk Pięknych ${ }^{7}$ (Verbandes Jüdischer Kunstliebhaber und Künstler) sowie die Judaica-Sammlung der ebenfalls aufgelösten polnischen Filiale des American Jewish Joint Distribution Committee, die nach 1945 entstanden war. Alle diese Objekte sind bis heute im $\dot{Z} I H$ - teilweise als staatliches

5 Dekret z dnia 1 marca 1946 r. o rejestracji i zakazie wywozu dzieł sztuki plastycznej oraz przedmiotów o wartości artystycznej, historycznej lub kulturalnej.przepis o wywozie zabytków, D.U. 1946 Nr 14 poz. 99. Entsprechend dieses Gesetzes wurden nicht nur strenge Ausfuhrverbote der Kulturgüter bestimmt, sondern auch eine obligatorische Ausfuhrabgabe in der Höhe von 22 Prozent für wertvolle Gegenstände, die man in begrenzter Zahl mitnehmen dürfte.

6 ŻIH ist 1947 entstanden, ausgestaltet von der Zentralen Jüdischen Historischen Kommission (CŻKH) des CKŻwP.

7 Abgekürzt ŻTKSP. 
Eigentum, teilweise als Eigentum des ŻIH-Vereins. Zweifel an ihrer Provenienz und Fragen über eine eventuelle Restitution erheben sich in beiden Fällen nicht. ${ }^{8}$

Von Anfang an ging der polnische Staat zynisch mit dem - einst sehr großen - Eigentum der israelitischen Kultusgemeinden der Vorkriegszeit und den mit ihnen zusammenhängenden Organisationen und Institutionen um. Er verweigerte ihnen den Status juristischer Personen und hat ihnen damit u. a. ihre Eigentumsrechte entzogen. Ein am 6. Februar 1945 veröffentlichter Runderlass über die vorläufige Regelung der Glaubensfragen der jüdischen Bevölkerung erlaubte die Gründung der Żydowskie Zrzeszenia Religijne (Jüdischen Religiösen Vereinigungen), die 1949 in Związek Religijny Wyznania Mojżeszowego (Religionsverband Mosaischen Bekenntnisses) umbenannt wurde. ${ }^{9}$ Sie hatten nur den Status kultureller Verbände, d. h. sie waren im juristischen Sinne keine Nachfolger der früheren Gemeinden. Um „Praktiken und Riten des jüdischen Kultus" aufrechterhalten und ausüben zu können, sollten sie unverzüglich passende Gebäude und Objekte zur Nutzung [sic!] erhalten, die dem vollständig unter provisorische Staatsverwaltung gestellten Vermögen der jüdischen Kultusgemeinden der Vorkriegszeit gehörten. ${ }^{10}$ Am 1 . Januar 1956 wurde dieses Vermögen "przewłaszczony“ (umgeeignet), ging also in den Besitz des Staates über. Zwar trat 1997 in Polen ein Gesetz in Kraft, das den jüdischen Kultusgemeinden nach fast sechzig Jahren den Status einer juristischen Person wieder zubilligte und eine Restitution ihrer Vorkriegsimmobilien garantiert. ${ }^{11}$ Aber die Rückerstattung beweglicher ritueller Gegenstände und jüdischer Bibliotheken wurde in diesem Gesetz nicht berücksichtigt. Sie blieben (und sind bis heute) Eigentum der öffentlichen polnischen Museen und Bibliotheken.

8 Es genügt, die Kataloge der ŻIH-Sammlungen und ihre Ausstellungen zu sehen, z. B. eine der letzteren: Ocalałe/Salvaged. Kolekcja malarstwa, rysunku i rzeźby ze zbiorów Żydowskiego Instytutu Historycznego / A collection of paintings, drawnings and sculpture from the holdings of the Jewish Historical Institute, Warszawa 2014.

9 Okólnik Nr 3 z dn. 6 lutego 1945 r. o tymczasowym uregulowaniu spraw wzyznaniowych ludności żydowskiej. In: Dziennik Urzędowy Ministerstwa Administracji Publicznej Nr 1, rok 1, Warszawa dnia 1 kwietnia 1945.

10 Ibidem.

11 Ustawa z dnia 20 lutego 1997 r. o stosunku Państwa do gmin wyznaniowych żydowskich w Rzeczypospolitej Polskiej, D.U. 1997 Nr. 41 poz. 251. 


\section{Kulturgut aus dem Privatvermögen der jüdischen Vorkriegseigentümer}

In Bezug auf das Privatvermögen sah das Dekret vom 8. März 1946 eine Frist für individuelle Ansprüche zum 31. Dezember 1947 vor (die später bis 31. Dezember 1948 verlängert wurde. $)^{12}$ Angesichts der Anzahl polnischer Opfer der Shoa, des Nachkriegschaos, der antisemitischen Stimmungen im Land sowie des am 8. Oktober 1946 eingeführten Gesetzes, das den Kreis der Erben auf die engsten Verwandten beschränkte, muss der eindeutige Profit des Staates aus dem übernommenen Privatvermögen der Juden nicht weiter begründet werden. ${ }^{13}$ Natürlich betraf er vor allem Grundstücke und Immobilien. Der materielle Wert der „herrenlosen“ bzw. „verlassenen“ beweglichen Güter, darunter auch der Kunstwerke, war unvergleichlich geringer, doch auch sie fielen im Großen und Ganzen an den polnischen Staat. Auf diese Weise fand eine volle Verstaatlichung des in Museen und Bibliotheken enthaltenen ehemals jüdischen Kulturguts statt. Neben Judaika und Werken jüdischer Künstler geht es großteils um Werke bekannter polnischer Maler der zweiten Hälfte des 19. Jahrhunderts bzw. Anfang des 20. Jahrhunderts, die vor dem Krieg im Besitz zahlreicher assimilierter Juden waren. Solche Bilder - schon ohne Provenienz - waren während der deutschen Besatzung Polens die Lieblingsware der polnischen Kunden des florierenden damaligen Kunstmarkts. Seit Jahrzehnten hängen viele von ihnen stillschweigend in polnischen Museen. Die Menschen, die sie einst mit Liebe und Stolz gesammelt haben, finden meist keine Erwähnung.

\section{Staatliche Übernahme der privaten jüdischen Kulturgüter}

Man kann unter diesem Gesichtspunkt über zwei Gruppen von Gegenständen sprechen. Die erste Gruppe besteht aus Sammlungen und Bibliotheken, die von den jüdischen Eigentümern im Jahr 1939 in polnischen Einrichtungen deponiert wurden. Alle anderen Objekte gehören zur zweiten Gruppe, unabhängig davon, ob sie von den Nazis konfisziert und in Museen und Bibliotheken gelagert wurden, oder nach dem Krieg gefunden, geschenkt oder erworben worden waren.

Im Falle der deponierten Sammlungen hatten die Museumsdirektoren bis ca. Mitte 1947 freie Hand, sie ihren Vorkriegseigentümern bzw. deren Erben zu-

12 Dekret z dnia 8 marca 1946 r. o majątkach opuszczonych i poniemieckich D.U. $1946 \mathrm{Nr} 13$ poz. 87; URL: http://isap.sejm.gov.pl (abgerufen am 20.05.2017).

13 Dekret z dnia 8 października 1946 r. - Przepisy wprowadzające prawo spadkowe., D.U. 1946 Nr. 60 poz. 329. 
rückzugeben - unter der Voraussetzung freilich, dass sich diese gemeldet hatten. Denn die Museen und Bibliotheken hatten - wie in ganz Europa - keinerlei Absicht, nach den verschollenen Besitzern zu suchen. Die Museen führten dabei nicht selten bestimmte interne Einschränkungen zur bevorstehenden Rückgabe ein - selbstverständlich besonders gerne bei den wertvolleren Werken. Darüber hinaus erwartete man in der Regel ein - mehr oder weniger freiwilliges - „Geschenk“ für das Museum als Zeichen der Dankbarkeit des Besitzers. Andernfalls versuchte man, die besten Werke als langjähriges Depositum im Museum zu behalten.

Seit 1948 war eine derartige einfache Rückgabe von Werken an ihre rechtmäßigen Eigentümer ausgeschlossen. Fortan mussten die Eigentümer eine amtliche Genehmigung für die Wiederbeschaffung ihres Eigentums vorweisen, was einer Zusage des Kulturministeriums bedurfte. In der Praxis bedeutete dies, dass die deponierten Gegenstände als verlassenes Vermögen entsprechend dem Dekret von 1946 behandelt wurden. Theoretisch blieb dem geschädigten Eigentümer noch der Rechtsweg, dieser war im kommunistischen Polen aber chancenlos. Im Laufe der Zeit haben die Sammler, die den Krieg überlebt, aber ihr ganzes sonstiges Vermögen verloren hatten, einige ihre deponierten Werke an die Museen verkaufen oder verschenken müssen, um andere zurück zu bekommen. Nach 1990 ist mir ein einziger Fall bekannt, wo die neun wertvollsten Bilder eines jüdischen Sammlers restituiert wurden, die seit 1940 im Museum gelagert waren und jahrzehntelang nicht zurückgegeben wurden. ${ }^{14}$ Allerdings wurden diese Werke nach einem langwierigen Gerichtsverfahren von einer Firma zurückerstritten, die der Witwe des jüdischen Eigentümers die Eigentumsrechte abgekauft hatte.

\section{Mauer des Schweigens}

In allen anderen Fällen ist eine Rückgabe der Gegenstände, die vor dem Krieg jüdischen Eigentümern gehörten, bis heute unmöglich. Es gibt einige Erben von im Holocaust ermordeten polnisch-jüdischen Künstlern wie Bruno Schulz (1892-1942) oder Ralf Immergluck (1892-vor 1944), die nach dem Fall des Kommunismus versucht haben, zumindest einige Werke zurückzubekommen. ${ }^{15}$

14 Roman Olkowski: Kolekcja Rotwandów. Losy polskich zbiorów w dobie bezprawnych praktyk. In: Adlojada Prawo i Kultura. Hg. von Jaromir Brejdak, Dariusz Kacprzak u.a. Szczecin: Muzeum Narodwe w Szczecinie 2016, S. 193-209.

15 Nawojka Cieślińska-Lobkowicz: Who owns Bruno Schulz? The Changing Postwar Fortunes of Works of Art. by Jewish Arists Murdered in Nazi-Occupied Poland. URL: http://art.claims con.org/wp-content/uploads/2016/03/Cieslinska-Lobkowicz-Who-owns-Bruno-Schulz-21March-2016.pdf (abgerufen am 20.05.2017). 
Diese Versuche blieben erfolglos. Die Verstaatlichung des jüdischen Kulturguts und die damit einhergehende Missachtung ihrer Provenienz wurde nie in Frage gestellt - trotz der Washingtoner Konferenz von 1998 mit ihren Principles on Nazi-Confiscated Art, die Polen mitunterzeichnete, und trotz des steigenden Interesses an der Geschichte des polnischen Judentums und am Holocaust. ${ }^{16}$

In diesem Sinne kann man von einer „Kontinuität des Schweigens“ in Sachen Raubkunst polnischer Juden sprechen. ${ }^{17}$ Die Gründe dafür sind tief in der historischen Politik des Staates verankert. Die großen polnischen Kulturverluste während des Zweiten Weltkrieges lassen im offiziellen Narrativ keine Konkurrenz der noch größeren Kulturverluste der polnischen Juden zu. Der im Land nach 1945 gebliebene Restbestand des NS-entzogenen jüdischen Kulturbesitzes bleibt in den polnischen Museen und Bibliotheken meistens bis heute anonym und gehört zum nationalen Eigentum Polens. Auch das ŻIH zeigt bis heute kein Interesse, nach den Erben der jüdischen Künstler und Sammler zu suchen. ${ }^{18}$ Es versteht sich als völlig berechtigter Eigentümer der gesammelten Objekte und nicht als deren moralisch verpflichteter Depositar, der die Washingtoner Prinzipien vorbildlich umsetzen sollte. Die polnischen Museen suchen nicht nach der Provenienz ihrer nach dem Krieg erworbenen Kunstwerke. Sie konzentrieren sich lediglich auf die eigenen kriegsbedingten Verluste, nach denen das Kulturund das Außenministerium intensiv im Ausland fahnden. Dabei berufen sie sich gerne auf die Washingtoner Prinzipien von 1998.

Das in polnischen nationalen Museen und Bibliotheken verwahrte, anonymisierte jüdische Kulturgut wartet bis heute auf seine Entdeckung und Erforschung. ${ }^{19}$

16 Washington Conference Principles on Nazi-Confiscated Art. In: Washington Conference on Holocaust-Era Assets. November 30-December 3,1998. Ed. by J.D. Bindenagel, Washington 1999, S. 971-972 (Appendix G). URL: https://fcit.usf.edu/holocaust/resource/assets/princ. htm (abgerufen am 05.11.2017).

17 Zweimal in den vergangenen 15 Jahren hat man in Polen versucht, die Provenienzforschung in dem Sinne der Washington Principles in polnischen Museen zu implementieren: 2002 hat eine Gruppe Kunsthistoriker ein unabhängiges Advisory Forum on Displaced Works of Art beim Verband der Kunsthistoriker gegründet, das bald zur Auflösung gezwungen wurde. Nach der Terezin Declaration on Holocaust Era Assets and Related Issues vom Juni 2009 hat der damalige Stellvertreter des Kulturministers Tomasz Merta ein Provenienzforschungsteam gegründet, das die entsprechende methodologische Basis und Hinweise für Museen vorbereiten sollte. Nach Mertas Tod bei der Flugkatastrophe in Smolensk im Jahr 2010 wurde das Provenienzforschungsteam aufgelöst. Seine Ergebnisse wurden im Jahrbuch Muzealnictwo 53(2012), S. 10-37 veröffentlicht, URL: https://issuu.com/nimoz/docs/m_53_calosc (abgerufen am 05.11.2017).

18 Nawojka Cieślińska-Lobkowicz: Who owns Bruno Schulz? (Anm. 15).

19 Es gibt erste Forscher, die angefangen haben, sich mit den polnisch-jüdischen Sammlern zu befassen: Dariusz Kacprzak (Lodzer Sammler), Milena Woźniak-Koch und Roman Olkowski (Warschauer Sammler), Yagna Yass-Alston (Krakauer Sammler) und Magdalena Palica (Breslauer Sammler). 
Open-Access-Publikation im Sinne der CC-Lizenz BY-NC-ND 4.0

(c) 2018, V\&R unipress GmbH, Göttingen

ISBN Print: 9783847107835 - ISBN E-Lib: 9783737007832 


\title{
Lara Lempertienè
}

\section{Looted? Abandoned? Saved? The Provenance and Status of Jewish Documents in the State Document Repositories of Lithuania}

\author{
Zusammenfassung \\ Die Provenienz und der Status jüdischer Dokumente in staatlichen Archiven Litauens \\ Der Beitrag beschäftigt sich mit den Buchbeständen und anderen Dokumenten aus jüdi- \\ schem Vorkriegsbesitz, die in verschiedenen litauischen Bibliotheken und Archiven auf- \\ bewahrt werden. Diese Bestände haben einen unklaren Eigentumsstatus und gehören \\ jedenfalls nicht zu den vor dem Zweiten Weltkrieg legal erworbenen Beständen. In den \\ Jahrzehnten nach dem Zweiten Weltkrieg wurden mehrere Überlegungen und Abkommen \\ über ihren Status national und international präsentiert aber letztendlich wurden die \\ meisten Objekte nicht zurückgegeben. Der Beitrag beschreibt ihre gegenwärtigen Status, \\ sowie ihre Einbeziehung in internationale Projektpartnerschaften, die neue Zugänge für \\ die Wissenschaft und die breite Öffentlichkeit eröffnen.
}

Schlagwörter

Vilnius, Litauische Nationalbibliothek, Litauisches Zentralstaatsarchiv, Vilna Projekt

\begin{abstract}
The article discusses the holdings of books and other documents of prewar Jewish provenance in several Lithuanian libraries and archives. They are the documents of an ambivalent status, and not the ones that were legally acquired before the World War II. During the after-war decades different deliberations and changing agreements on their status were presented locally and internationally. However, most of the objects were not moved, and the article presents their current situation as to the status, processing, accessibility and inclusion in projects of international partnership that presented new ways of sharing with the academic and general public.

Keywords

Vilnius, Lithuanian National Library, Lithuanian Central State Archives, Vilna Project
\end{abstract}


The case of Jewish documentary materials in the libraries and archives of Lithuania (in the present article I will concentrate my attention on the capital, Vilnius) is complicated, and not only in a way that most post-war collections in repositories of Europe do not have straightforward ownership histories. I intend to draw attention to the case of extensive and diverse, but at the same time fragmented holdings of books and other documents of pre-war Jewish provenance in several Lithuanian repositories: the National Library of Lithuania, the Central State Archives and the Library of the Academy of Sciences. The Jewish holdings of the aforementioned institutions were not formed in a purposeful and planned way, as were the Jewish collections of the Historical Archives of Lithuania or the Library of Vilnius University, but rather haphazardly stored after surviving the Nazi occupation and cultural plundering, as well as later Soviet purges. If the documentary materials survived that way, they were nevertheless lacking proper storing, cataloging and study, and their immense cultural value for a long time was not acknowledged enough.

These are the remnants of the same huge conglomerate of printed books, periodicals and diverse documents that were not plundered and/or destroyed by Nazis and remained in the city, and they ended up not in one, but in several holding institutions that for decades acted as if there were no connection between the parts, and were unaware of the fact that other survived parts of the same conglomerate could be found yet in other repositories abroad.

The group of publications and documents I speak about is the holdings and documentation of several dozen Jewish institutions, public and private libraries that were uprooted by Nazis and partly looted partly annihilated and partly abandoned by them, as well as hundreds of personal documents. Such was the richness of Jewish social and cultural life in interwar Vilnius that the amount of just the abandoned materials found during post-war decades is almost overwhelming. For a very brief overview, in the pre-war Vilnius there were 3 public Jewish libraries (one of them - the children's library), the Yiddish Scientific Institute (YIVO) with its first-rate academic library in several languages, voluminous archives and several periodical publications, and the Historical-Ethnographic Society with its library of rare Jewish books and the archives. (In fact, the books were in such abundance that in 1923 a campaign was held in Vilnius for collecting books for the library of the Hebrew University in Jerusalem, and several thousand books were donated to it as a result). And of course, every Jewish school, synagogue and social organization had its own library, and there were many private owners of prominent book collections. Several Jewish dailies and many other periodical publications, as well as productive publishing houses, added to the plethora of Jewish documentary materials. Naturally, all of the mentioned institutions conducted and preserved its own extensive internal 
documentation and correspondence. In this regard, Vilnius resembled other major centers of Jewish life in Europe.

The first dramatic changes to the status of these collections and their owners were brought by the Soviet regime that came to Vilnius in June 1940 as a result of the Molotov-Ribbentrop pact of 1939. Most of the other Jewish organizations were closed or rendered inactive, some members of the staff arrested, killed or exiled, and their libraries and other documents made unavailable to use. The Jewish community as an organization was dismantled, its belongings confiscated, and its 2 major libraries, the Strashun and the Mefitse Haskala libraries, as well as the museum of the Historical-Ethnographic Society that also acted as a community institution, proclaimed transferred under the auspices of the Institute of Lithuanian Studies (later - the Academy of Sciences) of Lithuanian Soviet Republic ${ }^{1}$. But the collections and the documentation were mostly left in their respective places, because the Soviets did not have enough time to implement the planned transfers of the holdings prior to the Nazi invasion. In that way, the documents were, in a way, conserved and not dispersed ${ }^{2}$.

After establishing the ghetto in Vilnius a representative of the Rosenberg Stab, Johannes Pohl (1904-1960), identified Jewish intellectuals among the ghetto inmates: the YIVO director Zelig Kalmanovich (1881-1943/44) and members of the Institute's staff, the senior librarian of the Strashun Library Chaikl Lunski (1881-1943) and others. They were forced firstly to transfer the contents of Vilnius Jewish libraries and archives to one designated place - the premises of the Vilnius university (which collections also were looted), and after that sort them by "more" and "less" valuable materials - the former shipped to Germany, the latter were meant to end up, as much of them did, in paper factories. The structure of the prewar collections was totally deconstructed. The group of intellectuals (dubbed "the paper brigade") was obliged to carefully document their "progress". In addition, they were ordered to produce written works in German on a variety of subjects: Vilna Jewish past and heritage, Karaites etc., thus leading them to create new documents within the ghetto. ${ }^{3}$ Some of them were completed and typed, and some left in their preparatory stages, in manuscripts.

The biggest public Jewish library of the pre-war period, the Mefitse Haskala library, was located in the territory which now belonged to the ghetto. Since the

1 Lithuanian Central State Archives (LCSA), 401/2 /518; 401/2 /330; R-1001/ 1/ 32; R-1001/1/40.

2 There are indications that some of the YIVO institute documents were, in fact, taken to the Academy of Sciences, but for now, no records have been found that would specify the full amount and the exact nature of them. See: Manuscript Division of the Lithuania National Library (LNL MD), The Jewish Museum 1944-1949 [unprocessed].

3 On the Nazi looting policy in Vilna ghetto see: Maria Kühn-Ludewig: Johannes Pohl (1904-1960). Judaist und Bibliothekar im Dienste Rosenbergs. Eine biographische Dokumentation. Hannover: Laurentius 2000 (= Kleine Historische Reihe 10). 
Nazis were mostly uninterested in its holdings, which contained virtually no antiquities or bibliographic rarities, it wasn't plundered and functioned as the ghetto library. Through it, as well as through some other channels and contacts with non-Jewish comrades outside the ghetto, the members of "the paper brigade" aided by the director of the library, Herman Kruk (1897-1944), and the librarians, were able to conceal a part of the documents, printed materials and artifacts. ${ }^{4}$ It should be mentioned though that in their rescuing work, as well as in their forced labor of segregating Jewish materials for the needs of the Nazis, the people of "the paper brigade" inevitably further mixed the materials and deconstructed their provenance histories.

In September 1943 the ghetto in Vilnius was liquidated, most of the survived inmates murdered, and a certain number transferred to concentration camps where most of them perished. Nevertheless, several members of the former "paper brigade" survived as members of the anti-Nazi Soviet partisan resistance, which they joined before the liquidation of the ghetto, and in 1944, while participating in the liberation of Vilnius, they were able to recover some of the materials hidden by them. On the other hand, they witnessed a scene of chaos and scattering of many documentary materials abandoned by the Nazis, as well as bundles of books and documents prepared for annihilation at the paper mills, now overtaken by the Soviets. The most active among these former partisans were Yiddish poets Avrom Sutzkever (1913-2010), Shmaryahu Kacherginski (1908-1954) and Abba Kovner (1918-1987). They were allowed to establish the Jewish Museum of Vilnius, where they housed all their findings.

However, it shortly became clear that Jewish national life under the Soviets was not meant to develop and flourish, or, indeed, be allowed at all. Kovner, Sutzkever and Kacherginski left Vilnius. Sutzkever and Kacherginski took with them secretly some of the materials that they later have donated to the YIVO Institute that continued its work in New York and inherited, by international agreement, also other documents and books of Vilnius provenance found among the Nazi shipments from the occupied Eastern European cities. ${ }^{5}$ Indeed, in 1949

4 The most extensive research on the activities of the "paper brigade" and the post-war activities of its surviving members was conducted by David E. Fishman; see his newest monograph: The Book Smugglers. Partisans, Poets and the Race to Save Jewish Treasures from the Nazis. Lebanon, NH: ForeEdge 2017. On the ghetto library see: Dina Abramowicz: Die Bibliothek im Wilnaer Ghetto (1941-1943). In: Bücher und Bibliotheken in Ghettos und Lagern (1933-1945). Hannover: Laurentius 1991 (= Kleine Historische Reihe 3), S. 119-137; Herman Kruk: The Last Days of Jerusalem of Lithuania. Chronicles from the Vilna Ghetto and the Camps, 1939-1944. Transl. and ed. by Benjamin Harshav. New Haven-London: Yale University Press 2002; Hermann Kruk (1897-1944). Bibliothekar und Chronist im Ghetto Wilna (1941-1943). Translat. and ed. by Maria Kühn-Ludewig. Hannover: Laurentius 1990 (= Laurentius Sonderheft).

5 On the transfer of the Vilnius materials found in Germany to YIVO see, e.g.: Lucy S. 
the Jewish Museum was closed, and its exhibits offered to different cultural institutions. And so, the dispersing and muddling of the provenance continued.

It was a pivotal event for the Lithuanian National Library (then - the State Book Chamber), because it received most of the books collected by the Jewish museum as well as many unsorted documents, and so its Judaica holdings began to evolve. But even previous to the transfer, the director of the Book Chamber, Antanas Ulpis (1904-1981), and other dedicated members of its staff were so concerned about the fate of abandoned books, Jewish and non-Jewish alike, that they organized gatherings in Vilnius, as well as expeditions into the Lithuanian province with the task to rescue what was possible. Of course, the biggest part of their findings constituted Jewish materials, because the Jewish population of provincial towns and rural places was almost entirely annihilated. As a result of all this, after the 1949 closing of the Jewish Museum more than 50,000 books, hundreds of full or fragmented periodicals sets and about 170 boxes of documents were gathered at the Book Chamber.

Its librarians tried to save Jewish materials not only from loss, but also from further destruction: while observing that the Soviets closed the last existing Jewish institutions, destroyed Jewish cemeteries and implemented covert antiSemitic politics, they understood that Jewish books and documents were at risk to be sent, again, to paper mills. So, on Ulpis orders, they concealed materials in Jewish languages in a huge labyrinthine building of the former St. George Church, which then belonged to the Book Chamber and housed about 1 million books in different languages. This was a treasure with dubious status: saved, but impossible to process, even the materials transferred officially from the Jewish Museum, because the restrictions on any national life and culture gradually became more pronounced.

Restitution could not be mentioned; in fact, the international community was absolutely unaware of these holdings for several decades. Moreover, since the Book Chamber staff segregated materials in Jewish languages and hid them, while continuing to process other printed matter, the parts of former Jewish collections in non-Jewish languages were excluded from Judaica, and their provenance further muddled. Admittedly, this was the legacy of the National Library until recently; however, the Judaica Research Center that was established in the National Library of Lithuania in the 2017 has taken the first steps towards rectifying the situation.

In the 1950ies, some cultural workers from the Lithuanian province trans-

Dawidowicz. From That Place and Time. A Memoir 1938-1947. New-York-London: W.W. Norton\& Co 1989, pp. 314-326; Nancy Sinkoff: From the Archives: Lucy S. Dawidowicz and the Restitution of Jewish Cultural Property. In: American Jewish History, 100, no. 1 (January 2016), pp. 117-147. 
ferred abandoned Jewish materials from their towns to the Book Chamber, although few of these inputs were documented, at least not in detail. The most probable reason for the transfer, apart from the inner professional knowledge that the Book Chamber had such holdings, was the lack of specialists in Jewish languages, and maybe even the unwillingness to hold the potentially dangerous collection. Thus, in 1951, 2,320 Jewish books and bound manuscripts were transferred, without any lists, to the Book Chamber by the Telshiai City Museum. Such documentary materials joined the Judaica holdings and mixed with them, without regard to their different provenances.

Only at the end of the 1980s a small working group of people with knowledge of Jewish languages acquired in a course of pre-war Jewish education was officially invited by a new director of the Book Chamber to process the books and periodicals. The work of the group was lead by Esther Bramson (1924-2016). The gathered manuscript documents had been forgotten in their hiding place and were re-discovered only in 1994, when it was decided to divide them between the Lithuanian State Archives, the manuscript division of the Library of the Academy of Science and the manuscript division of the National Library that at the moment had absorbed the Book Chamber. With that decision, the controversial and misguided process of the physical saving of the documents while confusing traces of their provenance and losing the comprehensive picture, received a new turn. The printed matter and the documents, although closely connected in the past, now belonged to different housing institutions. This step was not considered extreme or even unusual: the absence of provenance research was a commonplace for Soviet-era repositories, and for the Jewish materials it was especially difficult in the light of a lack of exchange with world Judaica collections and no access to academic literature in Jewish Studies.

In the late Soviet period and after desintegration of the Soviet Union Western scholars began getting access to the Judaica holdings of the National Library, and they drew the attention of the international community to this phenomenon and motivated discussion of its status. Different deliberations were presented locally and internationally, including instances of campaigning for relocation of the documents to repositories abroad seen as more appropriate, thus suggesting that no Lithuanian institution has a right to own or keep in trust any documents of previous Jewish ownership; however, no legal claims were made. Those discussions were virtually put to an end by the 1997 visit of the delegation of the Judaica librarians from USA lead by Zachary Baker (then the Head librarian of the YIVO Institute for Jewish Research, later the curator of Judaica/Hebraica collections at the Stanford University) that presented to the National Library and the international specialists their recommendation. The delegation's report advised that the collections should be further preserved in Lithuania with regard 
to their diverse provenances and their significance for Lithuanian cultural life. ${ }^{6}$ (Lithuanian institutions themselves grew to recognize the true cultural value of their collections in almost two decades).

The only instance of restitution was the decision of the National Library to donate more than 300 Torah scrolls from its holdings to the world Jewish communities. It was implemented in 2002 through collaboration with the Heichal Shlomo Museum in Jerusalem, which took upon itself the mediating and actual distributing of the scrolls. In the process of the preparation the defected scrolls were localized and buried at the Jewish cemetery of Vilnius by an international group of rabbis in 1997, in the first such ceremony in the post-war Lithuania.

But if not returned, the books and periodicals were gradually made more accessible through several projects. In cooperation with the Library of Congress all periodicals printed in Lithuania were microfilmed at the end of the 1990s, and later included into the online digital database of the National Library. The project of the Jewish Theological Seminary, the Russian State University of Humanities and the Library brought several groups of students of that university to bibliographic practice in Vilnius, when they helped catalogue the books and were taught to work with Jewish printed materials. The attitude of insecurity on the part of the National Library that represented itself previously through its reluctance to publicize anything about its Judaica holdings changed to openness towards international scholars and institutions of documentary heritage. At the same time, the National Library never undertook an organized effort of provenance research. For about 20 years the bibliographers of the Judaica holdings simply carried on recording known personal and collective owners and doing provenance research for the materials without ownership marks. Until recently, the work with these holdings was not a priority in the Natioanl Library's strategy: the bibliographers were paid salaries, and the books were steadily catalogued, first manually, then digitally, but almost none of them were properly restored, so that their physical state remains mostly the same as it was at the moment of their inclusion into the holdings in the 1944-49. At least the conditions of the storage dramatically improved in 2016 when they were transferred, along with all library holdings, to modern storage facilities. Due to the constant attention of the international community of academics and heritage institutions the National Library came to value its Judaica holdings more and made the work with them an actual agenda. The staff of the Judaica Research Center regards provenance research as one of its main goals. Two collections from the Judaica holdings were nominated by the National Library for acceptance in the National

6 Zachary Baker: Judaica Librarians Visit Vilnius. In: Spoils of War 4 (August 1997), pp. 61-65. 
Register of the UNESCO program Memory of the World and received the nominations.

The biggest part of the documents that had been transferred to the Central State archives had been processed, microfilmed and catalogued through the cooperation with the YIVO Institute for Jewish Research by the end of the 1990s. But the project was not finished, and of course no ties and connections between the documents and the holdings of the National Library were established. As to the manuscript divisions of the National Library and the Library of the Academy of Sciences, the Jewish documents were never processed.

The vital change came in 2013, when the Lithuanian Central State Archives rediscovered the unprocessed part of the documents that arrived from the National Library (about 30 big boxes). This time, they immediately informed the YIVO Institute as their former partner. I was lucky and privileged to make the first assessment of the documents. It showed, not surprisingly, the same provenances as the holdings of the National Library. Later, similar assessments were made of the documents in the manuscript divisions of the National Library and the Library of the Academy of Sciences; the conclusions were the same. Since then, a major joint project of all four institutions called the Edward Blanc YIVO Vilna Collections Project (further - the Vilna Project) has evolved. It aims to process, restore, digitize and put in open virtual access all the documents and several large groups of the books in the aforementioned Lithuanian institutions, the YIVO library and its archives. The main research goal is to finally restore the severed connections between the haphazardly divided parts and put together a comprehensive provenance history. The YIVO Institute has created a website of the Vilna Project that shows the work flow and highlights major findings. ${ }^{7}$ At the end of the project an open database for the documents will be developed which will finally virtually unite them under necessary scholarly guidance. The database, in its turn, will provide international scholars and educators with materials and tools of utmost importance. The decision behind the project was not to undertake any moving of the documents but to virtually bring them together and to the broadest international audience possible. The status of the collections is not to be changed as a result of the project.

This new development will hopefully bring the documents to the fore of public and academic attention, and so undoubtedly broaden and enrich the knowledge of the destroyed Jewish life in Vilnius, Lithuania and other parts of Central and Eastern Europe.

7 See URL: https://vilnacollections.yivo.org/ (accessed on December 15, 2017). 


\title{
Ekaterina Oleshkevich
}

\section{Rediscovering the Schneerson Collection: Historical Aspects and Challenges of Provenance Research}

\section{Zusammenfassung}

Sammlung Schneerson als Fall von geraubtem und verlorenem Besitz: historische Aspekte und aktuelle Situation

Die Sammlung Schneerson - früher Eigentum der Familie Schneerson, die zu den hasidischen Rabbinern des Chabad-Lubawitsch gehörte - wurde nach der Russischen Revolution von 1917 verstaatlicht und ist nun ein wichtiges Beispiel für geraubtes Kulturgut. Die Chabad-Lubawitsch Vereinigung versuchte mit großem Aufwand die Bibliothek zurückzugewinnen, aber die Sammlung wurde nicht restituiert; die Gerichtsverfahren sind noch nicht abgeschlossen. Die russische Regierung ist unter keinen Umständen bereit, die Sammlung abzugeben, wobei auch jegliche rechtliche Grundlagen für eine Restitution fehlen. Was die Situation noch komplexer macht, ist, dass es kein authentisches Inventarverzeichnis für die ursprüngliche Sammlung Schneerson gibt und dass es nach der Verstaatlichung zu einer Vermischung mit anderen Bänden aus der Russischen Staatsbibliothek gekommen ist. Die Identifikation der tatsächlich zur eigentlichen Sammlung Schneerson gehörenden Bücher bleibt eine Herausforderung, auch wenn fortlaufend weitere Forschungsergebnisse hinzu gewonnen werden können, die als verlässliche Eigentümermerkmale für die Sammlung gelten können.

\section{Schlagwörter}

Schneerson-Sammlung, Chabad, Chabad-Dynastie, Provenienzforschung, Eigentümermerkmale, gedruckte Bücher

\begin{abstract}
The Schneerson collection - former property of the Schneerson family of the Chabad Lubavitch hasidic rebbes - was nationalized after the 1917 Russian revolution, and is now a prominent example of looted cultural property. The Chabad Lubavitch movement made great efforts to recover the library; however, the collection was not restituted and the legal proceedings are still ongoing. The Russian government is not willing to surrender the collection under any circumstances nor has it any legal basis for its restitution. What makes the situation even more complicated is that the collection had no authentic inventory and after being nationalized, the Schneerson books were mixed in with other volumes at the Russian State Library's main repository. The identification of the original
\end{abstract}


Schneerson books remains challenging; however, research on the signs of reliable ownership signs continues to bring results.

Keywords

Schneerson Collection, Chabad, Schneerson Dynasty, Provenance Research, Ownership Signs, Printed Books

The Schneerson collection is one of the most famous examples of looted cultural property, and has provoked much controversy, political debate, and legal proceedings between the Chabad Lubavitch movement and the government of the Russian Federation in the 1990s and 2000s. Chabad and the Russian government dispute the ownership of the collection and refuse to compromise. Neither party, however, displays much interest in resolving important pertinent questions, such as what constitutes the Schneerson collection. My article tackles exactly this question.

\section{History of the Schneerson collection before the Russian revolution}

The history of the Schneerson collection begins at the turn of the nineteenth century. The founder of the Chabad movement, a leading branch of Hasidism, and the first Lubavitcher Rebbe, Rabbi Shneur Zalman of Lyady (the Alter Rebbe, 1745-1813), began collecting books for the library. In 1801, his collection included more than 103 printed volumes and 14 manuscripts, according to the inventory compiled by Russian police officials when the Alter Rebbe was arrested. ${ }^{1}$ The inventory featured different classical Jewish books such as the Torah (Pentateuch), Tanakh (Hebrew Bible), Shulchan Arukh as well as multiple Sheelot $\mathrm{u}$-Tshuvot (rabbinical responsa) by different authors and Kabalistic books. ${ }^{2}$ The inventory did not include all the Alter Rebbe's books but only those examined by the Russian police investigators ${ }^{3}$, so it is likely that the collection included more books. However, only a few of these books survived the fires of 1810 and 1812.4

The library of the Alter Rebbe's son, Rabbi Dov Ber (the Mitteler Rebbe, 1773-1827) included 611 volumes arranged in four bookcases, also according to the 1826 police account of the Mitteler Rebbe's arrest and search of his home. ${ }^{5}$

1 Sifriyat Lubavitch [Lubavitch library]. Ed. by Shalom DovBer Levine. New York: Kehot 1993, p. 1 f. [in Hebrew].

2 Ibid. pp. 5-8.

3 Ibid. p. 11.

4 Ibid.

5 Barkussky Ilya: Biblioteka Schneersonov: Kak chastnoe sobranie stalo nazionalnim dostoyaniem [Schneerson library: How the private collection became state property]. In: 
As significant part of the Schneerson library was collected by the third Lubavitcher Rebbe, Rabbi Menachem Mendel (Zemach Zedek, 1789-1866). ${ }^{6}$ According to Rabbi Yoseph Yitzchak Schneerson (1880-1950), the sixth Lubavitcher Rebbe, his father, Rabbi Shalom Dov Ber (1860-1920), the fifth Lubavitcher Rebbe, recalled that Zemach Zedek had five unlocked bookcases with printed books in one room and seven in another; in addition, he had two more locked bookcases with manuscripts. ${ }^{7}$ Booksellers used to come to Lubavitchi twice a year - on the holidays of Shavuot (in summer) and Chanukah (in winter) bringing with them lists of new books so that Zemach Zedek could order books from these lists. ${ }^{8}$ Zemach Zedek usually ordered a large number of books - one order could easily fill an entire bookcase. ${ }^{9}$ However, many books from his library were destroyed in a large fire in Lubavitchi in the 1850 s. $^{10}$

Zemach Zedek's library was divided between his three sons, so the fourth Lubavitcher Rebbe, Rabbi Shmuel (the Maharash, 1834-1882), inherited only a third of his father's book collection. However, the Maharash inherited all the manuscripts collected by his father. ${ }^{11}$ The Maharash catalogued the manuscript collection and labeled most of the manuscripts, including bibliographic descriptions. ${ }^{12} \mathrm{He}$ also added his own books and manuscripts, which he collected from a very young age ${ }^{13}$, to the library and rebound many volumes. ${ }^{14}$

The Maharash's book collection was divided between his sons, while Rabbi Shalom Dov Ber (the Rashab), the fifth Lubavitcher Rebbe, inherited all the manuscripts. ${ }^{15}$ The Rashab and his son Rabbi Yosef Yitzchak Schneerson (the Rayatz) put considerable effort into the preservation and development of the Schneerson collection. They rebound many of the books and marked them with distinct bookplates (ex-libris). The Rashab's correspondence mentions the acquisition of books many times. In his letters, the Rashab asked his son to send him booksellers' catalogs so that he could appraise the books. ${ }^{16}$ Some letters

Lechaim 3 (263), March 2014. URL: http://www.lechaim.ru/ARHIV/263/kabinet-istorikabiblioteka.htm [in Russian] (accessed on June 15, 2017).

6 Sifriyat Lubavitch [Lubavitch library] (Fn. 1), p. 24.

7 Yosef Yitzchak Schneersohn: Likutei dibburim. V. 2. $6^{\text {th }}$ ed. New York: Kehot 1984, p. 457 [in Yiddish].

8 Ibid. p. $457 \mathrm{f}$.

9 Ibid.

10 Shalom Dov Ber Schneersohn: Torat Shalom. $5^{\text {th }}$ ed. New York: Otzar ha-Sfarim 2003, p. 81. Different sources give different dates, from 1856 to 1858. Sifriyat Lubavitch [Lubavitch library] (Fn. 1), p. $34 \mathrm{f}$.

11 Ibid. p. $36 \mathrm{f}$.

12 Ibid. p. 37.

13 Ibid. Schneersohn: Likutei dibburim (Fn. 8), pp. 456-458. [in Yiddish].

14 Sifriyat Lubavitch [Lubavitch library] (Fn. 1), p. 43.

15 Ibid.

16 Ibid. p. 53. 
contain lists of acquired books. ${ }^{17}$ Moreover, the Rashab carefully sorted and arranged his collection in order to find duplicate editions for sale or exchange. However, he preserved the duplicate copies that belonged to his father, the Maharash, which could be easily identified by his father's distinctive binding. ${ }^{18}$

In 1913, the Rashab invited bibliographer Samuel Wiener (1860-1929) from St. Petersburg to help him catalogue the Schneerson library. According to the Rayatz, at that time the collection included only religious Hebrew books, including more than one thousand editions of Sheelot u-tshuvot. ${ }^{19}$ In total, the library boasted about 5,400 editions of Hebrew books, or about 12,000 physical volumes. ${ }^{20}$

During the First World War, in the autumn of 1915, the Rashab and Rayatz, fleeing from the approaching German troops, moved the library from their residence in Lubavitchi to a safe storage place in Moscow (the Kokorevskie sklady, owned by Jewish merchant, book publisher and collector Zelig Persits (?-after 1918). After leaving the library in Moscow, the Rashab and Rayatz moved to Rostov-on-Don, taking with them some manuscripts ${ }^{21}$ and about sixty books. ${ }^{22}$

\section{The Schneerson collection during the Russian revolution and the early Soviet years}

In 1917, the Russian Empire collapsed and after two revolutions, the Bolsheviks seized power and established the Russian Soviet Federative Socialist Republic. This was accompanied by the complete destruction of the Tsarist legal system and the formation of a new legal system. One of the typical features of Bolshevik policy was the requisition of property, affecting libraries as well.

The first decree concerning libraries was issued on 17 July 1918 ("On the Protection of Libraries and Book Repositories") and stated that the care of libraries and books repositories was delegated to the People's Commissariat for Education (known by the abbreviation Narkompros, in Russian). This decree involved the book collections of liquidated and evacuated state offices in Petrograd as well as the libraries of emigrants and dismantled organizations. ${ }^{23}$

17 Ibid.

18 Ibid. p. $57 \mathrm{f}$.

19 Yosef Yitzchak Schneerson: Igrot Kodesh. Ed. and comm. by Shalom DovBer Levine. V. 1. New York: Kehot 1982, p. 538 (commentary) [in Hebrew].

20 Yosef Yitzchak Schneerson: Igrot Kodesh. Ed. and comm. by Shalom DovBer Levine. V. 11. New York: Kehot 1985, p. 82 [in Hebrew].

21 Sifriyat Lubavitch [Lubavitch library] (Fn. 1), p. 63.

22 Ibid. p. 65.

23 Dekret Soveta Narodnyh Komissarov Ob ohrane bibliotek i knigohranilishh [Decree of the 
The next decree, adopted on 26 November, stated that all requisitions of private and public libraries were under the control of Narkompros. ${ }^{24} \mathrm{~A}$ month later, on 27 December Narkompros issued instructions on the requisition of private libraries, stating that private libraries containing more than 500 volumes and belonging to citizens who did not need these books for their work were to be nationalized and declared state property. To keep a library consisting of more than 2,000 volumes, individuals had to obtain written permission from the Libraries Department of Narkompros. ${ }^{25}$

The decree of 5 September 1919 increased the severity of these instructions:

“3) confiscate all collections belonging to persons who fled to the Crimea and other areas of territory occupied by the Whites, or whose whereabouts are unknown, [...];5) deliver all books from all former private collections [...] that are of great historical, scientific and literary interest to special public repositories (the Rumyantsev Museum, the Historical Museum, etc.) [...] ; 6) revise instructions on the order of requisition of libraries relating to the definition of the number of volumes that can be left at the disposal of individuals, with the number decreased to the minimum required to meet the professional needs of their respective owners." ${ }^{26}$

According to the new Soviet laws, the confiscation of the Schneerson library was completely legal. Some of the legal reasons were formulated in official responses to the requests of Y. I. Schneerson in the 1920s: the Schneerson family did not receive written permission from the Libraries Department to keep their library as prescribed by the decrees mentioned above, and their books represented "great scientific and literary interest." 27 In addition, its owners resided on territory occupied by the Whites when the decree was issued: in 1915, the Schneerson family fled to Rostov that became one of the centers of the White movement during the Civil War. The Schneersons stayed in Rostov until the early 1920s.

Council of People's Commissars on the Protection of Libraries and Book Repositories]. In: Sobranie uzakonenij i rasporjazhenij pravitel'stva za 1917-1918 gg. [Compiled Governmental Laws and Decrees for 1917-1918]. Moscow 1942, p. $691 \mathrm{f}$.

24 Dekret Soveta Narodnyh Komissarov. O porjadke rekvizicii bibliotek, knizhnyh skladov i knig voobshhe [Decree of the Council of People's Commissars on the Requisitioning Procedure for Libraries, Bookstores and Storages and Books in General]. In: Sobranie uzakonenij i rasporjazhenij pravitel'stva za 1917-1918 gg. [Compiled Governmental Laws and Decrees for 1917-1918], (Fn. 23), p. 1207.

25 O porjadke rekvizicii chastnyh bibliotek: Instrukcija Narodnogo Komissariata po Prosveshheniju RSFSR ot 27 dekabrja $1918 \mathrm{~g}$. [On the Requisitioning Procedure for Private Libraries: Instruction of People's Commissariat for Education RSFSR from December 27, 1918], in: Instrukcija jemissaram Moskovskogo Bibliotechnogo Otdela [Instruction to the Emissaries of Moscow Libraries Department]. Moscow 1919, pp. 1-16.

26 Postanovlenie SNK o nauchnyh bibliotekah [Decree of SNK on Academic Libraries]. In: Dekrety Sovetskoj vlasti [Decrees of Soviet Government]. Moscow: Politizdat 1973. Vol. 6, p. 402.

27 Ibid. 
In 1919, Narkompros gave orders to the Rumyantsev Museum, which afterward became the Lenin Library and then the Russian State Library:

“1) to inspect the library of Rabbi Schneerson containing a fair amount of valuable editions of the Mishna, the Talmud and commentaries; 2) to remove all editions necessary for your book department and 3) to give an opinion concerning the rest of the books." 28

The first mandate for the transport of the Schneerson Library, packed in 35 crates, to the Rumyantsev Museum was given in February $1920 .{ }^{29}$ However, the library was only sealed in $1920^{30}$ and was not physically transported to the Rumyantsev Museum until June 1924, for technical and financial reasons. ${ }^{31}$

In 1920, R. Shalom Dov Ber passed away, and R. Yosef Yitzchak became his legitimate heir and the sixth Lubavitcher Rebbe. In 1922, he returned from Rostov and settled in Leningrad. He addressed multiple requests to different institutions demanding the return of the library. ${ }^{32}$ He added that in 1921 he had already obtained permission from the Department of Academic Libraries of Narkompros to retrieve the library, but had not been able to do so at that time. ${ }^{33}$

All his requests were refused with references to Soviet laws. It was stated that the Schneerson library must have been considered "property under abeyance", because Y.I. Schneerson did not register the library in the Library Department of Narkompros as the decrees of July 17, 1918 and November 26, 1918 prescribed, and in addition, he did not obtain a protection document for the library that would allow him to keep it, as required in the same November decree. ${ }^{34}$ As for the permission obtained by Y.I. Schneerson, it was not found in the files of the Rumyantsev Museum, and he did not attach the original permission or a copy of it to the request. ${ }^{35}$

28 OHID RGB (Archive of the Russian State Library), op. 14, d. 115, 1. 1. From RSFSR, People's Commissariat for Education, Department of Academic Libraries, 16.12.1919 № 4229/9, To Rumayntsev Museum, Department of Jewish books (copy).

29 OHID RGB, op. 14, d. 115, 1. 3. Mandat to comrade Popov P.S., 26.02.1920, № 642 (copy).

30 OHID RGB, op. 14, d. 115, 1. 5. Act about sealing of the Schneerson library on Sofiyskoe podvorye. 01.03.1920 (copy).

31 OHID RGB, op. 14, d. 115, 1. 19. Rapport of B.I. Lebedev, director of administrative department to the director of Lenin Russian national public library, 28.06.1924. Archive of the Russian State Library, op. 14, d. 115, 1. 20. Act № 186, 28.06.1924 (Copy).

32 OHID RGB, op. 14, d. 115, 1. 12. To the Director of Rumayntsev Museum, Request from Iosif Shalomovich Schneerson living in Moscow at the address M. Zlatoystyevskiy lane, 2, app. 27. 22.11.1922. Archive of the Russian State Library, op. 14, d. 115, 1.24. To Glavnauka, Request from Iosif Shalom Bernich Schneerson living in Leningrad at the address Mokhovaya str., 22/ 12, app. 10. 15.01.1925 (Copy).

33 Ibid.

34 OHID RGB, op. 14, d. 115, 11. 25-26. To the Scientific Department of Glavnauka, № 475, 26. 02. 1925.

35 Ibid. 
In a letter written in 1927, the Rayatz pointed out that the seizure of the library had not been carried out in accordance with Soviet laws, and he put personal responsibility for it on the head of the Jewish department of the Rumyantsev Museum. Samuil Aizenstadt (1886-1970), who immigrated to Palestine in 1925. The Rayatz assumed that this was Aizenstadt's personal revenge for the fact that the leaders of Chabad had argued against emigration to Palestine. According to the Rayatz, Aizenstadt was also responsible for the loss of the permission mentioned above: when Y.I. Schneerson's representative came to the $\mathrm{Ru}$ myantsev Museum to retrieve the books, the permission was taken from him and he was told to come the next day, however, when he came the next day, he was told that the permission had been lost and he needed to obtain a new one. The Rayatz assumed Aizenstadt did this for personal reasons. ${ }^{36}$

In 1927, Yosef Yitzchak was arrested and accused of counter-revolutionary activity and sentenced to death. The death sentence was later changed to deportation from the USSR. After settling in Poland, in a letter dated 1936, the Rayatz asked one of his Hasidim who had remained in the USSR (possibly Zalman Idel Zhislin) to find employment in the Lenin Library without anyone knowing who he was ${ }^{37}$ - probably to gain more information about the situation with the Schneerson books. However, even if he had succeeded in this task, there was no information to find.

When transferred to the Rumyantsev Museum, the Schneerson collection was not preserved separately as a collection, but was mixed with other volumes confiscated from different libraries owned by individuals or institutions. ${ }^{38}$ The collection did not have an accurate inventory - the Rumyantsev Museum staff accounted for it in crates, stating that they had transferred 35 crates of books with a total weight of 411 pud $^{39}$ (approximately 6,700 kg). Therefore, even if Schneerson's request had been satisfied, it would have been impossible to return the library to him because it was already unclear which books belonged to the Schneerson collection and which did not.

In 1925, realizing that restitution of the library would be a lengthy process, $R$. Yosef Yitzchak decided to buy the library of Shmuel Wiener, who was a famous bibliographer invited to Petersburg in 1887 to catalogue the Jewish collection of the Institute of Oriental Studies and then helped Lev Friedland (1826-1899) with his collection, which he later donated to the Institute of Oriental Studies

36 Schneerson: Igrot Kodesh. V. 11 (Fn. 20), p. 82.

37 Yosef Yitzchak Schneerson: Igrot Kodesh. Ed. and comm. by Shalom DovBer Levine. V. 13. New York: Kehot 1993, p. 244f. [In Hebrew].

38 OHID RGB, op. 14, d. 115, 1. 29. To the Scientific Department of Glavnauka. № 4449, 29. 11. 1927.

39 OHID RGB, op. 14, d. 115, 1. 20. Act № 186, 28.06.1924. 
("Bibliotheca Friedlandiana.") ${ }^{40}$ He was a famous book collector and his library included more than 13,000 books and manuscripts. ${ }^{41}$ As mentioned above, Shmuel Wiener was previously involved in the organization of the Schneerson library in Lubavitchi. The Wiener library was the basis for today's Chabad library in Brooklyn. ${ }^{42}$ When leaving the USSR for Riga and then Warsaw, Yosef Yitzchak took the Wiener books with him. ${ }^{43}$ In 1940, when fleeing Nazi-occupied Poland to the United States, he left the books behind but managed to retrieve them after the Second World War.

\section{The Comeback of the Schneerson collection in the 1990s: certain legal issues}

From the late 1930s to the late 1980s, the issue of the collection was not discussed. It was brought up again in 1988 when the Chabad delegation visited Moscow and learned that for all those years the Schneerson collection had been preserved in the Lenin Library. ${ }^{44}$ In 1990, Chabad filed a lawsuit demanding the return of the Schneerson library to its legitimate heirs, which ended up in multiple court proceedings in Russia and the U.S. The details of the legal argument are thoroughly described in other works ${ }^{45}$ therefore only several legal points that are important for the discussion will be mentioned here.

The main legal issue is that Russia has no law about the restitution of property nationalized within the former USSR after the Russian revolution. The reason for this is quite simple: the amount of nationalized property was huge. Not only Jewish collections were confiscated but also the property of nobles, emigrants, Tsarist institutions, the Orthodox Church etc. The adoption of a law on the

40 Semen Yakerson: Evreyskie sokrovischsha Peterburga. Svitki, kodeksi, dokumenti [Jewish treasures of Peterburg. Scrolls, codices, documents]. Peterburg: Arka 2008, p. 59 [in Russian].

41 Ilya Barkussky: Biblioteka Schneersonov do 1928 goda: sobytiya i ludi [Schneerson library before 1928: events and people]. In: Izrail drevniy i noviy. Trudi kafedri iydaiki [Israel Ancient and Modern. Works of Department of Jewish Studies]. Ed. by Arkady Kovelman. Moscow: Indrik 2015. V. 1, p. 179.

42 Sifriyat Lubavitch [Lubavitch library] (Fn. 1).p. 90.

43 Russian State Military Archive. F. 706, op. 1, d. 9, l. 1. Act., 03.10.1927.

44 Patricia Kennedy Grimsted: Beyond Cold War Over a Restitution Claim? In: Antiquity and Law. V. XVIII, Issue 4, December 2013, pp. 345-406, here p. 353.

45 Thorough research of the topic was conducted by Patricia Kennedy Grimsted, and specific subjects of the case were examined by other researchers as well. Patricia Kennedy Grimsted: Beyond Cold War Over a Restitution Claim? In: Antiquity and Law. V. XVIII, Issue 4, December 2013, pp. 345-406; Michael J. Bazyler, Seth M. Gerber: Chabad v. Russian Federation: A Case Study in the Use of American Courts to Recover Looted Cultural Property. In: International Journal of Cultural Property. Vol. 17, Issue 2, May 2010, pp. 361-386 and other. 
restitution of this property would mean the complete redistribution of a large amount of current state property, a very difficult and nearly impossible task.

Several decrees and regulations protect the cultural objects and the holdings of museums and libraries. A decree from 30 November 1992 prohibits alienating "the most valuable monuments of cultural heritage" 46 and a regulation of the Russian State Library declares its holdings "unalienable." ${ }^{\text {" }}$

Recently, in 2010, a new law concerning this issue was adopted - the federal law, "On the Transfer to Religious Organizations of Property of Religious Significance Held in State or Municipal Ownership" that allows the transfer to religious organizations of unmovable property of religious significance, but prohibits the transfer of movable property. ${ }^{48}$

Therefore, from the perspective of Russian law, the Schneerson library cannot be alienated nor restituted to Chabad Lubavitch.

In 2013, in an attempt to reach a compromise, it was agreed that the Schneerson collection of printed books (not including manuscripts) ${ }^{49}$ would be moved to a special branch of the Russian State Library (RSL) in the Jewish Museum and Tolerance Center in Moscow. However, Chabad was not satisfied with this decision, calling for the complete restitution of the library and its transfer to America. ${ }^{50}$

46 Postanovlenie ob osobo cennyh ob'ektah kul'turnogo nasledija narodov Rossijskoj Federacii ot 30 noyabrya 1992 goda № 919 [Decree on the Most Valuable Monuments of Cultural Heritage of the Peoples of the Russian Federation from November 30, 1992, № 919], [in Russian] Accessible online, URL: http://docs.cntd.ru/document/9005803 (accessed on June 15, 2017).

47 Ustav Rossijskoj gosudarstvennoj biblioteki [Regulation of the Russian State Library], [in Russian]. Accessible online, URL: http://www.rsl.ru/datadocs/doc_4825mu.pdf (accessed on June 15, 2017).

48 Federal'nyj zakon ot 30 nojabrja 2010 g. N 327-FZ "O peredache religioznym organizacijam imushhestva religioznogo naznachenija, nahodjashhegosja v gosudarstvennoj ili municipal'noj sobstvennosti” [Federal Law from November 30, 2010 № 327-FZ “On the Transfer to Religious Organizations of Property of Religious Significance Held in State or Municipal Ownership"] [in Russian]. Accessible online, URL: https://rg.ru/2010/12/03/tserkovnoedo bro-dok.html (accessed on June 15, 2017).

49 Manuscripts are preserved in the Department of Manuscripts in the RSL, and the agreement on the transfer of the Schneerson collection to the new RSL branch in the Jewish Museum and Tolerance Center concerned only printed books previously preserved in the Department of Oriental Literature. The private archive of Y.I. Schneerson, partly seized at the end of the Second World War in Poland and now preserved in the Russian State Military Archive, was not moved to the RSL branch in the Jewish Museum. Therefore, the discussion concerning signs of ownership below is based completely on the material of printed books. The detected signs may be valid for the manuscripts as well; however, research on this particular topic has not been conducted yet.

50 Grimsted: Beyond Cold War Over a Restitution Claim? (Fn. 45), pp. 345-406, here pp. 394-398. 


\section{What is the Schneerson collection?}

As mentioned above, there was no accurate inventory of the Schneerson collection, and when it was transferred to the Rumyantsev Museum, it was intermixed with other volumes. The collection was never even catalogued until 2013 when it was moved to the RSL branch in the Jewish Museum and Tolerance Center.

In the court proceedings, Chabad has been demanding the restitution of 12,000 books that allegedly were part of the Schneerson collection. ${ }^{51}$ An inventory serving as an exhibit in court listed 5,641 items and was allegedly prepared in 1914 in Lubavitchi. The origins of this inventory remain unclear, however, these numbers appeared much earlier - already in a letter dated 1927, the Rayatz mentioned that the library contained more than 5,400 "numbers" (i. e. editions - each Talmud was considered one edition, even if it consisted of 20 volumes), which meant about 12,000 volumes. ${ }^{52}$ In another letter dated 1932, he mentioned that he had the inventory from the very moment when the books were sent to Moscow. ${ }^{53}$

Although the numbers correspond, the inventory presented to the court cannot be considered conclusive evidence - besides its unclear origins, attempts to find the books in the inventory in the catalogue of the RSL as well as attempts to find the books that definitely belonged to the Schneerson collection (bearing unmistakable ownership signs) in the inventory were unsuccessful. The Chabad representatives also agree that many Schneerson books, for instance, mentioned as recently bought from the letters of the Rashab or Rayatz, are not present in the inventory, but they explain this by saying that the inventory was probably not complete and the books from the private library of the Rayatz were possibly not included in the list. ${ }^{54}$ However, in his letters, the Rayatz did not mention the inventory being incomplete and, as is possible to conclude from the context, definitely did mean the entire library when mentioning 5,400 "numbers" and 12,000 volumes. This fact additionally challenges the reliability of this inventory.

Therefore, what is the Schneerson collection preserved in the branch of the RSL in the Jewish Museum and Tolerance Center in Moscow? In 2000, Shalom DovBer Levine, the head of the Chabad Library in Brooklyn, together with RSL staff, identified 4,354 books that allegedly belonged to the Schneerson collection $^{55}$. He had two days to do this and was not allowed to go through all the Jewish

51 Ibid. p. 357.

52 Schneerson: Igrot Kodesh. V. 11 (Fn. 20), p. 82.

53 Ibid. p. 215.

54 Sifriyat Lubavitch [Lubavitch library] (Fn. 1), p. 62 f.

55 Now the Schneerson collection consists of 4,655 books. However, the number of physical copies did not increase. The reason is that technically the RSL recognizes as a book not a 
holdings of the RSL Department of Oriental Literature where the books in Jewish languages were preserved. Additionally, the principles used by Levine while choosing the Schneerson collection books remain unclear. ${ }^{56}$

These 4,354 books are considered now officially to be the "Schneerson collection" and are preserved in the branch of the RSL in the Jewish Museum. However, as it is already clear, this is a kind of "commonly agreed" upon phantom, and today's Schneerson collection is most likely a mixture of books owned by the Schneerson family and books they never possessed. Two days were certainly not enough for a thorough examination of thousands of books and everything was done in a hurry, and then, after the cataloguing of these books was over, the conclusion was disappointing: about half the books did not contain any signs of ownership and did not offer any means by which to deduce their owner. Some of the books bear ownership signs that make them highly improbable candidates for the Schneerson collection: for instance, books printed in the early $20^{\text {th }}$ century with the stamps of Latvian synagogues etc. Only about 2,000 books from this phantom "Schneerson collection" can be said for sure to belong to the Schneerson family.

\section{Deducing ownership}

What are the reliable signs proving Schneerson ownership? The research on this topic is far from complete, and cataloguing was only the first step, but for now, the following reliable ownership signs have been detected:

- Distinctive binding and ex-libris of R. Yosef Yitzchak Schneerson

- Stamps and ownership inscriptions of the members of the Schneerson family or of the Tomchei Tmimim Yeshiva

- Marginalia written by R. Yosef Yitzchak Schneerson

- Stamps and owner's inscriptions of other prominent Hasidim (especially Zalman Idel Zhislin)

The binding typical of the Schneerson collection features the Hebrew letters YudShin meaning "Yosef Schneerson" as super ex-libris on the spine. Even if the spine is damaged, the binding can be recognized by the typical embossed diagonal stripes on the front cover. The binding is usually green; however, there are

physical volume, but so called registration number: the code assigned to each edition or part of serial edition, and a physical copy can contain several registration numbers in case of a convolute. The number of books (i. e. registration numbers) increased, because during cataloguing new parts of convolutes were found, and additional registration numbers were assigned to them.

56 Grimsted: Beyond Cold War Over a Restitution Claim? (Fn. 45), pp. 345-406, here p. 371. 
some examples of blue and black bindings, as well as instances of the letters YudShin embossed on the spine of regular bindings, not the distinctive Schneerson ones.

The ex-libris of Y.I. Schneerson on the inside front cover also proves Schneerson ownership. Other Lubavitcher Rebbes apparently did not have bookplates with their names and got along just by either writing about their ownership on the title page and front cover or by putting their personalized stamp - or both. The owner's inscriptions of the Lubavitcher Rebbes or of their family members - even if not written by them personally but by their secretaries - prove Schneerson ownership as well as the stamps of the Lubavitcher Rebbes. For now, the stamps of the Maharash, Rashab and Rayatz were detected in the books, but the search goes on.

Stamps of the Tomchei Tmimim Yeshiva are proof of Schneerson ownership as well. Though the books of the Lubavitchi Yeshiva were not mentioned in documents or letters as being among the books sent to safe storage to Moscow, they were probably considered a part of the Schneerson dynasty library and therefore were not mentioned separately. Apparently, they found their way to Moscow together with the Schneerson library and could be considered part of it.

The hardest ownership sign to define is handwritings. As of now, only the handwriting of Yosef Yitzchak Schneerson has been identified and is considered a sufficient ownership sign.

The ownership inscriptions and stamps of Zalman Idel Zhislin (Zislin) as well as those of his father, Baruch Yehuda Leib Zhislin, are considered a sign of Schneerson ownership as well. Z.I. Zhislin was one of the prominent Hasidim who belonged to the intimate circle of the Rashab, copied out his works ${ }^{57}$ and later dealt with the publication of the "Torah Or" siddur in Rostov-on-Don in 1918, including an introduction of corrections. ${ }^{58}$ Apparently, he was responsible for the organization of the Rashab's library in Lubavitchi and/or for buying books for him as well. Later, in the 1930s, the Rayatz, who had already been deported from the USSR, asked one of his Hasidim - probably Zalman Idel Zhislin - to find out more information about his books in the Lenin Library. ${ }^{59}$ Afterwards, in 1936, Z.I. Zhislin wanted to print the "Torah Or" siddur one more time and to introduce several more corrections, and he wrote about this in a letter to the Rayatz asking if he should do it. ${ }^{60}$

57 Taarukhat Raboteinu Nasieynu [The materials of the exhibition "Our Rebbes"]. New York: Sifryat Agudas Hasidei Chabad 2017, p. 9. URL: http://www.col.org.il/files/0.8167346734_ 2100645.pdf (accessed on June 15, 2017).

58 Menachem Mendel Schneerson: Hagahot le-siddur rabeinu ha-zaken [Corrections of the Siddur Arranged by Alter Rebbe]. New York: Kehot 2006, p. 9 [in Hebrew].

59 Schneerson: Igrot Kodesh. V. 13 (Fn. 37), p. 244 f.

60 Schneerson: Hagahot le-siddur rabeinu ha-zaken (Fn. 59), p. 9. 
There are several questionable types of ownership signs:

- "Brown binding"

- "Librarian handwriting"

- Any stamps featuring the word "Lubavitchi"

Numerous books from the official Schneerson collection are bound in "brown binding" featuring dark-brown fabric-covered covers and a leather light-brown spine with a black-out in the middle and a silver-embossed book title on it. Several volumes bound in this binding feature the unquestionable ownership signs of the Schneerson library, but whether or not the binding itself can serve a sufficient proof of ownership, is still in question. If the binding with Yud-Shin on the bottom of spine could have been made for the private library of Y.I. Schneerson, this binding could define the library of his father, Shalom Dov Ber, who used to rebind the books in a personalized binding, or even the books Shalom Dov Ber inherited from his father, Shmuel, who was also known to have had the books in his library bound in a personalized binding. ${ }^{61}$

"Librarian handwriting" usually indicates bibliographical details about a book such as book title, author, place and date of publication, etc. (especially when it lacks a title page) or the particularities of a certain volume such as absent or damaged folios, handwritten pages etc., therefore it was called "librarian handwriting." In some cases, such notes are made in the hand of Y.I. Schneerson; in other by someone else, the so-called "librarian". Though the "librarian handwriting" appears in many books bearing unmistakable Schneerson ownership signs, the research team of the Schneerson library has not achieved consensus yet, on whether or not this handwriting can be considered a sign of Schneerson ownership. The added complications are, first, that in many cases these notes are rather short and do not provide enough written material for analysis and second, that the handwriting slightly differs in different books that provokes the question of whether it is actually one person's handwriting or several different ones.

The last questionable sign, the mention of "Lubavitchi" in a stamp or an inscription is the most doubtful one. Certainly, the city of Lubavitchi was not very large and its population consisted mostly of Lubavitch Hasidim who would present their Rebbes with books for some holiday, knowing that they liked to collect books (such cases were discovered). Still, there is a possibility that some Hasidim had their own libraries that never intermixed with the Schneerson library before becoming the Jewish collection of the RSL. However, a book with the stamp of Lubavitchi could with much higher probability belong to the Schneerson collection than a book without this sign.

61 Cf. section "History of the Schneerson collection before Russian revolution". 
Many other types of signs require further research. First, the handwriting of other Lubavitcher Rebbes needs to be examined and identified. Second, other owner's stamps and inscriptions must be researched and if the connection between the owners mentioned in the stamp or inscription with the Schneerson family is proven, that will provide us with additional ownership signs. However, many books lack any ownership signs, making their provenance extremely difficult if not impossible to determine. The letters of the Lubavitcher Rebbes and acquisitions of books mentioned in them may be of additional help in provenance research. A thorough examination of these letters may help in specifying the provenance of the books that lack any ownership signs.

Unfortunately, the administration of the RSL as well as Russian government do not show much interest in provenance research. The reasons for the lack of interest are multiple. On the one hand, the RSL considers books (Schneerson books as well as other ones) more in terms of their bibliographical value than in terms of provenance. On the other hand, the Russian policy concerning the restitution of looted property as well as the political controversy and legal proceedings provoked by the Schneerson collection prevent governmental organizations from dealing with provenance research in general and of the Schneerson collection specifically.

For the same reasons access to other Jewish holdings of the RSL required for searching for other Schneerson collection books is restricted. Therefore, what the Schneerson library staff are able to do is only to compile lists of the books that certainly did or did not belong to the original collection and to research available signs of the Schneerson ownership and provenance of the books comprising the formal "Schneerson collection".

As for now, the part of the formal collection that unambiguously belonged to the Schneerson dynasty consists of books written mostly in Hebrew and in rare cases in Yiddish and printed between 1547 and 1914. The thematic scope of this original part of the collection spans Hebrew Bible, Jewish religious law, theology, and Hasidism, however, Hasidic literature comprises a rather small part of it (in comparison with other parts).

Thus, the Schneerson collection issue is still far from being concluded for many reasons. First, Chabad still hopes to retrieve the books and the legal proceedings remain unfinished. Second, the books preserved in the branch of the RSL in the Jewish Museum and Tolerance Center in Moscow can very highly probably not be considered the authentic Schneerson collection; therefore, the signs of Schneerson ownership still require research and thorough examination. 


\section{Abbildungsnachweis}

Markus Stumpf / Christina Köstner-Pemsel / Olivia Kaiser, „Treuhänderisch“ Themenaufriss im Kontext der NS-Provenienzforschung

Abb. 1 Universitätsbibliothek Wien

Abb. 2 Joseph Krpelan/www.derknopfdruecker.com

Abb. 3 Joseph Krpelan/www.derknopfdruecker.com

Alexandra Caruso / Anneliese Schallmeiner, Das Bundesdenkmalamt und der Bestand der sogenannten „1960er Jahre Zuweisungen“

Abb. 1 Lisa Frank/Anneliese Schallmeiner

Abb. 2 Archiv des Bundesdenkmalamts, Österreich

Abb. 3 Archiv des Bundesdenkmalamts, Österreich

Jana Kocourek, „Offene Vermögensfragen“ - von der Suche nach sogenannten Schlossbergungsbeständen in der SLUB Dresden

Abb. 1 Sächsische Landesbibliothek - Staats- und Universitätsbibliothek Dresden (SLUB)/ Deutsche Fotothek

Abb. 2 Sächsische Landesbibliothek - Staats- und Universitätsbibliothek Dresden (SLUB)/ Deutsche Fotothek

Abb. 3 Sächsische Landesbibliothek - Staats- und Universitätsbibliothek Dresden (SLUB)/ Deutsche Fotothek 
Michal Bušek, Provenance Research in the Book Collection of the Jewish $\mathrm{Mu}-$ seum in Prague

Abb. 1 Michal Bušek/ Jewish Museum in Prague

Abb. 2 Michal Bušek/ Jewish Museum in Prague

Abb. 3 Michal Bušek/ Jewish Museum in Prague

Abb. 4 Michal Bušek/ Jewish Museum in Prague

Johana Prouzová, Die Sammlung „Pollák“ in den Prager Museen

Abb. 1 Nationalgalerie in Prag

Abb. 2 Nationalgalerie in Prag

Abb. 3 Nationalgalerie in Prag

Abb. 4 Kunstgewerbemuseum in Prag

Abb. 5 Kunstgewerbemuseum in Prag

Abb. 6 Kunstgewerbemuseum in Prag

Abb. 7 Kunstgewerbemuseum in Prag

Abb. 8 Kunstgewerbemuseum in Prag

Abb. 9 Kunstgewerbemuseum in Prag

Monika Mayer, „Treuhänderische“ Übergaben von Kunstwerken an die Österreichische Galerie im Kontext der aktuellen Provenienzforschung

Abb. 1 Belvedere, Wien

Abb. 2 Belvedere, Wien

Abb. 3 Belvedere, Wien

Monika Löscher, Die „1963er Zuweisungen“ an das Kunsthistorische Museum. Einige Fallbeispiele

Abb. 1 KHM-Museumsverband

Abb. 2 KHM-Museumsverband

Abb. 3 KHM-Museumsverband

Abb. 4 KHM-Museumsverband

Abb. 5 KHM-Museumsverband

Abb. 6 KHM-Museumsverband

Abb. 7 KHM-Museumsverband

Abb. 8 KHM-Museumsverband 
Christian Mertens, „[...] ich kann Sie versichern, daß ich Ihnen das Paket mit den biogr. Schriften mit dem größten Vergnügen aufhebe“. „Treuhänderisch“ übernommene Sammlungen in der Wienbibliothek

Abb. 1 Wienbibliothek

Abb. 2 Wienbibliothek, Handschriftensammlung, H.I.N. 232611

Johannes Gramlich, Kunstwerke aus NS-Besitz auf dem Weg in die Bayerischen Staatsgemäldesammlungen - Amerikanische Restitutionspolitik und bayerische Treuhänderschaft

Abb. 1 Johannes Gramlich/National Archives

Abb. 2 Bundesarchiv/Bundesrepublik Deutschland

Abb. 3 Bundesarchiv/Bundesrepublik Deutschland

Meike Hopp, "In Trusteeship" or "Guilty Secret"? The "Rudolf von Alt Aktion" 1938, the "Collection" of Martin Bormann and the "Fiduciary" Transfers of "Former Nazi Property" to the Bavarian State after 1945

Abb. 1 Staatliche graphische Sammlung, München Abb. 2 Staatliche graphische Sammlung, München Abb. 3 Staatliche graphische Sammlung, München Abb. 4 Staatliche graphische Sammlung, München

Stephan Kellner, Abgabe der Alliierten: Die Bibliothek der NS-Ordensburg Sonthofen in der Bayerischen Staatsbibliothek

Abb. 1 Bayerische Staatsbibliothek München/Bildarchiv 
Open-Access-Publikation im Sinne der CC-Lizenz BY-NC-ND 4.0

(c) 2018, V\&R unipress GmbH, Göttingen

ISBN Print: 9783847107835 - ISBN E-Lib: 9783737007832 


\section{Kurzbiographien der Autorinnen und Autoren}

Prof. James D. BINDENAGEL - Henry-Kissinger-Professor; Leiter des Center for International Security and Governance an der Universität Bonn

James D. Bindenagel blickt auf rund 30 Jahre Erfahrung im diplomatischen Dienst der Vereinigten Staaten von Amerika zurück. Von 1996 bis 1997 war er US-Botschafter in Deutschland. Als maßgeblicher Diplomat verhandelte er die Entschädigung der Zwangsarbeiter während des Nationalsozialismus, die Washingtoner Prinzipien über die von Nationalsozialisten konfiszierten Kunstwerke und das Abkommen über den „Kimberley-Prozess“, das dazu dient, den Handel mit so genannten „Blutdiamanten“ zu unterbinden. James D. Bindenagel war mehrere Jahre stellvertretender Leiter eines amerikanischen Think Tanks und Vizepräsident der DePaul Universität in Chicago.

James D. Bindenagel looks back on 30 years of experience in the diplomatic service of the United States of America. From 1996 to 1997 he was U.S. Ambassador to Germany. An authoritative diplomat, he has negotiated the compensations for forced workers during the Nazi Regime, the Washington Principles on NaziConfiscated Art and the Kimberly Process Certification Scheme, in ordert o prevent "conflict diamonds". James D. Bindenagel was deputy head of an American think tank and vice president oft he DePaul University in Chicago.

\section{Michal BUŠEK - Jewish Museum Prague}

Geb. 1975, Forscher im Jüdischen Museum Prag; forscht zur Identifikation von Vorbesitzern und bearbeitet die Vorbesitzer-Datenbank des Museums. Kurator von drei Ausstellungen und mehreren Katalogen zum Thema. Mitglied der Restitutionskommission des Jüdischen Museums Prag.

Born 1975, works as a Jewish Studies researcher at the library of the Jewish Museum in Prague. Here he oversees the database of "original owners of the books". He curated three exhibitions and catalogues. He is a member of the Restitution Commission of the JMP. 
Alexandra CARUSO - Kommission für Provenienzforschung - Bundesdenkmalamt, Wien

geb. in Wien, Übersetzer- und Dolmetschstudium / Uni. Wien, Kulturmanagementlehrgang / Uni. Linz.; Studium d. Kunstgeschichte / Uni. Wien; seit 2000 in der Provenienzforschung tätig, seit 2006 im Büro d. Kommission für Provenienzforschung; Publikationen u.a.: NS-Kunstraub in Österreich und seine Folgen bis heute (2005, gemeinsam mit G. Anderl), Tagebücher Erica Tietze-Conrat (2015). Born in Vienna, studied translation and interpreting as well as art history at the University of Vienna, completed a course on culture management at the University of Linz. Provenance researcher since 2000 - Member of the Commission of Provenance Research since 2006. Publications on topics such as Looted Art in Austria and Its Consequences (2005 together with Gabriele Anderl) and the diaries of Erica Tietze-Conrat (2015).

\section{Nawojka CIEŚLIŃSKA-LOBKOWICZ - Warschau}

Polnische Kunsthistorikerin und unabhängige Expertin für NS-Raub der polnischen und jüdischen Kulturgüter und Bibliotheken im besetzten Polen, Nachkriegsrestitutionen und Provenienzfragen. Zahlreiche Publikationen in Polnisch, Englisch und Deutsch. Lebt in Warschau und in Starnberg nahe München.

Polish art historian and independent expert on the history of Polish and Jewish looted art and libraries, post-war restitution and issues of provenance. She has published articles on those subjects in Polish, English and German. She lives in Warsaw and in Starnberg near Munich.

\section{Christian GEORGE - Universitätsbibliothek Mainz}

Studium der Geschichtswissenschaft an der Universität Bonn. 2010 Promotion mit einer Arbeit über Studenten in der Nachkriegszeit. 2009-2012 Archivar am Landeshauptarchiv in Koblenz. Seit 2012 Leiter des Universitätsarchivs Mainz. Seit 2013 zuständig für die Altbestände der UB Mainz.

Master of Arts in History at Bonn University. 2010 PhD with a thesis concerning student life after World War II. 2009-2012 archivist at Landeshauptarchiv Koblenz. Since 2012 head of Mainz University Archives. Since 2013 responsible for the rare book collection at Mainz University Library.

\section{Johannes GRAMLICH - Bayerische Staatsgemäldesammlungen München}

Dr. phil., wissenschaftlicher Mitarbeiter für Neueste Geschichte und Zeitgeschichte an den Universitäten Köln und München 2010-2012; Stipendiat des Instituts für Europäische Geschichte in Mainz 2013; Promotion LMU München 2014; wissenschaftlicher Mitarbeiter am Institut für Zeitgeschichte MünchenBerlin 2014-2016; seit 2016 wissenschaftlicher Mitarbeiter an den Bayerischen Staatsgemäldesammlungen. 
Research assistant at the Bavarian State Painting Collections (Provenance Research Unit); previously research assistant for Modern and Contemporary History at the University of Cologne, at the LMU Munich, and at the Institute for Contemporary History Munich-Berlin; Research on the international art market, on Nazi looted art and restitution as well as on the collections Thyssen and Gurlitt.

\section{Meike HOPP - Zentralinstitut für Kunstgeschichte (ZI) München}

Studium der Kunstgeschichte, Archäologie und Theaterwissenschaften an der Ludwig-Maximilians-Universität (LMU) München. 2008 Heinrich-WölfflinPreis der LMU München. 2012 Promotion zum Thema „Kunsthandel im Nationalsozialismus. Adolf Weinmüller in München und Wien“. Seit 2009 wissenschaftliche Mitarbeiterin am Zentralinstitut für Kunstgeschichte (ZI) in München, im Rahmen verschiedener Projekte zur Provenienzforschung u.a. in Kooperation mit der Staatlichen Graphischen Sammlung in München und dem Projekt „Provenienzrecherche Gurlitt“ der Stiftung Deutsches Zentrum für Kulturgutverluste (DZK). Siehe: http://www.zikg.eu/institut/personen/mhopp. 2008 M.A. in art history, archaeology and theatre history at Ludwig-MaximiliansUniversität (LMU) in Munich. 2008 Heinrich-Wölfflin-Prize, LMU Munich. 2009-2011 postgraduate studies. Received her Ph.D. from the University of Munich in 2012 for her thesis Kunsthandel im Nationalsozialismus: Adolf Weinmüller in München und Wien (Cologne: Böhlau 2012). Since then she has worked on several projects within the field of the art market, looted art and provenance research at the Zentralinstitut für Kunstgeschichte (ZI) München, e.g. in cooperation with the Staatliche Graphische Sammlung Munich as well as the project Gurlitt Provenance Research of the German Lost Art Foundation. See: http://www. zikg.eu/institut/personen/mhopp.

\section{Olivia KAISER - Universitätsbibliothek Wien, Universität Wien}

Studium der Geschichte und Cultural Studies in Wien und Madrid. 2009-2011 Mitarbeit bei Namentliche Erfassung der Ravensbrück-Häftlinge aus Österreich am Institut für Konfliktforschung Wien, seit 2009 Mitarbeiterin der Universitätsbibliothek Wien in diversen forschungsunterstützenden Services, 2010-2011 Projektmitarbeiterin bei www.ns-quellen.at, 2011-2012 Projektmitarbeiterin in der Provenienzforschung in der Parlamentsbibliothek, seit 2013 Provenienzforscherin an der Universitätsbibliothek Wien; Mitglied der Arbeitsgruppe NSProvenienzforschung der Vereinigung Österreichischer Bibliothekarinnen und Bibliothekare (VÖB).

Studied History and Cultural Studies in Vienna and Madrid. 2009-2011 project staff at "Namentliche Erfassung der Ravensbrück-Häftlinge aus Österreich" at the Institute of Conflict Research in Vienna, since 2009 staff member at Vienna University Library in different research supporting services, 2010-2011 project 
staff at www.ns-quellen.at, 2011-2012 project staff at NS-Provenance Research in the Library of the Austrian Parliament, since 2013 staff member of the NS-Provenance Research at the Vienna University Library; Member of the Working Group on NS-Provenance Research of the Association of Austrian Librarians (VÖB).

\section{Stephan KELLNER - Bayerische Staatsbibliothek München}

Landes- und Kunsthistoriker. Leiter des Referats Bavarica der Bayerischen Staatsbibliothek (BSB). Inhaltlich zuständig für die digitalen Angebote der BSB für Bayern (Bayerische Landesbibliothek Online, Literaturportal Bayern, Historisches Lexikon Bayerns, bavarikon). Seit 2003 in der NS-Raubgutforschung tätig, d.h. für die Suche in den Beständen der BSB nach unrechtmäßigen Erwerbungen zwischen 1933 und 1945 und in der Nachkriegszeit. Bislang konnten über ca. 500 Bände restituiert werden.

Studied History and Art History. Works as head of Bavarica Department of the Bavarian State Library. Is responsible for the digital offers of the BSB for Bavaria (Bavarian Landesbibliothek Online, Literaturportal Bayern, Historisches Lexikon Bayerns, bavarikon). Since 2003 working in research into unlawful acquisitions between 1933 and 1945 and during the post-war period in the Bavarian State Library. So far, more than 500 books have been returned to the rightful owners.

Jana KOCOUREK - Sächsische Landesbibliothek - Staats- und Universitätsbibliothek Dresden

Studium Germanistik und Geographie (TU Dresden), Bibliotheks- und Informationswissenschaften (HU Berlin); ab 2009 Provenienzforschungsprojekte an der SLUB Dresden (Bodenreform, NS-verfolgungsbedingt entzogenes Kulturgut), seit 2016 Leiterin der Abteilung Handschriften, Alte Drucke und Landeskunde.

Studied German Language and Literature and Geography (Technical University Dresden), Library and Information Science (Humboldt University Berlin); since 2009 projects for provenance research at the Sächsische Landesbibliothek -Staatsund Universitätsbibliothek Dresden (land reform, Nazi looting cultural property), since 2016 head of the department of manuscripts, Rare and Precious Prints and Saxonia.

\section{Christina KÖSTNER-PEMSEL - Universitätsbibliothek Wien, Universität Wien} Bibliothekarin und Provenienzforscherin an der Universitätsbibliothek Wien. 2003-2005 FWF-Projekt Geschichte der Österreichischen Nationalbibliothek in der NS-Zeit (Dissertation). Kuratorin der Ausstellung "Geraubte Bücher. Die Österreichische Nationalbibliothek stellt sich ihrer NS-Vergangenheit“ (gem. 
mit Margot Werner). Zuletzt Masterarbeit „Die Lust zur Ordnung“. Bibliothekarinnen an wissenschaftlichen Bibliotheken in Österreich (2015).

Academic Librarian and provenance researcher at the University Library Vienna. 2003-2005 Austrian Science Fund (FWF) project about the history of the Austrian National Library during the Nazi period. Curator of the exhibition "Geraubte Bücher. Die Österreichische Nationalbibliothek stellt sich ihrer NS-Vergangenheit" (together with Margot Werner). Masterthesis about Women in research libraries in Austria.

Lara LEMPERTIENE் - National M. Mažvydas National Library of Lithuania Leiterin des jüdischen Zentrums an der Litauischen Nationalbibliothek. Forschungsschwerpunkte sind Kulturgeschichte der europäischen Juden, speziell der Klassischen jüdischen Texte und ihre Integration in die jüdische Erziehung unter verschiedenen Gesichtspunkten; jüdische Bücher und Presse; Forschungen zum intellektuellen, sozialen und Alltagsleben der litauischen Juden und des jüdischen Erbes Litauens.

Head of the Judaica Center at the National Library of Lithuania. Her field is the cultural history of the European Jewry, more specifically - Jewish classical texts and their integration in Jewish education in various settings; Jewish book and press; intellectual, social and day-to-day life of Lithuanian Jewry; and Lithuanian Jewish heritage.

Monika LÖSCHER - Kommission für Provenienzforschung - Kunsthistorisches Museum Wien

Seit 2009 Provenienzforscherin im Auftrag der Kommission für Provenienzforschung im Kunsthistorischen Museum.

Since 2009 Provenance researcher on behalf of the Commission for Provenance Research at the Kunsthistorisches Museum.

Monika MAYER - Kommission für Provenienzforschung - Belvedere Museum Wien: Österreichische Galerie Belvedere

Historikerin, Leiterin des Archivs der Österreichischen Galerie Belvedere in Wien, Mitglied der Kommission für Provenienzforschung. Publikationen zur Museumsgeschichte, Provenienzforschung und zur Kunstpolitik im Austrofaschismus und Nationalsozialismus.

Historian, director of the archive of the Austrian Gallery Belvedere in Vienna, member of the Commission for Provenance Research. Publications about museum history, provenance research and art politics in the period of Austrofascism and National Socialism. 


\section{Christian MERTENS - Wienbibliothek im Rathaus}

Mag., Studium der Geschichte und Politikwissenschaft in Wien, seit 1999 wissenschaftlicher Mitarbeiter der Wienbibliothek (u.a. Mitarbeit am „Wien-Geschichte-Wiki“, Provenienzforschung), Mit- und Alleinkurator mehrerer Ausstellungen, zahlreiche Veröffentlichungen.

Studied History and Political Science in Vienna, since 1999 research assistant at the Vienna City Library (responsible i. a. for "Wien-Geschichte-Wiki", provenance research), curator of several exhibitions, numerous publications.

Philipp METTAUER - Institut für jüdische Geschichte Österreichs, St. Pölten Historiker, wissenschaftlicher Mitarbeiter des Instituts für jüdische Geschichte Österreichs. Projekte und Publikationen zur österreichisch-jüdischen Emigration nach Argentinien, Shoah und Nationalsozialismus im Familiengedächtnis, Psychiatrie und NS-Euthanasie sowie Provenienzforschung an der Österreichischen Nationalbibliothek.

$P h D$, associate researcher at the Institute for Jewish History in Austria. Scientific Projects and Publications on Jewish-Austrian exile 1938-45 in Argentina, National Socialism and Shoah in family memory, National Socialist psychiatry and euthanasia and Provenance research at the Austrian National Library.

\section{Ekaterina OLESHKEVICH - Russian State Library, Moskau}

Studium der Judaistik und Geschichte der Asiatischen und Afrikanischen Länder, Leitende Bibliothekarin und Forscherin der Sammlung Schneerson der Russischen Staatsbibliothek im Jüdischen Museum und Toleranzzentrums.

M.A. in Hebrew and History of Asian and African Countries, chief librarian and researcher in the Schneerson collection (branch of the Russian State Library at Jewish Museum and Tolerance Center).

\section{Johana PROUZOVÁ - Documentation Centre for Property Transfers of Cultural} Assets of WW II Victims, Prag

Studierte an der Karls-Universität Prag, wobei sie schon in dem „Documentation Centre for Property Transfers of Cultural Assets of WW II Victims“ tätig war. Jetzt arbeitet sie im Zentrum als Forscherin.

Studied at Charles University in Prague. Prior to graduation, she worked for the Documentation Centre for Property Transfers of Cultural Assets of WW II Victims and today she continues her work there as a researcher. 
Anneliese SCHALLMEINER - Kommission für Provenienzforschung - Bundesdenkmalamt, Wien

Mitglied der Kommission für Provenienzforschung in Österreich seit 1999. Archivarin des Bundesdenkmalamts in Wien. Publikationen zu jüdischen Sammlern, Provenienzforschung in Österreich und Bergung von Kulturgütern. Member of the Commission for Provenance Research in Austria since 1999. Archivist at the Bundesdenkmalamt in Vienna. Publications on topics of provenance research such as Jewish collectors, Provenance Research in Austria and Storage of cultural assets.

Sebastian M. SPITRA - Institut für Rechts- und Verfassungsgeschichte, Universität Wien

Universitätsassistent und Doktorand am Institut für Rechts- und Verfassungsgeschichte der Universität Wien. Fellow der Vienna Doctoral Academy „Communicating the Law".

Research Fellow and doctoral student at the Department for Legal and Constitutional History of the University of Vienna. Fellow of the Vienna Doctoral Academy "Communicating the Law".

Julia STEPNOWSKA - Faculty of Law and Administration of the University of Gdańsk

Masterstudium für Kunstmanagement (Neoma Business School, Rouen, Frankreich; Thesis: „Restitution von während des Zweiten Weltkrieges in Polen geraubten Kunstwerken“), Doktorandin an der Fakultät für Rechtswissenschaften und Verwaltung der Universität von Gdańsk, Polen (eingereichtes Doktoratsthema: Gebührende Sorgfalt als Mittel bei der erfolgreichen Kunstrestitution). Arbeitet als Kunstberaterin für die Espace Dali and Galerie Montmartre in Paris. Spezialisiert auf Gegenwartsmalerei, Skulpturen und moderne Drucke. Interessiert sich für Art Nouveau.

MSc in Arts Management (Neoma Business School, Rouen, France; thesis: "Restitution of Artworks Looted From Poland during WW II"), PhD Student at the Faculty of Law and Administration at the University of Gdansk, Poland (thesis proposed: "Due Diligence as the Means towards Effective Art Restitution"). Spoke at national conferences on cultural heritage. Worked as an art consultant for Espace Dali and Galerie Montmartre in Paris. Specialised in Contemporary painting, sculpture, and Modern prints. Passionate about Art Nouveau.

\section{Marcela STROUHALOVÁ - Czech National Library Prague}

Studierte zwischen 2007 und 2012 Kulturgeschichte an der Universität von Pardubice. Seit 2012 Doktoratsstudium ebenda. Seit Januar 2015 Historikerin an der Tschechischen Nationalbibliothek. Verantwortlich für die Archivrecherche 
und Dokumentation des Projekts „Books Discovered Once Again“ von Januar 2015 bis April 2017, gefördert von EEA Grants.

Studied between 2007-2012 Cultural History at the University of Pardubice. Since 2012 she has been working on her doctoral thesis at the same university. Since January 2015 she has been working at the National Library of the Czech Republic as a historian. She is responsible for the archival research and specialized outputs of the project Books Discovered Once Again which was carried out from January 2015 to April 2017 and funded by EEA Grants.

\section{Markus STUMPF - Universitätsbibliothek Wien, Universität Wien}

Seit 2000 in diversen Funktionen an der Universitätsbibliothek Wien, Leiter der Fachbereichsbibliothek Zeitgeschichte und Leiter der NS-Provenienzforschung an der Universität Wien. Mitherausgeber der Reihe Bibliothek im Kontext. Zahlreiche Veröffentlichungen, u. a.: Guido Adlers Erbe. Restitution und Erinnerung an der Universität Wien. Hrsg. von Markus Stumpf, Herbert Posch u. Oliver Rathkolb. Göttingen: Vienna University Press | V \& R 2017 (= Bibliothek im Kontext 1), Open Access: http://www.v-r.de/_uploads_media/files/978384710 7217_stumpf_adler_wz_120822.pdf und Stefan Alker, Bruno Bauer, Markus Stumpf: NS-Provenienzforschung und Restitution an Bibliotheken. BerlinBoston: de Gruyter Saur 2017 (Praxiswissen).

Academic Librarian, Vienna University, Contemporary History Library, and head of NS Provenance Research at Vienna University Library. Co-editor of the series Bibliothek im Kontext (BiK). Numerous publications, e.g. NS-Provenienzforschung und Restitution an Bibliotheken. Berlin/Boston: de Gruyter Saur (coauthor 2017).

\section{Leonhard WEIDINGER - Kommission für Provenienzforschung - Österrei- chisches Museum für angewandte Kunst (MAK), Wien}

Historiker; tätig für die Kommission für Provenienzforschung (Recherchen im MAK, Online-Projekte etc.), das Getty Research Institute und das Zentralinstitut für Kunstgeschichte; Vorsitzender des Arbeitskreises Provenienzforschung e.V. Historian; working for the Commission for Provenance Research (research at the $M A K$, online projects etc.), for the Getty Research Institute and the Zentralinstitut für Kunstgeschichte; head of the board of Arbeitskreis Provenienzforschung e.V.

\section{Michael WLADIKA - Wien Museum}

MMag. Dr., geboren 1961 in Wien; Jurist und Historiker; seit Juli 1999 als Provenienzforscher für die Museen der Stadt Wien tätig; unter anderem 1999-2003 wissenschaftlicher Mitarbeiter der Historikerkommission der Republik Österreich; mehrere Projekte zum Thema (Ursprünge des) Nationalsozialismus, NSKunstraub und Rückstellungsrecht; seit Mai 2008 von der Republik Österreich 
und der Leopold Museum Privatstiftung bestellter Provenienzforscher für die Leopold Museum Privatstiftung.

MMag. Dr., born 1961 in Vienna; jurist and historian; since July 1999 provenance researcher for the museums of the City of Vienna; 1999-2003 research fellow of the Historical Commission of the Republic of Austria; several projects on the subject (origins of) National Socialism, Nazi art looting and restitution legislation; appointed im May 2008 as a provenance researcher for the Leopold Museum private foundation by the Republic of Austria and the Leopold Museum private foundation.

\section{Kamil ZEIDLER - Faculty of Law and Administration of the University of Gdańsk}

Professor für Rechtwissenschaften am Institut für Theorie und Philosophie von Staat und Recht, Fakultät für Rechtswissenschaften und Verwaltung der Universität von Gdańsk, Polen. Autor von mehr als 400 Publikationen zum Rechtsschutz von kulturellem Erbe, der Theorie und Philosophie von Recht, Internationalem Recht und Europäischem Recht. Vom polnischen Kulturministerium wurde er zweifach zur Erforschung der Rechtsfragen bezüglich kulturellem Erbes beauftragt $(2003,2007)$; u. a. DAAD Stipendium an der Friedrich Wilhelms Universität in Bonn (1997). Mitglied folgender internationaler wissenschaftlicher Vereinigungen: Internationale Vereinigung für Rechts und Sozialphilosophie (IVR), Polish Branch of International Law Association (ILA), Polnish National Committee of International Council on Monuments and Sites (ICOMOS), Polish National Committee of International Council of Museums (ICOM); Direktor des Zentrums für Ostasienstudien der Universität Gdansk. Professor of Law at the Department of Theory and Philosophy of State and Law, Faculty of Law and Administration, University of Gdansk (Poland). Author of more than 400 publications on legal protection of cultural heritage, theory and philosophy of law, international law and European law Gained scholarships from Ministry of Culture of the Polish Republic for cultural heritage legal research twice (2003, 2007); DAAD scholarship at Friedrich Wilhelms University in Bonn (1997). Member of international scientific associations: Internationale Vereinigung für Rechts- und Sozialphilosophie (IVR), Polish Branch of International Law Association (ILA), Polish National Committee of International Council on Monuments and Sites (ICOMOS), Polish National Committee of International Council of Museums (ICOM); director of the Centre for East Asia Studies at the University of Gdansk. 
Open-Access-Publikation im Sinne der CC-Lizenz BY-NC-ND 4.0

(c) 2018, V\&R unipress GmbH, Göttingen

ISBN Print: 9783847107835 - ISBN E-Lib: 9783737007832 


\section{SCHRIFTENREIHE DER KOMMISSION FÜR PROVENIENZFORSCHUNG}

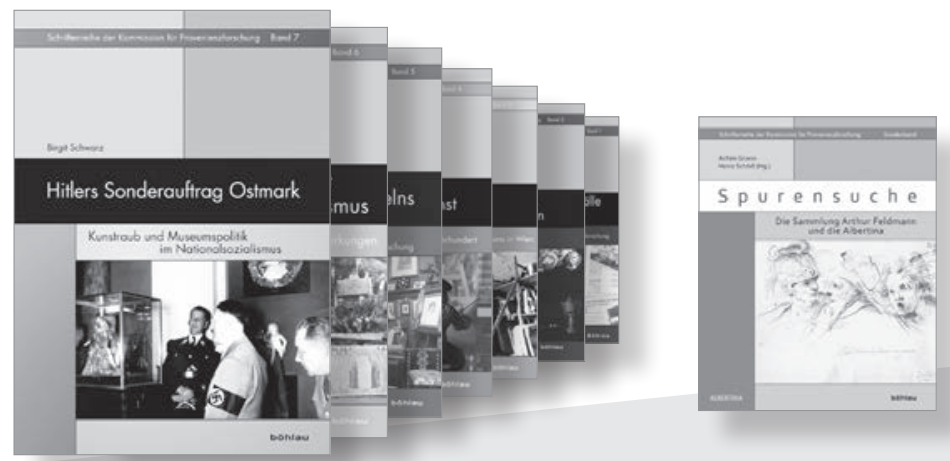

Die Diskussion um Arisierung und Rückstellung wurde in Österreich unter anderem durch die Beschlagnahme zweier Bilder von Egon Schiele aus der Stiftung Leopold in New York ausgelöst. Aus diesem Anlass wurde im März 1998 die Kommission für Provenienzforschung eingerichtet und im Dezember desselben Jahres das Kunstrückgabegesetz beschlossen. Die Kommission erforscht systematisch die Provenienzen in den Sammlungen des Bundes und legt dem Kunstrückgabebeirat Dossiers zur Entscheidung für oder gegen eine Rückgabe an die ehemaligen EigentümerInnen vor. Die Schriftenreihe wurde im Jahr 2009 begründet, um darüber hinaus gehende Forschungsergebnisse einer interessierten Öffentlichkeit zugänglich zu machen.

\section{Band 7}

Birgit Schwarz

\section{Hitlers Sonderauftrag Ostmark}

Kunstraub und Museumspolitik im

Nationalsozialismus

2018. 236 S. mit 57 s/w- u. farb. Abb. gebunden mit SU.

$€ 35,-D \mid € 36,-A$

ISBN 978-3-205-20621-7

\section{Band 8}

erscheint November 2018

Eva Blimlinger, Heinz Schödl (Hg.)

\section{... (k)ein Ende in Sicht}

20 Jahre Kunstrückgabegesetz in

Österreich

2018. Ca. 400 S., gebunden mit SU.

ca. $€ 35,-\mathrm{D} \mid$ ca. $€ 36,-$ A

ISBN 978-3-205-20808-2

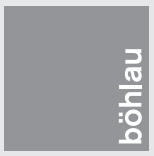

Vandenhoeck \& Ruprecht Verlage

www.vandenhoeck-ruprecht-verlage.com 
